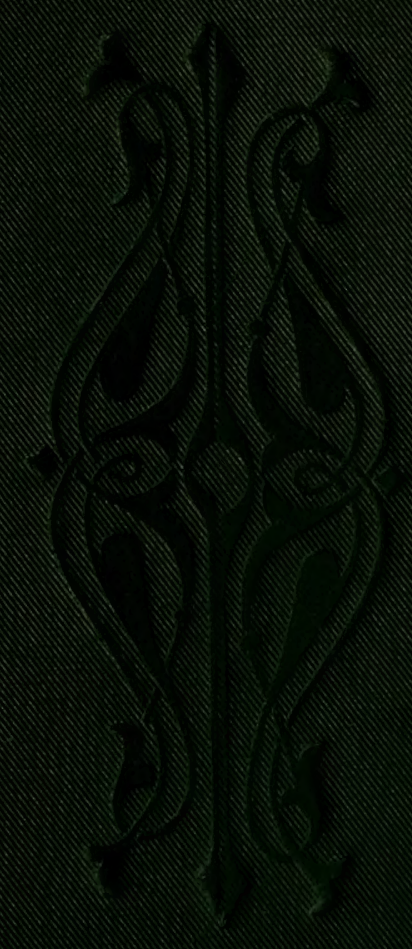




$$
\%
$$






\title{
POULTRY DISEASES
}

Including Diseases of Other Domesticated Birds

\author{
With a Chapter on the \\ Anatomy of the Fowl
}

\author{
by \\ B. F. Kaupp, B.S., M.S., D.V.M.
}

Pathologist and Poultry Investigator, Animal Industry Division, North Carolina Experiment Station and Agricultural College. Formerly Director of Anatomy Laboratory Kansas City Veterinary College. Formerly Pathologist for the Colorado Experiment Station and Agricultural College. Formerly Veterinary Inspector B. A. I., etc.

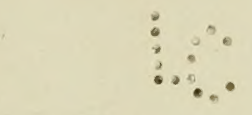

\section{THIRD EDITION}

Revised and Enlarged

\author{
CHICAGO
}

ALEXANDER EGER 


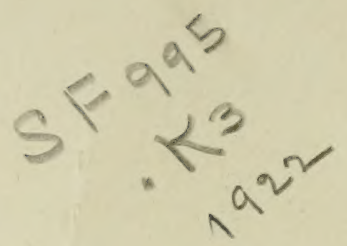

Copyright,

1922,

by

Alexander Eger

$\because \vdots$
$\therefore$

SEP 151922

(C) CI.A681862 


\section{FOREWORD TO THIRD EDITION}

This book was written to fill a need from veterinary students, students in poultry husbandry courses at agricultural colleges, veterinary practitioners and others interested in the scientific treatment of poultry diseases.

An effort has been made to make the language so plain that all can comprehend the subject matter, which is a summary of the results of experimental research in the laboratory of pathology of the author and of many other investigators.

For the purpose of simplification, the synonyms are given for the various names of diseases. Then follow, in order, the cause, the symptoms, the conditions found upon post-mortem examination, and lastly the treatment and means of eradication for each disease.

The first and second editions being exhausted in so short a time is evidence that it has met the expectations of persons interested in the scientific treatment of poultry - that it fills a real need. The third edition has been thoroughly revised and brought down to date. Much new information has accumulated as a result of continued and persistent efforts of investigators in the field of poultry pathology.

State College Station, Raleigh, N. C. B. F. KAUPP.

September, 1922. 



\title{
CONTENTS
}

List of Illustrations

\section{Chapter I}

\begin{abstract}
Anatomy
The skeletal anatomy of the fowl; head and neck, trunk and limbs; visceral anatomy; digestive tract, genito-urinary tract, organs of respiration, organs of circulation; ductless glands and accessory glands of digestion; blood of a normal fowl; the lymph vascular system; nervous system; organs of special sense; reproductive organs of the hen.
\end{abstract}

\section{Chapter II}

Sanitation

Site for the poultry plant; buildings and runs; immunity or natural resistance; health of the baby chick as influenced by incubation; effect of atmospheric surroundings; water supply: disinfection of buildings; disinfection of yards; disposal of sick and dead birds; mode of performing autopsy.

\section{Chapter III}

\section{External Parasites}

Definition; lice of birds; how and where lice multiply; effects of lice upon the fowls; dealing with louse infestation; scabies of fowls; air sac disease; red mite infestation of the hen house; fleas affecting birds; tick infestation; the bedbug of poultry; bee stings; fungi affecting fowls; white scale of the face, wattles and comb; mycosis of pigeons; baldness of fowls and canaries; blastomycosis of the goose.

\section{Chapter IV}

\section{Internal Parasites}

Definition; important round worms; the gap worm; unimportant round worms; tapeworms; thorn headed worm; flukes; worms of canaries; diseases due to protozoa; coccidiosis; coccidian white diarrhea; coccidiosis of adult fowl; coccidiosis of wild duck; protozoal hepatitis of pigeon; sarcocystis; leucocytozoon of duck; trypanosomiasis of birds; malaria of fow1; enterohepatitis or blackhead. 


\section{Chapter V}

Diseases of the Digestive Tract of Fowls .

Obstruction of the beak; parched tongue or pip; stomatitis; croupous inflammation of the pigeon; impaction of the crop; impaction of the intestines; tympany of the crop; enlarged crop; gangrene of the crop; catarrh of the crop; depraved appetite; indigestion; distension of the bursa of fabricus; clocaitis; peritonitis; ascites; nephritis; suppurative inflammation of the gizzard; calculi; eversion of the cloaca and oviduct; volvulus or gut tie in a fowl; intussusception of the intestine of the fowl; intestinal diseases of canaries; constipation; diarrhea diseases of fowls.

\section{Chapter VI}

Diseases Due to Bacteria .

Infectious blood diseases; fowl cholera; fowl typhoid; myeloid leukemia; bacillary white diarrhea; adult fowl infection; apoplectiform septicaemia of fowls and pigeons; sleeping disease of fowls; epiornithologic pneumo-pericarditis in turkeys; infectious enteritis of pheasants; hemorrhagis septicaemia of the dove; cholera of swans; septicaemia of parrots; septicaemia of pigeons; grouse and quail diseases; fowl pest; septicaemias of canary birds; inflammation of the joints of geese and ducks; spirochetosis; infectious constitutional diseases; tuberculosis; pseudo tuberculosis; aspergillosis.

\section{Chapter VII}

\section{Constitutional Diseases}

Asthene; gout of fowls.

\section{Chapter VIII}

\section{Diseases of the Respiratory Passages}

Obstruction of the trachea; catarrh; asthma; bronchitis; contagious bronchitis; congestion of the lungs; pneumoniainflammation of the lungs of fowls; contagious nasal catarrh of fowls; contagious inflammation of the air sacs of geese; roup, avian diphtheria; chicken pox; contagious epithelioma; diphtheric sore eye; respiratory diseases of canaries; asthma of canaries.

\section{Chapter IX}

\section{Diseases of the Liver}

Fatty degeneration; fatty infiltration; rupture of the liver; congestion of the liver; inflammation of the liver; inflammation of the bile ducts and gall bladder; enterohepatitis; other diseases of the liver. 


\section{Chapter X}

\section{Diseases of the Ovary and the Oviduct}

Prolapse or eversion of the oviduct; obstruction of the oviduct; rupture of the oviduct; broken eggs in oviduct; abnormal eggs; obstruction of the oviduct due to ulceration of the anus; dwarf eggs; epiornithologic abortion in birds.

\section{Chapter XI}

\section{General Diseases}

Purulent inflammation of the abdomen; purtulent inflammation of the air sacs; contagious inflammation of the air sacs of geese; abscess of the foot; dry gangrene of the feet of chicks; pericarditis; endocarditis ; myocarditis; hypertrophy; productive inflammation of the soft structures of the shank, comb and wattles; rupture of the heart and large blood vessels; thrombosis; gangrene; inflammation of the oil gland; swell head of young turkeys; pox of turkeys; diphtheric inflammation of the eyes of ducks; ulceration of the cornea; frozen comb; broken beaks; arthritis terminating in osteitis of the femorotibial articulation of the cock; open joint in fowl.

\section{Chapter XII}

\section{Tumors}

Definition; classification of tumors; adult epithelial tumors; adenoma; papilloma ; carcinoma ; epithelioma ; lipoma ; chondroma; osteoma; fibroma; myoma ; angioma; lymphoma ; myxoma; hematoma ; sarcoma ; compound tumors; cyst-lipoosteo-chondro-adeno-carcinomata; lympho-sarcoma; myxosarcoma ; sarco-chondro-osteomata ; teratoma; horny growths ; cysts; cystoma; caseous abdominal tumors or cysts.

\section{Chapter XIII}

\section{Diseases of the Brain and Nerves of the Fowl.}

Leg weakness; paralysis of the domestic fowl; rheumatism; paralysis of the wings of pigeons; dizziness, vertigo; hemorrhage of the brain; epilepsy; myelitis; polyneuritis; paralysis of the auditory nerve; paralysis of the cocklear nerve; paralysis of the vestibular nerve.

\section{Chapter XIV}

Bacteria of the Intestinal Tract of the Fow1 . . . 285

\section{Chapter XV}

Composition of the Egg

Composition of the egg shell; animal parasites in eggs; bacteria of eggs; bacteria in fresh eggs. 


\section{CiIAPTER XVI}

Malformations

\section{Chapter XVII}

Fractures-Wounds-Anaesthesia

Fractures; repair of the fracture of bone in the domestic fowl; the structure of bone; reparative process of bone; the treatment of fracture and care of the fowl; wounds; anaesthesia and restraint of the fowl; injury to the sternum or breast bone; curvature of the spine.

\section{Chapter XVIII}

Castration or Caponizing .

Ovariectomy of the pullet.

\section{Chapter XIX}

Foods Poisonous to Fowls

The rose chafer; arsenical poisoning; salt poisoning; other mineral poisoning; ptomaine poisoning; limber neck or botulism; corn cockle poisoning; ergot poisoning.

\section{Chapter XX}

Poultry Remedies

Temperature, respiration, pulse and blood pressure of the domestic fowl; poultry materia medica; list of dosage of drugs for adult fowls. 


\section{LIST OF ILLUSTRATIONS}

Fig

Page

1. The Bony Skeleton of the Domestic Fowl............... 4

2. The Visceral Organs of the Hen................... 6

3. Visceral Organs of the Right Side of the Fowl.......... 23

4. Visceral Organs of the Left Side of the Fow1............. 24

5. Section Through the Median Line of a Two Pound Pullet.. 25

6. Transverse Section Through the Thoracic Region.......... 27

7. Heart of Fowl With Its Main Branches................ 33

8. Blood Vessels of the Pelvis and Thigh of a Fowl........... 35

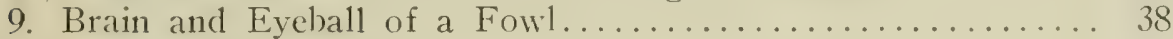

10. Section Through the Eye of a Hen.................. 41

11. Cochlear Labrynth of a Hen..................... 41

12. The Pelvic Organs of a Pullet................... +4

13. The Functionating Pelvic Organs of a Hen............... 45

14. Photomicrograph of a Section of the Ovary of a Hen....... 46

15. Diagramatic Structure of the Egg.................. 48

16. Gencrative Organs of a Cockerel................. 51

17. Reproductive Organs of a Cock Showing Functioning Testes 52

18. Photomicrograph of Spermatozoa from a Single Comb White

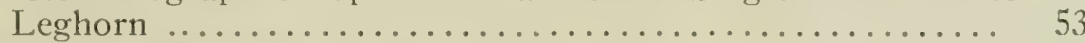

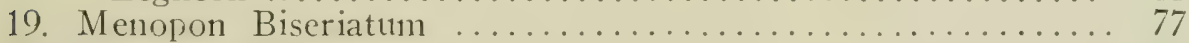

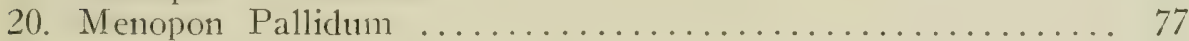

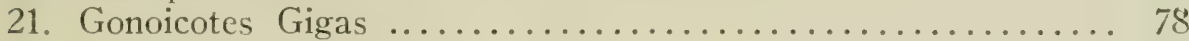

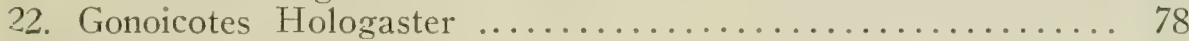

23. Goniodes Stylifer ............................ 79

24. Lipeurus Baculus ............................ 79

25. Eggs or Nits of the Goniodes Stylifer............... \&0

26. Lipeurus Infuscatus .......................... \&

27. Seven Weeks Old Single Comb White Leghorn Cockerel Infested by Head and Neck Lice.................. 81

28. Lipeurus Variabilis, Depluming Louse................... 82

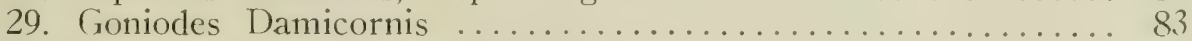

30. An Inexpensive Spray Pump..................... 86

31. Sarcoptes Mutans Variety Gallinae.................. 88

32. Scaly Legs or Scalifes.............................. 89

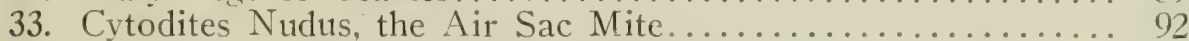

34. Trombidium Holosericeum or Chicken Mite............. 03

35. Dermanyssus Gallinae ........................ 98

36. Pulex Avium ............................. 99

37. Sarcopsylla Gallinaceate ......................... 100

38. Argas Miniatus ................................. 102

39. Acanthia Inodora ............................. 104

40. Ascaris Inflexa ............................ 110

41. Heterakis Papillosa .......................... 110

42. Heterakis Papillosa ......................... 110

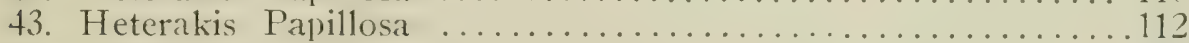

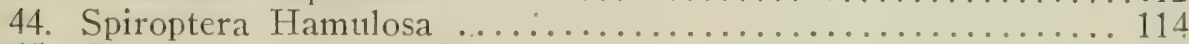

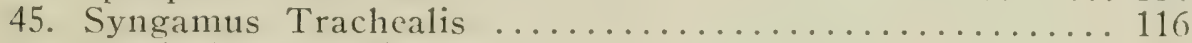

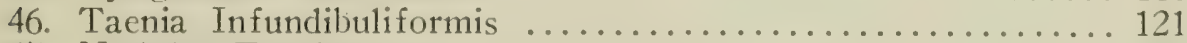

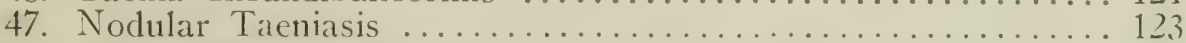

48. Section Through Caecum of Chick Dead of Coccidiosis........ 129 
Fig.

49. Showing Various Stages of the Development of the Coccidiae 129

50. Pulmonary Coccidiosis of a Wild Duck................. 131

51. Intestinal Coccidiosis of a Wild Duck................. 131

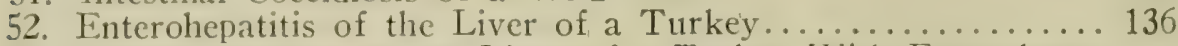

53. Cloudy Swelling of the Liver of a Turkey With Enterohepa-

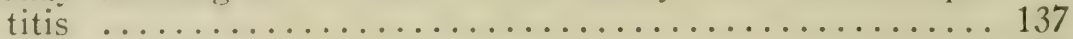

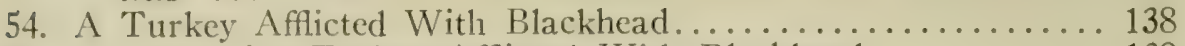

55. Caecum of a Turkey Afflicted With Blackhead...................

56. Photomicrograph of a Section of the Liver of a Turkey With

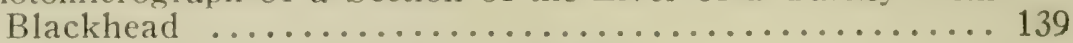

57. Blood Smear from a Case of Blackhead................. 140

58. Section of a Kidney of a Case of Blackhead.............. 141

59. Photomicrograph of a Liver Showing Ameba Meleagridis.... 141

60. Croupus Inflammation of the Throat of a Pigeon........... 148

61. Pendulous Crop in a Barred Plymouth Rock Hen............ 152

62. Suppurative Inflammation of the Gizzard of a Fowl......... 158

63. Eversion of the Cloaca and Oriduct of a Hen .............. 1.59

64. Volvulus or Gut Tie in a Fowl.................... 160

65. Invagination of the Bowel of a Fowl................. 161

66. Hemorrhagic Enteritis of a Hen.................. 163

67. Blood Smear from a Case of Fowl Cholera............... 165

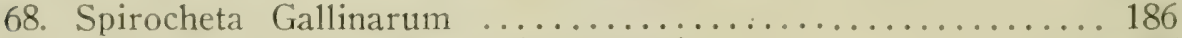

69. A Fowl With Acute Spirochetosis.................. 186

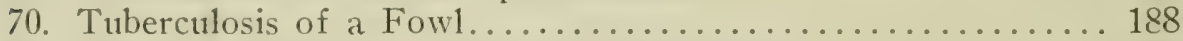

71. Gout of the Feet of a Barred Plymouth Rock Capon......... 198

72. Obstruction of the Trachea of a Chick................. 201

73. Obstruction of the Larynx of a Hen.................. 202

74. Ulcerative Pharyngitis of a Fowl................... 204

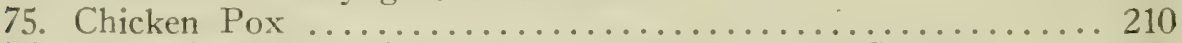

76. Contagious Epithelioma of the Heads of Two Chicks....... 211

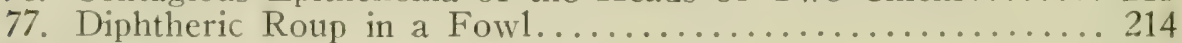

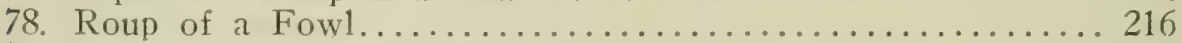

79. Obstruction of the Oviduct Due to Constriction......... 226

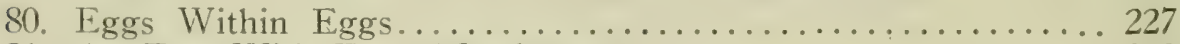

81. An Egg With Four Membranes.................... 228

82. Three Dwarf Eggs and Their Causes.................... 229

83. Photomicrograph Showing Pus Cells and Chains of Strep-

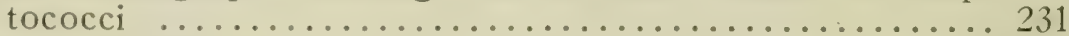

84. Purulent Inflammation of the Abdominal Air Sac.......... 232

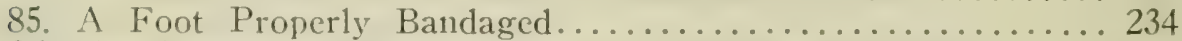

86. An Abscess of the Foot Due to a Russian Thistle....... 235

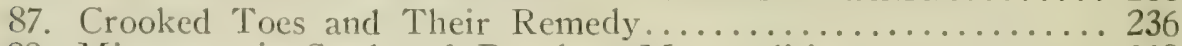

88. Miscroscopic Study of Purulent Myocarditis.............. 238

89. Hypertrophy of the Cecum of a Cock.................. 240

90. Productive Inflammation of the Shanks of a Cock........... 240

91. Thrombosis of the Blood Vessels of a Hen............... 241

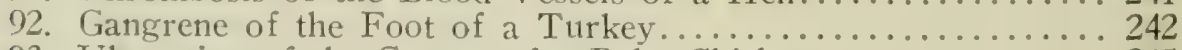

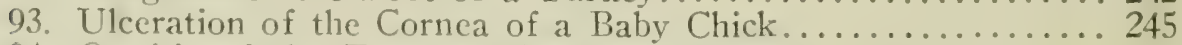

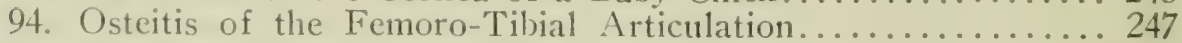

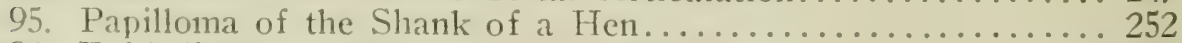

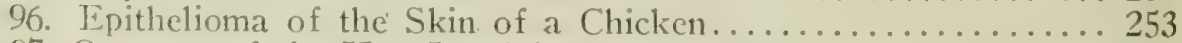

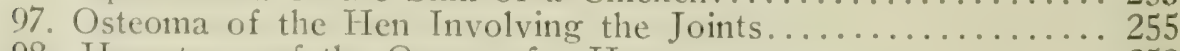

98. Hematoma of the Ovary of a Hen.................. 258

99. Spindle Celled Sarcoma of the Skin of a Hen........... 259

100. Cysto-Lipo-Chondro-Osteo-Adeno-Carcinoma of a Cock.. 260

101. Lympho-Sarcoma of a Single Comb White Leghorn Hen..... 261 
Fig.

102. Lympho-Sarcoma of a Single Comb Rhode Island Red Hen.. 262

103. Myxo-Sarcoma ................................ 263

104. Photomicrograph of a Myxo-Sarcoma of a Hen........... 264

105. Sarco-Chondro-Osteomata of the Thigh of a Hen....... 265

106. A Horny Growth from the Thigh of a Fowl............... 266

107. Multiple Tumors or Cysts of the Ovary of a Hen........... 268

108. A Single Comb Rhode Island Red Hen With an Abdom-

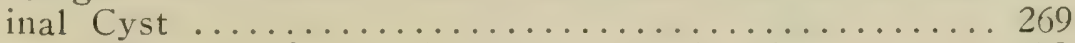

109. Section Through Mass of Arrested Formative Eggs......... 270

110. Paralysis of a Single Comb Rhode Island Red Hen......... 275

111. Apoplexy or Hemorrhage of the Brain of a Mottled Houdan. . 278

112. Paralysis of the Auditory Nerve of a Silver Campine Hen.... 282

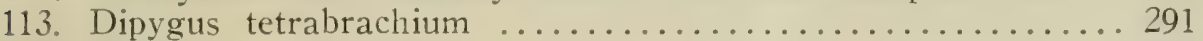

114. Polymelus .................................. 292

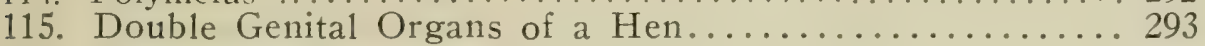

116. Holoacardius Acephalus ......................... 294

117. Fowl Supernumerary Hind Limbs................... 296

118. Double Head and Neck......................... 297

119. Duck With Two Necks............................ 297

120. Supernumerary Hind Limbs........................ 298

121. Dipygus, the Supernumerary Hind Limbs Are Ventrally At-

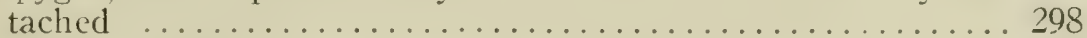

122. Two Bodies With Legs, Wings and Anus................. 299

123. One Leg Only................................. 300

124. Umbilical Hernia of a Baby Chick.................. 301

125. Sections Through Fractured Bones.................. 304

126. Photomicrograph Showing Repair of Bone............... 305

127. Roentgenograph of Fractured Bones.................. 306

128. Broken Leg of Rhode Island Red Pullet Properly Set.... 307

129. A Convenient Poultry Operating Table................. 310

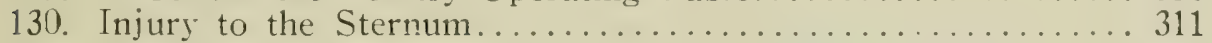

131. Curvature of the Spine of a Young Developing Chick....... 312

132. Top of a Barrel Used as an Operating Table............. 315

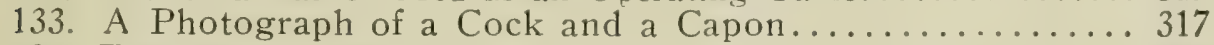

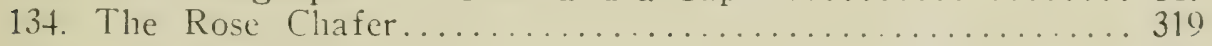




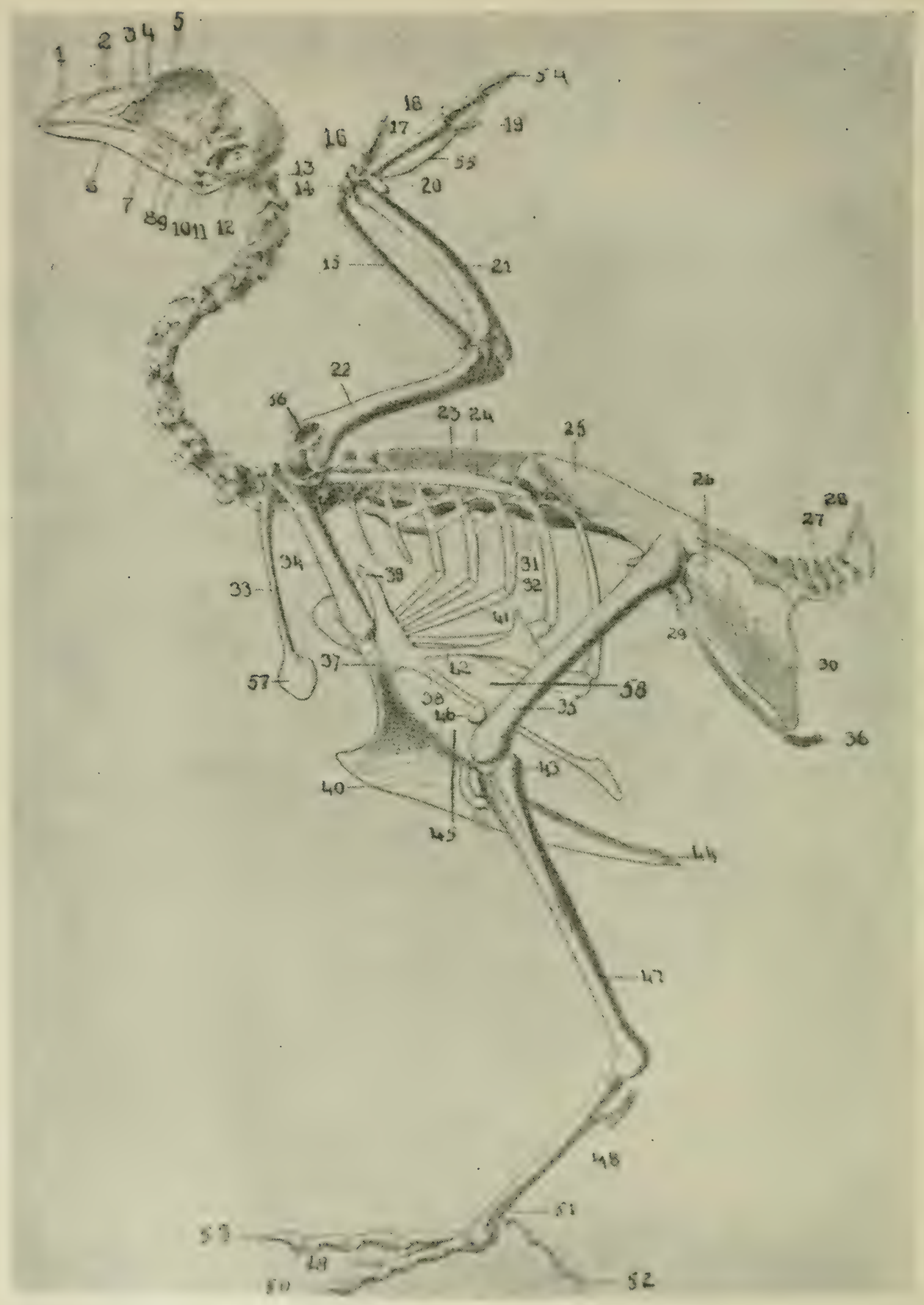

Fig. 1. Tine hony Skeleton of the Domestic Fowl. 


\section{EXPLANATION OF FIG. 1}

\section{Head and Neck}

1, os incisivum or premaxilla; 2, anterior nasal opening; 3 , os nasale or nasal bone; 4, os lacrimale or lacrimal bone; 5, lamina perpendicularis of the ethmoid bone or perpendicular plate between the eye orbits; 6 , os maxillare or inferior mandible or the inferior jaw; 7 , os palatinea or the bone supporting the hard palate; 8 , os quadratojugal; 9, os pterygoideum or pterygoid bone; 10 , os quadratum or quadrate bone; 11 , os articulare, the articular portion of the inferior jaw; 12 , external auditory canal; 13 , atlas or the first cervical vertebra.

\section{Trunks and Limbs}

14, os carpi radiale also called the scapho-lunar and located between the radius and metacarpus and constituting one of the two wrist or carpal bones; 15, the radius, which in the chicken, is smaller than the ulna; 16, the first digit or thumb also called the fal'se or bastard wing; 17, metacarpus, two in number, fused at either end; 18 , the first phalanx of the second finger; 19 , the third, small rudimentary digit or finger; 20, os carpi ulnare, also called the cuneiform, is anvillike in shape and is located between the ulna, radius and metacarpus; 21 , os ulnae or ulnar bone, the largest bone of the forearm; 22, os humeri or humerus constituting the arm bone; 23 , the dorsal or thoracic vertebrae; 24 , os scapulare or scapula; 25 , os ilii or ilium, the long pelvic bone; 26 , foramen ischiadicum through which the ischiadic nerve passes; 27 , coccygeal or tail vertebrae; 28, pygostyle or plough share, the last bone of the tail; 29 , foramen obturatorum through which the tendon of the internal obturator muscle passes; 30, os ischii or ischium, one of the pelvic bones; 31, uncinate or hook-shaped process of the ribs, this gives greater surface for attachment of muscles; 32 , vertebral portion of the rib, ribs in birds are divided into a vertebral and a sternal portion; 33, os clavicula or clavicle which come together at the bottom, forming the hypocledium, this represents the collar bone; 34 , os coracoideum or coracoid bone; 35 , os femori or femur, the thigh bone; 36, the pubis or lay bones; 37, the sternum or breast bone which is expanded into the keel shown in figure 40 , also provided with lateral sternal processes as shown at figure 38, and costal processes shown at figure $39 ; 41$, sternal or inferior portion of the rib attaching to the breast bone or sternum; 42, the lateral external process of the sternum; 43 , the fibula which in the chicken articulates with the femur and tibia; 44 , the posterior process or xiphoid portion of the sternum; 45, the internal notch of the sternum; 46, the patella often called the knee cap; 47, the tibia or drum stick; 48 , the metatarsus or shank; 49,50 , and 53 , the principal digits; 51 , the rudimentary digit or toe; 52 , the toe nail; 58 , the external notch of the sternum.. 


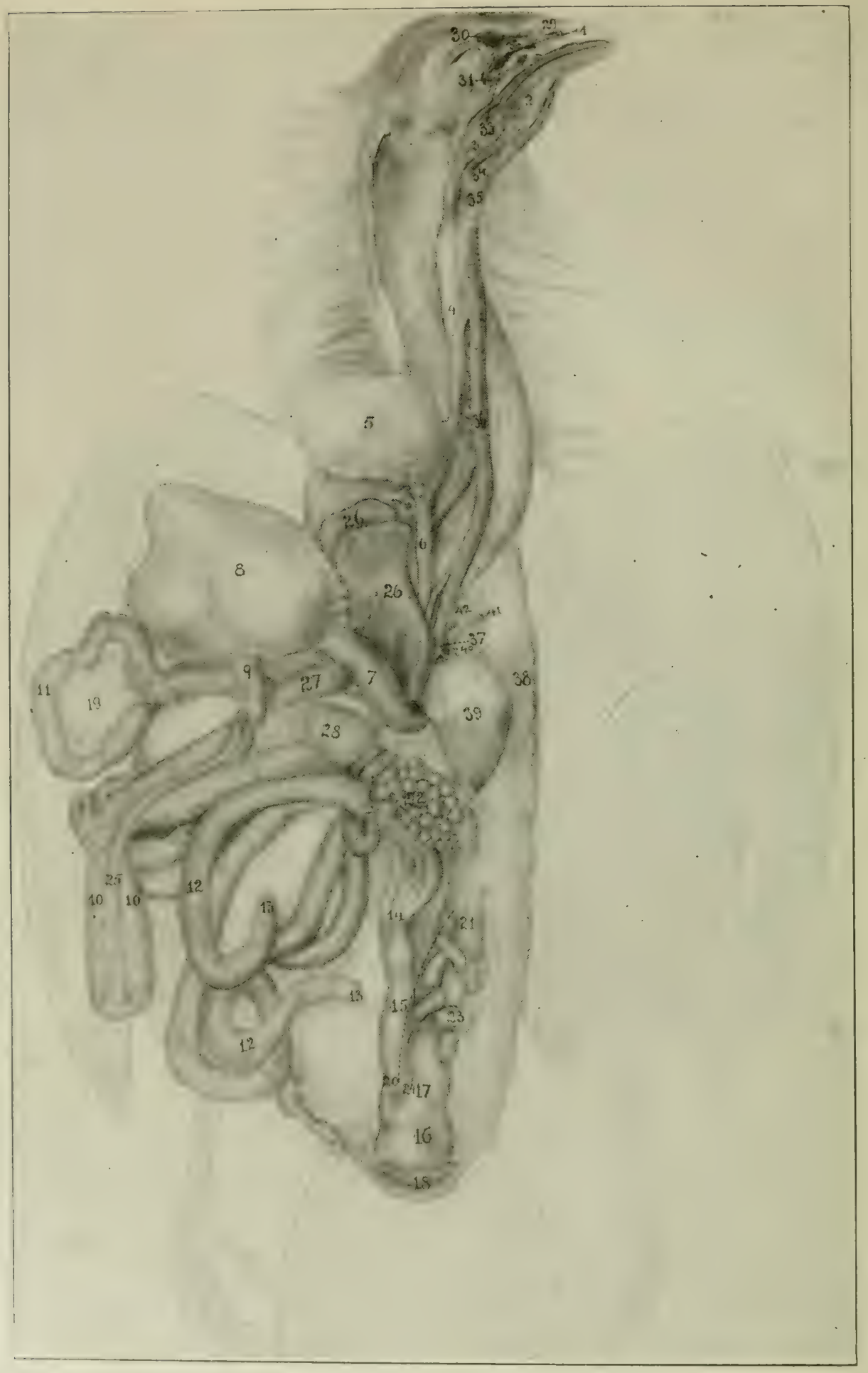

fiig. 2. The Visceral Organs of the Hen. 


\section{EXPLANATION OF FIG. 2}

\section{Digestive Tract}

1 , the beak; 2 , the tongue; 3 , the pharynx or throat; 4 , the first portion of the esophagus or gullet; 5 , the crop or storehouse for food; 6 , the second portion of the esophagus; 7 , the proventriculus or glandular stomach; 8 , the gizzard or muscular stomach; 9 , the duodenum or first portion of the small intestine; 10 , the duodenal loop, between which lies the pancreas; 11 , the floating portion of the small intestine; 12 , the caeca or blind pouches; 13 , the blind extremities of the caeca; 14 , the juncture of the small intestine, caeca, and large intestine; 15, the large intestine or rectum; 16, the cloaca or cesspool; 18, the anus; 19 , the mesentary, a web-like structure which supports the free portion of the small intestine; 25 , the pancreas which is located between the limbs of the duodenal loop; 26 , the liver; 27 , the gall bladder; 28 , the spleen.

\section{Genito-urinary Tract}

17 , opening of the oviduct into the cloaca; 22 , the left ovary; 23 , the egg canal; 20, opening of the ureter or canal from the kidney in to the cloaca.

\section{Organs of Respiration}

29 , the nostrils; 30 , the frontal sinus; 31 , the infraorbital sinus; 32 , the turbinated bones; 33 , the posterior nares or posterior opening of the nasal passage; 34 , the glottis or opening into the larynx; 35 , the superior larynx; 36 , the trachea or wind pipe; 37 , the true or inferior larynx; 38, the left lung.

\section{Organs of Circulation}

39, the heart pulled down to bring into view the inferior larynx; 40 , the aorta; 41 , the left brachial artery; 42 , the carotid artery. 



\section{ANATOMY OF THE FOWL*}

\section{THE PECULIARITY OF THE BONES OF FOWLS}

In the young bird the bones, for the most part, contain cavities filled with red marrow. In the adult bird these cavities are largely filled with air. The air passes from the respiratory organs into these cavities. This air is gradually renewed in the process of respiration. In the running bird most of the bones contain marrow. Separate bronchial tubes of the lungs are continued by air sacs and these through extensions communicate with the air spaces of the bones. The air spaces are more abundant in the larger bones as the humerus, ulna and radius.

The air goes into the air passages and bony cavities of the head, then through the superior larynx, trachea, and inferior larynx into the bronchi. From air tubules here, the air passes into the air cells of the body cavities and then into the air cells of the bones. In the process of breathing the air is drawn out of the air sacs located in the body and bone cavities. Fresh air in turn is forced through the small communicating tubules which enter the hollow bones through small openings in their bony walls.

\section{DIVISIONS OF THE SKELETON}

For study of the skeleton it may be divided into three regions: head and neck, trunk, and limbs.

\section{THE HEAD AND NECK}

\section{(The numbers refer to Fig. 1)}

The bird's head is rather small and conical in shape in front and is composed of several bones as shown in Fig. 1. The os incisivum or premaxilla (1) forms the anterior part of the upper jaw. It is elongated anteriorly into a v-shaped structure in the chicken, turkey, guinea, and pigeon and is flattened in the water fowl as the goose and duck. This portion constitutes the beak. This bone, together with the inferior maxilla (6) forms the prehensile organ or means of picking both solicls and liquids. This organ contains the anterior nasal opening (2) through which air passes on its way to the lungs. The lamina perpendicularis of the ethmoid bone (5) forms a thin wall between the two orbits or eye cavities. It contains an opening

*For a detail study the student is referred to Anatomy of the Domestic Fowl by B. F. Kaupp and published by W. B. Saunders Co,, Philadelphia, Pa. 
or foramen for the passage of the optic nerve and a smaller opening. the ethmoid foramen, for the passage of the ethmoid nerve. The os palatinum or palatine bone encircles the guttural opening of the nasal passage and forms the greater part of the hard palate or roof of the mouth. It articulates with the pterygoid, superior maxilla and premaxilla. The os pterygoideum or pterygoid bone (9), articulates with the sphenoid and os quadratum (10). The os quadratum articulates with the temporal, maxilla, zygomatic, and pterygoid. The os articulare (11) is interposed between the maxilla and quadrate bone. The atlas (13) or first cervical vertebra articulates anteriorly with the condyle of the occipital bone and posteriorly with the axis.

There are fourteen cervical vertebrae in the fowl, fifteen in the duck, eighteen in the goose and twelve in the pigeon.

The long column of cervical or neck vertebrae serves as a sort of balancing pole, and by changing in form and direction it varies the center of gravity. In flying, the bird changes the center of gravity from the region over the legs to the region of the wings. When at rest the head and neck are thrown backwards, carrying the center of gravity back over the legs. The first cervical vertebra is the atlas and is the smallest of the vertebrae. It is ring-shaped and articulates anteriorly with the single condyle of the occipital bone. This anterior articular surface is half-moon shaped and forms a (ceep articular joint called a ba!l and socket joint. This type of joint makes possible, movements in all directions. The condyle of the occiput also touches an articular end of the odontoid process of the axis or second cervical vertebra. Posteriorly there are found two small lateral wings possessing articular facets which articulate with similar facets on the lateral wings of the axis. The posterior part of the body is provicled with an articular facet which articulates with a similar articular surface on the anterior portion of the body of the axis.

The body of each succeeding vertebra articulates with the one immediately anterior and posterior to it. Between each two is a pard of fibro-cartilage. Above these bodies and below the neural spines. We find a large neural canal which is occupied by the spinal cord. Between each two vertebral segments in the cervical region, the neural canal is exposed, due to the meural arches incompletely bridging the canal in that lucation. This spatce is protected or bridged over by an intervertebral ligament.

The dorsal spines of the cervical vertebrae are very small, 
the ventral are more distinct. The anterior oblique spines are provided on their under surface with articular facets, which articulate with similar articular facets on the upper side of the posterior oblique process of the vertebral segment in front. The ventral spine on the last cervical segment is well developed. The transverse processes on either side of the cervical vertebra are penetrated by a vertebral foramen. Through each of this series of foramina passes a vetrebral artery, vertebral vein, and a spinal nerve. The transverse processes of the last cervical vertebra are provided with ridges and excavations for muscular and tendinous attachments.

\section{THE TRUNK}

(The numbers refer to Fig. 1.)

The dorsal or thoracic vertebrae form the roof of the chest cavity (23) number seven in the fowl and pigeon, and nine in the duck and goose. These bones are usually fused or consolidated, giving great support and stability to the wings. The dorsal vertebral region is short. The first and sixth vertebrae articulate similar to the cervical vertebrae, that is, by the bodies and transverse processes of the dorsal vertebrae from the second to the sixth; are well developed and bridged over with a thin wing of bone. The ventral spines are well developed and are partly fused to form a continuous ridge.

In the bird, the lumbar and sacral vertebrae are consolidated. In the embryo there are fourteen distinct vertebrae which soon consolidate with each other and with the ribs. With these vertebral segments are fused the last dorsal and first coccygeal vertebral segments. This fusion is so complete that the segments are indicated only by the intervertebral foramina through which the spinal nerves extend. This fused lumbosacral region forms the roof of the pelvic region. The lumbosacral vertebrae and ilia are fused. The dorsal spines of the vertebral segments are indicated only in the anterior portion.

The joints of the coccygeal or caudal vertebrae (27) are freely movable since in the birds of flight the tail is used as a rudder. The terminal bone (28) is called the ploughshare bone.

There are seven coccygeal or tail vertebrae. The last segment is the larger and is supposed to have originated from the fusion of several segments. It is shaped like a ploughshare and is called the pygostyle. It supports that part which gives rise to the twelve main tail feathers.

The ribs are divided into the true and the false. 
The true ribs articulate with the sternum or breast-bone by means of an intermediate bone. The false ribs do not articulate with the sternum, but are floating.

The first rib articulates with the quadrate portion of the last cervical vertebra and the first dorsal segment. The last ril, articulates with the under side of the ilium at its anterior alar portion. This rib is situated similar to the true ribs, but instearl of articulating directly with the sternum the second portion lies against the second portion of the rib just anterior to it. The rib is provided with two articular facets on the dorsal vertebra. The true rib is called a sternal rib, and each succeeding sternal rib is longer than the one preceding it. Nearer and nearer these ribs assume a horizontal position. The proximal end of the sternal rib articulates with the distal end of the vertebral rib. The sternal end is provided with two small ridges.

From the posterior border of the second, third, fourth and fifth vertebral ribs and near their middles, are flat uncinate or hook-like bony processes projecting upward and forward overlying the succeeding ribs, giving greater stability to the thorax. These processes are absent from the first and the last two ribs. The vertebral portion of the rib (32) articulates inferiorly with the sternal portion (41) joining it to the sternum or breast-bone, with which it articulates. The two portions of the rib are joined by a diarthrodial articulation.

The sternum or breast-bone is a large, four-sided plate of bone, the posterior portion of which overlaps the ribs on the outer side. On the inferior portion of the sternum there is a leaf-like ridge called the crista sterna or breast ridge, commonly known as the keel. This bony expansion gives a greater surface for the attachment of muscles.

The sides are provided with an external and an internal process forming an internal and an external notch. These notches are bridged over by a broad ligament, to which the hreast muscles are attached. In the poor flyers, as the fowls, these notches are large. The sternal end posteriorly is called the xiphoid process, is pointed, and in the young fowl consists of cartilage, but in the mature bird become bony. Anteriorly. the loreat-lone is provided with lateral external processes, the costal processes. In the center of the anterior part is the episternal process.

\section{THE LIMBS}

The os scapulare or scapula (24), is elongated, narrow and presents smooth surfaces. Anteriorly, it forms a portion of 
the glenoid or shoulder joint cavity and is united to the fork of the coracoid by means of fibro-cartilage.

The os clavicula or collar-bone, often called the wish-bone (33), terminates below into an enlargement, the hypocledium (57), and is attached to the breast-bone by means of a ligament, the claviculo-sternal ligament. The superior extrenity' of the clavicle rests within and opposite the glenoid cavity; against the scapula (24) and coracoid (34). These three bones form a passageway through which extends the tendon of the levator muscles of the wing. This bone is called the furculum or "wish-bone." Its forks are elastic and prevent the wings from coming toward each other during contraction of the depressor muscles. The os coracoideum (34), with the scapula, forms a glenoid cavity at its proximal or upper end, in which articulates the head of the humerus. Inferiorly, it articulates with the sternum or breast-bone. The os humeri (22) presents an articular head inferiorly, which occupies the glenoid cavity. It articulates superiorly with the radius (15) and ulna (21). The os ulnae, or ulnar bone, is larger than the radius. This bone articulates inferiorly with the humerus and superiorly with the carpus or wrist bones in company with the radius and is provided with a short olecranon inferiorly. The two bones meet at both extremities but bow apart in the middle. A strong ligament binds the ends so that pronation and supination is impossible. This limits movements to a gliding motion lengthwise. The carpus, or wrist, is made up of two bones, the os carpi radiale (14) and the os carpi ulnare (20). The metacarpus (17), consists originally of two bones but they are fused or consolidated at the extremities in the mature bird. The first digit, thumb or false wing-bone (16), is a styloid-shaped phalanx. The second, middle or large finger (18), consists of two phalanges, the second phalanx (34), and the third or rather ruidmentary finger (19).

The pelvis is voluminous and very strong. The three pairs of bones composing it are: the ilium (25), the ischium (30) and the pubis (36).

The ilium is long and is consolidated or fused with the last two dorsal, the lumbar and the sacral vertebrae. It is excavated on the internal face to receive the three lobes of the kidneys.

The ischium forms a part of the sides of the pelvic cavity.

The pubis, commonly called the lay-bone, is long and thin and extends along the inferior border of the ischium.

The femur or thigh-bone (35), articulates stuperiorly with the ilium by the cotyloid or hip-joint cavity. This cavity is 
provided with a foramen or opening at its bottom which passes through the bone. The femur articulates inferiorly with the patella (46), the fibula (43), and with the tibia (47). The fibula articulates superiorly with the external condyle of the femur and the head of the tibia and inferiorly is consolidated with the tibia. The tibia, or drum-stick bone, terminates below in two condyles which articulate with the proximal end of the metatarsus (48). The metatarsus, or shank-bone, terminates inferiorly with three articular surfaces or facets which articulate with the three principal digits (49), (50) and (53). A conical eminence is noted near the inferior third which turns backward and is the base of the spur. Most fowls have four digits. The os metatarsale, or rudimentary digit (51), projects backward and is made up of three phalanges. The second or internal toe (49), is provided with three phalanges; the third or middle toe (53), is provided with four phalanges. and the external or fourth toe $(50)$, is provided with five phalanges.

\section{VISCERAL ANATOMY OF THE HEN}

\section{THE DIGESTIVE TRACT}

\section{The Mouth}

The mouth cavity is characterized by the changing of the jaw-bones into a beak. There are no teeth. The tongue is shaped like an: Indian arrowhead, with prominent, rather hard and sharp-pointed papillae, along its posterior border.

The beak is divided into an upper and a lower portion. The upper portion of the beak is provided with sharp free edges. There are no lips or cheeks. The upper jaw forms the base of the upper portion of the beak, and the lower jaw forms the base of the lower portion of the beak. The beak is covered by a continuation of the epiclermis. The beak is formed of horn-like material that is rather hard and resists wear to which it is subjected.

In many water birds, as geese and ducks, a thin clermoid structure is formed over the edges of the beak, in which numerous branches of the trigeminus or fifth pair of cranial nerves terminate in taste-buds.

In chickens, the beak terminates in a sharp point, while in water fowls, as geese and ducks, the beak is flat, spatulalike, and grooved transversely at its free margin. The roof of the mouth (hard palate), is provided with a slit that cxtends antero-posteriorly, and is about one inch in length; this is the posterior nares or opening of the nasal passage into 
the throat. There are, on the hard palate, four or more crossbars, each of which is provided with hard filiform papillae that point backward. The tongue presents a flat surface superiorly, and is covered by a thick strata of corneous epithelium. The upper or dorsal surface is provided with many small filiform papillae, that point backward. The body of the tongue is made up of muscles and connective tissue. The tongue of swimming bircls is thinner than that of chickens. The tongue is an organ possessing both of the senses, taste and touch. The transverse row of filiform papillae of the posterior portion of the roof of the mouth marks the border between the oral and pharyngeal cavities. Both palatine and sublingual salivary glands are present. The parotid and submaxillary when present in birds are rudimentary. They have ducts or canals through which their secretions are discharged into the mouth.

\section{The Pharynx}

The mouth cavity terminates in the pharynx, or throat. The pharynx is covered by a mucous membrane. There is a transverse ridge, provided with filiform papillae pointing backward and located on the supero-posterior portion of the larynx that marks the posterior edge of the pharynx.

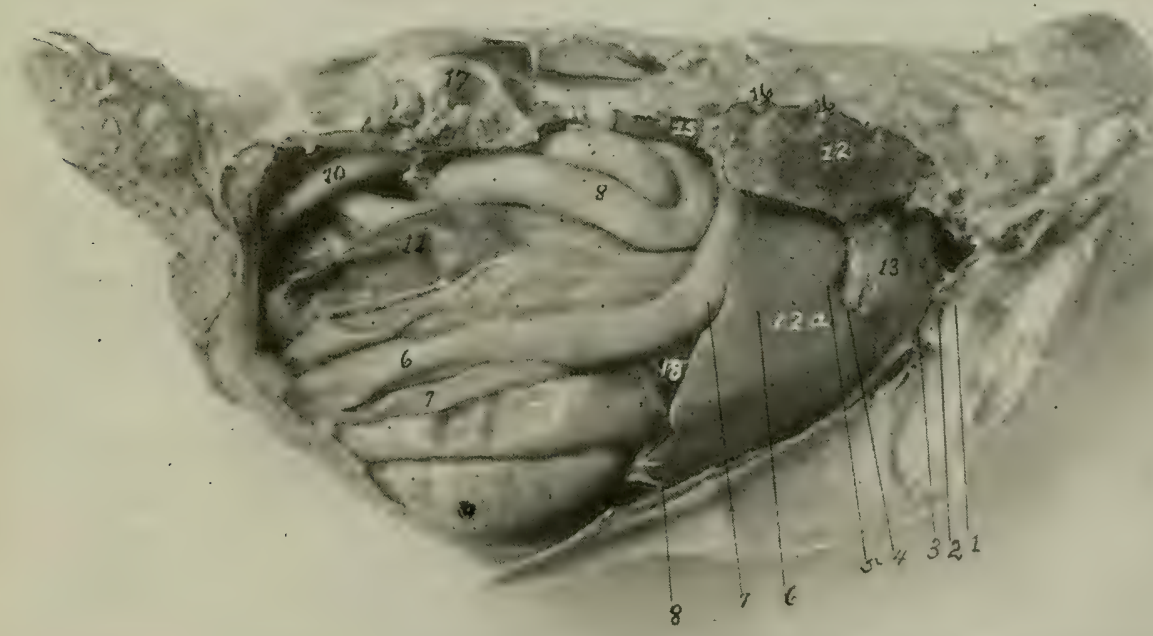

Fig. 3. Visceral Organs of the Right Side of the Fowl.

The right abdominal and thoracic walls have been removed; 6, duodenal loop; 7 , pancreas; 8 , small intestine; 10 , rectum; 11 , caeca; 12 , right lung; $12 \mathrm{a}$, right lobe of liver; 13 , base of heart; 15 , kidney; 16 , ends of the broken ribs; 17 , upper part of the cotyloid or hip joint cavity; 18, the gall bladder located on the right lobe of the liver. The gizzard is not visible from the right side. 
The pharymx communicates anteriorly with the mouth cavity and posteriorly with the esophagus and larynx.

\section{The Esophagus}

The esophagus is a muscular tube lying posterior to the trachea. The esophagus is divided into two parts, a first portion and a second portion. The first portion passes over to the right side as it reaches the anterior surface of the breast, where it forms an expansion called the crop. The second portion, springing from the under side of the crop, enters the thorax through the anterior thoracic opening and occupies a space between the lungs and passes over the base of the

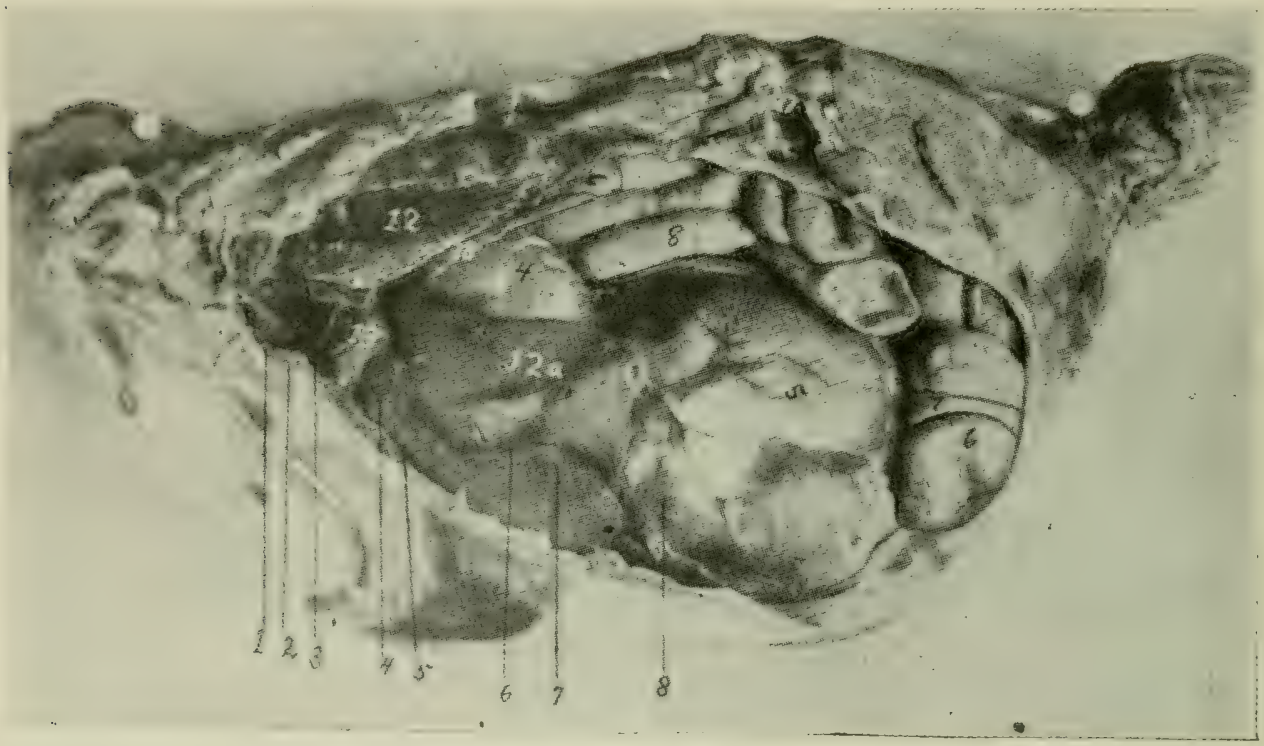

Fig. 4. Visceral Organs of the Left Side of the Fowl.

The left abdominal and thoracic walls have been removed; 4, proventriculus or glandular stomach; 5, gizzard; 6, extreme end of duodenal loop; 7, pancreas; 8 , small intestine; 12 , left lung (it will be noted that the lungs occupy the superior part of the thoracic cavity and that there is no distinct pleural sac, but that the lungs push up between the ribs; in fact, the ribs are covered on the inner and lateral sides by the lungs); $12 \mathrm{a}$, left lobe of the liver; 13, base of the heart; 17, hip joint just above the kidney; 18, diaphragm.

heart. Some areas of the mucous membrane of the crop contain mucous glands. The crop is globular and simple in the fowl. fusiform in the goose and duck, and in the pigeon forms two symmetrical sacs. In the pigeon, during and shortly after hatching time, in loth sexes, it produces a milly fluid which the pigeon mixes with grain and serves as nourishment for the young. The second portion of the esophagus terminates in the proventriculus or glandular stomach. 


\section{The Stomach}

Some anatomists call the proventriculus, the pars glandularis and the gizzard, the pars muscularis. The pars glandularis, or proventriculus, lies clorsal to, and midway between, the two lobes of the liver, taking an oblique course to the left. It terminates in the gizzard. Its mucous surface is studded by papilla-like projections, which furnish openings to glands of the type of those in the fundus of the stomach of the horse and other quadrupeds.

\section{The Gizzard}

The gizzard, or pars muscularis, is round, muscular and flattened laterally and lies to the right and posterior to the proven-

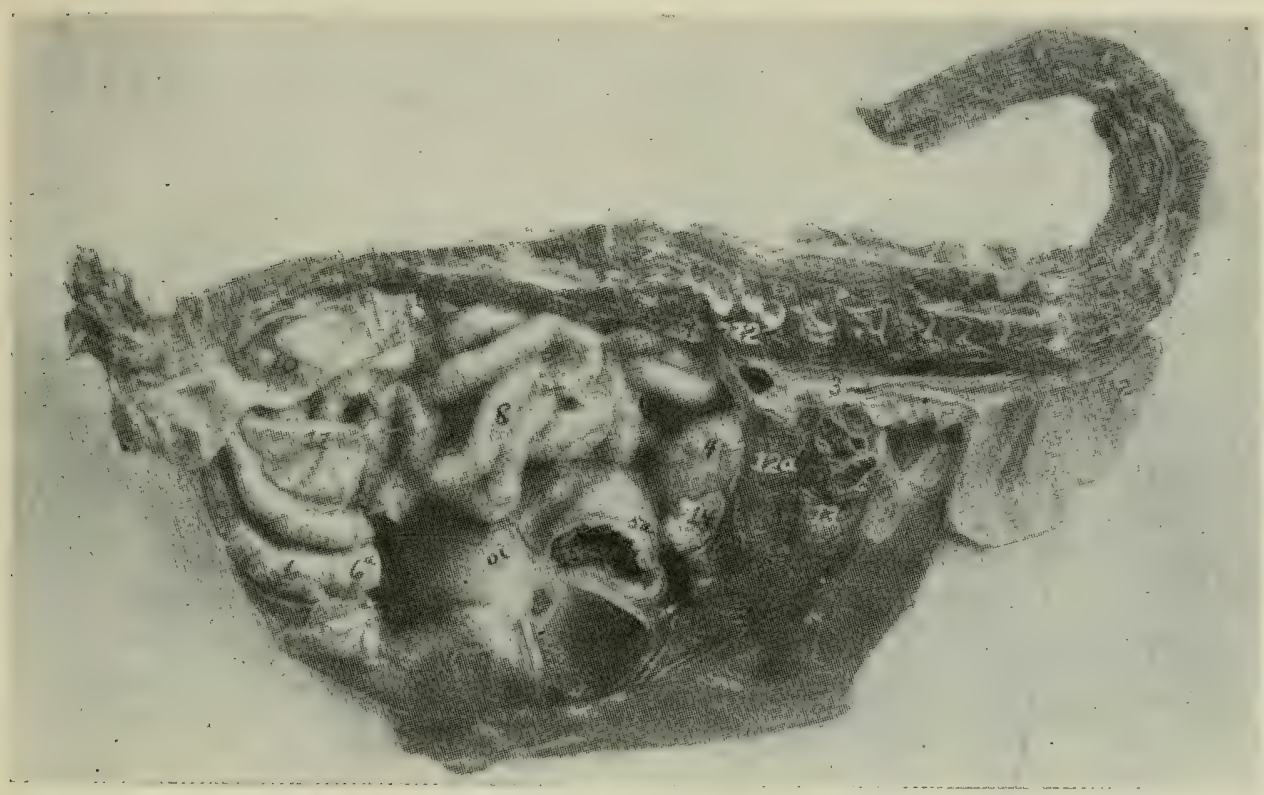

Fig. 5. Section Through the Median Line of a Tivo-Pound Pullet.

1, stump of the first portion of the esophagus; 2 , left wall of the crop; 3 , second portion of the esophagus located just below the bodies of the vertebrae; 4 , proventriculus lying antero-laterally to the gizzard, superoposteriorly to the liver, and to the left of the spleen; 5 , gizzard; 6 , duodenal loop; $5 a$ and $6 a$, points where duodenal loop has been cut away; 7 . pancreas: 8 , floating portion of small intestine; 9 , ovary; 10 , rectum; 11 , caeca; 12 a, liver; 13 , heart; 14 , spleen.

triculus. It lies behind and partly between the lobes of the liver and consists of a hollow organ. It is somewhat contracted at the sides and stands obliquely in the abdominal cavity. In gramniverous birds the walls are very thick, being made up for the most part of two powerful muscles. The cavity is lined with a very thick stratified epithelium. The heavy horny pads form grinding surfaces. Certain portions 
of the mucous lining contain glands which by some anatomists have been regarded as modified pyloric glands of mammals.

The small intestine originates on the same side of the gizzard that the proventriculus enters and about one-half inch distant.

\section{The Small Intestine}

The first portion of the small intestine is called the diiodemm, which forms a long loop, called the duodenal loop. This loop passes obliquely to the right side and proceeds along the outer right abdominal wall, where the terminal portion of the loop rests. The remaining portion of the small intestine forms a thick coil supported by a mesentery and occupies the space between the two large abdominal air sacs.

\section{The Large Intestine}

The large intestine is very short. The large intestine, in fact, in the fowl, is the rectum or straight gut. At the point where the small intestine terminates in the large intestine, there is given off two long blind pouches-the caeca. These blind pouches are directed toward the head and then double backward toward the posterior abdominal quadrant. The caeca in the pigeon, are comparatively short, while in fowls, ducks and geese, as well as in turkeys, they are quite long. The major portion of the caecum is narrow, becoming larger in calibre at the blind extremity and constricted at its point of origin. From the point of origin of the large intestine, it extends backward in a straight line just under the bodies of the vertebrae and terminating in the cloaca.

The entire length of the alimentary tract in fowls is five to six times, and in geese and ducks, four to five times, the length of the body.

\section{The Cloaca}

The cloaca, meaning cosspool, is a sacculation with greater diameter than the large intestine and communicating anteriorly with the large intestine and opens to the external world through the anus. In this receptacle, everything is dumped. It receives the feces, urinary secretion and the egg from the oviduct of the female. The vas deferens of the male opens through a papilla on the anal mucous membrane. At the point where the large intestine or rectum empties into the cloaca, there is a strong, oblique fold of mucous membrane. The ureters empty at the summit of small papillae midway between the vas deferi. The mouth of the oviduct is represented by a slit. In the males of geese, ducks and swans, there is present a copulatory organ, somewhat resembling the penis of mammals. 
A small round pear-shaped sac, called the lursa of Fabricus. is located in the dorsal or ton wall of the cloaca. The cavity communicates, through a short canal, with the posterior border of the cloaca close to the anal wall. The mucous membrane lining the fursa contains glands. The bursa is large in the young. lout decreases in size with age. It apparently reaches its greatest size in chickens at about four months of age, at which time it may measure two or three centimeters by one and one-half centimeters. By ten or eleven months of age, it has become quite rudimentary. Its function is not known. Glands similar to those of nammals are located in the intestine.

\section{The Liver}

The liver is the largest gland in the body and consists of two lobes-a right and a left. The right lobe is somewhat

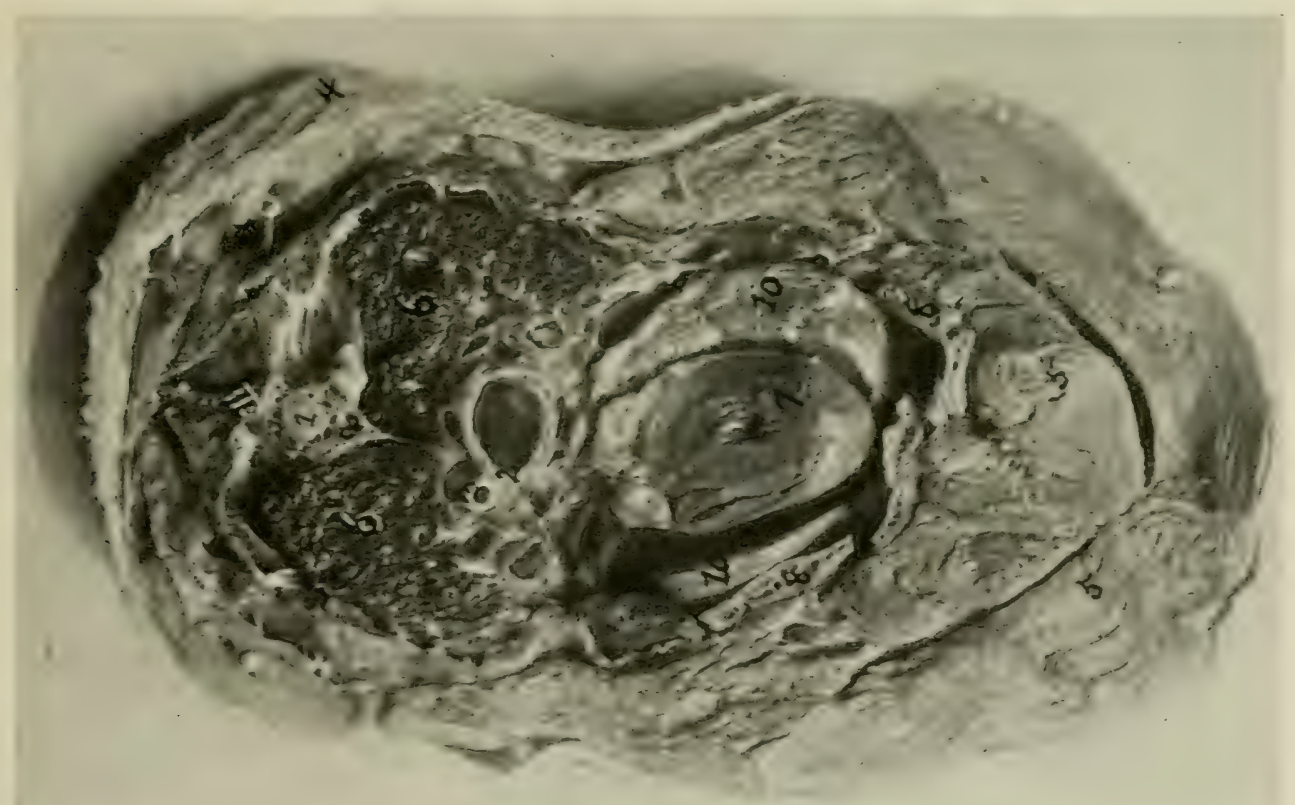

Fig. 6. Traxsterse Section Throtgh the Thoracic Regiox. Lookixg Backiard. 1, spinal cord: 2 , esophagus: 3 , trachea; 4 , skin; 5, pectoral muscle; 6 , lungs; i. heart: 8 . breast bone: 10 , liver.

larger than the leit, in most birls. except in the turley and guinea. The liver lies behind the heart, the apex of which extends into the fisture between the anterior jortion of it: two lobes. The inferior surface of the liver is convex and covered hy a layer of visceral peritoneum. The two lobes are held together by a -mall ligament. A filiform liganent extends from the inner -urface of the sternum and beconnes lost in the 
serous covering of the liver. This ligament assists in holding the liver in position. Most species of birds are provided with a gall bladder, which is locater on the posterior surface of the right lobe of the liver. In birds without a gall bladder, as pigeons and guineas, the main gall ducts of the two lobes of the liver unite and empty as one duct into the duodenum. In birds having a gall bladder, the ductus hepaticus passes from the left lobe of the liver to the duodenum, while the bile from the right lobe empties into the gall bladder, from whence the gall is carried to the duodenum through the ductus cysticus.

In fowls, the ducts of the liver and pancreas empty into the duodenum in the following order: First, the ductus pancreaticus, from the pancreas; second, the ductus hepaticus, directly from the left lobe of the liver ; and, third, the luctus cysticus, from the gall bladder of the right lobe of the liver. In ducks, the ductus hepaticus and the ductus cysticus are united. This common duct empties into the duodenum near the pylorus. The two branches of the pancreatic duct empty close behind the hepatic duct. In the pigeon, the ductus hepaticus is double and extends from the left lobe, while a third duct carries the bile from the right lobe. There are apparently three pancreatic ducts in this species.

\section{The Pancreas}

The pancreas is a pale, long, yellowish, lobulated gland, sometimes called the abdominal salivary gland, and lies between the iwo branches of the long duodenal loop. In some species of birds, the gland is divided into three distinct lobes, each provided with its own duct, which carries the secretion to the duodenum.

\section{The Spleen}

The spleen of the fowl is reddish-brown in color, shaped like a horse chestnut and lies to the right in an angle formed by the proventriculus, liver and gizzard. Its shape varies in the different species of birds. In some it is globular. while in others, it is lenticular.

\section{THE RESPIRATORY TRACT}

\section{The Nasal Cavities}

The nasal cavities in the bird are short and narrow. The two nasal passages are separated by a cartilaginous septum and, in part, ly a bony wall. In ducks and geese the external nasal opening is located toward the base of the beak. Through the openings, one can see through from one nostril to the other (nares perviae). The external openings are either round or 
slit-like. They are often surrounded by a thin dermoid structure and a border of peculiar feathers. Each nostril is provided with three turbinated bones. The middle turbinated bone is the largest and the upper one the smallest. A small, flat gland, peculiar to birds (fowls, lucks and geese), lies on the frontal bone in close proximity to the mesial corner of the eye. A duct extends from this gland forward and empties into the nostrils. The posterior nares, or posterior nose opening, communicates with the mouth cavity through a slit-like opening in the roof of the mouth.

\section{The Larynx}

The superior larynx is a musculo-cartilaginous valve located at the superior extremity of the trachea and receives the air from the nostrils and pharynx. The bird is not provided with an epiglottis as observed in mammals. A ring-like cartilage, the cricoid, is located at the base of the larynx. This cartilage forms the principal support. It is divided into four parts, namely, two side parts, one unequal ventral part and an unequal dorsal part. Some of these parts at times and in some species of birds, are fused. The arytenoid cartilages, three in number, are flexible and joined to the superior part of the cricoid. These cartilages bound supero-posteriorly the entrance of the larynx. At times, the arytenoids are partially bony.

The walls of the superior larynx are smooth and are not provided with vocal cords. This organ is sometimes called the larynx cranialis, in contradistinction to the true larynx or larynx caudalis. The true larynx is located at the bifurcation of the trachea into the right and left bronchi; it is provided with delicate vocal cords. This organ is absent in voiceless birds. It is sometimes called the larynx bronchio-trachealis. In fowls, it is flattened laterally. The last rings lie close together in fowls, but are connected in pigeons and more or less completely fused in geese. This structure is called the tympanum or drum. A bony, arrow-like passage is found between the tympanum and the opening into the bronchi. This bears a small half-moon-shaped fold, which is concave from above. ()n either side of this passageway, there is located an elastic membrane, the membrana tympaniformis interna, which forms the inner wall of the adjacent bronchus. Laterally and between the typmpanum and the two bronchial rings on either side, there is a membrane called the membrana tympaniformis externa. Singing birds possess a well-cleveloped muscle which in fowls, ducks and geese is rulimentary. In the water fowls, the inferior extremity is enlarged into a large sac, called the 
bulla tympaniformis. The left bronchus has a perceptible share in the formation of this bulla. This bulla is a resonance box.

\section{The Trachea}

The trachea varies in length, according to the kind of bird and length of neck. It is made up of closed rings, round in shape and connected by short connecting bands or ligaments. In fowls, these rings are cartilaginous, while in singing birds they may be partially bony. The trachea is moved by means of two lateral tracheal muscles.

\section{The Lungs}

The lungs are two in number, red to pink in color and firmly connected with the costal or inner chest wall. The ribs indent the lungs, allowing a part of the outer surface to project slightly between them. The ventral free surface is turned toward the body cavity and is covered by the rudimentary diaphragm. The rudimentary diaphragm contains some muscular structure and is attached to the ribs and vertebrae. The pointed anterior lobe extends to the first rib. The posterior part terminates in a broad surface and extends back as far as the anterior end of the kidneys. The bronchi communicate with the air sacs by openings from the posterior border. The two bronchi are broadened after they enter the lungs, which is at the beginning of the second, third and on the ventral surface. They lose their cartilaginous rings and continue as duct-like channels to the extreme posterior edge, where they terminate into cartilaginous rings called the ostium caudale, from which point they communicate with the ventral or large abdominal air sacs. Each bronchus gives off a ventral bronchus, called the bronchus diaphragmaticus caudalis, through which the air is conducted to the ostium intermedium caudale and into the caudal cella thoracica diaphragmatica. Each bronchus gives off lateral bronchi which extend to the ventral surface of the lung. They form blind pouches or air sacs (alveoli) near the surface of the lung.

Two bronchial systems take their origin from the main bronchus, the ventral and the dorsal. The bronchus clavicularis arises from the dorso-medial wall of the main bronchus, a short distance after it enters the lung and before it broadens out. This bronchus gives off a large branch, called the bronchus cervicalis. It bends round the base of the main bronchus and the pulmonary artery and supplies the dorsal surface of the lung, and through the ostium claviculare it supplies air to the air sacs on the respective side (dorsal air sac). It termi- 
nates close to the middle of the lung, where it communicates, through a small opening, with the diaphragmatic air sac.

The bronchus cervicalis extends forward in the direction of the original trunk and anteriorly communicates with the cervical air sac through the opening the ostium cervicale.

There is also given off from the main bronchus the bronchus cervicalis, dorsalis and medialis. The first extends with a few bronchi into the clavicular air sac. The bronchus diaphragmaticus cranialis extends laterally, dorsally and medially, and terminates in the thoracic air sac through the ostium intermedium craniale. The bronchus caudalis originates from the dorso-median wall and extends to the caudal and middle portions of the lung. There is given off another bronchus called the bronchus lateralis from this lateral bronchus.

There extends from the main bronchus several bronchi in the medial half of the lung. There are six to ten dorsal bronchi (bronchi dorsales). Numerous perpendicular, narrow, thick-walled tubes lying close to each other are given off from the dorsal wall of the main bronchus and other bronchi mentioned. These tubes have been called the "lung pipes," which by lateral pressure become five- or six-sided tubes. The mass of the lungs are made up of these tubes. These tubes terminate near the surface of the lung. The ends of these tubes, which lie at right angles to the main channel, form sacculations somewhat simulating the air sacs of mammals. Connective tissue, through which pass blood vessels and nerves, fill in the space between these lung tubules.

\section{The Air Sacs}

The air sacs are bladder-like structures with delicate walls. The air sacs are lined with a mucous membrane which is a continuation of the mucous membrane of the bronchi. The air sacs are connected with the bronchial tubes, but do not communicate with each other. They also communicate with air spaces in many of the bones of the trunk and limbs. Bronchial tubes extending to the surface of the lung communicate directly with these air sacs. The air sacs send extensions into the bones and line the walls of these air spaces. These air spaces, some of which are of considerable size, add to the bulk of the body of the bird without increasing its weight. They reach their highest state of development in the fliers and swimmers and are less developed in running birds. There are nine air sacs, as follows: The anterior thoracic air sac, from which arises the axillary extension which goes into the humerus, breast-bone and ribs. This is the only air sac that 
is single, the balance existing in pairs. The cervical air sacs lie on the cervical vertebrae and send extensions into the cervical vertebrae. The infero-posterior thoracic air sacs, also called the rentral, phrenic, or diaphragmatic. The superoposterior thoracic air sacs, also called the dorsal, phrenic, or diaphragmatic. These latter two pairs of air sacs lie between the lungs and heart and the abdominal viscera at the rudimentary diaphragm and aid in keeping separate the thoracic and abdominal viscera. The abdominal air sacs, which are by far the larger of all the air sacs, extend from the anterior to near the posterior border of the abdominal cavity. Part of the abdominal viscera lies between them. They extend to the posterior part of the pelvis and the thigh bones.

The air spaces of the bones of the head are supplied by air by extensions from the eustachian tube.

\section{The Thymus Gland}

The thymus gland is present in young birds and is located along each jugular vein in the neck. This gland is elongated and lobulated. In old birds, it is lobulated.

\section{The Carotid Glands}

The carotid glands, two in number, are round or oblong glands abundantly supplied by blood ressels and lie at the base of the carotid arteries.

\section{The Adrenal Glands}

The adrenal glands are small reddish-like bodies which lie at the anterior end of the kidneys. They produce an internal secretion which has an effect upon the circulation.

\section{THE CIRCULATORY SYSTEMS}

The circulatory systems consist of two, namely: the blood vascular system and the lymph vascular system.

\section{The Blood Vascular System}

The blood vascular system consists of the heart, the propelling engine; the arteries extending from the heart, the veins, and the capillaries connecting the arteries and the veins.

The heart is located in the thoracic cavity with the base directed upward and forward. The apex is directed downward and backward and lies in the anterior portion of the fissure formed by the two lobes of the liver. It is surrounded by a pericarlial sac, which is often attached by means of connective tissuc to the adjacent air sac. The structure of the heart 
is similar to that of mammals, except that the tricuspid valves of the right auriculo-ventricular opening are replaced with a strong double muscular plate which extends from the outer chamber wall. The papillary muscles are absent in the right chamber. The aorta is given off from the left ventricle and the pulmonary artery from the right ventricle as in mammals. The bicuspid valve frequently is provided with three points. The venae cavae, two anterior and one posterior, empty into the right auricle. The pulmonary veins empty through one common opening into the left auricle.

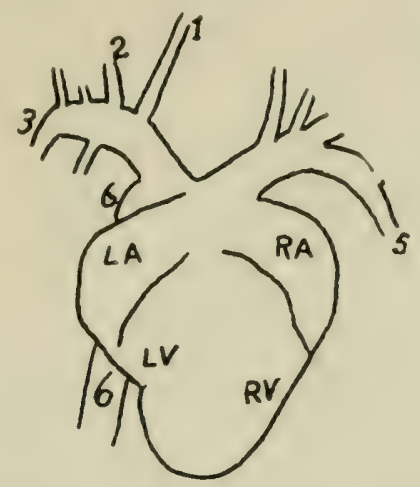

Fig. 7. Heart of Fowl with Its Marn Branches,

1, carotid artery; 2, sterno-clavicularis artery; 3, axillary artery; 4, thoracic cranialis artcry; 5 , thoracic caudalis artery; 6 , posterior aorta; L $\Lambda$, right auricle; $\mathrm{RA}$, left auricle; $\mathrm{LV}$, right ventricle; $\mathrm{RV}$, left ventricle.

The structure of the blood vessels are the same as in mammals. The pulmonary artery emerges from the right ventricle, is short and divides into a right and a left branch, which go to the respective lungs.

The aorta emerges from the left ventricle, is short and gives off the right and the left coronaries and bifurcates into the brachiocephalic dexter, the right branch, and the brachiocephalic sinister, the left branch. The brachiocephalic dexter gives off the posterior aorta which winds round the right bronchus and passes along the lower portions of the vertebrae. It then bifurcates to form the right carotid and right subclavian arteries. The brachiocephalic sinister bifurcates into the left carotid and the left subclavian arteries.

In some species of birds, the right and the left carotids spring from a common trunk called the carotis primaria. The carotic arteries, as they pass up the neck, give off branches to the adjacent parts. A vertebral artery is given off, which supplies the vertebrae. At the level of the head, each carotid artery divides into two branches, one supplying the brain, eyeball and adjacent parts, and the other the remainder of the cranial structures. The two subclavian arteries each give off 
a sterno-clavicularis which supplies the anterior sternal region up to the shouller, where it divides into two arteries, which supply the breast and the arm. The thoracic cranialis is also given off from the brachio-cephalic as well as the thoracic caudalis, the latter supplying the large breast muscle. At about this point, there is also a branch, the internal mammary, given off and which follows along the inner border of the sternum. The axillary artery may be consiclered a continuation of the brachiocephalic; it supplies the muscles of the wing.

The posterior aorta extends along the lower borders of the vertebrae as far as the pelvis. It gives off on its way intercostals, which pass, one along the posterior border of each rib, and are disposed of similar to those of mammals. It also gives off a celiac axis supplying the stomach, liver, and spleen, two mesenteric branches, the mesentericus cranialis or anterior mesenteric, and the posterior mesenteric or mesentericus caudalis, supplying the mesentery and small intestine. The posterior aorta also gives off lumbar arteries, and renals, the latter supplying the kidneys, also, in the male, arteries to the testes, called the testicular artery; and in the female the ovarian, supplying the ovary. At the hind extremities there is given off the external iliac at a line near the juncture of the anterior and micldle thirds of the kidney. This supplies the pelvic and outer muscles of that region. The aorta divides into two branches, the ischiatica and also sends an artery back along the under side of the caudal vertebrae, the middle sacral. The ischial artery, in company with the ischial nerve, passes through the foramen ischiaticum, giving off branches to the muscles of that region.

The pulmonary veins, two in number, originate from a short stem, the pulmonary artery, which springs from the right ventricle. These veins furnish the lungs with functional blood, which is returned to the heart through pulmonary veins entering the left auricle.

There are three venous trunks carrying the venous blood from the body and extremities. These are the left and the right anterior vena cava, the vena cava cranialis and the posterior vena cava, the vena cava caudalis. These three vessels (mpty into the right auricle. Each anterior vena cava receives the jugular and subclavian vein of its respective side. The right jugular is larger than the left. They are located ventrally to the skull. where they anastamose through an oblique vein. It the inferior portion they receive branches from the head, the cranial veins, also neck and back veins. The subclavian vein receives the blood from the veins of the breast and wing, 
the sterno-clavicularis, and the thoracic-cranialis, caudalis and axillary veins. The subclavian empties into the anterior vena cava.

The posterior vena cava is short and receives the blood from the external and internal iliacs, hepatic, renal hypogastric and the coccygeal veins. It also receives the blood from the porta hepatis, which collects blood from the abdominal viscera, especially the intestines.

\section{The Blood of the Normal Fowl}

The blood of the fowl is made up of organized and unorganized parts. The unorganized part is the plasma and the organ-

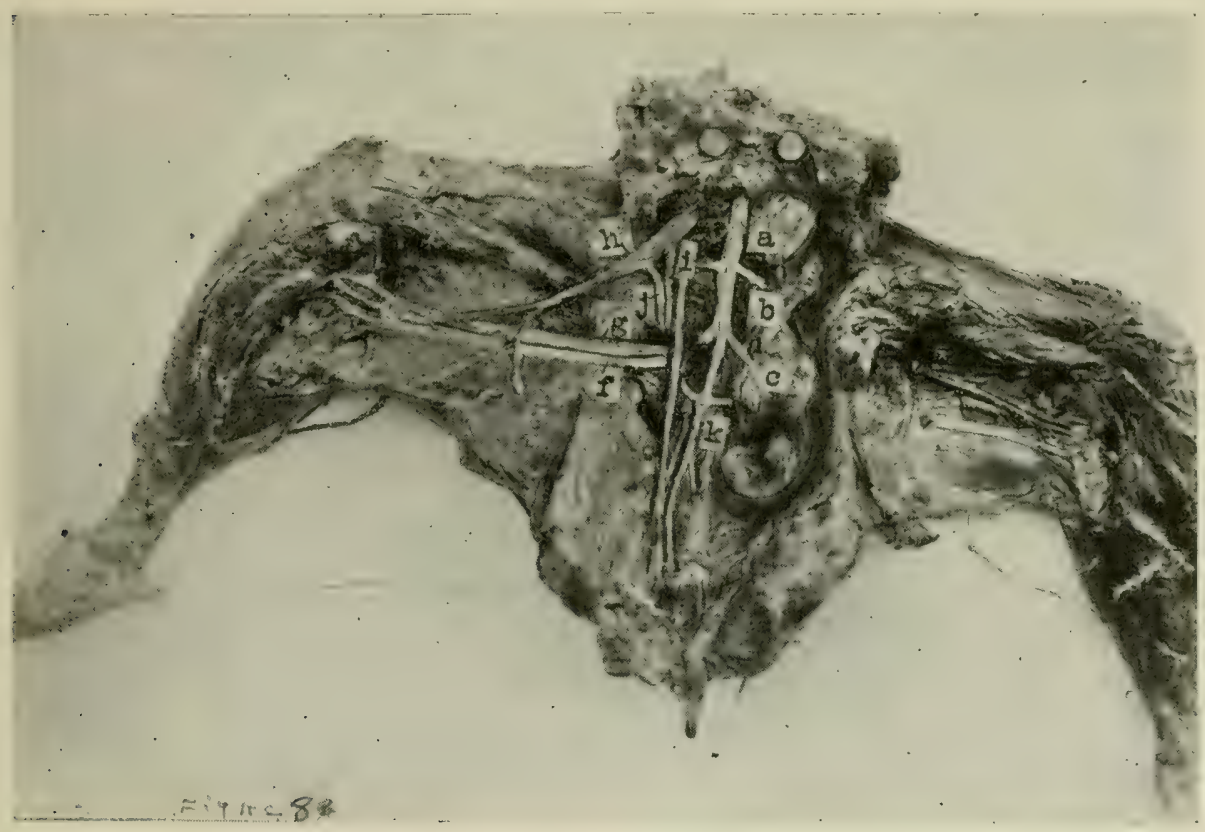

Fig. 8. Blood Vessels of the Pelvis and Thigh of the Fowl.

a, posterior aorta; a, b, external iliac artery; c, ischiatic artery; e, the ureter; $f$, the ischiatic nerve; $g$, the main artery of the thigh; $1 r$, the femoral vein; $i$, the internal iliac vein; $j$, the external iliac vein; $k$, the middle sacral artery.

ized is the cells. The cells are of three kinds, namely: red blood cells or erythrocytes, white blood cells or lencocytes, and the thrombocytes, corresponding to the platelets of mammals. The red blood cells are elliptical-shaped with an ellipticalshaped nucleus located centrally. The red blood cells measure $7 \times 12$ microns. The thrombocytes are oval with a round centrally located nucleus. There are several varieties of white blood cells, these are as follows: Mast cells or basophiles, eosinophiles, some of which contain round eosinophilic granules and others rod-shaped eosinophilic bodies, these are all 
polymorphonuclear. There are also small and large lymphocytes and mononuclear leucocytes.

The red blood cells number about 3,500,000 per cmm., the white blood cells number 25,000 to 30,000 per $\mathrm{cmm}$., and the thrombocytes about 50,000 per $\mathrm{cmm}$. The lymphocytes constitute forty-five per cent, the large mononuclear leucocytes eighteen per cent, the polymorphonuclear leucocytes with eosinophile rods 30 per cent, polymorphonuclear leucocytes with eosinophile granules six per cent, and the mast cells one per cent.

\section{The Lymph Vascular System}

The lymph vessels collect lymph from the tissues of the body and, carrying it forward toward the head, finally empty it into the large veins shortly before they empty their contents into the heart. The lymph vessels are numerous. Those of the hind extremity empty into the veins on the border between the tail and the pelvis. There are very few lymph glands. At the entrance of the thoracic cavity there is located two or more small lymph glands.

\section{THE NERVOUS SYSTEM}

The nervous system consists of two parts, namely: the cerebro-spinal sy'stem and the sympathetic sy'stem. The cerebrospinal system includes the brain, spinal cord and the nerves that spring from them. The sympathetic system consists of a chain of ganglia or nerve centers that extend along either side of the spinal cord and give nerves to the viscera, blood vessels and other involuntary parts.

The brain and spinal cord are surrounded by three membranes as in mammals. The brain is divided into three parts, as follows: cerebrum, cerebellum and medulla oblongata. The pons varolii, well developed in mammals, is either lacking or consists of but a few narrow oblique fibers. The cerebrum is the fore part of the brain and is divided into two hemispheres, which are separated superiorly by a deep longitudinal fissure. In mammals, there are deep fissures or sulci and the greater the intelligence of the animal the deeper is the fissure. There are no fissures or convolutions in the fowl. The fissure lateralis is well marked and is located laterally about the posterior border of the anterior third of the cerebrum. The epiphysis, a small body, is located between the hemispheres and at the posterior portion of the longitudinal fissure. The olfactory nerve consists of two conical bodies projecting forward from between the anterior portion of the hemispheres. The hypophysis or infundibulum, the cruri cerebri, and the optic chiasm 
are all similar to those of mammals. The corpus callosum is lacking or at most marked by only a few diagonal fibers. The hippocampus and the septum lucidum of mammals are lacking. The lateral sinuses are well developed. The lateral walls are thin and the gray matter is small in quantity. At the base of each of the sinuses there is found an eminence which corresponds to the corpus striatum of mammals. At the posteroinferior portion of the cerebral hemispheres, there are round eminences called the sight eminences. They are bound on the dorsal side by the bridge of Sylvius. Their cavity communicates with the aqueductus cerebri, which connects the third and the fourth ventricles.

The cranial nerves number twelve, as in mammals. The first cranial nerve is the olfactory and leaves the cranial cavity through a foramen which represents the perforated plate of mammals. It passes through the dorso-median part of the eye cavity, thence into the nasal cavity, where its fibers terminate in the mucous membrane.

The second cranial nerve is the optic nerve, which forms the chiasm by crossing at the base of the brain. At this X, the two nerves of sight cross. The motores oculorum, is the third pair of cranial nerves and supplies impulses to the muscles of the eye. The fourth cranial nerve is the pathetic, which supplies the superior oblique muscle of the eye. The fifth pair of cranial nerves is the trifacial, which is divided into three main branches that supply the muscles of the head and the eyes. The sixth pair of cranial nerves is the abducens, which is distributed to the muscles of the eye. The seventh pair of cranial nerves represents the seventh and eighth of mammals, the facialis and acusticus. The facialis is distributed to the face, including the tongue, and the acusticus is distributed to the internal ear and is the nerve of hearing. The ninth nerve is the glossopharyngeal and supplies the tongue and pharynx and gives fibers to the tenth pair of cranial nerves, which is the pneumogastric. The pneumogastric is sometimes called the vagus because it is a so-called wondering nerve in that it furnishes impulses to the neck, lungs, heart and some to the sympathetic system of the abdominal cavity. The eleventh pair of cranial nerves is the spinal accessory supplying nerves to the neck. The twelfth and last pair of cranial nerves is the hypoglossal, which supplies the tongue and other adjacent structures.

The cerebellum or lesser brain is located in the posterior part of the cranial cavity. Numerous oblique fissures mark the upper surface, dividing it into a leaf-like structure. The 
fourth ventricle is located under the cerebellum and on top of the medulla oblongata. Lateral pedicles attach the cerebellum to the medulla oblongata. The medulla oblongata broadens as it extends forward, being broader than the spinal cord. The medulla oblongata joins the spinal cord with the brain.

The spinal cord terminates posteriorly in a thread-like termination without forming a cauda equina as in mammals. There are dorsal and lumbar segments from which are given off nerves for the wings and the hind extremities. The dorsal
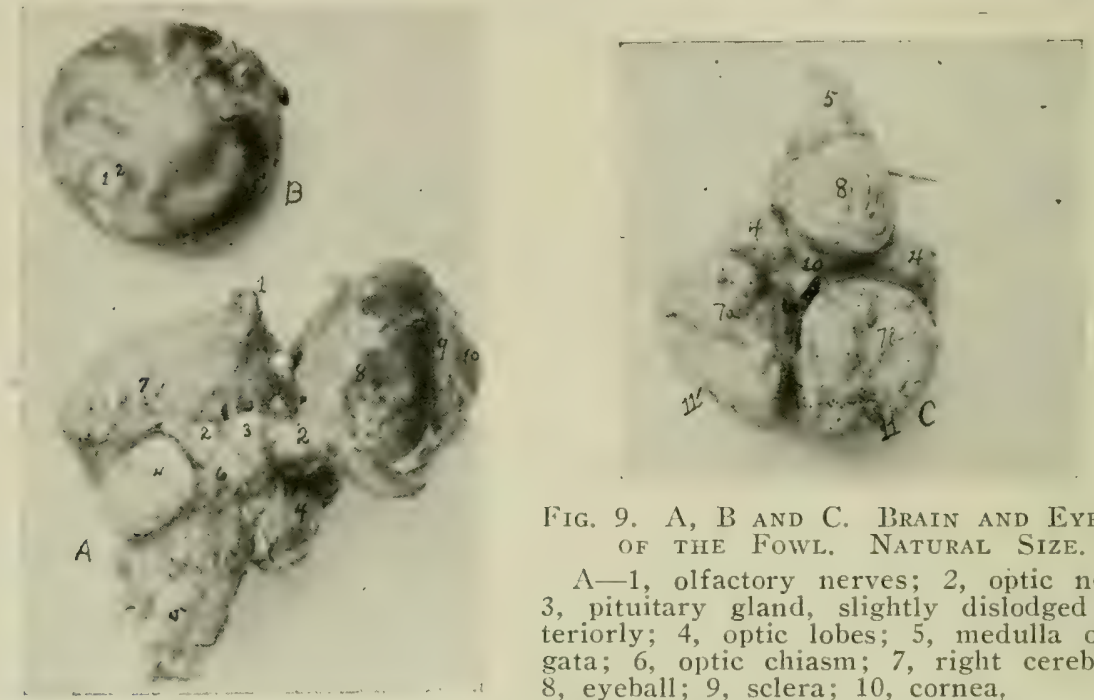

Fig. 9. A, B and C. Brain and Eyeball of the Fowl. Natural Size.

A-1, olfactory nerves; 2 , optic nerve; 3 , pituitary gland, slightly dislodged posteriorly; 4, optic lobes; 5, medulla oblongata; 6, optic chiasm; 7 , right cerebrum; 8 , eyeball; 9 , sclera; 10 , cornea,

b-1, optic nerve; 2 , its sheath.

C-4, optic lobes; 5, medulla oblongata; $7 \mathrm{a}$, right and $7 \mathrm{~b}$, left cerebral hemispheres; 8 , cerebellum; 9. fissure longitudinalis; 10 , fissure transversalis.

segment is marked by an enlargement. The central canal, as well as the mode of entrance and exit of the fibers, is the same as in mammals.

The number of nerves that are given off from the spinal cord depends upon the number of vertebral segments. The spinal nerves are given off in pairs; each nerve originates from one clorsal and one ventral root, one nerve for each side of the body. The spinal ganglion is located on the dorsal root. Each spinal nerve divides into a small dorsal nerve supplying the muscles and other structures in the region above the level of the spinal columm and a larger ventral branch supplying the body wall below the level. The spinal nerves have been placed in four groups, as follows: cervical, those of the neck; dorsal, those of the back or chest region; lumbo-sacral or those of the lumbar and sacral regions, and the caudal or those of the tail. The wings receive their nerve supply from the brachial plexus. The brachial plexus is made up of the last two or three cervical nerves and the first one or two dorsal 
nerves. The structures in the pelvic region are supplied from branches of the ventral divisions of the lumbo-sacral nerves. The nerves of the shoulder and pelvis are essentially the same as in mammals. The nerves of the skin and tail are small.

The sympathetic nerves have their origin at the cranio-cervical ganglion, which lies at the base of the skull. From this ganglion, fine filaments are sent to most of the cranial nerves, as well as connecting branches. The sympathetic trunk extends along the cervical vertebra, occupying an oblique canal. It exchanges filaments with the cervical nerves. It continues on either side of the base of the vertebrae through the thoracic and abdominal cavities. Branches from these trunks go to form the splanchnic nerve system supplying the visceral organs of the abdominal cavity. The right and left trunlis finally unite, forming the ganglion coccygeum or ganglion, which presides over the functions of the tail.

\section{THE ORGANS OF SPECIAL SENSE}

The Eyes

The eyeballs of the bird are rather large for the size of the body. The sense of sight in birds is well developed. 'The eyes stand to the side of the head in domesticated birds. The orbital or eye cavities in which the eyeballs rest are separated laterally by a bony partition or septum. This is the septum interorbitale. The eyeball is not completely surrounded by bone. The lower eyelid is larger than the upper and often incloses a small cartilaginous plate. The lower eyelid is more freely movable than the upper. The third eyelid or membrana nictitans is well developed. Two special muscles operate this third eyelid. These are the pyramidalis and the quadratus. The third eyelid is located in the inner angle or canthus of the eye and the muscles operating it pull it over the eyeball and again retract it. There are no tarsal glands as in mammals. The muscles of the eyelids are of the smooth variety and in the place of eyelashes, there may be found minute feathers. The feather follicles from which the feathers are developed are similar to the hair follicles of mammals. The gland of the eyelid lies on the inner or nasal sicle and is often larger than the lacrimal or tear gland. Its secretion is poured out onto the third eyelid. The tear or lacrimal gland lies in the angle close to the temple. The tear gland is small and has one or more ducts emptying into the conjunctival sac at the temple side of the eyeball. The lacrimal sac and lacrimal duct carrying tears to the nasal cavity are similar to those of mammals. The eyeball is made up of three coverings. The cornea, or front part, through which the bird sees, covers the 
anterior part of the eye and the sclera, the outer remaining portion of the eyeball. The sclera is made up of small overlapping scales, connective tissue and a cartilaginous cup extending from the optic nerve at the posterior pole to the equator of the globe. The scleral ring is at the anterior border of this cartilage. The anterior chamber of the eye is large. The middle layer of the posterior portion of the eyeball is called the choroid, and is rich in blood vessels and pigment. The retina forms the inner coat and is rich in a dark pigment. The ciliary body consists of many folds. The ciliary muscle consists of oblique fibers arranged in three bundles. Numerous wedge-shaped folds rich in blood vessels and containing pigment are found at the point of entrance of the optic nerve. In some birds, these folds extend forward and are attached to the lense capsule. The iris forms a partition between the anterior and posterior chambers, and is perforated in the center with a round hole, the pupillary opening, through which hole the bird sees. The iris on the posterior or lens side, contains a dark pigment which gives color to the eye. In most breeds and in the present-day fowls ancestor, the Gallus Bankiva, the pigment is red. The yellow coloration of the eye is caused by lipochrome, a fat pigment. This iris is made up of circular and of longitudinal muscles which govern the size of the pupillary opening. The circular muscles contracting make the opening smaller and the longitudinal muscular fibers contracting and the circular fibers relaxing make the opening larger. The size of the opening is governed by the brightness of the light the bird happens to be in. The lining of the vitreous or most posterior chamber does not contain blood vessels and its structure is similar to that of mammals. The crystalline lens in birds is rather flattened on the anterior sicle except in birds of nocturnal habits, in which it is very convex. The lens of birds differs from the lens of mammals, in that the epithelium develops into fibers in the equatorial portion and are arranged almost perpendicular to the axis of the eyeball. These are located near the ring pads. The portion for the passage of light is relatively small.

\section{The Organs of Hearing}

The car in mammals, with which we compare that of birds, consists of an outer, a middle and an internal ear. The outer ear is lacking in hirds. A skin fold surrounds the external opening of the ear in the fowl and is called the ear lobe. It also may be surrounded and partially covered by a peculiarly formed tuft of feathers. The owter canal is short and contains an ear gland. This canal is lined with the continuation of the 
skin and connects with the ear drum. The tympanic membrane is convex externally and is stretched in a bony ring. The ear drum forms an irregular cavity which is in relation to the air cavities of the skull and with the bony and cartilaginous ear trumpet in connection with the pharyngeal cavity. The columella only is present, which may be compared to the stirrup of mammals. Therefore, instead of three ear bones as in mammals, there is but one. The inner ear consists of a bony labyrinth surrounded by a spongy bone substance. In it is distinguished the vestibule, the three half-circled canals of the cochlea. The vestibule is a small irregular cavity which communicates with the cochlea and through the fenestra vestibularis with the drum cavity. The endolymph of the vestibule contains microscopic crystals of calcium carbonate. The semicircular canals are relatively larger and thicker-walled than in mammals. The ampullae are the upper and back canals with bony walls. The cochlea is a tube thinly covered at the blind end and contains the cuticular cochlea. At its point it is broadened for the formation of the lagena. The cavity of the cochlea is divided by the spiral walls, the scala vestibuli and the scala tympani. These walls are visible in the vestibulum so that they may be seen at the beginning of the cochlea.

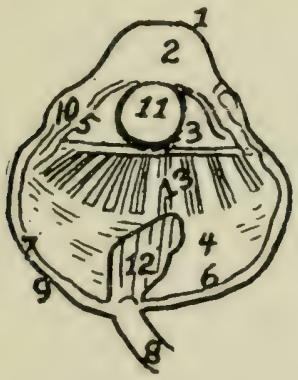

Fig. 10. Section Through the Eye OF A HEN.

1, cornea; 2, anterior chamber; 3 , posterior chamber; 4, vitreous cham. her; 5 1ris: 6 , retina; 7 . chornid coat; 8, optic nerve; 9, sclerotic coat; 10, scleral ring; 11 , crystalline lens; 12 , pecten; 13 , corpus ciliaris.

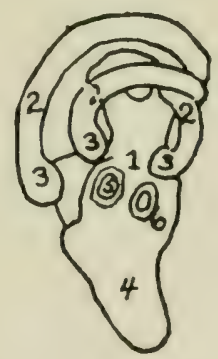
IFig. 11. Cochlear Labyrinth OF A

1 , vestibule; 2 , semicircular canals; 3, ampullae; 4 , cochlea; 5, fenestra vestibularis; 6 , tenestra cochlearis.

\section{The Organs of Smell}

The olfactory nere is the organ of smell. After emerging from the cranium through the olfactory foramen, the olfactory nerve passes down and spreads out, terminating in filaments on the mucous membrane of the nasal passage. It here receives the impressions of smell and transmits them to the brain. 


\section{The Organs of Taste}

The tongue is considered the organ of taste. In most birds, the thick, stratified squamous epithelial dorsal surface is little adapted for taste perception. In birds, the ninth nerve is the nerve of taste, the lingual or tongue branch of the trigeminus or fifth pair represented in animals is not present in the bird. Filaments of the first and the second branches of the trifacial, which is broadened in the mucous membrane of the hard palate, furnishes taste filaments to that part. Taste buds are, therefore, found in the tongue and in the hard palate.

\section{The Organs of Touch}

The organs of touch in the fowl are the skin and the feathers. The skin consists of an cpidermis and a dermis. The dermis is the true skin and the epidermis a protective layer. The skin of birds does not contain sweat glands. Fowls are provided with a tail or rump gland, called the glandula uropygii, or oil gland. This is a tubular gland which secretes an oily substance that is carried to the surface through a common duct. The bird, by obtaining some of this oil substance on its beak, oils the feathers. This oil preserves the feathers from becoming dry and brittle and prevents loss from weather conditions. In certain kinds of birds, special sense of touch and taste perception is provided by the edges and point of the beak. The dermis or corium is well developed in the bird and furnishes ample muscular means, through dermal muscles, for raising and lowering the feathers. This structure is thinner than in mammals. Papillary bodies are present only in a few areas, as in the region of the eyes and on the toes. Thickened epithelial elevations are noted on the ventral portion of the tocs, where there is great wear as a result of contact with the ground. The dermal muscles, which govern the movement of the skin, are well developed in certain parts of the body. They are livided into primary and secondary muscles. The primary muscles are the true dermal muscles and originate and terminate on the under side of the skin and control the movement of the skin for that region. The secondary muscles are the dermo-osseous muscles and are branches of the skeletal muscles having their origin on the bone and terminating on the under side of the skin.

The feathers grow in tracts, that is there is a space with no feathers then a space in which the feathers grow in definite rows, the feathers covering over the unfeathered space or tract. ()n the feathered parts of the skin, the epidermis is thin, rather dry on the surface and is provided with continuous scale-like 
layers. The stratum corneum is thick on the horny sheath of the beak, on the dorsal surface of the toes, the spurs of the cock, and the scaly plates of the shanks. The feathers covering the surface of the body of birds develop from papillae similar to the hair on manmals. Feathers occur over the whole surface of the body except on certain parts of the under surface, and the neck, shanks and toes of certain kinds of birds. Some breeds of birds are provided with a row: of feathers on the outer side of the shanks and outer toes. The chorium is not very rich in blood vessels. Blood vessels form a thick net-like structure in the comb, gills or wattles, and similar appendages of chickens and turkeys.

The feathers develop from the papillary structure of the corium. The feather is divided into three main parts, as follows: a quill, a midrib or shaft and primary and secondary barbs or the barbs and barbules. The free end of the quill occupies the papilla of the skin. The quill is nearly round, usually being slightly rounded laterally. The quill is hollow with thin dividing septa extending crosswise in all directions. At the free extremity of the quill, there is a cup-shaped depression, in which a fleshy mass is found during the development of the feather and through which, during growth, the feather gains nourishment. The shaft is provided with four sides and four edges. The central core is a spongy material. From the sides of the shaft, the primary barbs extend out, and in most breeds on either border of these primary barbs we find secondary barbs or barbules which dovetail into the barbules of the adjacent barb, thereby making the feather's web. The barbules of the fluffy portion of the feather are imperfectly formed and do not dovetail with their opponents, hence do not make a web. In silky breeds of fowl, the barbules are either absent or are, at most, imperfectly formed.

Fowls change their feather coat twice a year, either in late summer or fall, and in spring or early summer. The one in the fall or later fall is complete. A chick moults four times before growing its adult feathers.

\section{THE REPRODUCTIVE ORGANS OF THE HEN}

The female organs of reproduction in the fowl is the left ovary and the left oviduct. The right ovary and right oviduct are alssent, due to the fact that they degenerate during embryonic life.

The orary is located in the sublumbar region of the abdominal cavity and just to the left of the median line. It touches the left adrenal gland and lies just anterior and below the 
anterior portion of the kidney. It is located superior to the liver and at the juncture of the abdominal and thoracic cavities. In the pullet, the ovary appears as a miniature cluster of grapes, as seen in Fig. 12, letter a. In the adult hen, there is found from 900 to 3.500 ova, each capable of forming, in its turn, a yolk. Fig. 13, letter a, represents an active ovary from a three-pound bantam hen. This hen was developing one egg a day, having laid an egg only three hours before being killed; b represents the yolk of an ovum, which would

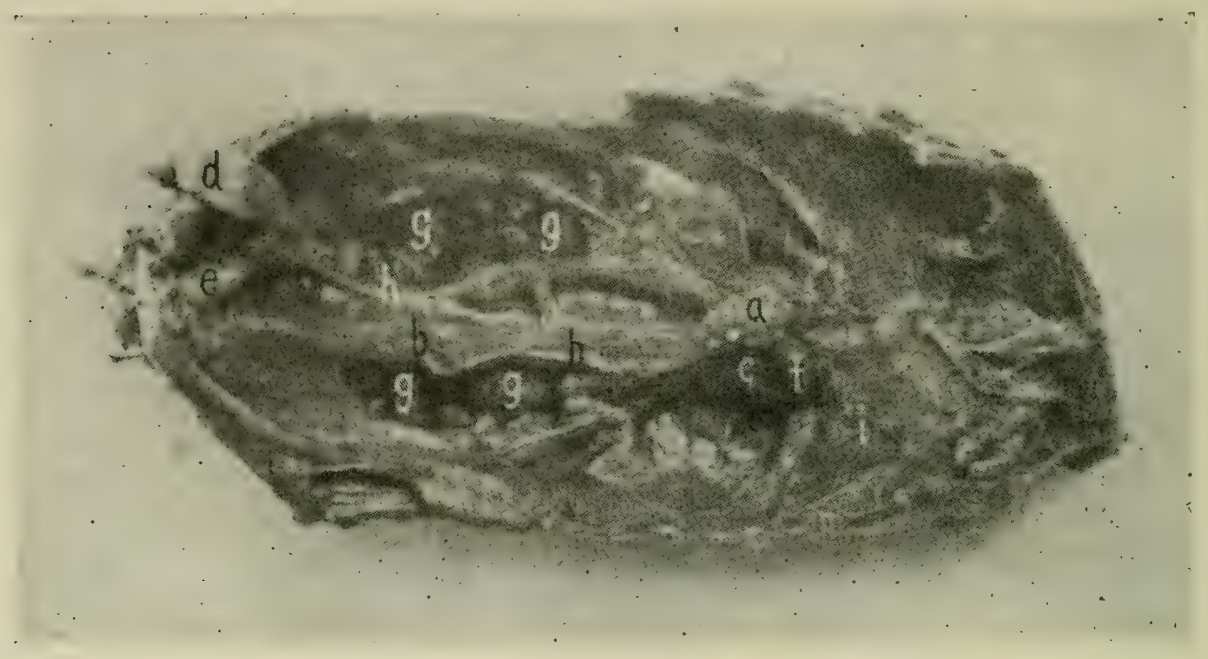

Fig. 12. The Pelvic Organs of a Pullet.

a, ovary; b, oviduct; c, infundibuliform portion or origin of the oviduct; $d$, rectum; e, cloaca into which the oviduct empties; $f$, adrenal gland; $g$, kidney; $h$, ureter carrying the secretion of the kidney to the cloaca; $i$, lung.

probably have been fully developed in less than twenty-four hours. The ovum or yolk, is surrounded by a thin membrane or capsule, which is very vascular and is shown in Fig. 13. This capsule is continued back onto the stalk, which attaches it to the central fibrous supporting portion of the ovary. This portion is attached to the structures of the back. When the yolk portion of the ovum is fully developed, the capsule ruptures and the yolli falls into an expanded portion of the oviduct at c, Fig. 13, called the ovarian pocket. This portion of the oviduct is very thin and gradually merges into a thick wall, in which portion the mucous membrane is thrown into folds. The yolk is surrounded by a delicate membrane called the vitelline membrane, which holds the yolk mass intact, thus giving it its spherical appearance.

After the stigmen ruptures and the yolk is discharged into the ovarian pocket, there remains a cup-like cavity attached 
to the ovary which is called the calyx, and which gradually disappears.

During the laying period of the hen, one by one, these yolks are developed to full size or to maturity from the mass of andeveloped ova of the ovary, as illustrated in Fig. 12, letter a. The ova in the process of formation are illustrated in Fig. 13 , a. In letter $\mathrm{b}$, there is seen an ovum or yolk reaching full

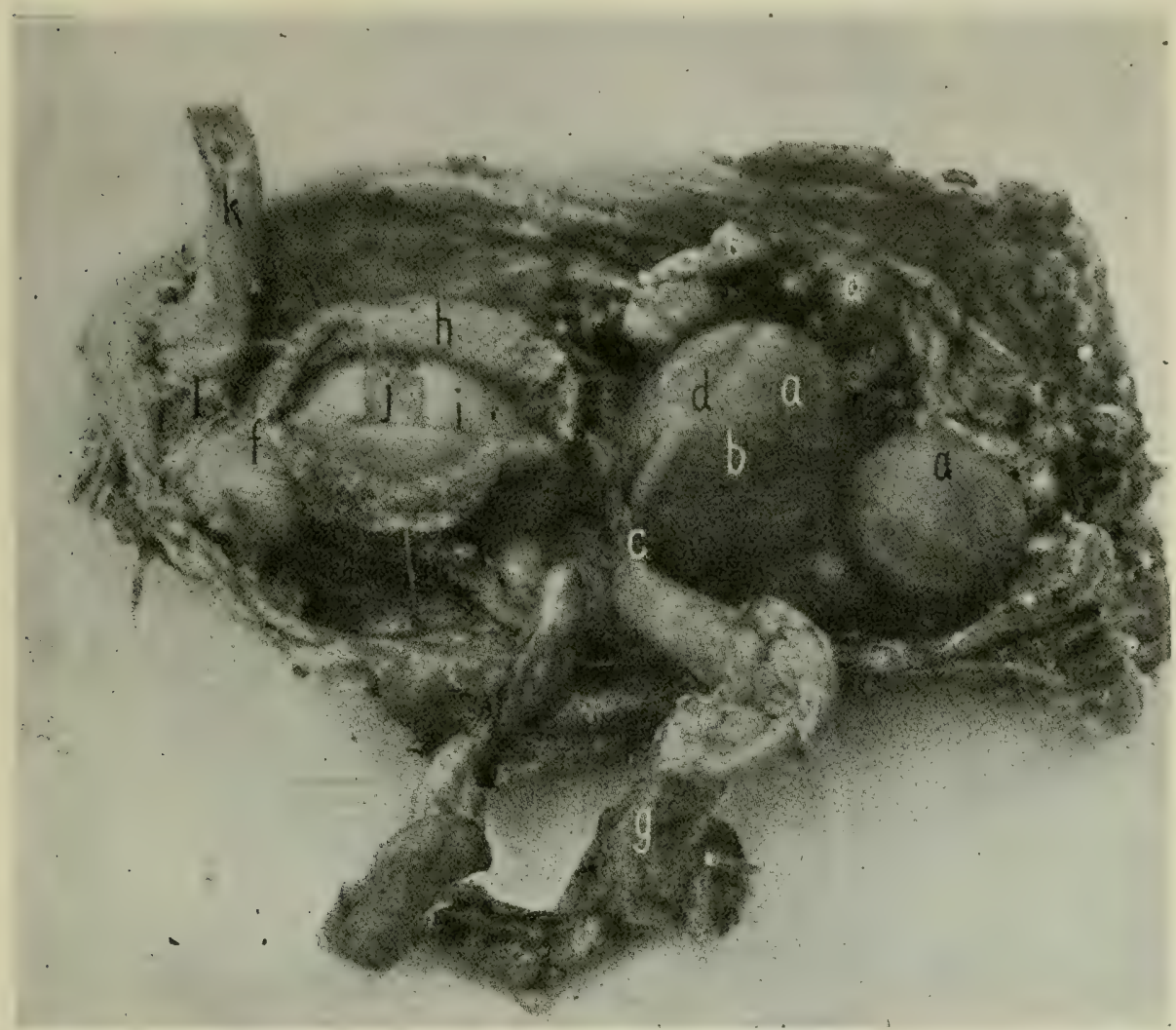

Fig. 13. The Functioning Pervic Organs of a Hen.

a, ovary; b, ovum fully developed; c, infundibuliform portion of the oviduct; d, stigmen or point of rupture of the follicle containing the developing yolk; e, a degenerated ovum; $f$, portion of the oviduct in which the shell is formed; $g$, a portion of the oviduct torn from its ligaments and laid over to one side; h, showing the villus-like mucous membrane; $i$, albuminous portion of newly formed egg; $\mathrm{j}$, the yolk of same; $\mathrm{k}$, rectum; 1 , cloaca.

development, and d shows a non-vascular line, the stigmen where the follicular wall is becoming thin preparatory to discharging the yolk into the oviduct. The discharge of the yolk into the oviduct is sometimes spoken of as ovulation. The blood vessels are noted to separate at the stigmal line so that ovulation or the discharge of the fully-formed yolk into the ovarian pocket should occur without hemorrhage. When derangement of this part occurs, and hemorrhage occurs, then 
there will be a blood clot on the surface of the yolk which is objected to by the consumer.

The yolk has its origin in a minute microscopic sphere containing a nucleus as shown in Fig. 14, letter a. This is a typical cell with its nucleus, nucleolus and protoplasm. The nucleus marks the point of development of the embryo chick

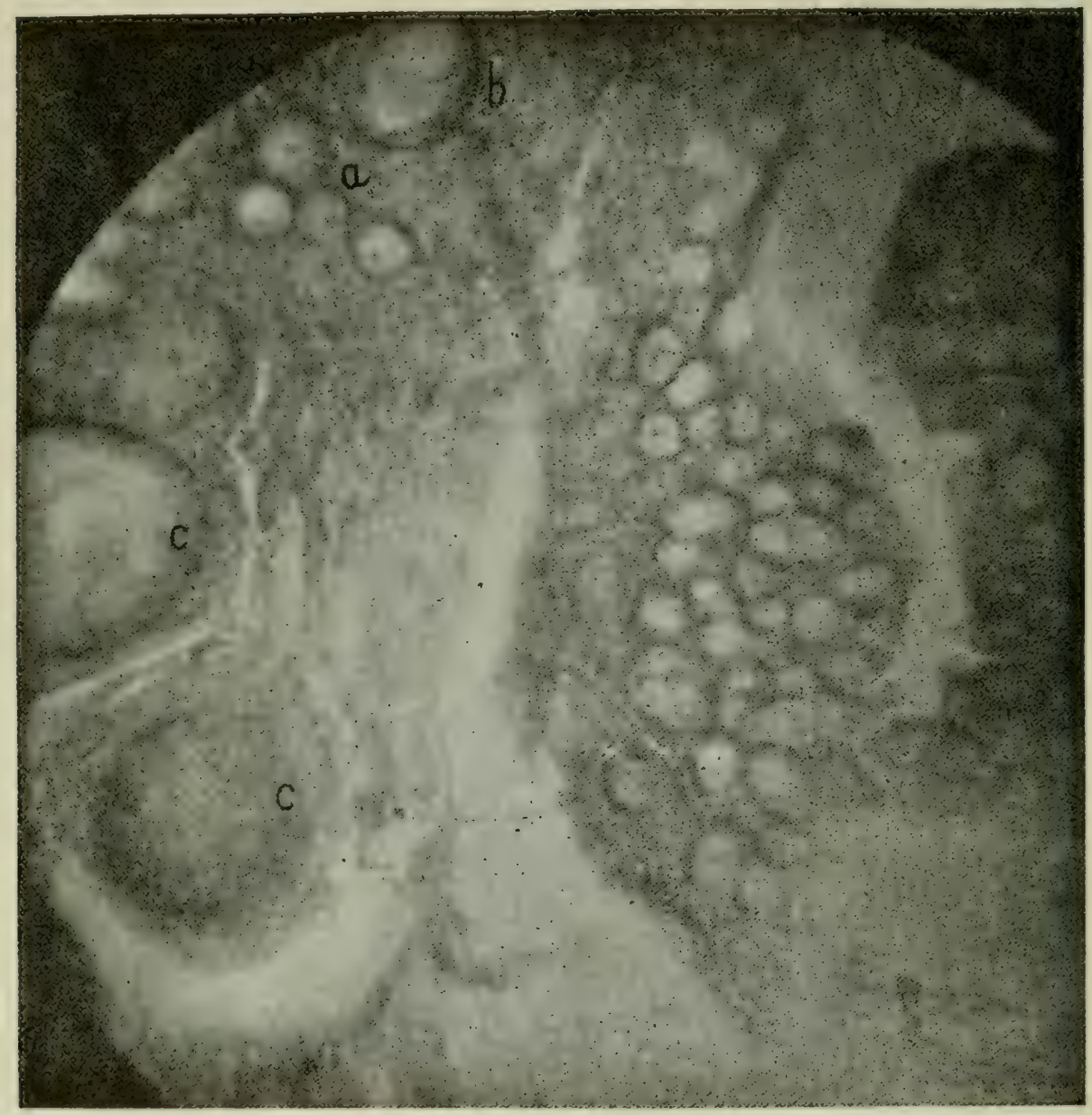

Fig. 14. A Photomicrograph of a Section of an Ovary of a Hen. a, undeveloped ova showing their nuclei; $b$, an ova in which deposits of yolk has begun; c, a section through the center of the developing ova showing the germinal vesicle.

after fertilization by the spermatozoon or male element. The nucleus is located in the central part of the cell. When the coll begins the development of the yolk there is noted first a deposit of fine yolk granules around the central nucleus. These granules of yolk material gradually extend toward the cell wall. This deposit is known as the latebra or flask-shaped mass of white yolk, forming thin layers of yellow yolk.

Later, when the ovum has reached the size of about 0.66 
millimeters in diameter, the nucleus occupies a position just under the vitelline membrane and at the end of the flaskshaped mass, as seen in Fig. 15, letter a.

Still later there are formed several layers of yellow yolk deposited around the central mass of white yolk, apparently brought about through the secretion of the peripheral layer of protoplasm.

The male element or spermatozoon is seen in Fig. 18, letter a. The spermatozoon makes its way by aid of its terminal flagellum or tail through the oviduct and fertilization takes place as soon as the yolk is discharged into the ovarian pocket and oviduct. While as many as fourteen spermatozoa have been found to have penetrated the germ cell, only one spermatozoon is utilized in this fertilization process. The body of the spermatozoon becomes the male pronucleus which unites with the female pronucleus and thus fertilization takes place. The balance of the spermatozoa perish.

After the yolk passes into the oviduct, albumin is formed around it in the second, or upper portion, by specialized columnar epithelial cells.

The contraction of the muscles of the walls of the oviduct forces the contents along. When the albumin formation is completed, the newly-forming egg passes into the isthmus or third portion, where, through the activity of other specialized cells, two membranes are formed around the mass.

In the lower portion of the oviduct, Fig. 13, letter $\mathrm{f}$, the calcium layer or shell is formed to protect the delicate mass within from external violence. In the fifth portion of the oviduct or vagina, the tint is placed on the outer portion of the shell, if the egg is a tinted one.

The formation of albumen around the yolk in the upper portion of the oviduct is probably accomplished in about three hours. The membranes surrounding the egg mass are formed in the isthmus in about the same length of time. The formation of the shell and the expulsion of the egg will be accomplished in from twelve to eighteen hours.

The yolk is of less specific gravity than the alloumen and therefore it gradually rises to the surface with the blastoderm uppermost; if allowed to remain, the blastoderm or germ may, in the course of a few weeks, become adherent to the egg membrane and cause death of the embryo. However, we have found by experiments that this will not take place in the twenty-one days in which eggs are likely to be kept for hatching purposes. In fact, eggs should be set just as soon after being laid as possible as hatchability gradually goes down after the 
fourth day and rapidly so after the twenty-first day. If possible eggs should be set before they are ten days old.

There is just as high production of eggs from an individual without the services of a male as with such service. In other words, the spermatozoa have no influence on the rate and number of eggs developed, they have to do with fertilization of the germ only.

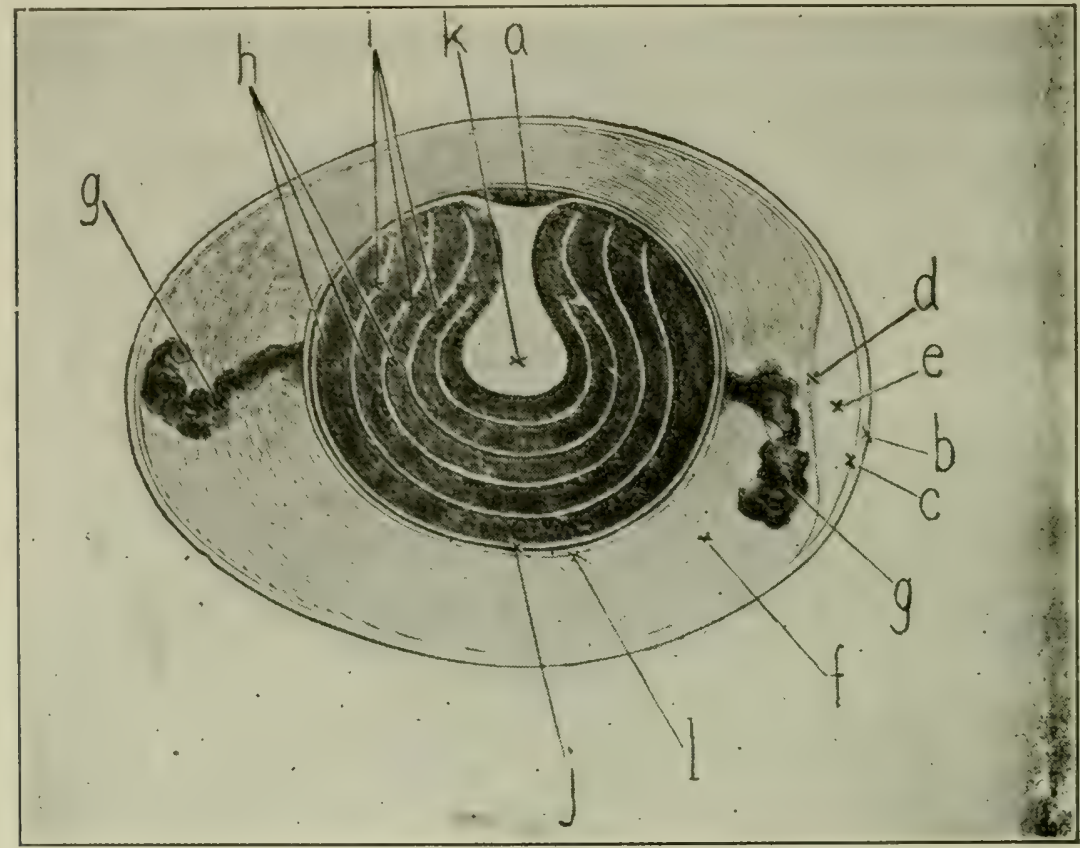

Fig. 15. Diagrammatic Structure of the Egg.

a, blastoderm; b, shell; c, outer shell membrane; d, inner shell membrane; e, the air cell at the large end; $f$, albumen; $g$, chalaza; $h$, dark yolk material; $i$, white yolk material; $j$, vitelline membrane; $k$, flash-like white yolk the latebra; 1 , a thick fluid albuminous layer which immediately surrounds the yolk.

The normal egg consists of an outer shell coating giving it a gloss or the so-called bloom, which may be considered as a protective coat. The shell consists largely of lime salts. See Chapter XV. The two shell membranes are designated as the outer and the inner shell membranes. The inner shell membrane is located just inside the shell, to which it is closely adherent. At the time the egg is laid, the egg material fills the entire shell space, but as the egg cools from body temperature, which is about $107^{\circ}$ Fah., the inner shell membrane dips across at the large end of the egg, forming an air cell or air space. The balance of the membrane is intimately adherent to the outer shell membrane. The air chamber becomes larger as the egg becomes older and evaporation of moisture from the egg takes flace. Also in incubation, the air chamber becomes 
larger as incubation goes on, in order to meet the respiratory needs of the developing embryo or, as we may say, the fetus, as the head is always at the large end if the egg has been kept on its side during incubation. The shell membranes consist of a fibrous structure, the fibers of which extend in all directions. The albumen and a portion of the yolk become appropriated for the formation of the embryo chick, as well as some of the calcium or lime salts from the inner surface of the egg shell.

Immediately surrounding the yolk there is a dense layer of albumen and outside of this a thin or less dense layer.

The albumen at either pole of the yolk contains a long mass of clense and partly twisted albumen, apparently adherent to the vitelline membrane or yolk sac, and to the inner side of the inner shell membrane by the other end. By some authors, the chalazae have been regarded as stays, which to a certain extent, prevent violence to the delicate structures within the central part of the egg.

The albuminous portion or white of the egg consists of 86.2 per cent water, 12.3 per cent protein, 0.2 per cent fat and 0.6 per cent ash and possesses a caloric value of 250 . The egg yolk consists of 15.7 per cent protein, 33.3 per cent fat, 1.1 per cent ash and 49.5 per cent water, with a caloric value of 1705 .

The hen egg corresponds to the ovum developed in the ovary of the female of higher animal life, where, after fertilization of the ovum, development of the fetus takes place normally in the uterus of the mother. The ovum of mammals is made up of a male and a female pronucleus as in the hen egg and protoplasm and deutoplasm. The deutoplasm being nutrient material for the embryo till it has developed sufficiently to draw on the nutrients of the blood of the mother's uterus. In the case of the bird, there is no uterus in the sense that we speak of it in higher animal life, hence no uterine placenta, because there is no fetus developed in the bird, but to take the place there is stored up an abundance of food, taking the place of the deutoplasm and material nutrients of higher animal life. The embryo of the bird develops outside the body, in the egg shell, and is surrounded by three similar membranes as found surrounding the fetus as it developes in the uterus of the female mammal. These membranes serve as a means of exchange of gases and for absorption of the nutrients the eggs contain and from which the embryo chick is developed. Nature has been elaborate in storing up food for the embryo and the baby chick, for the yolk is apparently almost wholly 
intended to be drawn upon as food for the first seventy-two hours of the chick's life after emerging from the shell. An examination of a newly-hatched baby chick will show this yolk in the ahdominal carity and much still unabsorbed. About 47 per cent remains in the abdominal yolk sac at hatching.

The active and functionating oviduct is a rather large, tortuous tube, varying in size and length according to the size of the hen and filling a large part of the left half of the abdominal cavity as illustrated in Fig. 13. In a single comb Rhode Island Red pullet weighing six pounds and producing an egg a day, the oviduct measured twenty inches in length. In a White I'yandotte pullet weighing five pounds and whose ovary and ovicluct had not yet become active, the oviduct measured but five inches in length. An active ovary and oviduct is illustrated in Fig. 13, and an inactive ovary and oviduct is seen in Fig. 12. When a hen goes from a laying to a non-laying period, the ovary absorbes the material of the developing yolks not yet discharged and the ovary greatly shrinks in size. The oviduct also greatly shrinks in size, however, in neither case do they quite reduce to the size they were as a pullet. It has also been found that the intestines increase 30 to 40 per cent in bulk when a hen goes from a non-laying to a laying condition. Igain shrinking when the laying period ceases. As a hen goes from a non-laying to a laying period, as a result of the increased bulk of intestines and reproductive organs, the keel is forced downward and the lateral sternal processes are forced downward and outward, and the pubic or lay bones become wider apart. This is for the purpose of giving greater capacity and hence we take advantage of these increased measurements and use them in judging the laying qualities of the hen. With this crowded condition of the laying hen, it can be readily seen that in a very fat hen, with the intestines, liver and other organs and a functionating ovary and oviduct, as illustrated in Fig. 13, the abdominal cavity would be crowded. When this crowded condition arises, there may be a partial or a complete cessation of the function of the ovary and oviduct, and the hen ceases laying.

The oviduct originates at the anterior portion of the abdominal cavity, Fig. 12, letter c, by an expansion at the ovary in such a way as to receive the yolk when it is discharged from the yolli sac of the ovary. This portion is anatomically known as the funnel, ostium abdominale, or infundibulum. The oviduct may be divided into five parts, as follows: First, tne funnel or infundibulum; second, the albumen secreting portion; third, the short constricted portion known as the 
isthmus; fourth, the shell gland portion or uterus, and fifth, the vagina. The vaginal or posterior portion of the oviduct is guarded by a rather well-developed sphincter muscle. The oviduct is attached to its surrounding structures by two ligaments, one situated above called the dorsal ligament, and one located below called the ventral ligament.

The oviduct consists of three main coats, namely: an external serous, a middle muscular being made up of an outer longitudinal and an inner circular layer, and an internal mucous coat which is thrown into folds, both primary and secondary, and provided with columnar epithelial cells. The oviduct has great power of dilatation, but tears easily if traction is too much in one direction. A rupture of the oviduct sometimes occurs.

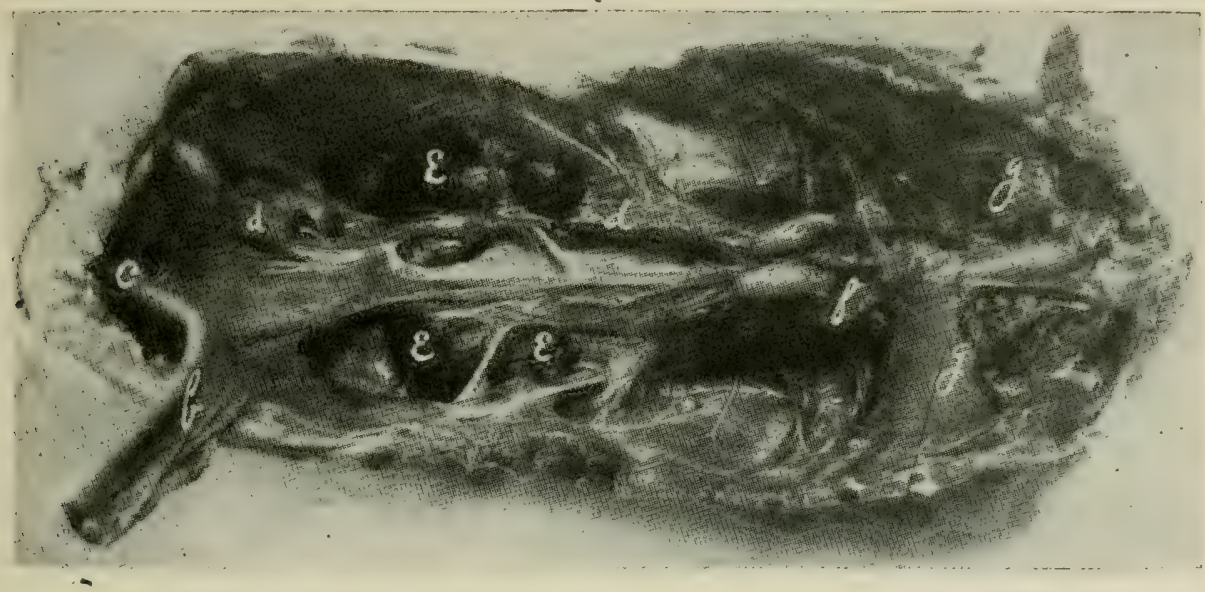

Fig. 16. Generative Organs of a Cockerel.

$a$, testes; b, rectum cut and turned back; c, cloaca; $d$, vas deferens; e, kidneys; $f$, adrenal glands; $g$, lungs.

\section{THE MALE REPRODUCTIVE ORGANS}

The generative organs of the male fowl are the testes, two in number, with the ducts which lead from them, the ras deferens.

In the cockerel, before sexual maturity, which is denoted in physical appearance by the male bird crowing and well development of the comb and wattles, the testes are very small, measuring only about one-half inch long and scarcely one-fourth inch in diameter. They resemble, in shape, a navy bean and are yellowish-white in color. Fig. 16 illustrates the testes at a and at d may be seen the undeveloped vas deferens, sometimes called the seminal tubule.

As the male bird becomes sexually active, the testes develop to enormous size, measuring two inches in length and seven- 
eighth inch in diameter, as illustrated in Fig. 17, which is from a Single Comb White Leghorn cock one year old.

The testis is made up of two parts, the globus major and the globus minor, the latter being rudimentary. The globus major forms practically all the bulk of the testis. The globus minor or epididymis, is short and from it originates the vas deferens as shown in Fig. 17, letter d. The testicular tissue is made up of fine interwoven sperm canals, united by a web of connective tissue. The canals are broad. They are the secreting tubules in which are formed the spermatozoa, Fig. 18 , and a quantity of fluid in which the spermatozoa are trans-

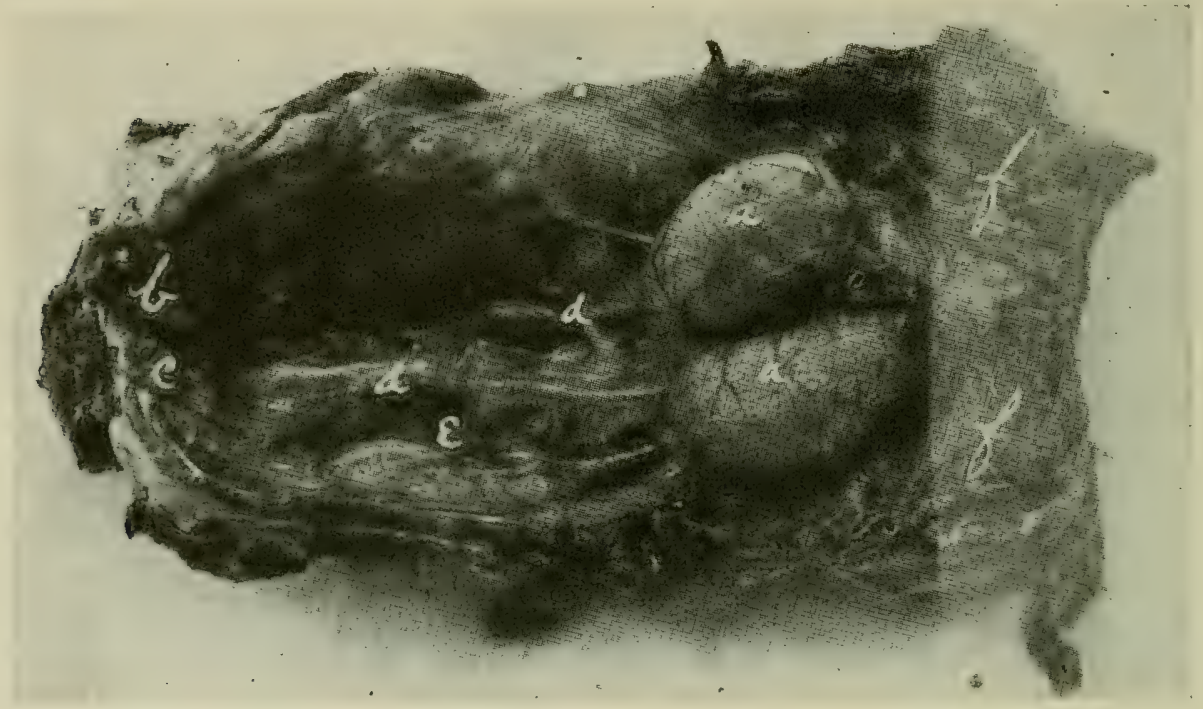

Fig. 17. Prelvic Organs of a Cock Showing the Fully Developed and Active Testes.

a, testes; b, rectum cut and turned back; c, cloaca; d, vas deferens; e, kidneys; $f$, lungs.

ported and an internal secretion which gives to the male his masculine appearance.

The left testis is usually larger than the right. In wild bircls they increase in size during the breeding season and shrink again out of breeding season.

The testis is surrounded by a delicate thin membrane, which is very vascular, as is shown in Fig. 17, letter a. The testes are located just back of the lungs in the region of the adrenal glands and below the anterior part of the kidneys and between the three last ribs. They are attached by means of loose connective tissue to the alodominal aorta, veins and bodies of the vertebrae.

The tube carrying the fluid or semen from the testes is 
called the vas deferens and originates in the globus minor or epididymis, which is very short and is located on the upper and inner surface of the testis and extends backward, attached by connective tissue to the roof of the lumbo-pelvic cavity and to the inner side of the kidney. This tube, at first small, gradually becomes larger and is tortuous as it reaches the cloaca. It empties its contents at the summit of a small eminence in the cloacal mucous membrane.

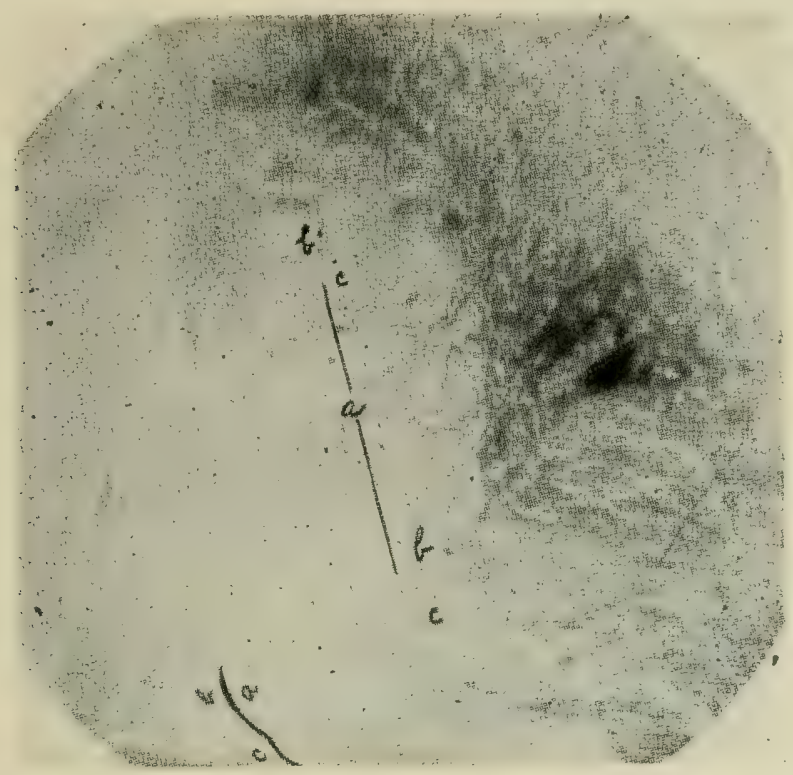

Fig. 18. Photomicrograph of Spermatozoa From a Smear From Fluid of the Vas Deferens

of a Single Comb White Leghorn Cock.

$A$, the spermatozoa; $b$, the head; $c$, the tail.

That an internal secretion is manufactured in the testes is proved by the physical changes which take place after the testes are removed. The bird loses his vim, energy and masculine appearance and ambitions and becomes sluggish, lays on fat and is hated by males and females alike. It often shows some femininity in that it will take a brood of chicks and mother them. The meat becomes more tender and more palatable. In short, there is the same change that is noted in other animals that have been castrated.

\section{THE URINARY SECRETION}

The urinary secretion or urine is formed by the kidneys. The kidneys are elongated and lobulated, measuring 2.5 inches long in the average size adult fowl and occupy irregular cavities in the lumbo-pelvic roof. Each kidney has three distinct lobes. Each lobe is divided into lobules. The minute tubes 
carrving the urine are called uriniferous tubules, which terminate on the surface of the kidney, forming the ureter. The ureter extends along the under surface of the kidney, receiving the contents from the various tubules which empty into it. The ureter empties into the cloaca. The kidney tissue is very soft and of a reddish-brown color. There are two kidneys.

The urinary secretion or urine of the fowl is very thick and at times pasty or creamy in consistency, filling the ureter lumen. The salts are abundant. In many cases, the material solidifies on exposure to the air in less than one minute. The salts dry on the outer surface of the droppings and appear as white. chalky material.

The urine of the fowl in reaction is acid to litmus. 


\section{SANITATION}

Where any considerable number of birds are brought together on limited ground, disease is certain to appear among them sooner or later. The greater the number of birds kept on any given area, other things being equal, the sooner disease will appear, the more rapidly will it spread, and the greater will be the loss from it.

All intelligently directed measures to prevent or delay the appearance of disease in a flock, all sane measures to limit its sprearl and encompass its eradication, constitute sanitation. Neasures, the purpose of which are to cure the sick birds or relieve suffering, come under the head of therapeutics or administration of drugs.

On farms of considerable size, where attention is given chiefly to general crops, and but few fowls are kept on a practically unlimited range, the loss from disease may be small, where indifferent or even bad sanitation prevails; but in intensive poultry plants, where the number of birds is large for the size of the range, there can be no continued exemption from devastating epiornithics, if reasonable sanitation is not carried out. Any attempt to operate such a plant in insanitary buildings and yards, or under conditions that do not permit of sanitation, while it may succeed for a time, will result in loss of tener than otherwise, and in the end, must inevitably fail.

\section{SITE FOR THE POULTRY PLANT}

A rolling, or even moderately steep, plot of ground is desirable for the location of the poultry houses and runs for the fowls. Good drainage is a necessary requirement and must be provided for artificially if the location is such that natural drainage is not perfect.

The surface of the poultry yard must be free from unevenness, so that water will not collect in small pools. Dust bath places or "waller holes" must be kept filled.

The poultry runs and buildings should have a free exposure to sunlight, though some shade must be provided for protection during excessively hot summer days. For shade, trees are the coolest on account of evaporation of watery vapor given off from this form of regetation. The trees best suited 
for poultry runs are figs, china berries, mulberry, willow, plumbs and peach. Birds must have shade in excessively hot days or they suffer and this may lower resistance and disease appear. Tin should never be used for shade as it deflects heat more than boards and best of all improvised shade can be made of a frame on which has been stretched burlap. Shade should always be on a grassy plot. In cold climates, if the house can be placed so that it will be protected from cold winds by other buildings, a hedge, or an orchard, it is advisable to do so. The house should not be placed where it will be shaded at all times as it is likely to become damp and unhealthful.

Soil best suited for poultry runs should contain a goodly proportion of sand. It is very desirable that it be of such a nature that the run will not readily become muddy during wet weather, and such that they will dry very quickly after rains. The runs should be thoroughly grassed over, and the kind of grass will depend on the location. In some sections bermuda grass will be found very satisfactory and a sod can be held where the number of birds do not exceed one to fifty square feet. In other sections, blue grass and clover, or alfalfa can be used where the area to hold a sod will amount to about 150 square feet per bird. In England, the practice is to keep but 100 breeding birds or growing chicks to the acre of pasture, and in the case of breeding hens the houses are fenced and cows used to pasture the ground. The houses must be widely distributed over the pasture with one house and only 100 fowls on each acre. If the run is too limited to hold a sod, then the double yardage system can be used and one yard sown in rape, cow peas, or oats, depending on the season, while the other yard is being grazed. Rye, wheat or oats is best for winter pasturage, rape in the spring and fall when plenty of moisture is present, and cow peas in the more dry part of the year. The continued ploughing or spading up of the ground and the growing of crops has a tendency to use up the organic matter or droppings deposited on it and a tendency to prevent the ground from becoming fowl-sick. If a contagious disease appears it is often necessary to change to new quarters and even dispose of the old flock for food and start a new flock. This has been found necessary in such diseases as fowl cholera, blackhead and paralysis of adult fowls.

\section{THE BUILDINGS AND RUNS}

Fowl must be provided with green feed the year round and the green feed of the runs either in the form of crops or of 
grass will furnish this in the warmer months of the year, besides helping to keep the yard pure. Animal food and exercise is also essential. The exercise can be provided by keeping litter in the house and throwing the grain feed in the litter, make them exercise for it. The animal food on the farm can in part be supplied in the summer time by allowing the birds to range over the farm. Some of the smaller breeds as the Leghorns, will forage for a quarter of a mile in search of waste grain, bugs, insects and other waste products which she converts into growth or into eggs. No more than fifty to one hundred birds should be kept in one flock on the farm and these allowed to run out into the fields of larger crops as corn, cotton, tobacco and sugar beets. Commercial flocks sometimes consist of 500 birds to the unit, but so many birds in one flock running over a farm will kill some of the vegetation or crop closer to the building. A poultry house of portable style can be moved from one part of the farm to another as the crops are rotated from small grains to larger grains. In this way, young growing birds, and breeding and laying birds, can be scattered over the farm, protecting the crops against bugs and insect pests, improving the fertility of the soil, and making a return in eggs or in growth of the waste grain, bugs and insects. It has been shown that fruit trees and nut trees of all kinds are protected by fowls running in the orchard. The fowls devour the insects and worms that are harmful to the trees and at the same time furnish themselves with needed animal protein. Fifty birds can be kept with very little extra feed on a four-horse farm living, for the most part, on the waste around the barn and fields. The same protection is furnished to the smaller fruits as grapes, currants and gooseberries. Birds may be kept among these shrubs except in the season of the development of the blossom and fruit. Fowls rid stubble fields such as wheat and oats after the crops have been removed, of bugs and other insects, and thus make more favorable the growing of another crop the succeeding year. Often the youngsters on range can be used for this purpose. housing them in portable poultry houses as stated above. These houses have sled runners or they may be mounted on low wheels, and are easily moved from place to place. Grasshoppers are gotten rid of by this means. At the same time. the fowls are provided with feed and make satisfactory growth and thus profit. The slogan "fence the garden and not the fowls," should be carried out.

All modern poultry houses are provided with an open front. This open space should be at least thirty inches wide and nearly 
as long as the house. In cold countries, where it is necessary to licep the birds shut up for a considerable time each winter, a window should be provicled to give light while they are shut in the house. In this sort of climate the open front is provided with a (lrop) curtain made of burlap or ducking to keep out the wind and much of the cold, but at the same time always insure proper ventilation. The house should face the south, so that the sun can gain access to the interior at all times. The ground should slope from the house so that water does not accumulate around the building. The floor may be made of dirt, cinders, gravel, crushed rock, boards or of cinders. Cement is the most sanitary and advisable, but is the most expensive. If of cinders, there is needed ten inches of cinders or crushed rock as a base and for underdrainage to ensure a dry floor surface. On top of this base, there is placed from two to four inches of rough concrete made of one part of cement to seven of crushed rock and sand. On top of this rough concrete there is placed one-half to one inch of finish coat made of one part cement to two of sifted sand. The surface coat should be troweled out smooth. On top of this is placed the litter in which the birds scratch for their grain feed. The cement floor top is above the ground so as to ensure good drainage from the top of the floor. Birds cannot be successfully housed in buildings with wet floors. Nothing reduces the resistance of the bird so quickly as a wet floor from which cold damp vapors arise. Birds are a dry weather individual. If underdrainage is not provided as outlined above, then the floor during rainy times will be wet and is a fruitful source for such diseases as roup.

If the floor is of dirt, gravel, or cinders, it should be several inches above the level of the outside ground surface and the ground should slope from the building. If of cinders or rock, they should be thorcughly tamped. Dirt is objectionable because the birds make dust baths and the surface is kept more or less uneven. Litter on such a floor is objectionable because of the dust that arises when the birds are scratching for their grain. Birds without this exercise do not lay as well as with exercise. Board floors are best constructed about eighteen inches above the ground and made of tongued and grooved flooring. The north, east and west sides should be boxed so that the cold winter winds cannot sweep under the house and make the house colder.

The moderin poultry house equipment includes a removable (ropping hoard huilt horizontally and about thirty inches above the floor. The removable dropping boards allow of their removal so that they can be sprayed on all sicles and thus rid 
of mites and infection. The perch poles are located horizontally above the dropping boards and about fourteen inches apart and ten to twelve inches from the back wall of the house and the same distance from the front of the dropping boards. The perch poles should be about ten inches above the dropping boards. At one end of the dropping boards is built a broody coop in which to break up the broody hens. The nests are constructed according to the size of the breed of birds. For the small breeds as the Leghorns, twelve inches square is of right size, and for the utility breeds, as the Reds, Rocks or Wyandottes, the nests should be fourteen inches square and for the larger breeds, as the Brahma, the nests should be sixteen inches square. The nests are placed either under the front end of the dropping boards or at one side of the building and above the floor. By this arrangement the entire floor is available for scratch material such as shredded stover, straw or leaves, and in this scratch material the grain ration is thrown. During the hot part of the summer, ventilation is needed in the back of the house so as to get a draft through the house. In the winter time, this ventilation is closed and ventilation is then by diffusion and the cold air moving through the house slower makes a warmer house. The back ventilator is constructed high enough so that there will be no draft on the birds as cold damp drafts lower the birds' resistance and such diseases as affect the respiratory tract may appear. Among these diseases are swollen eyes, colds and roup.

In constructing the poultry house, except for the open front and back ventilator, the walls and roof are made tight, so that no wind or drafts come through and rain is kept out.

Sunlight and especially sunshine in the building ensures a dry house, which is essential to the general health of the birds and also makes an unfavorable place for the parasites and bacteria. It has the advantage also of revealing filth in the building which might otherwise escape the eye of the attendant, and remain to breed disease in the flock.*

The scratch material or litter in which the grain is thrown is usually cleaned out once every three months, at which time the house should be thoroughly cleaned and disinfected, using a four per cent solution of some standardized coal tar disinfectant dip, such as Zenoleum, with a spray pump. Whitewash gives a clean appearance to the house and also gives longer feeding hours to the birds as the building will not become dark as soon as otherwise. This is worthy of con-

* For further study of poultry house construction see Poultry Culture Sanitation and Hygiene by 13. F. Kaupp, I'ub. by W. B. Saunders Co., Philadelphia, Pa. 
sideration by the poultryman since we have found that the length of feeding hours is a factor in egg production since the longer the feeding hours, the more mash the hen will consume, and the more mash the hen consumes the greater number of eggs she will lay, provided it is the right season of the year for her to lay and she has been bred for high egg production. Poultrymen object to whitewashing poultry houses and it has practically gone out of use. They argue that lime is hard on the feet and legs and that particles flying in the air may get into the eyes of the birds and cause inflammation of the eye. Lime in a dry state has no destructive action on mites and lice so that from that standpoint we will not consider it. Lime whitewash then can be considered from but two standpoints, that is, in making a lighter room for the birds, and in making a clean white appearing wall.

The perch poles should be made proof against mites. This can be done by giving them, occasionally, a coat of creosote or pure Zenoleum. New perch poles will need about three coats about one week apart and after that time once or twice during the warm weather will make them mite-proof. Kerosene or a four per cent solution of any standardized coal tar disinfectant dip will kill all the mites it gets on but it will not keep the mites away. As soon as the perch pole dries, the mites can swarm over them again. The perch pole should be free from cracks and crevices. It is better to swing the perch poles by chain from the ceiling so that they do not touch the wall or any part come in contact with the dropping boards. Dropping boards should be cleaned once and better twice a week. Mites multiply in the droppings as well as in cracks of the perch poles and nests and when in large numbers may be found swarming all over the house.

\section{IMMUNITY OR NATURAL RESISTANCE}

Immunity literally means proof against disease, that is, it is the name of the condition that enables a fowl to resist the action of pathogenic or disease-producing germs, or to be unaffected by their products. Immunity is only a relative term, the condition is not absolute and permanent, neither is it constant and continuous.

Whenever a bird is unable to adjust itself to its environment. it becomes susceptible to the effect of the environment, it becomes susceptible to the effects of the causative agents of disease, that is, its immunity, at least acquired immunity, is suspended. 
The term acquired immunity is ordinarily used in reference to infective diseases, that is, diseases caused by germs, or bacteria, those diseases resulting from the invasion of miscroscopic parasites called germs; although it may be used in designating the resistance to the action of zootoxins, or animal poisons, such as snake venom and possibly, also, of the poisonous substances ejected by centipedes and scorpions, as well as the vegetable poisons, ricine, abrin, crotin and robin, obtained from the proteins of grains.

Immunity or proof against disease is then of two kinds: acquired and natural.

Acquired immunity is a resistance in the form of antitoxin built up in the body as a result of being vaccinated as in the case of fowl cholera, or of sore head; or of a bird that has passed through an attack of the disease.

Natural immunity is a resistance resident in the body of the bird from hatching time or without being the result of a stimulus as in the case of vaccination, or of the bird passing through an attack of the disease.

When a bird recovers from a contagious disease it does so by the fact that the cells of the body of the bird are stimulated to form an antitoxin as a result of the injury done these cells by the toxin or poison given off by the germs causing the disease. The bird then does not again, or at least for a considerable time, contract a second attack of the same disease. Each kind of disease producing germ causes a formation of a protective substance by the cells which will kill that germ and none other. In every outbreak of contagious disease we note some birds that do not contract the disease. These birds have a natural immunity or resistance. We also note that where birds have poor sanitary surroundings contagious diseases are more prevalent; that is, the natural resistance of all birds is lowered. All birds have more or less natural resistance but the susceptible ones, that is, those that contract the disease have less resistance than those that do not contract it under good sanitary conditions. A good example of lowered natural resistance is given in the classical test for anthrax germs on fowls and is as follows: The fowl is naturally immune to anthrax germs and if in good physical condition will not develop anthrax after being inoculaterl with the germs. But on the other hand if the bird be subjected to a chamber kept at a high temperature for several hours and inoculated with the anthrax germs it will clevelop the disease. In this treatment the natural resistance is lowered. The bird contracts the disease on account of having 
its natural resistance lowered by being kept under unnatural surroundings.

Insanitary buildings will have a tendency to lower the average resistance of the fowls and favor the attack by contagious diseases. Such conditions as dirt floors allowed to become uneven and below the level of the outside ground allowing pools of water to accumulate and the floor becoming wet and remaining so after protracted rain. The dirt constituting the floor becoming foul and stinking by not being cleaned for several months at a time and no replenishing of the top soil. Perch poles placed at an angle from front to back instead of horizontal, with no dropping boards to catch the droppings. Such dirt floor is invariably wet and many times we have found leaking roofs so that the water ran down into the house during a rain. The sides and back as well as the top had large holes between the boards. These cracks allow drafts upon the birds and this lowers their resistance and impairs their health. Under these conditions resistance to disease is sure to be lowered and the birds may become sick at any time. When contagious disease attacks such a flock the death rate is always greater than when kept under proper hygienic conditions.

\section{HEALTH OF THE BABY CHICK AS INFLUENCED BY INCUBATION}

The question which has exercised the minds of poultry breders ini all countries where the poultry industry has made considerable progress, and also of those engaged in investigation, is the enormous embryonic mortality, or "dead in the shell" chicks during the period of hatching. Such observations are of especial interest to those who are hatching on a large scale and use incubators almost exclusively. It has been stated that such embryonic mortality is in the neighborhood of 40 per cent, of fertile eggs, taking a whole season's breeding. That cannot be regarded as satisfactory. It may be due entirely to the fact that incubators are second best, and that artificial methods are entirely distinct from that of the hen. If so, we will need find how far it is possible to remedy such a state of affairs. We know that the heat as applied to an egg from the body of a hen is by contact and totally different to that of an incubator. One is by actual contact and the other is by radiation. In one experiment eggs all from one breed and one flock were taken. The eggs hatched under hens had only 10.7 per cent dead germs, and the other eggs from the same stock placed in an incubator had 33.4 per cent dead germ in the shell.

A flock of chicks hatched by an incubator using a water circulation method of applying heat, in which the water pipe 
leaked to an extent that too high a humidity attained, resulted in all such chicks being weak. This weakness retarded growth and produced a flock of chicks showing weakness in constitution throughout their entire growing period and the pullets coming into laying were weak as a result of the defect in incubation.

Chicks hatched under hens irregular in attention to their nests and in incubators that are not held at a constant temperature are weaker than where incubation has gone on properly. Such chicks are puny and readily succumb to improper care or to abuse as being run over and knocked about by stronger chicks. An incubator that has carried a temperature at one time 95 degrees, another 105 degrees, and still another 103 degrees, will have more embryos die in the shell after the 18th day and the chicks will be weaker and this weakness will be reflected through their developmental possibilities. In many cases the chicks die while pipping out of the shell. This is due, in part, to undue drying of the shell. The hen places on the egg shell a very light coat of oil which is rubbed off of the skin of the hen and this prevents the shells from becoming too dry. Best results from a health standpoint in hatching is a temperature of 103 degrees Fahrenheit throughout the hatch. Eggs should be turned at least six times a day and even more will be beneficial as a ten turns her eggs about nine times a day. This prevents the adherence of the fetal membranes, a fertile cause of cripples and a source of injury to the yolk sac and death of the chick. Chicks at hatching have 47 per cent of the egg yolk in the abdominal yolk sac and should not be fed till the chick is seventy-two hours old as this abdominal yolk acts as a food for this length of time. It is noted that where chicks are thus handled they are stronger and healthier and do better and more of them are reared. The first day the chicks are taken out of the incubator they should receive only sour milk or butter milk. The second day they should receive the same kind of milk and two small feeds of wet mash and the third day may be placed on full feed. Injury may be done by being over kind in the feeding of baby chicks. The three days supply of a perfect food in the abdominal yolk sac makes shipping of baby chicks long distances possible and the chicks arrive in good physical condition. 25 chicks to the compartment, is all that should be allowed. Too much crowding lowers their vitality or physical resistance.

Never allow baby chicks to become chilled. Chilling for only fifteen minutes may result in a fatal bowel trouble. Diarrhea will appear in three of four days after chilling and the diarrhea 
due to chilling usually appears from the tenth to the fourteenth day after hatching.

That diarrhea leaves the chick in a weakened constitutional condition is shown by the fact that in one flock the chicks suffering from liarrhea weighed, as an average, at the end of eight weeks, 0.28 pound each, and in the same flock those chicks not affected with diarrhea averaged 0.47 pound each in weight. In another flock those chicks that had an attack of diarrhea weighed, at the end of eight weeks, 0.56 pound each and those not attacked by diarrhea weighed as an average 0.74 pound each. In these two tests all birds were Single Comb White Leghorns and all of one flock.

\section{THE EFFECTS OF ATMOSPHERIC SURROUNDINGS}

The health of birds suffer and their resistance is lowered if the poultry house is located on low flat ground where the mists, fogs, and vapors settle and remain. The currents of atmospheric moisture naturally follow down the slopes of land and come to rest in the inclosed valley and swamps. In these places one notices at night the cool, and often chilly, moist, heavy air which has settled and stagnates there. When fowls are required to live in such stagnant air saturated with moisture they tend to become mopish, debilitated indicating a lowered resistance and readily succumb to roup, sore head, and other devastating diseases.

The ground selected for poultry yards therefore as pointed out above should be high and dry, slope to the south, and have good drainage, and preferably gravelly or sandy soil. Sickness lurks where stagnant, saturated, foul soil exists. Such yards dry off soon after rains. Where grass or some vegetation is not grown to take up the organic matter chicks are likely to become sick and mortality run high and also these conditions are unhealthful for adult fowls. Another thing that is essential for the health of birds, and that is fat soluble vitamine. Since fat soluble vitamine is found only in certain organs of animal life as the liver, kidneys, blood, and in animal and bird food elaborated for their young, such foods as eggs and milk. and in the leafy vegetables, it is necessary for birds, for the most part, to secure this substance from green feed though it is possible that fish meal, and meat scrap contains some of this substance. This green stuff grown upon the yards will make good this shortage. For good health of fowls not only must the birds receive a sufficient quantity of fat but also of water soluble vitamine, but also sufficient linds and amounts of minerals, a ration not excessively acid, protein high in certain 
amino acids, and of proper nutritive ratio, or to put it another way, a certain percentage calories protein calories. This varies with the purpose for which the birds are fed, but usually runs between 12 and 16 per cent. Certain minerals are not only an aid to construction of tissues but also to the function of organs of the body.

The health of chicks is affected by the kind of food some feeds being injurious to poultry in any quantities, such as velvet bean meal, either ground beans alone, or beans and pods. Cotton seed meal in quantities over 10 per cent may be injurious to birds and especially to growing chicks and pullets in their first laying year. The injury is greater where the birds are kept in confinement. Environment is a greater factor in the health of birds. The fewer birds in a unit, and the more widely distributed these birds are over pasturage, the more robust health the birds will be in. Inherited weakness of many kinds is a factor in health.

\section{THE WATER SUPPLY}

To avoid great suffering among the fowls in hot weather they require an abundance of water. Eggs are sixty-five per cent water and the flesh is as high as eighty-five per cent water. Birds drink every few minutes during hot weather and if water is not kept constantly before them at all times ill health is likely to result, such diseases as going light appear, growing birds become stunted and laying hens fall off in the yield of eggs.

The water should be fresh and the supply replenished three times a day, and in vessels so arranged that the birds cannot get into them and thus contaminate the water with the filth from the yard carried on their feet. Various kinds of infections and parasitisms are spread in most cases, not by direct contact between the sick and well birds, but, indirectly through the medium of the soil and the roosts on which the birds live, the food that they eat, and the water they drink. Any means of feeding birds in which a contagious disease is present to prevent contamination as in birds stepping into feed or water with their dirty feet, will tend to lessen the chances of other birds becoming infected.

The vessels containing the drinking water should, uncler normal conditions, be thoroughly cleaned and disinfected daily in hot weather, and once a week the remainder of the year. When disease is present in the flock, the ressels for drinking water should be cleaned and clisinfected daily, regardless of the season; and this practice should be continued for several days after all symptoms of the disease have ceased to appear 
in the flock. Vessels containing water for small chicks should be cleaned daily and disinfected.

The cleaning of the drinking vessels is mainly a matter of thorough washing: and the disinfection of the vessels can best be accomplished with a four per cent solution of some standardized coal tar disinfectant dip as Zenoleum. Twelve tablespoonfuls of the dip to each gallon of water will make the required strength.

Fowls tolerate certain antiseptics internally very well and do not resent the taste of them in the drinking water to the extent that other animals do and it is a wise plan to use antiseptics in the drinking water whenever an infectious disease is present on the premises or when the purity of the water is under suspicion.

The most desirable antiseptic to use in the drinking water is potassium permanganate. This is best used by keeping a stock solution. 'To make this stock solution place a quantity of the crystals of potassium permanganate in a large bottle or jar and fill with water; of this solution use sufficient in the drinking water to give it a red color stopping just short of a purple tinge. This solution is best given in enamel ware or in glass or glazed earthern ware as it has an effect upon metal as galvanized iron water containers. More water can be added to the stock solution from time to time, as needed, also crystals, care being taken to keep an excess of the permanganate crystals in the bottom of the jar.

Permanganate of potash may be used to an advantage in water containing a large amount of organic matter.

Other antiseptics such as pure carbolic acid may be used. A one-half of one per cent solution, or five teaspoonfuls to the gallon of water, will meet the requirement. Do not use carbolic acid and permanganate of potash at the same time.

Under many conditions, especially when certain intestinal diseases as those caused by the coccidium tenellum or protozoa, mercuric chloride or corrosive sublimate may be used. Bichloride of mercury is used in a solution of $1: 10,000$. To make this solution it requires six grains of the mercury to the gallon of water. To put the mercury in solution it will be necessary to add three grains of citric acid.

Both mercuric chloride and carbolic acid are very poisonous and must be handled with care. On this account the comparatively harmless potassium permanganate should be used.

Ipecac is also used as an agent against protozoan diseases of fowls. 


\section{DISINFECTION}

The removal or destruction of parasites is called disinfestation and the removal or clestruction of germs is called disinfection. Because of the ability of a single germ to multiply, and a single pair of parasites to multiply, and start trouble again the work of destruction of these enemies must be thoroughly done.

The first step in any disinfection is the removal of all visible filth. A small lump of manure behind a next box or a single grain of dirt in a crack in the floor or on the roosts may furnish the hiding place from which will emerge the parasites or germs to reinfest or reinfect the whole building, and spread disease anew among the flock, thus undoing the whole of the disinfection.

\section{Disinfection of the Buildings}

The first operation in disinfecting a poultry house is the thorough removal of all manure, trash, and litter. If the nests and roost poles are removable they should be taken from the house and thoroughly disinfected. After they have sunned and the balance of the fixtures have been thoroughly cleaned and disinfected they should be returned; if left in the building during the disinfection, they must be as thoroughly cleaned as the remainder of the building, and the disinfectant used must be applied to them as carefully as to other parts of the building.

The floor should be scraped and the walls and ceiling thoroughly swept. All parts of the interior of the building should then be thoroughly scrubbed with water, to which lye has been added, and a broom or stiff brush used and then the building flushed, using plenty of water. The building is then ready for use of the disinfectant.

There are three different classes of agents that may be used in disinfection, but only one of these can successfully be used in the modern poultry house. These three kinds are as follows: First, gaseous form; second, liquid; third, heat.

A gas may be used in disinfecting only when the building can be closed tightly enough to prevent its ready escape. This excludes the great majority of poultry houses; but in such as it can be used, all floors, windows and other openings must be tightly closed and sealed with sealing paper for several hours after the application of the gas.

Of the gases that are used, two may be recommended. These are formaldehyde for germ infection and sulphur fumes for parasites, as mites and lice. 
Formaldehyde is used as a gas. It may be procured at drug stores in a forty per cent watery solution known as formalin, from which gas may be readily generated.

After tightly sealing all openings into the building except the door, place in an earthen or metal vessel, two quarts of formalin for each 1,000 cubic feet of space in the building, place this ressel in a much larger one and set on the floor, then empty into the formalin one-half pound of the crystals of potassium permanganate for each quart of formalin and leave the building at once, closing and sealing the door from the outside.

The temperature of the room, during the disinfection, should be above 50 degrees Fahrenheit, and the more it is above this temperature, the better. Moisture in the air is an aid in this sort of disinfection; it may be secured by sprinkling the floor just before starting the generation of the gas. The building should be kept closed a minimum time of six hours and better still twenty-four hours. The building must be thoroughly aired before the fowls are permitted to re-enter it.

For disinfection with sulphur fumes, the ordinary commercial flowers of sulphur should be used. The sulphur must be burned in the building to generate sulphur dioxide, which is effective in disinfection only in the presence of water vapor; therefore, some means for providing the necessary moisture in the building must be provided. This may be accomplished by spraying the walls and ceiling until they are dripping, just before the beginning of the disinfecting, or by boiling a large ressel of water in the building during the generation of the sulphur fumes.

Fire is required to generate the sulphur fumes and care must be taken not to endanger the building with it. A large iron vessel partly filled with live coals may be used; set the vessel on the floor, or if the floor is of wood, place several bricks under the kettle so that it does not come in direct contact with the wood and endanger the building from fire. Ilace live coals in the iron vessel and pour two pounds of sulphur on the coals for each 1,000 cubic feet of space in the building. Before leaving the building and closing the door be sure the sulphur is burning.

The building should remain tightly sealed for from twelve to twenty-four hours and then be thoronghly aired before the fowls are admitted.

The disinfection of drinking fountains is discussed under "Water Supply:" (See page 65.) Closed drinking fountains 
that cannot be gotten at on the inside to remove slime and to properly scrub with disinfectants should not be used.

Disinfectants that can be applied in liquid form are best suited for disinfecting the ordinary poultry house. It requires longer to apply them than it does to prepare for disinfection by gas, and germs and parasites protected in crevices and in decayed surfaces of the wooden walls cannot be reached as by the gaseous disinfectants. Fowls need not be shut out of the building for several hours, as is the case when the gas is used. This is often a considerable advantage. Furthermore, the germs and parasites hidden in the walls and roosts and buried in the decayed surface of wooden buildings can in a great measure be covered up and rendered harmless by use of whitewash. If whitewash be used, there should be placed in it sufficient disinfectant dip to make four per cent solution to make it destructive to all parasites it comes in contact with. Lime alone is not sufficient.

Liquid disinfectants are best applied with a spray pump, and all the force possible should be used in throwing the spray on the walls. In this way, the liquid should reach all parts of an uneven surface better than when applied with a brush and much time will be saved in its application.

Disinfectants will act more vigorously when applied hot, and solutions should always be at least warm when they reach the surfaces to be disinfected. A copious quantity should be used. The solution may cost but a fraction of a cent, or at most a few cents a gallon, and it is a poor policy to economize by using an insufficient amount. Every part of the surface of the interior of the building should be thoroughly wet and completely covered with the disinfecting solution when the work is complete; great care should be observed that no part is skipped.

There are a great number of disinfectants that may be used in solution for disinfecting poultry houses, but none are superior to the standardized coal tar disinfectant dips. Formalin, for example, is exceedingly irritating to the eyes and respiratory passages of the one doing the spraying. Potassium Permanganate needs to be applied in almost saturated solution to be effective, and thus becomes expensive. A solution of copper sulphate is not fatal to all parasites. Crude petroleum leaves the building unsightly and the odor persists unduly long, and so it is with many others.

Of the coal tar disinfectants, crude carbolic acid perhaps stands at the head on account of its low cost; however, it is quite variable in comparison, because it is not constant in its 
antiseptic properties. It should be used in at least a five per cent solution. Use two pints of crude carbolic acid to each five gallons whitewash. The other coal tar dips are numerous and a dozen or more can be found on the market, each under its own trade name. The dip should have a coefficient of at least 5 to 6 , meaning that its strength is five to six times as great as carbolic acid.

The coal tar disinfectant dip when mixed with water produces a soapy emulsion. The alkalinity of the soap is a factor that assists in its penetration, the soapy emulsion helps to bring into activity the cresols and hydrocarbons, the active principles of the product.

In our tests with coal tar dips of a coefficiency of 5 to 6 , we determined that a four per cent solution will kill mites and lice in about two minutes.

While spraying is in progress, all eggs should be removed from the nests, as the fat of the yolks readily absorb objectionable odors.

Heat is one of the most effective and reliable disinfectants. It may be utilized in poultry houses, after thoroughly cleaning, in the form of a flame from a gasoline torch. Every portion of the walls, ceiling, floor, roosts, nests and boxes must be carefully flamed. This method, though tedious, is effective. Used with ordinary care, it is devoid of danger to the operator or building.

\section{Disinfection of the Yards}

A complete disinfection of poultry yards and runs, that is, a destruction of all the disease germs and parasites and parasite eggs with which the premises may be contaminated by an infected flock, is scarcely possible by the ordinary means employed in poultry house disinfection.

When it is remembered that the germs of nearly all diseases, and the eggs of nearly all internal parasites of poultry are eliminated in the droppings of affected birds, the danger from contaminated runs will be better appreciated, and with the realization that each mature hen produces nearly thirty pounds of droppings a year, approximately one-third of which is reposited on the runs, the importance of the yard as a factor in the spread of disease is seen to be very great.

The problem of having clean, non-infected ground as yards for poultry can be solved by the double yardage system; spading or ploughing and seeding one yard while the other is being grazed, or by having a permanent sod to utilize the 
organic matter deposited on it, or by a change of ground from time to time. Ground, after a while, may become fowl-sick, so that birds do not do well on it and it finally becomes impossible to raise chicks and finally old birds cannot successfully be kept on it. As mentioned before, the movable poultry housc offers many sanitary advantages and solves the problem for the farm. Ploughing or spading the yard, thus exposing surface layers of the soil to the disinfecting actions of the rays of the sun, and keeping the birds off till a few crops have been raised, offers the most practical means of disinfecting it. Growing crops in yards while idle tend to use up the organic matter deposited in the droppings.

The ground can also be aided in purifying itself by applying quite a coat of lime each year, as lime aids in splitting the organic matter and particularly liberates the ammonia. This should be applied just before ploughing the ground.

Where the construction of the poultry buildings is such as preclude a change of location, the two-yard system can in most cases be installed. It offers many advantages; while one yard is being used, the other may be ploughed and a crop grown. This may be a crop on which the birds may be turned for half an hour each evening to allow them a feed of green forage.

In any system of yardage where the area of the ground is small for the number of birds, the yard should receive frequent attention at the hands of the cleaner. If the yard is grassed, and the grass is short, it should be swept weekly, gathering the manure in piles and carting it away, as street cleaners do. A yard that is bare of vegetation can be cleaned in the same way, even more easily and effectually. This will lengthen the sanitary life of a yard to many times its duration without cleaning.

All mashes should be fed from a mash hopper and kept inside the house, or an outdloor mash hopper, which is protective against rain, may be used. The grain feed should be given in litter inside the house so that the birds will have to work two or more hours a day. Without this exercise, the birds will not lay near so well, as experiments have shown that by the sudden removal of the litter and feeding the birds their grain feed where they will have no effort in securing it, results in a sharp declining curve in egg production. Daily exercise is essential for good health. 


\section{DISPOSAL OF SICK AND DEAD BIRDS}

A strict adherence to the rules of sanitation would require that the well birds be removed from the buildings and enclosures in which sick birds are found, or in which birds have died of an infectious disease, and that they be not returned until after thorough disinfection of the buildings and the grounds. Such a procedure is not often practicable, and the poultryman is left the alternative of removing the sick or dead birds from the flock to prevent as far as possible an extension of the infection.

Whenever an ailing bird is discovered in any flock, it should be immediately isolated. An ailing bird cannot take care of itself and will be overrun and imposed on by its mates. When the flock is fed, a moment's pause to check up all the birds to see that they are all eating and apparently healthy is the best plan, and any bird not feeling well can then be given attention by placing it in quarters where the other birds will not molest it and given attention needed and in most cases soon be returned to the flock in good health. Do not wait to discover what is the matter with the bird before removing it from the flock, whether it is an infectious disease or a disease at all, or to decide as to its treatment. The fact that it is not acting normal is sufficient reason to have it removed and an examination made. Remove it from the well birds and decide upon further measures afterward. The same directions apply with equal force to the finding of dead birds among the well ones. Remove the carcass immediately, and unless there is conclusive evidence that death was not due to disease, disinfect the place where it has lain.

Sick birds should be placed by themselves, where they will not be molested by other birds or animals. They should be given as comfortable quarters as possible and be disturbed only for treatment. Unless the poultryman is very positive that he knows what ails the sick bird, and what means should be taken to prevent others in the flock from acquiring the same discase, he will usually find it best to call in a veterinarian for consultation and leave the matter with him; particularly is this true if there are a large number of birds on the premises or if the flock be one of high value, because of pure breeding.

Immediately after removal of the dead bird from the flock. the poultrymen should satisfy himself as to the cause of its death. If it is obviously due to accident or if it is due to some discase already recognized as present in the flock, such action should be taken as the conditions seem to warrant, but 
if there is any doubt as to what has occasioned the death, a careful autopsy should be held. Since a post mortem examination means very little to one without at least some fundamental training in pathology, and a veterinarian is close at hand, the poultryman will ordinarily find it advantageous to take the dead bird to a veterinarian for examination. This should be done immediately, before the changes incident to decomposition have masked the lesions which disease may have produced, or before parasites that may have caused death have changed their location or escaped from the body.

\section{THE MODE OF PERFORMING AUTOPSY}

Lay the carcass on its back, head from you, and remove the feathers from the under sicle of the body. With a sharp knife, open the abdominal wall, commencing close to the anus, passing the knife forward between the ribs and the breastbone to a point just back of the wishbone or clavicle. In a like manner, open the left side, being careful not to injure any of the organs in the cavities. Now grasp the breastbone or sternum, pulling upward, forcing it forward, and it will break so that it will be easy to remove it. This will lay the cavities open so that all the organs can be observed, as illustrated in the drawing shown in Fig. 2, to which refer for further description.

The final disposal of carcasses of birds, whether dying from known or unknown causes, should be carefully attended to. The habit of throwing dead birds onto the nearest manure pile or into an unoccupied field, or in the weeds out of sight, cannot be too severely condemned.

Among many people, there is a belief that if the body of a person that has died is not properly buried, the spirit of the departed will haunt its living relatives, and if they do not heed its warnings, bring great disaster to them. If poultrymen entertained a similar belief regarding the disposal of dead birds, it would save them much loss from disease and parasites among their flocks. The carcass of a bird that has died of an infectious disease or of parasitism may be the means. of infecting grounds and spreading disease among the flock many months later, or portions of it may be carried to neighboring farms with disastrous results to neighboring flocks.

The carcasses of birds found dead in a flock should be burned, whether or not they have died of some canse other than disease; the chances are that they harbor intestinal parasites which are capable of being spread from the carcass to live birds. Where time cannot be taken to properly burn the 
dead birds, they should be buried deeply, so that they cannot be dug up by dogs, skunks or foxes, and so that worms may not carry infection from the carcass to the surface of the ground. 


\section{EXTERNAL PARASITES}

A parasite is an organism either animal or vegetable that lives with or upon another organism, either animal or vegetable. Bacteria are vegetable organisms that live upon an animal organism, as example, the bacterium pullorum causing white diarrhea of baby chicks. Lice upon a hen is an example of an animal organism living upon an animal.

Parasites are divided into external and internal groups. The group of external parasites live upon the skin surface of the body, as for example, lice. Or external parasites may visit their host only for food, as for example, the mites or mosquito. Internal parasites live in the tissues or organs of the body, as for example, the intestinal worms or the gape worm.

More than thirty species of external parasites infest fowls; their economic importance is very great; fowls heavily infested with any of them are unprofitable, and many of these parasites are so injurious as to kill the infested birds; especially is this so with mites and lice.

It is necessary to know something of the life history of these parasites and their habits and especially where they breed to intelligently combat and eliminate them from the poultry and their houses.

The external parasites affecting poultry are many kinds and varieties. Some of these are insect parasites, as mosquitoes, maggots of flies, as the blow fly, lice, chigger, fleas and the chicken bug. Other parasites are in the class of acari and include the scab or scaly leg parasite and a degraded acarus, the air sac mite, which while it infests the air sacs and from that standpoint would belong to the internal parasites yet from a classification standpoint, we are placing it with the external parasites. The bird does not escape the vegetable parasites as mold, as it is subject to ring worm.

\section{LICE OF BIRDS}

Infestation by lice is scientifically known as phthiriasis from a Greek word meaning a Louse. It is commonly known as lousiness. 
In its ordinary sense, the term louse comprises all the apterous insect parasites, or parasites without wings, which do not jump like fleas, and only accidentally quit their host.

Lice of birds are placed in the family Philopteridae, of which we are interested in six genera, as follows: Menopon, Gonoides, Gonoicotes, Lipcurus, Docophorus and Nirmus.

The members of the family Philopteridae have a mouth parts on the under side of the head. The mandibles are strong, maxillae wanting, tarsi short, of one or two joints, the claw meeting a tooth at the apex of the tibia; mesothorax apparently wanting; abdomen having nine segments.

The group is a large one, the species being so numerous that there is scarcely a bird but harbors one, and sometimes several, species of this family.

The genera are, for the most part, easily separated; Docophorus, by the presence of a movable appendage in front of the antennae; Nirmus, by the presence of an immovable tooth in front of the antennae and the generally entire terminal segment of the abdomen of the female. Goniocotes and Goniodes are robust forms, usually with large heads strongly curved in front. They differ from the former, having simple antennae in both sexes, while in the latter that are modified in the male. The former are also usually much the smaller. In Lipeurus the body is generally long and slender, the antennae of the males large and often with a complicated structure, while the terminal segment of the female is bilobed.

Lice of birds embrace a group of biting lice; their bodies are flat and their mouth parts are arranged for biting and cutting. They live upon feathers, epidermis or scales of the skin, and secretions of the body of the bird. As may be noted in Fig. 19, the mouth parts are located just back of the antennae and are not always visible. The antennae consist of five articles or joints each. The thorax in some species is long and narrow, in others short and globular. The thorax is provided with three pairs of legs, which are attached to the thorax. The free extremity of the legs is provided with two hooklets or claws, which enable them to hold on to their host. The body and legs may be covered with a greater or less quantity of hair or bristles.

As to the region in which lice infest, we may say that there are three groups. First, those that infest the body and called body lice; second, those that infest the head and neck, and called head lice, and third, those that infest the feathers, called the depluming lice. 


\section{LICE THAT INFEST THE BODY}

The most common lice which infest the body are those of the genera, Mcnopon, Gonoides and the Goniocotes, which are as follows:

Menopon biseriatum.-This is the large body louse and is seen in Fig. 19. This is the largest louse we will find upon the chicken. It is about one-twelfth of an inch in length. It is light in color. In Fig. 19, the small drawing indicates the actual size of this louse. This louse will also be found on the heads of small chickens as well as on the bodies of the hen. Its most favorite place is in the vent fluff.

Menopon Pallidum.-This is the small body louse of the chicken. It is the smallest of the two Menopon and is the most common louse infesting the fowl. The annoyance this one species causes poultry is probably equal to that of all other species that infest fowls. It occurs in great abundance and on almost every fowl we have examined. This louse is easily distinguished from the other common species infesting the hen by its light color and its great activity among the feathers and over the body of the bird it infests. It moves so fast that it is rather difficult to catch one and they readily leave the bird and crawl in numbers upon the hands of the person holding the bird. Fig. 20 is a picture of the Menopon pal-

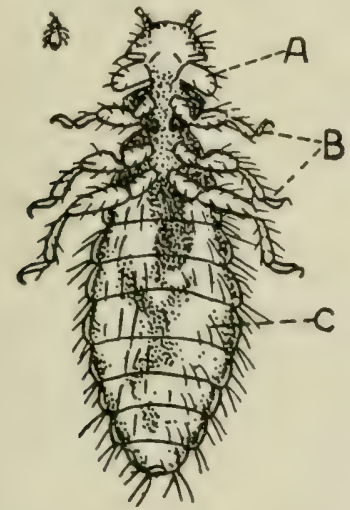

Fig. 19. Menopon Biseriatum.

A, head provided with mouth parts for biting, feelers or antennae, and eyes; B, legs attached to the thorax; C, abdomen.

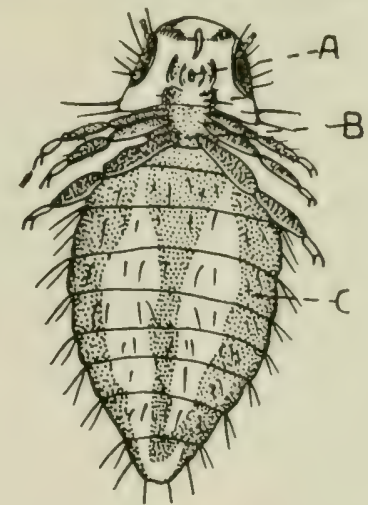

Fig. 20. Menopon Pallidum.

$a$ head; b, thorax provided with three pairs of legs; c, abdomen bearing hairs.

lidum and the difference in shape of head, thorax and body can be seen between this and the Menopon biseriatum and the other kinds of lice. This louse is reported to spread from birds to other animals where it causes temporary parasitism, causing great discomfort of the newly infested animal. Where poultry houses are built on to barns there is grave danger of the birds becoming infested with mites or lice and these temporarily infesting animals kept in the barn. Great care is always necessary to keep the poultry free from parasites as they will not give satisfactory performance, or results when infested with parasites that irritate them and sap their lives.

Goniocotes gigas.-Is the largest species of the genus Goniocotes and is recognizable by its large size and full rounded head as seen in Fig. 21. In color it is a light yellow with bands and outlines along the outer border of the abdomen. The female may reach the length of $4 \mathrm{~mm}$. and the male $3 \mathrm{~mm}$. This specie has been found 
rather common in North Carolina, but not apparently so in the west and middle west. It is also regarded as rather common in Australia and is also reported from England. This louse we have found on both body and neck, in the latter case where we observed them feeding on the barbs and barbules of the neck feathers.

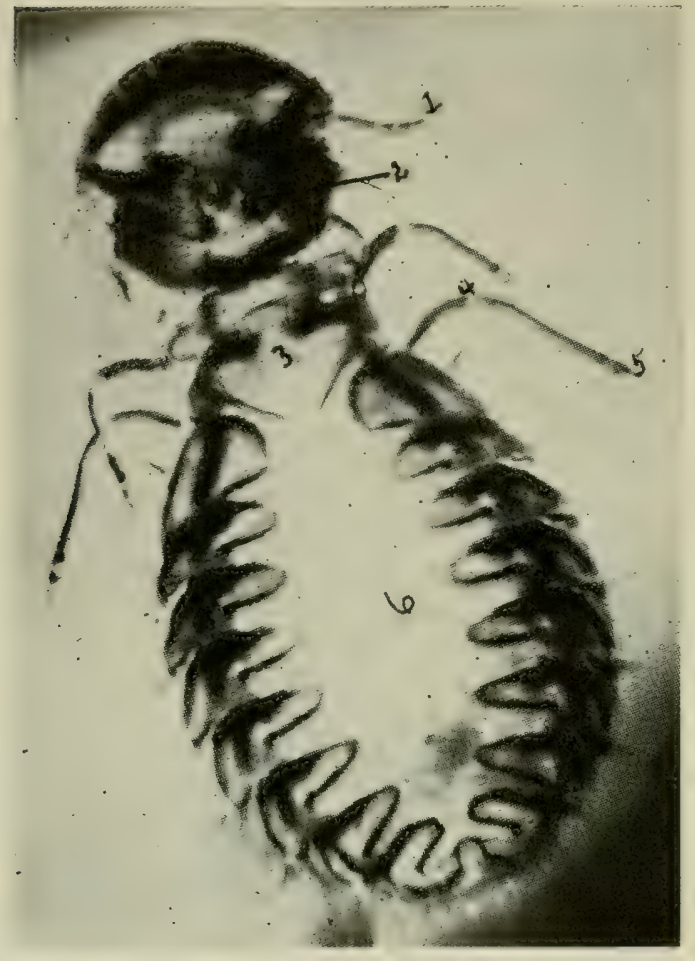

Fig. 21. A Photomicrograph of the Goniocotes Gigas.

Head showing antennae or feelers at $1 ; 2$, the mouth parts; 3 , thorax with legs provided at extremity with small hooks at $5 ; 6$, abdomen.

The Goniocotes hologaster.-This louse is somewhat more common than the previous one and is illustrated in Fig. 22. Its head is nearly quadrate, the thorax narrow and the abdomen short and globular.

Fig. 22. Goniocotes Hologaster.

a, mouth parts; b, antennae; $c$, hooklets on the free extremity of the leg.

\section{LICE OF TURKEYS}

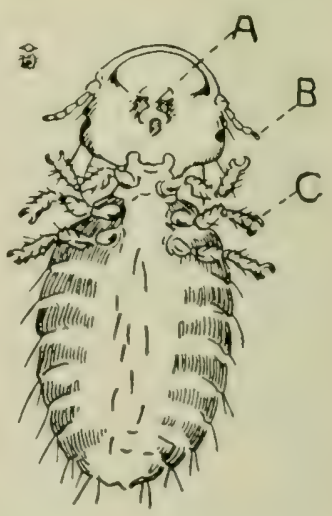

Goniodes stylifer.-This is the common body louse of the turkey. Its head is well rounded in front, rather square cut, with scallops behind; the thorax is narrow and the abdomen large and globular. This louse is shown in Fig. 23. 
Lipeurus polytrapesius.-This is a long, slender louse, with two or three bristles extending from each segment of the abdomen. Its head is well rounded in front and the thorax is rather broad and long.

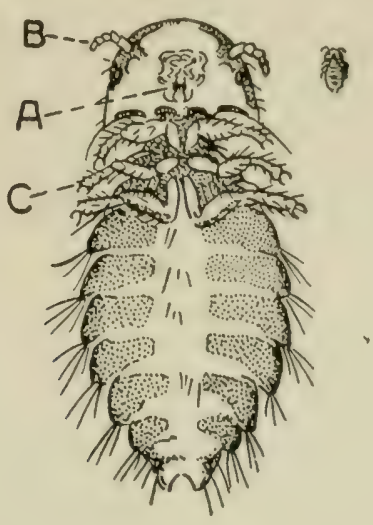

Fig. 23. Goniodes Stylifer.

a, mouth parts; $b$, antennae or feelers; $c$, legs; the drawing to the right of the head indicates the actual size.

\section{LICE OF DUCKS}

Menopon obscurum.-The head is crescent-shaped in front and the abdomen has dark, lateral bands. It is dark fawn-colored.

Lipeurus squalidus.-The head is narrow and somewhat elongated in front. There are six hairs on the front part of the head. This louse is reported as being common in some localities.

\section{LICE OF GEESE}

Lipeurus jejumus.-This louse is slender, pale yellowish-white. It is probably the most common of all the lice of geese.

Trinoton continumm.-This is a fairly large louse. Its body is covered by hairs. It is quite common.

\section{LICE OF PIGEONS}

Lipeurus baculus.-This is the most common louse of the pigeon. It is seen in picture 24. This louse is long and slender. It is lightcolored and the abdominal segments are provided with two or three hairs on each side.

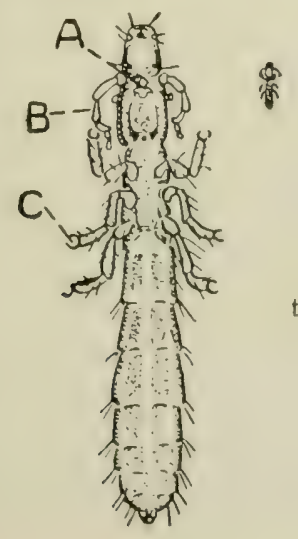

Fig. 24. Lipeurus Baculus.

a, mouth parts; b, antennae; c, legs; drawing to the right of head indicates actual size. 


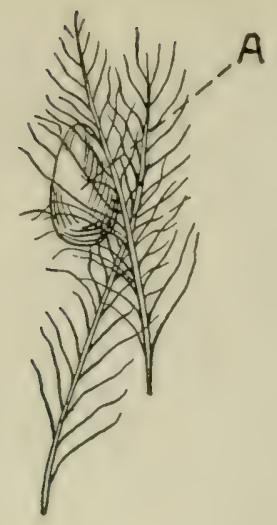

Fig. 25. Eggs or Nits of the Goniones StyLifER (GREATLy MAGNified).

a, egg cemented to the barbes of the feather.

Goniodes damicornis.- This louse is found most abundant on the body close to the oil gland at the base of the tail and upon the back, or they may be imbedded among barbules of the large wing feathers. The long slender Lipeurus is found over the surface of the body especially darting under cover, then on the surface, and are not apparently confined to one certain place. The Goniodes is rather common and is of a bright-brown color. The head is very rounded in front as can be seen by Fig. 29, which is a photomicrograph of same. The head is strongly angular behind. In the male the abdomen is rather short and globular.

\section{LICE OF CANARIES}

The gray louse.-This louse is provided with a slender, elongated body and a large head, provided with strong jaws. It lives upon the feathers of the bird. It does not suck blood. Its sharp mouth parts irritate the skin and cause a discomfort to the bird. The eggs of the gray louse are cemented to the feathers and are easily removed. Fine sodium fluoride worked well into the feathers is best treatment.

\section{LICE THAT INFEST THE HEAD AND NECK}

Some species of lice infest the head and neck of the adult fowl, as well as of the baby chick and range birds. These lice cannot be found on any other part of the body. To find them one must part the feathers in the region of the neck, and the eggs, somewhat different from those of the body louse, are attached to the feathers, close to the roots. There is a tendency to deposit these eggs singly. The lice will be found close to the skin.

Lipeurus infuscatus. - This louse is found only on the head and neck of fowls and is rather common. The louse is moderately long

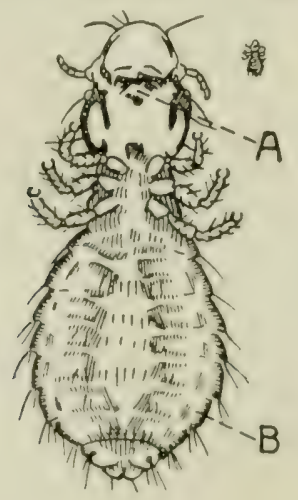
and slender and is seen highly magnified in Fig. 26. The front part of the head is rounded, the thorax is a trifle narrower than the head and the $a b$ domen is long and rather thin.

Fitg. 26. Iipeurus Infuscatus.

a, mouth parts; $b$, abdomen; drawing to right of head indicates actual size. 
Fig. 27 shows a seven-weeks'-old Single Comb White Leghorn cockerel infested badly with the head and neck lice. This bird, forty-eight hours before this picture was taken, was the strongest and most vigorous bird of the flock, crowing and master of the household. Note his dejected, partially comatosed condition, bluish colored comb turned over to one side, bluish face and wattles. $\mathrm{He}$ no longer presents a sleak coat he formerly had. He refuses all food. A nicotine powder thoroughly dusted among the neck feathers, to the skin, and a little care caused this bird to recover but the balance of the flock grew better and were stronger for several weeks after this attack. Lice stunts the growth of chickens and kills many. An examination of the body and vent fluff did not show lice as they were confined to the head and neck. In examining birds it is essential to examine the head and neck as well as the body and vent fluff.

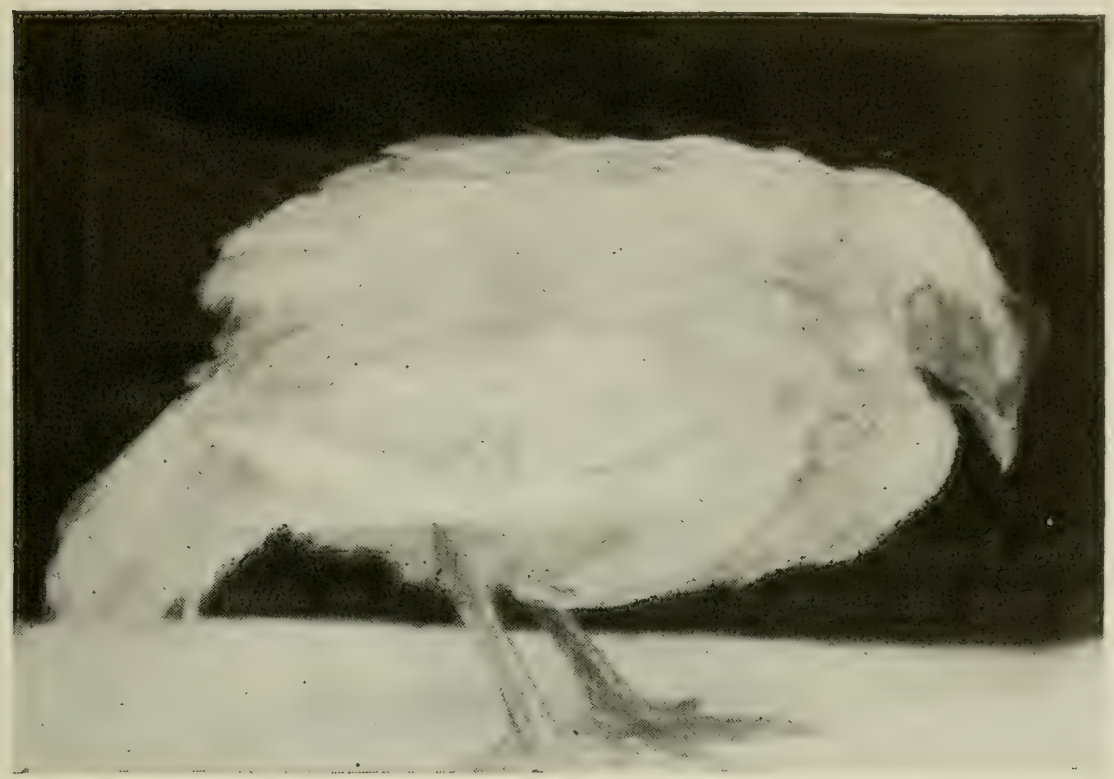

Fig. 27. A Photograph of a Seven Weeks Old Single Comb White Legiorn Cockerel Badly Infested With the Head and Neck Lice.

Note his dark comb, face, and wattles and the ruffled unkempt feathers. His drowsy condition.

\section{THE DEPLUMING LICE}

There are certain lice which live upon and destroy the large flight and tail feathers of fowls. These lice are easily seen, as they are dark in color, slender and long, and in prominent places.

Lipeurus variabilis.-This is the depluming lonse of the fowl. This louse is about one-twelfth of an inch in length, whitish to dark in color as seen in Fig. 28. It is smooth and shining and the margins of the body have a tendency to be black. The head is large, rounded on the anterior margin, and the whole appearance is sufficiently distinct from any of the species infesting the fowl that with the 
aid of the photomicrograph given in Fig. 28, there will be no difficulty in distinguishing it at a glance. They move with great rapidity among the webs of the flight and main tail feathers.

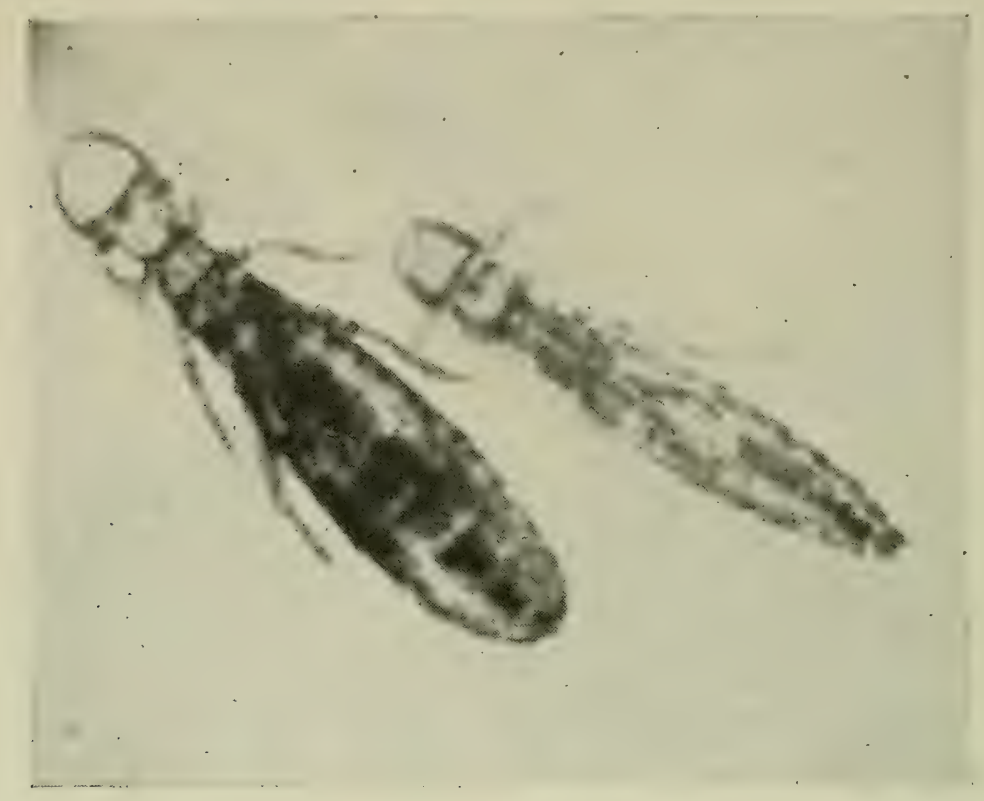

Fig. 28. Lipeurus Variabilis, the Depluming Louse, Male and Female.

\section{HOW AND WHERE LICE MULTIPLY}

The female lice are slightly larger than the males. The eggs vary in shape in some being oval as shown in Fig. 25, while others are of different shape, as for example, those of the head and neck lice, are lemon-shaped. The eggs are whitish or whitish-yellow in color and are commonly called nits. The eggs are cemented to the feather barbes and barbules with a kind of cement-like material with which the female is provided. The length of time for the lice eggs to hatch depends upon the temperature and weather conditions. If the weather conditions are favorable, the eggs may hatch in from ten days to three weeks. Lice live for a considerable period if on birds, several months under favorable conditions. Lice off the body of the bird will live but from seven to ten days. When the egg hatches, the young louse emerges from the egg shell by breaking open the end of the egg as a cap, in much the same manner that a chick emerges from the egg. The young have much the same shape as the adults, ustrally the abdomen is rather undeveloped, which soon expands. The young lice can be distinguished from the adults by the fact 
that they are smaller and are lighter in color. The males are usually less numerous than the females.

Lice breed with great rapiclity; it has heen computed that the unhindered reproduction of a single pair would reach the enormous total of 125,000 individuals in the third generation, which may mature in eight weeks! The time which elapses from the time a louse is in the egg stage till it is mature and reproducing will vary from two to four weeks, depending upon weather conditions. Lice multiply and develop very rapidly in warm or hot weather.

\section{EFFECTS OF LICE UPON THE FOWLS}

Chicks hatched in the incubator are free from lice and stay so until they are placed with lousy hens or chicks, or in quarters in which lousy hens and chicks have recently been kept. Lice produce much irritation; the effect of large numbers upon birds is quite marked. The lousy birds scratch, pick at the feathers, show signs of being drowsy as seen in Fig. 27, may refuse to eat, and, in growing birds, development is retarded.

Young chicks infested with lice often sit around, moping, with wings hanging down, and in a week or two may die. For

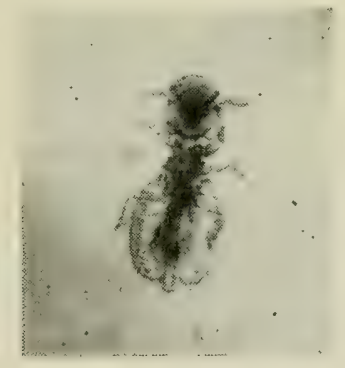

Fig. 29. Goniodes Damicornis of the Pigeon (GREATLY ENLARGED).

this reason, brooder chicks sometimes thrive better, grow faster and are freer from certain ailments than chicks hatched by the hen. It has been said that lousy birds show a greater tendency to wallow in the dust than those not infested.

The effect of lice on older birds is not so severe as upon younger ones, but is noted in conditions of flesh and in the production of eggs. The irritation is sometimes so severe that setting hens desert their nests. Their comlss may iecome dark or even black, shriveled, dry, hard and in upright combs they àre noted to lop over to one side and laying hens soon fail in their egg yield. The birds are unable to rest day or night, become emaciated, and die. 
In looking for body lice, part the feathers in the vent fluff region or of the body and the lice will be seen running over the slin at the base of the feathers and among the feathers; for the neck and head lice part the feathers of the neck and the lice will be seen as described above for the body lice. Unless the different locations are carefully examined, lice may be present and overlooked, since certain lice live on certain parts of the body of the fowl. During the months of July and August, lice will be found most abundant because weather conditions are most favorable for their multiplication and life at this time of year.

\section{DEALING WITH LOUSE INFESTATION}

\section{The Head and Neck Lice}

In setting a hen, dust her thoroughly, over body, vent fluff, neck and head, with some good nicotine lice powder, at the time of sitting. Dust her again thoroughly when she is taken from the nest and watch the heads of the baby chicks for the appearance of lice. Usually they appear in the region of the head and neck, on the baby chick, and if not attended to at once will kill the whole flock in very short order. For the head and neck lice, once they are on the baby chick, thoroughly work down in the skin of the baby chick, either some nicotine lice powder or sodium fluoride. Sodium fluoride because of its being a very fine powder works very nicely. The application should be repeated every four days till the lice and their nits or eggs all disappear. For the head and neck lice of adult birds, dusting similar to the baby chicks is required. We do not like grease in any form on the heads of baby chicks. The powder does not give them a "smeared-up" appearance and is nicer to apply and we have seen ill effects by the application of too much grease and allowing the baby chicks to run out in the hot sunshine.

\section{The Body Lice}

The most common lice of fowls are the body lice. Either a blue ointment or dusting powder may be used. In using the blue ointment, secure a pound can of fifty per cent mercurial ointment and a pound can of vaseline. Thoroughly mix the two together and apply by rubbing a piece as large as a pea or larger into the rent fluff. One good application will usually rid the fowl of infestation by the body lice. If it is the desire to nse a powder, then we recommend either a nicotine lice powder or plain powdered sodium fluoride. Secure a pan sixteen inches in diameter and about four inches deep, grasp- 
ing the hen firmly by the legs with head down, hold her over the pan. Commence to work the lice powder through the feathers down to the skin by beginning at the vent fluff and gradually work toward the head. The pan will catch the excess powder, which can be used again. Repeat this dusting every four days till all lice have disappeared. At the same time the hens are dusted the house should be thoroughly cleaned, burning the litter, and thoroughly spray the house with a four per cent solution of some standardized coal tar disinfectant dip having a coefficiency of at least five or six.

A third method not mentioned above is that of dipping. This can be done only in warm weather. Secure a washtub and fill with a four per cent solution of coal tar dip, such as Zenoleum, and dip the hen by grasping her firmly by the legs and head, dragging her backward through the liquid, taking care not to get the head under the water or the solution in the eyes. It is better for two or three to handle the bird. This will allow of brushing the feathers the wrong way and agitating them in such a way as to get the dip to all parts of the body. One dipping usually rids the flock of lice. If the head gets under the solution, usually the bird will breathe and get some of the liquid into the lungs and a mechanical pneumonia result which often is fatal to the bird. If the dip, in this strength, gets into the eyes, it may cause an inflammation. Sodium fluoride, one ounce to the gallon of water, is also used.

It is not advisable to dip birds of delicate constitution or which lack vigor, as considerable loss by deaths may result.

At the North Carolina Experiment Station, we have used a lice powder for a number of years made in the following manner :

When the treatment of individual birds for lice becomes necessary, some kind of powder thoroughly dusted into the feathers seems to be, on the whole, the most effective and advisable remedy. The powder used must be of such a nature, however, that it will give off an effective poisonous gas. The following powder will be effective in dealing with lice infestation and can be made at a low cost of five cents a pound. In using any lice powder on poultry, it should always be remembered that a single application of it is not sufficient. When there are lice present on a bird there are always unhatched lice eggs or nits present and these are not killed by the lice powder and will later hatch. It is necessary to make the second application to catch these, and it is often necessary to follow up with still more applications to catch those lice that were off the birds at the time of dusting, and later after the dust has shaken from the feathers, find their way on the bird again and reinfest her.

In making this lice powder, take three parts of gasoline and one part crude carbolic acid, or if the crude carbolic acid cannot be obtained in its place use the same quantity of any standardized dip. 
Mix these together and add gradually, while stirring, enough plaster of Paris to take up all the moisture. As a general rule, it will take about four quarts of plaster of Paris to one quart of the liquid. The exact amount, however, must be determined by the condition of the powder in each case. The liquid and dry plaster should be thoroughly mixed and stirred so that the liquid will be uniformly distributed through the mass of plaster. When enough plaster has been added the resulting mixture should be a dry, pinkbrown powder having a fairly strong carbolic acid odor and a rather less pronounced gasoline odor. Do not use more plaster in mixing than is necessary to take up the liquid. When the mixing is complete it is well to pass the powder through a fly screen sieve so as to break up any large lumps that may have formed and to make a uniformly fine powder. If the bird is infested with body lice a demonstration can be made by thoroughly working the powder into the feathers to the skin. The bulk of the application should be in the fluff around the vent and on the lower side of the body and in the fluff under the wings. Its efficiency, which is greater than that of any other louse powder known to the writer, can be very easily demonstrated by anyone to his own satisfaction by this test. After a lapse of about five minutes, shake the bird, loosing its feathers with the fingers at the same time, over a clean piece of paper. Dead and dying lice will drop on the paper in great numbers. Anyone who will try this experiment will have no further doubt of the wonderful efficiency and value of this powder.

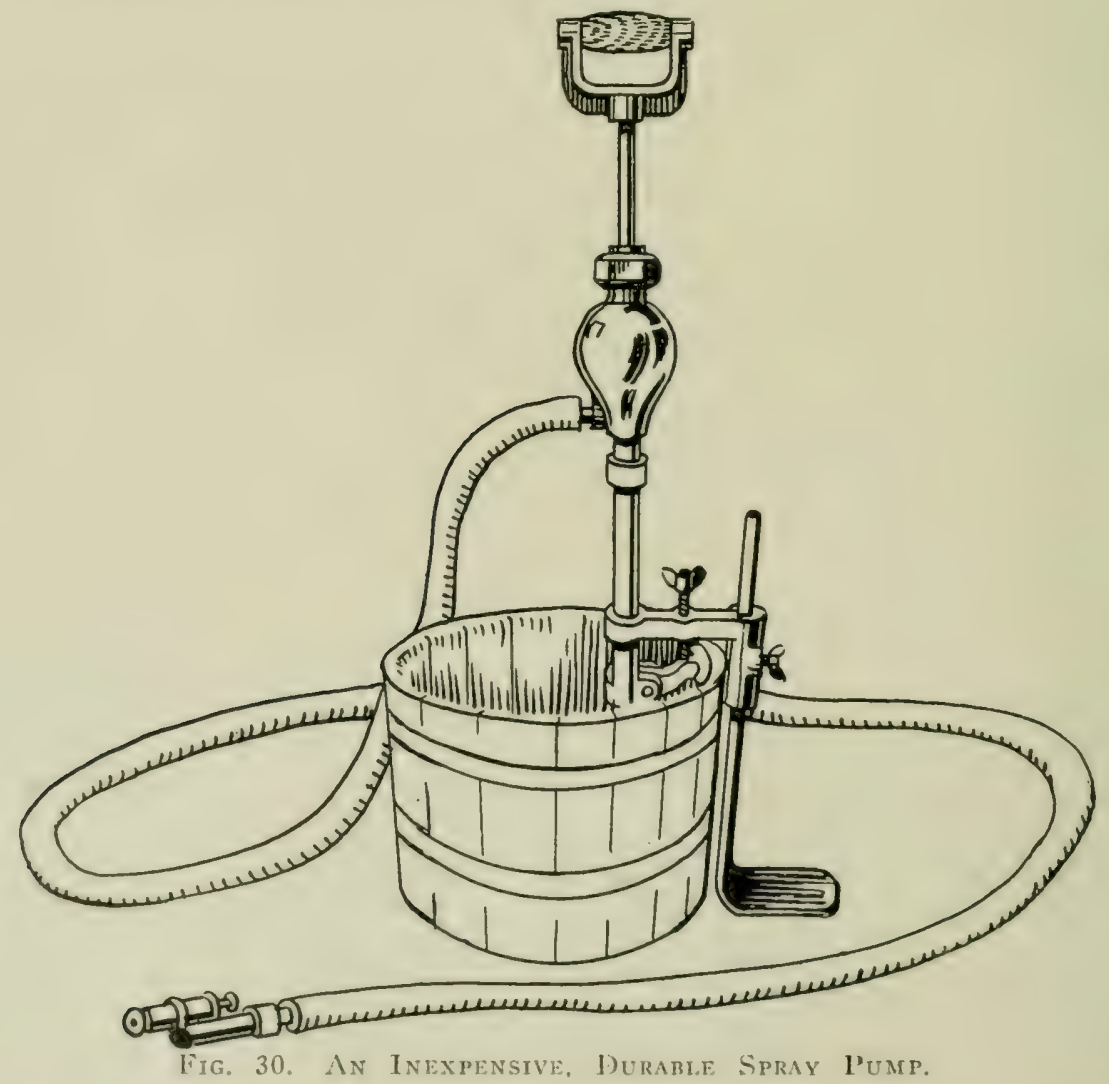


After freeing the flock from lice, care should be exercised that a reinfestation is not brought about by the introduction of lousy birds. When birds have been to the show, or, when new birds are being introduced into the flock, they should be thoroughly examined for lice and dusted as a precaution against some that might have passed unobserved. In case lice are found present, keep the birds in quarantine till two or more dustings can be made and you are sure all danger of live lice are past.

The infested henhouse should be thoroughly and frequently cleaned and the walls sprayed. The spray should contain some destroyer of parasites as carbolic acid, five per cent; coal tar dip, four per cent; or corrosive sublimate one to onethousand. The perch poles should be scrubbed in boiling water and after drying in the sun should be saturated with kerosene. The litter and straw should be removed from the nests and burned and the nest boxes sprayed with coal tar dip or better painted with creosote. After the boxes dry, they should be refilled with nesting material consisting of straw or excelsior. The spraying should be done with a spray pump as seen in Fig. 30, or some other equally good spraying apparatus so that the liquid will be driven into the cracks and crevices and destroy all the parasites.

\section{SCABIES OF FOWLS}

Lice are a group of insect parasites having six legs. We now come to another kind of parasites that belong to another group and, in the adult stage, are provided with eight legs. This group of parasites are the acarids, or mites, as they are commonly called on account of their extremely small size many of which are scarcely visible to the eye. Lice and the acari are widely distributed and are of great economic importance.

There are numerous species of mites that infest fowls. Some live on the feathers and scales of the skin, others bore into the skin, and still others inhabit the interior of the body and from this standpoint could be considered internal parasites.

There is one form of scabies called dcpluming scabies that is very rare, and so far as the author knows has not been reported in this country. It affects the body of both chickens and pigeons. The one on chickens is the Sarcoptes laevis variety gallinac, and the one on pigeons is the Sarcoptes laevis variety columbae.

The acarid parasites for birds are placed under the following genera: sarcoptes, cytodites, trombidium, and dermanyssus. Unlike the various genera of lice, the scab parasites differ greatly in the effects which they produce, and therefore a separate discussion of each one will be given. 


\section{SCALY LEGS OF FOWLS}

Scaly legs of fowls is also called foot mange, or scabies of the legs.

Scabies, mange, or scaly legs are three words to indicate the piling up of scales caused by the irritation of the parasite, the oozing out of minute quantities of a straw colored fluid called serum, from the injured skin, the drying of this serum, and the gradual accumulation of scab material till large areas or patches of scab is visible.

Scaly legs is a condition that is very common and especially where the fowl's sanitary condition is not closely looked after. Scaly legs constitutes leg scabies, and is caused by a parasite called the Sarcoptes mutans variety gallinae.

\section{THE SARCOPTES MUTANS VARIETY GALLINAE}

Description.-This parasite is one of the same family that infest horses, cattle, hogs, sheep, dogs and cats. That particular branch of the family affecting chickens is distinguished by calling it "variety gallinae"; gallinae being a Latin word meaning "of the chicken." Owing to the small size of the parasite, it is often called a mite, though the term mite as ordinarily used refers to the red mite of the perch poles-the Trombidium holosericeum. Fig. 31 illustrates the parasite causing scaly legs. It is magnified 100 times; the actual size of the parasite is shown by the small dot in the square at the upper right-hand side of the drawing. In the drawing it will be noted that the legs are short and strong and its mouth parts arranged for biting the skin. They live upon serum that seeps out at the point attacked and this small quantity of serum drying adds to the gradually accumulating scabs. For a picture of scaly legs, see Fig. 32.

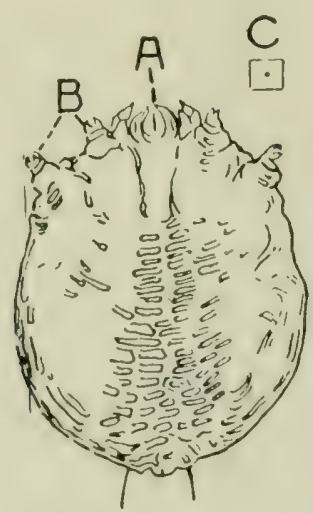

Fig. 31. Sarcoptes mutans, Variety Gallinae. 


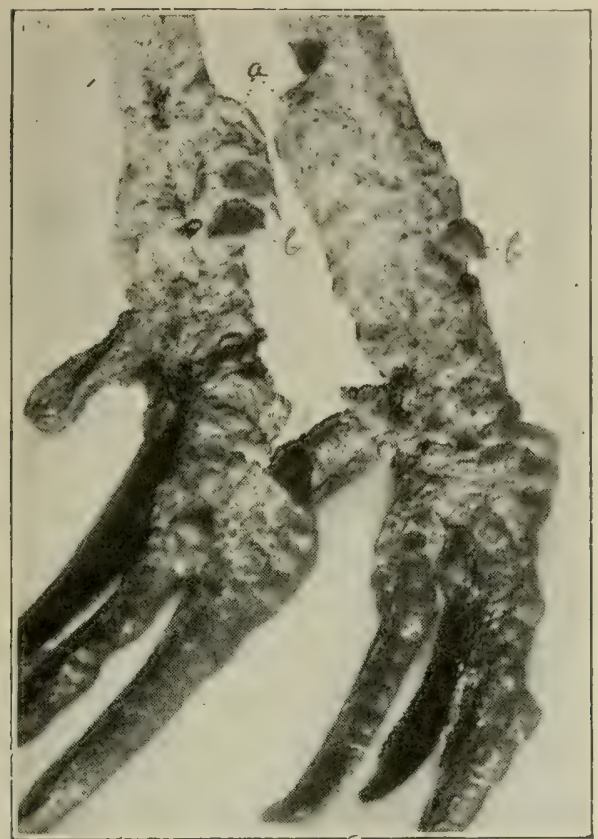

Fig. 32. Scaly Legs or-Scabies.

a, mass of scabs due to dried serum that seeps from the injured parts; $b$, scale of leg forced up and out of place by accumulating aricd serum.

Life History.-The female lays her eggs under the scabs, where in about ten days they hatch, if conditions are favorable. The young mites called larvae are provided with only three pairs of legs and are not provided with sexual organs. These young scab parasites pass through several moults, in which each time the skin is shed, and are finally developed into the mature or adult stage. In the adult stage they are provided with four pairs of legs and are sexually mature.

How the Parasites are Spread.-The tearing off of the scabs favors the escape of the parasites, which in warm weather may live in the filth, roosts, nests, or other parts of the building for at least thirty days, and may in that time find their way upon other birds and infesting them produce scaly legs in them. Thus birds become infested by being placed in infested quarters, that is, in quarters in which birds suffering with scaly legs are or have recently been kept, or by an infested bird being introduced into a healthy flock, or by the bird being transported in coops, or placed in coops, as at shows, in which infested birds have recently been kept.

Symptoms.-This parasite attacks chickens, turkeys and cage birds, but the writer has not observed it infesting water fowls as ducks and geese. The parasites always attack the 111feathered portion of the legs and feet, especially the shanks and upper portions of the toes. The minute parasite works its way under the scales of the legs and there irritates the tissue by attacking it with its strong mouth parts. Is a result of this irritation a vesicle or small blister appears. The blister is practically microscopic in size. Later this vesicle or blister ruptures and the small amount of serum it contains dries and adds to the already accumulating scabs. These scales finally accumulate in such large masses that the normal scales of the 
legs disappear and the shanks present an unsightly appearance as seen in Fig. 32.

The parasite can be found as a minute white speck in the serum between the scalss and the leg. Both legs are usually affected at the same time. Itching is present and the bird may pick at the affected part. Itching is more intense at night. The birds may become weak, stop laying, and even die from the effects of the irritation and loss of rest.

How to Treat and Rid the Birds and Premises of Scab Parasites.-The scabby shanks and feet should be soaked in soapy warm water to which has been added enough dip to make a four per cent solution. Many of the scabs can readily be removed, if one will take time, and in valuable birds it will pay. If the bird is of insufficient value to justify the expenditure of time and labor, kill the bird and burn the affected shanks and feet. In these cheaper birds it is good treatment to saturate the shanks and feet with pure kerosene repeating this treatment in two weeks. It is also good treatment to follow the saturation of the affected parts, at the end of four days, with carbolized vaseline and after three days more much of the scab material can be rubbed off. If the shanks and feet are scrubbed with the antiseptic material the normal scales of the shanks and upper parts of the toes can be preserved provided the disease has not progressed too far. After removal of all the scabs possible. scrub thoroughly using a nail brush and the flat end of a wooden tooth pick removing all scales possible. After this is done apply just a little oil of paraffine, or petrol, or even vaseline and rub thoroughly to remove all excess. Repeat the treatment in ten days and follow up with subsequent applications till the bird is well.

Kerosene Emulsion May be Used and is made as follows:Kerosene or coal oil, one-half gallon; common soap, two ounces; water, one quart. Dissolve the soap by boiling in the water, add this solution boiling hot, to the kerosene and stir with an egg-beater, or otherwise violently agitate. When ready for use take one part of the emulsion and add to this nine parts of water.

Lime and Sulphur Dip may be used.-It is made as follows: Unslaked lime, one-third of a pound, sulphur one pound, water four gallons. This mixture should be boiled for two hours and the amount lost by evaporation made up by adding water. The slaking lime acts as a solvent for the sulphur; dissolved sulphur is one of our very best agents to kill parasites. This well-known parasiticide used warm and scrubbed thoroughly under the scales is very effective. 
Commercial Disinfectant Dips may be used.-A four per cent solution in water of any of the standardized coal tar disinfectant dips with a coefficiency of at least five or six will give good results. They must be used warm and the affected parts scrubled thoroughly to remove all scabs possible and repeat this treatment every four days till a cure is effected.

Treatment of Houses and Yards.-The scab parasites that find their way off the infested birds will be found on the perch poles and nests and in other parts of the house and yard and a thorough disinfection as given under the discussion of lice will be effective in riclding the premises so that these or other birds that may be placed in these houses and runs will not become infested.

\section{AIR SAC DISEASE}

A disease of the air sacs, bronchi and lungs is caused by minute mites which in reality are degraded scab parasites. They appear to the naked eye like minute, rather yellowishwhite granules running over the surface of the walls of the air sacs, trachea and bronchi of fowls laid open, that have died of this air sac mite infestation.

The disease is rather wide spread as specimens have been studied, by the writer, in infested birds from Colorado, North Carolina, New York and Pennsylvania. This is a very serious disease of birds, as it is exceedingly difficult to obtain desired results in treating affected birds.

Air sac disease is caused by Cytodites nudus, also called the Cytoleichus sarcopticles, and the Cnemidocoptes mutans, commonly known as the air sac mite.

\section{Cytodites Nudus}

Description.-As seen by Fig. 33, the body of this parasite is ovoid in shape, whitish in color, provided with a conical mouth part, through which it sucks fluid or serum from the surface on which it is found. The legs are rather short, conical in shape, and in both males and females the free extremity of each leg is provided with a cup-shaped sucker called an ambulacrum. These suckers aid the mite in holding on to objects and in moving about. The legs are each provided with five segments or articles.

Life History.-The ovigerous female lays eggs, as a rule, but at times has been observed to deposit eggs ready to hatch and even lay young larvae. The larvae are not provided with sex organs and have only three pairs of legs. The young mite goes through several moults and finally arriving at the sexually mature stage and provided with four pairs of legs.

Symptoms. - The air sac mite lives in the air sacs, lungs, bronchi, and air sac extensions of the bones of the fowl. At autopsy they are easily seen in the abdominal air sacs of 
infested birds, because these air sacs lying along either inner side of the abdominal wall are opened in cutting and exposing the abdominal organs and are then in plain view. Chickens appear to be the most often infested, though pigeons and other birds have been reported as being infested. If only a few parasites are present they may not cause any noticeable symptoms, but when they crowd the bronchi in large numbers they may cause fits of coughing by irritation of the mucous membrane lining these parts, reaching of the neck, partial asphyxiation with bluish comb, face and wattles. There may be a rattling in the throat due to the accumulation of mucus in the trachea and bronchi. The bird will become thin in flesh and even become emaciated, it will appear dull, stay away from the other members of the flock, and in the long protracted cases where the bronchi are not crowded, the comb may be pale in color. The wings will droop, there will be labored or difficult breathing and death finally ends the scene.

Postmortcm Appearance.-All internal organs will appear normal in size and color. By a careful examination of the abdominal and other air sacs as well as of the trachea and bronchi, the mites may be seen as minute yellowish-whitish bodies crawling over the surface. These parasites are scarcely visible to the eye.

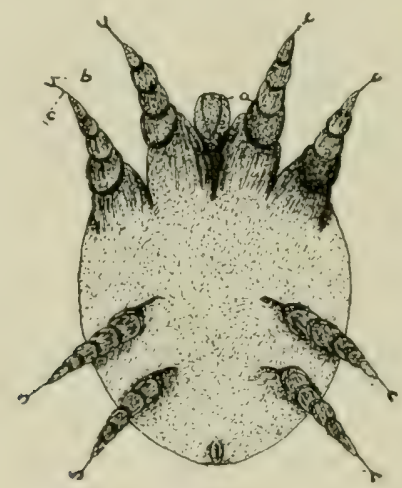

Fig. 33. Cytonites Nudus or Air Sac Mite.

a, rostrum; b, ambulacrum; c, pedicle of ambulacrum.

The air sac mite has also been reported as infesting the liver, kiclneys and other abdominal organs, in which cases they produce yellowish pearl-like nodules or tubercles.

Treatment.-If the flock shows numerous birds infested it is best to kill all the fowls. Great care should be exercised 
not to sell any of the birds as breeders or on a market where there is danger of them being bought for a family or farm flock. The houses and yards so infested should not be used again for birds for at least one year. All birds found infested should be cremated by burning. It is almost impossible to eliminate the disease from a flock once infested except by slaughter and a long time disinfection by not using the premises for several months for birds.

\section{RED MITE INFESTATION OF THE HENHOUSE}

Red mite infestation is caused by the chigger. Of the chiggers there are two kinds, namely, the Trombidium holosericoum, and the Dermanyssus gallinae. The red mite is one of the most persistent and injurious of the pests of the henhouse. They gather on the fowls, especially at night, and suck their blood.

\section{The Trombidium Holosericeum}

This parasite is the common chigger, often called jigger, of the henhouse.

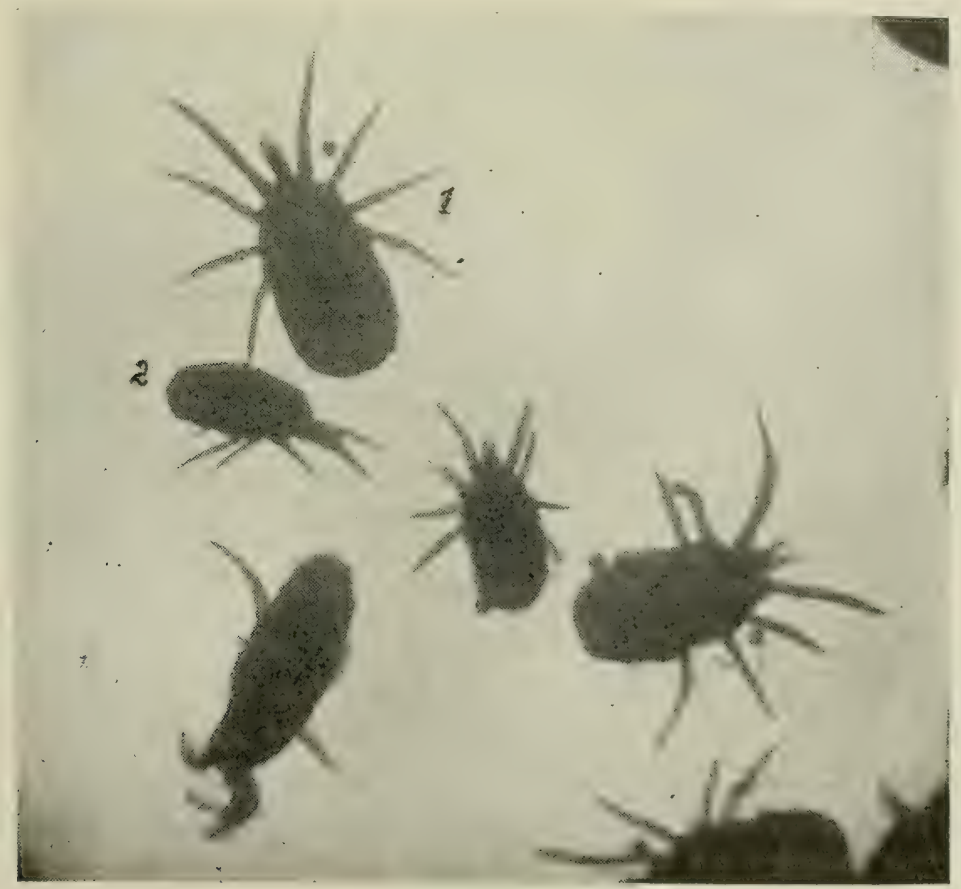

Fig. 34. $\Lambda$ Pirotomicrograpit of the Trombidium HoloseriCeum or Chicken Mite.

1 , a view of the chigger from top or dorsum; 2, a side view.

Description.-The full-grown mite is about $1 \mathrm{~mm}$. in length and a light-gray or whitish color, with dark patches showing through 
the skin, but when full of blood have a distinct red to bluish color. The body is oval as will be seen by Fig. 34 and in the adult stage are provided with four pairs of legs. In the young or larval stage they are provided with but three pairs of legs and no generative organs. The distal end of each leg is provided with two small hooks or claws, with which it clings to objects and which enable it to crawl about. Its mouth parts are conical in shape and arranged for wounding the skin and sucking the blood.

Life History.-Mites multiply by laying eggs. The swarm in the cracks and crevices of the henhouse, and especially on the perch poles, nests, dropping boards and finally on the walls and floor. When numerous they will be found over all surrounding objects and may prove a great pest to man and animals that come in their way. Rotten eggs are good sources and a broken egg will invite myriads to the nest. They come from the cracks and crevices at night to prey upon the fowls.

The eggs are laid in the cracks and crevices of the perch poles and the mites can be found in clusters, even balls, on the under side of the perch poles and where the poles rest on their supports at the ends. When the perch poles have been repeatedly painted with creosote so that mites cannot live upon them they have been noted to commence their infestation in the nests. Sparrows are known to have carried them from one premise and from one house to another. Sitting hens are apt to become attacked and the hen and her brood need be carefully looked after frequently as once infested the entire brood will soon succumb. Sitting hens leave their nests and in some cases are killed by the myriads of mites attacking them. In the cracks of the perch poles where the mites breed very fast in hot weather, a white fuzzy material accumulates. This is the moults of the young parasites and a keen observer is quick to note when these parasite signs are present. Poultrymen claim there is a peculiar odor given off by mites and that they can detect the odor as soon as stepping into the infested house. If the temperature is warm, the eggs laid in the cracks will hatch in a very few days. The young mite has six legs and no sexual apparatus. After going through a few moults they arrive at the adult stage at which time they are provided with eight pairs of legs and sexual organs and they are now ready to reproduce. The parasite multiplies very fast in the warmer parts of the summer, July and August, when conditions are more favorable for its propagation.

Symptoms of Mite Infestation.-To examine premises to see if mite infestation is present, examine the under sides of the perch poles, the nests, dropping boards and in sitting hens and brooding hens their roosting coops should be examined. The mites are found in swarms and are concealed during the day in the cracks of the perch poles, dropping boards, nests, floor and walls. The mites come out at night, swarming over the fowls to suck their blood. The mite by means of its conical shaped mouth parts, referred to above, wounds the skin and sucks the hlood of the fowl. The fasting parasite is white in color, but as it takes in blood its color changes to a bright red, the blood it engorges heing reflected through its skin. In 
the older mites, the color of the engorged ones may vary from a bright red to a bluish color. The symptoms as we have observed them are similar to those of lice. The birds become unthrifty, laying is interfered with and finally the hens may cease laying, sitting hens desert their nests with pale comb, face and wattles. The afflicted birds show an unkempt appearance of the feathers. Many hens are found dead under the roosts in the morning. Examination of such birds, and of the perch poles, nests, dropping boards, walls and floors, revealed millions of mites.

How to Rid Premises of the Mitcs.-The same treatment as for lice should be followed. Absolute cleanliness, thorough spraying with a four per cent solution of some standardized coal tar disinfectant dip, repeated every three days till all mites have disappeared. Painting the perch poles several times, three days apart with creosote. It is a good plan to also paint the inside of the nests, also sitting and brooding coops with creosote.

In order to know definitely the exact value of the many agents recommended for the destruction and prevention of mites and lice the writer made extensive laboratory tests and has recorded these results in the following paragraphs:

There is a common belief that dry tobacco clippings, sulphur, paris green, and a host of liquids, are great destroyers of the formidable foes of the poultry house, but no one so far as we could find, from the literature at hand, had actually made tests to prove it. It was therefore thought best to try a host of these agents.

Mode of the Tests.-The tests were run either in open tumblers or sauce dishes so as to have an abundance of air present and to have the tests as nearly under normal conditions as possible.

Agents Used.-The agents used fall into three classes, namely: dry powders which give off poisonous gases, liquids which may kill by coming in direct contact with the parasite, and liquids and powders that give off gases poisonous to the parasite. Tests were made with sulphur, air slaked lime, paris green, naphthalene, gasoline, carbolic acid, insect powder, tobacco stems from a tobacco factory, crude carbolic acid, five per cent carbolic acid, naphthalene in kerosene, one and two per cent kreso dip, ten per cent formaldehyde, pyrethrum, and a nicotine powder prepared in this laboratory.

Sulphur.-Flowers of sulphur was placed in the bottom of two tumblers and several hundred mites, some very vigorous, were placed on top of the sulphur. At the end of five hours the mites were still walking over the sulphur. Dry powdered sulphur has apparently no destructive action upon them.

Air Slaked Lime.-Air slaked lime was placed in the bottom of a tumbler. At the end of twenty-four hours the mites had all accumulated in a cluster in the center of the dry lime. Upon being poured out on a paper they were still found to be vigorous. Dry air slaked lime has apparently no injurious effect upon mites. 
Paris Green.-Dry paris green was placed in the bottom of a tumbler and several hundred mites were placed in the powder and stirred. At the end of forty-eight hours the mites had formed in a cluster in the edge of the powder. Upon being removed they were found to be as vigorous as before being placed in the paris green. Dry paris green apparently has no ill effect upon mites.

Naphthalene, Powdered Moth Balls.-A quantity of powdered moth balls were placed in the bottom of a tumbler and several hundred vigorous mites placed on the surface. At the end of thirty minutes motion was not so active and at the end of forty-five minutes all motion had ceased and upon being removed and placed upon paper and examined, all were found to be dead.

Tobacco Bits.-Bits of tobacco leaves, the sweepings from the floor of a tobacco factory, were placed in the bottom of a tumbler and several hundred very active mites were placed in this tobacco. Frequent observations were made and at the end of seventy-two hours the mites were as active as when they were placed in the tumbler.

Insect Powder.-A powder prepared in this laboratory consists of gasoline three parts, crude carbolic acid one part and plaster of paris sufficient to make a rather dry mixture. This is passed through a sieve allowing the sieved powder to drop onto a dry paper and after a few minutes it is placed into tight jars and kept till needed. It should be used in a few days after being prepared as the gasoline soon evaporates. A quantity of this powder was placed in the bottom of a tumbler and several hundred very active mites were placed in the material and mixed with it. At the end of one minute all mites were dead.

Five Per Cent Carbolic Acid Solution in Water.-A quantity of a five per cent watery solution was poured out into a saucer and several hundred very active mites were placed on one edge of the saucer and the dish then tilted till the mites were all wet, then the liquid drained from them, the mites remaining on the wet surface for observation. In thirty seconds the movements were retarded and at the end of sixty seconds all mites were dead.

One Per Cent Naphthalene in Kcrosene.-One per cent powdered moth balls dissolved in kerosene was tested. A quantity of this fluid was poured in a saucer and several hundred mites placed on the opposite side of the saucer, then immersed as in the preceding test. In thirty seconds all mites in the test were dead.

Crude Carbolic Acid.-Pure crude carbolic acid was poured in a saucer and several hundred mites placed on one side, were immersed as in the preceding test. In twenty seconds all mites in the test were dead.

One Per Cent Kreso Dip.-This liquid was poured in a saucer and several hundred mites subjected to a bath as in the preceding tests. At the end of four minutes motion slowed and at the end of ten minutes all mites in the test were dead.

Two Per Cent Kreso Dip.-The test was conducted as the preceding. At the end of two minutes motion was retarded and all mites in the test were dead at the end of four minutes.

Ten Per Cent Formaldehyde. The test was conducted as in the preceding. At the end of ten minutes all the mites in the test were dead. 
Pyrethrum.-Commonly called insect powder. Lice covered with pyrethrum powder were rendered inactive in six to ten minutes.

A powder also prepared in this laboratory as follows, kills lice in thirty seconds: Nicotine $1 / 4$ ounce, naphthalene 1 ounce, standardized coal tar disinfectant dip 4 ounces. Sufficient plaster of paris is mixed with this to make a slightly moist mixture, this is then passed through a screen and used at once.

There was also tried the following mixture: Nicotine, 40 per cent aqueous solution, 40 drops, water four ounces, plaster of paris one pint. As soon as these substances are mixed together they heat, due to the plaster of paris being converted back into gypsum. During this heating process the powder must be occasionally stirred. This powder kills lice in thirty seconds and has been found still effective after being prepared seven months. The sulphate of nicotine is also effective.

It was found that though sulphur in solution is an efficient destroyer of parasites, that although paris green in solution is a violent poison because of its arsenic content and although tobacco leaves contain nicotine which, when extracted is a destroyer of parasites, yet these agents in their dry state do not destroy mites and lice. From tobacco leaves and stems there is probably given off a very small amount of free nicotine, but it is not in sufficient quantities to in any way injure the parasites. The active principle of tobacco is nicotine. Of the nicotine present in tobacco usually 60 per cent is free nicotine and there can be secured the additional 40 per cent by the addition of an alkali such as caustic soda or concentrated ammonia. In the preparation of lice powder using the leaves or stems it is necessary to finely triturate the leaves and stems making it like snuff so that the nicotine will be liberated in large enough quantities to readily destroy the lice. Free nicotine is an extremely volatile substance and if such triturated tobacco is allowed to stand for an extended period of time there will be a material reduction of the nicotine content. Tobacco contains 0.6 per cent nicotine in high-grade tobacco to 8.0 per cent nicotine in poor-grade tobacco.

Naphthalene or powdered moth balls, on account of its volatile substance emitted, kills all mites in forty-five minutes.

Insect powder containing gasoline and crude carbolic acid, on account of its volatile substances given off, killed all mites in one minute.

The test solutions used killed the mites in the following length of time: Crude carbolic acid, twenty seconds; five per cent carbolic acid, one minute; one per cent naphthalene in kerosene, thirty seconds; one per cent kreso dip, ten minutes, and two per cent in four minutes; ten per cent formadehyd, in ten minutes. Formadehyd is a slow parasiticide and must be in quite strong solution. Its gas does not destroy flies. Free nicotine in one-fourth of one per cent kills lice in thirty seconds.

In order that parasiticides be effective in the destruction of the mites and lice they must either be in solution or be capable of giving off volatile gases which in themselves are destructive.

\section{Dermanyssus Gallinae-Dermanyssus Avium}

Description.-By referring to Figure 35 it will be seen that the body of this mite or chigger differs from the Trombidium holosericeum in that it is ovopyriform in shape instead of oval. 
The diameter of the posterior third is greater than that of the anterior third. The abdomen and legs are provided with rather short bristles. Its mouth parts are rather conical in shape and arranged for injuring the skin and sucking blood. The color varies according to the amount of blood contained within the intestinal tract, varying from yellow to a yellowish-red. The free extremity of the legs is provided with an apparatus which enables them to hold on or cling to objects and to move about rapidly.

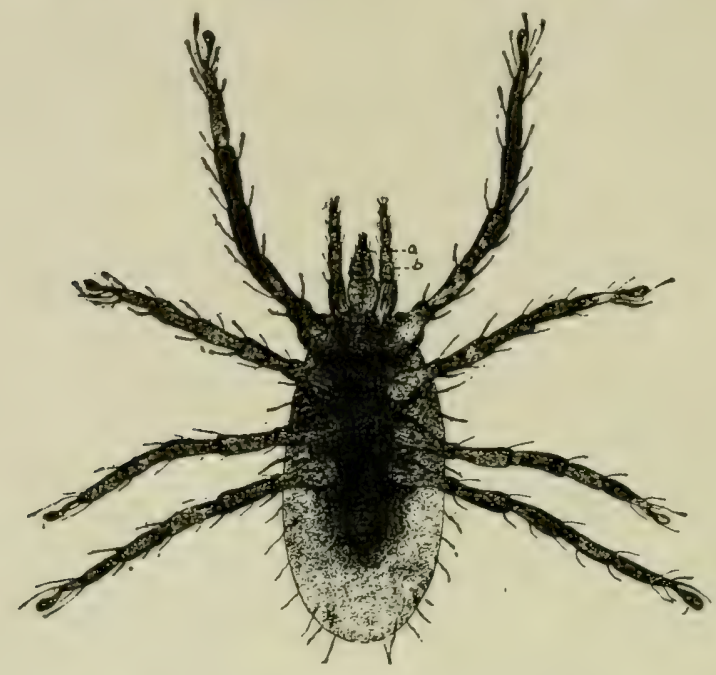

Fig. 35. Dermanyssus Gallinae.

a, conical-slraped rostrum; b, palpus.

Life History. - The female, like the female of the preceding parasite described, lays her eggs in the cracks and crevices and the filth of the floors and nests, where they hatch out in a few days, if the temperature is favorable. The young, six-legged asexual larva goes through several moults. Finally maturing into the adult, sexual, eight-legged parasite.

Symptoms of Dermanyssus Infestation.-This parasite lives in the poultry houses and dove-cotes, hiding in the straw of the nests, cracks and crevices of the roosts, and other places of concealment in the daytime. It comes out at night and makes its attack. Few of these parasites are to be found on the birds, chickens, or pigeons, in the daytime, but at night they may be numerous. Birds so harrassed at night cannot sleep or rest and soon become emaciated. The laying hens will leave their nests and even cease laying. Birds may be found dead under the roosts in the mornings from the attack of these mites.

These parasites may also attack horses and other animals kept close to the quarters of infested birds; they cause irritation, the animal scratches, rubs, and unable to rest at night, becomes thin in flesh and weak. Persons may be annoyed by them.

Treatment.-The same as has been ontlined for lice. (See page S4.)

\section{FLEAS AFFECTING BIRDS}

Fleas are jumping insects that affect the skin of birds, man and animals. There are two kinds infesting fowls, one tech- 
nically known as the Pulex Avinm has been observed in Western United States, and the second, the stick-tight flea, or Sarcopsylla Gallinacea is found in the sandy regions of the Southern and more warm states, and adlheres or sticks to the skin, especially in the region of the head, neck and under sides of the wings.

\section{The Pulex Avium}

Description.-This is the common chicken flea. It resembles to some extent the flea that infests dogs, cats and man; however a microscopic study shows it to be a distinct species. Fig. 36 illustrates this parasite. It is provided with antennae or jointed feelers. In the larval state its mouth parts are arranged for mastication and in the adult for wounding the skin and sucking blood. It is flattened laterally, the thorax being a trifle deeper than the head and is provided with three pairs of legs, of which the posterior pair is longer than the others, giving the insect great power to jump. The free extremity of the legs is provided with two hooklets or claws. In color, the chicken flea is light or dark brown.

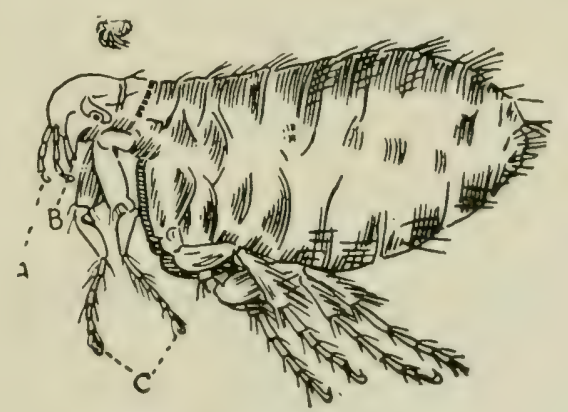

Fig. 36. Pulex Avium.

$a$, antennae; b, stylet; $c$, hooklets on free extremity of leg.

Life History.-The female lays about twenty oval brown eggs in some dirty, dusty place, such as the floor, cracks or crevices or nests. These eggs hatch in a few days, usually six to twelve, if the temperature is warm and from them come wormlike larvae composed of thirteen segments each. The mouth parts are arranged for mastication. The larval stage lasts about eleven days; they then pass through the pupa stage in a tough brown cocoon. The pupa stage lasts about fourteen days, when the six-legged adult flea emerges from the cocoon.

Symptoms.-The presence of fleas in the flock may first be noticed by the insects getting on and attacking persons who enter the poultry houses. Investigations under such conditions reveal large numbers. It has been noted that where this 
kind of flea attacks flocks that lice and mites usually disappear. The fleas attack the birds lay or night and suck blood. If in large number they continually harass the fowls. The fowls cannot rest at night, fall off in egg production, become emaciated and finally may die: Growing birds have stary unkempt appearance, do not make proper growth, become poor in flesh and may even die from the attacks.

Treatment.-Dipping the hens in a four per cent solution of any of the coal tar disinfectant dips with a coefficiency of four or five will be effective. This must be done only in very warm weather and the dip must be body temperature, that is, about 100 to $105^{\circ}$ Fah. Weak birds sometimes die as a result of the shock of dipping. After dipping, do not allow the birds to get into the sun until they are dry.

A dusting powder containing snuff or nicotine to the extent of at least one-fourth per cent nicotine, or sodium fluoride. One ounce sodium fluoride to the gallon of water or a four per cent coal tar disinfectant dip makes a reliable dip. The premises must be thoroughly cleaned and sprayed with a four per cent dip.

\section{Sarcopsylla Gallinacea}

(Stick-Tight Flea or Jigger)

The stick-tight flea is smaller than the flea just described.

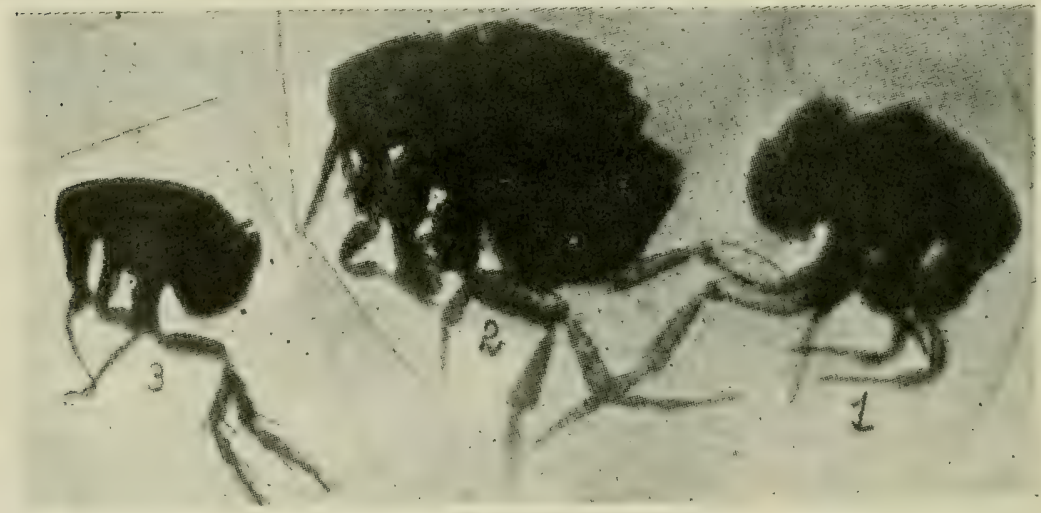

Fig. 37. Sarcopsylla Gallinacea.

1 , male; 2 , females; 3 , young.

By comparing Fig. 36 with that of 37 , the reader can readily sce the gross differences. The Sarcopsylla Gallinacea is much shorter and different in shape. These fleas are found particularly in sandy soil of the warmer parts of the country. They are commonly called stick-tight fleas because they stick tightly to the skin. 
Description.-The female is $0.75 \mathrm{~mm}$. to $1.0 \mathrm{~mm}$. in length and the male a trifle shorter. Its posterior legs are much longer than the anterior pair. The posterior angles of the metathoracic scales are angled. The eyes and antennae are located in the posterior part of the head. It is brown to brownish-black in color.

Habitat.-It lives in shady places, under old houses, on earthen floors; in filth. It is a veritable pest to adult birds and especially to young chickens and turkeys.

Life History.--It lays its eggs in dirty filthy corners or sand or while on the bird, under which conditions the eggs roll off on the ground, where they hatch out and go through the changes which bring them to the adult stage. The eggs are oval in shape and white in color, while the eggs of the Pulex Avium are brown.

Condition Produced.-It does not have the tendency to hop like the Pulex Avium, but implants itself on the heads, necks, and under the wings of fowls and especially the young. With its powerful proboscis it pierces the skin and sucks the blood and remains in one position, burying itself in the upper layers of the skin producing irritation and inflammation. When removed, they may jump like species of the pulex.

Treatment.-Grease the affected parts of the head and neck and the affected parts under the wings with lard or vaseline in which there has been rubbed a small quantity of sulphur. The vaseline or lard dissolves the sulphur and this is destructive to the fleas. The lard also by sealing the breathing pores of the flea is destructive. A small application of kerosene is also destructive to the fleas. Saturate the infested premises with kerosene or a four percent solution of some coal tar disinfectant dip with a coefficiency of at least 5 .

\section{TICK INFESTATION}

In some parts of the Southern United States and in Africa and India, reports are to the effect that chicken ticks are quite troublesome. In some parts of the Southern United States one variety, the Argas Miniatus, is quite common and quite troublesome to poultry raisers.

\section{The Argas Miniatus}

Description.-The body is flat and thin. The posterior end is more bluntly rounded than the anterior. The broadest portion of the adult tick is posterior to the fourth pair of legs. It has an overreaching dorsal surface that hides the mouth parts. The hood projects above the head or capitulum and spreads out on the level of the dorsal or top surface. The lateral margins are thin or only slightly thickened. The mouth parts are provided with mandibles. which have hook-like teeth or denticles at the free extremity and an under part or hypostome provided with six rows of irregularlyarranged, toothlike denticles. With this apparatus the tick holds on to its host. At either side of this apparatus there is a palpus, an articulated, finger-like structure taking the place of antennae as found in insect parasites. The surface of the tick is slightly roughened by irregular zigzag wrinkles or folds, which are absent only at certain points occupied by nearly circular pits; the latter are more or less numerous and scattered; the larger pits form a radiating series on the dorsal and the ventral surface, the median posterior row being the longest. The eyes are absent. 
This tick is a blood sucker making its attacks at night. After feeding upon the blood of the fowl it leaves the bird and retreats to the cracks, crevices, or other darkened hiding places where it remains during the day. In its hiding place, off of the bird, it lays its eggs in masses containing many hundreds.

Life History.-After the eggs are laid they hatch in from three to four weeks. The young, or larvae are six legged and very active, attacking the fowls as readily in the day time as at night. The larvae attach themselves to the skin and feed for five or more days, remaining firmly attached all this time. At the end of this feeding period the young detach themselves and crawl away from their host, hiding in some crack, or crevice near by. The larva molt in about a week, and are now provided with four pairs of legs; this is the first nymphal stage, appearing like miniature adults. Night feeding now takes place and in ten to twelve days they molt again and the second nymphal stage is reached. Upon reaching this stage it again attaches itself to the fowl becoming engorged in about an hour and at the end of another week molts a third time reaching the adult stage. The adults are able to engorge themselves in twenty to forty minutes.

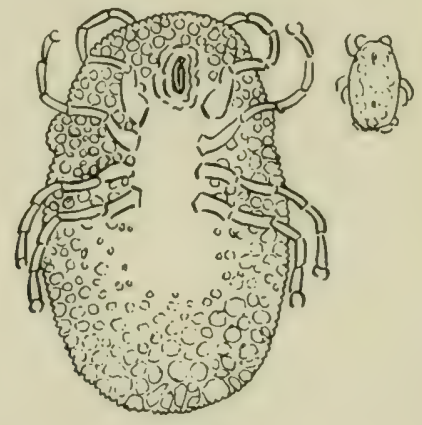

Fig. 38. Argas Miniatus.

The drawing at upper right hand corner is natural size.

Symptoms.-Large numbers attacking the fowl extract considerable quantities of blood. They also cause much irritation and the birds are not able to rest at night. As a result the birds become unthrifty, laying hens fall off in the yicld of eggs, and growing chickens do not make satisfactory gains. Sitting hens will leave their nests, and the lowering of resistance lays the fowls open to attack of disease. The birds become dull, refuse food and in many cases die in three or four days after being attacked by large numbers of these parasites.

The Argas Miniatus is a carrier of the Spirocheta gallinarum, the cause of a blood disease, spirochetosis of fowls. This disease has been reported from Brazil, and from Africa.

Treatment.-If the hen house is of little value, burning it will save time and trouble. If the house is of modern structure thoroughly clean the house burning all the litter and nesting material and thoroughly spray with crude petroleum, or a strong kerosene emulsion. or full strength coal tar dip. This must be driven into the cracks of the building and equipment as nests, feed hoppers, and broody coops. Hot tar driven into the cracks eliminates them sealing in the parasites. One application may not rid the premises of the pests as they are very resistant and some of the eggs or even the 
parasites may be missed as the remedy must come in actual contact with the tick. Owing to the fact that the young ticks attach themselves to the birds for a few days makes it necessary to continue the attack of the pests. Ordinary dusting powder as used for lice will not kill the parasite. Lard in which has been rubbed a small quantity of sulphur and this applied to the parts attacked by the young ticks will kill all that it comes in contact with. Metal buildings are not recommended as they are extremely hot in the hotter parts of the year. Thorough soaking with crude petroleum, creosote, and sealing cracks with hot tar is preferable.

\section{THE BEDBUG OF POULTRY}

The chicken bug or dove cote bug is known as the Acanthia Inodora. It is especially found in the warmer parts of the country around unclean roosts and filthy dove cotes. It is closely allied to the bed bug, the Cimex Lectularia, from which it requires a microscopic study to differentiate it.

\section{Acanthia Inodora}

Description.-The Acanthia inodora is provided with a large oval abdomen, narrow thorax bearing three pairs of legs, and a rather broad head bearing a pair of eyes, and a pair of antennae. The third and fourth segments of the antennae are much thinner than the first and second. The rostrum or anterior head parts are short. The body is covered with short hairs. The thorax is crescent shaped on the anterior border and is much wider than the head. The abdomen is segmented.

Life History.-The female deposits 100 to 200 eggs in the cracks, and crevices of the perch poles, and building, and in the filth of the floor and dropping boards, where they soon hatch, if the weather is warm, and rapidly develop to adult size. The eggs are oval and pearly white. The young escapes from the egg by lifting the end cap or operculum. The hatching under favorable conditions occurs in one week, the nympha stage six weeks, and the entire time from the egg to the age of reproduction is from seven to eight weeks.

Symptoms.-The poultry bedbug is quite a pest in warm countries as Southern United States, Mexico and Central America. The bugs hiding in the cracks and other darkened protected places in the daytime come out at night and make their attack in swarms, overrunning the legs and bodies of the fowls and suck their blood. After feeding the engorged bugs leave the fowls and seek a hiding place, where they remain for several days, during which time the meal is being digested. Finally they may be seen in enormous numbers swarming over the roosts and nests, specking the eggs with their excrement. The effect of the bites depends upon the susceptibility of the fowl attacked. In some, as with the ticks, it causes marlied irritation with marked swelling, while little effect may result in other attacks. The biting organ, consisting of four piercing filaments within the lips or labium, is closely applied to the point of puncture as the blood is drawn up. The effects upon 
the sitting hen, laying fowls and growing birds are similar to that of the chicken tick above described.

Trcatment.-Similar treatment as described for the chicken tick is advised. The attack against this bug, in countries where it occurs, must be directed early in the year as it at times is a formiclable foe, even invading dwelling houses and proving

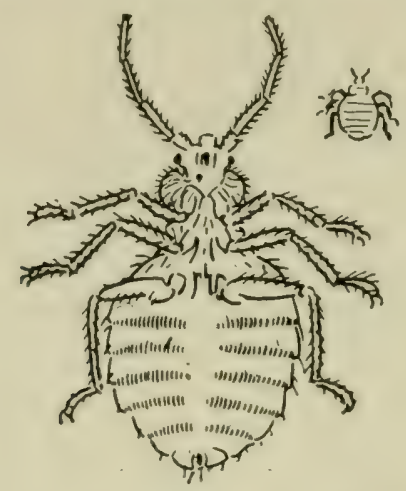

Fig. 39. Acanthia Inodora.

The drawing at the upper right hand corner is actual size.

more troublesome than the common bedbug, Cimex Lectularius. They begin to appear about the middle of April and after that time it may be necessary to keep the chickens entirely out of doors.

The bugs may live for many months in the filth about a dove cote or henhouse and the disinfection must be thoroughly done to eradicate them.

\section{BEE STINGS}

Young chickens, and especially young ducklings, are prone to catching flies and other insects. Young ducklings have been known, where they were kept in a yard with bee gums sitting on the ground, to catch the bees that in hot weather settle on the outside of the gum. In many of such cases the ducklings were attacked by the infuriated bees and so severely stung that many died. Severe swellings follow the bee stings.

\section{FUNGI AFFECTING BIRDS}

The fungi constitute a complex group of organisms which inclucle the molds, grain rusts, mildews and smut. They range in size from single microscopic cells to systems of entangled threads made up of series of cells. These entangled masses are called mycelia and are made up of threads and spores. The threads consisting of chains of cells are called hyphae and the spores are round or oval microscopic bodies from which the hyphae develop or which propagate the mold. 
Many of these fungi, among them some of the molds, do not cause disease, but live on dead and decaying matter, both vegetable and animal. These are called saprophytes. Others may live in or upon the body and cause disease and these are called pathogenic fungi.

Of the pathogenic fungi, four are harmful to chickens. The Oidium Albicans causing sore mouth, the Tinea Favosa causing honey-comb ringworm, the Aspergillus Glaucus causing a skin disease of pigeons, and the Aspergillus Fumigatus causing a fungus pneumonia of fowls.

\section{Thrush—Aphtha-Sore Mouth}

This is a condition affecting the mouth and is due to a lowgrade fungus called the Oidium Albicans, or Saccharomyces Albicans. The sore is covered by a mat-like structure or mycelium. This mycelium consists of hyphae or fine threadlike processes, which in some instances show well-marked chains of cells. It reproduces by forming oval spores. These spores are microscopic in size and are mixed with the hyphae in the mycelial mat.

Symptoms.-The symptoms are those of dullness, loss of appetite, emaciation, convulsions and death. The mucous lining of the mouth and esophagus are affected by whitish or brownish yellow deposits adhering to the mucous surface. These are composed of the filaments or hyphae with oval spores. The disease not only affects fowls, but has been observed in other birds among which are the turkey.

Trcatment.-If the patches can be seen, it is best to cauterize the area with lunar caustic, or nitrate of silver. Pure iodine applied with a swab, made by twisting a small piece of cotton around the end of a stick, and dipping this in pure iodine, and then rubbing the sore, is also effective treatment. Care must be exercised so that some of the iodine does not run down onto other adjacent tissue. Intestinal antiseptics are also indicated such as are given in other intestinal disorders as fowl cholera.

\section{Tinea Favosa-Honey-Comb Ringworm}

This is a disease of the head and upper neck of fowls and is due to a low-grade fungus, the Achorion Schocnlcinii. The fungus somewhat resembles the Oidium Albicans appearing as a mat or mycelium consisting of hyphae or threads, and reproducing by spore formation.

The hyphae are three to five microns thick, forming ramifying branches with tapering ends. The hyphae are matted together, forming mycelia or mat-like masses. Spores vary- 
ing from three to six microns in diameter are found in the meshes of this mycelia. These spores are eggs or seed, and are egg, ball or biscuit-shaped.

Like the oidium albicans, the fungus may be grown on artificial media. Upon artificial medium it appears as a moss-like growth. It grows best at twenty-five degrees C.

Symptoms.-This disease has been called favus, baldness and white comb. It is a disease that is highly contagious and attacks the comb, face and upper portion of the neck. If not treated, but allowed to spread and go on uninterrupted, it may later involve the entire neck and extend to the body.

The disease first appears on the comb or face as whitish or light gray, small, roundish patches, which vary from the size of a millet seed to a half-inch in diameter. Later these patches may coalesce and form large areas.

The diseased area is covered with a scale which may be depressed in the center and turned up at the edges, giving each area a cup-shaped appearance. In the course of four to six weeks, the crusts may be one-fourth inch in thickness.

The feathers become dry, erect, brittle and break off at the surface, leaving large denuded areas. A disagreeable odor is given off by the diseased areas which odor has been likened to that of moldy cheese. As the disease progresses the bird loses its appetite, becomes gradually emaciated, weakened and finally dies.

Treatment.-In the early stage this disease yields to treatment readily. The crusts should be soaked with a soapy water containing four per cent solution of a disinfectant dip. The disinfectant dip used should have a coefficient of at least five. The premises should be thoroughly cleaned and disinfected with a four per cent solution of the dip. Lard or vaseline in which a small amount of sulphur has been incorporated, and this thoroughly rubbed into the affected part once every third day, is also effective treatment.

\section{Pneumomycosis-Aspergillosis}

Pneumonycosis is a pneumonia of fowls due to a fungus the Aspergillus Fumigatus. This fungus is somewhat similar to the green mold. It affects the lungs and will be discussed under, "Diseases of the Respiratory Organs."

\section{MYCOSIS OF PIGEONS}

Mycosis of pigeons is a disease of the skin of pigeons due to a fungus, the Aspergillus Glaucus. The fungus attacks the skin of the pigeon causing yellowish crusts or scabs. These patches may be located on any part of the body of the bird. 
The scabby patches give off an offensive odor. The birds suffer a loss of appetite, gradual emaciation, unkempt appearance of the plumage and may finally die of exhaustion.

Treatment.-The same treatment as indicated under honeycomb ringworm is recommended.

\section{BLASTOMYCOSIS OF THE GOOSE}

Blastomycosis is a disease caused by the blastomy'cetes, a yeast-like organism. The disease has been recorded as occurring among geese. The symptoms are those of a chronic disease among which may be mentioned weakness, partial loss of appetite, emaciation and death.

Postmortem Findings.-At autopsy, there is found small cysts attached to the capsule of the liver or other structures. The cysts vary in size up to a pea. They are seldom, if ever, found in the liver substance. The cysts are yellowish-white in color, fluctuate and resemble somewhat the small tape worm cysts. Upon microscopic examination yeast-like bodies are observed which somewhat resemble the Oidium Albicans. Some may be found in the process of budding. The liquid of the cyst is of a yellowish color and of a gelatinous consistency. The cells are provided with a capsule and contain a homogeneous protoplasm in the center of which is observed a nucleus.

\section{WHITE SCALE OF THE COMB, FACE, AND WATTLES}

This is a condition among fowls which affects the comb, face and wattles. The disease manifests itself as very thin, white scales, covering large areas of the comb, face and wattles and in some respects simulates white comb, due to a fungus, but in these cases laboratory examinations have failed to reveal a fungus and inoculations of other birds from the scales fail to reproduce the disease. It never attacks the feathered parts of the head or neck. The disease may attack many members of a flock and suddenly, after a few weeks or at most, months, spontaneously disappear.

Treatment.- The best results have been attained by applying a sulphur ointment made by rubbing in five parts of sulphur in 100 parts vaseline or lard.

\section{BALDNESS OF FOWLS AND CANARIES}

Baldness of fowls and canaries is a loss of feathers of the top or crest of the head. Baldness may sometimes be due to lice or mites. A loss of feathers about the head, especially in canaries, may also indicate old age or even general debility. 
Look carefully with a hand lens for mites. One fowl may sometimes eat the feathers from the head of its mate.

Treatment.- At the natural time of moulting, the growth of feathers may be aided by warmth and a well-regulated diet. In addition to the usual feed, twice a week give to the canary a little bread moistened with milk which has been dusted with a mixture of two parts sulphur and one part potassium chlorate. At the same intervals rub a little carbolized vaseline on the bald spot. 


\section{INTERNAL PARASITES}

Internal parasites are those parasites that live within the body of the bird. They may infest the intestinal tract, liver, trachea, lungs and air sacs, or the subcutaneous structures.

Parasites infesting the intestinal tract of fowls are very common and serious results are often met with where large numbers occur. These parasites are commonly spoken of as worms. Other internal parasites, such as gape worm and the air sac mite, while not so common as the intestinal worms, are by no means unknown and have the same possibilities of serious infestations.

Intestinal parasites in small numbers infest the majority of fowls without doing perceptible harm, but there is always the possibility that conditions for their propagation may become so favorable as to turn the mildest infestation into a devastating parasitism. Indeed, this very thing has occurred numberless times and not a few flocks have been entirely destroyed by it. The death of any bird from the effects of internal parasites should be looked upon with apprehension.

Flocks infested with large numbers of round worms are unprofitable in the extreme. The birds are unthrifty, appear unkempt and suffer from diarrhea and constipation. Young fowls are most severely affected.

Internal parasites may be classed under four orders, as follows: Nematoda, or round worms; Cestoda, or ribbonshaped segmented worms; Acanthocephala, or thorn-hearled worms; Trematoda, or flat leaf-like worms, called fukes.

\section{IMPORTANT ROUND WORMS}

Round worms are the most common of the internal parasites. The small round cecal worm may be found in the ceca of more than eighty per cent of the fowls, and these worms are known to transmit the blackhead protozoa. Usually in other portions of the bowel and especially the small intestine may be found the large round worm. When numerous, these worms may seriously interfere with digestion and nutrition, and by their irritation of the intestine cause a stubborn diarrhea. Rarely they become so plentiful in the intestine as to wholly obstruct it. If in large numbers they may cause a loss of weight, stunted growth of range chickens, and in laying hens a loss in egg production. 
The round worms include four important internal parasites of birds; the large, round, intestinal worm, the Ascaris inflexa; the small, round intestinal worm, the Hetcrakis papillosa; the gizzard worm, the Spiroptcra hamulosa, and the gape worm, the Syngamus trachealis, besides a number of rare, or for other reasons, unimportant worms, all of which will be described in turn.

\section{THE ASCARIS INFLEXA}

(The large, round worms of fowls.)

This parasite, sometimes called the Heterakis perspicillum, is commonly known as the large round intestinal worm. It is very common, having been observed in twenty-four out of eighty-seven autopsies.

Description.-This intestinal parasite is round in shape and whitishyellow to white in color, varying from one to two inches in length. There are two sexes, male and female, the female being considerably the larger. Fig. 40 shows a drawing, actual size of the young male and female. Some few specimens and especially the adult are much larger than the ones shown.

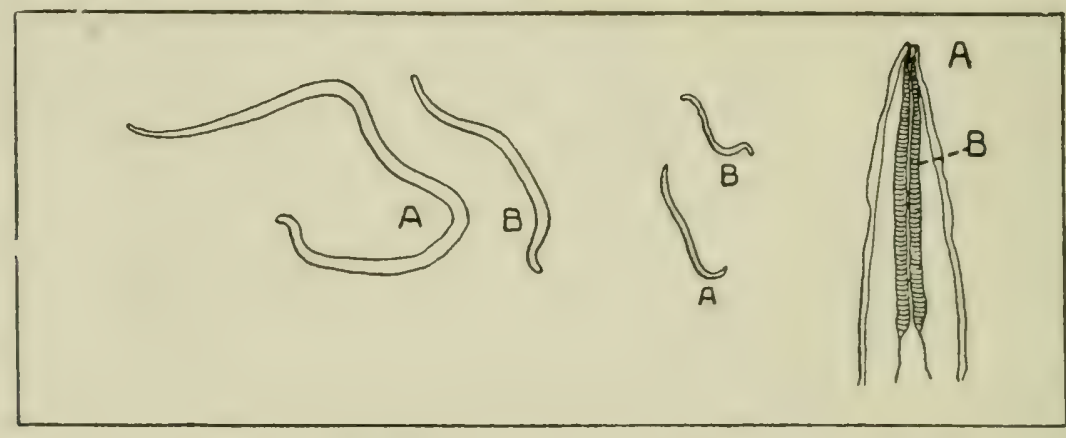

FIG. 40.

FIG. 41.

FIG. 42

Fig. 40. Ascaris Inflexa (natural size).

$a$, female; $b$, male.

Fig. 41. Heterakis Papillosa (natural size).

$a$, female; b, male.

Fig. 42. Heterakis Papillosa, Head Extremity (Magnified).

a, mouth parts; b, esophagus.

Life History.-The adult worms deposit large numbers of eggs in the intestines of the infested fowls. The eggs pass out with the droppings and ordinarily do not hatch till they are taken into the alimentary tract of a fowl. These eggs are very minute, microscopic in size, and can be seen only under a high power microscope. In the droppings they are very resistant to drying and may remain alive for at least a year. There is some evidence, however, that the eggs may hatch under certain conditions in the droppings. Birds become infested by drinking water, or eating feed, that is contaminated with egg-laden droppings. Thus the introduction into a flock of one infested bird may result in the infestation of an entire flock. The younger worms are found in the small intestine particularly in the duodenal loop or gizzard end and the larger worms 
farther down the small intestine. The development from newly hatched larvae to full grown males and females is attained in three to four weeks. If infestation has lasted the required length of time the droppings of an infested fowl will be seen to harbor great numbers of tiny worm eggs.

Symptoms.-These parasites harm the fowl by ingesting food during its digestion by the host, thus robbing the bird of food nutrients. A few worms may produce no noticeable ill effect upon the health of the bird, but if present in large numbers, they may cause serious trouble. It has been observed that the excrementitious, or waste matter, given off by these and other intestinal worms is poisonous. It is absorbed by the bird and has a deleterious constitutional effect, similar to that of like infestations by parasites in the larger animals and in man.

At times the worms are found in large masses, partially obstructing the bowel, causing constipation and possibly irritation and diarrhea result, or, the irritation may be sufficient to cause inflammation. There may be a loss of appetite, unthrifty condition, unkempt appearance of plumage, dullness, languor with droopy wings, emaciation, loss of color from the comb, face and wattles and mucous membranes, followed in a few weeks by death. Large numbers of these worms in laying hens cause a falling off in egg production, and stunted growth in developing chickens.

By careful examination of the contents of the digestive tract of the birds killed for food purposes, the poultry raiser may keep informed as to whether these parasites are present in his flock. If these worms are present in members of the flock close observation will occasionally discover them passed in the droppings.

Treatment.-It is necessary to thoroughly clean the henhouse and yard and burn all litter. Scatter with a sprinkling can, a salt brine solution over the entire yard, floor and dropping boards. A saturated salt solution coming in contact with the worms and eggs will kill them. The water for the birds should be kept in clean fountains and the food in mash hoppers. These utensils should be so arranged that the bircls cannot step into them. The water container should be frequently disinfected with a four per cent solution of a coal tar dip. If at all possible, birds should be moved upon new ground. The parasites' eggs in the droppings removed from the henhouse may be destroyed by mixing the manure with unslaked lime. Or, in case it is the desire to save the fertilizer value, the droppings may be hatuled far out in the field out of reach of the fowls. 
Fincly chopped tobacco leaves or stems may be steeped in hot water and given in the mash. One-half pound finely chopped tobacco is one dose for fifty adult birds. Fast the birds over night and give, on an empty crop, in the morning. Follow this with four ounces epsom salts to each fifty birds, giving the salts dissolved in water, and the water mixed with mash. The salts should be given as a physic, two hours after giving the tobacco. In two more hours, the birds may be put back on regular feed.

Another treatment consists of giving each bird one teaspoonful of turpentine followed by a tablespoonful of olive oil. Care must be taken lest the liquid finds its way into the trachea and a mechanical pneumonia result. If the crop is full it will require larger doses. It is best to give all medicine to fowls on an empty crop.

Five to ten grain doses of areca nut, per bird, is also effective treatment. The areca nut can be mixed with soft feed and given in clean troughs. This drug acts as both a parasiticide and a cathartic. One grain doses of thymol, also two grain doses of santonin, per bird, have also been recommended.

\section{HETERAKIS PAPILLOSA}

\section{(The small round worm of fowls.)}

The Heterakis papillosa, or small round intestinal worm, is quite common and is found in the blind gut, or caeca. It has been found present in about fifty per cent of the adult birds autopsied.

Description.-This worm is much smaller than the Ascaris Inflexa, being only about one-fourth to one-half inch long. It is white in color. Fig. 41 shows a drawing, natural size, of the male and

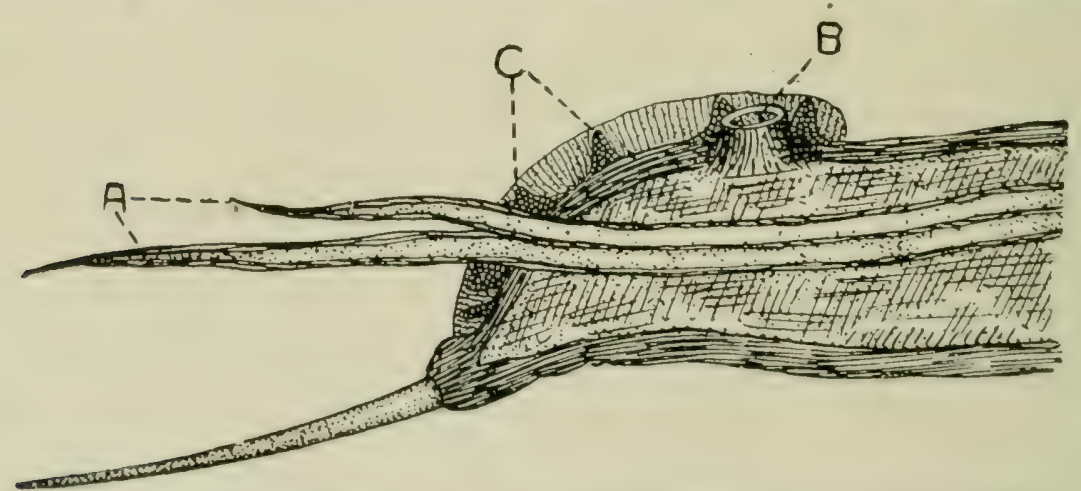

Fig. 43. Heterakis Papiliosa, Tail Extremity (greatly magnified). a, spiculae; b, preanal sucker; $c$, papillae.

female. Fig. 42 shows a drawing of the head parts, magnified sevcral times, and Fig. 43 the tail, or caudal, extremity of the male, magnified several times. 
Life History.-So far as is known the life history of the Heterakis papillosa is the same as the Ascaris Inflexa described above. The eggs are elliptical in shape and measure 74 to 78 micra long by 41 micra broad. The shell is thick, measuring 3.5 micra. The embryo under favorable conditions will develop in seven days. The egg, containing the embryo, taken into the digestive tract, will hatch and, in the caecum, reach the mature state in 57 days. The entire cycle is 64 days. The embryos measure from 290 to 340 micra long and 14 to 15.6 micra thick. The embryo develop slowly, in the egg, at $13{ }^{\circ} \mathrm{C}$. About $30{ }^{\circ} \mathrm{C}$ appears to be the optimum temperature for development, the embryo reaching its final stage in 7 to 12 days. Freezing does not kill the ova or embryos. Fully developed eggs resist drying for 18 days. Embryos in physiological salt solutions, at room temperature, were alive at the end of 12 months. Fully developed ova kept in soil outdoors under circumstances simulating natural conditions contained living embryos after a period of eight months. The ova hatch in the small intestine, pass into the large intestine, to the ceca where they develop to adult stage.

Symptoms.-When present in large numbers this worm may cause considerable irritation and results in digestive disturbances, diarrhea, inflammation, unthrifty condition, falling off in egg production, interference with growth in developing birds, emaciation and possibly death. The worms are found lying on the mucous membrane and to the outside of the fecal mass of the blind gut. As with the ascaris, they rob the fowl of nourishment:

Treatment.-The Heterakis papillosa or caecal worm-infested bowel can best be treated by forcing the medicine through a syringe into the cloaca and rectum. In the normal function of the bowels, the caeca are filled from the large intestines through reversed peristalsis, the caecal valves acting as flood gates, forcing the liquid to go into the caeca.

Sanitary measures for the prevention and eradication of this parasite and directions for its treatment are the same as for the Ascaris Inflexa. Powdered areca nut, powdered pomegranate root bark, turpentine, gasoline, iron sulphate and tobacco, given alone or in various combinations in the form of pills or mixed with food and on an empty crop are effective.

Tobacco stems when finely chopped, steeped in water for two hours and the stems and liquid mixed with the mash are readily eaten by the fowls and give uniformly good results. The fowls which are very badly infested with round worms are in most instances entirely freed from these parasites after two doses. 


\section{SPIROPTERA HAMULOSA}

(The gizzard worm)

This is the gizzard worm of the fowl. Specimens have been secured from outbreaks in the middle west.

Description.-The male measures about one-half inch in length and the female about three-fourths of an inch. Fig. 44 shows a drawing natural size, of this worm.

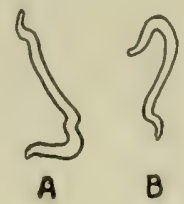

I'ig. 44. Spiroptera Hamulosa (natural size). $a$, male; $b$, female.

Symptoms.-The economic importance of this worm is due chiefly to the loss of weight, the stunted growth of range chickens, and in laying hens a loss in egg production.

The infested bird becomes anemic, loss of flesh and finally emaciation, extremely lazy, and has a ravenous appetite. The worms are imbedded in the mucous lining of the gizzard where they may cause nodules or tumor-like formations. The fowls become infested by eating feed or water which have become soiled with the droppings of infested birds. One infested bird introduced into the flock may result in infestation of the entire flock.

Treatment.-Effective treatment is difficult, owing to the fact that the worms are imbedded in tumefactions in the walls of the gizzard.

Give turpentine and olive oil as directed for the treatment of the Ascaris Inflexa as outlined above. The treatment should be repeated three or four times at intervals of one week.

Eradication of Worms.-A campaign to control the round worms of all kinds infesting the intestinal tract involves both treatment of the fowls in order to expell the worms and disinfection and sanitation of the coops, houses and yards, to destroy the expelled worms and worm eggs and larvae to prevent a reinfestation of the birds.

Some bircls do not like mash in which there is incorporated turpentine, or areca nut. Tobacco stems finely chopped and steeper in hot water for two hours and this mixed with mash, gives uniformly good results and is readily eaten by the fowls. Badly infested birds expel large numbers of worms and are entirely freed from the infestation. Two doses should be 
given three days apart. For each fifty fowls, one-half pound finely chopped tobacco stems should be used. The birds should be fed this mixture in the morning, on an empty crop. In two hours, give to each fifty fowls, four ounces of epsom salts dissolved in water and this water mixed with mash. Do not give any other feed till the physic has acted. For chicks, give doses in proportion to the size of the bird. This treatment will cost about one cent for each ten birds.

After the treatment the birds should be moved to yards and houses free from worm infestation. In yards where infested fowls have been kept, it has been observed, upon microscopic examination, that loose soil may be infested by the eggs of the round intestinal worms to a depth of two inches below the surface. For disinfecting the yards, a corrosive sublimate solution 1 to 1,000 may be used. This applied by aid of a sprinkling can after all rubbish has been swept up and removed. One gallon of the solution should be used for each ten square feet. Another effective treatment is to use the same quantity of a strong salt brine. This solution is very destructive to the worms, their eggs and larvae, but care must be exercised to not allow this solution to accumulate in small pools as fowls are easily poisoned with salt. Young birds are poisoned by a one and one-half per cent solution of salt.

The houses should be thoroughly cleaned and every square inch saturated with the corrosive sublimate or salt brine solution. The litter should be removed from the yard and house and hauled out and scattered on a field used for raising crops and out of reach of the range of the fowls, or the litter may be burned.

Corrosive sublimate, or mercuric chloride is poisonous and care must be taken not to allow the birds to drink it or the feed and water to become contaminated with it. After the feed and water troughs have been thoroughly scrubbed inside and out with the solution, they must be carefully rinsed with clean water.

\section{SYNGAMUS TRACHEALIS}

(The gape worm)

Gapes in young chickens is due to the gape worm of the trachea. This worm is sometimes called the Silcrostoma Sy'ngamus, and popularly the forked worm or gapeworm. There is another variety of this worm slightly larger than this one that infests the bronchi and trachea of water fowls, as ducks, geese and swans. It is called the Syngamus Bronchicalis.

Description.-The male is very much smaller than the female upon which it is usually firmly and permanently attached by its caudal or 
tail clasping apparatus. Thus giving to the united form the appearance of a single worm with a forked appearance which in reality is two worms, a male and a female, as shown in Fig. 45.

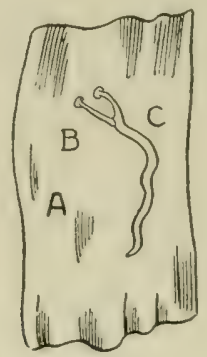

Fig, 45. Syngamus Trachealis (NATURAL SizE).

a, mucous membrane of the trachea; b, male; $c$, female.

A, illustrates a section of the esophagus to which is attached a male and a female in permanent copulation. B, is the male, and $C$, is the female. Note how tiny, both in length and thickness, the male is as compared to the female. The male is only about one-fourth inch long. The female is about one inch long and is quite thick as compared to the male. The mouth parts are surrounded by a capsular structure by which it holds firmly to the mucous membrane of the trachea, or "windpipe," or to the mucous lining of the larger bronchi. The mouth parts are provided with chittinous teeth, with which it wounds the mucous membrane; from this wound it sucks blood.

Life History.-The adult female worms are expelled by the infested chick by its coughing. The female produces eggs which escape from her body only after she is expelled from the chick and her body disintegrated. The eggs or embryos (young worms) thus escaping from the decomposing and disintegrating female may lie taken up by other chicks through soiled feed or water containing them. Or if the chick should pick up an expelled female containing mature eggs, the embryos would be liberated in the stomach of the chick, in which case they migrate to the air passages as the bronchi and trachea and develop to adult size to again reproduce. At this stage the young worms are microscopic in size. It is not thought that an intermediate host is necessary though it has been shown that the feeding of earth worms may cause the disease among chicks otherwise protected against infestation. The earth worm is thought to contain the young embryo gape worm in its intestinal tract taking it up with its food from the ground without playing the role of an intermediate host. The young worms may live for a considerable time in water, and wet soil. Damp soil thus favors its spread. Ground contaminated by gapeworms is likely to remain infective for at least a year after further infection of the soil has ceased.

In examinations made of 635 market chickens, during the winter months, none of which were fowls less than 6 months old, no infestation with syngamus trachealis was detected. Traceas of 679 turkeys from the same market, all of which birds were at least 6 months old, gave an infestation of 22.5 per cent. In artificial infestation experiments in which gape worm eggs containing fully developed larvac were fed to incubator chicks there resulted an 
infestation of 87 per cent in chicks up to the age of 4 weeks, 84 per cent from 5 to 8 weeks of age, 66 per cent from 9 to 20 weeks of age, and 29 per cent from 21 weeks of age to adult. It is possible that the turkey is therefore the natural host of the gapeworm and that both old and younger turkeys may harbor these worms without showing any symptoms. Gapes appears more prevalent on farms where turkeys are kept.

Symptoms.-Wild, as well as domesticated birds may develop gapes, due to this worm, as it has been observed in chickens, turkeys, pheasants, partridges, pea fowls, magpies, black stork, starlings, crows, parrots, swifts, woodpeckers and martins.

The poultryman's trouble with this worm is usually with young chicks and young turkeys as it does not ustally produce harmful effects in adult birds. The small immature gapeworms or eggs containing the tiny worms find their way to the intestinal tract of the young birds as described above, and from the intestines the embryos migrate to the trachea and its branches or bronchi and attach themselves, usually in clusters, where owing to their small size they do not cause serious trouble at that time, but as they grow in size they gradually obstruct the air passages to the lungs and as a result the bird, day by day, finds breathing more and more difficult and after a while gasps for air, finally becoming asphyxiated. The presence of the worms in a bird is indicated by the peculiar wheezing cough, gasping and the expulsion of frothy saliva from the mouth. The bird appears dull with ruffled feathers, loss of appetite, rapid emaciation, gaping, opening the mouth and extending the head high in the air, and death. Usually a lump may be found by feeling along the trachea, if the worms be lodged in that part of the trachea which is palpable. The sick bird coughs and shakes its head frequently. The bird frequently opens its mouth, making a wheezing sound and extends its head far up into the air. A definite diagnosis may always be made upon autopsy by the presence of the worms in the trachea, where. if present, they will be found in pairs attached to the mucous membrane.

Prevention.--Hatch the eggs in an incubator. Do not allow chicks to run out in wet grass, where they may find earth worms or contaminated water or on ground that has been exposed to chicks with gapes within at least a year. Feed only from containers which are constructed for the purpose and kept clean. Burn all extracted gape worms and the bodies of chicks dead of the disease. The allowing of chicks dead of gapes to lie around or the burying of them or throwing on the ground of the extracted worms, is a fruitful 
source of the perpetuation of this disease. If turkeys are kept on the premises, exclude them from the chick range, since they are possible carriers.

Treatment.-By grasping the bird in the left hand and forcing the mouth open, a doubled horse-hair may be run down the trachea, and, by twisting and again withdrawing, the worms are usually dislodged. Gentle pressure over the region of the mass may so injure the worms as to cause them to loosen their hold and be expelled by the bird during the coughing which this causes. Care must be exercised lest the trachea be injured. A feather from which all the barbes except those on the extreme tip have been removed may be dipped in turpentine, or oil of cloves, thinned to twice their volume with olive oil, forced down the trachea, and when the tip passes the mass of worms it may be twisted as it is withdrawn. This usually results in the removal of the worms. By referring to Fig. 2, the location of the opening of the trachea, 34, through the superior larynx may be seen. The syngamus bronchialis affects the bronchi of water fowl where it causes a catarrhal condition and at times abscess formation.

\section{UNIMPORTANT ROUND WORMS OF FOWLS}

There are other round worms that may infest the intestinal tract, but they are not common, or important, to the poultry raiser. The list follows:

\section{Heterakis Differens}

This is a slightly larger worm than the Heterakis Papillosa to which it is closely allied. Its mouth has no apparent lips; the pharyngeal bulb is distinct; there are two unequal spiculae. It is found in the posterior portion of the intestine of the fowl.

\section{Heterakis Compressa}

This is a round worm of about the size of the large round worm or Ascaris Inflexa. The tail ends in a sharp mucro. It is found in the small intestine of the fowl.

\section{Trichosomum}

Several species of the whip worm, or the genus trichosomum have been reported from various parts of the world, but have not been observed in the United States. They are shaped something like the old-fashioned blacksnake whip. They are blood suckers, and in the adult stage live in the small intestine.

\section{Heterakis Maculosa}

This is a round worm, white in color, and is found in intestinal vesicles of the pigeon. The male is about three-fourth of an inch in length and the female about one inch. At times this worm is a serious menace to the flock, killing many birds. The symptoms are similar to those produced in fowls by the round worms. 


\section{Dispharagus Nasutus}

(Filaria Tite, Dispharagus Spiralis, Dispharagus Laticeps)

These round worms infest the crop and stomach of the fowl and when in large numbers may be of serious consequence. They are slender and thread-like and sometimes cause catarrh, and if in very large numbers the bird becomes emaciated and dies.

\section{Trichosoma Contortum}

This worm infests the crop of water fowls as ducks and geese. When in large numbers it causes catarrh, dilitation of the crop, and emaciation. The bird appears dull and may show nervous symptoms, even epileptiform symptoms. Aggrevated symptoms appear in about ten days after severe infestation. Obstruction of the crop often follows. A positive diagnosis may be made by irrigating the crop and washing out the small thread-like worms. Both macroscopic and microscopic examinations should be made of the material washed from the crop. The macroscopic examination to see if worms are present and the micropscopic examination to see if the worm eggs are present.

Treatment.-In this disease treatment has been rather unsatisfactory. Keep well birds away from infected water. Give each bird one grain thymol and one teaspoonful epsom salts. The tobacco treatment may also be tried.

\section{TAPEWORMS}

\section{Flat Segmented Worms}

Tapeworms are flat segmented worms resembling a segmented tape line, and inhabit the intestinal tract of all species of birds, as well as of animals and man. More than thirty different kinds of tapeworms have been reported for poultry.

Tapeworms differ from the round worms just described, in that they have no mouth or digestive tract, are flat and segmented and have no distinct sex; that is, the male and the female are combined in each segment, in a single individual, constituting a hermaphrodite. The tapeworms all live in the intestinal tract, in their adult stage and absorb, through their body, nutrients taken in by the bird and digested; thus they rob the bird of food nutrients. The entire worm is divided into a head, neck and body. The head is provided with four suckers and in some species one or more circular rows of hooklets. This constitutes its fixation apparatus by which it attaches itself to the mucous membrane lining the intestinal tract and floats in its contents. In some species, the neck is long, in others short, but always unsegmented. The body is composed of segments. These segments grow from the neck. At first the segments are short and narrow, but become longer and wider as the distance from the head increases. At varying distances from the head the segments become mature, that is, fully developed sexually and ready to propagate. Each segment is really a separate animal and possesses both 
male and female generative apparatus, that is, a hermaphrodite. Each segment impregnates itself, after which the eggs are developed. As soon as the segment is filled with fully developed or mature eggs, the segment detaches itself, passes out with the dropping and falls to the ground. Thus at times, we may find on the droppings of an infested bird the segments, white in color and possessing the power of movement; that is, they may contract and expand, showing them to be live matter. This is especially noticeable if the segments are placed in water. Before it is detached, each segment absorbs its own food nutrients through its integument or body. This nutrient consists of the food eaten and digested by the fowl. New segments are constantly being developed by the neck of the tapeworm, growing down, becoming mature, then ripe, that is, filled with mature eggs and detached. If not interfered with, this process goes on almost indefinitely.

Upon disintegration of the segment shed from the worm, and passed out with the droppings, the eggs become scattered. The life history of many worms from this point is not well understood. It probably has an intermediate host, by which the egg is taken up and within which the embryo passes through a cystic stage in which is formed a larva, consisting of a head with its suckers and small hooks and a neck. When this cyst reaches the intestine of the bird, it is digested and the larva liberated and the larva becomes attached to the mucous lining of the intestine and develops to the adult stage just described. This is the case of most of the different kinds of tapeworms listed below as infesting fowls and turkeys, as well as water fowls, as ducks and geese. In some of these the common fly acts as the intermediate host, in others the slug and common snail, and in some of the varieties of the water fowls, fresh water crustaceans and molluscs. The eggs are taken up by these intermediate hosts through their food soiled with droppings of the infested fowl and these eggs hatch in the digestive tract of the intermediate host, and penetrate into its tissues where it develops into the larval or cystic stage, above described, in which condition it is capable of reinfesting another fowl. Fowls eating such insects or molluscs containing larval tapeworms become infested.

The larva consists of a head with its fixation apparatus, namely, the suckers and hooklets, if such be present in the adult stage, and a neck. By means of this fixation apparatus, it attaches itself to the mucous membrane or lining of the intestine of the fowl. It now absorbs digested food from the intestine of the fowl and begins to develop segments from the 
free portion of its neck. In a few weeks the worm has attained its adult length consisting of a series of segments, which from the head posteriorward, become wider and longer as the distance from the head grows greater. Finally, ripe segments filled with fully matured eggs begin to be cast off at intervals. Under proper conditions, each segment contains several hundred eggs, and each egg is capable of developing into a single tapeworm. A single tapeworm may live throughout the life of the fowl.

\section{Tapeworms of Domestic Fowls}

\begin{tabular}{|c|c|c|}
\hline $\begin{array}{l}\text { Name } \\
\text { Davainea proglottina } \\
\text { Davainea tetragona } \\
\text { Taenia infundibuliformis } \\
\text { Dicranotaenia sphenoides } \\
\text { Echinocotylus rosseteri }\end{array}$ & $\begin{array}{l}\text { Host } \\
\text { Fow1 } \\
\text { Fow1 } \\
\text { Fow1 } \\
\text { Fow1 } \\
\text { Fow1 } \\
\text { Duck }\end{array}$ & $\begin{array}{l}\text { cystic stage. } \\
\text { Slug (Limax cinereus) } \\
\text { Snail (Helix) } \\
\text { Fly (Musca domestica) } \\
\text { Fly (Musca domestica) } \\
\text { Earth worm (Lumbricales) } \\
\text { Fresh water crustacean }\end{array}$ \\
\hline $\begin{array}{l}\text { Dicranotaenia coronula } \\
\text { Drepanidotaenia gracilis }\end{array}$ & & \\
\hline $\begin{array}{l}\text { Drepanidotaenia tenuirostris } \\
\text { Davainea echinobothrida } \\
\text { Davainea cesticillus } \\
\text { Hymenolepis carioca } \\
\text { Davainea echinobothrida } \\
\text { Davainea cesticillus }\end{array}$ & $\begin{array}{l}\text { Duck } \\
\text { Fowl } \\
\text { Fowl } \\
\text { Fowl } \\
\text { Turkey } \\
\text { Fowl }\end{array}$ & $\begin{array}{l}\text { Crustacean (Cypris viriens) } \\
\text { Crustacean (Cyclops agilis) } \\
\text { Not known } \\
\text { Not known } \\
\text { Fly (Stomoxys stabulaus) } \\
\text { Not known } \\
\text { Fly (Musca domestica) }\end{array}$ \\
\hline
\end{tabular}

\section{The Taenia Infundibuliformis}

The taenia infundibuliformis, also called the Choanota Infundibuliformis, and also the Drepanidotacnia Infundibuliformis, is a common tapeworm infesting the small intestines of fowls.

Description.-The Taenia Infundibuliformis varies in length from one and one-half to three inches. Fig. 46 illustrates a mature worm. Its head is oval, the neck short, and the segments shorter in length than in width. The head is provided with four sucker-

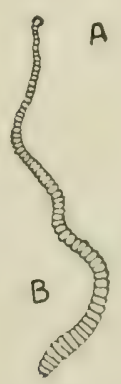

Fig. 46. Taenia Infundibuliformis

(NATURAL SIZE).

a, head; b, segmented body. 
discs and a crown of from sixteen to twenty hooklets (tiny hooks), which cannot be seen except by aid of a microscope. The anterior border of the segments is a trifle shorter than the posterior border, giving the edge of the worm a serrated appearance. The male and female genital pores irregularly alternate.

Life History.-The eggs, from the disintegrating segments shed from the worm, pass out with the droppings and flies feasting upon the fresh feces devour, with their food sucked up, the tiny microscopic eggs, of the tapeworm, in which they hatch out into single celled embryos. These embryos penetrate the abdominal cavity of the fly and become encysted. Each cyst contains the larva of the tapeworm or Taenia Infundibuliformis and a fowl devouring the fly containing the cystic young tapeworm becomes infested. In the stomach of the fow 1 the cyst is digested liberating the live larva which now attaches itself to the lining of the small intestine and soon develops into an adult worm consisting of a head, a neck, and a long chain of segments.

Sy'mptoms. - If a bird be infested by large numbers of tapeworms, it is robbed of much food, as related above, and the fowl becomes unthrifty, shows an unkempt appearance of the feathers and possibly a loss of flesh. As a result of the irritation produced by these worms, there is a loss of appetite, derangement of digestion, catarrhal condition of the bowels and in laying hens a loss in egg production, and in growing birds, a stunted growth. Birds two to three months old may harbor many adult tapeworms. This tapeworm often causes death of the infested bird. In the later stages of infestation, the bird appears dull, emaciated, and there may be a complete loss of appetite.

This, one of the most common tapeworms, has been observed to be transmitted by the house fly, musca domestica. Young birds are more active in catching flies than older ones and are often more greatly infested. The degree of harmfulness depends upon the number of tapeworms infesting the bird. Fowls with slight infestations may not show any symptoms. They may devour large quantities of feed and appear ravenously hungry. The irritation caused by these worms may canse diarrhea and the feed rushed through the intestinal tract before digestion and absorption of its nutrients can be properly accomplished. Though birds eat ravenously at first, their intestines are practically empty. The tapeworms rob the birds by absorbing digested nutrients. In the earlier stages, the bird appears restless. Heavily infested growing birds show a lack of proper development; they are usually slender, poor in flesh, the head thin and the face, comb and wattles pale.

Treatment.-Give to each fowl one teaspoonful of epsom salts dissolved in warm water, by the mouth or mix in bran, making the mash wet; follow with one-half teaspoonful of 
turpentine. A few teaspoonfuls of a decoction of pumpkin seeds usually rid fowls of tapeworms. This should be followed by a teaspoonful of epsom salts or of olive oil. Powdered areca nut in three-grain closes given in dough ball by the mouth or in wet mash is also effective. Thymol in onegrain doses is said to rid the digestive tract of worms.

Mix one tablespoonful of concentrated lye with four quarts of grain, as wheat, oats and corn and feed to the infested flock. It is better to fast the birds for twelve to eighteen hours. Give water as soon as the grain has been given as it aids in diluting the lye. Repeat the dose in twenty-four and again in forty-eight hours. The lye acts to some extent as a purgative.

\section{Davainea Tetragona}

The Davainea Tetragona is the tapeworm that causes nodular taeniasis or nodular tapeworm disease. This disease has been observed and reported as occurring in some of the eastern states and causing quite a loss to poultry raisers.

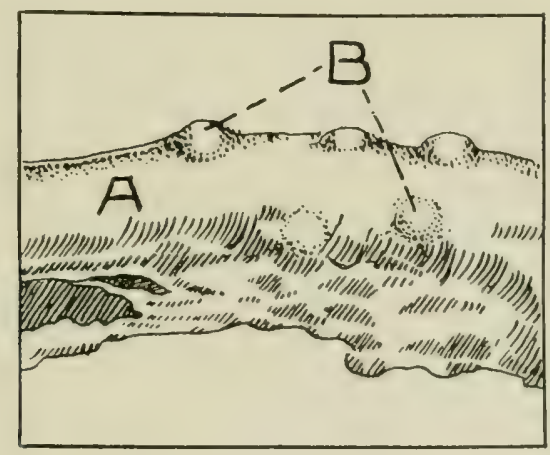

Fig. 47. Nodular Taeniasis or Tapeworm DISEASE.

a, section through the intestine of fowl, natural size; b, nodules; natural size.

In Fig. 47 may be seen a drawing, natural size, of an intestine showing this disease. This is a drawing of the outer or serous surface of the intestine, which presents a nodular appearance that might be mistaken for tuberculosis. Note that the nodule pushes the serous covering out. The mucous or inner lining of the intestine is similarly elevated and protruding from the nodules into the intestine may be seen a portion of some of the worms. In later stages, these nodules may show ulceration on the mucous surface. There may be seen in these nodules a greenish-yellow necrotic or dead tissue. A secondary invasion, with pus germs, may take place, in which case, pus will be present. Before the nodules are 
formed, these worms may be seen, under the microscope, to be lodged between the villi or miniature teat-like projections from the inner lining of the intestine.

Life History.-The intermediate host is the snail which becomes infested by eating the eggs of the tapeworm with its food that has been contaminated from the droppings of an infested fowl. In the tissue of the body of the snail the cystic or larval stage develops and the fowl becomes infested by eating the snail containing this young larval tapeworm.

Symptoms.- The symptoms have been observed to be similar to those described as occurring in infestation by the Taenia Infundibuliformis described above:

Treatment.-The treatment should be the same as for infestation by the Tacnia Infundibuliformis, or, mix with the feed one teaspoonful of powdered pomegranate root bark for every fifty adult birds. Follow with a physic. The physic may consist of four ounces of Epsom salts dissolved in water and mixed with mash or given as a drink.

\section{Davainea Echinobothrida}

The Davainea Echinobothrida is one of the common tapeworms that infests the small intestines of turkeys. Its head is slightly larger than its neck, is rounded in front and is provided with four suckers and a circular rostellum of hooklets. These miniature hooks are arranged in a double row. The hooks number about 200 and surround a pit-like cavity. This constitutes the fixation apparatus by which means it holds on to the mucous lining of the bowel. These worms may reach the length of six inches. The mode of spread of this worm and the symptoms and treatment are the same as in the fowl.

\section{Other Tapeworms}

Many other species of tapeworms infest fowls, turkeys, ducks and geese, but these will serve to give a general idea of their appearance, conditions they produce, their economic importance, and means of combating them.

\section{THE THORN HEADED WORMS}

The thorn headed worms belong to the class of Acanthocephala, and infest the intestinal tract of fowls including ducks and geese. The body of the worm is cylindrical. It is not provided with a digestive tract, as in the worms classed as round worms and belonging to the family Nematoda. It has transverse markings and, like the tapeworm, lives by absorption through its body, nutrients eaten and digested by the fowl, thus, to a degree, robbing its host. Furthermore, when present in great numbers, these parasites cause digestive derange- 
ment and emaciation of their host. They are provided with a globe-shaped proboscis, in the place of a head, armed with miniature hooks, which enables it to attach itself to the inner lining of the bowel. It embeds these hooklets in the mucous lining of the intestine; thus attached by its head, it floats in the intestinal content from which it absorbs digested nutrients. There are two distinct sexes, a male and a female, the female being the larger.

\section{Echinorynchus Polymorphus}

The Echinorynchus Polymorphus is one of three species of the thorn headed worms that live in the intestine of the duck. It is also found in the goose.

Description.-.-The Echinorynchus Polymorphus varies in length from one-fourth to one inch. The body is orange-red in color. It has a neck-like constriction, just back of the hooked, globe-shaped structure. Its proboscis is provided with eight or nine rows of hooklets. It is round in shape with transverse marking giving it somewhat the appearance of being segmented.

Life History.-This worm reproduces by laying eggs. The intermediate host is certain fish, as the shrimp, and cray fish. Ducks and geese become infested by eating fish infested by the larval or cystic form. The shrimp and fish become infested by eating the eggs of the worm, in its food that has been contaminated by droppings of a fowl that harbors the adult thornheaded worms. This parasite has not been reported from the United States.

\section{FLUKES \\ (Flat Leaf-like Worms)}

The flat leaf-like worms belong to the family, Trematoda. Flukes of birds are harbored, for the most part, in the intestinal tract. It is believed that this worm is exceedingly rare if found at all in the States as we have no authentic case reports.

\section{Notocotyle Verrucosum}

The Notocotyle Verrucosum is perhaps the most commonly described fluke as infesting the intestinal tract of fowls. The body of this worm is white or reddish-white and from onetwelfth to one-fourth of an inch long. It is oblong in shape, narrow in front and rounded behind. It is found in the intestine, principally the caecum or blind gut. It has not only been reported as infesting the duck, but also the fowl.

No serious results have been attributed to the flukes of poultry, although it is well known that they cause serious diseases of other animals. There have been three or four other similar worms described which closely resemble this one. 


\section{INTESTINAL WORMS OF CANARIES}

If the bird is infested with worms, these may at times be noted in the droppings. Place in the drinking water ten drops tincture gentian to each ounce of the liquid. After two days, give two drops olive oil by the mouth by means of a medicine dropper.

\section{DISEASES DUE TO PROTOZOA}

(Single Celled Animal Organisms)

Trichomoniasis

Trichomoniasis is a disease of young chicks caused by a single celled animal organism, the Trichomonas pullorum.

The baby chicks are attacked at the age of 10 to 14 days. They are rarely attacked after the chicks are four weeks old.

Symptoms.-The affected chicks may droop, huddle together, usually the appetite remains normal, and in later stages, the wings drag the ground due to weakness of the wing muscles, the eyes are closed, heads pale, temperature usually subnormal, $102^{\circ} \mathrm{F}$.

In the earlier stages, diarrhea may be absent, but is always present in later stages.

Treatment.-Intestinal antiseptics are indicated, such as bichloride of mercury 1 to 10,000 which is made by placing in one gallon water 6 grains bichloride of mercury and three grains of citric acid. Also ipecac, five grains to the pint of water, may be given. Sulphocarbolates compound, five grains to the pint of water, is indicated. Or permanganate of potash in drinking water, making the water deep red.

Postmortem Findings.-Upon opening the abdominal cavity, after death, there may be noted no sign of disease except an anemic condition. The caecum, or blind gut, may be slightly enlarged and its contents slimy and upon microscopic examination the crypts in the mucous lining are observed to be filled with the germs, Trichomonas pullorum.

\section{Coccidiosis}

Coccidiosis is a disease caused by some species of the genus coccidium, a single-celled animal organism. The coccidia may not only infest the intestinal tract, but also the liver, kidneys, and even the lungs. It is a serious disease, once it gets a foothold and may infect young chicks, pigeons, turkeys, geese and wild birds.

The variety most common is the Coccidium Arium, sometimes called the Eimcria Avimm, and belongs to a class of protozoan parasites known as sporozoa.

The resistant stage is called the oocyst and is most easily 
recognized on microscopic examination of the intestinal content, the parasite generally appearing as an oval body containing a central granular mass surrounded by a highly refractile zone with a double limiting border. The size varies from 25 to 35 micra in length, and from 15 to 20 micra in breadth.

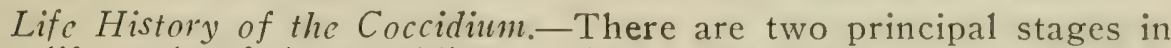
the life cycle of the coccidium. First, a stage of asexual reproduction called schizogony in which male and female elements unite to form resistant bodies for life outside the animal cells and outside the body of the bird. In the asexual stage the newly formed parasites pass from one cell to another, and it is during this cycle that the great destruction of intestinal mucosa occurs.

The oocyst referred to above reaches the intestine of the bird through food or water contaminated with droppings of an infected bird. In the intestines of the bird the tough cyst wall is softened and there is liberated four small bodies or spores, which are slender with one end more pointed than the other. These young coccidiac are called the sporozoits, are very actively motile, and pass into the epithelial cells of the mucosa lining the intestines. It now begins to grow in size finally destroying the cell. This reaches the trophozoit stage. When fully developed in the trophozoit stage it measures 10 to 12 micra in diameter. The nucleus now divides into a number of daughter nuclei which arrange themselves in a zone at the periphery or outer portion of the cell. This constitutes the schizont stage and represents the beginning of asexual multiplication or schizogony. Each nucleus becomes surrounded with protoplasm and assumes a long narrow form with pointed ends. The length measures from 6 to 10 micra. These bodies, known as the merozoits, differ from the sporozoits in that their nuclei contain a small particle of chromatin, the karyosome. From 8 to 20 merezoits are formed from one schizont. On separation from one another, the merezoits work their way into other cells, lining the intestinal tract and may repeat the process of development just described, or there may take place a differentiation into sexual forms within the newly invaded cell. This latter process is called gametogony.

Second, a stage of reproduction called gametogony. Two forms of the organism are here produced, the macrogametocyte, or female mother cell, and the microgametocyte or male mother cell. Both forms are oval in shape, the macrogametocyte being larger and more granular than the microgametocyte. The macrogametocyte gives rise only to one daughter cell or macrogamete, measuring 11.8 to 17.5 micra long. The microgametocyte produces a large number of minute, biflagellate, actively motile male cells called microgametes, 3 to 4 micra long.

Fertilization takes place through the entrance of the micro. gamete or male cell into the structure of the female cell or macrogamete at the thin spot at one end known as the micropyle. After the entrance of the male cell the macrogamete forms protoplasm which plugs the opening and prevents the entrance of other microgametes. The nuclei of the two cells now unite forming a zygote which proceeds to spore formation or sporogony.

In the sporogony or zygote state it is surrounded by a tough double membrane which is extremely resistant. This is the oocyst stage. The contents is at first granular and grayish in appearance. 
Later the granular material is gathered into a compact circular mass in the center of the oocyst. The nucleus imbedded in the central mass now undergoes division into two and these in turn divide, forming four daughter nuclei. These are surrounded by cytoplasm and are separated from each other. There are now four rounded bodies formed within the cyst. These are the sporoblasts. Later the sporoblasts lose their rounded form, become oval and are inclosed within cell walls. In this form it is called a sporocyst and from it develops a spore which contains two young parasites or sporozoits which are capable of invading an epithelial cell of the mucous membrance of the intestinal tract. The life cycle of the parasite is from eight to ten days.

\section{Coccidian White Diarrhea}

Coccidian white diarrhea is caused by coccidae, a singlecelled animal organism, and occurs in chicks:after the tenth day and usually runs a rapid course. The chicks become infected through feed or water contaminated by the excreta of infected birds. An infected bird may be introduced in the flock, or the chicks may be placed in coops or on ground on which infected birds have recently been kept, or they may be shipped in infected coops. Infection may also be carried on the feet of men, $\log s$, cats and other animals and by wild birds. Sparrows, ever a pest of the poultry house and yard, carry many infectious disease germs, as well as parasites, as mites. It may be possible that a chronic type of coccidiosis occurs in older birds and thus perpetuate and disseminate the coccidiae.

Simptoms.-In young chicks, the disease develops rapidly and the death rate is high. The chick is weak, unkempt feathers, wings droopy due to muscular weakness, loss of appetite, or in some cases, birds may continue to eat. The birds stand in bunches, and peep much of the time. They appear stilty and the droppings are fluidy, or a whitish or a whitish-brown color and paste up the rent region and may prevent bowel movement. The discharges may be streaked with blood.

Postmortem Findings.-Upon opening the abdominal cavity of the chick dead of coccidian diarrhea one may find the intestines congested and ulcers of the intestinal tract, especially of the caeca. The caeca may be filled with a bloody semi-solid mass which distends the organs. This mass consists of blood cells, exfoliated mucosa, fecal matter, and numerous coccidae in the oocyst stage. See Figs. 48 and 49.

Treatment.-Give one-third teaspoonful catechu to each gallon drinking water, or ipecac may be used in the same proportion. Sulphocarbolates compound may also be tried. One to ten thousand bichloricle of mercury has also given most excellent results. Permanganate of potash is also indicated. 
A cathartic should be given once a week. Give one tablespoonful of epsom salts in the drinking water for each 100 baby chicks, or to fifty chicks four weeks old, or to each twelve older chicks.

\section{Coccidiosis of Adult Fowls}

Adult fowls may also be infected with coccidia, becoming infected in the manner described above for chicks.

Symptoms.-The symptoms depend upon the age of the fowl and the degree of infection, usually the disease develops

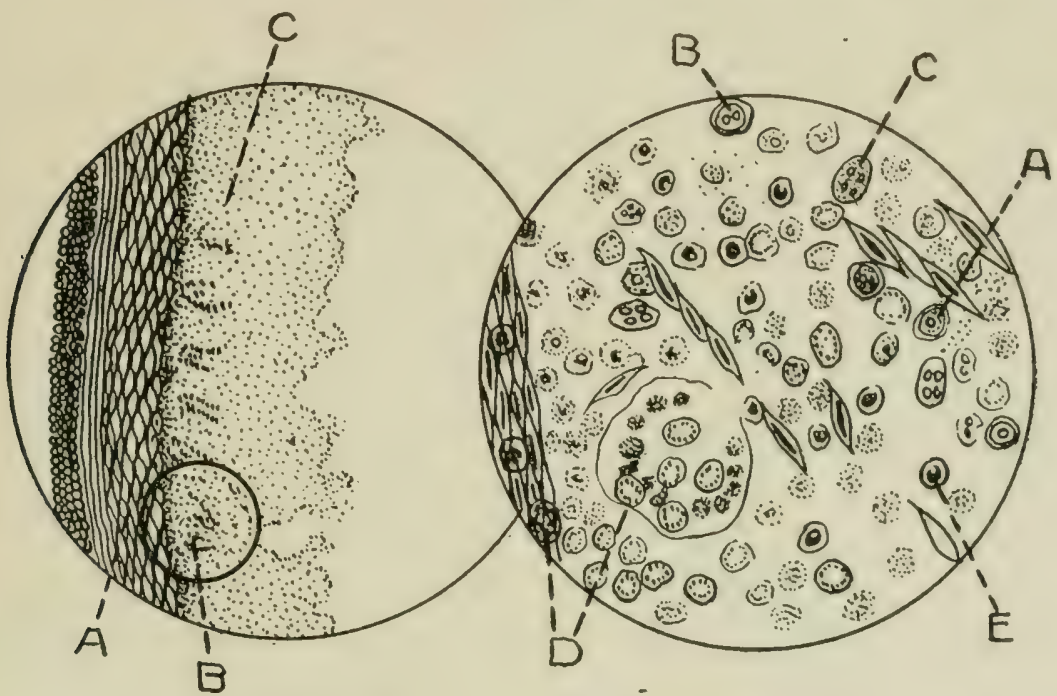

FIG. 48 .

FIG. 49.

Magnified 100 T'imes.

From a chick that had died from coccidian white diarrhea. a, muscular layer; $b$, remnant of gland; c, degenerating mass. There is complete destruction of the mucous membrane.

Fig. 49. Section B in Fig. 48, Magnified 900 TIMES.

Shows various stages of the coccidium avium. a, oocyst; b, sporoblast; c, sporozoit; d, schizont, merozoites within, surrounded by a distintegrating cell mass; $e$, polymorphonuclear leucocyte.

rapidly. At times, it may assume a chronic type. The affected birds, in the acute type, appear droopy, stand around away from the balance of the flock, loss of appetite and gradual emaciation. In semi-acute cases, the fow 1 develops a gradual and progressive listlessness and loss of activity with emaciation. The comb becomes pale and periods of dejection, during which the bird stands in an isolated position, increase as the disease progresses. The appetite may remain normal and even may be ravenous when fowls are aroused at feeding time. During intervals between regular feeding, there is little effort to scratch for feed. Death may occur in from one to 
three weeks after the appearance of the first symptoms. Death may occur suddenly or the bird may be in an unconscious state for many hours preceding its, death. Leg weakness and paralysis may accompany the disease.

Affected geese show great weakness and rapid emaciation. When compelled to move, after walking a few steps they may fall and after a struggle roll on their backs, a position which is frequently assumed.

Treatmont.-The same as outlined above for chicks is indicated.

Postmortem Findings.-The intestinal tract and principally the caeca are the ones involved.

In the caecum the mucous membrane may be completely degenerated and forming a part of a caseated mass. On microscopic examination, the necrotic material appears as an amorphous mass in which cells in various stages of degeneration, mucous, food material and many coccidia. The affected mucous membrane is denuded, congested, and even small hemorrhages.

Preventative Measures.-In considering preventative measures we must regard the means by which the infections matter is distributed and the source from which it comes. The coccidia are eliminated from the body of the bird through the droppings by way of the bowel, since the mucous lining of the intestines are affected. Infected premises, then, will be all places in which sick fowls have been kept and all objects, living beings, water, feed, litter, houses, ground, etc., which can be soiled with the droppings of the sick birds and considered as carriers.

The drinking water must be from a source where it has not been possible for contamination, and infected ditch water must be avoided. If it is not certain that the supply is free from contamination, then the water should be boiled. The feed should be dry, distributed in clean, non-infected containers, and placed in disinfected troughs. (ireen feed is always dangerous and must be from a field known not to be infected and given cut fine in a trough.

Close the poultry house in such a manner that no small wild birds can get in. If new birds are to be introduced into the flock, they should be from sources known to be free from infection. (Juarantine them for several weeks in a house some distance from the resiclent fowls before allowing them to mix with the other birds.

Ilatching egges should be plunged, immediately after arriving, into a strong solution of alcohol. Take only thoroughly healthy broody hens.

Keep the poultry houses, incubators, feeding buckets, tools and other equipment clean, lieep the soil dry and frequently 
thoroughly spray the house and utensils with a four per cent solution of some standardized disinfectant dip of a coefficiency of at least five or six.

Each bird affected with diarrhea should immediately be separated from the balance of the flock. Burn the dead bircls. Kill and burn all birds considered incurable. The litter of the house and yard should be frequently cleaned and the cleanings burned.

Never move the chickens from an infected house to another house, not even when the disease has disappeared from the house in question. Consider the house as infected and the chickens as carriers of the infectious matter and keep the whole severely isolated. Once the house having leen cleaned by killing off the remaining birds, disinfection should again be carried out in a thorough manner. The soil should be removed to quite a depth, at least six inches, from the floor of the house and fresh non-infected soil be put in its place. The outside yard can be flooded with 1 to 1,000 bichloride of mercury, using one gallon to each ten square feet. Do not use the house again till at least one year. These measures can be successful only in the case of birds in confinement, because in this case the propagation of the disease can be easily controlled.

\section{Coccidiosis in Wild Ducks}

(Coccidiosis of wild ducks is a disease catused by Coccidiae)

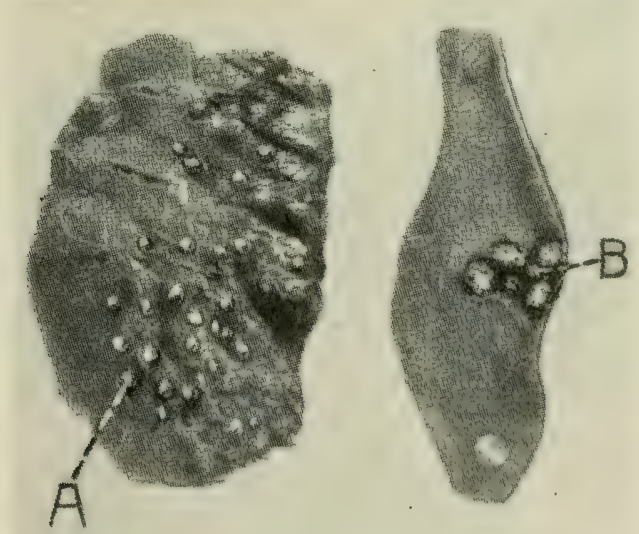
$\begin{array}{ll}\text { Fig. 50. } & \begin{array}{l}\text { Fig. } 50 \\ \text { Pulmonary Coccidiosis }\end{array} \text { OF } \\ \text { Wild Duck. }\end{array}$

a, nodules in lung caused by the coccidium, natural size.

Fig. 51. Intestinal Coccidiosis of A WILD DUCK.

b, ulcers caused by the coccidium, natural size. 
Wild ducks may be attacked in considerable numbers. Such birds are found dead around the lakes in which they are accustomed to inhabit. The birds become weak, unable to procure food, emaciate and finally die.

Postmortem Findings.-Small peral-like nodules may be observed throughout the lungs. Ulcerations of the mucous lining of the intestinal tract is also observed. This has been observed as extending through the entire length of the intestinal tract. Upon microscopic examination these lesions, as well as the lung nodules, show coccida.

\section{Renal Coccidiosis}

Renal coccidiosis is a disease of the kidneys caused by the coccidiae, a single-celled animal organism.

Raillat observed the invasion of the kidneys of a goose with coccicla in which the symptoms were those of progressive emaciation, weak, staggery gait. The bird was observed to roll over on its back, thus assuming a dorsal position. There is a loss of appetite, gradual emaciation, unthrifty appearance and finally death.

Postmortem Findings.-At autopsy the kidneys showed small whitish nodules, varying in size up to a pinhead. At times the lesions are found rather diffused. A microscopic examination of the nodules show them to be filled with masses of the encysted coccidae.

\section{PROTOZOAL HEPATITIS OF PIGEONS}

Protozoal hepatitis is an inflammation of liver due to proto $\approx o a$, single-celled animal parasites, invading that organ.

A variety of protozoa, the Carcomonas hepatis, has been observed to have caused an inflammation of the liver in a pigeon. The microscopic parasites are provided with one or two flagella or hair-like tail appendages, which enables them to move about in the liquid it happens to be in. Upon postmortem examination it was observed that the liver appeared large and studded with multiple foci or small areas of dead liver tissue.

Young pigeons may die suddenly without showing symptoms of any disease.

\section{SARCOCYSTIS}

Sarcocystis is a condition in which the fow is affected by a protozoa or single-celled animal organism belonging to the genus sarcocystis. It affects the striped and unstriped muscular tissue, connective tissue and at times the visceral organs.

The disease formerly described under the head of disease of the subcutem, is thought to be due to calcified sarcocysts. There is found, at times, peculiar dise-shaped yellowish or yellowish-gray bodies in the loose connective tissue that 
attaches the skin to the underlying structures. These bodies are only one or two millimeters in diameter and upon being sectioned and examined under a microscope show by their microchenical action that they contain calcium or lime salts. This condition is found in fowls of any breed and in any condition, but is rather more common in those fowls thin in flesh. There is no evidence of it being an acute reaction of the tissues and is apparently of no constitutional significance. All efforts to isolate an organism or to reproduce the condition have been negative. There is a possibility that it may be a calcification of a parasitic invasion as stated above.

\section{LEUCOCYTOZOON OF DUCKS}

Lencocytozoon disease is a disease among young ducks in which a single-celled protozoon parasite invades the leucocytes or white blood cells of the blood.

The leucocytozoon or organism causing this disease is spindle-shaped and 35 to 60 microns in length and 10 microns in breadth. It has an elongate oval, or irregular-shaped nucleus, with dark chromatic band extending along one border. At each end the parasite terminates in an acute angle.

Symptoms. - The disease runs a rapid course, with few wellmarked symptoms. There is partial loss of appetite and the bird does not come when called at feeding time and in later stages the sick duck lies in a semi-comatose condition with the neck bent backward and the head resting upon the dorsal portion of the spinal column. When roused from this stupor, the bird experiences a period of intense excitement during which remarkable movements are made. The head is held in various positions, or describes circles in the air, or at other times swings to and fro. In some cases, the neck is completely turned on itself with the head resting on the ground in an upright position. The power of equilibritum is lost and the bird turns over backward until exhausted. In recovered birds, there may be lameness and difficulty of locomotion. An inflanmation of the eye may be observed in which case there is an accumulation of pus. Only 25 to 30 per cent of the affecter birds recover.

Postmortem Findings.-At death there is found an acute hemorrhagic inflammation of the large intestine. Other lesions are not described as being observed.

\section{TRYPANOSOMIASIS OF BIRDS}

Trypanosomiasis of birds is a condition in which the blood of the bird is infested by a protozoon belonging to the genus trypanosoma. 
The blood of a large number of wild birds is infested by trypanosomes without producing any noticeable disease. Nine species of trypanosomes have been observed in birds. Among the birds are included fowls and pigeons.

\section{Malaria of Fowls}

Malaria of fowls is caused by a protozoon parasite called the Plasmodium danielcwskyi. This parasite has many points of resemblance to the malaria parasite of man. The parasite occurs in large numbers in wild birds. The only domestic bird to have been reported as infested by this parasite is the pigeon. As high as 90 per cent of the affected birds die.

Symptoms.-The affected bird is mopish, plumage losing its lustre, and the bird becomes emaciated and dies.

Postmortem Findings. - The blood of sick birds may be watery and less abundant. The blood may be pale and coagulates slowly. The spleen is of chocolate color and swollen, being twice its normal size.

\section{ENTERO-HEPATITIS-BLACKHEAD}

Entero-Hepatitis or blackhead, is a disease of the liver and intestine caused by a protozoon, the Amcba Melcagridis, and is hence placed under the protozoal diseases. The first part of the word refers to the intestine and the latter to the inflammation of the liver. It has been commonly called blackhead because it was supposed that the head always turned a dark purple or black. This is, however, seldom the case.

This disease affects mostly turkeys, though the author has observed 42 out of 43 Silver Spangled Hamburg chiclis die of the disease at the age of about five weeks. The lesions of the chicks were the same as those of turkeys and the Ameba meleagridis present in the liver and affected caecum. Among young turkeys, it is quickly fatal. It has partially annihilated the turkey industry in sections where it was formerly profitable and carried on extensively.

Canse.-This disease is claimed, by Dr. Theo. Smith, formerly of the Bureau of Animal Industry, to be due to a protozoon, the Ameba Mcleagridis, a microscopic organism. This germ is always found in the diseased areas in the cecum or blind pouches and in the liver of the affected birds, which are chicfly young turkeys and less common in young chicks, especially of the more delicate breeds. ()thers attribute the disease to a protozoon belonging to the genus coccidium, but this confusion has, perhaps, come about by finding coccidiae in the cecal lesions as a secondary invader. The protozoon is purely a 
connective tissue parasite and does not enter epithelial cells at any time, as does the coccidium.

Mode of Spread.-As will be seen later, the protozoon escapes from vlcers in the ceca or blind gut and passes with the droppings to the ground. Feed or water contaminated with such droppings transmit the disease germs to other susceptible birds. Chronic carriers or cases in older turkeys or in chickens may keep the premises infected for a long time. These germs entering the liver and the mucous membrane lining the ceca, cause inflammation and degeneration. Usually the cecum becomes infected first and later the liver is invaded and inflammation of its structures ensues.

The period of incubation, that is, the time from which a bird is infected till the first symptoms appear, is usually from ten to twelve days.

The exposure of young incubator turkeys to older birds from infected flocks on infected ground produces the disease within two weeks. The exposure toolder infected birds on fresh ground also produces blackhead, but after a somewhat longer period. Blackhead may be complicated with coccidiosis. Blackhead may appear in an isolated group of turkeys and suggests that bircls other than turkeys, as hens, may be carriers of the parasite. Young diseased birds may fail to carry the disease. Turkeys fed with infectious material contract the disease. Spontaneous outbreaks among turkeys penned with incubator chicks has occurred. In our experiments, turkeys hatched and brooded with hens on new uninfected ground contracted the disease and died. Field experiments show a steadily increasing concentration of the infection on the ground. where infected turkeys are reared even though the ground be ploughed and seeded before use. The growth in the intensity of the disease may be in part ascribed to an accumulation on the soil of infectious agents during any given season after they had been introduced. There is a possibility that other birds are carriers of this disease and the attraction of wild birds in large numbers and greater variety on account of the food supply in the turkey inclosures and the more intensive cultivation of the land, may be a cause of the spread of the disease. It was observed that the intensity of the outbreak due to the confining of young turkeys with birds over a year old, which had been infected cluring the preceding year, or on ground previously occupied by them, was in all instances much greater than in the spontaneous outbreaks. It was observed that soil on which turkeys had been kept was infective for young ones. The protozoa parasite 
invading the walls of the cecum and liver multiply very rapidly when once started. Smith and Graybill have shown that living embroys of the Heterakis Papillosa transmit the disease to susceptible turkeys. They collected from the ceca of healthy chickens these worms, cut them to pieces in a small quantity of a normal salt solution, 0.85 per cent sodium chloride, to liberate the eggs or ora. The suspension was kept in small dishes, Petri dishes, at room temperature and distilled water added from time to time. After the cultures had been

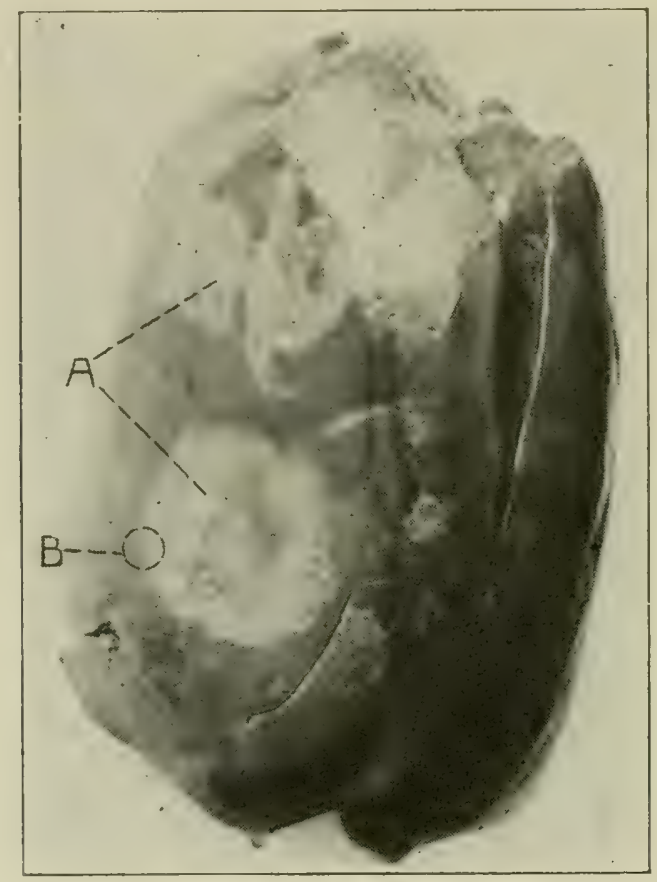

Fig. 52. Enterohepatitis of the LIVER OF A TURKEY.

a, yellowish-white necrotic areas. This liver weighed 452 grams, nearly one pound.

liept for seventeen days, examination showed that the ova contained living embryos. These embryos fed to young turkeys produced the disease. The rukeys dying of blackhead in one to two weeks' time. The worms, when invading the cecum in large numbers, lreak down the resistance of the bird and the invading Imeba gain entrance and develop the disease much more rapidly. Turlieys should be hatched in an incubator and bronded artificially and liept away from both turkeys and chickens and from infected ground.

Symptoms.-The first symptoms noted in the poults are that they lag behind the balance of the flock-a sign of weakness. 
This is soon followed by more pronounced weakness as shown by the slow careful gait with droopy wings and a tendency to stand in one spot. There is now a loss of appetite and loss of weight. The temperature taken of several turkeys with blackhead and the same number of controls does not show an elevation of temperature, that is, there is apparently no fever.

Entero-hepatitis is common in turkeys between the ages of one month and one year, although I have seen the disease in

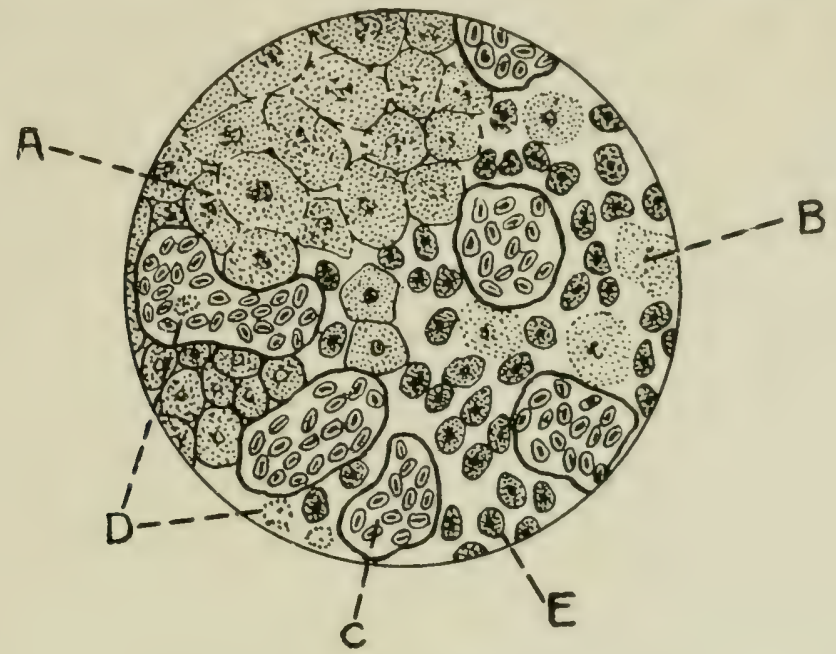

Fig. 53. C'loudy Swelling of the Liver of a TURKEy DUE to ENTEROHEPATITIS.

This is a section from b in Fig. 52, magnified 900 times. a, liver cells in a state of cloudy swelling; b, liver cells undergoing disintegration, necrosis; $c$, congested blood vessels; $d$, white blood cells, eosinophiles abundant in this disease; e, Protozoa causing the disease.

birds that were much older. Several outbreaks have been studied in this laboratory. Only one case was observed in a hen, but it has been observed among chicks from four to five weeks of age, in which the mortality was close to one hundred per cent. The disease is also reported in a peacock and in wild birds, as pheasants.

The symptoms are not manifest till the disease has progressed to a considerable extent. The bird as stated above appears dull, later the wings and tail may (roop; the feathers become ruffled and the bird sits around much of the time; diarrhea supervenes, the discharge being of a greenish-yellow color; there is a loss of appetite; the bird grows gradually weaker, showing muscular weakness, droopy wings and usually dies in from three to ten days after the first symptoms appear. 


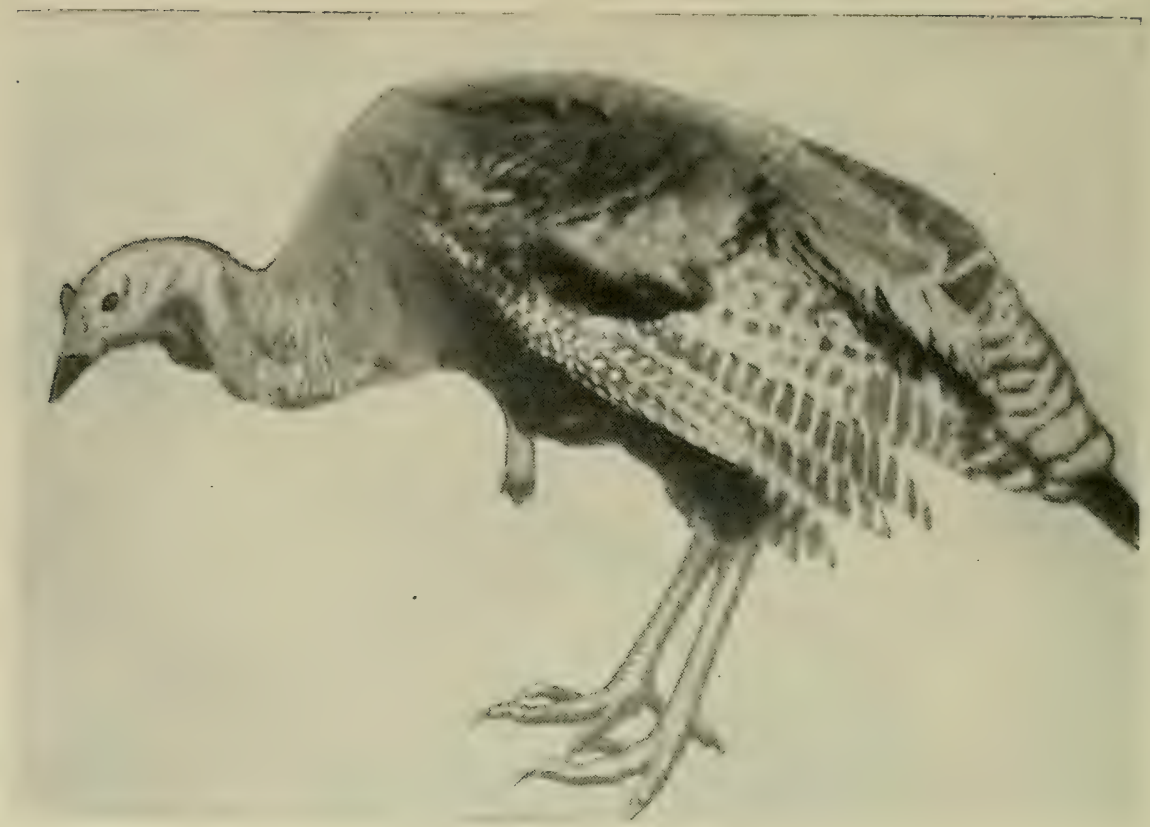

Fig. 54. A Turkey Affected With Blackhead.

Note the droopy wings, dejected appearance, tail lowered, and general signs of weakness. The ceca from this turkey are shown in FIG. 55.

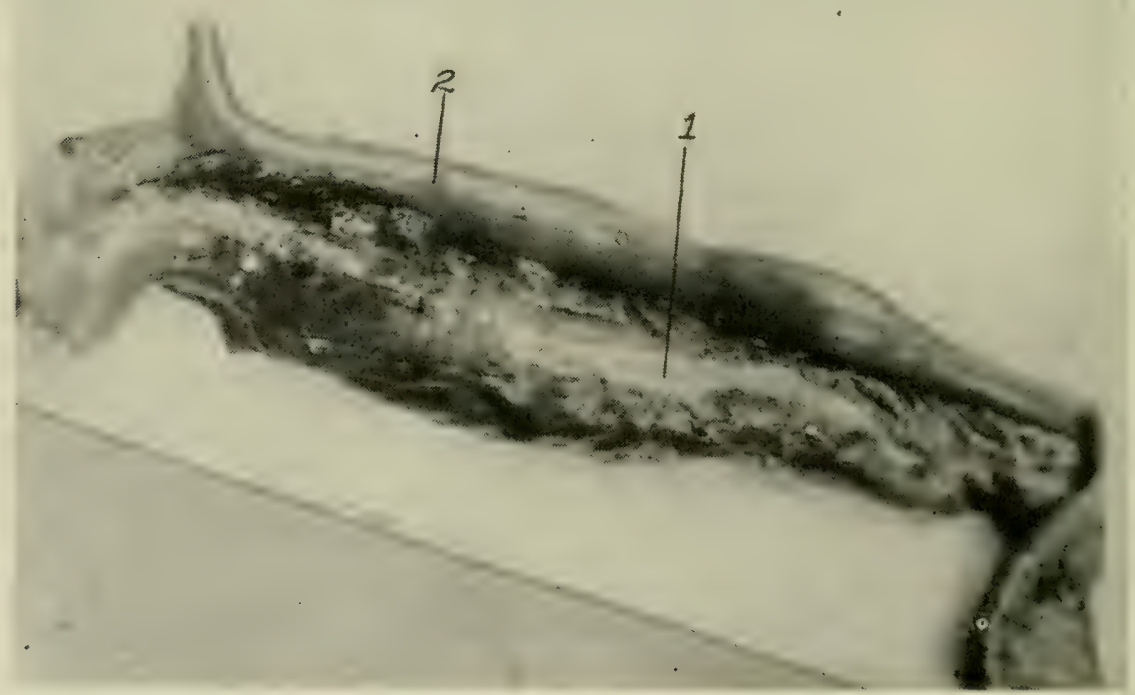

lifg. 55. Ceca of the T'urkey Shown in Fig. 54 That Died of Blackmead, 1, affectcd cecum showing it to be much enlarged and covered with cheesy pus; 2 , normal cecum. 
In the cases that run longer the bird hecomes emaciated. The head may or may not turn purple. From the cases in which the head turns purple the disease gets its name-blackhead.

Although turkeys of all age are susceptible to blackhead, youth suffers most. Cases seldom appear before the thirteenth day. The most critical period begins at about the thirty-fifth

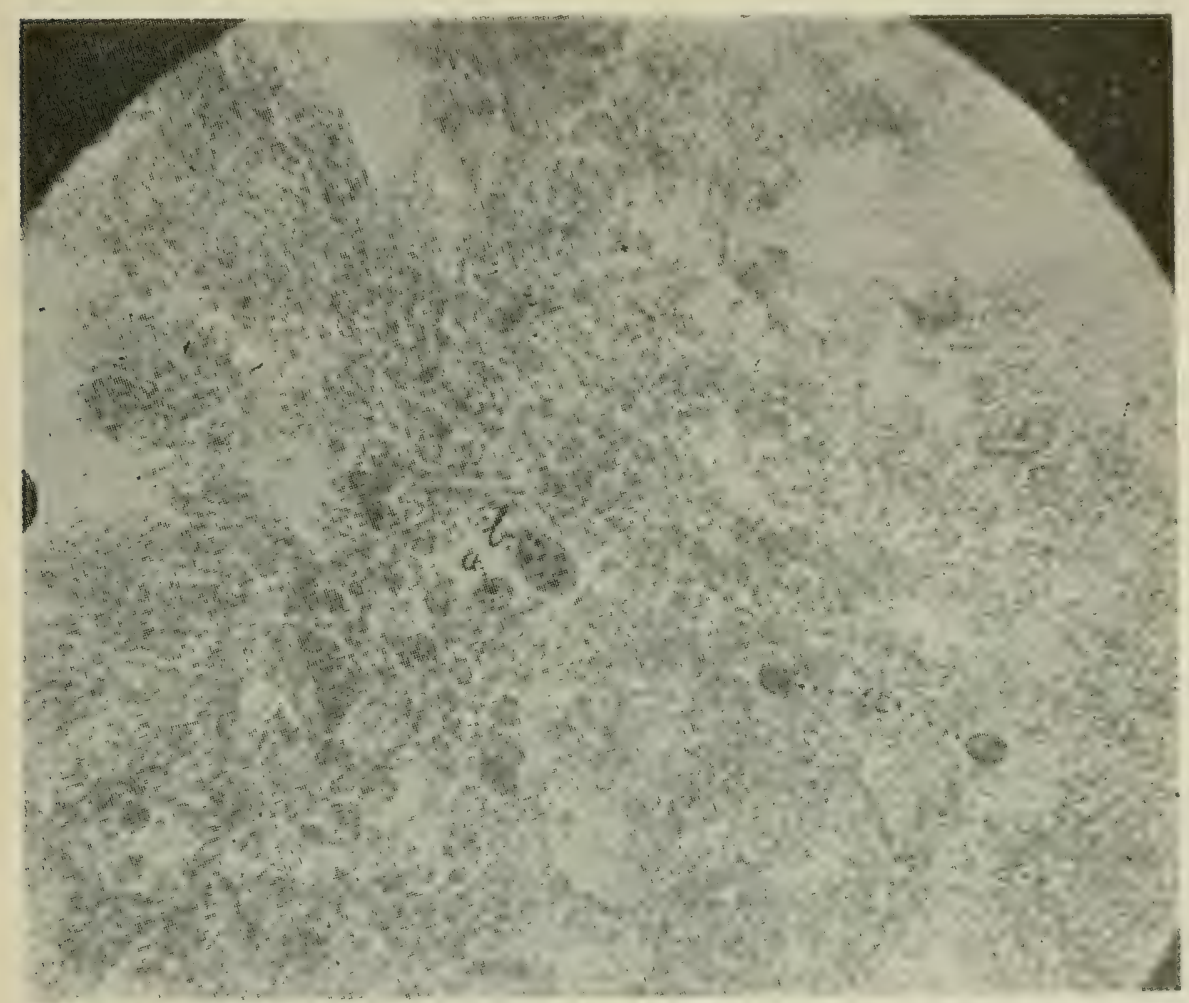

Fig. 56. Photomicrograpit of A SecTION OF THE Liver From a CASE OF BLACKHEAD.

a, protozoa causing tike disease; b, giant cell.

day after hatching. Young poults are observed to be sick no more than four to six days. While old birds may recover, young birds probably never do.

Postmortem Findings.-Upon opening the abdominal cavity one's attention is attracted by the enlarged liver with areas of dead or necrotic tissue. This is a caseation necrosis, and is shown in Fig. 52 , of the liver of a turkey dead of blackhead.

The ceca or blind pouches, are also affected. Usually only one is diseased as shown in Fig. 55. The enlargement is usually a short distance from the blind end or point. Upon opening the ceca, 
ulcers and areas of dead tissue, caseation necrosis, are observed in the mucous lining. There will also be noted a straw-colored fluid, edema, in the loose tissue about the heart.

Fig. 53, taken from an area in the edge of the necrotic portion marked $\mathrm{B}$, in Fig. 52, illustrates the condition of the cells of the affected part. A, illustrates the liver cells as they are first affected in a state of cloudy swelling. B, shows the cells farther along in the disease process, in which it may be noted that the nucleus has entirely disappeared and the cell is in a state of disintegration, or necrosis. C, shows a congested blood vessel which is in a state of passive congestion. D, shows white blood cells or eosinophiles referred to above. There is also noted in these areas giant cells.

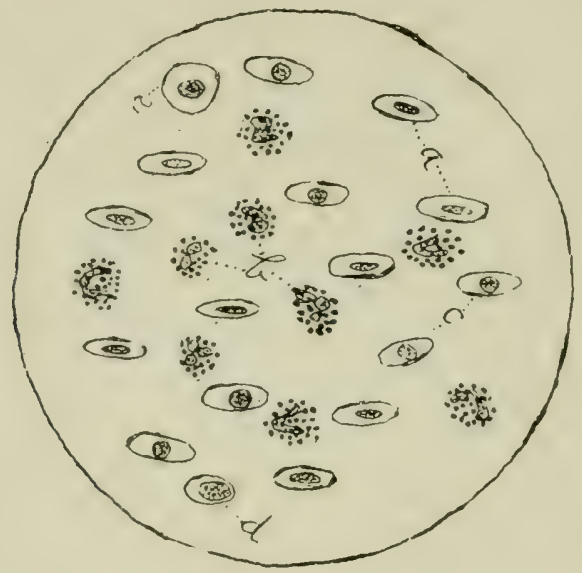

Fig. 57. Blood Smear From a Case of BLACKHEAD.

Showing intense eosinophilia; a, red blood cells; b, eosinophiles; c, mononuclear leucocytes. The blood of birds normally contains a ligh percentage of eosinophiles.

Fig. 56 b, illustrates a giant cell; a, protozoa causing the disease. A like microscopic examination of sections from the kidneys indicates that poisonous products have been taken up by the blood, for in these sections 'we find degenerative changes-congestion, cloudy swelling, and focal necrosis.

Fig. 57 shows a microscopic field from a blood smear from a turkey affected by entero-hepatitis. It will be noted that there is an intense eosinophilia. Fig. 58 shows a field from a portion of the kidney, in a state of cloudy swelling and focal necrosis-evidence of absorbed poisonous substances. Fig. 55 shows one of the ceca, the inner wall shows ulceration, the wall is thickened, and its tissues forced apart by masses of the protozoa-the ameba melagridis.

A microscopic study of the blood showed the following: Hemaglobin, 73 per cent; red blood cells, 2,000,000; white blood cells, 73,000 . The differential count showed: cosinophile white blood cells, 86 per cent; neutrophile white blood cells, 1 per cent; lymphocyte white blood cells 11 per cent; mononuclear white blood cells, 1 per cent; mast cells, 1 per cent. 


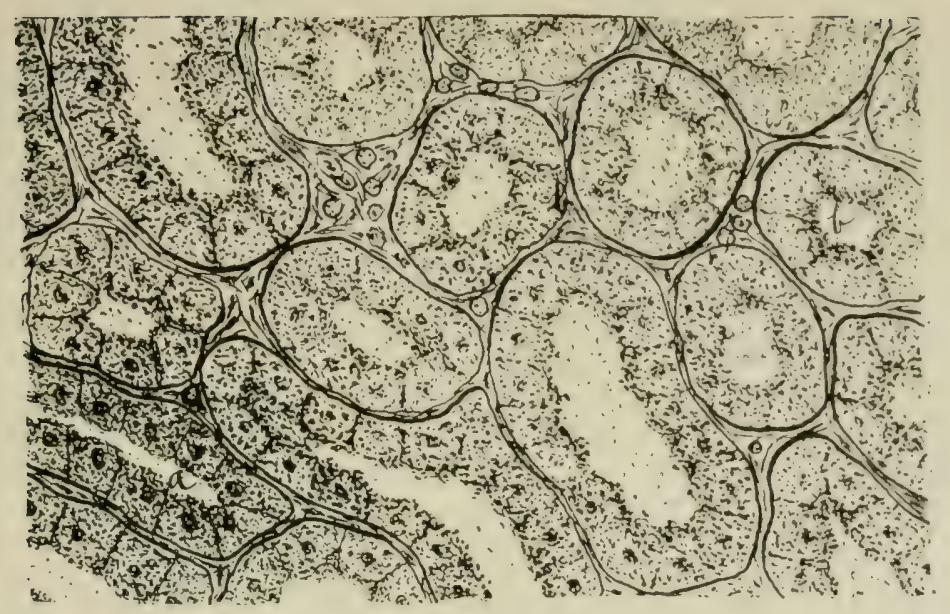
Fig. 58. SECTION OF A KiDNEy, From a
TURKEy THAT HAD DiEd OF BLACHEAD.

$a$, cloudy swelling; $b$, area of focal necrosis. Note in the dead areas the absence of nuclei.

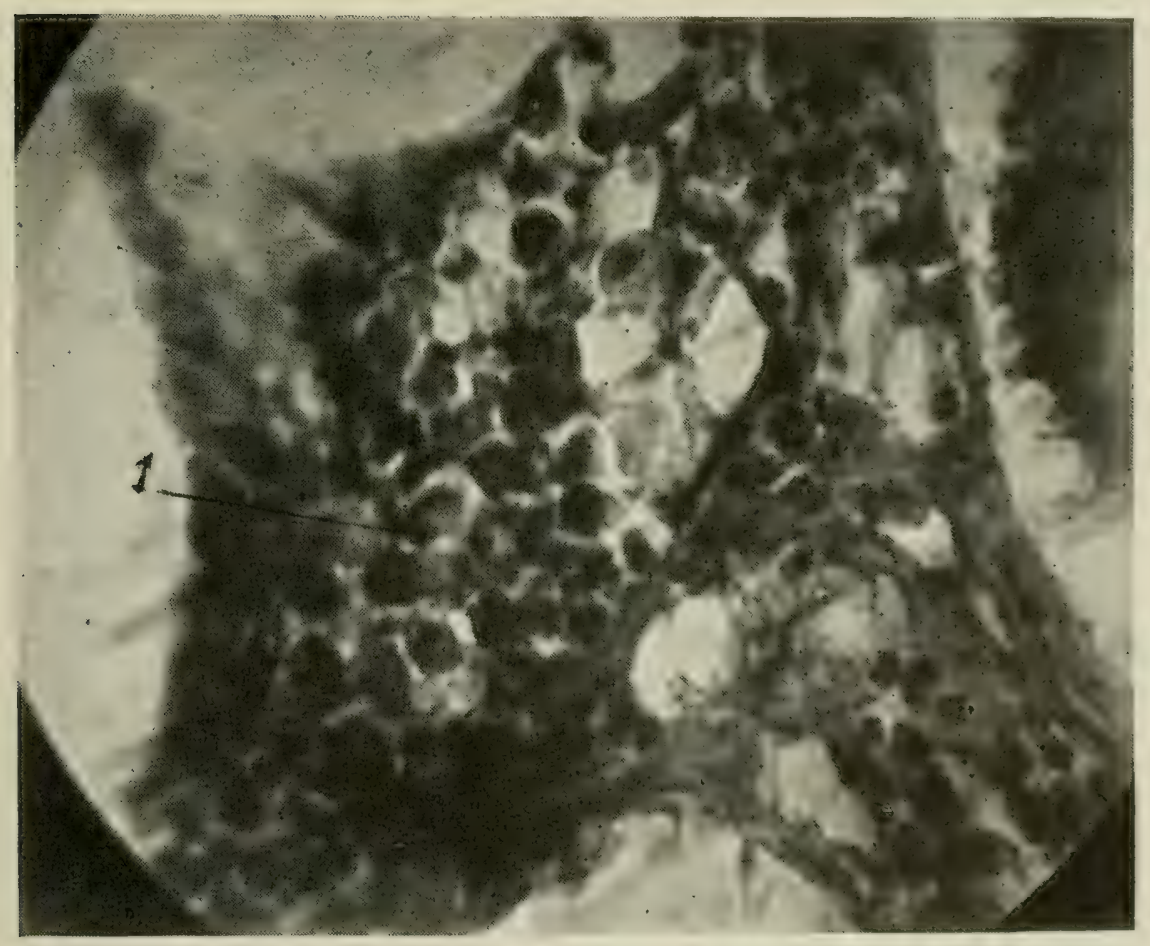

Fig. 59. A Photomicrograpi of a Section Silowing Masses of time Ameba MELEAGRIDIS.

1 , shows a typical parasite with its small nucleus. 
Treatment.-Thorough cleanliness of the henhouse and yards followed by careful disinfection as outlined under coccidiosis. Care as to feeding and watering, and intestinal antiseptics are indicated as recommended for fowl cholera. In this condition ipecac has given good results. Use 5 grains to the pint of water. Also the triple sulphocarbolates compound. Give one-half grain per bird twice a day in the case of large birds, or with young poults and chicks five weeks old give onethird this dosage.

It has long been known that milk feeding stimulates rapid growth in poultry as in all other animals. It has also been shown that milk feeding of chicks prevents to a large extent certain intestinal disorders. The same stimulating principle applies to turkeys and there is a possibility that acidity plays an important part. Since the greater death rate is among young poults. Hadley has recommended a limited feeding as follows. believing that overfeeding or crowding young poults favors the development of this disease: First 48 hours no feed; third day, chopped egg, including the shell, is given at the rate of two grams a day (32 grams make an ounce). The amount of egg is gradually increased till the end of the tenth day, then gradually decreased till the end of the second week, when egg feeding is discontinued. The fourth day green chopped feed such as lettuce or sprouted oats is given in increasing amounts. On the fifth day rolled oats are added and discontinued at the end of the sixth week. On the ninth day mash is given as follows: corn meal 6 parts, wheat bran 4 parts, wheat middlings 2 parts, and linseed meal 1 quart. Sour milk to drink should be given throughout.

The following scheme of feeding poults has been suggested as a means in warding off blackhead where the hens are known to be free from the organism:

1. Hatch the turkey eggs in an incubator, in the meantime having hens set on china eggs in nest boxes or brooders on the permanent range.

2. Remove the poults from the incubators about twenty-four hours after hatching, and distribute them at night among the hens, giving each hen from twelve to fifteen. Be sure and see that the hen accepts them before leaving them.

3. Give no feed before the poults are two days old. Each brood may then receive two teaspoonfuls of egg chopped fine with some green feed such as nettles, rape, lettuce, dandeline, or onion tops. A little cracker may be added to take up the surplus moisture so that the mixture will not be pasty but crumbly dry.

4. This feed mixture may be repeated for the remainder of the feedings upon this day, or bread soaked in sour milk may be substituted for the meal.

5. During the first three or four days of feeding the poults receive four meals each day, at about eight o'clock, eleven, two, and five. After this but three meals are given. 
6. On the second day of the feeding about the same rations are given, but one of the meals may be of chick grain, and some rolled oats may be added to the chopped egg mixture.

7. The third day of feeding is like the second. The poults are allowed to run in their inclosure.

8. On the fourth and fifth day of feeding, the number of meals may be reduced to three, at eight o'clock, twelve, and five. The amounts are slightly increased and a little grit may be added.

9. When the poults are about a week old they may be allowed to run free with the old hen on the range on pleasant days when the grass is dry. Chopped egg in the ration is reduced and omitted by the seventh day of feeding.

10. On the sixth day, the feeding is put on a time basis. Several spoonfuls of feed are put on the tray and well distributed, but the poults are not allowed to eat for more than about three minutes at any one meal.

11. By the end of the second week, the time limit is reduced to two and one-half minutes, since the poults are now obtained more feed on the range in the form of insects.

12. About the same time sour milk is introduced. The whey and curd well mixed, is put in shallow pans or in troughs, scattered about the range. It is at first given each morning and night at the rate of about one quart to each forty poults, and is gradually increased in amount until by the beginning of the fourth month one quart may be given for each twenty birds, each morning and night.

13. During the second month, which is the critical period for young birds, the feeding is continued about as in the latter part of the first month. But, after the age of about six weeks, the number of meals per day may be reduced to two. Green feed in the form of chopped carrot tops, onion tops, or lettuce should be given in abundance; it should comprise at least one-half of the ration for each meal. The time limit remains at about two and one-half minutes.

14. When the poults are about six weeks old the brooder coops should be replaced by larger houses made of lath and covered partly with roofing paper. Such a house will accommodate them till the poults are three or four months old. These should be constructed about three by five feet, and three feet high. Adult houses should then be provided and all the poults, with their mothers, be brought together in a single roosting shed.

15. The feeding for the third month is like that of the second except that the amount of milk is gradually increased and that grain mixture of equal parts of cracked corn and wheat may be gradually substituted for the chick grain.

16. As the autumn months advance and insect life disappears, the time limit may be lengthened to three or three and one-half minutes. In rainy weather the noon-day meal may be added and a four minute period allowed. Rolled oats may be omitted and the ration made to consist of grain mixture with an occasional feed of rolled oats or bread and milk. A mash may now be allowed containing some beef scrap or fish meal.

17. Before Thanksgiving the breeders for the coming season should be selected and marked. Their feeding for the winter may consist of the following grain mixture given at the rate of one quart for six or seven turkeys each night and morning: cracked corn 3 parts, barley 2 parts, wheat 2 parts, oats 1 part. 



\section{CHAPTER V}

\section{DISEASES OF THE DIGESTIVE TRACT OF FOWLS}

Birds are not subject to the manifold ills of the digestive system that prevail in higher animals and man, at least the list of digestive ailments which we recognize in fowls are not so numerous as they are in higher animals. Beginning with the anterior portion of the digestive canal, the mouth, we find its part in digestion relatively unimportant compared to that of the same organ in mammals, and its ailments correspondingly fewer and less important.

The food is not masticated in the mouth as in higher aninals, but is swallowed whole, passing into the crop, where it is softened by the action of the fluids secreted by that organ and perhaps also by the action of bacteria swallowed with it. After masceration in the crop is accomplished, the food passes into the proventriculus, or first portion of the stomach, where the processes of digestion are carried still further by the secretions, or juices, of that organ. The thoroughly soaked and softened food is next received into the gizzard and ground, with grit consisting of pebbles, always present in that organ, to a paste by the action of its strong muscular walls. Grit is continually being taken in by the fowl, the surplus being passed out with the droppings, thus there is a tendency to a residual or normal amount, though this amount may vary in different individuals.

From the gizzard the food passes into the small intestine. where digestion is carried on much as it is in mammals, by the action of the secretions of the intestine, liver and pancreas.

Domestication has affected the feeding habits of fowls much as it has the feeding of horses. In the wild state birds, like horses, eat most of the time, but they secure their provender more slowly. Under domesticated conditions they are fecl nutrients in a highly concentrated form, two or three times a day, and are required to exercise but slightly to get it. Frequently disturbances of digestion, largely due in one way or another to engorgement, is the result. Later feeding methods include a litter on the floor from which the fowls are required to scratch and exercise in getting their grain feed, this exercise in conducive to good health. 


\section{OBSTRUCTION OF THE BEAK}

This condition is very rare. Cases have been noted in which an object, such as a sunflower seed, has become wedged between the branches, or rami, of the lower jaw or beak, and serious trouble has resulted from this pressure: for example, paralysis of the tongue, inability to eat, starvation due to the inability to take in food, and death follows unless relieved.

Symptoms. - A fowl with obstruction of the beak will shake its head and scratch at its beak. Upon noticing such symptoms in a fowl the caretaker should examine its mouth and remove the obstruction. In fact a good poultry keeper will always, after feeding his fowls grain, stop to observe if any birds are eating and if one hesitates it is caught and examined, and, if it cannot well take care of itself it should be removed from the flock, as abuse by other birds of one of the flock in a weakened state soon become a serious matter.

\section{PARCHED TONGUE OR "PIP"}

Among poultrymen one often hears of "pip" as a disease of fowls, particularly of chickens. It is one of those names like "hollow horn" or "loss of cud" in cattle, which signifies no specific disease or condition, but merely a symptom of some ailment, real or fancied.

In some of the respiratory diseases, particularly in roup and chicken pox, the nostrils may be closed by an exudate and the fowls compelled to breathe through the mouth, and if, as is usually the case, the bird has an abnormally high temperature, or fever, at the same time there is a tendency for the mouth to become very dry and the mucous membrane may crack and bleed. Owing to its dryness, the epithelium of the tongue may not exfoliate, or be cast off normally, and, being retained, may form a transparent horn-like material on the end of the tongue. This dryness of the mouth and the resultant changes are what is known as "pip." This dried end of the tongue should not be picked off, as often this causes death of the bird.

This condition may also accompany diseases of the mouth or of the respiratory passage, in which, on account of the difficulty in breathing the bird holds its beak partialiy open. The surface of the mucous membrane may become dried and catarrhal inflammation follow. The fowl will be noted to hold its mouth partly open and at intervals emit a shrill sound, accompanied by a jerk of the head.

() pen the mouth and observe the tongue and mouth cavity. The tip, borders and attached portion of the tongue are observed to be covered hy a hard, dry coating, which may also 
extend to the mucous membrane lining the mouth. Forcible removal of this membrane results in bleeding as the surface uncler the membrane may be ulcerated as a result of infection and death of the bird is likely to follow.

Trcatment.- In such cases the treatment consists in the first place of measures directed at the primary cause; that is, the condition which is producing the dryness of the mouth. The hardening and drying of the membranes of the mouth may be relieved by the application, several times a day, of a mixture of equal parts of glycerine and water.

If cracks and ulcers have formed they should be bathed in a solution of potassium chlorate water, twenty grains of the former to an ounce of the latter. This is best accompanied by dipping the bird's beak into a vessel, containing this solution, five or six times and repeating every hour or two. If pus has formed in the ulcers, they may well be cleaned with a few drops of hydrogen peroxide before the potassium chlorate solution is used.

\section{STOMATITIS-SORE MOUTH}

Stomatitis is an inflammation of the mouth. There are many different kinds of sore mouth due to many causes. The ulcerative form of sore mouth is one of the most common forms and may be caused by a fungus described on page 105 . It also accompanied chicken pox or roup when it is called arian diphtheria which is described under respiratory diseases on page 213 . In the ulcerative type of sore mouth the diseased areas appear covered with a yellowish-white cheesy material which is the result of the products of inflammation being thrown out on the surface.

Sore mouth may also be caused by injury due to foreign bodies, bites, chemicals as strong acids, lye, corrosive sublimate, or hot foods.

Simple catarh or acute inflammation may also affect fowls. It is catised by some irritant, or to bacterial infection of the part.

Treatment.-In the ulcerative kind scrape off the cheeselike material and rub the part with lunar caustic or stick nitrate of silver till all parts turn white. Repeat in five to seven days if necessary.

In the acute inflammation kind wash the mouth with a saturated boric acid solution. Repeat three times a day.

\section{CROUPOUS INFLAMMATION OF THE PIGEON (Canker)}

Croupous inflammation of the pigeon is a disease affecting the throat of young squabs in which there appears a cheesy 
mass in the upper part of the esophagus. The squabs become affected early, in fact, while still being fed by the parents. Infection apparently comes from the adult birds. Certain pairs of pigeons are noted to invariably have this trouble with their squabs. The disease or tumor-like area becomes larger, due to the accumulation of the croupous exudate, from an inflamed area.

Symptoms.-The young squab is unable to swallow. If in birds old enough to eat grain they find it impossible to eat.

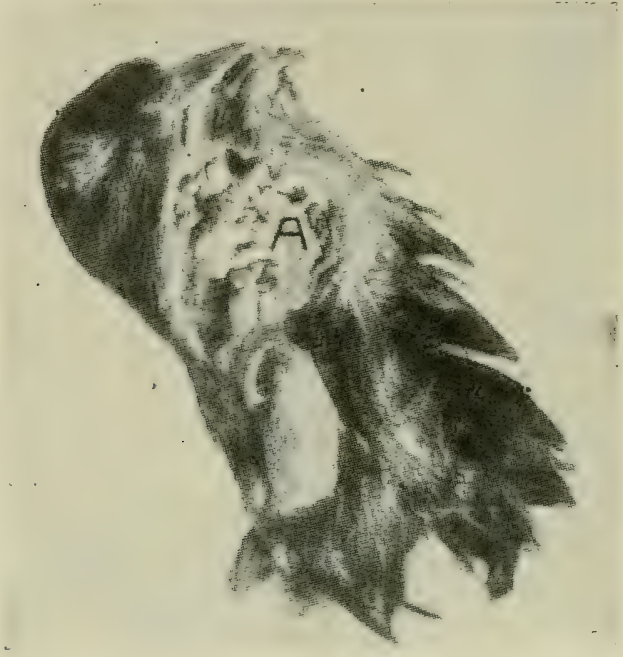

Fig. 60. Croupous Inflammation of the Throat of a Pigeon.

a, necrosing mass made up of pus cells, fibrin, and debris.

The squab rapidly becomes emaciated if not relieved. The loss to some lofts is quite a serious matter. The lump in the throat is easily seen and felt.

Postmortem Findings.-On opening the esophagus in the region of the disease the cheesy mass appears. A microscopic examination of the part shows an inflammation, the surrounding tissue being filled with polymorphonuclear cells, the cells of inflammation. The mass thrown out on the surface as a result of this inflammation is made up of pus cells and fibrin. The condition is of an infectious nature. The disease does not confine itself to the upper part of the esophagus but may affect the structures at the entrance of the chest or thorax, again it has been observed in the region of the eyes, and in the roof of the mouth, in the structures of the region of the neck, and again internal lesions of the abdominal and chest cavities affecting the lungs, liver, and eren the kidneys. It has also been observed in the crop, and in the pharynx, and in the bronchi and involving the chest wall. In such cases the bird shows symptoms of going light, appears languid, dull, stupid, losing appetite, gradually becoming emaciated and finally death. 
Treatment.--In the case of the squab with throat affection, grasp the squab forcing its mouth open, and with a very small pus scoop or curette, gently remove the cheesy mass. Then with a swab made by twisting cotton around the end of a small stick, and this swab dipped in pure indine gently swab out the parts. This must be thoroughly done. The first treatment is often successfu1.

A similar disease has been studied in fowls in which cases the croupous exudate or mass is removed and the parts swabbed with pure iodine as with pigeons.

\section{IMPACTION OF THE CROP-CROP BOUND}

The crop becomes impacted with food when its passage is obstructed. Obstruction of the crop is generally due to swallowing bodies that cannot pass readily from the crop through the second portion of the esophagus to the proventriculus, that is, to an obstruction of the outlet of the crop and the commencement of the second portion of the esophagus. Hog bristles, small feathers, straw, etc., are usually the cause of such obstructions. An interesting case was studied some years ago in which brooder chicks, just beginning to feather, were given potato parings, after which they died. There was found in each crop a potato paring extending from the crop through the second portion of the esophagus into the proventriculus.

By referring to Fig. 2 these organs and their relations can be seen.

Low vitality of the bird may cause impaction of the crop. It may accompany acute diseases as fowl cholera, or improper nourishment, the thin muscular walls of the crop may become paralysed or so weakened as to be unable to force its contents onward into the proventriculus. Depraved appetite as often seen when fowls are fed too heavily on cottonseed meal may cause them to eat litter and thus obstruction of the outlet of the crop.

Symptoms.-The fowl becomes dull, occasionally opening its beak and fetid gases are sometimes emitted. There is an obsence of appetite. The bird stays away from the balance of the flock, is inactive, usually stands off in a corner with crop protruding. The crop thus appears enlarged, the walls tense. doughy to the touch and foreign bodies may be felt through its walls. Obstruction of the crop rarely disappears without treatment and if no relief is given the bird may die in a few days. Death is usually due to exhaustion and starvation. Foreign bodies in the stomach of fowls often cause perforation of its wall. Such a case has been observed in the stomach of a goose. The foreign body was a pointed piece of metal. We 
also, in a fowl, observed a piece of small wire about one inch long that had penetrated the wall of the proventriculus and extended to the base of the heart, and in still another case a pin that had penetrated the wall of a gizzard of a hen.

Treatment.-Where the obstruction is due to weakened walls of the crop water may be forced into the mass within the crop and by massaging the material may be forced out through the gullet or esophagus. An antiseptic solution should be used as there is usually considerable fermentation of the obstructed food.

Where the cause is due to foreign bodies as hog bristles, feathers, and large pieces of straw it will be necessary to open the crop and remove the material. To do this operation provide a needle, a half curved surgeon's needle is preferred though an ordinary sewing needle will do, and white cotton thread. A small, sharp pointed knife. Have an assistant hold the fowl on its left side and pluck the feathers from over the (rop). Make a bold cut through the skin and crop wall, all at one stroke, and about two inches long. Make the cut in the skin slightly longer than the cut through the crop. With the index finger remove all the crop content and observe that all has been removed from the outlet into the second portion of the esophagus. Wash out the crop with a weak antiseptic solution and wash off foreign matter from the wound. Then sew the wound passing the needle through the skin, and crop wall on one side and passing the needle through the crop wall, and skin on the other side, draw tight and tie. After the first stitch has been made make the stitches about one-half inch apart and in a continuous manner not cutting the thread till all stitches have been made. The crop wall and the skin must be sewn with the same stitch.

After the operation place bird in a comfortable coop and give only slightly moistened bread for the first three days, after which may be given light feed and limited amount of water.

\section{IMPACTION OF THE INTESTINES}

By impaction is meant the accumulation in hard mass of food in such a manner as to obstruct the passageway of the intestines.

Impaction of the intestine is due to an obstruction of the lowel thus preventing the passage of the normal bowel content. This condition is rare in fowls but has been observed. It may be due to masses of the intestinal worms, especially the Ascaris Inflexa and to tape worms. A case was observed in which the fowl had eaten coarse hay, similar to bermuda or prairie, and this had obstructed the gizzard and protruded out 
into the small intestine. Large pebbles, pieces of rags, and feathers may likewise obstruct the intestine.

Canaries and other cage birds at times suffer from impaction caused by sluggish peristalsis due to the sedentary life or lack of exercise, and heavy, indigestible feeds. Or from the lack of grit. Impaction of the caecum due to improper feeding may occur.

The matting together of the vent fluff in diarrhea of baby chicks may prevent defecation.

Symptoms.-There may be depression of the bird, listlessness, loss of appetite, repeated straining, as though trying to pass droppings, small amount of (lroppings passed at long intervals. Death may occur from necrosis of the wall at the point of obstruction and from exhaustion.

Treatment.-Give to adult fowls a cathartic as castor oil. in one teaspoonful doses. Epsom salts in one-half to one teaspoonful doses.

\section{TYMPANY OF THE CROP_GAS OF THE CROP}

Gas of the crop is a condition in which the crop is distended by gas. This is either due to unnatural fermentation of the crop contents, or, is due to gas forming organisms. There may be present putrefaction of the contents of the crop. It is usually accompanied by an inflammation, or to a catarrh of the crop which interferes with its normal function. Fowls, at times, have been noted to have enormously distended crops, which, upon examination, was observed to be filled with gas. Usually these crops contain very little food. This condition more often affects chicks than older birds.

Treatment.-Give intestinal antiseptics, such as one part carbolic acid to two hundred parts of water, or mercuric chloride, corrosive sublimate, one part to ten thousand parts water. To make this put 6 grains bichloride of mercury, and 3 grain citric acid, in one gallon of water. The critic acid aids the bichloride of mercury to go into solution. The sulphocarbolates compound are also indicated, also a permanganate of potash solution, making water deep red.

Immediate temporary relief may be given the fowl by liberating the gas through an aspirating needle or a small cantula. The crop may then be irrigaterl, through the canula, with a mild antiseptic solution. Follow with two teaspoonfuls of castor oil and feed sparingly on easily digested food.

\section{ENLARGED CROP_PENDULOUS CROP}

Pendulous crop is a condition where the walls of the crop do not properly contract when the crop is empty and it langs 
down as a pendulous bag. Fig. 61 shows a Barred Plymouth Rock hen with a pendulous crop.

Pendulous crop is due to weakening and stretching of the crop walls and may be due to catarrh, worms as the Filaria Tite, and to injudicious feeding.

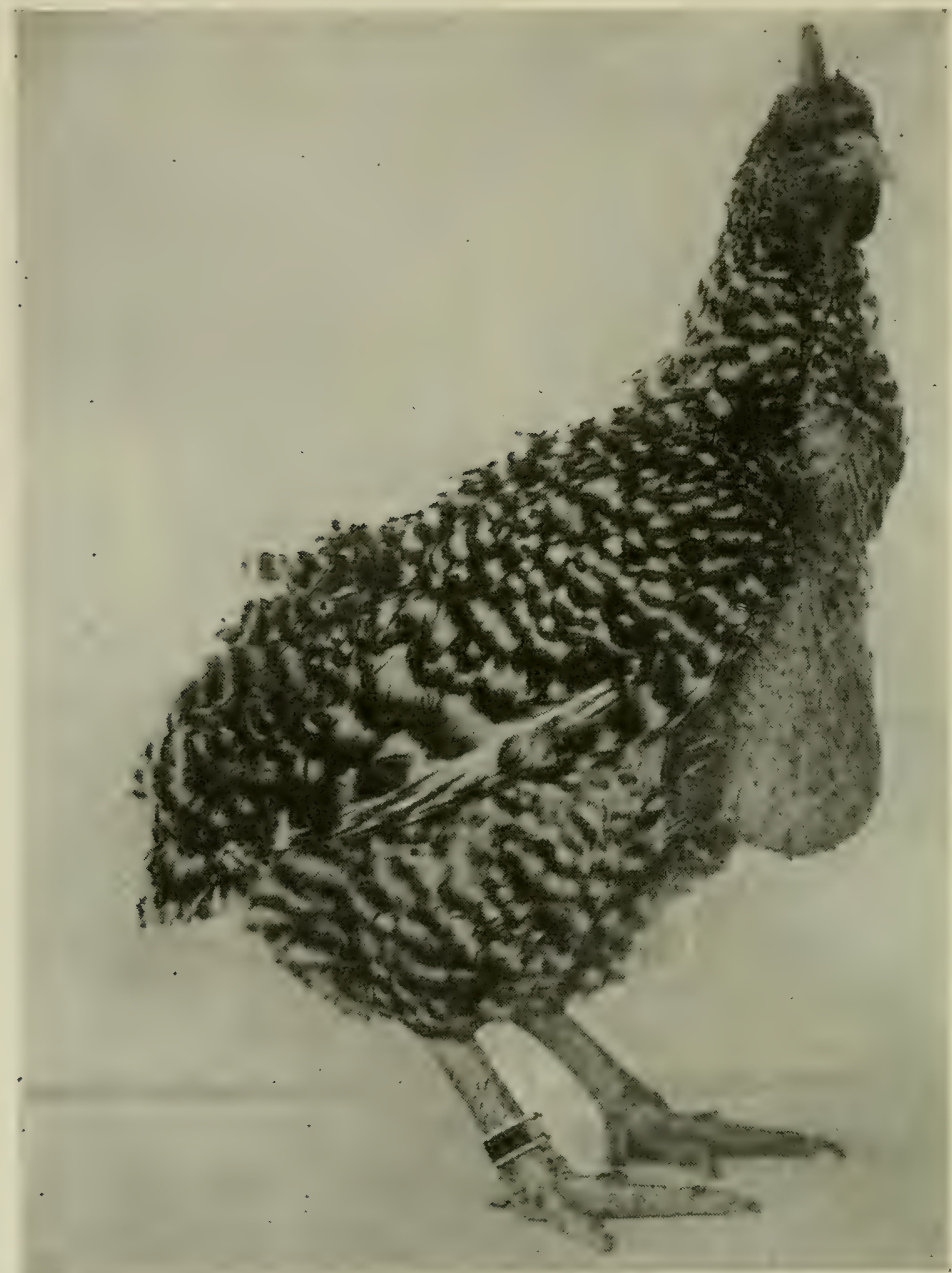

Fíg. 61. Pendulous Crop in a Barred Plymouth Rock Hen.

Treatment.-Pendulous crop causes little inconvenience to the bird and is incurable except by operating which consists of cutting out a piece of the crop wall. The operation is an easy one and similar to operation for crop bound described above.

\section{GANGRENE OF THE CROP}

Gangrene of the crop is a condition accompanied with death, or sloughing, of the crop wall and is a moist gangrene. The 
mucous membrane alone is affected and it results fatal to the affected birds.

Symptoms.-The bird appears dull, unkempt plumage, loss of appetite, may stay apart from the balance of the flock, and may develop impaction of the crop. Upon opening the crops a very offensive odor is noted, the mucous lining will he observed in a necrotic, or sloughing state, and appears as a dark, sometimes a greenish, caseous mass.

Treatment.-In the earlier stages there may be given in the drinking water or in the feed intestinal antiseptics as salol, subnitrate of bismuth, or sulphocarlolates compound. If the condition becomes prevalent in a flock, the runs, yards, and hen houses should be thoroughly disinfected or the birds completely changed to new ground, and in any case given clean feed and water. The sick fowls should be seprarated from the well ones and the dead fowls should be burned.

\section{CATARRH OF THE CROP-INGLUVITIS}

Catarrhal inflammation of the crop of fowls may be caused by irregular feeding, a distended crop and irritating and indigestible feed, such as feathers, putrid meat, and irritating chenicals, may be mentioned as causes of this condition which is essentially a more or less chronic inflammation of the mucous membrane, lining the crop. If the crop be overdistended the strain on the muscles may be so great that paralysis results. In these cases there is noted a crop filled with a pulpy, soft, more or less gascous mass.

It may be noted after the ingestion of pointed objects. In pigeons, by too early removal of the squabs from the parent birds. In this case the glands of the parent bird that were active during the feeding of the young suddenly cease being used and become inflamed.

Symptoms.-There is a loss of appetite. The fowl appears dull, repeatedly stretches its neck and swallowing becomes difficult. The crop is tender to pressure, soft to the touch, and at times, gaseous. Pressure on the crop may cause feticl gases to escape from the mouth. Repeated attacks cause the development of pendulous crop.

Treatment.- If the crop is distended with a dough-like mass. grasp the fowl by the legs, holding with head down, gently press out the mass; then by introducing water through the mouth and forcing it out as before, the crop, in this way, may be washed out.

Give bland substances, such as gruel and mild antiseptics, such as salol, subnitrate of bismuth, or sulphocarbolates compound. 


\section{DEPRAVED APPETITE-PICA}

A depraved appetite is one where the bird eats litter as straw, sticks, feathers, etc., in addition to feed and in many times in preference to their normal feed.

This condition may he due to disease of the digestive organs or it may be a vice learned from others. Hens learn to eat eggs by finding them brokien or by seeing an egg-eating hen and copying as a cribbing horse acquires the habit from his mate, or as one hog may learn to eat chickens from seeing another eating one.

Feather eating, or plucking the feathers of its mate, is another habit that may be acquired from mimacry. The only thing to do with a feather eater or an egg eater is to kill them as the habit cannot be broken. Obstruction of the gizzard, lack of grit, insufficient or unsuitable feed and catarih of the crop are factors of greater or less importance in causing a depraved appetite. Heary fesding of cotton seed meal and especially to pullets in one of our experiments resulted in eight per cent developing depraved appetite, eating litter followed, and crop impaction resulted.

\section{INDIGESTION}

Indigestion is the lack of power of the bird to digest and assimilate the food eaten.

Indigestion of fowls may result from a lack of quality or of appropriate volume of feed or by the ingestion of foreign bodies. Pigeons which are normally gramnivorous, or grain eating, will suffer if they cannot get grain and are forced to eat herbage or green feeds. Likewise it is reported that birds in zoological gardens are liable to suffer from lack of appropriate food. Storks, swans, herons, geese and ducks in the winter and spring failing to obtain their natural food, such as slugs and molluscs, eat water mosses to allay their hunger. These foods are to them true foreign bodies and cause indigestion which in some cases is fatal. It is the same in the small insectivorous or semi-insectivorous birds. When it is attempted to feed them exclusively on grain or vegetable mashes they succumb to the diet.

Grains too large for the digestive organs of a bird act like a foreign body and cause fatal indigestion. Young pheasants may be killed in this way by grains of corn. And one should be careful in feeding grain to very young baby chicks.

Overloading the digestive organs is a general cause of indigestion of hircls. Dry grain which is palatable to fowls is less suitable to others such as palmipedes which are accustomed to soaking their food in water. For this reason we 
give our geese and ducks wet mashes instead of grains. Such birds may readily overload their stomachs with dry grain which may sivell and cause death.

Indigestion may be due to lack of sufficient grit in the gizzard. This material is indispensable to grain-cating birds. Omnivorous or mixed feeders also need grit. If grit is not present grain may accumulate in the gizzard and catse a fatal indigestion. Grit lasts a long time, as experiments at the North Carolina Experiment Station have shown that grit may remain in sufficient quantities in the gizzard to grind feed for at least a year.

Treatment.-See that the fowls have sufficient grit. Give cathartics as epsom salts one tablespoonful to each six birds. Dissolve the epsom salts in water and mix mash with the solution or give as drink.

The treatment in parrots which is shown by the bird yawning, refusing food, and nervous, give grit and castor oil, also put 6 grains bicarbonate of soda in the drinking water each day.

\section{DISTENSION OF THE BURSA OF FABRICUS}

The Bursa of Fabricus is a gland located in the superior wall of the cloaca. Occasionally this bursa may become enormously distended into a cyst-like structure containing a clear, colorous fluid.

\section{CLOACITIS}

Cloacitis is an inflammation of the cloaca of the fowl. Ulceration of the anus and cloaca is an apparent contagious disease and is frequently observed.

Symptoms.-The anus and cloaca become inflamed, red in color, and protrudes, and later may ulcerate. From the ulcerative form there has been isolated an acid fast bacallus which was thought to have something to do with the disease. There is a very stinking odor accompanying this condition.

Postmortem Findings.-Upon autopsy the inflammation may be found to extend the entire length of the large intestine, or rectum. If the inflammation extends the entire length of the rectum it would be a proctitis. Thus there may be a catarrhal proctitis or an ulcerative proctitis. The condition may extend to the lower portion of the oviduct. Such hens become sterile and an occlusion, with scar tissue, has been observed in the lower portion of the oviduct. The disease is thought to be communicated from bird to bird, and especially by copulation.

Treatment.-Apply with a swab pure iodine. Repeat every three days till cured. Antiseptics are indicated. Ciood results have been obtained, in some cases, with a one per cent solution of sulphate of iron where the ulcers extend into the bowel. The liquid is injected, three times a day, with a syringe. 


\section{PERITONITIS}

Peritonitis is an inflammation of the peritoneum or smooth, delicate, shiny membrane, lining the abdominal cavity and reflected over the viscera, the liver, intestines, gizzard, etc.

Peritonitis may be acute or chronic. The exudative or purulent variety is the most common in fowls and especially heavy laying hens.

Canse.-There may be various causes of this condition. Streptocicci and a variety of bacilli have been observed in these cases. There are numerous specific diseases of a septicaemic nature in which the involvement of the peritoneum is of secondary consideration. Other causes are infected yolk concretions or deposits, ovarian infection, extension of inflammation of the oviduct, or rectum, as a result of rupture, perforation of the digestive tract by foreign bodies, or as a result of ulcerative changes in the intestinal wall such as sometimes occur in coccidiosis of fowls.

Symptoms.-Diagnosis is difficult except by postmortem examination. In the acute peritonitis, which is the more rare type, the bird may die shortly after showing signs of being sick. The fowl shows a loss of appetite, unkemped plumage, elevation of temperature, weakness and depression. Diarrhea may be present, especially when the outer covering of the intestine is involved. In case of rupture of the rectum the droppings will be scanty owing to the passage of a part of the intestinal content into the abdominal cavity. The abdomen may show a pendant position. On palpation or feeling the abdomen, the fluctuation of liquid may be felt. The presence of concretions may be determined by their dense consistency, free, and of rounded contour.

Postmortcm Findings.-Peritonitis in fowls is not usually marked by extensive congestion of the serous lining of the abdominal cavity and abdominal organs. There may be localized areas of congestion and inflammation and hemorrhages. The purulet variety is most common especially among laying hens. There will be found an exudate and collections of more or less cheesy material among the intestines. The serous exudate may be straw colored, or more often cloudy and putrid. The septic condition is generally accompanied with a cheesy exudate covering the viscera. Yolk concretions may be found. When rupture of the oviduct occurs there may be found many eggs in the abdominal cavity.

\section{ASCITES, DROPSY}

Ascites is an accumulation of liquid in the abdominal carity. Ascites is common in fowls and especially hens and has been (o)served in canaries. No specific causative agent is responsible. The accumulation of fluid results from a filtration of blood serum through the serous lining of the abdominal cavity. 
It may be present in chronic diseases of the liver, kidneys, heart, and in chronic suppurative inflammation of the peritoneum. It may also accompany chronic cases of enteritis or inflammation of the bowel, sarcomatosis, tuberculosis, or other debilitating diseases.

Symptoms.-The abdomen may be distended and in some cases it will be greatly distended. Upon feeling the abdomen it will appear tense. Shaking the fowl will cause the sound of liquid.

Treatment.-Pass a trocar and canula or a hypodermic needle through the abdominal wall, being careful not to injure any of the vital organs. Allow the liquid to drain. This will give temporary relief.

\section{NEPHRITIS}

Nephritis is an inflammation of the kidneys. Acute nephritis is frequently met with in fowls. Baby chicks which are allowed to be exposed to cold rains and become chilled or which are not properly cared for and improper heat supplied, suffer with acute congestion and inflammation of the kidneys.

Chronic indurative nephritis has been observed in the fowl.

Acute parenchymatous nephritis has been observed in hens dying of purulent inflammation of the air sacs.

Symptoms.-Acute and chronic inflammation of the kidneys interferes with the appetite, arrests growth in young fowls, and at times there is a loss of co-ordination. Gradual emaciation and death results.

Postmortem Findings.-The kidneys are increased in size; the tissue is rather firm in consistency in the chronic indurative type. The organ may be of a grayish-yellow color. There is hypertrophy of the interstitial connective tissue. The sectioned surface in acute parenchymatous nephritis is gray, due to the cloudy swelling, and is soft. The uriniferous tubules are partly destroyed and the epithelium in many parts may be in a state of fatty degeneration.

\section{SUPPURATIVE INFLAMMATION OF THE GIZZARD}

Suppurations or pus formations on the surface or in tissues in the form of abscesses are common in the fowl. Pus in the bird is almost always of a cheesy nature. Suppuration and abscess formation has been observed in the fowl.

Symptoms.- In the case that has been observed there was at first a slight diarrhea, mopishness, and no interest in life. Gradual emaciation and finally death.

Postmortem Findings.-The carcass was poor, being destitute of abdominal fat. There is an inflammation of the gizzard wall with an accumulation of a cheesy pus in one end of the gizzard. The testes are in an atrophic condition. If in a hen the ovary and oviduct will be shrunken and inactive. There may be secondary 
changes as shown in one case in which the liver weighed 60 grams, 35 grams being the normal weight. The liver presented a grayish mottled appearance. In a microscopic study of the wall of the gizzard through the part affected with suppurative inflammation there was noted a portion of the adjacent muscle to be densely packed with polymorphonuclear leucocytes, the cells of inflammation as well as newly formed connective tissue. The pus consisted of fibrinous material and pus cells.

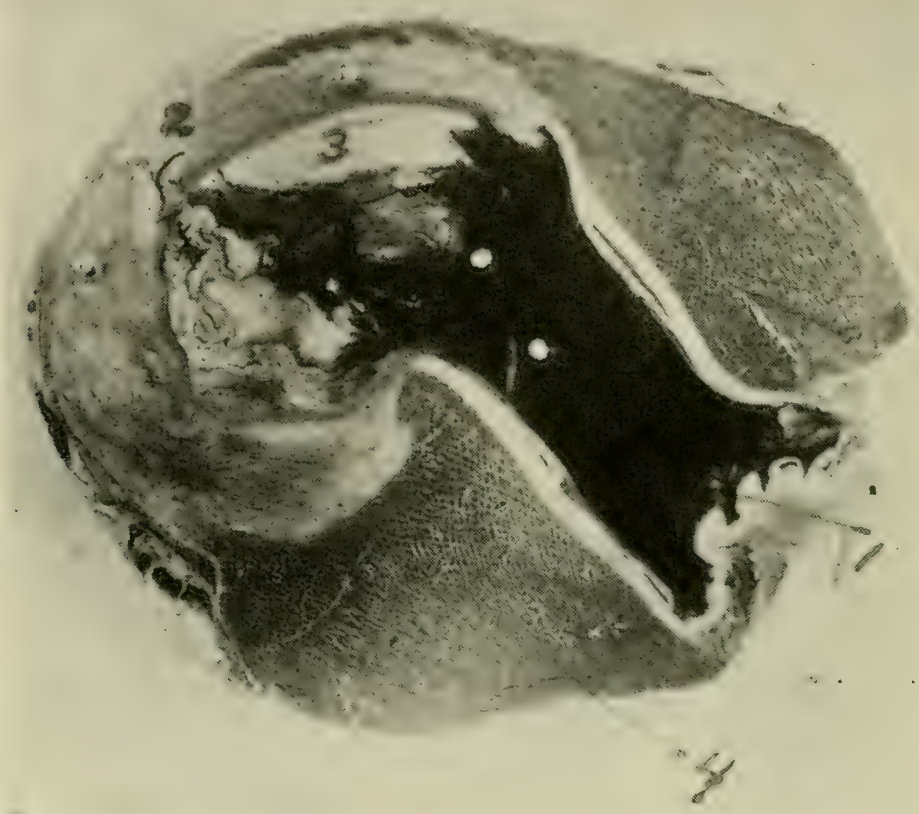

Fig. 62. Suppurative Inflamation of ' the Gizzard of A Fowl.

1 , cavity of the gizzard; 2 , wall in state of suppurative inflammation; 3 , cheesy pus in cavity of gizzard; 4, normal gizzard wall.

Sections of the liver showed passive congestion with hemaciderosis. There was an acute inflammation, the inflammation cells, or polymorphonuclear leucocytes appearing more densely packed in the perivascular spaces and immediate adjacent tissue. In some areas the inflammation seemed rather diffused while in other parts of the liver there appeared a tendency toward focalizing areas of inflammation.

\section{CALCULI}

Calculi are earthy concretions which have been settled out of liquid and become a small stone. Urinary sediment in the form of calculi has been found in the fowl. These deposits may be located in the small collecting and transporting tubules of the kidney tissue and may consist principally of uric acid. These deposits appear as small nodules or stones. 


\section{EVERSION OF THE CLOACA AND OVIDUCT OF A HEN}

An eversion of the cloaca and oviduct, commonly called a prolapse, is the turning outward of the cloaca and the lower portion of the oviduct. The condition may be so severe that even the posterior portion of the large intestine or rectum is turned "inside out."

Causes.-Among the causes of such a prolapse may be mentioned stimulating feeds, aggravated constipation, and large eggs causing considerable straining. It may also be seen in

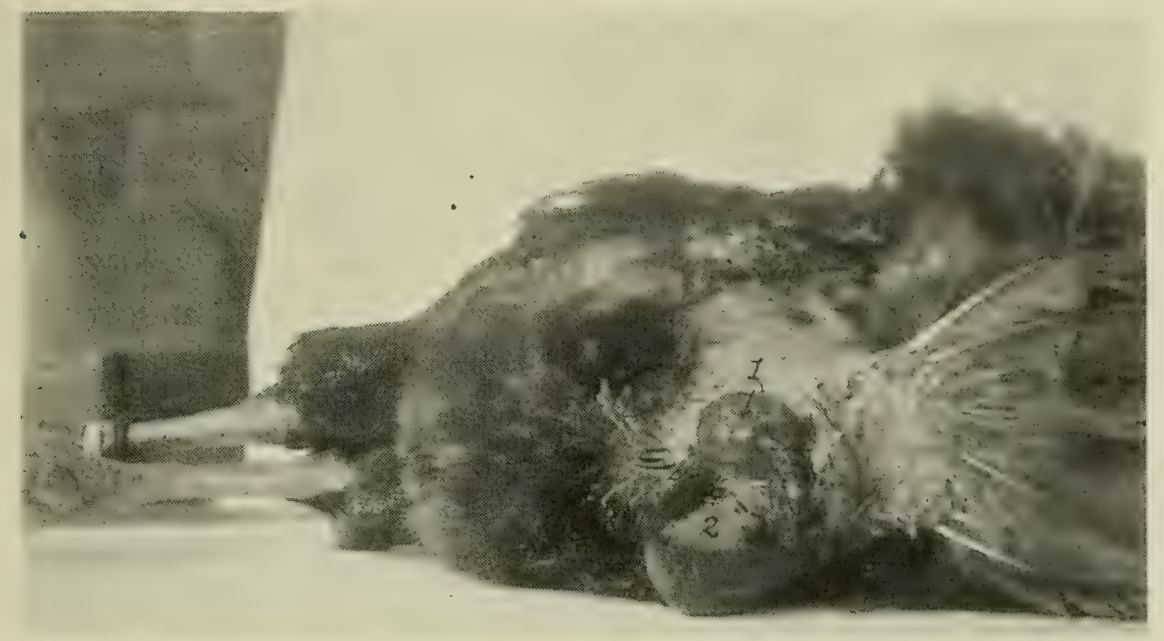

Fig. 63. Eversion of the Cloaca and Oviduct of a Single Comb Rifode ISLAND RED HEN.

1 , eversion of the vaginal portion of the oviduct; 2 , eversion of the cloaca, also the posterior portion of the large intestine.

inflammation of the mucous membrane of the oviduct when this membrane may be seen protruding through the cloaca. In constipation the bowels may become gorged, and this in addition to the obstruction when the egg canal contains one or more developing eggs, and the ovary, being active, is larger and added to the bulk, predisposes to this condition. It is also noted in heavy laying pullets that are being crowded by very stimulating feeds and especially in the earlier part of winter.

Symptoms. - This condition is most often seen in heary laying hens. The hen will be noted to strain as though trying to pass droppings. The mucous membrane will be noted to protrude through the anus. If not treated at once and the mucous membrane is allowed to continue to protrude through the anus, it soon becomes inflamed from exposure to the air and infection may take place. Later the parts may become ulcerated as a result of mechanical injuries or attack by germs.

Treatment.- Wash the protruding parts with warm water in 
which has been dissolved enough permanganate of potash to make the water a deep red. If congested and swollen tincture of belladonna application may aid in reducing the swelling. After the parts are thoroughly cleaned gently replace the protruding parts, being careful that the hands are clean and using on the fingers a small quantity of carbolized vaseline. Put the bird on light food for a few days. Do not give laying mash as it is the desire to stop, at least temporarily, egg production till the parts can have a chance to recover. Put the hen in a comfortable coop and give only a grain ration so that laying will cease and give the parts a rest and till she regains her strength and the parts become normal when she may be again placed with the balance of the flock.

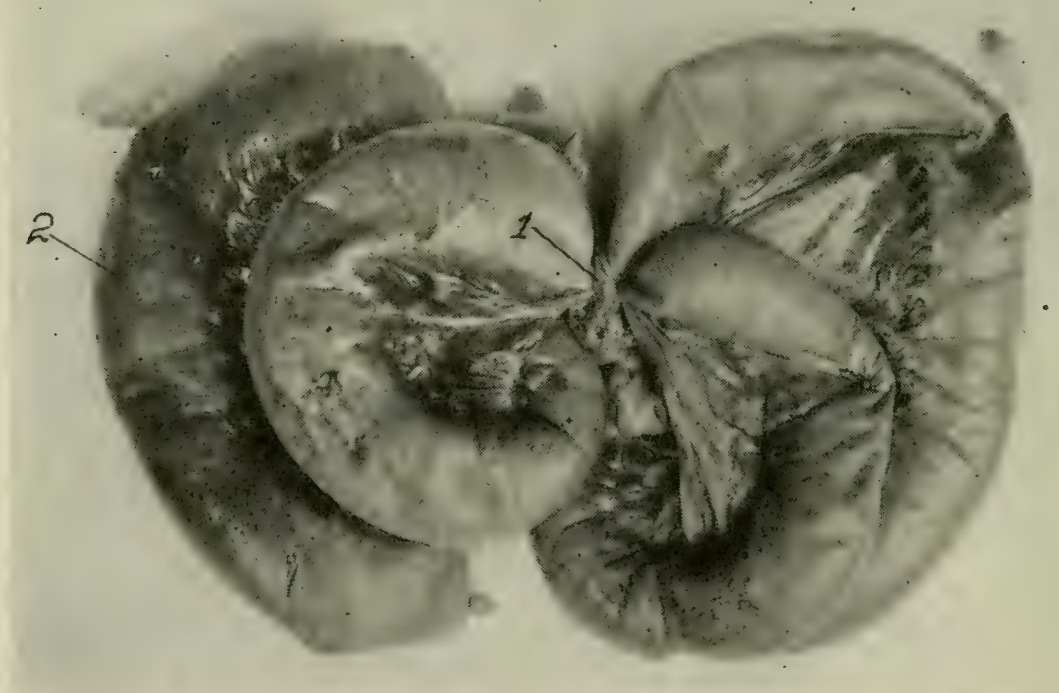

Fig. 64. Volvulus or Gut Tie of the Free Portion of the Small Intestine of a Single Comb Rhode Island Red Hen.

1 , shows the tie strangulating the bowel; 2 , passive congestion due to interference of the return flow of blood.

\section{VOLVULUS OR GUT TIE IN THE FOWL}

Volvulus or gut tie is a condition in which the bowels become displaced and a loop forms so as to tie a portion of the intestine in a knot.

Volvulus has been observed in the free portion of the small intestine of the fowl. Fig. 64 shows a case of this kind. This was observed in a Single Comb Rhode Island Red hen, one year old. In this case note that the bowel is distended with gas. The picture shows that four loops are involved. Pas- 
sive congestion was present in the strangulated portion of the intestine and it will be noted that this portion of the bowel is very dark. This tie was brought about by a part of the free portion of the small intestine passing through a rupture of the mesentery, the loop gradually growing tighter, causes an interference of the circulation and passive congestion follows which is indicated by the very dark condition of the bowel, later the circulation of the strangulated part is entirely cut off and deatin of the strangulated part follows.

\section{INTUSSUSCEPTION OF THE INTESTINE OF THE FOWL}

Intussusception is an invagination of the bowel of a fowl. Among the causes are excessive and irregular peristalsis under

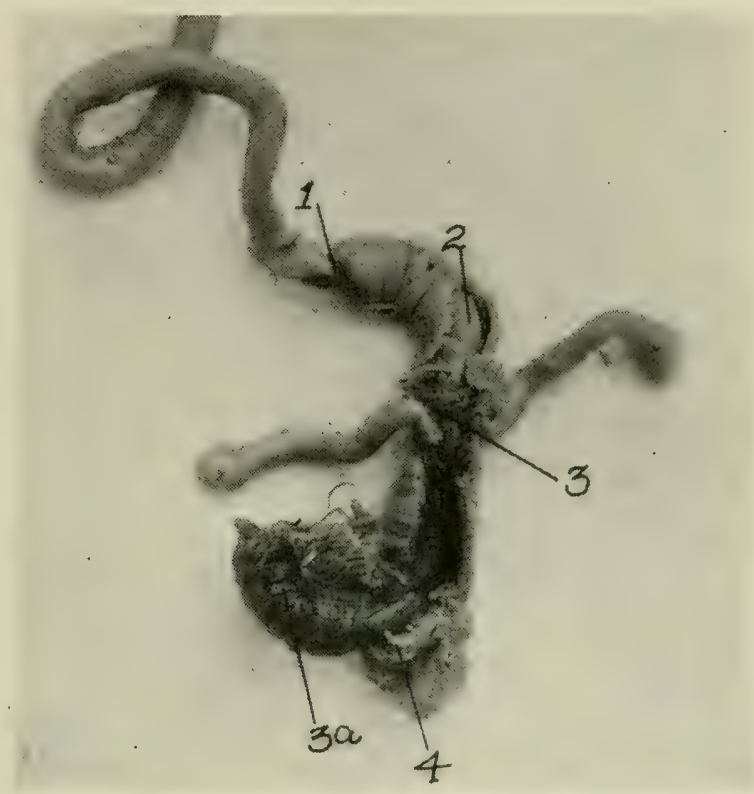

lig. 65. Invagination of the Bowel of a Four-Weeks-Old Buff plymouti Rock CHICK.

1, point of invagination; 2 , slrowing invaginated part surrounded by the invaginating portion; 3 , juncture of small and large intestine; $3 \mathrm{a}$, inflamed protruding portion of small intestine passing through the arms at 4 .

which condition there may be quite a length of the small intestine become telescoped, the outer or invaginating portion strangulating the inner invaginated portion, and disturbs circulation; the onward movement of the food becomes stopped.

Symptoms.-Little is known of the symptoms of this condition in the fowl. If the invagination takes place in the posterior portion of the intestines a portion of the invaginated bowel may protrude out the cloaca.

There is little that can be done in this condition and the fowl dies. 


\section{INTESTINAL DISEASES OF CANARIES}

Intestinal disturbances of canaries are usually due to errors in feeding and watering. In diarrhea remove all green feed and do not give soft feed. Give just the normal seed supply. Feed the bird with a small piece of wet bread on which has been sprinkled subnitrate of bismuth. If the case does not yield to this treatment place in the drinking water one ounce of clear water in which has been placed four drops of tincture of opium and fifteen drops of whiskey or brandy.

In case of constipation give plenty of green feed such as lettuce, apple or chick weed. Add a pinch of epsom salts to the drinking water. Make the water so that it has a faintly saline taste of the epsom salts.

\section{CONSTIPATION}

Constipation is a common ailment of fowls and it is shown that where epsom salts are given to fowls once a week either in drinking water or the salts dissolved in water and the water used to mix mash that the birds do much better as it acts as a regulator for the eliminative organs.

Fowls confined in small quarters without sufficient exercise are liable to suffer from constipation. Lack of green feed tends to constipation. Following diarrhea there may be a temporary or slight constipation.

\section{DIARRHEA DISEASES OF FOWLS Simple Diarrhea}

A simple diarrhea may be described as a frequent liquid passage from the bowels not caused by a contagious disease.

A condition of mild diarrhea is chronic in many fowls throughout life. This is especially true of certain breeds and varieties, especially the Barred Plymouth Rocks and especially if fed on rich food. In these cases there are no symptoms of the disease other than the softness or fluid condition of the (lroppings. Though this condition is probably due to a mild form of incligestion and the birds may not thrive or fatten or lay as well as those not so affected, the condition is not serious and ordinarily the poultryman pays little or no attention to it.

It is when the soft, pasty or liquid excrement has an offensive odor, and adheres to the feathers of the vent fluff region. staining them yellowish, greenish or brownish, that the matter becomes serious and interferes with the health of the bird. Young fowls are more susceptible to diarrhea from unfavorable conditions, of which the commonest are improper feed, and exposure to cold, than are adult birds.

When this reaction to external influences, cold, or when the 
irritation from indigestible matter within the intestine becomes severe enough to set up an inflammation of the mucous lining of the small intestine, it is termed enteritis. When it extends to the large intestine and the evacuations are accompanied with straining and some blood in the liquid discharge it is called dysentery. In both conditions there is an increased thirst, loss of appetite, high fever, and fluid discharge from the bowel, and in dysentery the discharges are streaked with blood.

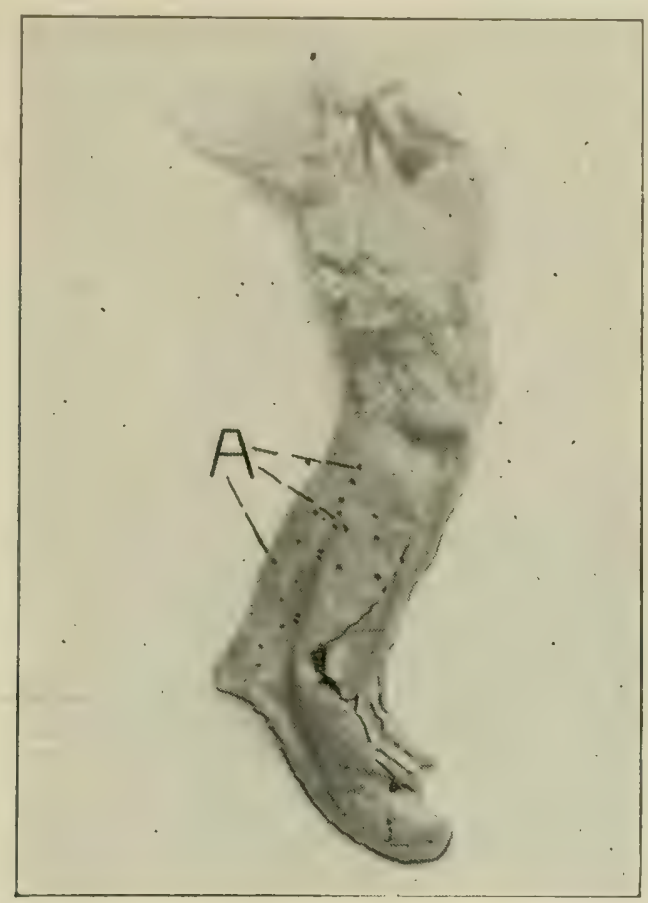

Fig. 66. Hemorrimagic Enteritis of a Hen $\Lambda$, small hemorrhages, natural size.

Cause.-Among the causes which may produce a catarrhal condition of the intestinal mucous membrane and result in a simple diarrhea are digestive disturbances, sudden change of feed or an unnatural large supply of green feed, too much meat scrap or fish scrap, moldy, putrid, or too stimulating food, drinking water which contains much organic matter and hence is filthy and putrid, foul drinking ressels, drinking from stagnant pools, and exposure to certain unfavorable atmospheric conditions are contributing factors, as is also the ingestion of irritating substances, such as lye, paint, spray-mixtures, unslaked lime, etc. Baby chicks being allowed to become chilled. - fifteen minutes chilling may do it. 
Along with diarrhea due to these causes may be mentioned a like condition sometimes caused by the presence in the intestinal tract of certain species of worms and of irritating foods. Exposure in damp coops, cold rains, or draughts often result in digestive derangements of this nature. Birds being yarded on damp low ground. A bird, during molting, has poor protection against inclement weather, from lack of feathers, and requires more care than at other times.

Symptoms.- The plumage loses its smooth, well kemped appearance; the bird is depressed and not inclined to move about as much as usual; there may be loss of appetite; the crop is full; digestion is slow; the cloaca may be inflamed, appearing red and sensitive; the evacuations from the bowels are frequent, the discharges being fluid, offensive and varying in color from whitish-yellow to greenish. In later stages the evacuations are quite spasmodic and forcefully ejected in a squirting manner, and the vent fluff feathers are soiled with the droppings. The affected bird gradually becomes weaker and there is a rise in temperature. It may eat little or nothing; thirst is extreme in some cases. The fowl may die in two or three days or it may live two or three weeks.

Postmortem Findings.-In opening the abdominal cavity after death the most noticeable alterations are in the intestinal tract and the liver. Upon opening the small intestine, areas of inflammation are noted, and occasionally a small hemorrhage is observed in the wall of the intestine. Microscopic examination of stained sections from the vital organs as the liver, kidneys, etc., reveal degenerative changes; as cloudy swelling which is usually the most marked.

Treatment.-Intestinal antiseptics are indicated such as the sulphocarbolates compound. Give to adult hens in one-half grain doses. Dissolve the compound in water and mix with mash. Repeat the treatment twice daily. Also permanganate of potash may be given in the drinking water. Use sufficient of the crystal to make the water a deep red color. One teaspoonful of catechu in each gallon drinking water is also indicated. 


\section{CHAPTER VI \\ DISEASES DUE TO BACTERIA \\ Infectious Blood Diseases \\ FOWL CHOLERA}

Fowl cholera; also called chicken cholera, is a disease of fowls, turkeys, ducks and other birds, and is caused by a germ belonging to the rod-shaped nommotile, polar staining, bacilli. It is placed in the Pasteurella group. It is called the Bacillus Ariscpticus and lives, and rapidly multiplies, in the blood, thus causing a septicaemia.

The bacillus avisepticus is rather short, plump and with aqueous fuchsin stains deeper at the poles or ends, than in the

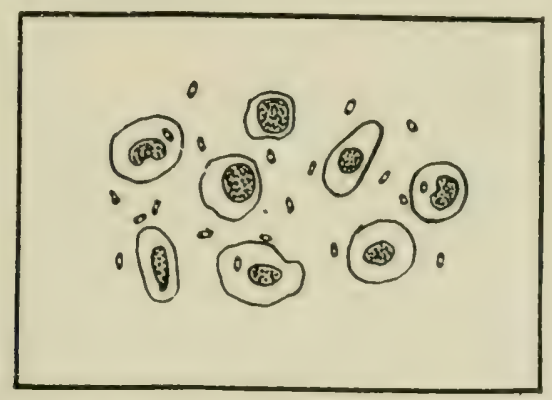

Fig. 67. A Blood Smear From a Case of Folvi Cholera.

Showing red blood cells, thrombocytes, mononuclear leucocytes, neutrophiles, and many polar staining germs, the Bacillus Avisepticus, the germ causing fowl cholera.

middle. Hence it is called a polar staining bacillus. Fig. 67 shows the germ, magnificd 1,000 times. This drawing was made from a blood smear from an outbreak among turkeys and chickens, which was one of several outbreaks we have studied. The large objects are various kinds of blood cells. One of these, a white blood cell, a phagocyte, has taken up one of the germs.

The germ of this disease can easily be isolated by ordinary laboratory methods from the heart, blood, the liver, spleen or kidneys of a fowl that has died of the disease. The colonies on plain nutrient agar at the end of twenty-four hours appear round, smooth, thin, shiny, with entire border and measure about $2 \mathrm{~mm}$. in diameter.

A blood smear made from the blood of a fowl sick of the 
disease and stained will show, under the microscope, the polar staining bacilli.

The virulency of the fowl cholera germ varies greatly. Thus inoculations of from four to fifty of the organisms into the breast muscles of the fowl, of the most highly virulent strain, caused death. Other tests run by feeding the dead carcases of cholera fowls to susceptible healthy birds resulted in infection in from eighteen hours to many days.

Mode of Spread.-The germs are eliminated from the body by all the natural discharges. Eggs have been proven to be infected with the germs when produced by hens coming down with the disease.

Birds become infected by eating or drinking water that has been contaminated by the droppings of infected fowls. The germs may be introduced through a wound, or in the eye.

The virulency of a strain can be increased by repeatedly passing it through birds or through guinea pigs.

The death rate in an outbreak of fowl cholera runs from 90 to 95 per cent.

Birds often contract this disease from others at shows, and when taken back home infect the remainder of the flock and the premises, or a bird recently purchased from an infected flock, or eggs from an infected flock, or chicks recently hatched in infected surroundings, or infected droppings carried on the feet of men, animals, or by wild birds as sparrows, by rats, mice, dogs and cats, from henhouses where the disease exists, or carried by streams or irrigation ditches, or dried and carried by the wind. Even insects have been known to carry the contagion. Buzzards are common carriers of this disease.

The germ of fowl cholera retains its power to produce disease for weeks, and even months, about premises where it has occurred, unless they be thoroughly disinfected. The germs have been kept in test tubes, experimentally, for two years and still prove virulent and capable of producing the disease. It resists, for a long time, both drying and zero weather.

The period of incubation, or time elapsing from the time the bird is infected, or takes in the germs, till the first symptoms appear, is from twelve to forty-eight hours in the case of the virulent strains though may be many days as indicated above in case of weaker strains of the organism.

In our experimental work, in which the virus or germs, were introduced into the peritoneal cavity, this period was six to twelve hours; when the virus was given by the mouth it required twentyfour to thirty-six hours to produce the first symptoms of the disease. The birds died twelve to seventy-two hours later.

Symptoms.-In the very acute cases the bird may suddenly 
drop dead during the day without showing marked signs of the disease. Igain birds may drop off the perch at night dead. Such symptoms may be the first intimation to the poultry keeper that his birds are seriously affected. In the less severe cases the first noticeable symptom is the yellowish or yellowishgreen droppings. This is, however, not positive that the birds have fowl cholera as noncontagious bowel diseases cause yellowish-green droppings. The discharge varies considerably in color and consistency. It may be a greenish mass of pasty consistency, a brownish red mucous, or a sticky transparent fluid. The yellow color of urates is the most constant character. The sick bird appears in an unnatural attitude, there is a loss of appetite, great prostration, stairy feathers; the bird mopes about, or sits around with tail and head down, giving it a "ball" appearance, the comb becomes dark, the gait swaying, and there is trembling, convulsions, thirst is great, standing at the trough of water for a long space of time, severe diarrhea. There is a high fever, the temperature varying from $109^{\circ}$ to $112^{\circ}$ Fahr. The bird becomes rapidly emaciated.

The chronic form of the disease usually appears at the end of an outbreak, occurring in the birds of the flock having the greatest natural resistance. The birds are dull, depressed and show persistent intermittent diarrhea. The mucosae are pale and emaciation is present. Inflammation of the joints may develop.

The percentage of loss in the flock, if not treated, is very great. The disease spreads rapidly through a flock. Purebred birds, if badly inbred, have lower resistance and hence are more susceptible than farm run scrubs. It was observed in an outbreak of fowl cholera among ducks that the disease progressed very slowly. Only one to five dying in the course of each week, in a flock of 500 ducks.

Postmortem Findings.-There may be observed in some cases a reddening of the skin of the breast and abdomen. The comb is usually very dark red to black, or, at times it may be rather pale. Upon examining the abdominal organs one notes a general congestion of the blood vessels. Small pin point hemorrhages may be observed in the heart and there may be present in the heart sac or pericardium a gelatinous exudate. Small hemorrhages may be noted over the small intestine and serous lining of the abdominal cavity. The contents of the bowel may be streaked with blood and may be frothy, pasty, and of a greenish, creamy, or brownish-red color. The liver is usually of normal size, dark in color, and may be studded with small necrotic areas. The liver is in a state of congestion, cloudy swelling, and focal necrosis. There is congestion of the lungs with possible pneumonia, a croupous hemorrhagic pneumonia, under which conditions the lungs are hepatized appearing liver-like on the sectioned surface. A serofibrinous exudate may occur in the air sacs. The kidneys are con- 
gested, grayish on sectioned surface, a cloudy swelling, and parenchymatous nephritis. The blood does not coagulate readily and upon microscopic examination is found teaming with the germs of the disease.

Blood Study.-A study of the blood of a fowl sick of cholera shows the hemaglobin, 90 per cent; red blood cells, 2,520,000; white blood cells, 6,000; thrombocytes, 184,000 . In this case there was very little reduction of hemalobin, with some reduction of red blood cells or erythrocytes. There is a great reduction of white blood cells or leucocytes, a hypoleucocytosis.

The differential blood count of the white blood cells showed 37 percent eosinophiles, 2 per cent neutrophiles, 52 per cent small lymphocytes, 5 per cent large lymphocytes, 4 per cent mononuclear leucocytes, with no mast cells observed.

Treatment. - The germs are eliminated from the body of the sick fowl through the droppings and are carried by the feet of men, dogs, cats, rats, sparrows and by utensils taken from infected quarters to susceptible noninfected flocks, such utensils may be watering pans, buckets, dropping board scrape, bags, nests, shipping coops, feed hoppers, dropping boxes and innumerable other ways, all of which must be avoided and infected utensils thoroughly and frequently disinfected, using a four per cent solution of some standardized coal-tar disinfectant dip. The house should be gone over several times with thorough cleaning and disinfection. The germs live two years or more so that unless thorough disinfection and cropping of the yards is carried out diligently it will not be safe for many years to bring susceptible birds on the premises. If the yard is small disinfection by use of bichloride of mercury, one to one thousand, using one gallon of the disinfectant to each ten square feet of surface, should be effective.

Sick birds should be immediately removed from the flock and dead birds immediately burned. All birds that are incurable should be destroyed and their carcasses burned.

In spraying the house a type spray pump shown in Fig. 30 , page 86 , may be used. Not only must the disinfectant, which may consist of a four per cent solution of a standardized coal tar disinfectant dip of a co-efficiency 5 or 6 , be driven into all cracks of the house and the dropping boards soaked, and if a ground floor the dirt removed and replaced with new dirt, thoroughly sprayed, but also the nest boxes, drinking fountains, feed troughs and all utensils used about the hen house. The droppings and all litter must be burned. Do not take this infective material to the field as fertilizer as wild birds, as sparrows, may spread the infection.

For the birds intestinal antiseptics are indicated. Permanganate of potash in the drinking water, using enough of the crystals that when dissolved, will make the water a deep red. 
The triple sulphocarbolates using one-half grain per fowl, in hoi mash, twice a day.

Vaccine is prepared for use against this disease. The germs of the disease are grown in pure culture on plain nutrient agar, washed off with sterile physiological salt solution, heated in sterile flask in water bath at $60^{\circ} \mathrm{C}$ for 60 minutes and preserved in 0.25 per cent trikresol and tested on guinea pigs for toxicity and in culture for spores and is then ready for use.

Serum against fowl cholera, both as a curative and as a preventative, has been prepared. The serum is prepared either from the blood of horses or from the blood of cattle. The animal is injected either subcutaneously or intravenously, usually in the latter way, with cultures of the organism causing the disease. The cultures consist of broth in which the organisms are grown. These injections are made weekly, in the beginning a very small quantity is injected, which quantity gradually increases and at last the animal is inoculated with very large quantities of the virulent cultures. After a period of about four months the animal is ready for the production of the serum from its blood as it now contains antibodies against the poison of the germs and when the serum of this horse is injected into the body of the fowl the antibodies it contains, neutralize the poison of the fowl cholera germs, should the bird be infected, and will also kill any germs present as it contains other bodies with bactericidal power, or power to destroy bacteria. The power then, to neutralize the poison or toxin is an antibody which has been manufactured by the cells of the horse in which the germs were injected and as a result of the injurious effects on these cells.

From the horse thus immuned its blood is drawn into a sterile flask and the fibrin whipped out and removed and the serum thus prepared is bottled and kept stored in a cool place till ready for use. The immunity produced by this serum is effective for at least two weeks and possibly, in the case of high potency serum much longer.

Some use a combination of the vaccine and serum under which conditions the immunity or resistance of the bird against the disease is much more lasting.

It has been shown that the blood of chickens immunized against fowl cholera has immunizing properties for other chickens. The white of the egg and also the yolls of eggs of immunized fowls possess similar properties.

\section{FOWL TYPHOID}

\section{(Infectious Leukemia, or Klein's Disease)}

Fowl typhoid or infectious leukemia is an infectious blood disease due to a short, plump, germ with rounded ends called the Bacterium sanguinarim, and is easily isolated from the liver, heart blood, spleen or kidneys of a bird dead of the disease. It reproduces the disease in inoculated birds, multiplying in the blood.

In 1889 Klein working in England, in the county of Kent. identified this disease in that country and later on the contin- 
ent in the Netherlands, and from this time in Europe the disease has been called Klein's disease and the bacillus causing the disease Klein-bacillus.

The Dutch investigators report that the most of the cases of the disease appear in the spring and early summer, and disappear completely at no definite season of the year. The disease is more common in adult than in young fowls and assumes an acute type. Chronic cases are seldom seen.

The mode of spread is similar to that of fowl cholera.

Symptoms. The first symptom is diarrhea, consisting of a thinnish, greenish-yellow color. There is a loss of appetite. The bird is very thirsty standing at the drinking fountain and drinking much of the time. Death usually comes on in a paroxysm. The course of the disease is very rapid, the sick vird dying in from a few hours to five days.

'The germ isolated from the sick bird's blood is observed to be destroyed at a temperature of $50^{\circ} \mathrm{C}$. for fifteen minutes. A one per cent solution of carbolic acid is fatal to it in five minutes. It resists drying when in a thin layer for from seven to fifteen days. Animal inoculations show it to be fatal to fowls, ducks, pigeons, rabbits, guinea pigs and mice. The temperature of a fowl begins to rise on the second day after inoculations of small quantities of the bouillon culture and reaches $109^{\circ}$ to $114^{\circ}$ Fahr.

Other symptoms of the sick bird are those of an anemic or blanched appearance of the mucous membrane of the head, with a dull appearance and great prostration. There is a decrease in the red blood cells or erythrocytes and an increase in the white blood cells or leucocytes. The latter increase is usually in the polymorphonuclear leucocytes.

The fowl stays apart from the balance of the flock and may sit on the perch poles much of the time. The bird has a "drooped" appearance with head and tail down giving it the socalled "ball appearance." The feathers are ruffled and in later stages the bird appears drowsy.

It is not easy to differentiate by the appearance of the sick bird, between fowl typhoid and fowl cholera.

Postmortem Findings.-The mucous membrane of the intestine is observed to be in a state of catarrhal inflammation. The catarrh, however, is very different as to degree, place, and development; it is often localized in a few spots, in other cases it extends from the stomach to the cloaca. or throughout the entire digestive tract. There may be an inflammation of the ceca or blind pouches. The character of the catarrh is either mucus with abundant exudation or rather purulent with little exudation. The congestion which is generally not very prominent, manifests itself by a greater filling of the vessels of the mesentery and of the intestinal wall, and by the red color of the affected parts of the mucous membrane. 
Hemorrhage may appear in the form of pin point bleeding in the mucous membrane. Hemorrhagic inflammation of the ceca or blind pouches also occurs.

The liver is enlarged and mottled, with grayish patches due to areas of invasion by white blood cells or leucocytes. The liver is congested and blood may drip from the cut surface. There may be pin point areas of dead tissue, focal necrosis. The liver is easily torn, greasy, and degenerated. The color of the sectioned surface varies between brown and yellow. The bronzy discoloration of the surface is characteristic. When the liver is exposed to the air for a long time the bronzy appearance becomes more pronounced.

Microscopic study of sections of the liver show an engorgement of the blood vessels and a breaking down of the liver cells. This degeneration extends from the commencement of cloudy swelling to complete necrosis as indicated above.

The spleen is usually enlarged, and of a dark red color. The consistency of the pulp differs but little from the normal one. In some cases there is no trace of lesions.

The kidneys show congestion which may be recognized by the minute red lines. Parenchymatous degeneration is present which is shown by the grayish color of the cut surface. The kidneys may be enlarged and acute nephritis present.

The heart may appear normal or pale in color with grayish spots of necrosis. The blood is lighter in color and does not clot readily. The red blood cells decrease to a marked degree as the disease advances and the white cells become greatly increased. In stained preparations of the blood numerous red corpuscles are observed undergoing degeneration. 'I here may be edema around the heart and an increased amount of pericardial fluid.

Muscular tissue changes, in some cases, is quite marked. This has been observed in chicks but one to two months old. It is also observed in adult fowls. These are most marked in those cases assuming a more chronic form. All the muscles of the body show white specks on their exterior. In the proventriculous, as well as in the heart muscle above described. These white knot-like areas are due to infiltration of the point with white blood cells. The infiltration also contains a great amount of the bacterium sanguinarium or germs causing the disease.

Treaiment.-It is important that proper methods be employed in combating this disease. Measures of a general character aim at combating the spread of the infectious matter. Birds from a sick flock should not be allowed to be sold on the market or even removed from the poultry yards. Birds too sick to recover should be killed. All birds dead of the disease should be burned. Birds should not be allowed to be sold for breeding purposes from a flock in which the disease has recently made its appearance, also eggs should be prohibited from sale for hatching purposes. (ieneral cleaning of the yarls and houses and the burning of the litter is indicated, also thorough disinfection as outlined under Fowl, Cholera, which see.

Intestinal antiseptics may be given such as permanganate of potash in the drinking water, using enough of the crystals to make the water a deep red. Also the sulphocarbolates com- 
pound and catechu. One teaspoonful of these latter to each gallon of water.

Vaccine made by the following method is useful in this disease: The bacteria, grown during 17 hours at $37^{\circ} \mathrm{C}$. on plain slant nutrient agar, is washed off with sterile $0.85 \%$ salt solution, and heated in a water bath at $60^{\circ} \mathrm{C}$. for one hour. Each slant is washed off with $50 \mathrm{cc}$. solution and the dose when prepared is $3 \mathrm{cc}$. Vaccinated chickens resist the infection and stay healthy when placed in infected yards. The Rotterdam Serum Institute report that the use of vaccine, in which over 10,000 fowls were vaccinated in an outbreak of Fowl typhoid or Klein's disease at Amersfoort, the disease was completely controlled. We have found the vaccine to contain some curative value.

Serum, made from the blood of a horse that has been repeatedly injected with increasing quantities of the virulent cultures, has proved effective in combating the disease. The disease can, therefore, be successfully combated by means of proper sanitary measures, vaccination, and serum-therapeutics.

\section{MYELOID LEUKEMIA}

Leukemia is a condition of the blood consisting in a superabundant development of white blood cells or leucocytes. It is a disease that is accompanied by enlargement of the spleen and liver, and by increased size of the lymph glands.

Spontaneous leukemia occur in fowls. It is transmitted by the intraveneous or intraperitoneal injection of an organic emulsion.

The clinical picture and changes produced in the blood and organs of the fowl are analogous to those which occur in human leukemia.

In a case of spontaneous leukemia the following differential blood count was found:

Polymorphonuclear with eosinophilic rods......... 8 per cent Polymorphonuclear with eosinophilic granules........ 0 per cent Lymphocytes .......................... 2 per cent Large mononuclear cells........................... 30 per cent Mast cells ............................. 0 per cent Mononuclear myelocytes with eosinophilic granules.. 52 per cent Unclassified cells ...................... 8 per cent

100 per cent

It is associated by an infiltration of large mononuclear lencocytes. Atrophy of the bone marrow is noted. There is noted an accumulation of white blood cells in the bone marrow, spleen and liver. The bone marrow appears greyish-red in color. In these cases the liver is enormously enlarged also the spleen is enlarged.

Treatment.- Prompt isolation of the well from the sick birds and sanitary measures for the fowl should be observed. 


\section{BACILLARY WHITE DIARRHEA}

Bacillary white diarrhea is a blood disease of baby chicks due to the Bacterium Pullormm. The disease is manifested by a frothy, white to whitish-brown, pasty discharge from the bowel which adheres to the vent fluff.

The loss to the American poultry raiser from white liarrhea is greater than from any thing else, perhaps greater than from all other contagious diseases combined. It strikes at the root of the poultry industry; no one can successfully conduct the business if he is unable to rear a reasonable number of chicks annually.

Without treatment and control the resulting mortality, when white diarrhea has secured a foothold in a poultry plant, is extremely high, often reaching ninety per cent of the season's hatch. The loss from white diarrhea in dollars and cents is enormous, almost beyond calculation. It is widesprearl throughout the United States and causes the loss of perhaps ten per cent of all chicks hatched in the country. By proper measures the disease is fairly easily preventable and a large number of the affected chicks will recover under proper treatment.

The Bacterium Pullorum or organism causing the disease is a long, slender bacillus, with slightly rounded ends. It is nonmotile, does not liquefy gelatin, does not produce pigment in culture media, and lives best in the present of oxygen. It somewhat resembles the typhoid bacillus and stains readily with ordinary aniline dyes. It does not stain with Gram's method and the organism does not produce spores. On nutrient agar, in twenty-four hours, the colonies appear small and white and increase in size slowly. They seldom reach more than two $\mathrm{mm}$. in diameter.

In artificial media the Bacterium Pullorum produces a toxin which is fatal to rabbits. When bouillon filtrate is injected intraveneously into a rabbit the animal dies in two hours, or if the rabbit has great resistance there is noted marked difficult breathing and death over night. In a rabbit dead from the toxin there is noted congestion of the liver, spleen, kidneys. adrenals, lungs and mesenteric lymph glands together with hemorrhage into the gastric mucosa.

Mode of Spread.-Ovaries of laying hens, diseased, but still functioning, may be infected by the germ. The germ can be isolated. particularly from the yolk, of at least some of the eggs, and as a result the chicks have the disease more or less developed when they are hatched, as conditions which favor hatching also favor the multiplication of the germs to an extent that toxins or poisons have already been produced in the young in sufficient quantity for the disease to at least manifest itself in a few hours after hatching, 
although ordinarily they do not begin to die until they are about three days to a week old.

The whitish, frothy, pasty bowel discharges, more or less sticky and having a tendency to "paste up the vent," from these chicks is laden with the germs, and others of the flock soon become infected from contaminated food picked up from the ground. In the former case, chicks may begin to die soon after hatching; in the latter, in from three to four days, a few dying each day.

The death rate is high, reaching in many cases as much as seventy-five per cent or more. Those that recover are stunted and do not make satisfactory growth. The greatest loss is from the first few days to, in some cases, two or three weeks. It is probable that the carriers are chicks that have recovered, but which still carry the organisms, especially in the ovary, as the human typhoid carriers carry the germs of typhoid fever, in the infected kidneys and in bowel ulcers. These "carriers" having established an immunity, do not themselves succumb to the disease, and they rarely show any outward symptoms of it.

Insanitary conditions, spoiled feeds, dirty, stagnant water, improperly ventilated incubators, brooders, and buildings, or badly regulated heat, are factors in weakening the physical condition of chicks and favor ravages of diseases.

Should one or more chicks be hatched, harboring the disease, the others of the lot are exposed early and nearly all may become affected. The chicks are especially susceptible during the first forty-eight hours of life, but are practically immuned after the fifth day. Brooders and incubators that have previously held infected chicks may be a source of the spread of the disease. Day-old chicks frequently carry the disease to other points and contaminate brooders or infect other chicks with which they come in contact.

In this disease then, there appears for the most part, to have a well defined cycle. The bacterium passes from the infected ovary of the hen through the egg to the chicks and back to the ovary of the pullet which has survived the disease as a chick.

Preventative or Eliminative Measures.-Knowing the cycle through which the bacterium passes we may begin the elimination of the infection from the flock by two methods. First, test all hens and males of the flocks on the premises with the agglutination method to determine the hens and cocks that are infected and sêll such birds as food as they will transmit the infection to the eggs and thus perpetuate the disease. The agglutination test is applied in the following manner: a sample of the blood of the fowl is secured; a 24 hour bouillon culture is made, a dilution of 1:50 and 1:100 is made with the serum of the blood and the bacterial culture. If bacterium is present in the fowl from which the blood was secured there will be present in that blood an antibody called agglutinin and this will cause the germs to form in clumps and this is called agglutination. Second.-Remove from the flock all chicks that do not develop white diarrhea and raise them under uninfected brooders, house, and yard and from these start a new flock. Experience has shown that this has given most excellent results.

Symptoms.-The wings are droopy, the feathers ruffled, and the bird presents a sleepy appearance. The chicks are seen to huddle together, they have little or no appetite, the abrominal yolk is not properly absorbing; there is a whitish or whitish- 
brown frothy discharge from the bowel which adheres more or less to the vent fluff; the eyes are closed much of the time and apparently the bird has no interest in life. They peep much of the time, the appearance in many is stilty, and the abclomen is prominent behind. In these cases after death one finds the yolk unabsorbed or only partially so. The intestines are more or less full. Late fall, winter or early spring hatched chicks are freer from the disease than summer hatched. This may be explained by the fact that hens with diseased ovaries gradually become poorer layers as the disease progresses, and hence, only lay in late spring or early summer, when nature intends reproduction of birds. Finally the hen may cease laying.

The disease may show itself within two or three days after the chicks are hatched and spread rapidly through the brood. The period of incubation is four to ten days. Deaths begin to nccur soon after hatching and may continue to the third or fourth week. Chicks which survive, remain stunted and unthrifty for some time. In two series conducted at the North Carolina Experiment Station to determine the effect of diarrhea on the growth of chicks for the first eight weeks of their lives the results were as follows: In the first flock the birds without diarrhea weighed, at the end of eight weeks an average of 0.47 pound each and the chicks that were affected by diarrhea, during the first few days of their lives, weighed on an average but 0.28 pound each. The chicks that had suffered an attack of diariliea weighed 19 per cent less than those that did not go throtigh an attack. In the second series the chicks that did not suffer an attack of diarrhea weighed, at the end of eight weeks, 0.74 pound each, and those that had suffered such an attack weighed but 0.56 pound each. The chicks that suffered an attack of diarrhea weighed 21 per cent less than those not attacked by diarrhea.

Treatment.- Intestinal antiseptics are indicated as in other forms of diarrheas. Permanganate of potash in the drinking water. Bichloride of mercury $1: 10,000$ in the drinking water. Catechu one teaspoonful in a gallon of water. Sulphocarbolates compound in similar quantities are recommended.

\section{ADULT FOWL INFECTION}

Bactcrimm Pullormm infection of hens has occurred and caused considerable loss. In from two to six weeks after infection of the adult birds with a strain of the Bacterium Pullorum virulent for adult fowls the first simptoms appear. The bird has a loss of appetite, sluggish, appears droopy, feathers ruffled and unkemped appearance, the comb and wattles are 
pale, and more or less diarrhea is present. The bird may live for several days after the first symptoms appear and die in an emaciated condition.

At autopsy there is found cloudy swelling and focal areas of necrosis of the liver, spleen and kidneys. The natural yolk color have changed from a dark brown to a greenish color. The ovary is shrunken, and on cut section the ova are of a tough cheesy consistency, with a reddish-yellow, or greenish tinge. Some of the ova appear as cysts with a darkened fluid content.

Intradermal Test to Detect Bacterium Pullorum Infection.-To make the intradermal test a culture is made from several strains of the Bacterium Pullorum, from widely separated sources, grown in bouillon at a temperature of $37^{\circ} \mathrm{C}$. for a period of thirty days to two months. The product is then sterilized in a water bath at a temperature of $60^{\circ} \mathrm{C}$. for 60 minutes. To this product there is added 0.5 per cent carbolic acid as a preservative. This is then concentrated by evaporation to one-fifth its original volume. 0.2 cc. or about three drops is injected into the wattle of the suspected bird. The injection is made with a hypodermic syringe graduated in tenths of a cubic centimeter. The needle must be very fine and the injection is made in the skin and at the lowest point of the wattle. The injected wattle should be checked twenty-four and again at the end of thirty-six hours for the reaction swelling.

\section{APOPLECTIFORM SEPTICAEMIA OF FOWLS AND PIGEONS}

Apoplectiform septicaemia is a highly infectious blood disease of fowls and pigeons due to the Streptococcus gallinarum, which grows in the blood in long or short chains. The organism is readily isolated from the liver, spleen or kidneys of a bird dead of the disease. It will grow upon ordinary laboratory media and does not liquefy gelatin. Experimental inoculations killed the following animals: chickens, mice, rabbits and swine. It does not kill guinea pigs or dogs.

Symptoms.-Apoplectiform septicaemia is rapid in its course. The bird at first appears dull and depressed, there is a loss of appetite, ruffled feathers, stays apart from the balance of the flock, does not respond when feeding time comes, there is a staggery gait, great prostration, at times diarrhea and death. The bird may die in from twelve to twenty-four hours after the first symptoms appear. Birds in which symptoms have not been observed may be found dead under the roost in the morning. This disease is reported to have caused large losses to pigeon fanciers.

Postmortem Findings.-The spleen is enlarged, dark and soft, cloudy swelling and focal areas of necrosis are noted in the kidneys, spleen, and liver. Pneumonia may be present. There may be hemorrhagic discoloration of the breast, and neck, due to diffused hemorrhages in the structures under the skin. The abdominal 
cavity may contain an abundance of sero-sanguinous fluid. A serosanguinous exudate may also be observed in the pericardium. The blood vessels of the mesentery are congested. The small intestines are congested in patches. The contents of the intestines is stained with blood and mucus. Upon opening the brain cavity there is observed profuse subdural exudation. The coverings of the brain are congested and the ventricles or brain cavities contain an abnormal amount of discolored serum.

Treatment.-Observe the same sanitary precautions as outlined under Fowl Cholera which see.

If possible, separate the well hircls from the sich. Vaccination with a raccine made from the Streptococcus gallinarum has given good results. A serum has been tried and is very effective.

\section{SLEEPING DISEASE OF FOWLS}

Sleeping disease of fowls is a disease caused by an encapsulated streptococcus which lives and rapidly multiplies in the blood of the fowl and causing as the chief symptom sleepiness of the fowl. The disease has been described in Europe by the Germans and by the French. The organism is called the Streptococcus capsulatus gallinarmm. It differs in length of the chains and in the size of the cocci making up the chains. according to the kind of animal in which it appears as a natural disease or into which it is inoculated. The streptococci are stained by the ordinary aniline stains. They are Gram positive. It is very sensitive to drying and to heat. Bouillon cultures are killed at a temperature of $80^{\circ} \mathrm{C}$., for five minutes. At $100^{\circ} \mathrm{C}$. it is killed in one-half minute. One per cent carbolic acid kills in two minutes and a similar solution of lysol. creolin and liquor cresoli saponatus, kills in three minutes.

The period of incubation as given varies from six days to 30 and even 70 days. The organism will kill fowls, pigeons, rabbits, mice and lambs.

Symptoms.- The most prominent symptom is the desire to sleep. The hen sits for hours at a time with eyes closed, feathcrs ruffled, and head bent backwards with beak buried in the feathers. Occasionally the fowl awakens and opens wide its beak as though panting. The mucous membrane of the eye may become congester and inflamed. Usually but one eve is affected. The comb and wattles become pale. Diarrhea occurs and the bird dies in an extremely emaciated condition. Birds may die suddenly after being sick for one or at most a few days.

Postmortem Findings.-There is observed in the abdominal cavity a bloody, shiny, viscid fluid. The peritoneum is diffusely reddened and appears swollen. The mucous membrane of the small intestine is diffusedly red and swollen and this condition may extend, 
more or less, into the gizzard and proventriculus. The liver is brownish yellow in color, enlarged and easily torn. Dark-red hemorrhages may be seen over the surface. The spleen is enlarged, the capsule tense, and the splenic pulp tears easily. The kidneys are enlarged and of a dirty yellowish color. The lungs may be congested and edematous and studded with small, dark red hemorrhages. The outer surface of the lung may be covered with a fibrinous exudate. The heart is distended with coagulated blood and small pin point hemorrhages may be noted on the outer surface of the heart. There may be covering the outer surface of the heart a thin layer of fibrinous exudate. Chains of streptococci may easily be obtained from smears from these exudates or from the blood.

\section{EPIORNITHOLOGIC PNEUMO-PERICARDITIS IN TURKEYS}

Epiornithologic Pneumo-pericarditis of turkeys is an infectious disease of turkeys affecting principally the lungs and the heart sac. The disease is infectious for turkeys alone as it does not produce disease of fowls, geese and pigeons. Rabbits and guinea pigs when inoculated with pure cultures die of the disease. The germ causing the disease is a small oroid rod closely resembling that causing fowl cholera. It is Gram negative and stains readily with ordinary laboratory stains. It does not liquefy gelatin. or produce gas in sugar-agar shake cultures. It does not produce acid or coagulate milk. There is no visible growth on potato under either aerobic or anaerobic conditions.

Symptons.-The turkey is first noticed to be dull, an apparent stiffness of the mouth, and death in a few days.

Postmortcm Findings.-At autopsy there is observed a severe pericarditis, there may be extensive adhesions of the heart wall, and there may be a fibrinous exudate of a yellowish color. Pneumonia may be present and the lung may be completely hepatized. Degenerative changes are noted in the liver and kidneys. The liver may also appear pale in color and enlarged with fatty degeneration. The bipolar rod can easily be obtained from the blood or from any of the internal organs.

\section{INFECTIOUS ENTERITIS OF PHEASANTS}

Infectious enteritis of pheasants is a disease of pheasants caused by a short bacillus measuring 0.7 microns in diameter and 1 to 2 micra in length. In cultures the organism forms chains. On gelatin it produces a gray color and on potato, after 48 hours, the growth is of a yellow color, which later changes to a brown with the erlges a rose brown. The culture has the appearance of honey. It attacks only pheasants, the males appearing the most susceptible. Inoculations of the cultures does not kill rabbits and guinea pigs. 
The disease is transmitted through contaminated food and water.

Symptoms.-The birds are at first depressed, do not appear as lively as usual. Later the gait is uncertain and there is a loss of appetite, diarrhea in which the bowel discharge is of a yellowish-green color, there is prostration, coma or unconsciousness and finally death. The sick bird dies in from five to eight days.

Postmortem Findings. - The mucous membrane of the intestine is congested, is reddish brown and covered with a viscid croupous exudate. Small hemorrhages are found on the serous membranes. The liver is enlarged and tears easily. The spleen is enlarged.

\section{HEMORRHAGIC SEPTICAEMIA OF THE DOVE}

I Iemorrhagic septicaemia of the dove is a disease which has been described in the Ring Dove and is caused by an ovoid rod shaped bacillus which show's polar staining with fuchsin, and is Gram negative. It does not liquefy gelatin. Rabbits and guinea pigs die of septicaemia following inoculations of pure cultures of the organism. Fowls do not contract the disease. Doves and pigeons alone contract the disease.

The symptoms as given are those of general septicaemias described above as occurring in other kinds of birds.

The postmortem finding are those of a hemorrhagic inflammation of the intestines.

\section{CHOLERA OF SWANS}

Cholera of swans and other waterfowls is due to germ, the Bacillus coscoroba. The organism is an ovoid, motile rod, showing polar staining, but does not stain with Gram's method. Wilk is coagulated and indol is formed. The fowl does not contract the disease.

Symptoms.-The bird remains in a sitting position, is dull, loss of appetite, diarrhea and emaciation followed by death.

I'ostmortcm Findings. - The lungs may show congestion. The liver is enlarged, dark in color, and may show small white spots. The spleen, in some cases, is slightly enlarged.

Hemorragic Septicaemia of Sa'ans. - Under this heading there has been described a condition among swans accompanied by edematous infiltration of the lungs. The disease apparently affects, by preference, young swans and the affected swan may die suddenly. There are small hemorrhages on the serous membranes. There is congestion of the intestines and a cloudy swelling of the liver and kidneys. Older birds may live several days. The lungs of the older birds show pneumonia often in the stage of gray hepatization. The liver of the older birds is congested, there is a thickening of the liver capsule, and at 
times a fatty degeneration as well as cloudy swelling. There may be small hemorrhages on the serous membranes, and a congestion of the intestines. The organism is easily recovered from the blood of the dead bird and from the internal organs as the liver, spleen and kidneys.

The shape of the organism is similar to that of fowl cholera but larger. It stains by Gram's method, and is motile. The organism may be four micra long. On potato it produces a brown color, with offensive odor.

\section{SEPTICAEMIA OF PARROTS}

Under this name there has been described a disease called by various names such as Psittacosis of Parrots and Parrot Septicaemia. It is due to a germ belonging to the hog cholera group. It is motile, Gram negative, and grows well either in the presence or absence of oxygen. It is rod shaped and does not liquefy gelatin. Its growth on gelatin is at first shiny, transparent, iridescent, later a porcelain white. The organism is readily isolated from the bone marrow, blood, spleen and other organs. The disease is spread through contaminated food or water. The discharges from the parrot contain the germs.

Among the causes predisposing to this disease are crowding, filthy cages, climatic conditions, and general hardships during shipping as it appears to be more common in parrots brought from the tropics and shortly after their arrival.

Symptoms.-The first signs of the disease are those of shivering, loss of appetite and a diarrhea, the droppings being of a frothy greenish appearance. There is great thirst, dullness, the sick bird sits in one position, eyes closed much of the time, feathers ruffled, and head turned on the shoulder. Emaciation ensues, convulsions and death. The sick bird usually dies in from eight to nine days. The period of incubation as given is from three to five days.

Postmortem Findings.-The intestines are congested and there is present small hemorrhages. The spleen is enlarged and soft in texture. The liver and kidneys may show small grayish spots. The muscles may show a spotted appearance. The heart muscle may be dark and hemorrhagic. Small hemorrhages may be found on the lining of the heart and also on the covering of the same organ. Pneumonia may be present and the air sacs may contain a fibrinous exudate.

\section{SEPTICAEMIAS OF PIGEONS}

An outbreak among pigeons has been described as due to an organism closely related to that of the Bacillus suipestifer.

Symptoms.-The disease has been popularly called megrims 
and is characterized by spells of aimlessly flying around, and a peculiar turning of the head to one side. There is emaciation and finally death.

Postmortem Findings.-No visible lesions of discase is observed. There may be an exudate of a grayish yellow color in the brain cavity. The organisms may be obtained in pure culture from the brain lesions. The germs may also be obtained from the blood and from the liver of a bird dead of the disease.

Infection of the Pigeon due to Bacillus Paratyphosis B.An infection of pigeons with the organism, the Bacillus paratyphosis B. has been described. The organism was isolated from the blood and from the muscular structures. Experimental inoculations of mice proved fatal.

The birds became emaciated, and the liver may be yellowishgray in color, and covered by hyperenic areas. In other cases there was observed yellow, round, compact knots varying in size from a grain of wheat to a bean, which gives the surface a roughened appearance. Hemorrhages may appear in the intestines. The greatest degenerative changes are in the kidneys. A study under the microscope shows a parenchymatous nephritis. Cloudy swelling and focal areas of necrosis may appear in the liver.

\section{GROUSE AND QUAIL DISEASES}

Several workers have reported outbreaks of disease among fowls and other birds due to the colon bacillus.

Under the heading of grouse disease there has been described a pneumonia caused by an organism belonging to the B. coli group.

Under the heading of quail disease there has been described a condition due to a germ belonging to the colon group. The disease affects the lungs causing congestion, cloudy swelling with focal necrosis of the liver, and ulcerations of the intestines. The disease has been called Colobacillosis tetraonidarum. The disease has especially appeared among those birds shipped for stocking purposes, the disease appearing during the transportation period or immediately afterwards. When inoculations of the organism, in pure cultures, are made it has been shown that the disease is not fatal to fowls, pigeons or rabbits, but kills guinea pigs and mice.

Symptoms. - The first symptoms shown by the bird is that of dullness, loss of appetite, ruffled feathers, emaciation, and death in from two or three days to several days.

\section{FOWL PEST}

Fowl pest is an acute infectious disease of fowls that attacks chickens, geese, turkeys, pheasants, sparrows, parrots, 
pigeons and owls. The infective agent is contained in the blood, discharges from the nose and in the droppings. It may also be recovered from the nervous system.

Fowls become infected through contaminated food and water. The disease has been transmitted experimentally to chickens by inoculation of emulsions from these sources from infected fowls. These emulsions were filtered through porcelain filters and the liquid passing through the filter was injected into a chicken and produced the disease. The disease is therefore produced by a filterable virus, that is, a germ too small to be seen under a microscope or to be grown in visible quantities upon artificial media. The blood cells are said to be more virulent than the blood serum alone.

The virus kept sealed in glass tubes retains its virulency or power to produce disease for at least three months. $65^{\circ} \mathrm{C}$. kills the virus at once and $55^{\circ} \mathrm{C}$. for thirty minutes kills it. Exposure to direct sunlight kills it. The virus remained active in dried liver and spinal cord for 200 days, and in blood mixed with glycerine 270 days. Drying in thin layers kills it. It is destroyed with five per cent creolin, three per cent chlorate of lime, and one per cent corrosive sublimate. Wounds inoculated with the virus proved fatal.

The disease is spread by contaminations from the droppings and the nasal discharges. The feet of man and animals and of wild birds as sparrows, utensils and clothing coming in contact with the contagion may transmit the disease. Birds introduced into the flock or placed in infected runs or houses may serve as a source of infection. Mascerated parasites removed from the skin of sick birds and this injected into susceptible birds did not produce the disease.

Symptoms. - The period of incubation is from three to five days. The bird first appears depressed with loss of appetite: later dull and sleepy. The comb and wattles appear dark red and the bird sits with droopy wings, and gives a peculiar lond cry when picked up. The walk is irregular and staggery. Toward the end of the disease grayish scales sometimes develop on the skin, especially on the face and around the eyes. The nasal discharge is of a reddish gray color and rather viscid in consistency. Similar secretions are noted in the mouth and throat. The bird if distressed by obstruction from mucous accumulation may throw its head causing a discharge of a quantity of the secretion. The droppings are gray or greenish in color and occasionally diarrhea accompanies the disease. The disease runs a rapid course and is fatal in the majority of the cases in from two to three days, the more chronic cases may live seven days. The time of cleath and 
rapidity of the course of the disease depends upon the resistance of the bird and the virulency of the organism. The temperature may run from $110^{\circ}$ to $112^{\circ}$ Fahr. A diphtheric type has been observed in which fibrinous exudate occurs in the nasal, mouth and pharyngeal or throat regions. Inflammation of the eyes may occur.

Postmortem Findings.-In very acute cases lesions may be absent or only slightly marked. There may be hemorrhages in the serous lining of the abdominal cavity and over the intestines, gizzard, and proventriculus. Hemorrhages may be observed in the heart sac, and in the mucous membrane of the trachea, larynx, and pharynx. The kidneys are swollen and the cut surface appears grayish in color indicating cloudy swelling. The kidneys are congested. There is also congestion of the liver and spleen. In less acute cases there may be edematous swellings of the neck and breast. The heart sac may contain a quantity of pale yellow, slightly cloudy fluid. Fibrinous exudate has becn noted in the pericardium and peritoneum. There is a catarrhal swelling of the mucous membrane of the eye; conjunctivitis. The mouth and nasal passages contain a quantity of mucous. The lungs may be congested. The intestines may be congested or there may be inflammation.

Treatmont.- Steps should be taken as in other contagious diseases before mentioned. It has been shown that blood of a goose that has recovered as well as hyperimmunized goose blood has curative effects. Immunity to the disease has been conferred upon chickens by intravenous inoculation of heated virulent blood. Chickens thus hyperimmunized furnished an active microbian serum which protected the birds against the virus injected 21 hours later. By mixing the virulent serum with washed globules of rabbit blood the alexin of the serum was destroyed. A mixture of this virus with the serum of the hyperimmunized fowl, heated to $56^{\circ} \mathrm{C}$., was capable of conferring immunity when injected into pigeons.

\section{SEPTICAEMIAS OF CANARY BIRDS}

\section{Infectious Necrosis of Canaries}

This clisease is also called bird ferer, bird plague and Scptic ferer. This disease is due to a non-motile bacillus which take the polar staining and is Gram negative. The culture produces an offensive gas, and on potato the growth is of a brownish color.

Symptoms.-The bird appears droopy with loss of appetite. Singing birds cease to sing and sit upon the perch pole much of the time. Death usually occurs in 24 to 36 hours. Upon opening the mouth there may be observed diphtheritic areas covered with the diphtheritic membrane.

Postmortem Findings.-Small necrotic areas are noted in the liver and spleen. These dead areas are of a yellowish color. The liver and spleen are enlarged. The spleen tears easily. The mucous 
membrane of the pharynx contains yellowish nodules. The surface of the lungs are covered with a yellowish exudate, and the lungs may be congested and even areas of disease. Inflammation of the intestines may also occur. The necrotic lesions somewhat resemble those of tuberculosis of the fowl. A microscopic examination of these small lesions show them to be made up of necrotic or dead tissue with masses of the bacteria.

General sanitation as observed in other contagious diseases is recommended.

\section{SEPTICAEMIA OF CANARY BIRDS}

This is an infectious disease due to a small rod shaped bacillus, Gram positive, and non-motile. It liquefies gelatin in about 48 hours, and on potato, at first grayish-white, later it becomes yellowish and even brownish-yellow.

Experimentally it kills hens, pigeons, rabbits and guinea pigs. Sparrows and mice are susceptible and may carry the disease.

Simptoms. - The bird is first noted to have ruffled feathers, sits much of the time, not active, peeps occasionally giving signs of difficulty of breathing. The appetite remains the same but the bird suffers from thirst. The disease is rapid in its course and usually dies in about two days after the first symptoms appear.

Postmortem Findings.-The mucous lining of the first part of the intestine is swollen and diffusely red. The liver is congested, yellowish in color, and tears easily. The spleen may be swollen.

Similar treatment is suggested as in other infectious diseases.

\section{CHOLERA OF CANARY BIRDS}

Cholera of canary birds is due to a non-motile bacillus that does not take the polar staining. In cultures it produces an offensive gas. On potato it produces yellowish colonies.

Symptoms.-The bird shows great weakness, ruffled feathers, diarrhea, loss of appetite and sits on the perch much of the time. The disease runs a rapid course.

Chickens and pigeons inoculated with pure cultures die.

\section{TYPHOID OF CANARY BIRDS}

\section{(Septic Fever of Cage Birds)}

Septic fever of cage birds is due to the B. paratyphosis B. The organism is short and plump with rounded ends. It is actively motile and Gram negative. Inoculations with pure cultures kill canary birds, mice, guinea pigs and rabbits.

Symptoms. - At first the bird is noted to be dull and sits on the perch with ruffled feathers. Later the wings droop, there is difficult breathing, debility and diarrhea. There is a loss of appetite and the eyes are closed much of the time while sitting on the perch. The breathing may be as high as 150 
times a minute. The bird may peep hoarsely. The course is very rapid and the bird usually dies.

Postmortem Findings.-When examined after death there is observed an acute gastro-intestinal catarrh, and a fibrinous inflammation of the serous membranes of the abdominal cavity. There is cloudy swelling of the liver and kidneys. The spleen is enlarged, at times, five times its normal size. There is congestion with enlargement of the liver and kidneys. Smears from the heart blood show great numbers of short, thick, rods in pure culture.

Treatment.-Place in the drinking water of the sick bird 5 grains sulphate of iron per pint of water. Separate sick birds from well ones. Disinfect the cages in which the birds sick of the disease have been kept.

\section{INFLAMMATION OF THE JOINTS OF GEESE AND DUCKS (Osteo-Arthritis)}

Inflammation of the joints of geese and ducks may be cansed by the common pus producing germ the Staphylococcus pyogenes aurcus. The organisms are observed in large numbers in the pus of the affected joints. The affection consists of a serous or sero-firinous inflammation or arthritis. Hemorrhagic inflammation of the bone marrow may be present. There may accompany this disease an intestinal catarrh.

Symptoms.-Young geese and ducks are susceptible. As many as 90 per cent of the flock may become affected. The disease manifests itself in two forms, either an acute attack with rapidly fatal results, or a chronic form terminating in death or recovery after a long time.

In the acute type the young geese and ducks have a loss of appetite, are dull and lame in one or both legs. The joints of the leg are swollen, hot, painful and fluctuating. Usually only a few joints are affected and attacks by preference the hock. toes and wing joints. The affected wing droops. There may be a slight mucous catarrh of the conjunctiva. Birds with acute symptoms die in from two to four days.

In the chronic form the inflammation of the joints is the most prominent symptom. There is slight diarrhea and a partial loss of appetite. Emaciation follows. The bird may die in about fourteen days or the bird may improve and gradually recover. Painless swellings of the joints may last for several weeks. Growing birds are stunted in their growth and are not suitable for fattening.

Treatment.-Local counter irritants as pure iodine may be tried or two drams of powdered cantharades rubbed in one ounce of lard or vaseline and applied twice with an interval of ten days. Follow the blister in twenty-four hours with pure 
lard. Disinfection of the premises as described under fowl cholera is indicated.

\section{SPIROCHETOSIS}

This is a blood disease or septicaemia of fowls due to a spiral-like miscroscopic organism that is carried from bird to

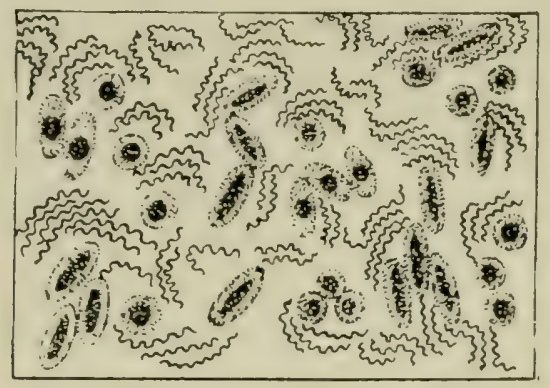

IiIg. 68. SPIRocheta Gallinarum.

This drawing made from a blood smear shows red blood cells, oval with oval nuclei; thrombocytes and leucocytes, round; and the corkscrew-like spirochetcs.

bird by the chicken tick illustrated in Fig. 38. Fig. 68 shows a drawing of the germ among the blood cells. It is the Spirocheta gallinarum; the slide from which this drawing was made was kindly sent to the author by Dr. Belfour, of Khar-

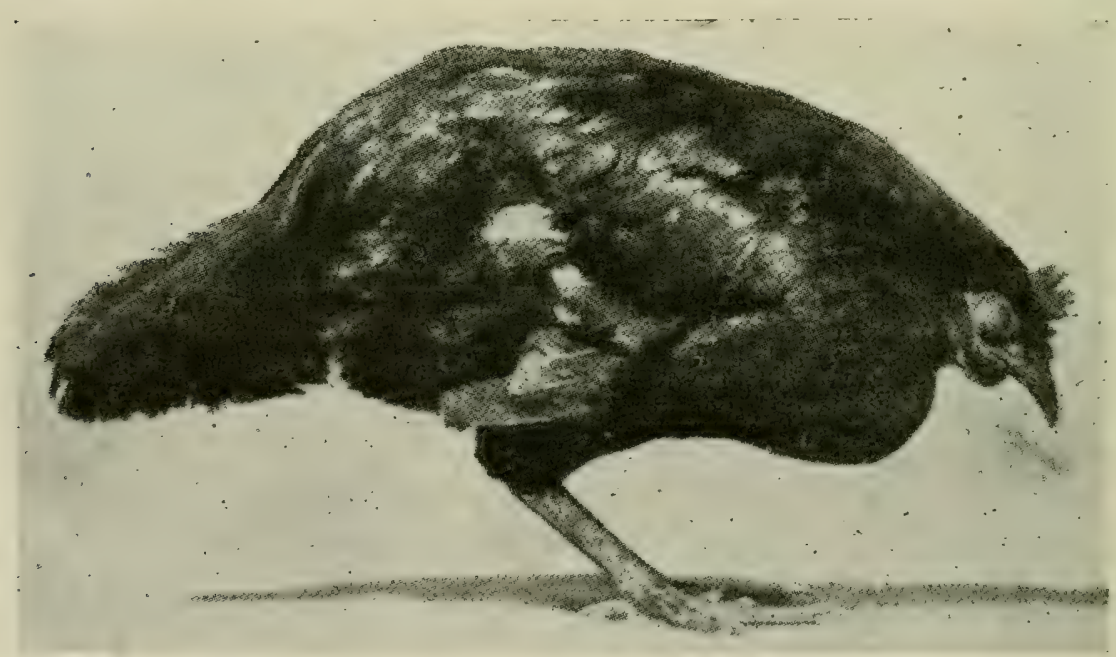

Fig. 69. A Fowr. With Acute Spirochetosis. Photograph by Belfour.

toum, Soudan, Africa. This organism may infect chicliens, geese, larks and other birds.

The disease was first recognized in Brazil: it is found in Africa and Europe. i disease occurs in the southern part of 
the United States, where the chicken tick is abundant, that presents symptoms similar to those of spirochetosis; so far as the author knows, no definite work has been done to determine the true cause of it.

Spirochetosis is most common among chickens, but it also infects geese, ducks, pigeons and sparrows.

Symptoms. - The onset of the disease may be so sudden that birds apparently healthy at night are found dead under the perch pole in the morning. There is noted dullness, ruffled feathers, loss of appetite, rapid emaciation, stands in corner or on roost with head and tail down, diarrhea, comb pale, coma and death. Fig. 69 shows a photograph of a fowl with typical symptoms.

Another form of septicaemia in chickens is caused by a comma-shaped germ-the Spirillum Metchnikorii or Vibrio Metchnikovii.

The symptoms are similar to those of fowl cholera, except that there is no, or at most but slight, elevation of temperature. Diarrhea is constantly present. Inflammation of the bowel, enlarged liver and spleen. The liver shows fatty degeneration, cloudy swelling and areas of focal necrosis. In chronic cases the liver and spleen may be smaller than normal.

\section{INFECTIOUS CONSTITUTIONAL DISEASES}

\section{Tuberculosis}

This is a discase of great importance to the poultryman, not only on account of its destructfulness to his flock, but also on account of its relation to the health of himself and family; for while fowls are not very likely to contract tuberculosis from domestic animals or from man, yet fowls that have the disease are a serious menace to the other animals on the farm as well as to the poultryman and his family.

Canse.-The Bacillus tuberculosis, which was discovered by Koch in 1882, is the cause of this disease. There are four principal types of this organism. The one most commonly infecting man is designated as the human type. The one peculiar to cattle is designated as the bovine type and this is the type most often affecting hogs. The one peculiar to fowls is designated as the avian type. There is still another variety which affects fish and other cold blooded animals and this has been designated the picean type.

While there are some differences in the shape of the organism grown for a considerable time in the various animals and some differences, biochemically, when grown in artificial media after isolation, yet the type peculiar to any of the warm blooded animals will grow in any of the other warm blooded animals, that is, the types are interchangeable, which means that the bovine type may cause tuberculosis in man and the human type may cause tuberculosis in birds, etc. Most authors consider that while the chicken has considerable resistance to the human type, it will and does be- 
come infected by this type. The avian type is more easily isolated from the disease tissues and grown on artificial media. The avian bacillus is somewhat shorter and stains more evenly than those of the mammalian type. The guinea pig is very susceptible to the mammalian types but inoculations with the avian type may result in emaciation with no visible lesions, or in slight lesions with a non-progressive character.

It has been found that a large percentage of hogs fed swill from houses where tuberculous persons have expectorated into it, become tuberculous, and when slaughtered, there is a considerable loss from condemnation of those badly affected (Busman).

Tuberculosis among chickens is rare in some portions of the

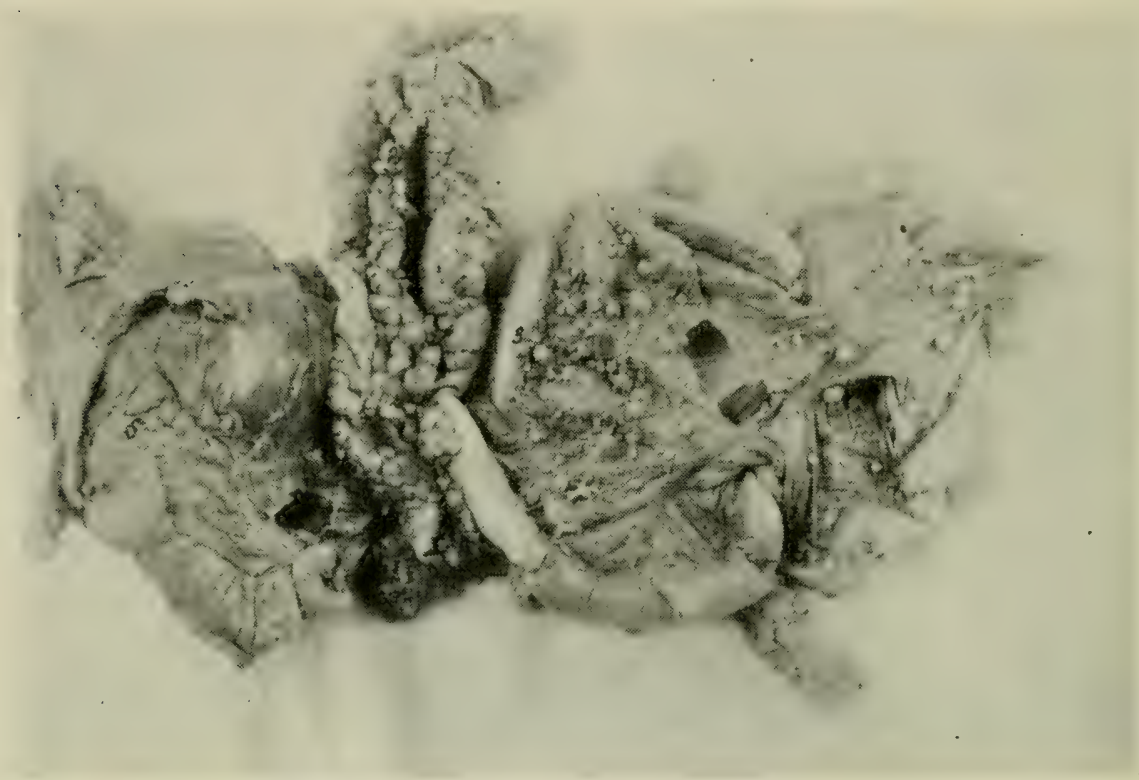

Fig. 70. Tuberculosis of a Fowl.

1 , tubercles of the duodenal loop; 2 , of the pancreas; 3 , of the free portion of the small intestine; 4 , of the mesentery; 5 , of the gizzard.

United States, and is very common in others. Although it is widespread throughout the United States and Canada, it was first reported in this country in 1900 and received but slight attention until 1903. Tuberculosis also occurs in turkeys, pigeons, and pheasants, and two cases of wild geese were reported at the Ontario Agricultural College.

From an economic standpoint it may be said that the loss from this disease is on the increase. The disease being of a slow going chronic type of a disease contributes to the unconcern of the owner of the infected flock. Deaths will not occur till the flock has extensively infected and when they occur, are so scattered in point of time that they do not cause the same concern on the part of the owner as would be occasioned by the same number occurring in a short time. It is not till the bird is in advanced stages and the disease is quite generalised or extensive that the bird dies. It usually dies in a state of emaciation.

Tuberculosis has been produced in sparrows experimentally in 
one test 12 sparrows were fed chopped tuberculosis hen liver mixed with feed and all contracted the disease.

Mode of Spread.-In the progress of tuberculosis of chickens, at times, there is noted a diarrhea. In these cases there are tuberculous ulcers of the mucous lining of the intestinal tract and the spread is very rapid through the flock, as birds are continually picking feed from the ground and floors where contamination is sure to have taken place.

If scraps be fed to which tuberculous sputum has found its way or if birds are allowed to devour parts of an animal dead of the disease, there is a liability of their contracting tuberculosis.

They may also contract the disease by the introduction of a tuberculous bird into a flock. Tuberculosis among wild birds has been mentioned as a possible fact, and has been known in wild birds in captivity. There is also danger of spread of the disease from eggs from a tuberculous hen where the eggs are purchased for hatching-prenatal infection.

There is also a possibility that birds, by following tuberculous cattle, may become infected, as do hogs. It has been argued that the temperature of the bird is so high, $106^{\circ}$ to $108^{\circ} \mathrm{F}$. with an average of $107^{\circ} \mathrm{F}$., that it furnished an unfavorable field for the human and bovine types of germs, which thrive at temperatures close to $98^{\circ}$ to $101^{\circ} \mathrm{F}$. though hogs carry a temperature between this and the temperature of fowls. It has, however, been observed that these germs soon adjust themselves to such changes in tempera. ture and to a certain degree to differences in food.

One case, a hen, was sent to the laboratory with the history of having had access to the sputum of a person afflicted with tuberculosis. Upon autopsy small pearl-like bodies were observed throughout the liver, in the lung substance, and over the serous lining covering the intestines and abdominal cavity. A microscopic examination of the lesions revealed the bacillus of tuberculosis. It more closely resembled the human than the avian type.

Symptoms.-There are no well marked initial symptoms or signs of the disease. As the disease progresses it makes itself manifest by general symptoms such as a loss of weight with gradual emaciation, the bird becomes weak and moves about very little, the disinclination to move about may be due to general weakness or to an affection of the joint by tuberculosis. The eyes appear bright and the appetite remains good. The skin and visible mucous membranes are pale, and the feathers appear ruffled. From these general symptoms it is impossible to make a positive diagnosis during life. The symptoms observable are common to many diseases, especially in the more early stages when there are no positive external symptoms by which it may be recognized. The comb becomes pale and the bird appears sleepy and remains apart from the balance of the flock. If the joints become affected there will be lameness, in case the affection is in the legs, the swollen joints, and often in affection of the skin and visible mucous membranes there is ulcerations or sores. This latter condition has been observed especially in parrots. These skin lesions are made up) 
of a cheesy material, a caseation necrosis, which is covered by a thick, rather hard crust, whitish in color. At times these crusts become rather horny in nature.

The red blood cells in tuberculosis of fowls may be greatly reduced, as low as 1,000,000, and the hemaglobin as low as thirty-five per cent. The white blood cells are slightly increased in numbers.

Tubcrulin Test.-Both subcutaneous and eye tests on birds with tuberculin, made from avian and from mammalian cultures of the tubercle bacilli, have been without success. Intradermal tests of tuberculin made from the avian type of tubercle bacillus has proven satisfactory. Only one to three drops are injected into the skin in the wattles with a very fine needle. A doughy swelling will be noted in 36 to 72 hours after injection if tuberculosis is present in the bird. Of 600 tests 97.77 per cent were accurate.

Postmortem Findings.-Owing to the fact that most birds are infected with tuberculosis through contaminated food, we find most of the lesions in the abdominal organs. Of these the liver is the most often diseased. Next in frequency may be named the spleen, peritoneum or lining of the abdominal cavity, ovaries, gizzard, intestines, lungs, kidneys, and bones.

As indicated above, the areas may appear as pearly, grayishwhite nodules varying in size from a pin head to a pea, or even larger. In these larger nodules there will be noted a cheesy mass which, as the lesions become older, seldom if ever become impregnated with lime salts so that the tubercular lesions of fowls do not cut "gritty" as in mammals. Usually the diseased organs are enlarged.

Upon opening the intestines of a tuberculous bird there may be noted ulcers, usually small in size, and a thickening of the intestinal wall. Small tubercles may be observed in the lung tissue and adjacent structures.

Like in mammals, the bones may become tuberculous, there is noted swelling, tubercles, and caseation.

Treatment.-Treatment of the affected bird is not to be thought of. As shown above, the germs of the disease are so often spread through the droppings that the only sure means of eradicating the disease from a flock is to kill all the birds in the flock and if possible move the hen house to a new location and provide new runs. The hen house should be thoroughly disinfected, in fact, it would be better to use for some other purpose and build a new house. The building may be disinfected with a five per cent solution of carbolic acid or the same per cent of a standardized coal tar disinfectant dip).

The tubercle bacillus is resistant to external influences. A contaminated poultry yard may remain infected for a long time, many months and possibly years. The action of disinfectants on the tubercle bacillus is slow. Direct sunlight on the surface of infected material kills the bacillus in a few hours. 
Birds from an infected flock should not be sold for breeders, and the birds from such a flock that are killed for food should be inspected by a competent veterinarian, so that none may be used for food purposes that are diseased to such an extent as to render the food unfit for use.

All birds in a flock infected with tuberculosis that die should be burned to prevent further spread of the disease from that source. All droppings and cleanings from the hen house and runs should be disinfected with calcium chloride, a five per cent solution of carbolic acid or other reliable disinfectant before spreading on the fields.

\section{INOCULATION EXPERIMENTS IN BIRDS WITH MAMMALIAN STRAINS}

Auclair observed that pigeons injected intraperitoneally with pure cultures of human tubercle bacilli, died after one to three and a half months, without showing any signs of tuberculous lesions. In a second series, pigeons were infected in a similar manner with tubercle bacilli from a similar source. At the sixth, seventh, and fourteenth days afterward, the pigeons were killed and the livers, lungs, and blood were injected into guinea pigs. A few of the pigs died without any evidence of tuberculosis. Only two died of local tuberculosis.

From this Auclair concludes: First, that pigeons infected with human tubercle bacilli die without any observable tuberculous changes; second, that tubercle bacilli may retain their vitality and virulence in the body of the pigeon, for at least fourteen days; third, that the tubercle bacilli localize themselves in the pigeon by preference in the liver and in the lungs, but not; so far as could be shown, in the blood; and fourth, that the tubercle bacilli passed through the pigeon gave rise to a slowly developing tuberculosis.

Van Es and Schalk after experimenting with many chickens came to the following conclusions:

A considerable number of birds into which mammalian tubercle bacilli are introduced, either by ingestion or by inoculation, die in an extremely emaciated state.

As a result of the incorporation of such bacilli into the bodies of birds, the latter may retain the oiganisms for a long period of time with their pathogenic characteristics preserved.

In consequence it is well within the range of possibilities that birds may serve as intermediary carriers and transmitters of mammalian tuberculosis.

\section{PSEUDO (FALSE) TUBERCULOSIS}

Pseudo or false tuberculosis manifests symptoms similar to true tuberculosis. Upon autopsy the lungs may be noted to be thoroughly studded with nodules varying in size up to that of a pea. The nodules are firm to the touch and when sectioned are found to be dense and appear homogeneous.

The lesions may be mistaken for tuberculosis, parasitic cysts, nodular tape worm disease, aspergillosis or coccidiosis. 


\section{ASPERGILLOSIS}

\section{(Pneumomycosis or Brooder Disease)}

Aspergillosis is a pneumonia caused by a fungus, the Aspergillus funigatus. The organism is similar to the common green mold. The disease is characterised by the formation of an exudate, usually of a moldy appearance, on the mucous membrane of the air passages, principally the air sacs of the abdominal cavity.

Symptoms. - The symptoms or signs of the disease vary in different cases. Like in tuberculosis, evidence of the disease is not manifested by symptoms, till it has progressed to a considerable extent. The affection of the air sacs does not cause visible symptoms. As the disease progresses the bird becomes dull, sluggish, staying apart from the balance of the flock, may sit on the perch poles much of the time, or stand in a corner. Later the bird is very weak and is finally unable to stand. There is a loss of appetite, the feathers have an unkemped appearance, the wings are drooping, and the eyes partially closed. The respiration is accelerated and there is a rattling of mucus in the trachea and bronchi. Fever is present, and there is usually thirst. The affected bird dies after a prolonged illness.

All species of birds are apparently susceptible to infection. Predisposing causes appear to be those birds kept under conditions in which they are exposed to moldy organic matter. These include especially chickens and pigeons as well as young brooder chicks and cage birds, especially those of zoological gardens are especially susceptible. The disease has been observed in the ostrich.

Postmortem Findings.-The disease is principally confined to the respiratory system which includes the trachea, bronchi, lungs, and their air sacs. Mold may develop at any point on the surface of the respiratory mucous membrane. The membrane affected is thicker and whitish or yellow nodules, varying in size up to a pea, may be noted in the affected parts; which may be the trachea, bronchi, lungs, and the various air sacs. The fungus may grow upon the surface of the mucous membrane forming, at first, a felt-like, whitish mass which takes on color according to the species of the fungus as it fruits or forms spores. This membraneous material, to the naked eye, resembles a fribopurulent exudate. The obstruction of the air sacs causes the difficult breathing and asphyxiation.

Inflammation is evident in the diseased areas. Sections through these areas of disease show the mycelia or thread-like branches of the mold, and the characteristic spores. Focal necrosis, preceded by cloudy swelling, is noted in the kidneys and other vital organs. A secondary invasion of pus producing organisms may take place and on autopsy abscesses may be observed in the liver, kidneys, 
spleen, and other organs. The bronchioes may be completely plugged with the mycelia.

Treatment.-Successful treatment is very difficult. Place the affected bird in a closed box and smoke them with tar being careful not to suffocate them. Efforts should be made to eradicate the disease from the premises by thorough cleaning and disinfection as described for other contagious diseases as fowl cholera. 



\section{CHAPTER VII}

\section{CONSTITUTIONAL DISEASES}

Under the head of constitutional diseases there will be discussed a condition or disease little understood and called "going light" or asthenia, and gout of fowls.

There is much doubt if the condition called asthenia should be classed as a disease; certainly this term as usually applied refers merely to a symptom of a disease, often tuberculosis or enteritis, or a condition in which there is a progressive loss in the weight of the bird.

\section{ASTHENIA \\ (Going Light)}

Those who look upon going light as a specific disease consider it as one that affects chickens and pigeons. It may affect young or old birds. It is called going light because the bird becomes gradually lighter until emaciated. It is a disease that is found in all parts of the United States. A germ called the Bacterium asthenia has been isolated by one investigator from the intestines of sick birds. It resembles the Bacillus coli communis always present in the intestinal tract of chickens.

The affected birds have a good appetite; in fact, at times a ravenous one. The loss of flesh is continuous for a few weeks, when the bird dies.

In eleven cases of going light examined by the Pathological laboratory of the United States Bureau of Animal Industry three were found to be infected by the Bacillus enteritidis. This germ is dangerous to man. It affects cattle and has resulted fatally to those persons eating infected meat.

Postmortem Findings.-Usually on autopsy all organs appear normal so far as gross appearance goes, but extreme emaciation as described above is noted.

Blood Study.-Two outbreaks were investigated by the author. One in a flock of Rhode Island Reds, in which flock there were about two hundred birds which should have weighed about two pounds each. The disease affected gradually increasing numbers. The feed consisted of grain, insects picked up from the fields, and plenty of green grass. As it was irrigation time, the birds had access to the irrigation ditches. The hen house and yard were kept clean. It was advised to change the run and continue giving a variety of good green feed and grain with a good supply of water. The disease finally disappeared from the flock. All efforts at the laboratory to isolate any germ which might have been the cause of the disease were unsuccessful.

The second flock were hatched from eggs that had been produced 
by birds in which roup had appeared the preceding winter. Five birds about four months old, with the following history, were sent to the laboratory for study: The birds had good hygienic surroundings, were moved from place to place, fresh water kept before them at all times, good quality of feed, plenty of green feed, under which conditions the birds continued to lose flesh, became emaciated and many died. The appetite remained normal, in fact in some, ravenous.

Chick No. 3.-Hemoglobin, 65 per cent; red blood cells, 2,920,000; white blood cells, 28,000. Differential count: polymorphonuclear neutrophiles, 39 per cent; eosinophiles, 30 per cent; lymphocytes, 29 per cent; mast cells, 2 per cent.

Chick No. 4.-Hemoglobin, 65 per cent; red blood cells, 2,600,000; white blood cells, 14,000. Differential count: eosiniphiles, 31 per cent; mononuclears, 8 per cent; lymphocytes, 60 per cent; basophiles, 1 per cent.

Chick No. 5.-Hemaglobin, 75 per cent; white blood cells, 34,000; red blood cells, 3,000,000. Differential count: polymorphonuclear neutrophiles, 4 per cent; eosinophiles, 50 per cent; basophiles, 3 per cent; mononuclears, 4 per cent; lymphocytes, 39 per cent.

Chick No. 6.-This bird was about four months old, stunted in growth, "going light," and had contracted roup from another bird. The blood study showed the following: hemaglobin, 60 per cent; white blood cells, 16,000; red blood cells, 3,600,000; thrombocytes, 436,000. Differential count: eosinophiles, 3 per cent; mononuclears, 4 per cent; lymphocytes, 89 per cent; mast cells, 4 per cent.

All efforts to isolate germs from the liver, spleen, kidneys, and heart blood were unsuccessful.

Treatment.-Best results, in flocks of young birds, have been obtained by changing their runs to pasture runs, and giving one tablespoonful of epsom salts to each 12 birds. Dissolve the epsom salts in water and give as drinking water or mix with mash. Repeat twice weelily. The flock must have ample shade as hot stun's rays with a shortage of water favors such conditions. Water must be kept before the birds at all times as more than 65 per cent of the flesh is water.

The birds should receive one-fourth grain doses of ferrous sulphate once a day in soft feed. The prognosis or final results is not very encouraging.

With our present knowledge we look to sanitary surroundings for the control of this condition, clean coops and yard, soorl feecl, water. A tablespoonful of powdered nux vomica to each dozen hens may be given with advantage once or twice a day in soft feed.

In going light in addition to a cathartic of epsom salts the following given once a day in mash has yielded good results: calcium carbonate, 120 grains; magnesium sulpluate, 240 grains; soflium bicarbonate, 360 grains: sodium chloride, 60 grains; sodium sulphate, 120 grains. This is a dose for 120 birds. 


\section{GOUT OF FOWLS}

Gout of the joints is an inflammation of the fibrous and ligamentous parts of the joints. It is accompanied by an excess of uric acid and deposits of urates of sodium in and around the joints.

Gout may also attack the internal organs and cause deposits of sodium urates in them. This type is called visceral gout.

Gout attacks man and some of the lower animals such as fowls and dogs.

The histology of urate deposits, both experimental and gouty, have been studied. These results all inclicate that uric acid and urates excite slight inflammatory reactions, cause a slight local necrosis, and seem to act as a week tissue poison. However, they may be deposited without causing necrosis.

That urates may cause necrosis in the tissues has been definitely established, and this may lead to connective tissue formation and contraction.

Gout is more common in birds force-fed and given rich nitrogenous diet and in old birds where the eliminating action of the kidneys is more or less impaired. Birds normally excrete large quantities of uric acid, which appears on the outer surface of the droppings as a whitish liquid or semisolid.

It would appear that the preponderance in the blood of substances which are of acid reaction favors the precipitation of uric acid.

Uric acid is converted into sodium urate by the salts in the blood in two ways: First, by breaking down of the nucleoalbumins of the tissue and especially perhaps of those contained within the leucocytes; and second, from similar substances contained in the food.

Excess of uric salts in the blood may be produced either by increased formation of the substances or by diminished excretion of them or by failure to utilize and destroy them in the metabolic processes in the tissues.

Typical gout has been produced in fowls by feeding them for several months exclusively on horse flesh. Gout is caued by chronic poisoning by oxalic acid, carbolic acid, corrosive sublimate, aloin and acetone.

If the ureters of the bird be ligated, gout may occur; hence, it is argued that perverted function of the kidneys or ureters may be a contributing factor in the production of gout. Lack of exercise predisposes to gouty conditions.

In visceral gout the chalky or mortar-like deposits may be observed in the air sacs, pericardium, peritoneum, heart or liver. 
In the articular form the joints of the feet are more often affected. Birds suffer especially in the tarsal, metatarsal and phalangeal joints and at times in the joints of the wings.

In birds there is, in the early stages, extreme tenderness as manifested by the bird standing on one limb or resting on the breast and moping around, staying away from the balance of the flock. The affected limb causes the bird to be lame in that leg. At first the affected limb is swollen, soft, hot and tender

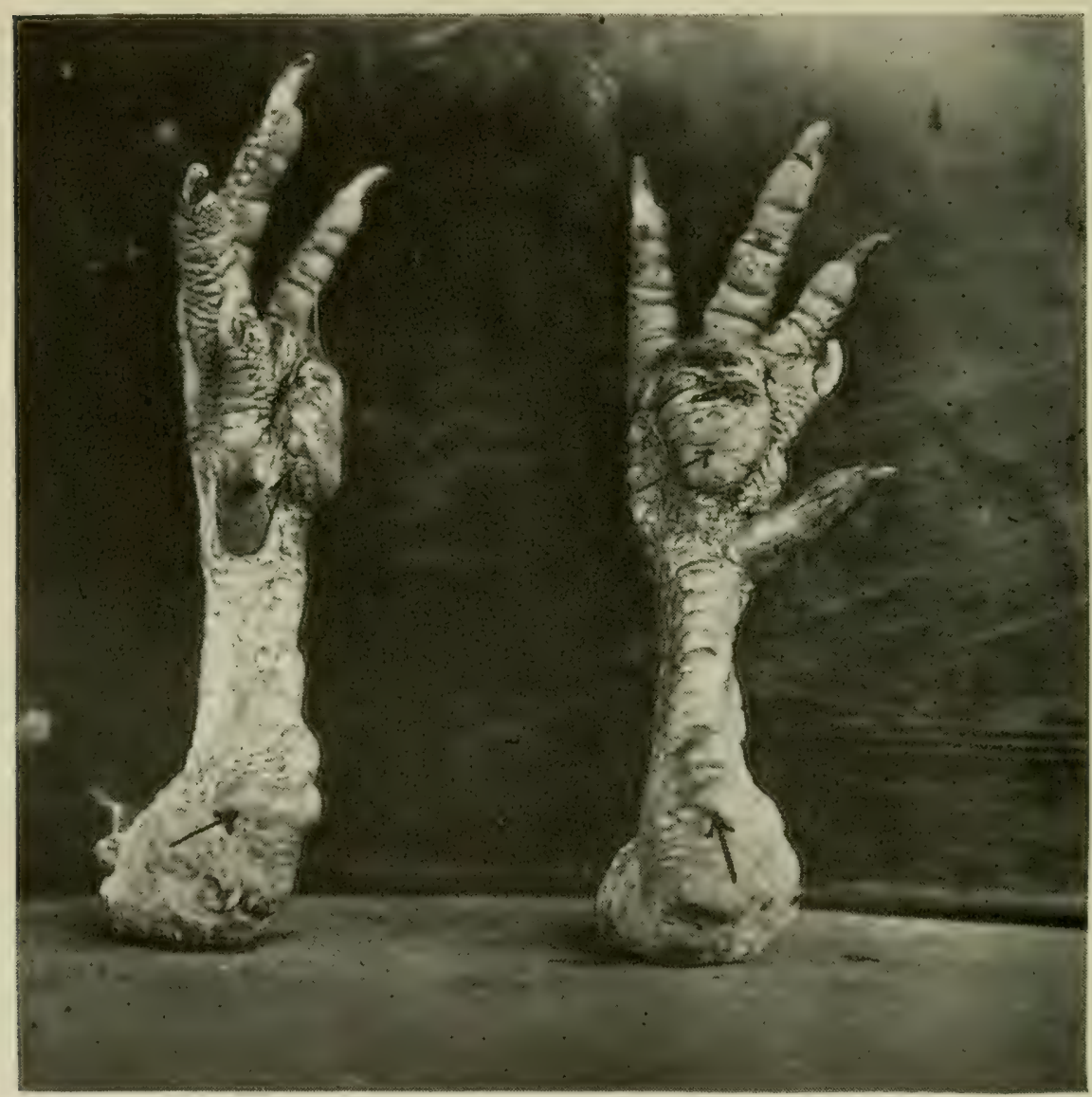

Fig. 71. Gout df the Feet and Shanks of a Five-Year-Old Barred Plymouth Rock Capon.

Gout lesions indicated by the arrows.

and may pit on pressure ; later the parts may become extremely hard. The enlargements may attain the size of an English walnut and the parts may undergo necrosis. When necrosis occurs there may he discharged a granular yellowish-gray material similar to talc. The bones may become deflected from their normal direction as a result of the process. The diagnos- 
tic symptom is the finding of deposits of sodium urate in the lesion.

This disease has been observed in capons five years of age. The capons were Barred Plymouth Rocks and Single Comb Rhode Island Reds. The birds were allowed to run at large and did not wander far from the barn, where there was, at times, an abundance of grain and especially more or less sprouting grain.

The birds with gout were quite lame, sitting around, much of the time, in comfortable places and only moving when necessary. They were often noted to stand alternately on one foot or the other apparently to ease the pain. The swellings were irregular in shape and hard to the touch. The nodules measured three-quarters of an inch in the largest diameter. The mobility of these joints were limited. Fig. 71 shows the feet and shanks of one of these birds.

In these birds the feather coat was rather rough; the comb and wattles as well as the face were pale.

When gout assumes a chronic type, the ultimate outcome may be considered unfavorable. When ulcers are present it is observed that they do not entirely heal. The birds finally become unable to move about and care for themselves, become emaciated, exhausted and die. 



\section{CHAPTER VIII}

\section{DISEASES OF THE RESPIRATORY PASSAGES}

In the fall, winter, and spring, these diseases are a source of trouble to the poultry raiser, unless strict sanitation is observed.

\section{OBSTRUCTION OF THE TRACHEA}

Obstruction of the trachea or "windpipe" quite frequently occurs in both chicks and adult fowls. Obstruction due to the gape worms has been discussed under the chapter on internal parasites. These obstructions quite often occur in chicks, due to the lodgment of a small piece of cracked corn or other

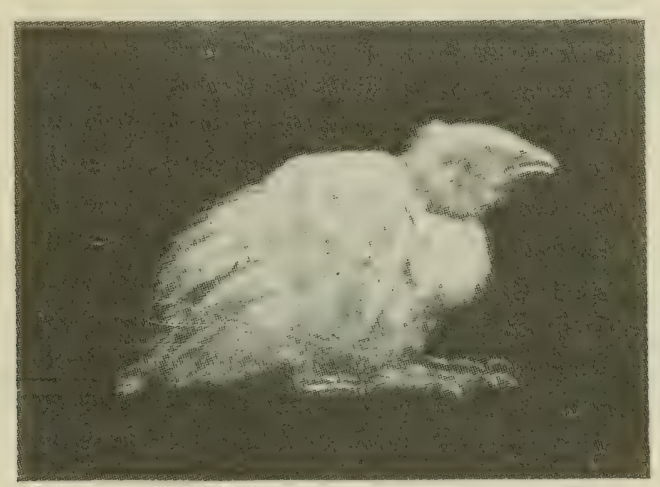

Fig. 72. Obstruction of the Trachea. False GAPES.

Showing depression, droopy wings, inability to stand, sleepy, mouth open.

small seed and is known as False Gapes as the word gapes as used is taken to mean that condition cat1sed by gape worms the Syngamus trachealis. Fig. 72 illustrates a case of suspected gapes. This bird was afflicted with false gapes and would extend its head high into the air, gasping for breath as one whose trachea is obstructed by gapeworms; it was weak and unable to stand square upon its feet. It was destroyed, after photographing, for examination. A piece of corn grain was found in the trachea, surrounded by an accumulation of mucus due to the irritation its presence in the trachea had caused. The foreign body and the accumulated mucus were obstructing the passage of air to the lungs; hence, the asphyxiation. In some cases a whole grain of corn may become lodged in the trachea of adult fowls. Fig. 73 illustrates 
a case of this kind. Number 2 shows the larnyx or cartilaginous box, laid open. This larynx is located at the upper end of the trachea. Number 3 is the trachea. Note that the trachea is made up of cartilaginous rings which are held together with connective tissue or fibrous structure. Number 1 is the whole corn grain which has become lodged in the upper larynx. There was a rattling sound in the trachea during life of the hen. The hen was uncomfortable and there was a slight gasping for air from the start but, at first, not very aggravated till the presence of the grain of corn lying against the mucous membrane caused an irritation and a pouring out of mucus. As a result of this irritation and pouring out of mucus there was a gradual closing of the trachea or air passage. The retching or gasping for air by extending the head far into the air was much aggravated for some time

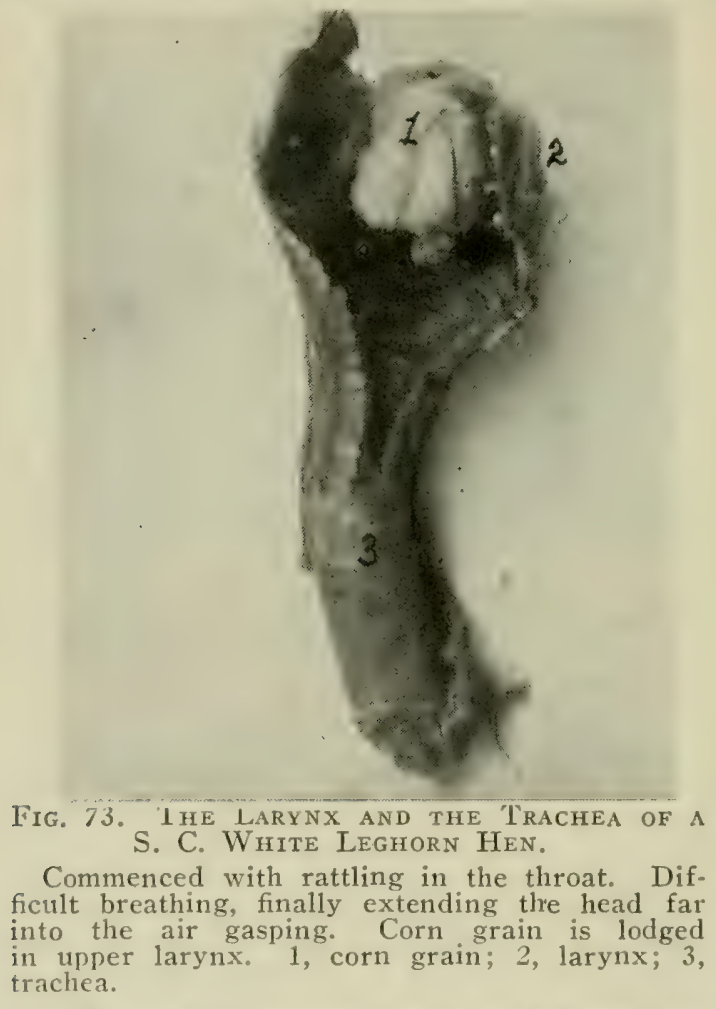

before death. If the obstruction had been observed soon enough it could have been easily removed by aid of a long pair of forceps.

\section{CATARRH "COLDS"}

Catarth is a discharge of fluid from the nose. This term is usually applied to simple inflammation of the mucus membrane of the nasal and respiratory passages, but is now extended to all mucous membranes, as of the stomach, oviduct, etc. 
Cause.--Sudden changes in the weather, cold, damp weather, roosting in drafts, and chilling by getting wet in cold rains are some of the factors in the production of catarrh among birds. Such affections are more or less contagious, but bad sanitation plays an important part in their spread. Weak stock and poorly nourished birds are predisposing to this contagion.

Symptoms.-The appetite may be somewhat diminished. The bird sneezes, throws its head and may expel some nucus The discharge at first is watery and later becomes more or less thick, muco-purulent. The eyes may show more or less inflammation, conjunctivitis, and the eyelid may become stuck together. The characteristic offensive odor of roup is absent.

Treatment.-The same treatment as outlined under nasal type roup has given uniform results. If the nostrils are syringed out with a warm solution of common baking soda, using one-half teaspoonful to a teacup of warm water; then injecting through the nose a solution of 30 drops each of oil of eucalyptus and oil of thyme, and ten grains crystals of menthol, in two ounces of oil, this bird will rapidly recover.

\section{ASTHMA}

True asthma is a chronic disease which may be inherited and is manifested by great difficulty in breathing, recurring at intervals, and accompanied with wheezing sound and a sense of constriction of the chest, and cough. There is a condition in fowls which has been described under the heading of asthma.

Cause.-Asthma in fowls and canaries may be due to a fungus, aspergillus. Hence aspergillosis of the air passages will give similar symptoms or it may be due to other catarrhal conditions of the mucous membranes of this passage.

Symptoms.-Wheezing sounds are noted and in expiration there is a creeking, whistling sound.

Treatment.-Good sanitary surroundings, clean coops, no drafts. Treat local condition of which there is a symptom.

\section{ULCERATIVE PHARYNGITIS}

Pharyngitis is an inflammation of the pharynx or throat. An ulcerative pharyngitis not accompanied by roup and called by some investigators diphtheritis may affect the pharynx. This condition is manifested by patches of coagulated exudate under which there is destruction of the cells of the mucus membrane.

Symptoms.-The bird may remain on the roost much of the time; there is an unkempt appearance of the plunnage; there is 
gradual loss in flesh when the appetite is interfered with, and the bird may find it difficult to swallow. The affected bird is noted, at feeding time, to not eat in a normal manner and may show signs of choking. In eating corn, wheat, and particularly unhulled oats the throat is irritated and swallowing is very difficult. The bird may suddenly stop picking up feed, gives its head a jerk, cough, and may even show signs of choking especially when the inflammatory product in any way interferes with breathing as often the laryngeal surface or even its opening may be affected. The bird may open its mouth and extend its head into the air, the comb, face and wattles may become bluish in color due to choking. The coughing sound is made as the bird throws its head. Standing tucked w1) while on the perch or around the yard, the bird may be noted to occasionally open its mouth, and in fact sitting or standing with its mouth partly open is a diagnostic symptom.

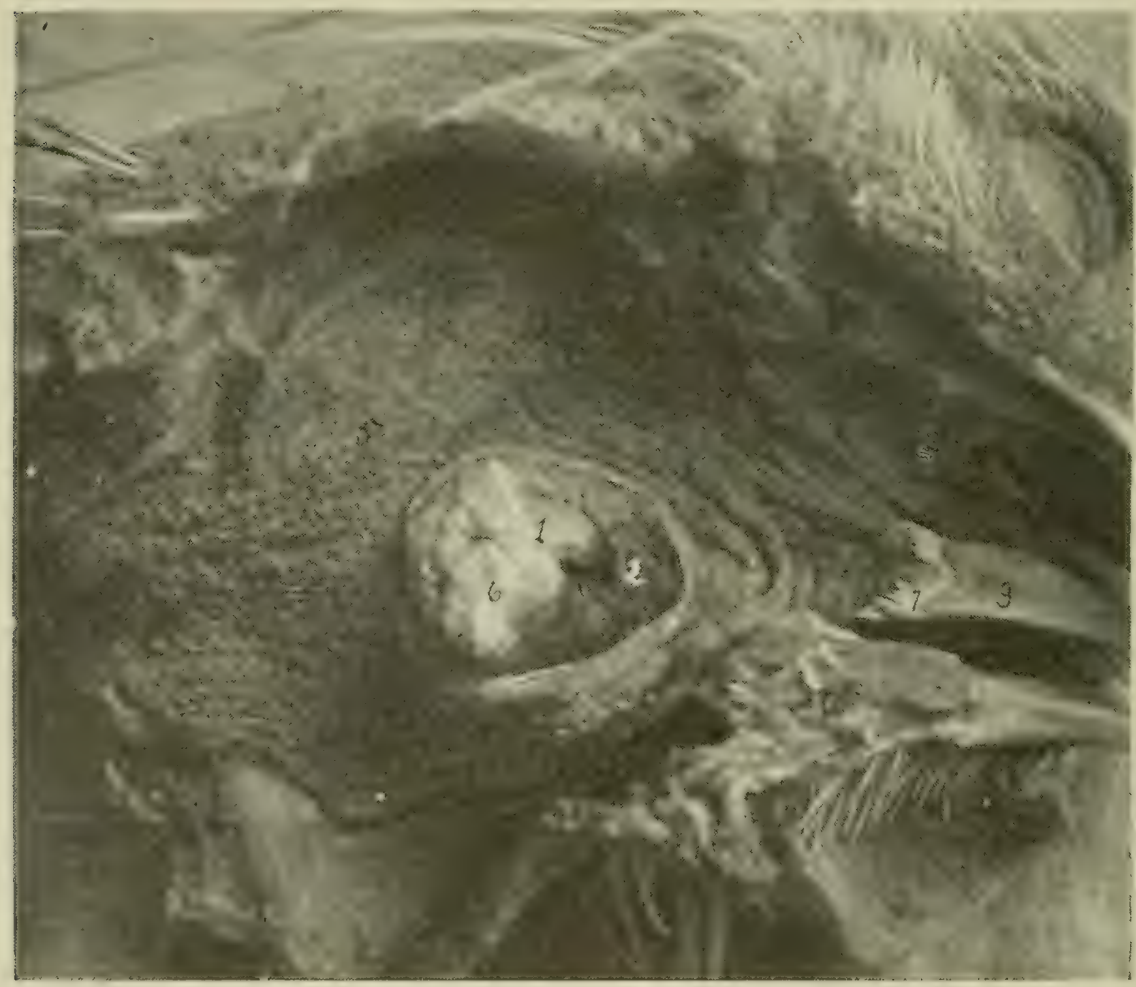

Fig. 74. Ulcerative I'inarygitis: Involving the Opening Into the Larynx, 1, top of larynx; 2, the ulceration with chcese-like mass

When the mouth is opened and the pharynx olserved it will le noted to be covered, to a more or less degree, with yellowish-white uleers varying in size from a wheat grain to as large as one's thumb nail as shown in Fig. 74. 
Microscopic Study.-In an examination, of the section from the diseased area, under a microscope one finds an ulceration of a diphtheric nature. That is, a destruction of the surface cells and a pouring out of a material which coagulates into a cheesy-like material.

Treatment. The parts should be swabbed out with pure iodine or a purple solution of permanganate of potash. Removing the cheese-like material in swabbing. To do this twist a small piece of cotton around the end of a stick, dip this in the solution for swabbing.

\section{BRONCHITIS}

Bronchitis is an inflammation of the lining membrane of the bronchial tubes, always more or less present in cases of pulmonary catarrh, accompanied by cough and rattling in the throat, difficult and uneasiness in breathing.

It has been noted in cases of catarrh of the nasal chambers that the inflammation may extend down and involve the pharynx and trachea, and even $t$ othe bronchi. Sudden changes in weather, dampness and roosting near a crack in the hen house so that a cold wind blows upon them, or, in fact, in any draft, are the principal causes of bronchitis.

Symptoms.-A rattling sound may be heard in the region of the trachea and bronchi-neck and anterior part of the thorax-and constitute mucus rales. This sound is caused by a mixture of air and mucus. The bird may be seen to gasp for air by opening the mouth and extending the head upward. This difficulty in breathing is due to an accumulation of mucus in the air passages which partially closes them, thus preventing the bird from getting enough air into the lungs. The affected bird coughs, and there may be dullness and partial loss of appetite.

The condition may pass off in a few days, may respond to treatment, or may last for several weeks and end in recovery or in death. In the latter case there is marked emaciation; in the former the bird coughs up mucus for a long time, but otherwise appears well.

Treatment.-A tablespoonful of castor oil to which 5 to 10 drops of turpentine have been added may be given, and if catarrh is present, treatment should be as outlined under roup. Give one grain doses of quinine sulphate three times a day. Place the bird in warm, clean, comfortable quarters, free from drafts. Give plenty of clean water and soft feed such as bread or middlings moistened in milk, to which has been added 2 grains of black antimony for each bird. Feed twice daily. 


\section{CONTAGIOUS BRONCHITIS}

There is a bronchitis and tracheitis observed among fowls that is apparently contagious. This disease appears in the same pen each year when young, apparently susceptible birds are placed in the flock in the fall, a certain per cent always develop the disease. Such pens apparently remain permanently infected.

Symptoms.-The affected bird manifests a rattling in the throat and trachea. The bird wheezes, coughs, the comb and face appear dark, and the bird loses flesh. Laying hens cease to lay and cocks cease to fertilize eggs.

Treatment.-The treatment that has given excellent results consist of injecting into the trachea some of the menthol and oil mixture mentioned under roup. To do this one attendant must hold the bird, a second hold the mouth open and the operator forcing the larynx upward with one hand, when the glottis opens, drops, with a medicine dropper, two or three drops of the medicine into the trachea. This treatment should be repeated every two or three days.

\section{CONGESTION OF THE LUNGS}

Congestion of the lungs is an engorgement of the blood ressels of the lungs. Congestion of the lungs may terminate in pnetmonia. Active congestion precedes pneumonia. It has been observed in young birds and in birds during their molting season, when they are poorly clad with feathers and exposed to bad weather.

Young chicks that are allowed to run out in the early morning and become wet with cold dew, and chicks allowed to become wet with cold spring rains and become chilled, are likely to suffer from congestion of the lungs and pneumonia.

A contraction of the blood ressels of the skin and periphery forces an abnormal amount of blood to the internal organs, and congestion is the result. Improper feeding and lack of exercise are also contributing factors. Birds having this ailment will be noted to be sleepy and stupid, and to breathe rapidly. In some cases the breathing is difficult. The comb becomes bluish and the bird may die because it cannot get enough air into the lnugs - asphyxiation.

Upon postmortem examination the lungs will be found engorged with blood.

The pressure of the blood in the engorged blood vessels of the lungs may close the smaller air passages which they surround, or may burst their thin walls and fill the bronchi with blood. In either case rapid asphyxiation occurs. 
Treatment.-Congestion of the lungs runs an exceedingly rapid course, terminating in recovery, pneumonia, or death. Treatment is impractical. The ailment should be prevented by good feeding and adequate protection from cold or wet weather.

\section{INFLAMMATION OF THE LUNGS-PNEUMONIA}

Pneumonia is an inflammation of the air vesicles and their supporting structure of the lungs. There are three chief varieties of pneumonia: 1 , croupous, which is always acute and involves the entire lung structure of the part attacked; 2 , catarrhal or broncho-pneumonia involving primarily the mucous membrane of the bronchi and air vesicles; and 3 , interstitial pneumonia involving the supporting air cell structure.

There are three stages of pneumonia: First, the stage of engorgement or congestion. The affected lungs distended; firmer, of a red color; and crepitates less than a normal lung. Second, red hepatization or consolidation; the affected portion becomes solid, brownish in color, resembling normal liver tissue; the air sacs and bronchi of the affected part are filled with solid exudate. Third, gray hepatization and resolution or purulent inflammation. The affected portion becomes gray by the disorganization of the blood globules, infiltration or absorption takes place; gangrene or calcareous encapsulation may take place.

Bronchitis described above, often terminates in pneumonia, forming the broncho-pneumonia or catarrhal type. It has been observed by the writer that broncho-pnetumonia, following an attack of bronchitis, is the most common form of disease in the fowl. The causes of pneumonia are the same as the causes of colds and bronchitis, except that the exposure is often more severe.

Symptoms.-There is an entire loss of appetite, with thirst and constipation. The bird stands with head drawn in, drooping wings and ruffled feathers; breathing is rapid and painful, and there may or may not be coughing. There is usually a discharge of thick, adhesive mucus from the nostrils; the eyes may be inflamed and water freely. The bird has every appearance of severe illness.

Treatment.-Except in the case of birds of unusual value, treatment is wholly impractical, owing to the amount of care and nursing necessary and because of the doubtful outcome.

If treatment is undertaken, the birds should be warmly housed and the best of rentilation maintained. Spirits of camphor, two drops, and brandy 10 drops, should be given 
hourly in a teaspoonful of warm milk; if the comb becomes dark, add one drop of fluid extract of digitalis to the medication.

Postmortem Findings.-Upon opening the bird that has died from pneumonia, the affected part of the lung will be observed to be dark red, and when cut through the sectioned surface appears like liver in texture. Yellowish colored serum and blood may pour out on the surface.

\section{CONTAGIOUS INFLAMMATION OF THE AIR SACS IN GEESE}

This disease is an inflammation of the air sacs caused by a slender bacillus.

Symptoms.-The bird shows great weakness, staggery gait, great depression, difficulty in rising, kicling at the head appears to be a diagnostic symptom, increased rate of. breathing, snoring sounds and opening the mouth and gasping for air. The affected bird usually dies in about six to eight days. The disease appears to be confined entirely to geese.

Postmortem Findings.-On autopsy the air sacs they are noted to appear yellowish in color, with their inner surface covered with a fibrinous exudate. Similar deposits are observed on the serous surfaces of the liver, spleen, intestines, and peritoneum.

\section{CONTAGIOUS NASAL CATARRH OF FOWLS}

This condition has also been called Coryza arium contagiosa and is manifested by a discharge from the nose, at first thin and watery, later thicker or muco-purulent.

Cause. - This disease can be reproduced by experimental inoculation. It occurs epiornithologically, mostly among young fowls, during damp, cold weather and more often in the fall or spring. It may attack old hens. Here it is of economic importance because of the loss in the egg yield of the laying flock. Many young birds succumb to its attack. A bacillus resembling in some respects the diphtheria bacillus of humans has been found accompanying the disease which Colon and others have termed the bacillus of fowl diphtheria.

Mode of Spread.-The mucus secretions of the head contain the virus. Food contaminated with these discharges are a fruitful source of its spread through the flock. Perhaps the most rapid method of the dissemination of the virus is through the clrinking water as an infected bird dipping its beak into the water will contaminate the whole ressel of water. A susceptible bird drinking from such a vessel will become inoculated and will in all probabilities develop the disease. The disease is also spread through contaminated utensils, man and animals.

Symptoms.-The contagium spread in the manner above described may result in the disease affecting all the members 
of the flock in from two to six weeks. The sick bird stays apart from the balance of the flock and sits around with ruffed feathers and droopy wings. There is a partial loss of appetite, tears may be seen to accumulate in the conjunctiva. The outer nasal passage becomes partially or completely closed and breathing is accomplished with difficulty. The mouth is held open as the bird breathes with difficulty or not at all through the nostrils. The bird shakes its head at the same time sneezing throwing the discharge laden with virus over surrounding objects. The eyes are kept closed and the eyelids may become stuck together so that the bird cannot open its eyes. The infra-orbital sinus or sinus just below the eyes may become filled with secretion and bulge out, much as is often observed in roup. The death rate may run as high as 95 per cent. The nasal secretions remain thin until later stages. It is differentiated from roup by the fact that diphtheric memlsanes never form in the mouth or eye and there are no sores on the head.

Treatmont.- The same treatment is recommended as for roup, namely, the injection of the oily preparation through the nostrils and the use of a solution of permanganate of potash in the drinking water, making the water a red color. All affected birds are immediately removed from the balance of the flock and if thought curable may be placed in clean, comfortable, warm quarters, and treated. Those that are thought certain to die should be killed and these together with the birds that die should be burned.

\section{ROUP, AVIAN DIPHTHERIA, AND CHICKEN POX}

The relationship between the various diseases of the head of fowls has been the subject of much controversy. The conditions are variously called chicken pox, sore head, contagious cpithelioma, avian diphtheria, diphtheritis, canker, colds, contagious catarrh, swolled head, swollcn eye, roup, etc.

The diseases here discussed will be those conditions of the head due to filterable virus or viruses. A filterable virus also called an ultramicroscopic organism is one that is so small that it cannot be seen under the microscope and cannot be grown in risible quantities on ordinary laboratory media and will pass through the fine filters visible germs will not pass.

Experimental inoculations, conducted in this and other research laboratories, tell us that one filterable virus will cause the diseases which have been termed chicken pox, contagious epithelioma, ulcerative sore eye, and canker or avian diphtheria.

\section{CHICKEN POX}

Chicken pox, also commonly called sore head, is a disease of the unfeathered portions of the head and its adjuncts, namely, 
the comb, face and wattles. Chicken pox may affect turkeys, pigeons and fowls. It has also been reported in geese.

Cause.-As stated above the disease is caused by a filterable virus.

Symptoms.-. The disease appears in late summer, fall and early winter among the young susceptible birds. The old bircls that have passed through the disease are immuned. Some have a natural resistance and do not develop the disease. The disease is universally found, and in some seasons is more severe than in others. It is one of the seasonal diseases. The first sign of the disease is a small red pimple on the comb, face or wattles, and must be differentiated from the small red pimples caused by the bites of mosquitoes. The nodules vary in size from a pin-head to the size of a pea, or even larger. Later these nodules ulcerate on the surface and it is this condition that it is commonly called sore head. The disease may be accompanied with roup and with canker of the mouth. It is this point that makes these cliseases hard to separate. It the same time the nasal discharge of roup will not reproduce, experi-

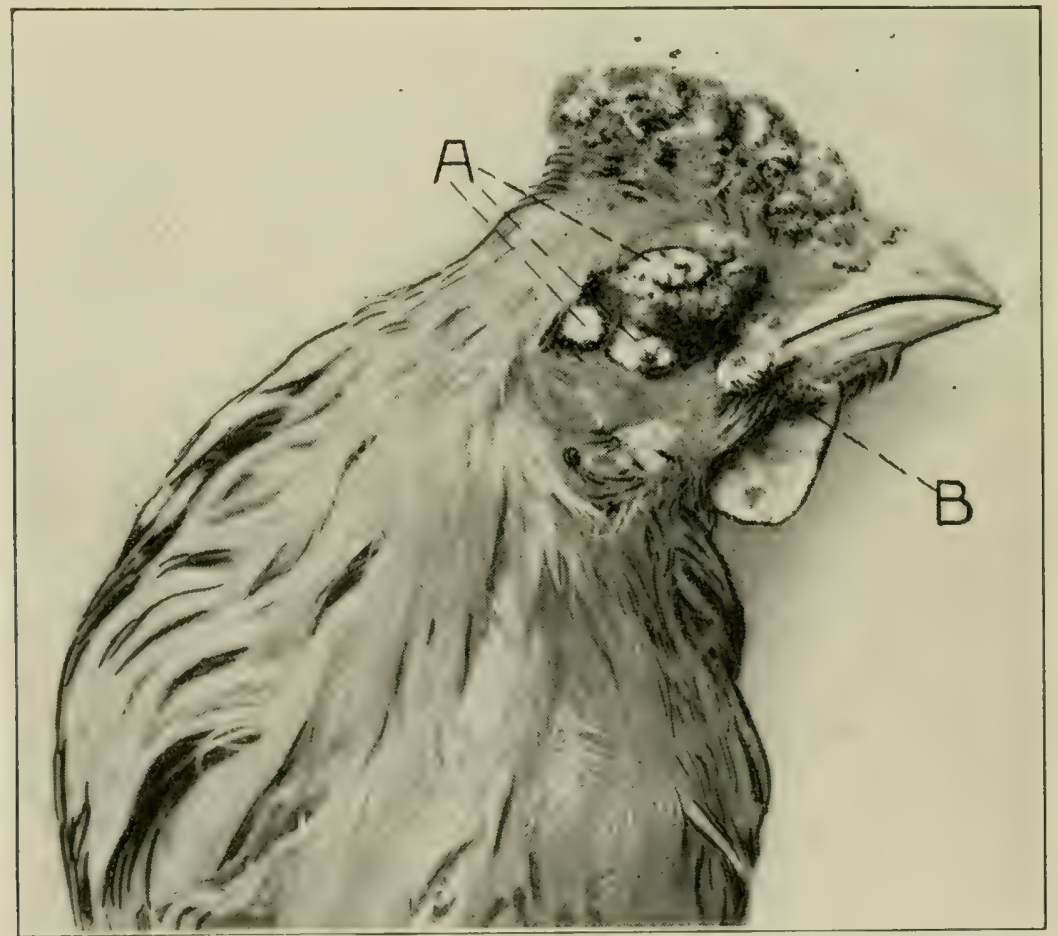

Fĩg. 75. CHicken Pox.

A, the pox or epithelial tumor-like nodule; $B$, a nodule that has ulcerated.

Microscopic Study.-A microscopic study of the nodule shows it to resemble in some respects an epithelioma. 
mentally, either chicken pox, canker, or ulcerative sore eye. Nor will the curettings containing the virus of chicken pox, canker, or sore eye cause roup. It thus appears as though there may be two different filterable viruses at work here though not being able to isolate such viruses it is to remain an tunsolved and disputed point. The pox nodules may be found on all parts of the head, comb, face and wattles. At times the sores may extend more or less down the neck. Fig. 75 shows chicken pox of the comb and face. As the nodule heals it appears as a dark, later a black scab-like mass. Finally the scab drops off leaving a scar.

\section{CONTAGIOUS EPITHELIOMA}

Contagious epithelioma is a disease of the head of young birds, usually birds of from 1 to 3 pounds weight, manifesting itself as a large nodule which microscopically is identical to epithelial tumors. The affected bird always dies. It is believed that the cause is the highly virulent pox virus described above.

Symptoms.-The epitheliomatous enlargements may involve any part of the head, and especially the unfeathered portions and may become as large as a hazel nut. Fig. 76 shows the carlier stages of the diseases. These are in two young chicks.

These epithelial growths first appear small, have a smooth surface and a red or hyperemic zone; they gradually increase in size till death of the chick. Necrosis may take place in various parts of the growth; the sloughing may leave ulcerative surfaces. The tumor-like body may obstruct the eye sight and even obliterate the eye.

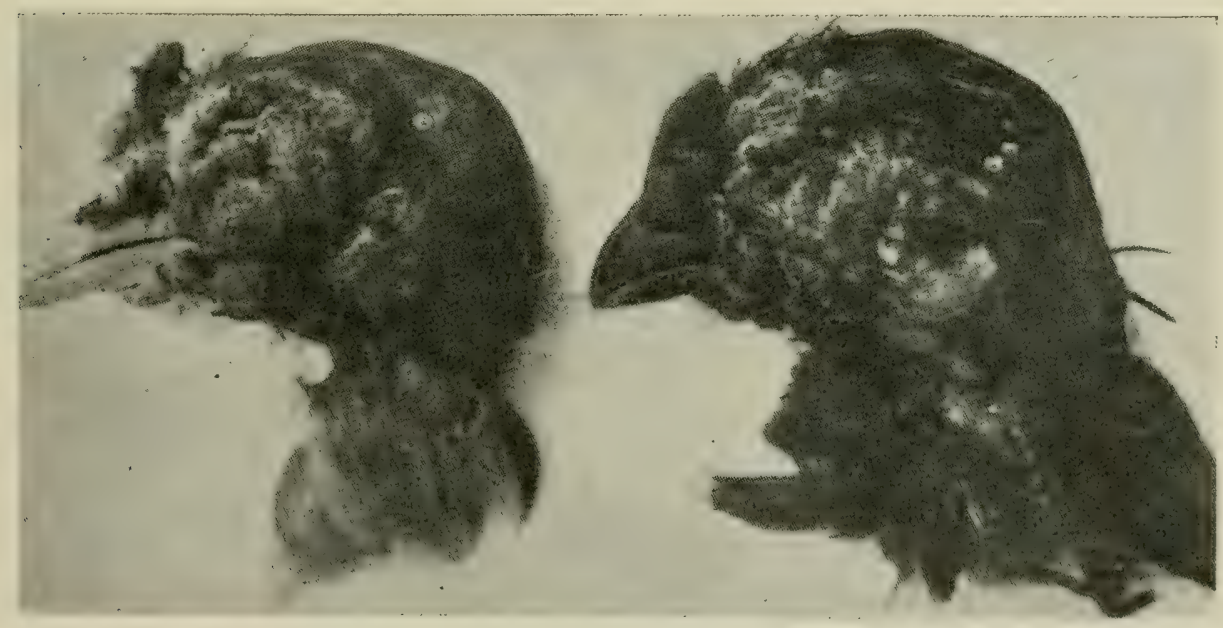

1iig. 76. Photograph of the Heads of Two Chicks Affected With Contagious Epitheliona.

Microscopic Study--A microscopic study shows the structure to be that of an epithelioma. The cells are arranged in more or less 
irregular rows supported by a network of connective tissue which is arranged in bundles between the nests of cells. The cells are of the epithelioma type. There may be observed necrosing foci.

Research has shown that there is a specific antibody developed in birds affected with chicken pox. By the use of the compliment fixation method the blood from the diseased fowl exhibits fixation of the compliment not shown by normal fowl's blood, thus showing that it is a specific germ disease. The antigen was prepared both from tumors on the head and from the liver of birds sick of the disease.

Treatment.-Treatment for chicken pox is of two kinds; namely, medical application and raccination. The best medical treatment consists of thoroughly rubbing on the affected parts some of the following preparation. Oil thyme, 30 drops; oil eucalyptus, 30 drops; menthol crystals 10 grains; neutral oil as paraffine oil 2 ounces. The germs appear to be aerobic and any oily preparation will be beneficial except in the rirulent type called contagious epithelioma.

All dead forls should be burned and the sick ones should he immediately removed from the flock for treatment or destruction.

Maintain sanitary conditions by frequently cleaning and disinfection of the hen house and ploughing the yards. Chicken pox rirus is very resistant to the action of disinfectants and this process must be thoroughly carried out.

All new stock brought into the premises should be quarantined for two weeks before being placed with other birds.

Carefully examine each fowl occasionally for evidence of the clisease. If a bird hesitates, at feeding time, to eat, catch her and examine the mouth and throat for disease.

Proper methods of housing and feeding are very important matters for successful poultry keeping.

The second method of attack is preventative measures by vaccination. The immunization of fowls against chicken pox has been practised by a number of investigators. It has not been possible to perfect a vaccine which will always produce immunity against severe artificial infections, but with highly potent strains of virus a vaccine may be prepared which will confer sufficient immunity to enable a flock to weather an outhreak of the disease without serious loss. The resistance is slow in developing, varying in different fowls from three to six weeks. One raccination is considered sufficient in healthy or slightly infected flocks. In hadly infected flocks a second treatment is advised for all fomls. In very severe outbreaks the injections should be continued at intervals of five to seven days until the disease disappears. The vaccine appears to have consiclerable curative properties when given to fowls sick of the disease. When the sick bird is of sufficient value to war- 
rant the trouble of handling, it should be isolated from the healthy fowls, and in addition to medical treatment be given one to two c. c. of vaccine every three days until improvement is noted.

Method of Vaccination.--The vaccine is given by injecting it beneath the skin with a hypodermic syringe. The dose is one cubic centimeter for each bird. The most convenient place for administration is beneath the skin of the breast under the thigh as the skin at this point is practically free from feathers. The bird may be held by the operator or by an assistant.

When the operator holds the bird the left wing is held back, the fowl is laid on its left side, and the right wing and leg and feathers are held back with the last three fingers of the left hand. The exposed skin is then cleaned with a piece of cotton saturated with a disinfectant solution, using a 4 per cent of some standardized coal tar disinfectant dip, and picked up with the thumb and forefinger of the left hand. Then with the right hand the syringe needle is inserted beneath the skin and the proper dose injected. With a little practice one man with an assistant to catch the birds can vaccinate 100 to 150 per hour.

When an assistant holds the bird both wings are grasped with the left hand and both legs with the right hand. The bird is then laid on its left sicle with the breast toward the operator. The operator standing a little to the rear of the bird, cleans the skin at the point of injection, picks it up with the thumb and forefinger of the left hand, and with the right hand, inserts the syringe needle beneah the skin and injects the vaccine. By this method a man with two assistants to catch and hold the fowls can vaccinate 200 to 300 per hour.

A syringe of six to twelve cubic centimeters capacity is well suited for this work. The needle should be 16 to 18 gagus and from $11 / 2$ to 2 inches in length. A screw needle is preferable.

Preparation of the Vaccine.-Young susceptible large comb cockerels as Single Comb White Leghorns are used for inoculation purposes. Scarify the comb and inoculate with a virulent virus secured from the sores of a virulent type of sore head. Sores will appear in five to seven days and develop sufficiently to permit the removal of scabs in 2 to 3 weeks. After the disease has developed and scabs have appeared curette the sore and dry the scabs in a dessicator over calcium chloride. After the scabs are water-free reduce to a fine powder in a glass mortar with a pestle. Place one gram of the powder in $100 \mathrm{cc}$. physiological salt solution and place in a water bath at $60^{\circ} \mathrm{C}$. for 60 minutes and place in it, as a preservative 0.5 per cent carbolic acid.

The liquid vaccine thus prepared deteriorates very rapidly and must be used at once. Until used the vaccine should be kept in a cool place as an ice box.

The injection must be just under the skin and bad results are likely to follow if given into the muscle.

\section{DIPHTHERIC ROUP-CANKER}

Diphtheric roup or avian diphtheria is manifested by sores in the mouth. These sores are covered with a yellowish white cheesy mass and when removed the underlying tissue is noted to be ulcerated. It is a true diphtheric inflammation. 
Fig. 77 shows the upper and lower walls of the mouth badly affected with these ulcers.

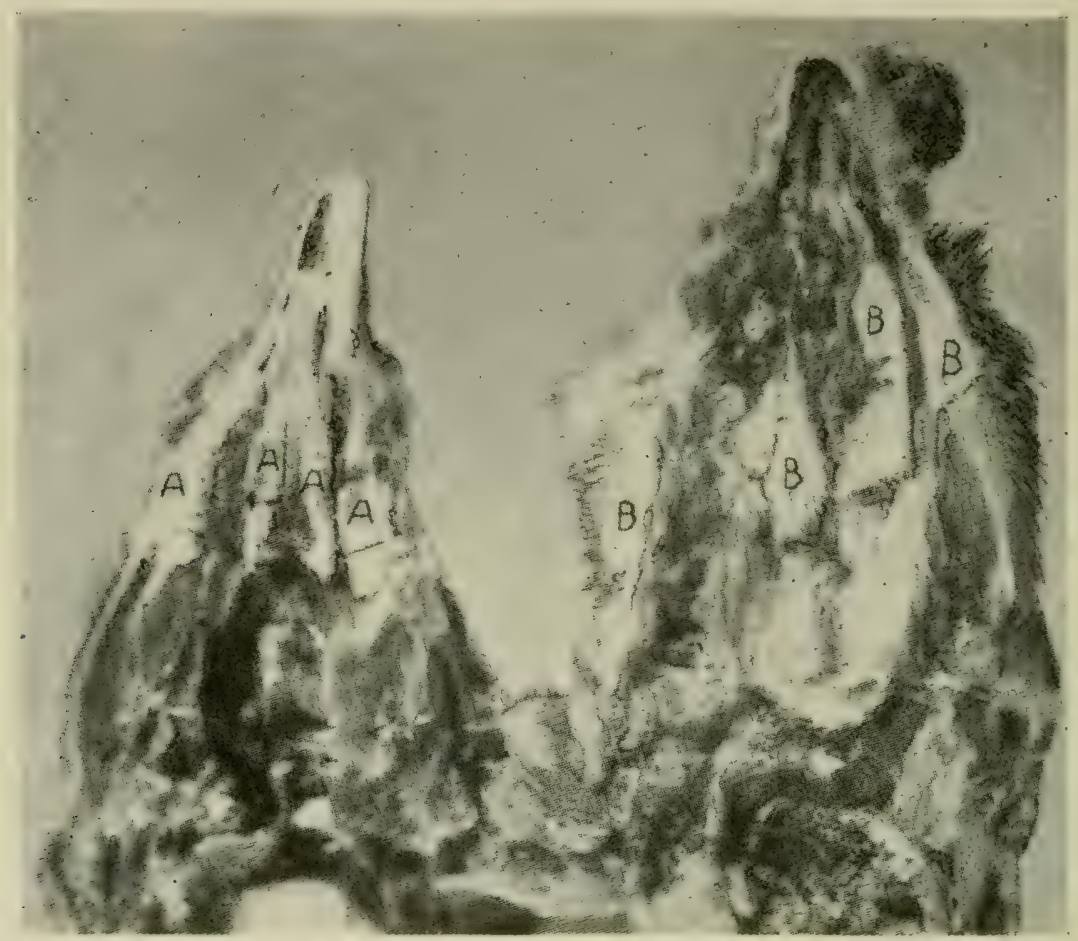

Fìg. 77. Diphtheric Roup in a Fowl.

$A$, the yellowish-white diphtheric patches on the upper surface of the tongue and lower jaw, natural size; $B$, diphtheric patches on the hard palate and the upper jaw.

Diphtheric roup may be spread by introducing affected birds into the flock, at poultry shows, or being shipped in infected coops, or by contaminated utensils, and by man and animals, as dogs, rats, etc. The disease may be spread through the flock through contaminated drinking water.

Diphtheric roup may appear as simple ulcers covered by thick cheesy crusts or it may assume a malignant type and gradually erode through the wall of the mouti. This usually occurs in the bony structure of the roof of the mouth finally destroying considerable tissue and making curetting and treatment impossible. Such malignant cases always die and treatment is not advisable.

Treatment.-With dull knife or curette scrape all of the cheesy accumulation and rub the sore with a stick of nitrate of silver till all parts turn white. Repeat in five days if necessary. Or pure tincture of iodine may be used. 


\section{DIPHTHERIC SORE EYE}

Diphtheric sore eye or occular type roup is an inflammation tion of the mucous membrane covering the anterior portion of the eyeball, the conjunctiva. As the diseases progresses, the catarrhal products accumulate as a watery, clot-like mass, whitish in color. The eyelids stick together and hold the material as it accumulates, till the part bulges ontward. The inflammation may involve the deeper structures of the eyeball and blindness occur and at times necrosis of the eyeball occurs, with a complete destruction of same. Keratitis or a white cloudy condition of the cornea precedes the more aggravated conditions.

There is loss of appetite, the bird appears weak, it sits around in corners or on the perch poles. Its walk is unsteady, and rapidly becomes emaciated. Ulcerations of the mucous membrane of the eye may occur. Death may follow in the course of a few days. This condition may be accompanied with ulcers in the mouth or with sore head or it may be the only affection of the fowl.

Treatment.-Press open the eyelids, remove with a piece of cotton, the white clot. Inject a few dops of the oily preparation mentioned for sore head. If it does not respond use two drops of a 1 per cent solution of Zenoleum once every three days with the oil preparation.

\section{ROUP}

Roup as generally understood is a catarrhal condition characterized by a discharge, of an offensive nature, from the nose. This discharge is at first thin and watery and later may become muco-purulent, gluing shut the nasal openings. The stinking odor is characteristic of roup. This disease may occur alone in a flock but is usually accompanied with canker of the mouth and sore head. It is caused by a filterable virus and is believed by most investigators to be a distinct disease from sorehead and canker, as the discharge from the nose does not produce these diseases when inoculated into other fowls.

Roup may assume a mild form in a flock or it may be very severe, killing many of the affected birds. In the early stages in very mild cases there are no or very few general symptoms. Secondary invaders may occur and the symptoms become more aggravated. In the acute severe cases the bird is dull, assumes a sitting posture, the wings are held pendent, the plumage is rough, and the patient shows much depression. The comb and wattles grow bluish-red in color, later they are pale and cold. In the colder climates the disease may assume a subacute or chronic form, while in warmer climates the 
acute form is most often olserved. Frequently, however, the clisease assumes the character of a chronic catarrh.

The disease usually makes its first appearance in the fall of the year and often occurs soon after the hirds have been placed in their winter quarters. The affected loird sneezes, the eres may be inflamed, the mouth held open for breathing as the nostrils may be partially or wholly closed. The feathers unkempt appearance, the bird refuses food, great depression, sits off in a corner or remains on the perch pole, and rapidly becomes emaciated and dies in a few days.

It must also be suspected when similar symptoms appear after adding new birds to a healthy flock or returned birds from a show.

Postmortcm Appearance.-The toxin, or poison, from the areas of disease is very destructive, as the rapid emaciation of the bird, following a severe attack, shows. Upon examination of the membranes that have formed in the mouth, it will be found that when they are removed there is left a raw, granular appearing surface. Upon microscopic examination, there may be seen cellular infiltration, with a destruction of cells of the mucous membrane underlying the diphtheric mass. An examination of the infraorbital sinus, see Fig. 2, No. 31, will reveal it to be filled with a purulent material, which is often cheesy in consistency. The wall over this part is very thin and can be easily opened with a knife.

A microscopic study of sections of the head, through the inflamed area, the mucous lining of the nasal passage, shows considerable thickening and an acute inflammation-invasion with polymorphonuclear leucocytes; at times the entire passage is plugged with mucus.

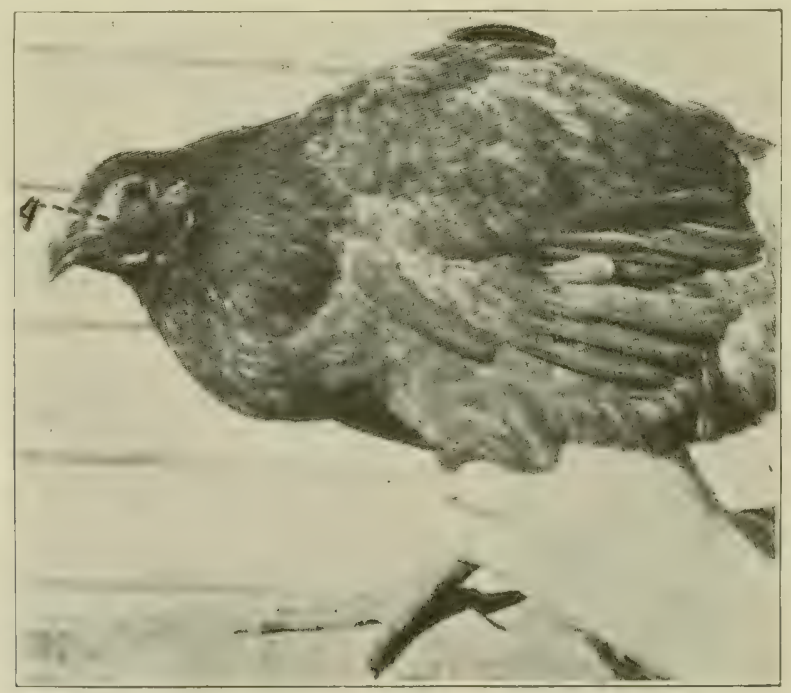

Fig. 7s Rour of a liowl SHowing bulging OF THE INFRAORBITAL SINUS, AT $\Lambda$.

Treatment.- In looking toward treatment and sanitation one takes into consideration the resistance of the organism to be 
combated. Fincly powdered virus, from this source and from sorehead, mixed in physiological salt solution and placed in sealed tubes and these tubes placed in a water bath at $60^{\circ} \mathrm{C}$. resulted in killing the virus in eight minutes. The finely powdered virus was lilled in 30 minutes when heated to a temperature of $56^{\circ}$. Suspension in water, at a temperature of $37^{\circ} \mathrm{C}$. killed it in eight days. Scabs exposed to sunlight in Petri dishes were active after two months. Exposed to 1 per cent solution of carbolic acid for one and one-half hours did not kill it.

Dispose of the chronic carrier. The chronic carrier may be known by its symptoms. The chronic carrier is usually a bird light in weight, pale face, comb and wattles, not in laying condition. Sits on the perch poles much of the time and is occasionally noted to sneeze. If this bird be caught and examined it will be noted to have a slight discharge from the nose. This discharge has a stinking roup odor. Such a bird will continually spread the germs of the disease which virus is very resistant as above shown. When the right conditions are present and susceptible birds an outbreak of roup may be expected. Wet floors, or concrete floors in which there is no underdrainage provided, in wet cold fall season, often is a fruitful source of roup. Correct any bad sanitation or hygiene, which may be a predisposing cause. The hen house should be well ventilated, but should allow of no drafts on the birds, and should be kept clean and free from dampness. It should be cleaned and disinfected daily with some standardized coal tar disinfectant dip using a 5 per cent solution. If the bird attacked is not a valuable one or if emaciation is rapid and there is no hope it should be killed and this and all dead birds should be burned.

As general preventative measures all diseased birds should he immediately isolated from the flock. Cleaning and disinfection of the house and yards, quarantine for two weeks all new birds or birds that have been to a show, careful examination of each fowl occasionally, if disease is present in the neighborhood.

The same treatment as prescribed for colds is indicated. Syringe out the nostrils with a soda solution. Place a half teaspoonful of common baking soda in a teacup of warm water and use this to syringe out the nostrils. This cuts the mucus so that the oily medicine that is to follow will come in direct contact with the inflamed mucous membrane. To make this injection procure a hard rubber syringe with a small nozzle so that the nozzle can be inserted into the anterior nose opening. Have an assistant hold the fowl with head down and force liquid through the nose till it comes out of the slit in the 
roof of the mouth. By holding the bird with head down the liguid will run out onto the ground and not have a chance of running into the trachea and lungs and causing further trouble. Following this a small quantity of the following preparation should be injected into the nostrils: Oil eucalyptus, 30 drops : oil thyme. 30 drops; menthol crystals, 10 grains; oil petrol, 2 ounces. All liquids injected into the inflamed mucous membrane should be warm. Repeat this treatment three times a day. Give an abundance of clean water and soft, easily digested food.

\section{RESPIRATORY DISEASES OF CANARIES}

Canaries are affected by similar respiratory diseases as described for fowls. As a preventative the cage of the canaries should be kept clean, free from drafts, and the birds should have a well regulated food supply. When the bird is first noticed to be ill, it should be isolated, and its diet regulated. Proper sanitary conditions of the cage and the cage kept in a well regulated temperature. Canaries suffer from cold drafts and it may be said that most of their common ailments come from this sort of exposure.

In ordinary colds there is noted difficult breathing, with some thin fluidy discharge from the nostrils. This may be accompanied with cough. As the cold progresses the symptoms become more aggravated. Breathing becomes more difficult and rapid. The catarrhal secretions may partly or completely block the nasal passage.

\section{ASTHMA OF CANARIES}

Asthma in canaries is a chronic affection. There is difficult hreathing, the greatest difficulty apparently, is with expiration. In severe cases there is a contraction of the abdominal muscles in forcing air into the lungs. The symptoms of asthma is more evident at night, and often birds apparently free from it during the day will wheeze when at rest.

False asthma may be caused by indigestion and overeating. Fanciers consider asthma as hereditary and do not recommend such birds for breeding. There is little that can be done for this condition except to give a light diet.

Treatment.-Place in the drinking cup one ounce of water to which has heen added 20 drops of syrup of tolu, 10 drops sweet spirits of niter, and 10 drops of glycerine. If the case is severe add 10 drops of whiskey or brandy. Pneumonia is quite often fatal. The birds become very weak and usually die in from two to seven days. 


\section{DISEASES OF THE LIVER}

Congestion, inflammation and necrosis of the liver as seen in many of the infectious diseases have already been referred to under the discussions of these different diseases, as chicken cholera, blackhead, tuberculosis, etc. Aside from disease of the liver due to infection, the commonest cause of ailments of this organ is improper feeding. It is with great difficulty that diseases of the liver can be recognized except upon postmortem examination.

\section{FATTY DEGENERATION}

Fatty degeneration of the liver is a degenerative and disintegrating change by conversion of the cell structure into fat. There is a disease process in the cytoplasm of the liver cells, by which the normal secreting cells of the liver are to a greater or less extent replaced by fat cells. The liver is smaller than normal, unless fatty infiltration is also present; the liver appears slightly yellowish, and when cut through with a knife, the blade of the instrument will have a greasy appearance, due to the fat that adheres to it.

Symptoms.-Birds affected with fatty degeneration of the liver show varicd symptoms, but ordinarily they are dull, eat little and the comb turns dark or even black. They gradually become thin in flesh and finally die. Usually the bird will live from two to three weeks to as much as three months after the symptoms first appear. An autopsy of all organs, as a rule, appear normal except the liver.

Treatment.-There is very little that can be done for this condition. Podophylin in one-grain doses repeated every three days may be given with some hope of relief.

\section{FATTY INFILTRATION}

Fatty infiltration of the liver consists in the addition of fat in the liver. It is a notable increase in the amount of fat normally present in the liver.

This condition may be a physiological or normal process until the accumulation of fat occurs in such quantities as to interfere with the function of the liver cells.

Overfed hens, or those closely housed and not forced to work, or fed too heavily on carbohydrates or starchy fceds, store up much of the surplus nutrition in the liver as well as in other portions of the abdomen, especially in the mesentery 
and in the abdominal walls. Geese are often fed large quantities of fat and carbohydrates to make fatty delicate livers for the table. This is a physiological process of excessive storage of fat in the liver.

It autopsy the liver will be observed to be enlarged, brownish or grayish-brown in color or mottles, friable, that is, tears easily, and when cut through appears "greasy," much fat adhering to the knife blade. In these cases rupture of the liver often occurs when the hen is stepped upon by a large animal, is thrown or jumps a long distance on hard ground or a concrete floor. Heavy hens with clipped wings are prone to be injured in this way.

In the liver, in which excessive fat is stored up, there is, after a while, an encroachment upon the protoplasm to such an extent that the cells cannot properly functionate and then death of the bird may occur. In these cases a microscopic examination shows the nuclei of the cells to be pushed to one side, and the protoplasm atrophied and disappearing. This is a pathological condition.

\section{RUPTURE OF THE LIVER}

The liver may rupture or tear and thus cause internal hemorrhage which is usually fatal. It may occur where the liver is excessively congested with blood or is overly filled with fat, as mentioned above, violence may result in rupture. From such internal hemorrhage the bird appears pale faced with pale comb and wattles.

Appearance at Autopsy.-There is considerable blood observed over the liver and among the intestines and other abdominal organs. Usually the blood is coagulated. An examination of the liver shows a tear which often is an inch in length. The organ may be considerably enlarged. A microscopic examination may show congestion with an occasional small hemorrhage into the liver tissue.

\section{CONGESTION OF THE LIVER}

Congestion of the liver is an engorgement or an abnormal accumulation of blood in that organ. It is an important condition in fevers, and other disorders; and may arise either from an extraordinary flow of blood by the arteries, or from a difficulty in the return of the blood to the heart by the reins.

There are therefore two kinds of congestion of the liver, active and passive. Active congestion precedes inflammation and is a state in which the capillaries, arterioles and arteries are engorged with blocel. It may he caused ly local irritation.

Passive congestion of the liver is usually due to a weak heart or a leaky valve between the two cavities of the right side. The blood backs up into the liver, and the central veins of the lobules and capillaries. hetween the columms of liver cells, be- 
come engorged. It gives the cut surface a peculiar yellowish mottled appearance called "nutmeg liver," from a fancied resemblance that it bears to the sectional surface of a nutmeg.

\section{INFLAMMATION OF THE LIVER}

Inflammation is a succession of changes that take place in a tissue as a result of an injury provided that injury is not sufficient to at once produce death of the part. That injury may be due to bacteria, or their toxins, or to protozoa, or mechanical, thermic as heat or cold, electric, or chemicals.

The process of inflammation comprises changes in the blood vessels and circulation, exudation of fluid and of blood corpuscles from the vessels, and changes in the inflamed tissue.

Inflammation of the liver may be the result of absorption of poisonous products from the intestines. These products or toxins, lodge in the liver, or the inflammation may be due to infection or germs, such as fowl typhoid. The irritation causes an active congestion followed by a migration of great numbers of polymorphonuclear leucocytes or white blood cells, and of thrombocytes, constituting inflammation. The liver is enlarged, dark, and easily torn; it appears very full of blood.

In many, and in fact most, of the contagious diseases and especially the septicaemias inflammation of the liver or hepatitis occurs.

Symptoms.--In general the symptoms of hepatitis or inflammation of the liver are those of a loss of appetite, weak in the legs, rapid emaciation, clark comb, face and wattles, finally unable to stand lies on its side. The bird may live from a few days to two or even three weeks.

Postmortem Findings.-The liver will be observed to be enormously enlarged, often weighing as much as 176 grams while the normal liver weighs but 35 grams. The surface has a grayish mottled appearance. Under the microscope these pale gray, irregular areas prove to be liver areas packed with white blood cells and thrombocytes.

\section{INFLAMMATION OF THE BILE DUCTS AND GALL BLADDER}

An inflammation of the bile ducts is called angiocholitis and an inflammation of the gall bladder is called chilecystitis. Both of these conditions may be met with in the bird. A postmortem examination of the gall bladder shows its contents to be rather mucilaginous in consistency and containing only a small amount of the biliary elements. 


\section{ENTEROHEPATITIS}

Enterohepatitis is a disease of turkeys and to a less extent of small chicks and other young birds, which extends from the intestine and involves the liver. It is discussed under diseases due to single celled animal parasites.

\section{OTHER DISEASES OF THE LIVER}

Abcesses and Tumors.-Abscesses and tumors of the liver appear to be very rare in the chicken and other birds with the exception of sarcoma of the liver, which we have frequently observed. Malignant tumors as sarcomas and carcinomas or cancer of this organ are usually associated with similar tumors of the other abdominal organs.

Jaundice.-Jaundice is a staining of the tissues yellow with bile. Jaundice may be caused by an obstruction of the bile in its natural channels, bile being absorbed into the blood. Jaundice in the fowl is very rare, and appears to result from a long continued mild congestion of the liver.

Cercomoniasis or spotted liver is a type of disease of the liver due to an infectious animal organism, the monocercomonas gallinarum, and is associated, at times, with severe diarrheas.

Aspergillosis is a disease due to the fungus Aspergillus fumigatus. It commonly affects the lungs but may, and occasionally does affect the liver. This disease has been discussed.

Amyloid degeneration.-Amyloid degeneration is a pathological condition in which a firm waxy substance is present in the tissues, not responding to chemical agents and resisting putrefaction. The substance resembles starch or glycogen. Amyloid degeneration of the liver, spleen and kidneys has been observed in birds afflicted with tuberculosis. In some cases the liver appears granular and brittle. Amyloid deposits in these organs have also been observed in this laboratory in hens dying of purulent peritonitis. 


\section{CHAPTER X}

\section{DISEASES OF THE OVARY AND THE OVIDUCT}

\section{PROLAPSE OR EVERSION OF THE OVIDUCT}

Prolapse or eversion of the oviduct is a condition in which the oviduct has passed through the natural orifice with which it is connected. This condition has been illustrated under the condition described under the heading of prolapse of the cloaca and oviduct.

Prolapse of the oviduct of the fowl is a common ailment of laying hens. It may be seen in pullets in early winter that are being force fed with highly stimulating feeds. Aggravated constipation has also been observed to be associated with this condition. When the eggs are large and considerable straining takes place during their passage, and in inflammation of the mucous lining of the oviduct or egs canal, prolapse or protruding of the nutcous membrane through the cloaca may be observed. In constipation, the bowels become engorged, and this in addition to the obstruction when the egg canal contains one or more developing eggs, and the ovary, being active, is larger and adds to the bulk, predisposes to prolapse.

This condition is most often seen in hens that are heavy layers. It perhaps occurs most often in pullets but may also occur in older hens. If the prolapsed or protruding mucous membrane is allowed to extend through the anus, it soon becomes inflamed from exposure to the air and infection takes place. Later the parts may become ulcerated as a result of mechanical injuries or the attack of germs.

Treatment.- Wash off the accumulated material on the vent feathers with clean, soapy warm water. After cleansing the hands, replace the protruding mass, using on the fingers carbolized vaseline, three to five per cent strength. Keep the hens on a light diet for several days so that the parts may have a rest and the irritation causing the trouble subside. It is best to give only soft feed and liquicls. Give the hen a tablespoonful of olive oil and plenty of clean water.

\section{OBSTRUCTION OF THE OVIDUCT (Egg Bound)}

An obstruction of the oviduct or egg canal is brought about by any condition that arrests the passage of the egg, during its formation. This is a common ailment of laying hens, perhaps the commonest of all discussed conditions of the oviduct. The poultry raiser knows this condition as "egg bound," by which 
is meant that there is something in the oviduct which the bird cannot force out.

The upper portion of the oviduct, or that part which receives the ovum or yolk as soon as it is fully formed in the ovary and delivered, is lined with secreting cells. In this part the albumin which surrotinds the yolk is formed. Further along the glands secrete the shell or calcium layer after forming around the mass the fibrous membranes or sacs. It can be readily seen, for all this to be brought about, there must be an abundant blood supply. An inflammation of the egg duct may be the result of infection from the digestive tract by way of the cloaca. Such inflammation means an arrest of function of these glands. There are other cells that secrete mucus which lubricates the passage way, and these too, are arrested in their function. The result is a stoppage of the egg:

Other causes are: eggs of too large size, exhaustion of the bird and atony and paralysis of muscular walls of the oriduct and vagina, volvulus or twisting and stricture of the oviduct. Weakened muscles, the result of disease, improper nourishment and overwork are contributing factors. Polypi of the oviduct have been found in which obstruction resulted.

Symptoms.-The hen goes frequently to the nest and repeatedly makes expulsive efforts but cannot lay. If the obstruction is well along in the egg canal the egg may be felt as a hard object in the posterior part of the abdomen. In many cases the obstruction is so far up in the oviduct it cannot be felt or seen and we must depend for diagnosis upon the action of the bird, which suffers acutely under these conditions.

Treatment.-First be sure that the bird will not lay the egg unaided. Allow her to remain quiet and alone for a couple of hours; she will often relieve herself maided. If it is evident that the bird must be given help, wash the hands carefully with soap and water, and luliricate the index finger with three to five per cent carbolized vaseline, which can be secured at any drug store, pass the finger through the anus and cloaca into the egg canal and remove the egg. Great care must be exercised not to tear the oviduct as the walls of this organ are very tender. At times the egg is large and it may be necessary to break the shell in order to remove it. If the egg is broken, make sure that all parts of the shell are removed. By referring to Fig. 2 the relations of these organs may be seen.

After removal of the egg give the hen a tablespoonful of olive oil or castor oil, taking care that it does not find its way into the windpipe, and place her on a light diet, as bread soaked in milk or grain, for a few days. Recovery ustally 
occurs in the simple uncomplicated cases which form the majority; in complicated cases, death is often the result.

\section{RUPTURE OF THE OVIDUCT}

Rupture of the oviduct is a tear in its wall and is observed in the hen. This condition is usually a complication of obstruction of the oviduct. It is frequently fatal in a very short time and in such cases can be diagnosed only upon postmortem examination.

Canse-Vigorous contraction of the muscular walls of the egg canal in expulsion efforts sometimes results in a rupture of the wall. When this occurs the usual sequel is peritonitis or an inflammation of the serous lining of the abdominal cavity, and death of the bird results. Disease processes sometimes so weaken the wall that it gives way under the stress of natural contraction.

Symptoms.-The hen ceases to lay, the abdomen becomes larger and often one or more eggs can be felt by palpating or feeling between the hands, the lower portion of the abdomen. Often the hen is noted to sit up penguin-fashion, walking with tail and posterior portion of the abdomen dragging the ground. There is nothing to do except to kill the bird. At autopsy there will be found many yolks in the abdominal cavity, possibly one or more with shells and possibly an inflammation of the lining of the cavity or peritoneum.

\section{BROKEN EGGS IN OVIDUCT}

Eggs in the oviduct, as well as ova still undelivered, are often found broken as a result of a kick of a large animal or of the hen being stepped upon. Death usually follows, if not immediately from the injury, which breaks the egg, after several days as a result of complicated obstruction of the oviduct resulting from the fibrous exudate thrown out about the broken yolk.

Rupture of the ovarian capsules containing the yolk may also take place especially in heavy hens roosting on high perchpoles and by jumping upon the hard floor, causing rupture of the larger forming yolks or ova or of eggs in the egg canal.

\section{ULCERATION OF THF ANUS. AND LOWER BOWEL AND OVIDUCT}

Symptoms.-The fowl is thin in flesh and feathers soiled around the vent. Upon examination of the vent region an ulceration of the anus is observed. A very offensive stinking odor is emitted. The appearance of the comb, face, and wattles shows such a hen not in laying condition. 
Autopsy.- Coon opening the aldominal carity the ovary is noted to be in an inactive state. See Fig. 79. In opening the cloaca, large intestine and oviduct it is observed that the ulceration has extended more or less along the mucous membranes of these tracts. Under these conditions constriction of the widuct by new formed tissue completely obstructs this passage.

Treatment.-Syringe out the parts three times a day with a solution of ferrous sulphate using fire grains to the ounce of warm water.

\section{ABNORIMAL EGGS}

Many linds of alinormal egegs are produced by fowls owing to various discased or other abnormal conditions of the generative apparatus. Because of the rarity of their occurrence such eggs are of little inportance to the practical poultry raicer, but they possess much interest for the scientific investigator.

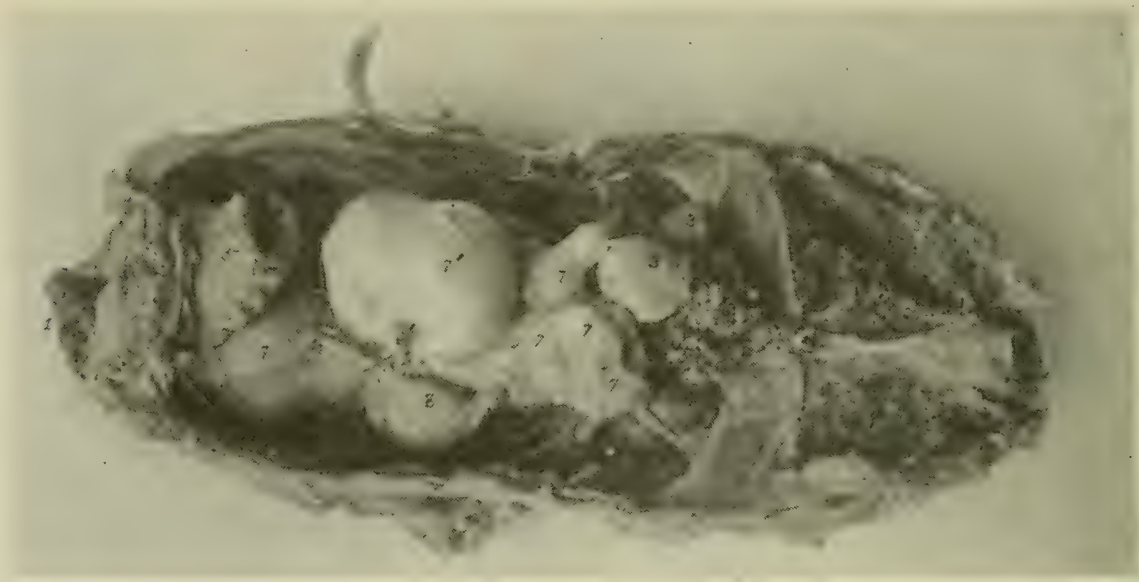

Fig. 79. Obstruction of the Oviduct Due to Ulceration of the Anus IVitil Neiv Formen Connective Tissue Obliterating the Opening of the Oviduct.

This obstruction stopped, the passage of 9 yolks. 1, ulceration of anus; 2 , the ovary; 3, two atrophied ova; 4 , the kidney; 5 , the cloaca; 6 sliows the fibroid obstruction; 7 , obstructed yolks; 8 , the only portion of the oviduct not occupied by yolks; 9, the lungs.

In any of the breeds of the domestic fowl, there are occasional individuals producing one or more small eggs which are from onetenth to one-half the size of normal eggs.

Soft-shell Eggs.-This is a condition where eggs are laid without a sufficient amount of shell substance covering the shell membrane. The commonest cause is overfeeding, another cause is the lack of sufficient shell-making material in the feed; still another cause is fright, which may cause a premature detachment of the yolk.

The cause should be remedied and the condition will disappear without further treatment.

Yolkless Eggs.-These are small eggs, in which the albumin and shell are formed about a small portion of detached yolk, a minute piece of hardened albumin or a bit of coagulated blood instead of the normal yolk. 
Double and Triple Yolk Eggs.-Eggs with two yolks are common. They are caused by two yolks getting into the oviduct and being enclosed together in the albumin and the shell. Three yolked eggs are rare, and have a similar origin.

Eggs with More than one Shell Covering.-Spasms or reversed action of the muscles of the oviduct may cause the egg to be passed back from the shell gland portion, after the shell has been deposited on it, to the ovarian pocket or starting point. This may be due to irritation of that part. After the spasm has subsided the egg again passes down the egg canal and another layer of albumin is deposited around it, then another shell membrane as it passes through the isthmus, and finally another shell. This is shown in the photograph in Fig. 80.

Eggs with More than One Membrane.-If there is a sensitive spot in the isthmus or immediately below it the eggs with its membrane may be forced back to the ovarian pocket or starting point, and

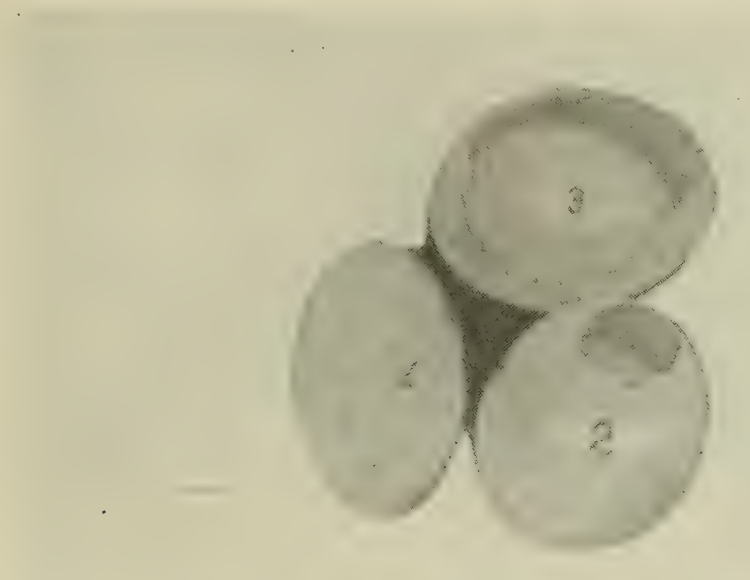

Fig. 80. EgGs Within EgGs.

1, the outside of a normal egg; 2 and 3 , showing the eggs inside their second shell coverings.

after the spasm has subsided, the egg will again pass down the oviduct receiving another coat of albumin as it passes through the albumin secreting portion and then another membrane over this. As many as four such membranes have been observed. Fig. 81 illustrates one of these cases.

Symptoms.- In such cases there may be a rupture of the parts and many eggs find their way out into the abdominal cavity and will show, at times, symptoms of running straddle legged, sitting down much of the time. Standing penguin fashion. The abdomen is very large, and on palpation or feeling the abdomen between the hands, it fluctuates as though it contained liquid.

Postmortem Findings.-Eggs in various stages of development will be found in the abdominal cavity. Some of the egg material may have been absorbed. A chronic peritonitis may be present. At times nature will have tried to encapsulate them and new formed connective tissue may be found around the individual eggs, finally 
the abdominal tumor-like mass when cut through will show areas of yolk surrounded with albumen in a more or less degenerative stage and among these the new formed connective tissue-nature's effort to heal or organize the part.

Bloodspecks, Blood Rings, and Egg Inclusions.-These conditions have little significance; particles of coagulated blood as shown in Fig. 82, No. 1, due to a hemorrhage when the ovum or yolk is discharged from the ovarian capsule where it develops, are most common, but lumps of bacteria, worms, fecal matter, etc., have been observed.

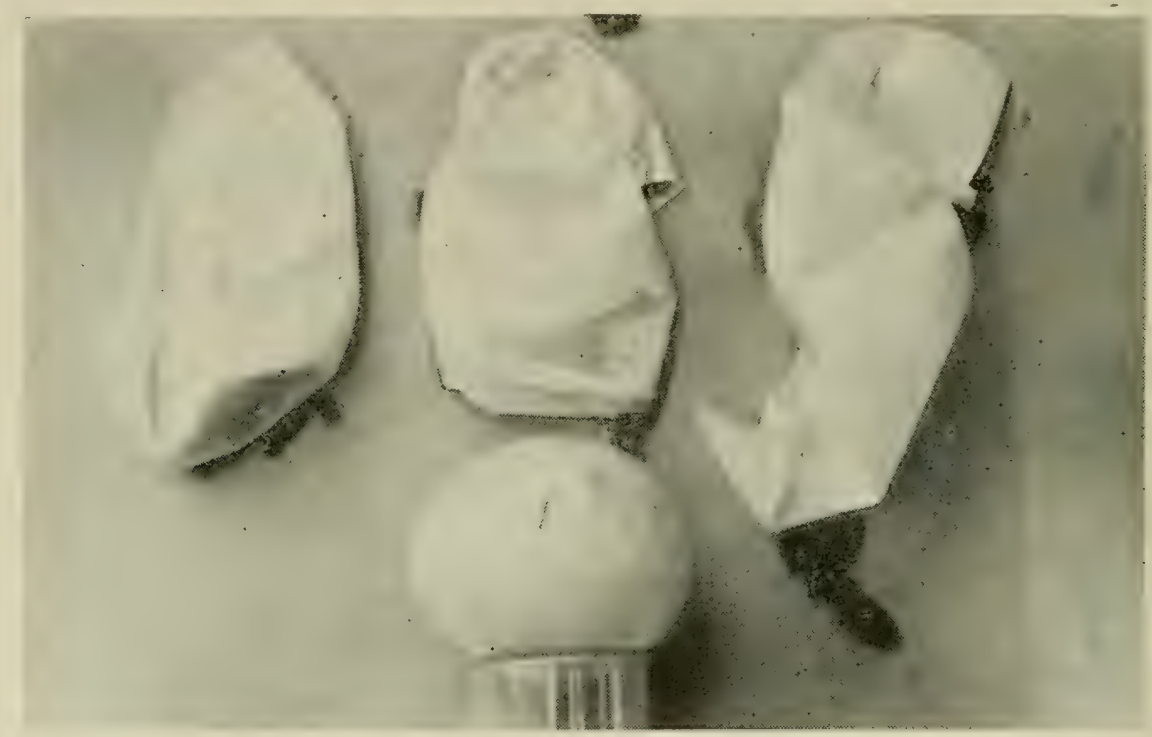

Fig. 81. An Egg With Four Miembranes.

1, the egg; 2, the second membrane; 3, the third membrane; 4, the fourth membrane.

Blood clots may be found in either the yolk or the white or albumin.

If hemorrhage occurs in the yolk, the clot has formed in the ovary before it was delivered into the oviduct. If the clot is in the white it has occurred in the upper portion of the oviduct, and if the blood is on the outer shell membrane or shell it has occurred in the vagina. The most common location is on the surface of the yolk and occurs from some capillary at the point of rupture of the ovarian capsule.

\section{DWARF EGGS}

Dwarf eggs of fowls vary greatly in size and shape. There are two distinct types: First, prolate spheroidal shape, similar to the normal egg; and second, the cylindrical type.

In one study of 27 flocks consisting of 579 birds there were produced in the twelve months 45,055 eggs of which there were 40 dwarf eggs. This is 0.00088 per cent, or one dwarf egg in every 1,127 eggs laid. It has also been observed that these small eggs may be developed at any time throughout the hens laying period. Trap nest records have shown that a normal egg may be laid on days immediately before and after these dwarf eggs. 
The internal structure of the dwarf egg varies in its makeup. Some dwarf eggs contain a small yolk surrounded by a membrane, others a small quantity of yolk without a yolk membrane, and still others no yolk. When yolks are present usually there is no germinal disc.

The albumin in the dwarf eggs differs in density. It may be dense and appearing like that of a normal egg. There is also found all gradations between these two extremes. The tendency is to a density greater than normal.

The size of the dwarf egg is apparently related to the size of the nucleus which by its presence gives stimulus to albumin secretion.

A bird may suffer a disturbance in her physiological functions of reproduction, and produce dwarf eggs since normal eggs are pro-

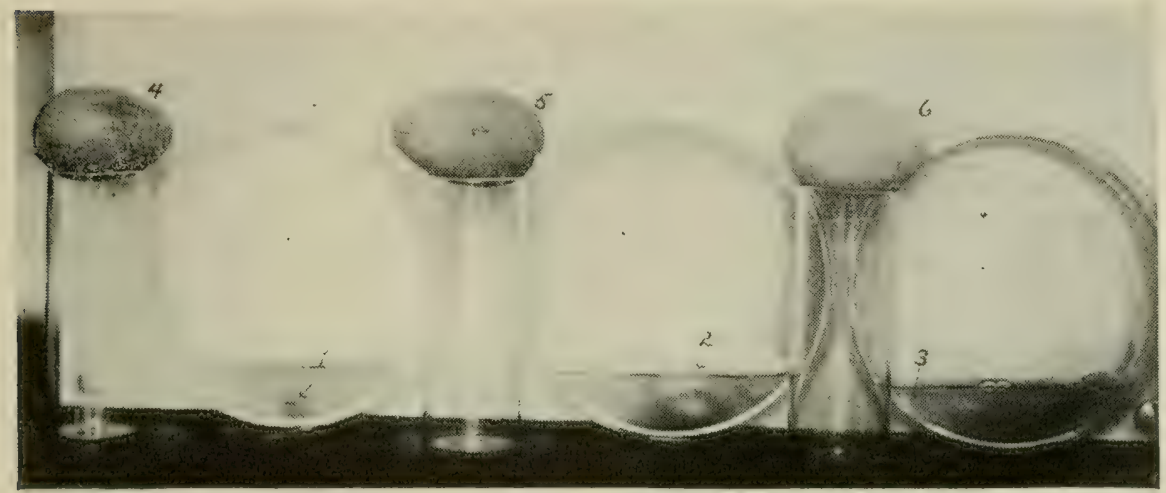

Fig. 82. Three Dwarf Eggs and the Cause of Their Formation.

1, blood clot causing dwarf egg No. 4; 2, a mass of inspissated albumin causing dwarf egg No. 5 ; 3 , a small piece of chalazae causing dwarf egg No. 6.

duced before and after dwarf egg formation and the cause of such dwarf egg production is of a temporary character. Dwarf egg production appears in both pullets and old hens and occur at any time during the laying period, but most often in the spring or early summer, perhaps because the bulk of the eggs are laid at this time of year.

The yolk of an egg constitutes, on an average, 24.37 per cent of the weight of the egg. and 33.91 per cent of double yolked eggs and 35.52 per cent of triple yolked eggs.

The shape of the egg is determined by the action of the circular and longitudinal muscular fibers of the oviduct wall.

The egg being a semifluid body has a tendency when free, to assume a globular shape, but at the time of the formation of the membrane it is larger than the oviduct lumen, hence there is a tendency under pressure exerted by the oviduct as this is the line of least resistance.

The degree of pressure will depend upon the size of the egg and the tonicity of the muscular coats of the oviduct.

The exact length as compared to the breadth will depend upon the tonus of the circular and longitudinal fibers. A strong tonus of the circular fibers and weak longitudinal fibers may greatly alter the normal shape of the egg. The two sets of muscles are independent in their action. 


\section{EPIORNITHOLOGIC ABORTION IN BIRDS}

Lnder this head, there have been mentioned by several writers, an affection in birks in which the eggs were expelled from the oviduct before the formation of the shell had taken place. We have noted this in flocks when crushed oyster shells were kept consantly liefore the hens, so that it could not have heen a lack of lime salts. The question arises is there any connection in the train of causes in bird and mammals? 


\section{GENERAL DISEASES}

\section{PURULENT INFLAMMATION OF THE ABDOMEN}

Purulent inflammation of the abdomen is a suppurative inflammation of the peritoneum or lining membrane of the abdominal cavity and is accompanied with pus formation. This condition is frequently met with in laying hens two years or more of age. It is usually due to a noninfective agent. Streptococcic infections have been observed as well as a bacillus.

Symptoms. - The unfeathered portion of the head is usually normal although it may become purplish-red at death. There, at this time, also may be noted a slimy mucus in the mouth. The bird may become thin in weight. There may, be a dullness

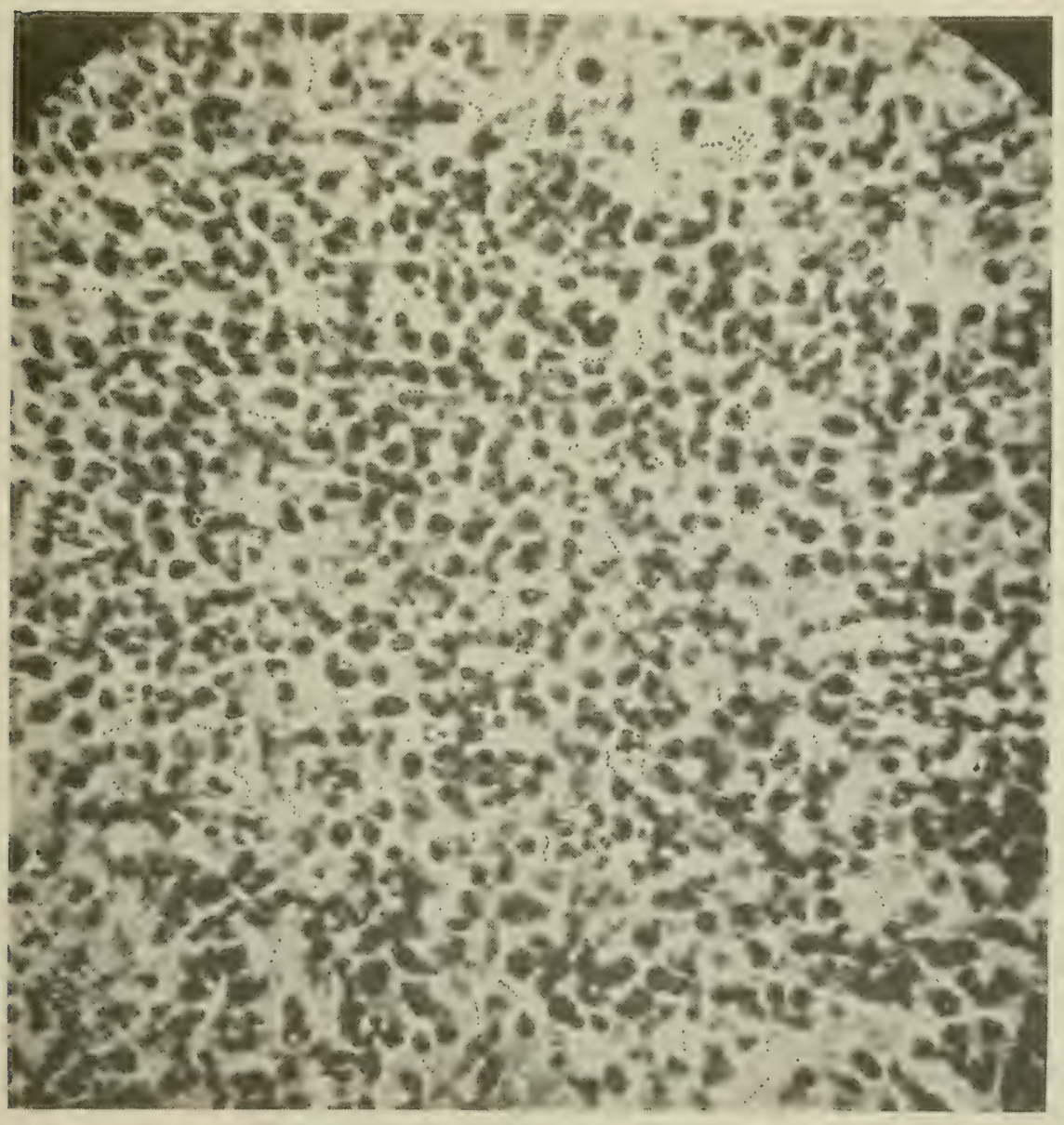

Fig. 83. A Microscopic Field Sifolving Pus Cells and Chains of Streptococci, Causing Purulent Peritonitis of a Hen. 
for several days before death. Laying hens finally cease laying and the comb begins to shrink, becomes pale, and covered with more or less scale as is the case when hens pass from a laying to a nonlaying period.

Postmortem Findings.-The liver may appear dark in color, but usually normal in size. The gall bladder may be full of bile. The peritoneal surface is more or less roughened and there is spread over the lining and intestines a purulent material which when it accumulates in considerable quantities may be more or less cheesy in nature. The spleen is usually normal in size. The intestines appear normal except for the above described condition. A small amount of food and gas may be observed in the intestines. The pancreas is normal. The kidneys may appear, on sectioned surface, a light mottled gray, and more or less swollen, indicating congestion and possibly more or less degenerative changes due to the toxic products absorbed from the abdominal cavity. Parenchymatous nephritis may be present under which conditions there is usually more or less changes in other parenchymatous organs as the liver. The kidneys, on section, may appear clogged with the pasty urinary sediment. Areas here and there may show localized nephritis as evidenced by the area being packed with cells of inflammation. The ureters are also clogged with the pasty urine. The ovary is inactive.

Microscopic Examination.-A micioscopic examination of the ureter content, in many cases, has shown besides the normal sodium urate crystals pus cells consisting of both mononuclear and polymorphonuclear cells. The milky fluid consisting of pus cells, liquor puris, detritis, and causative germs, may consist of five or more cubic centimeters. The microscopic examination of the liver and especially of the kidneys may show both active and passive congestion, with cloudy swelling, and possibly areas of focal necrosis.

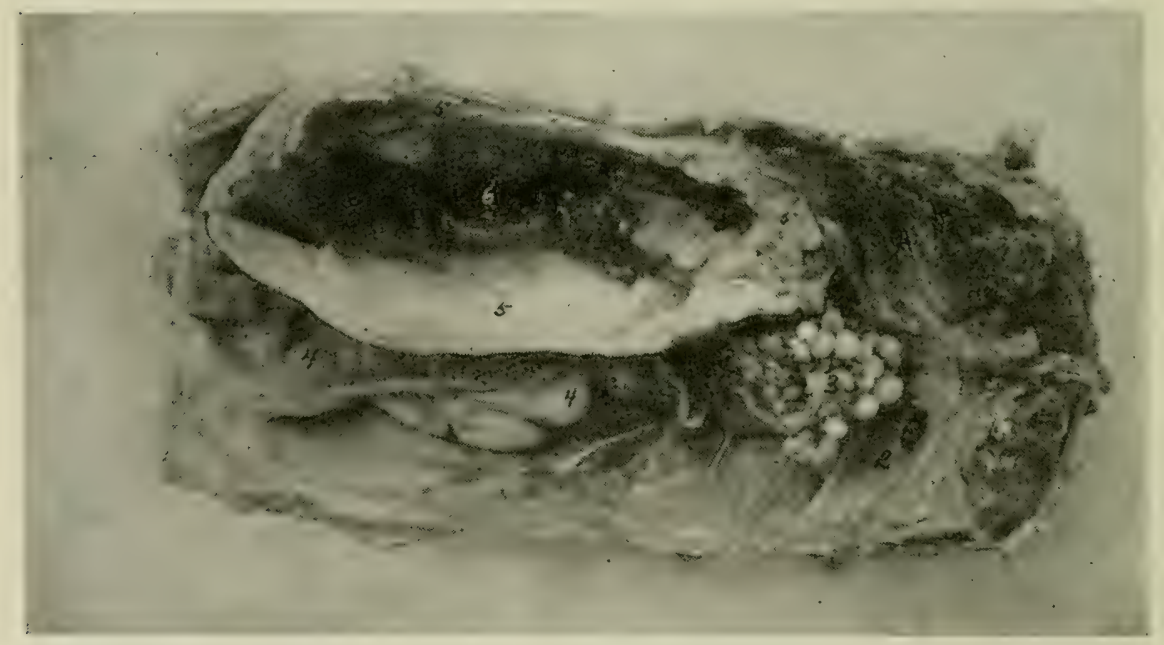

Fia. 84. Purulent inflamation of time Rigit abdominal air Sac of a S. C IVHITE LEGHORN HEN, 2 YEARS OLd.

1, sectioned surface of the lung; 2, the diaphragm; 3, ovary in a quiescent state; 4, oviduct in a quiescent state; 5, wall of right abdominal air sac in state of purulent inflammation; 6, a point through. which the abscess sacculates by the air sac extension which surrounds the hip joint. 


\section{PURULENT INFLAMMATION OF THE AIR SACS}

Purulent inflammation of the air sacs may occur and may be due to nonspecific germs.

Symptoms.-There are no definite symptoms before death. The symptoms are those common to many maladies of birds such as a progressive dullness, more or less loss of flesh, sluggishness toward the end, and loss of appetite and finally death.

Postmortem Findings.-At autopsy the air sac appears as a large tumor and fluctuates upon palpation or handling. The wall is thickened and when opened usually contains a liquid whitish in color and occasionally a flocculent material. Fig. 84 gives a photograph of a typical case involving the right abdominal air sac.

\section{CONTAGIOUS INFLAMMATION OF THE AIR SACS IN GEESE}

This is an infectious or contagious disease caused by a slender bacillus, and is confined exclusively to geese.

Symptoms.-The goose is weak, staggery gait, great depression, difficulty in rising, kicks at his head, accelerated breathing, snoring sounds and opening the mouth. Death usually follows in from six to eight days.

Postmortem Findings.-The air sacs appear yellowish in color. The inner surface of the air sacs are covered with a fibrinous material. Similar deposits may also be observed on the serous surfaces of the liver, spleen, intestines, and peritoneum.

\section{ABSCESS OF THE FOOT}

Suppurative processes resulting in abscess of the foot are common in the fowl. One form of injury resulting in abscess is due to thorns as from Russian thistles, or hedge, cacti, or similar structures. The thorn penetrates the soft parts between the toes as shown in Fig. 86. In this case $A$ represents the hole or opening through which the cheesy pus was removed by aid of a curette.

Another form of injury and pus formation is that observed in the heavy laying more delicate breeds as the Single Comb White Leghorns in the latter part of the summer or early fall. This form of abscess of the feet is usually found in heavy laying hens in the latter part of the summer when the long months of laying have reduced, to a certain extent, their vitality. This form of abscess of the feet is found to a less extent in the heavier breeds as Rhode Island Reds and Plymouth Rocks. Bruising of the soles of the feet, as jumping from a high perch pole to hard floors, favors abscess formation when the pus producing germs are present. In all these abscesses the pus is of a cheesy nature.

Symptoms. - The bird is noted to limp, the ball of the affected foot is swollen, and as the suppurative process prog- 
resses ulceration of the under surface of the foot is evident. There may appear a black piece of dead tissue and cheesy pus. In such abscesses and other suppurative processes as injury

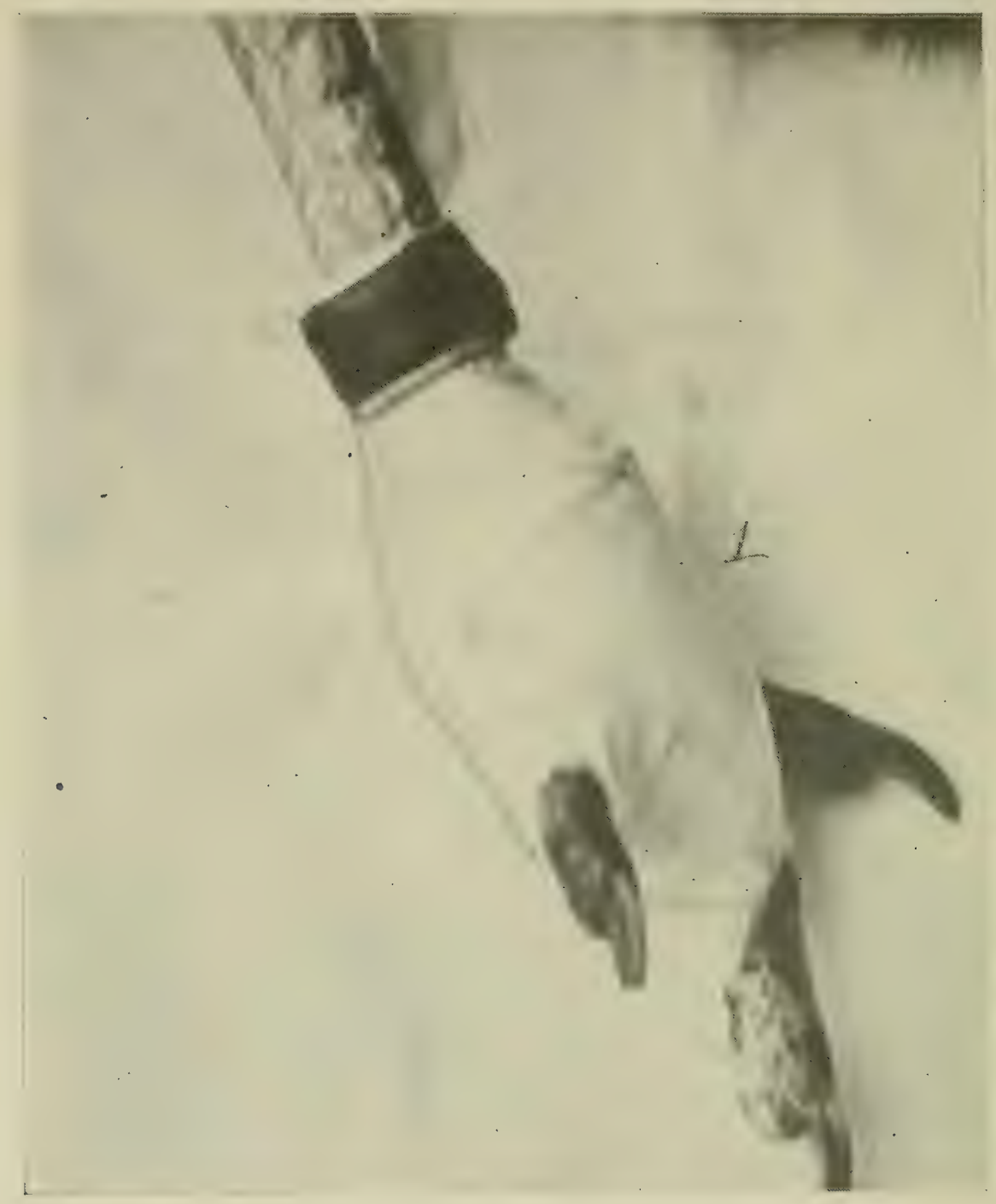

Fig. 85. A FoOt I'ROPERly liandaged.

Note that the banclage is passed diagonally across the ball of the foot and between the toes.

due to spirolettes, etc, the pus germ, staphylococeus pyogenes aureus has been isolated.

Treatment.-Lance the abscess and curette out or scrape out with a dull knife, the cheesy pus. Follow by swabbing out the wound with pure tincture of iodine. It is best to then bandage with a one inch bandage as shown in Fig. 85. The wound must be dressed each day, and as the pus will not be thin and drain- 
age impossible, the accumulating pus must be carefully removed with a swab in such a manner to not injure the granulation tissue.

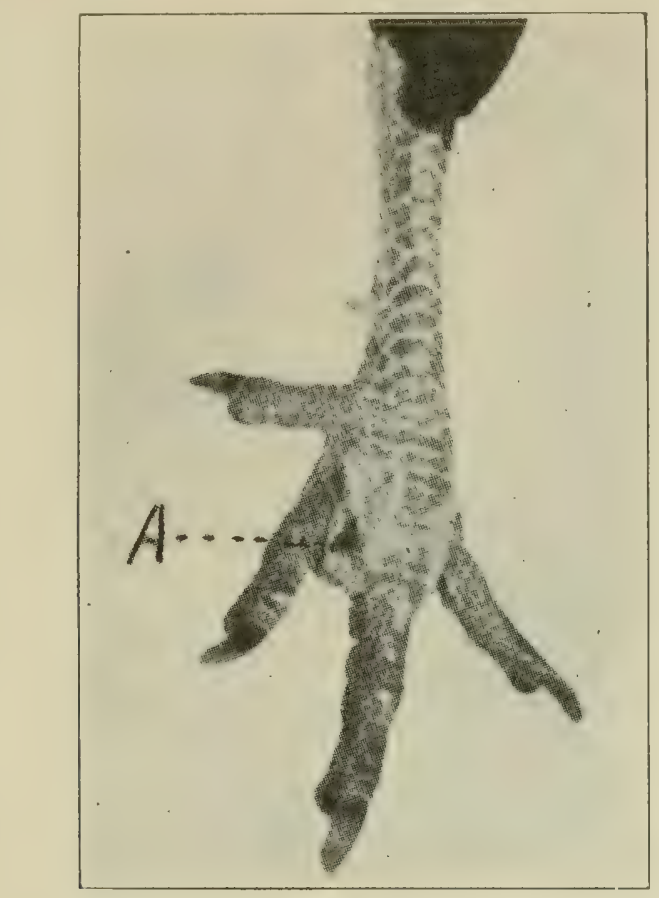

Fig. 86. Abscess Caused by a Russian Thistle THIORN.

a, opening through which the cheesy pus was removed.

After treatment of the foot, place the bird in a clean, dry place, preferably on straw, so as to keep dirt out of the sore.

After the foot has healed it will be found to be somewhat larger than normal. The sole of the foot will be somewhat tender for a while, and to prevent rebruising and reformation of an abscess it is well to place a leather pad on the sole of the foot as illustrated in Fig. 87.

\section{DRY GANGRENE OF THE FEET OF CHICKS}

A condition of dry gangrene of the feet has been described occurring mostly during the first month of the chick's life though it has been reported in older hirds. It occurs in the prairie regions of the west wherever the virgin sod is still unbroken.

Symptoms.-The first sign in the baby chick is that it lags behind the balance of the flock, there are blisters on the feet, some have ruptured. A scab has appeared at the seat of the blister. These scabs are now seen between the toes, on their 


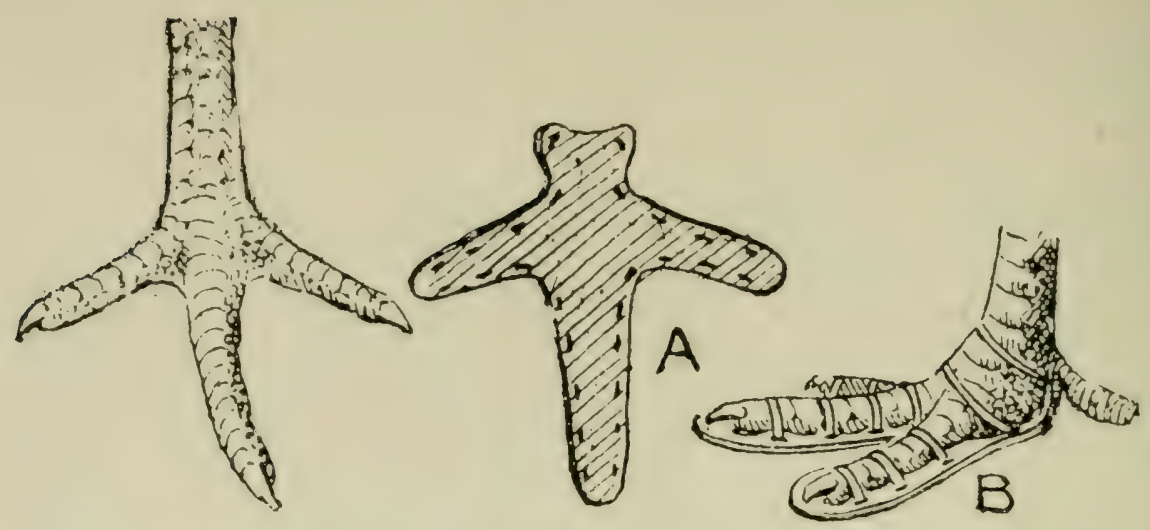

Fig. 87. Crooked Toe and Its Remedy.

To the left is the crooked toe. a, the pad. b, the pad adjusted. This pad can also be used on birds just recovering from abscess of the foot.

top, and up the leg. The foot may be swollen and tender to the touch. The chicken sits down much of the time. Toes may drop off or if recovery takes place the toes are distorted. These same conditions may attack the head. The disease may prove fatal in from one to two days to that many weeks. Mortality may reach 90 per cent. The feet become somewhat shrivelled or appear "dried up." The toes may become crooked and finally dry gangrene and death of the young bird result. These conclitions have been produced artificially by allowing the chicks to run over cacti thorns. The thorns entering the tender skin of the chicks causing blisters and sores.

Treatment.-Dip the feet and affected parts in kerosene. As a preventive it is advisable to rear the chicks on ploughed ground.

\section{PERICARDITIS}

Pericarditis is an inflammation of the pericardium or heart sac; there is usually a sero-fibrinous effusion about the heart, and it is often spoken of as dropsy of the heart sac or dropsy of the heart. It is, of course, not strictly a blood disease, but it is often associated with diseases of the blood and of the lungs, as a complication; further than this its cause is not known, but may result from exposure to cold and dampness. It may be a complication of fowl cholera, of acute tuberculous origin, or pneumo-pericarditis, the latter an extension of the inflammation from the lung structures to the pericardium. I'urulent pericarditis is also quite frequently observed in the fowl. Verrucose pericarditis has been observed.

Symptoms.-A diagnosis of pericarditis cannot ordinarily be made during life of the bird, but is easily demonstrated on autopsy. Among the symptoms are intense dyspmoea or very 
difficult breathing, the beak being held wide open. Tumultous heart action, extreme exhaustion on exercise may exist. The bird may fall if forced to move and death may occur from syncope, or heart failure. There may be a degeneration of the heart muscle and possibly of the pericardium.

Treatment.-Treatment is unsatisfactory; numerous cases occurring in the same flock should lead to the enforcement of better hygienic conditions, especially to better protection from cold and dampness.

\section{ENDOCARDITIS}

Endocarditis is an inflammation of the lining membrane of the heart, usually affecting the valves. Nothing is known of its cause, but it is of not infrequent occurrence during the course of certain diseases of the blood. It cannot be diagnosed during life, and therefore cannot be treated. From what we know of the cause of endocarditis in man and animals, we should expect exposure to cold and dampness to be a factor in the cause of this disease, and such conditions are to be avoided. Verrucose pericarditis has been found. The nodules form in a line or in an irregular manner in the vicinity of the valves. These nodules may become so large that they interfere with the action of the valves and a regurgitation of the blood results.

Chronic endocarditis is often observed. In old birds calcification of the aortic walls has been observed.

\section{MYOCARDITIS}

Myocarditis is an inflammation of the heart muscle and may be either acute or chronic, local or diffuse. Primary types may occur but it is probably nearly always secondary to some other affection. Both acute nonsuppurative and acute parenchymatous myocarditis may occur in fowls. Acute suppurative parenchymatous myocarditis has been observed and studied in these laboratories.

Symptoms.-The symptoms, as we have observed them, are general depression with possibly a partial loss of appetite and gradual emaciation. The head and external appearance show no specific sign of the dicease except for the unkempt appearance of the feathers. The comb may be dark and dried indicating that the bird is not in a laying condition.

Postmortem Findings.-The heart may be noted to be enlarged and the whole mass present a parboiled appearance. Upon opening the pericardium or pericardial sac it is noted to contain a small quantity of purulent material. The pericardium is thickened and the inner wall is rough as well as the outer surface of the heart. 
In sectioning the heart the heart muscle is noted to contain whitish areas not unlike neoplasms.

In these cases the liver may be enlarged, in fact sometimes it is three or four times its normal size. The outer capsule appears thickened, congested, and possibly hepatitis may be present.

The kidneys may be light gray in color, in fact mottled. Nephritis may be present.

Microscopic Examination.-The pericardial wall is thickened due to the infiltration of inflammation cells.

In some areas the heart muscle cells are losing their cross striations and further along these cross striations entirely disappear.

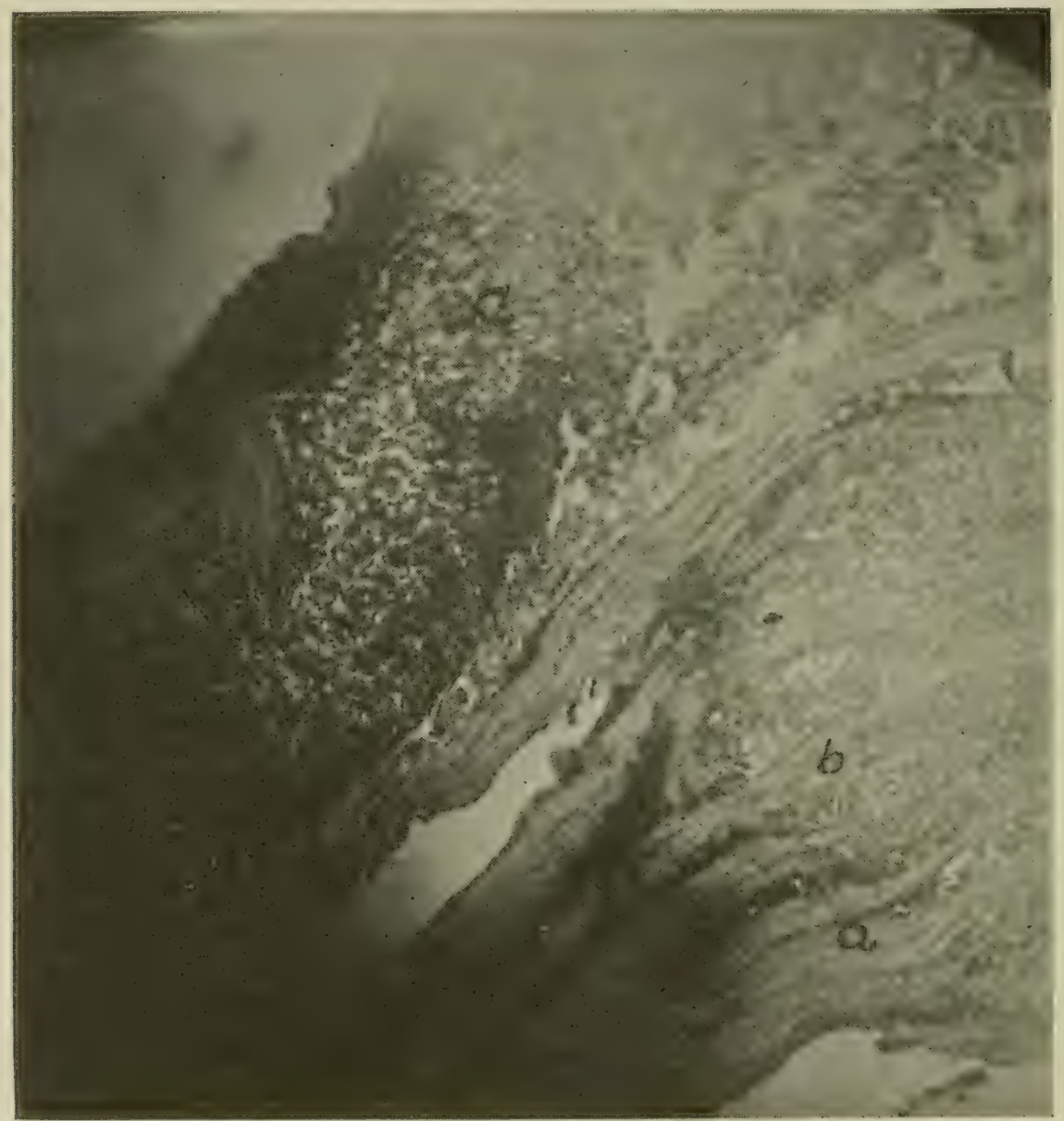

Fig. 88. Purulent Mtyocarditis of a Single Comb Wiitte Legiorn Hen. $a$, heart muscle structure; $b$, heart muscle fiber separated by masses of inflam. mation cells; c, a small abscess of the heart wall.

In these latter parts there is a cellular invasion in some areas these cells have pushed, in groups, between the bundles of the muscle fibers, as seen in Fig. 88. In still other places small focal abscesses have formed.

In suppurative myocarditis there may also be present in the kid- 
neys both active and passive congestion, cloudy swelling, and obliteration of the lumen of the convoluted tubules. Focal areas of inflammation and acute parenchymatous nephritis may be present. The same conditions may exist in the liver.

\section{HYPERTROPHY AND HYPERPLASIA}

Hypertrophy literally means excessive nutrition. By usage the term has come to mean an abnormal increase in the size of an organ or part. In a more restricted sense it is used to mean an abnormal increase in the size of the essential cells of the part.

Hypertrophy of the heart has been thought to be caused by obstruction of the circulation due to tuberculous growths in the liver or mesentery and also a sequel of gout and rheumatism. It is observed in both fowls and cage birds.

\section{HYPERPLASIA OF THE INTESTINES}

Hyperplasia indicates excessive formation and should be used to designate the condition resulting from an abnormal increased size of the part due to an increase in the number of cells of that part. Hyperplasia may be due to an increased number of parenchymatous cells, or an increased number of interstitial cclls. Interstitial hyperplasia is the more common form and is usually a sequel of chronic inflammation. It is supposed to be caused by long continued action of mild irritants.

Parenchymatous hyperplastic structures vary in appearance according to the amount of hyperplastic connective tissue. The part may vary from normal to dense, hard, pale irregularly lobulated masses of fibrous tissue.

Intestinal hyperplasia is often noted and in Fig. 89 is shown a drawing of caecal hyperplasia of a two-year-old Silver Penciled Wyandotte cock.

Symptoms.-The bird does not do well for some time before death and gradually becomes thin in flesh. There may he more or less loss of appetite.

Postmortem Findings.-Upon examination of the abdominal organs the hyperplastic intestine will appear to be enlarged and when cut through the walls will be observed to be greatly thickened. In the case shown in Figure 89 the walls were three millimeters in diameter. The lumen was very small and the entire portion of each of the caeca was uniformally involved.

The accompanying drawings show the relative thickness of the walls as compared to those of a normal caecum. These two drawings are made natural size. It will be seen that there is a uniform increase in the cellular structure of the external longitudinal and inner muscular coats, as well as of the mucosa and submucosa. In the last drawing there is illustrated a composite drawing of the various kinds of cellular elements. There will be noted the normal 
muscle cells, connective tissue cells, mononuclear leucocytes that have invaded, to some extent, these parts. There are also numerous coccidia in the various stages of development. These coccidia were observed invading the surface epithelial cells and in clusters in the subendothelial spaces, that is, between the muscles in the connective tissue spaces. There appeared to be a moderate number of them. There was no indication of necrosis in any part microscopically examined.

There is a possibility that these coccidia furnished the mild irritation causing this hyperplastic condition.

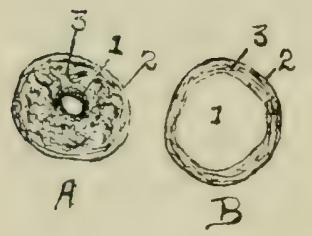

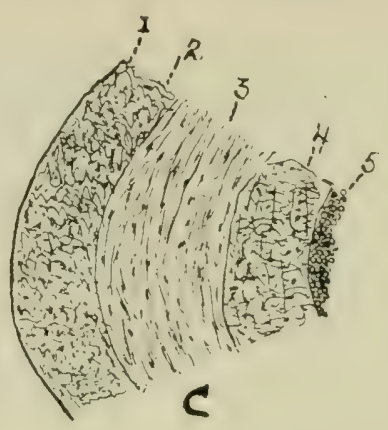

$6 x+10 x$

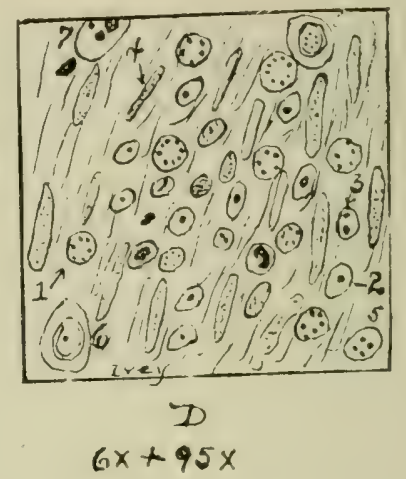

$6 x+95 x$

Fig. 89. ityperplasia of the Caecum of a Cock.

a, a tranverse section through the caecum showing at 1 : the mucous membrane; 2, the muscular layers; 3, the serous covering. b, same of a normal zaecum. c, a drawing of a higher magnification of the transverse section of the hyperplasia showing relative thickness of the various coats; 1 , serous membrane: 2 , outer longitudinal muscular coat; 3 , inner circular muscular coat; 4, muscularis mucosa; 5, the mucous membrane. d, a composite drawing from the mucosa; 1 , schizont; 2 , oocyst; 3 , sporoblast; 4, connective tissue cell; 5, sporozoit; 6 , epithelial cell; 7, epithelial cell just invaded by a spore.

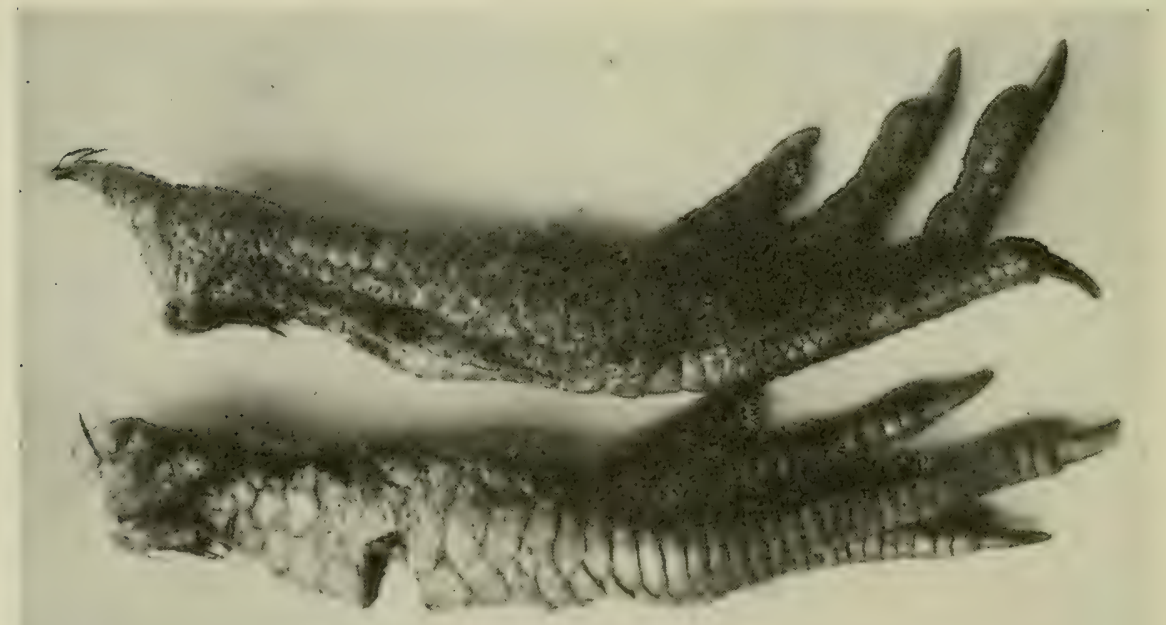

Fig. 90. Productive Inflammation of the Shank of a S. C. Rhode Island RED Cock. 


\section{PRODUCTIVE INFLAMMATION OF THE SOFT STRUC- TURES OF THE SHANKS AND COMB OF FOWLS}

A productive inflammation of the shanks or of the comb may be observed in both cocks and hens over two years of age.

Sy'mptoms. - The shanks or comb affected gradually becomes larger due to new formed connective tissue and the condition is apparently brought about without any signs of acute inflammation. The increasing enlargement is of the soft structures alone, the bony structures, in these cases, not being involved. The condition, as affecting the shanks, is more common in cocks than in hens. The bird may later have a stiff gait, and as the enlargement progresses the bird finally becomes lame, and unfit as a breeder and later dies. In some of these cases there is also observed, by microscopic study, nephritis and other changes of the parenchymatous organs. Fig. 90 shows a photograph of a typical case affecting the shanks of an S. C. Rhode Island Red cock.

\section{RUPTURE OF THE HEART AND LARGE BLOOD VESSELS}

Internal hemorrhage, or bleeding, due to rupture of the heart or large blood vessels is common in overfed fowls. It may be caused by any excitement or overexertion in such birds.

Symptoms.-There is a sudden blanching of the comb, wattles, face, and mucous membranes followed by great weakness, coma or unconsciousness and death. No treatment is practicable.

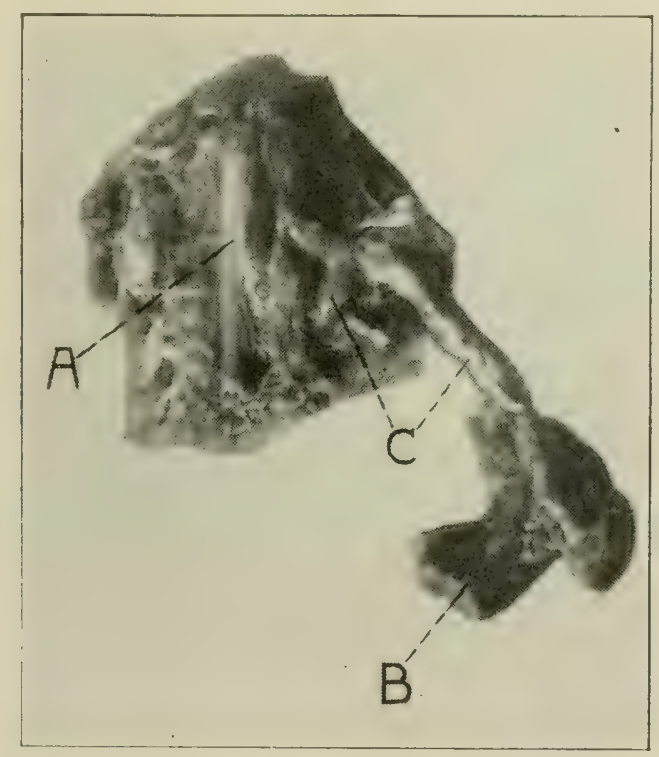

Fig. 91. Thrombosis in a Hen.

a, pelvic bone, central portion; b, muscle of thigh; c, blood vessel containing a white
thrombus. 


\section{THROMBOSIS}

Thrombosis is a blood clot in a vessel during life of the bird. This clot forms a solid mass consisting of fibrin, red blood cells, and thrombocytes and leucocytes.

Agony thrombi are those clots formed during slow death and may extend from the heart to many of the ramifying blood vessels. They are mostly white in color.

Thrombi have been studied in birds Fig. 91 showing a photograph of such a case.

Symptoms. - There is a partial loss of appetite, finally complete loss; the bird shows weakness and a gradual emaciation. The hen dies in about two or three weeks.

Postmortem Findings.-On autopsy there will be noted great emaciation. All organs appearing normal except the affected circulatory system. In the case of the specimen shown in Fig. 91 there was a complete plugging of the right brachial artery, the artery to the right wing, and the same of the large vessel of the liver, as well as of the iliac and femoral arteries of the left side and leg. Upon microscopic examination there was observed a white thrombus. The picture shows the blood vessels quite distended by the thrombi.

\section{GANGRENE}

Gangrene is death of a part. There are two kinds of gangrene, moist and dry. Death of a part en masse constitutes gangrene and death cell by cell, if on the surface, constitutes ulceration: If the part which dies has poured out in it serum, or it is in fatty tissue, or if the dead part becomes infected the

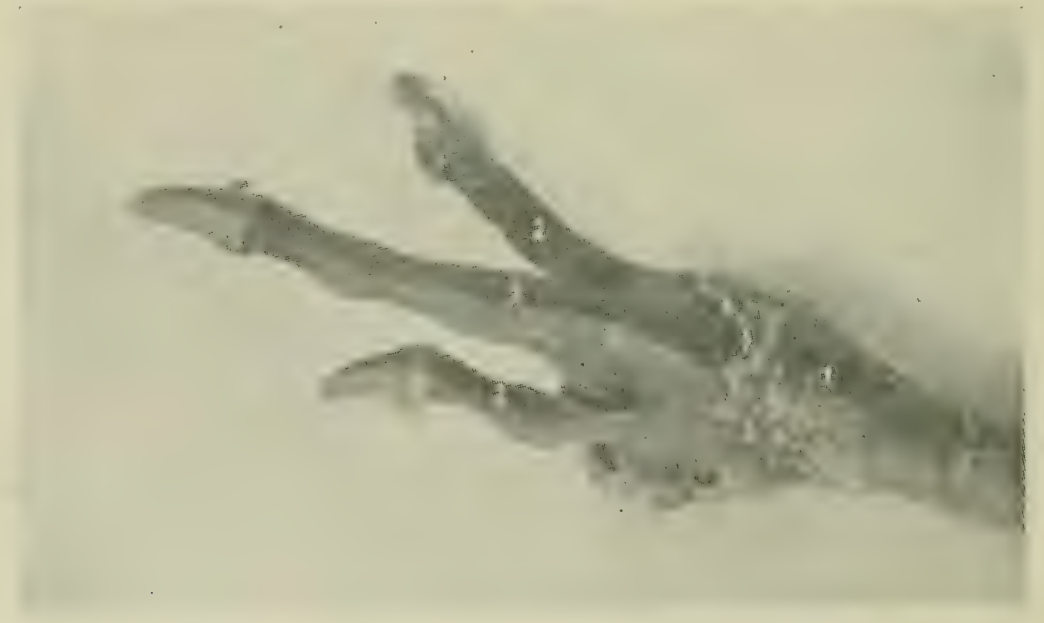

Fig. 92. Giangrene of the Foot of a Turkey. Commeneed with fluctuating abscess between the toes whicly when lanced gave off an offensive odlor and appeared somewhat gascous. Later the parts became in a state of clry gangrene. $\Lambda$ smear from the pus showerl many cosinophiles, lymphocytcs and polymorphonuclear leukocytes. 
condition will be that of moist gangrene. Certain kinds of gas forming organisms will cause an emphysematous or gasseous gangrene. If the part is poorly supplied with blood and there is no serum brought there dry gangrene may result. The part dries and shrinks in size and does not give off an offensive odor. Dry gangrene is likely to occur in dependent parts, as in the illustration, in the turkey's foot.

\section{INFLAMMATION OF THE OIL GLAND}

In the fowl there are no oil glands or sweat glands in connection with the feathers. This is compensated for by an oil gland located in the posterior dorsal region of the abdomen and at the base of the tail. The bird obtains the oil with its beak and stripping each feather seperately oils them.

Inflammation of this double lobulated tubular gland of ten occurs. The region of the gland becomes swollen, red, and painful to the touch. Miscroscopic examination shows a true acute inflammation. The ducts are occluded and the bird suffers considerable pain. Ifot applications are indicated. If possible by massage relieve the distended oil gland by pressing out the oil.

\section{SWELL-HEAD IN YOUNG TURKEYS}

The most characteristic symptoms of this ailment is swelling of certain parts of the head, especially in the region of the infraorbital sinus, which becomes filled with a gelatinous substance.

There has been isolated from these swellings a bacillus.

These swellings may disappear in a few days or weeks or they may remain for several months. In the latter instance the swelling may contain a cheesy foul smelling material, and in some cases they may cause death.

Treatment.-Open the swelling with a sharp knife and allow the gelatinous material to escape. In addition swab the cavity with pure tincture of iodine.

\section{POX OF TURKEYS}

A condition among turkeys has been described which apparently differs from chicken pox. It is, in some years, quite common in the southern states and in Cuba.

The condition was first observed by the writer in turkeys that had been kept in the back lot of a city residence. There were four turkeys in the flock and one after the other became affected.

From the standpoint of a field study it appears to be contagious. 
The pox are noted on the unfeathered portion of the head and neck. There is at first noted a small pimple-like elevation, which gradually becomes larger and in the course of a few days may appear four or even five millimeters in diameter and two or three millimeters in elevation. They do not appear as in the case of chicken pox; that is, they have no rounded bleblike appearance, but have almost perpendicular walls, with flat tops, and are shaped like an opera hat.

In the course of a few days, in many cases without any treatment the diseased areas become dry and when healed the side walls may be picked off, leaving a whitish, scar-like spot. The disease usually does not appear very virulent and little or no treatment is required. In some cases where we have advised the use of a five per cent carbolised vaseline, excellent results have been reported. Again. where we have had cases under observation, they recover without treatment. However, there can be little doubt but that at times the attacks are rather severe and may even cause death. While this has been reported to us from good, reliable sources, yet we have not personally made such observations. The cases studied did not appear as causing great suffering of the birds, as there was no loss of appetite and the birds were in good flesh.

Only one test was made to determine if the disease could be transmitted. The curetted material from a fresh nodule was rubbed in a scarified area of the comb of a three-year-old White Orpington cock. The results of this one test were negative. No opportunity was afforded to conduct experiments upon birds not exposed to the discase, though such procedure is contemplated.

\section{DIPHTHERITIC INFLAMMATION OF THE EYES OF DUCKS}

A diphtheritic inflammation in which there is formed a membrane over the affected part with destruction of the underlying cells occurs in ducks. In the diphtheritic inflammation of the eyes of ducks there is first noticed an itching of the eyes manifested by the duck rubbing them. There is noted later an inflammation of the conjunctival mucous membrane lining the eye. This membrane becomes thick, red, and there is noted a yellowish secretion. Diphtheritic areas may develop with ulceration of the cornea or front part of the eyeball. The discharge from the eye is caustic to the skin with which it may come in contact. The ducks become emaciated. The disease may last for several weeks. There appears to be no marked changes in the internal organs.

\section{ULCERATION OF THE CORNEA}

Conjunctivitis is an inflammation of the mucous membrane lining the front part of the eyelall. Tleeration is death of a 
part, cell by cell, and is always on a surface. At times this inflammation spreads to the cornea or front part of the eyeball. The inflamed cornea becomes cloudy and finally may totally obstruct the sight. There is frequently observed in connection with this an ulceration of the cornea with perforation of the eyeball. Fig. 93 is a photograph of ulceration of the cornea with perforation of the anterior chamber in a Single Comb Rhode Island Red chick which had developed conjunctivitis and keratitis when only three weeks of age. This condition is common in small chicks.

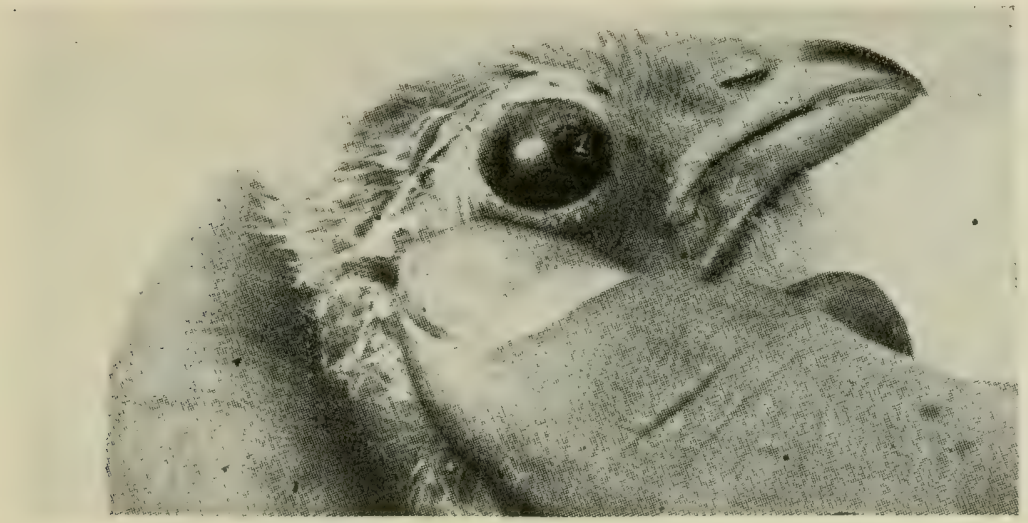

Fig. 93. Ulceration of the Cornea With Hemorrinage Into the Anterior, Posterior and Vitreous Chambers in a Three-WeEks-Old Chick.

The eye was enucleated, hardened in 40 per cent formaldehyde solution and sectioned for study. It was observed that there was an inflammation with hemorrhage of the entire eyeball-a pan-hemorrhagic condition.

Ulceration of the cornea is also sometimes observed in conjunction with the eye type roup. There is an intense conjunctivitis, an accumulation of quantities of purulent or catarrhal products in the conjunctival sac and a keratitis and later panophthalmia or an inflammation of the entire eyeball.

\section{FROZEN COMB}

The degree of cold at which the comb and wattles freeze depends upon many factors. The humidity in the house and the physical condition of the bird and its heart action are the most important factors. A weak heart means a sluggish circulation and the more sluggish the blood flows the slower it passes through the dependent parts and the quicker the comb freèzes. 
Wattles usually freeze more readily than combs because when the bird drinks it usually gets its wattles wet.

Birds in open front houses can stand lower temperatures than when the house is tightly closed, becanse the nore free circulation of air allows the watery vapor from the lungs of the birds to disseminate more rapidly and humidity is lessened.

When wattles and comb are frozen, remove the bird to a wamer room but not one heated by a stove. Apply carbolised vaseline twice a day.

\section{BROKEN BEAKS}

The beak often becones broken through fighting through a wire fence or by other violence. Such a fowl needs attention. It may starve through its inability to pick up feed or its beak may grow deformed.

The bird should be given feed in such a manner that it can easily pick it up, or the new and developing horny material may become injured. Mash, both wet and dry, given in a cup, is recommended. The cup should not be allowed to become so nearly empty that the bird strikes the bottom with its broken beak.

Often chicks are hatched with cross beaks, making the act of taking up feed a difficult one.

\section{ARTHRITIS-TERMINATING IN OSTEITIS OF THE FEMORO-TIBIAL JOINT OF THE COCK}

Quite often when cocks fight through a wire netting fence there results injuries to the hock or femoro-tilial joint or both. This results in inflammatory changes in both the soft and bony structures, a chronic osteitis. There may result infection of the injured part. Acute inflammation with, or without pus formation may result.

The early changes are congestion and exudation in the synovial menuluane, congestion and exudation in the marrow spaces with widening of these, and degeneration of the articular cartilage. These changes may take place at the same time ir they may occur separately. The process may become quiescent at various stages.

A traumatic injury may cause an ossifying periostitis which may later spread to the synovial membranes and then to the joint.

Symptoms.-In a fight with a cock in an opposite pen the livel injuring the femoro-tibial or other joints will he noted to be lame. He will not tred hens and is of no further use as a hreeder. The hird will be continuously lame when such proccsecs as a rarefying ostitis occur and after several months will 
die. The bird may retain a fairly good appetite but may gradually lose flesh. The joint will appear enlarged as a result of the chronic inflammatory processes. Fig. 94 shows the results of a chronic ostitis of the femoro-tibial articulation in a state of chronic inflammation. The bird died after eight months.

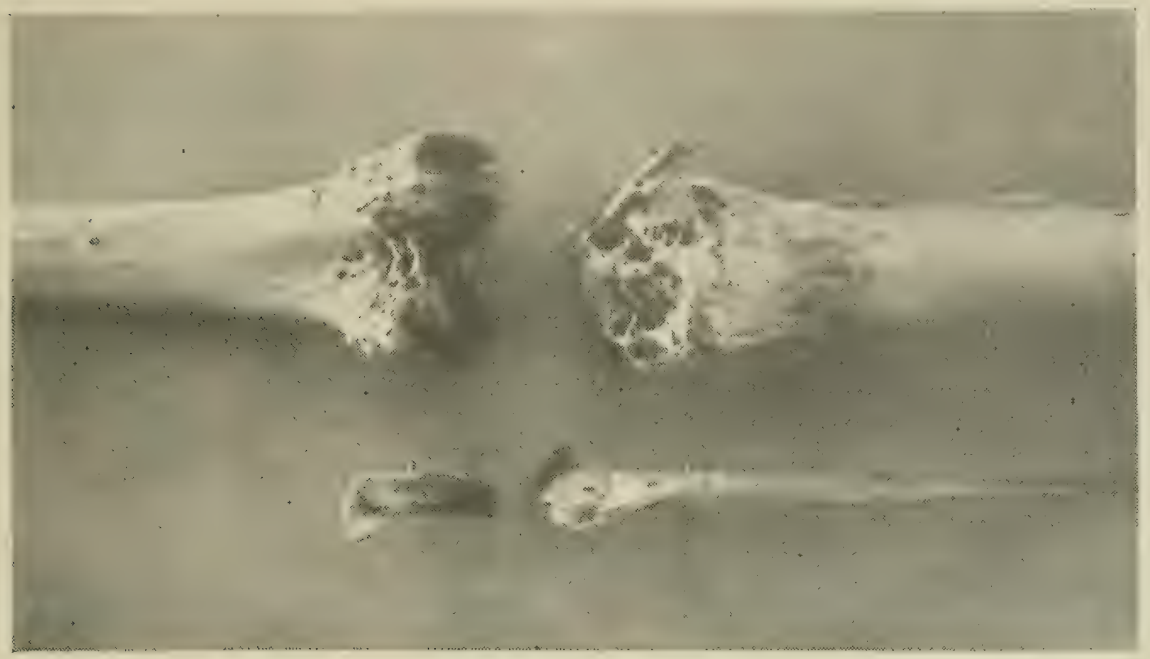

Fig. 94. Ostitis of thi: Femoro-Tiblal Articulation of a Cock.

1 , distal extremity of the femur; 2, proximal end of the tibia; 3 , proximal cnd of the fibula; t, the patella.

\section{OPEN JOINTS IN FOWLS}

The joint of the fowl, like that of mammals, consist of the articular surfaces of two or more bones making up the joint, and a capsular ligament lined with a synovial membrane. The articular ends of the bones are covered with a hyaline cartilage. The joint synovial membrane secrets a serous fluid which lubricates the joint to prevent friction during movement of one bone on the other. When the membrane is opened the fluid escapes and air enters the joint carity, causing much pain to the individual. Movement of the joint is impaired, and, in fact, becomes impossible for the bird to bear the weight of the body upon the injured leg. The constant escape of synovial fluid prevents complete healing of the punctured or lacerated wound and a fistula results. Following this, is a result of a chronic inflammation, the joint becomes enlarged, and the patient becomes thin in flesh from constant pain and finally dies or has to be destroyed for humane reasons. Chronic inflammation which often extends to and involves the bone may result. Rarefying ostitis has been observed in such cases of several months standing. Upon examining the ends of the bones a rarefying ostitis, as shown in Fig. 94, is observed. 



\section{CHAPTER XII}

\section{TUMORS}

The word tumor, which means swelling, has been variously applied in medical and veterinary literature. Some writers include among the tumors cellular collections clearly inflammatory in origin; to each aggregation of cells is given the name inflammatory tumors, granulation tumors, etc. Later writers exclude all such masses and restrict the term tumor to; first, neoplasms or new growth of tissue developing independently in any tissue of the animal body and atypical structures in function, and, cysts.

Tumors of various kinds affect birds, but are less common than in higher animal life. There is almost no literature on the subject.

The Maine Experiment Station reported, through the Journal of Agricultural Research, the results of several hundreds of autopsies. It was observed that of 880 birds autopsied 79 , or 8.96 per cent had tumors; that is, there were 90 cases of tumors per 1,000 birds.

There was no significant difference in frequency. of occurrence of tumors between birds that died from natural causes and apparently normal birds that were killed.

There was a significant correlation between age and the occurrence of tumors. Only 7.37 per cent of the birds under two and one-half years of age had tumors, while tumors were present in 19.17 per cent of those that were over that age.

In birds which have died from natural causes, with tumors, the tumors were the probable cause of death in from one-third to onehalf of the cases.

There was a tendency to the association of hypertrophied liver, spleen, or kidneys, with the presence of tumors in other organs.

Death often resulted from internal hemorrhage from the tumors, the underlying tissue, or the hypertrophied liver or spleen.

The tumors can be classified into cystic and tissue tumors; 22.78 per cent. of the tumors are cystic and 74.68 per cent. are of solid tissue structure. There are two cases of tissue tumors to which cysts are attached.

In the females the organs most frequently affected are the genital organs; 37.76 per cent. of all tumors are in the ovary and 18.36 per cent, are in the oviduct and oviduct ligament.

In most cases the tumors are confined to one organ. In fifteen cases, however, in these studies the tumor had evidently undergone metastasis, since tumors of similar nature occurred in from two to four organs. 


\section{CLASSIFICATION OF TUMORS}

Tumors are of two kinds; first, benign; and second, malignant.

A benign tumor is one that does not necessarily involve danger to life, while the malignant tumor, if undisturbed will eventually prove fatal.

The benign or so called innocent tumor may cause death of the birds from its location, as for example, a fibrous tumor growing on the inside of the skull.

Tumors are composed of cells and usually, an intercellular substance. The cells may be similar to normal embryonic cells or to adult cells. The embryonic tumor cells differ from normal embryonic cells in that the former have no tendency to become matured while the latter have.

Tumors having a mesodermal origin usually have an intercellular substance closely resembling that of normal connective tissue and hence may be mucous, fibrous, cartilaginous, or osseous. Tumors of an ectodermal or an entodermal origin may appropriate the pre-existing tissue framework for their stroma. Some tumors, like some normal tissue, are practically devoid of intercellular substance. Anatomically the intercellular substance or stroma is an integral part of a tumor and its function corresponds to the function of intercellular substance of normal tissue. The cells and intercellular substance of tumors may be so arranged that the resulting structure approximates that of normal tissue, but is never identical to a normal tissue. Tumors may be composed of structures derived from all three germ layers grouped indiscriminately but having some resemblance to an embryo-teratoid tumors.

Like normal tissue, tumors are usually nourished, by blood and lymph. The blood and lymph vessels may be structurally the same as normal vessels, or they may be composed entirely of tumor cells. The vessels have their origin from pre-existing vessels in the tissue from which the tumors are developed.

The form of tumors is largely determined by the location and the kind of tissue in which they occur. They may be spherical, oroicl, ellipsoid, nodular, miliary, tubercular fungoid, polypoid, tabular, elongated, cylindrical, etc. Where there is no resistance there is a tendency to be round or spheroid. In outline they may be regular or irregular, smooth, nodular, or even granular and in some cases the exact outline cannot be determined.

The color of the tumor depends upon; first, the kind of tissue composing it; second, pigmentation; third, degeneration; and fourth, the amount of blood they contain.

In consistency some tumors are soft and spongy, jelly-like, 
and from this type there are all variations up to that of a hard and resistant tumor consisting of bone formation. The consistency depends upon the kind of tissue composing them.

As to number tumors may be single, that is, only one occurring in the bird's body. Most single tumors are benign and, as a rule, do not cause serious trouble. The bird may be afflicted by many tumors, in fact, multiple tumors. Tumors may form by metastaces, that is, tumor cells may be carried by the blood from one malignant tumor and ceposited in another part of the body and a secondary tumor occur.

The growth of tumors varies, some growing very slow as in most benign kinds, or the growth may be more rapid as in the sarcomas - the malignant kind.

Tumors are subject to the same degenerative changes that normal tissues are, such as hemorrhage, necrosis, and degeneration.

There appears to be no satisfactory method of classifying tumors. Tumors have been grouped as follows:

\section{Occurrence :}

Primary.-The original or first tumor.

Secondary.-Metastatic tumor.

Recurrent.-A newly developed tumor at the point from which one has been removed.

\section{Structure:}

Histoid.-Simple tissue tumor.

Organoid.-Organ-like tumor.

Teratoid.-Mixture of various tissues resulting in a structure resembling an embryo.

Clinically :

Benign.-No tendency to kill.

Malignant.-Having a tendency to kill.

The following diagramatic schome shows the general structure and relations of the various tumors:

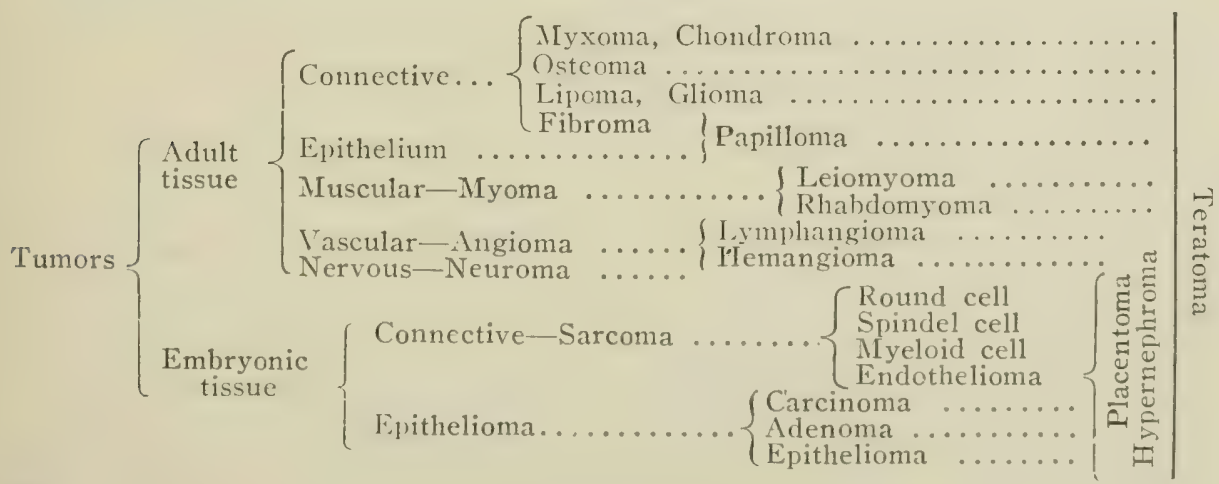




\section{EPITHELIAL TUMORS ADULT EPITHELIAL TUMORS PAPILLOMA-WARTS}

These growths are formed from the epithelia of the skin, or mucous surfaces. If the wart occurs on the skin it is made up of a connective tissue core covered by stratified squamous epithelia. If they occur on the mucous surface they are rather soft while those on the skin surface are hard.

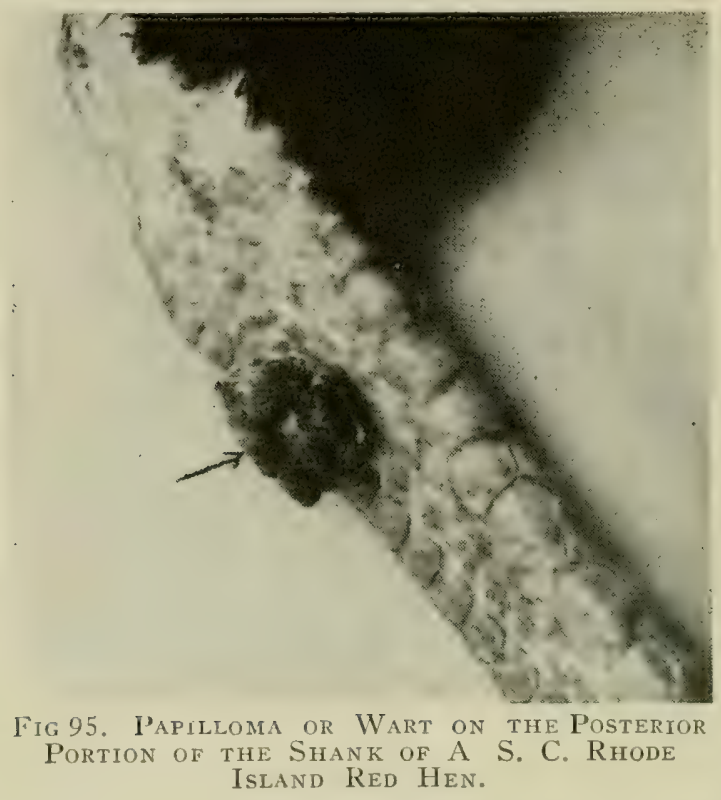

\section{ADENOMA}

An adenoma is a tumor constructed upon the type of a gland. Adenomata are of slow growth, and are believed to have their origin in some quiescent, congenitally displaced cell. They are as a rule completely encapsulated. They are benign. The tumor is made up of connective tissue and asini, or cavities, lined by columnar or cuboidal cells. They do not have communicating ducts. Such tumors have been observed in the fowl, the writer having studied one in the spleen.

\section{EMBRYONIC EPITHELIAL TUMORS CARCINOMA}

Carcinomata or cancers are made up of epithelial cells grouped in nests or alveoli. These tumors have no selective action for any tissue. They may be observed in any part of the body. They are usually diffused, that is, no limiting membrane or fibrous capsule as is usually seen in the benign tumors. They are malignant spreading into surrounding tissue and their cells 
being carried by the blood or lymph current and starting secondary centers. When cut out they recur. The color of a cut section of a carcinoma is usually gray, dirty-white, or pale pink. Studies under the miscroscope show them to be made up of nests of embryonic epithelial cells surrounded with connective tissue. The cells vary in size and shape and may be squamous. cylindrical or spherical. The nucleus is usually much smaller to the size of the cell than the nucleus of the sarcoma cells. True carcinomas do not appear to be common in the fowl.

\section{EPITHELIOMA}

This type of tumor is the result of an ingrowth of epithelium into the underlying structures and has been classed by some as

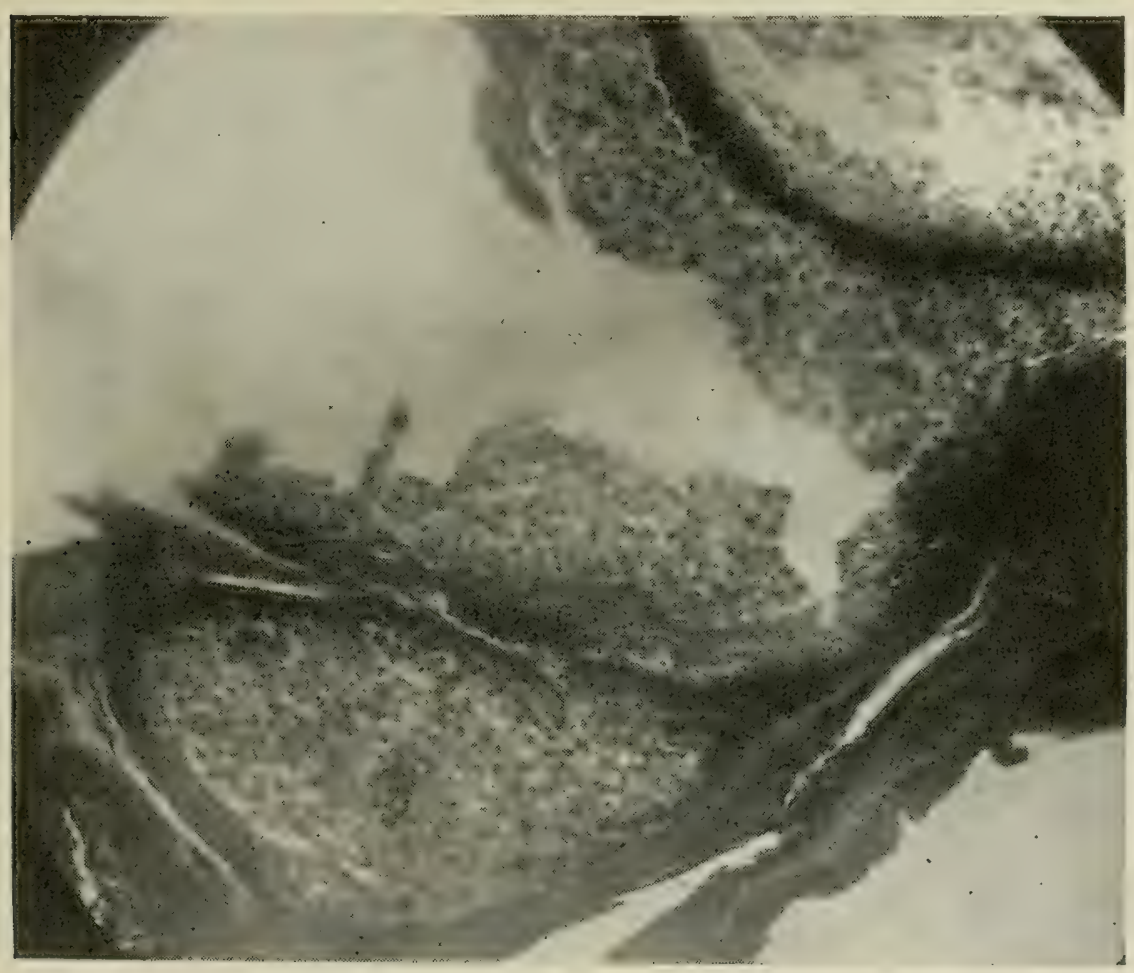

Fig. 96. Epithelioma jirom time Skin Surface of a Hen. $a$, the connective tissue; b, the epithelial cells.

a sub-variety of carcinoma. In this variety there is considerable evidence that they are seconclary to surface injuries. This form of cancer is referred to as skin cancer. It is often observed in the fowl.

Contagious epithelioma of young fowls, in which there are tumors form, varying in size, from a pea to a large philbert, show cells in long nests in true epithelial form. Fig. 96 is a typical illustration of a photomicrograph from such a study. 


\section{CONNECTIVE-TISSUE TUMORS ADULT CONNECTIVE-TISSUE TUMORS LIPOMA}

A Lipoma is a fatty tumor and is localized, more or less circumscribed, new formation of fat. Lipomata are usually lobulated, soft, doughy, and elastic. As to shape they are ovoid, spheric, or flat. If encapsulated the capsule is very thin. Nonencapsulated fatty tumors may be diffused, the margins often not being well defined. Lipomata often occur in the fowl.

\section{CHONDROMA}

Chondromata are masses of new tissue, composed of hyaline, elastic, or white fibrous cartilage. Chondromata may develop from pre-existing cartilage, such as cartilage from the larynxes, or trachea and may assume a pollypoid shape. They may develop from bone or in tissue not normally containing this element, the cartilaginous tumors are often spoken of as enchondromata.

Microscopically, the cells of the tumor resemble those of true cartilage; the intercellular substance may be hyaline, faintly or distinctly fibrous, or, in rare instances, mucoid. They are not as a rule composed of dense cartilaginous tissue, but of islands of cartilage surrounded by fibrous septa.

\section{OSTEOMA}

An osteoma is a tumor-like mass of bone developed without occurrence of inflammation or incident to the process of repair; usually such tumors occur at the point of juncture between a bone and cartilaginous tissue. The tumor may be marle up of compact bone tissue or of spongy bone tissue and are designated as exostosis, or those growing from the exterior of the bone, and, enostosis, or those growing from the interior of the bone. They may derelop entirely independent of any bony or cartilaginous structure.

Osteomata usually occur singly in the body, that is, they are localized.

New bony growths not attached to the skeleton may be classified as follows: Those which have their seat in the periostemm but are separate from the bone are called movable periosteal exostoses; those lying near the bone are called periosteal osteomata; those located some distance from the bone, in muscle and tendon and disconnected, osteomata; and those situated in other organs, as the lungs, mucous membrane of the trachea, the skin or abdominal walls, are heteroplastic.

(Ostermata may oceur either single or multiple. Frequently there are found multiple, circumscribed bony growths in great 
numbers on the bones of the extremities and trunk. The favorite seat of these new bony growths appears to be on the ends of the bones and the point of insertion of tendons, or both may be involved in the same individual and at the same time. It is probable that such growths are to be referred to as

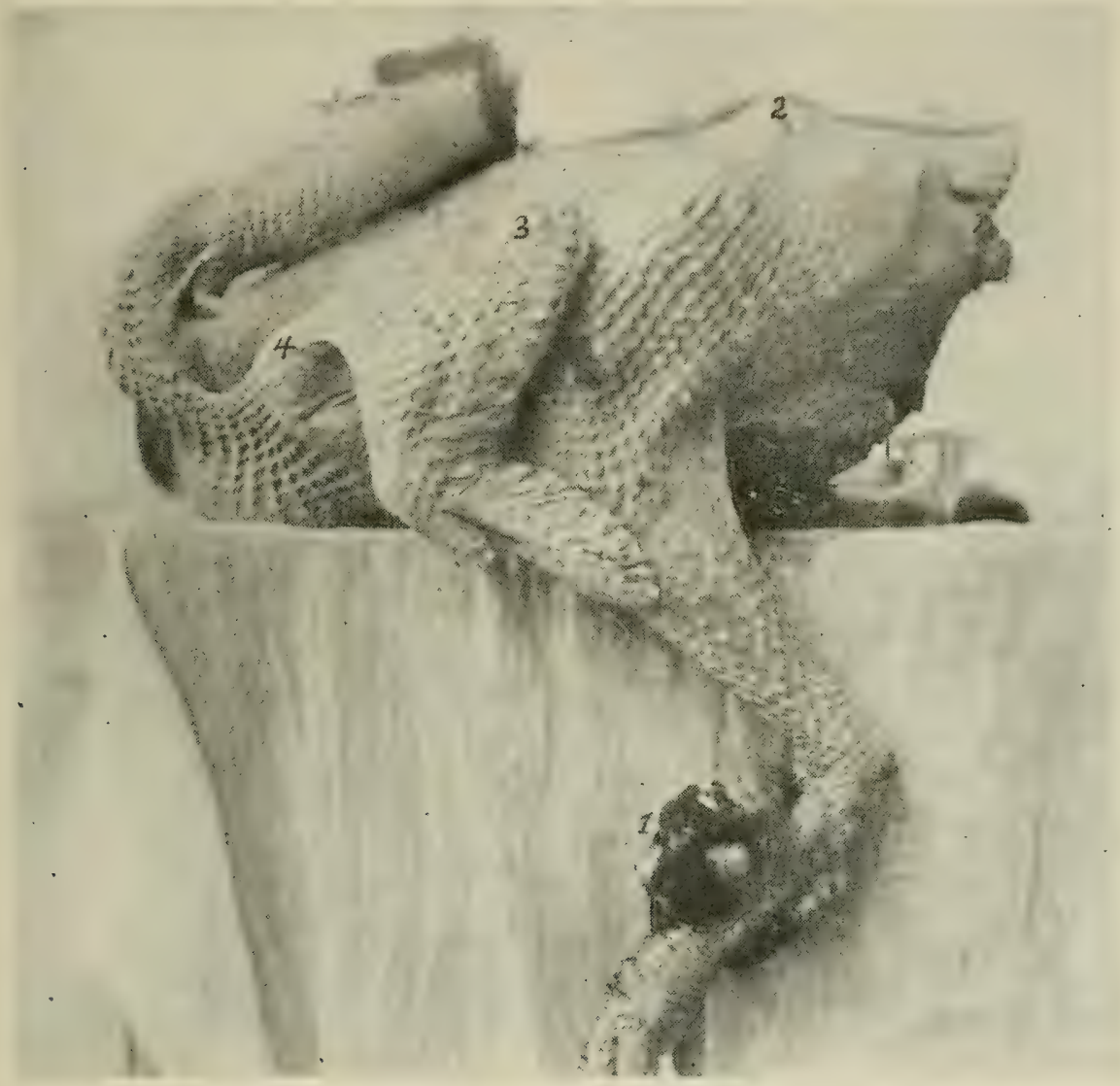

Fig. 97. Osteona of a Hen Jnvolving Many Pirts.

1, tmmor of the leg; 2 , tumor of the lumbo-pelvic region; 3 , tumor of the clbow; $t$, tumor of the shoulder joint. Note that the most of the bony tumors are at the juncture of the cartilage and bone.

inherited predisposition of the part affected to overgrowth, or to a disturbance in the development of the skeleton. The bony plates and spicules, which in rare cases develop in the lungs or in the mucous membrane of the air passages may occur in large numbers. Osteomata have been observed in the fowl involving many parts in the same individual.

The development of the bone takes place partly through the formation of osteoblasts, and partly through metaplasia of formed tissues. The matrix is formed chiefly from the connective tissue of the periosteum, as well as that of the tissue 
from which the osteoma arises; and also from that of the perichondrium and endosteum. If an exostosis develops in such a manner that cartilage is first formed from the proliferating periosteum or bone marrow, and from that cartilage, bone is later developed, it is called a cartilaginous exostosis; when the exostosis is formed directly from the proliferating periosteum without an intermediate stage of cartilage, it is known as a connective tissue exostosis.

If the connective tissue in a bony tumor is abundant, and in fact a predominating substance, it is called an osteofibroma. This form of tumor appears quite common among bony tumors.

An abundant of bone in a chondroma leads to the formation of an osteochondroma. These latter are usually observed in the long bones.

A condition in which the muscles become bony in nature is called myositis ossificans. The tendons of birds and especially those of the leg often become ossified, especially in old birds. This is also the case in some of the vertebral muscle tendons.

\section{FIBROMA}

Fibromas are tumors composed of wavy bundles of fibrous tissue and are observed in the fowl. The skin and subcutaneous areolar tissue is their most frequent location. They may also be observed in connection with the visceral organs. What few fibroma the writer has observed in the fowl have been small in size. The tumors are usually single, that is, one tumor occurring in the individual. They are usually oval in shape. It is surrounded by a more or less thick capsule. If on the surface they are not difficult to remove. Osteoma, and chondroma, are benign. They do not recur when removed.

In color the sectioned surface appears pearly white with grayish white areas. If the fibers lie close together it is called a hard fibroma, and if loosely arranged a soft fibroma.

\section{MYOMA}

A myoma is a tumor composed of muscle tissue. Two forms of myoma are recognized, depending upon the kind of muscle that the tumor simulates. I tumor composed of nonstriated muscle-fibre is called a leiomyoma, and, a tumor made up of structure simulating striped muscle-fibre is called a rhabdomyoma.

Myomas are not common in the fowl. The leiomyoma is found in connection with involuntary muscle structure, while the rhabdomyoma are observed in connection with striated muscle structure. 


\section{ANGIOMA}

Angioma is a tumor formed of blood vessels and may be formed of either blood or lymph vessels.

Henangioma is a tumor consisting of blood-vessels bound together by a small amount of connective tissue. Some of these may be composed of newly formed blood-vessels, while others consist of more or less altered pre-existing vessels.

Lymphangioma is a tumor made up dilated lymph-vessels. The affected vessels may form distinct caverns or sacs.

\section{LYMPHOMA}

A lymphoma is a tumor made up of lymph-node tissue. It is made up of lymphatic structure.

\section{MYXOMA}

A myxoma is a tumor made up of mucous tissue, which is not, strictly speaking, an adult structure; it is, at least, the lowest grade of adult connective tissue. The tissue may be identical with that surrounding the vessels of the umbilical cord, and resembles the vitreous humor of the eye. The tumor always contains a certain amount of fibrous storma, and may 1 esemble an edematous fibroma. In embryonic life myxomatuus tumors are met with in those subcutaneous tissues from which fatty tissue later develops. The sectioned surface of the tumor appears like a homogenious, structureless, gelatenous mass. The majority of the cells present are angular and stellate, with long anastamosing prolongations; others are indistinct, oval, spherical, or fusiform in shape.

\section{HEMATOMAS}

A hematoma is a blood tumor caused by a hemorrhage into a part. Occasionally consiclerable hemorrhage takes place in the ova as they are in process of formation or in those nearing tegenerative changes. These fail to find their way into the oviduct and become hematomas as shown in Fig. 98.

Exciting causes, like those that cause inflammation and congestion, are present. A rupture of a small, congested vessel causes a clot. Ergot in small doses may be given to combat this condition.

Hemorrhages may take place in other parts of the body so that the escaped coagulated blood becomes encapsulated and thus a hematoma. 


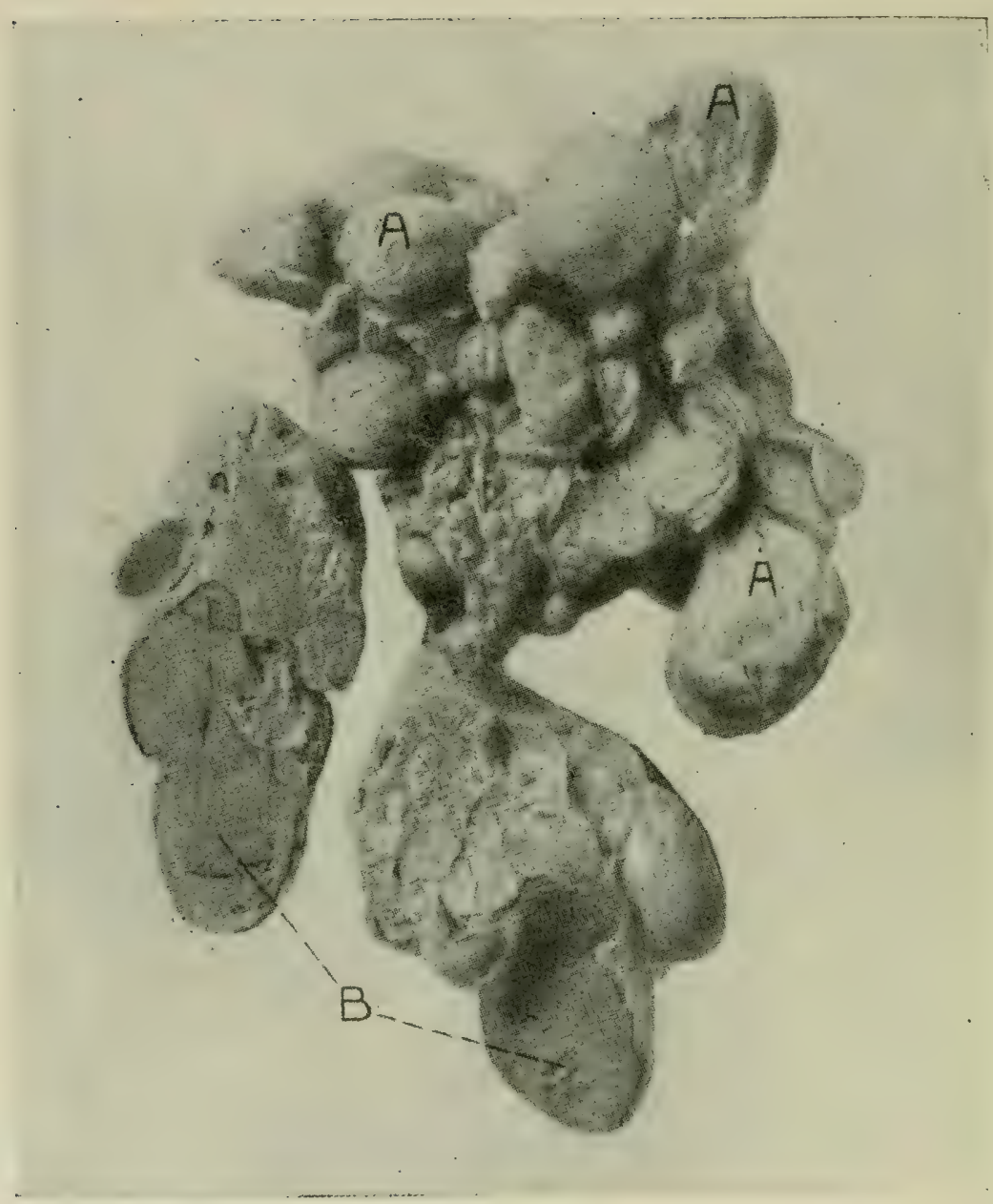

Fig. 98. Hematoma of the Ovary of a Hen (natural size). $\Lambda$, diseased ova undergoing caseous degeneration; $B$, sectioned surface of two of the blood tumors. 


\section{SARCOMA}

A sarcoma is a tumor of embryonic connective tissue cells. The cells have no tendency to become mature but constantly appear as undifferentiated embryonic cells. The sarcoma is a malignant type of tumor. They spread into surrounding tissue and the cells may be carried by the blood stream or lymph current and secondary tumors in other parts of the body result. The flesh of birds with sarcoma should not be eaten.

The embryonic cells tend to infiltrate the surrounding tis-

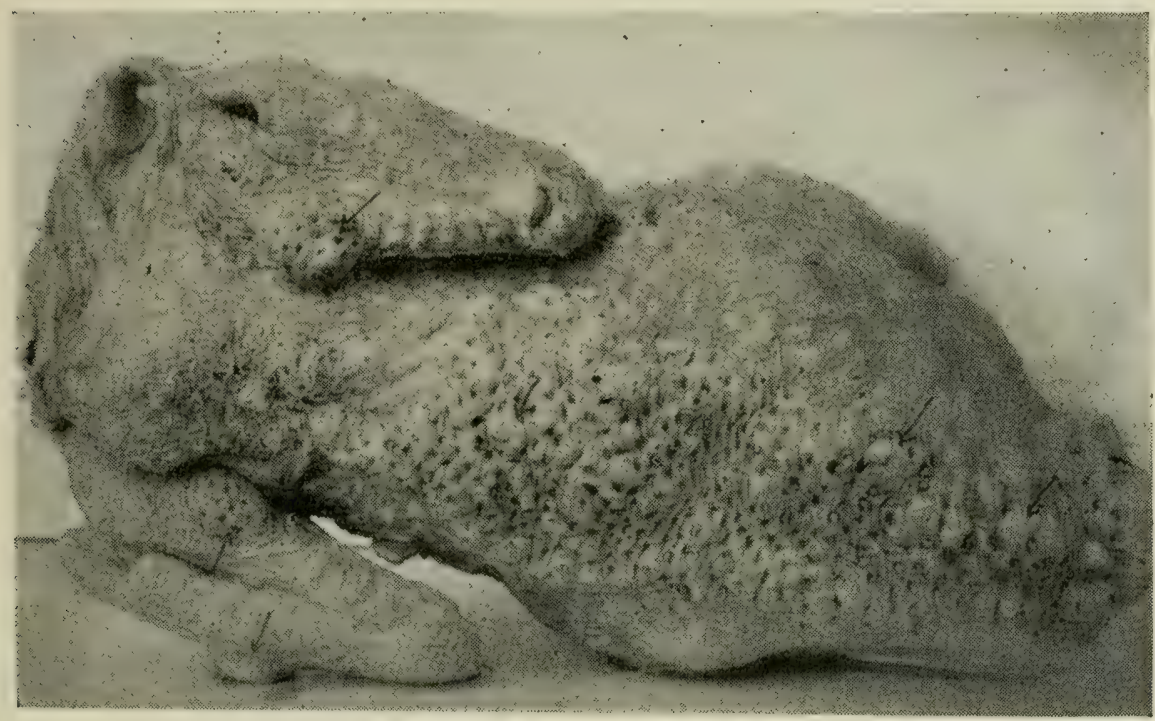

Fig. 99. Spindle-Cell Sarcoma of the Skin of a Buff Orpington Hen. The arrows point out some of the numerous tumors.

sues, as a result of which sarcoma are rarely, if ever, encapsulated.

The records of this laboratory show that of 730 autopsies held upon hens and cocks ranging in age from twelve months to three years, 8 , or a trifle over 1 per cent, were affected by generalized sarcomatosis. Round cell sarcoma is the most prevalent type, and round-cell lympho-sarcoma is frequently observed.

The sarcoma cells may be of the small, or of the large, round cell variety, or they may be of the small, or of the large, spindle cell variety. Or small amounts of connective tissue may divide the tumor making the cells in nests when it is called an alveolar cell sarcoma. Sarcomas quite frequently are found associated with other kinds of tumors making compound tumors.

Sarcomata develop either in previously normal tissue or belong to the connective tissue group - as for example, in the skin, subcutaneous tissue, intermuscular connective-tissue, 
periosteum, spinal cord, meninges, connective tissue of glands, and other similar locations. It may also develop in some preexisting connective tissue tumor as in fibroma, myoma, or chondroma. Fig. 99 shows a generalised case of alveolar sarcoma of the skin of a hen.

Round cell sarcomas are probably the most common type in the fowl though spindle cell sarcomas are by no means rare.

Sarcomas have been transplanted, using a portion of the tumor and imbedding it into the breast muscles of another fowl. Thus one such successful transplant. was made of a spindle cell sarcoma of a Brown Leghorn hen to a Barred Plymouth Rock hen. The filtrate also produces sarcoma but the tumors do not appear till several months after the inoculation of the filtrate.

\section{COMPOUND TUMORS}

While simple tumors, that is, tumors of but one kind of tissue, are the prevailing kind in both benign and malignant types, yet it is not unusual to find tumors made up of two or more kinds of tumor tissue. The tumor cells always resemble some normal body cell or cells either adult or in embryonic formation.

\section{CYSTO-LIPO-CHONDRO-OSTEO-ADENO-CARCINOMATA}

A tumor containing distinctly the above six tumor tissues was studied in a two-year-old Golden Wyandotte cock. The cock, during the breeding season had failed to fertilize the eggs of the hens with which he had been running. His appetite and general appearance remained good, and no serious physical condition was noted till shortly before death.

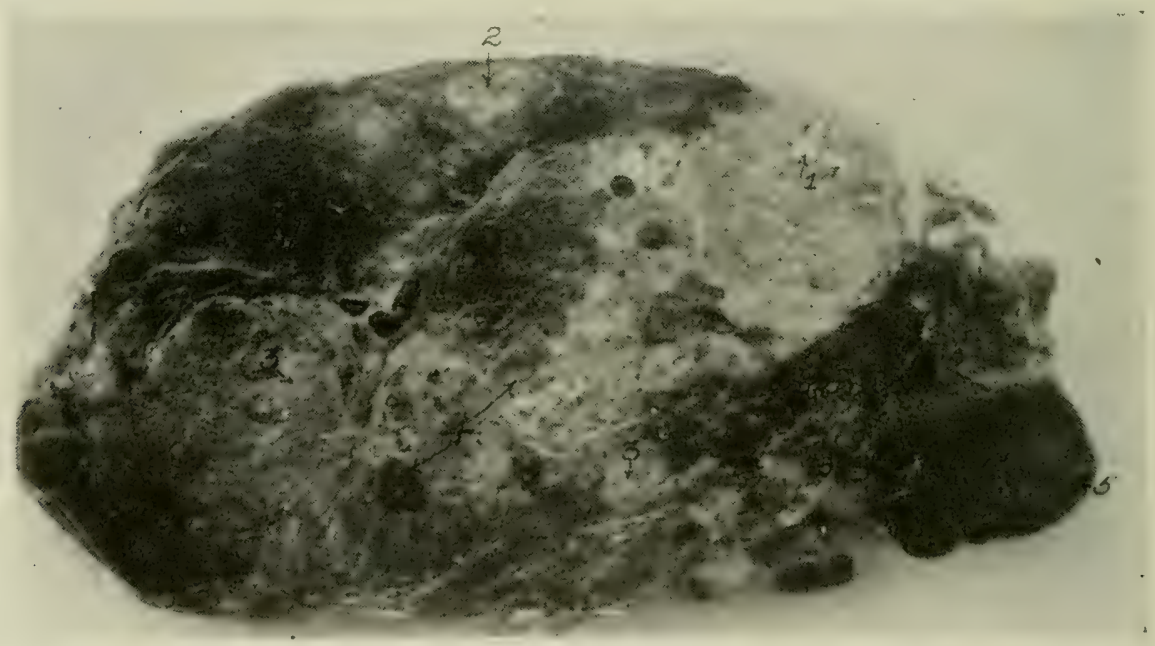

Fig. 100. Cysto-Itpo-Chondro-Osteo-Adeno-Carcinomata of a Golden WYANDOTTE COCK.

1, Cartilage and bony area; 2 , fatty tissue; 3 , adenomatous and carcinomatous zones; 4 , cysts; 5 , aneurysm. 
Upon opening the abdominal cavity there was noted $100 \mathrm{cc}$. of a straw-colored fluid, and a very large tumor, involving the right abdominal air sac. There was also a small tumor on the left lobe of the liver. This is a most unusual tumor, both in size and in general make-up, that we have ever studied. The tumor was placed in a 10 per cent solution of formalin for several days and then sectioned. The tumor measured $15 \times 10 \times 9$ $\mathrm{cm}$, and the sectioned surface indicated a compound tumor. Islands of bone surrounded by hyaline cartilage were observed. Fatty areas were also present. Cysts, varying in size up to 13 $\mathrm{mm}$. in diameter and containing a gelatinous substance more or less the color of serum were observed throughout the sectioned surface. Lining these cysts were columnar cells. Here and there are whitish areas, which contain more or less connective tissue and cellular substance, which proved to be carcinomatous areas. At the extreme right hand portion of the photograph will be seen a small aneurism, which plainly shows the coagulated blood laid down in layers. The photograph plainly shows the whole tumor section lacks uniformity in appearance, looking as though there were many kinds of tissues making up the whole tumor mass. Surrounding the whole tumor there is a thin fibrous capsule.

\section{LYMPHO-SARCOMA}

Lympho-sarcomas are quite common in the fowl. Several cases have been studied in the heart of fowls. In one case of generalized lympho-sarcoma there was contained in the abdominal cavity about $25 \mathrm{cc}$. of serum containing considerable flocculi. In addition to the tumor masses covering the visceral

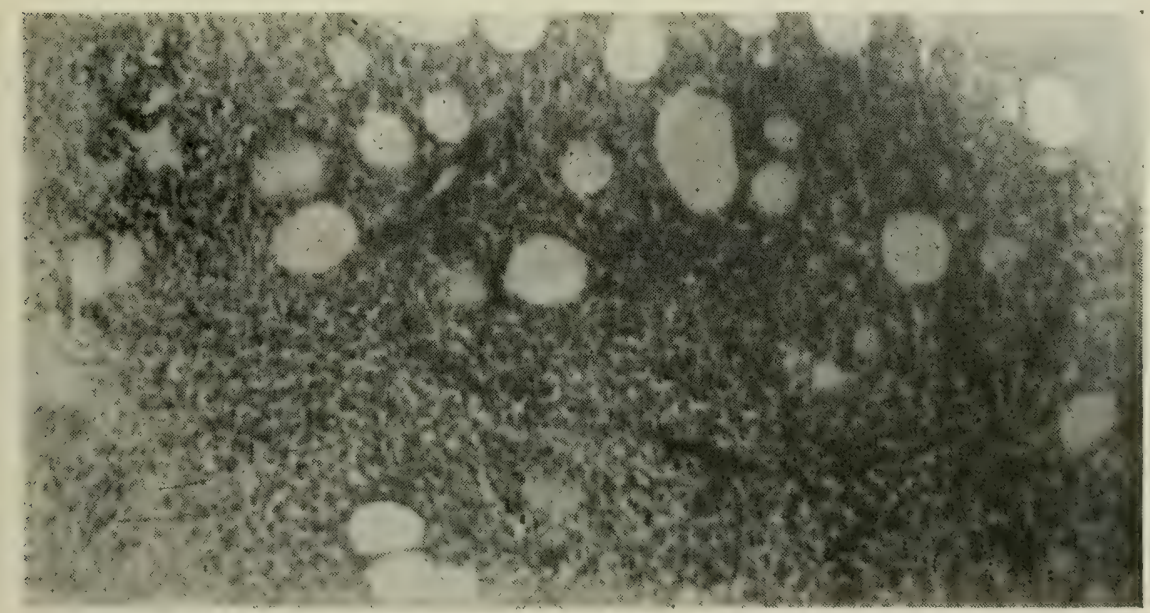

Fig. 101. Lympho-Sarcoma of a S. C. White Leghorn Cock.

Note among the round cell mass the transverse sections of the lympli vessels. 
organs there was present cloudy swelling of the liver and kidneys. Fig. 101 shows a photomicrograph of a section of the lympho-sarcoma.

Another case of lympho-sarcoma studied was in a S. C. Rhode Island Red hen, two years old. The hen had the appearance of being in excellent health, except for a slight diarrhea, up to about ten days before she was sent to the laboratory. At this time she

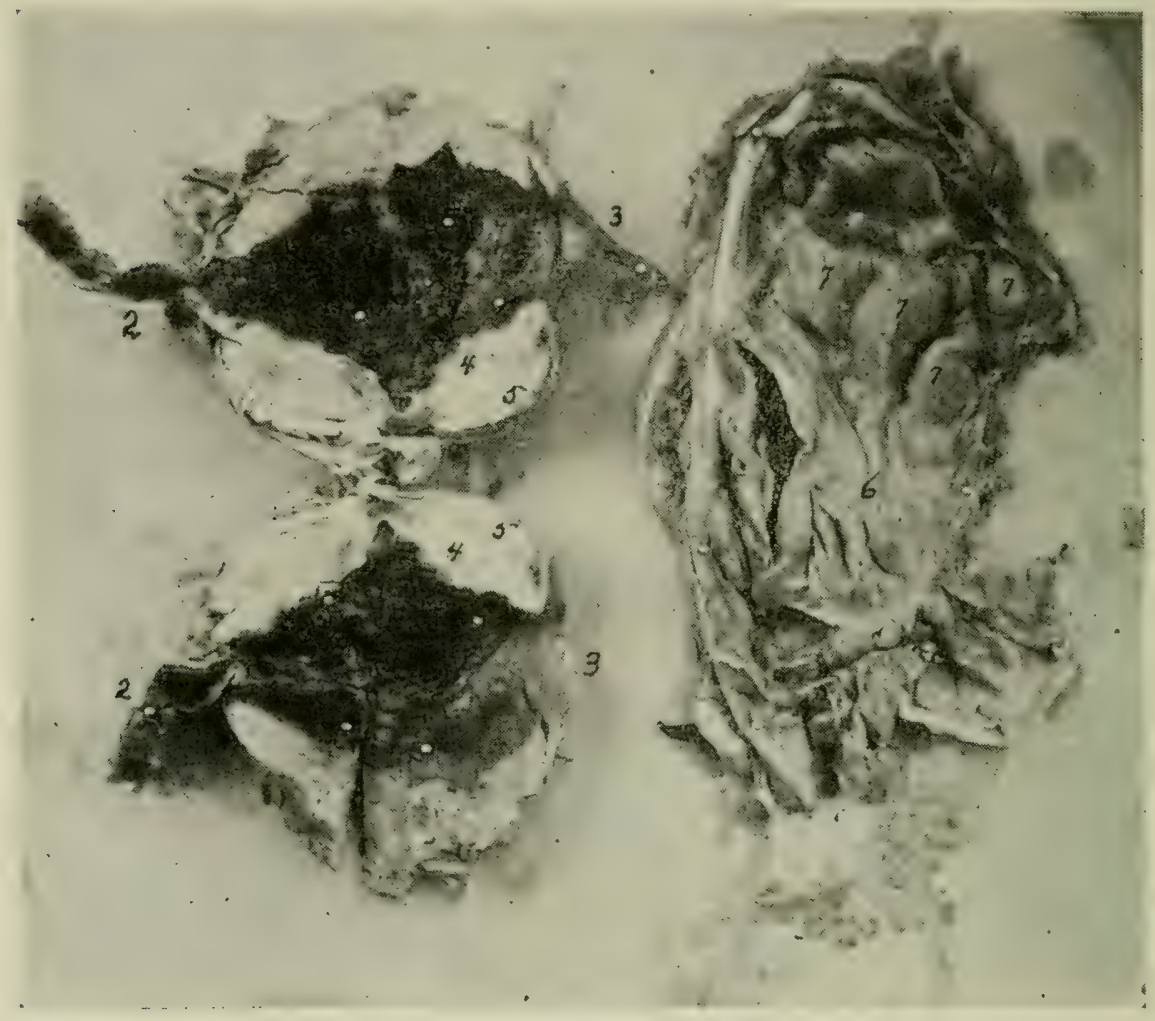

Fig. 102. Lympho-Sarcoma of a Rhode Island Red Hen, Involving the MESEntery and ReCtum.

1, lumen of large intestine-inside of tumor; 2, uninvolved portion of the rectum; 3 , a portion of the cloacal mucous membrane; 4, caseation necrosis; 5 , lympho-sarcomatous elements; 6 , the mesentery; 7 , five of the tumors.

had a loss of appetite, diarrhea, and was thin in flesh. The comb and wattles and facial regions were pale and the plumage in a rather unkemped condition.

After death, upon opening the abdominal cavity there was noted a complete loss of retroperitoneal fat. The liver was rather under size and of a dark reddish color and weighing but 25 grams. There was noted just anterior to the cloaca and involving the whole rectal wall, a tumor measuring $7.5 \times 7.5 \times 4 \mathrm{~cm}$. Upon opening the tumor it was observed that it involved the entire rectal wall giving passage to that viscus through its middle. The sectioned surface of the tumor wall was $1.5 \mathrm{~cm}$. in diameter as shown by Fig. 102. There was an ulceration of the lumen of the intestine, a mass of necrotic tissue appearing suspended from the 
wall of the cavity. The cavity was fusiform in shape, measuring $1 \times 3 \mathrm{~cm}$. in the widest place. Anterior to this point and involving the intestinal wall were two other tumors which measured $2 \times 1$ $x 1 \mathrm{~cm}$. At the anterior point of the kidney and overlying the adrenal gland was a fourth tumor measuring $3 \times 3.5 \times 4 \mathrm{~cm}$. The sectioned surface revealed a solid though soft whitish structure.

In the mesentery of the free portion of the intestine there were observed ten small, rather flat whitish-yellow appearing tumors. They varied somewhat in shape but the majority will fall within the limits of the measurements of the two following tumors: $5 \times 8 \times 2$ $\mathrm{cm}$., $5 \times 6 \times 1 \mathrm{~cm}$.

\section{MYXO-SARCOMA}

A myxo-sarcoma is another malignant tumor being made up of elements of both a sarcoma and of a myxoma. It is largely

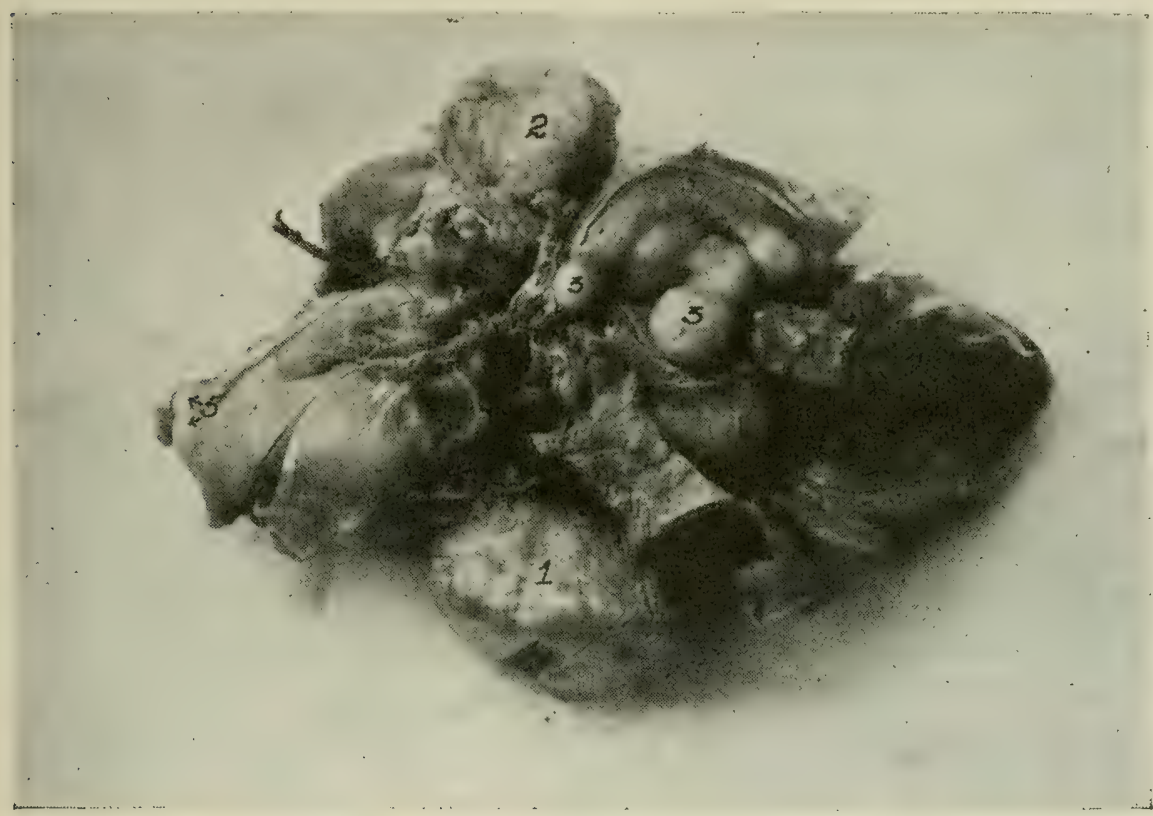

Fig. 103. Myxo-Sarcoma of a Barred Plymouth Rock Hen.

1,2 , and 3 , are tumors of the liver; 4 , are small tumors developing in the liver substance; 5 , are two small tumors of the pericardium at the apex of the heart.

composed of cells; the basement substance, though a constant and important factor, being much less conspicuous than in adult connective tissue. They closely resemble, in general, the developing connective tissue of the embryo or granulation tissue of inflammation.

The sarcoma cells may be varied in size and shape. They may be flat, fusiform, spheroidal, or branched, and even cubical or cylindrical; they may be multinuclear, that is, have more than one nucleus, and very large, or they may be small and spheroidal, resembling leucocytes. The fibrillar basement substance may be present in such small quantities as entirely to 
escape a superficial observation, covered as it may be by the abundant cells, or it may be so abundant as to give the tumor the general appearance of a fibroma.

Mucoid degeneration is frequent in the various forms of sarcoma. A combination of myxoma and sarcoma, myxo-

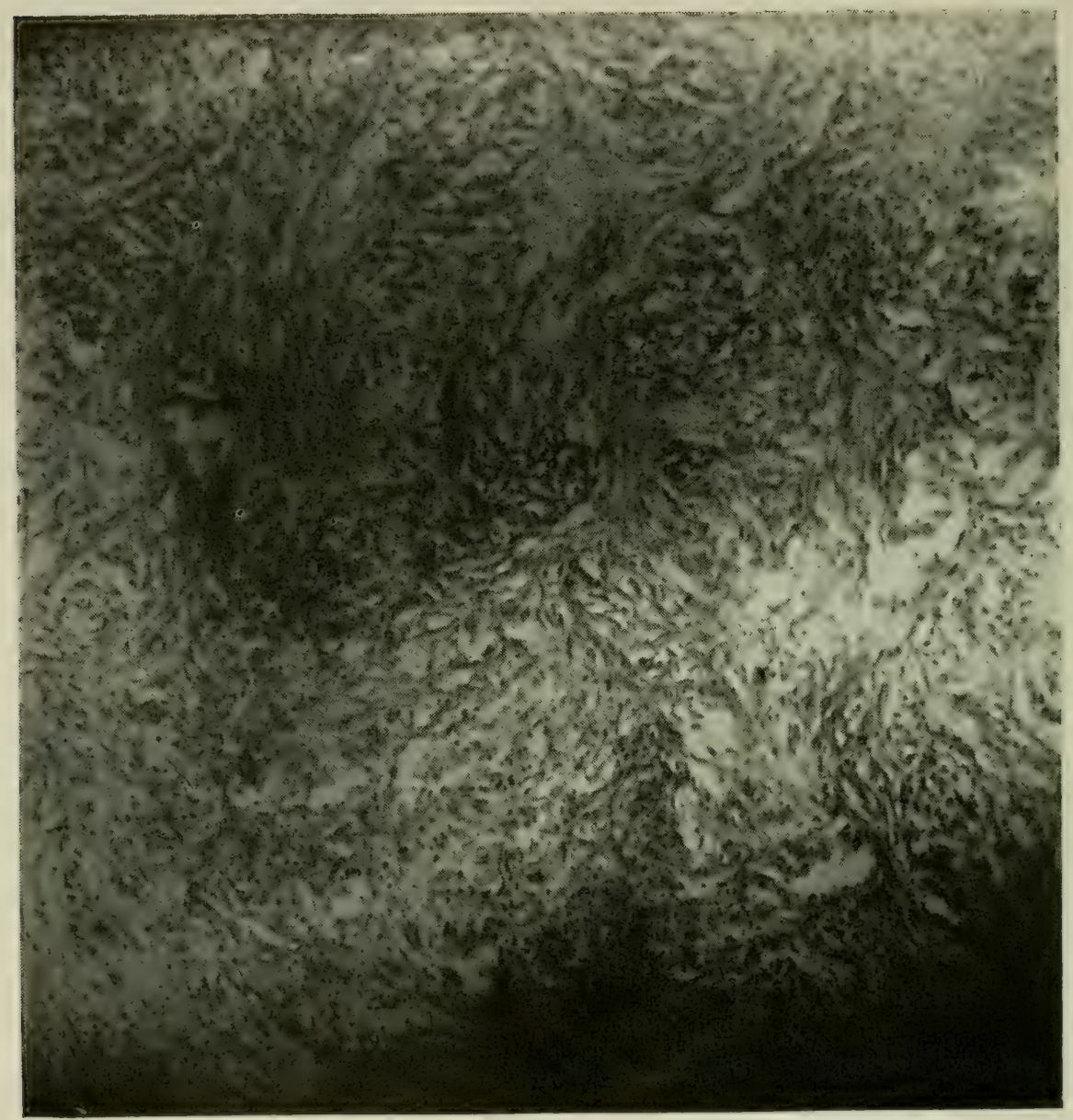

Fig. 104. A Photomicrograph of a Section of One of the Tumors Shown in Fig. 103, Showing Myxomatous Areas.

Note the spindle and stellate cells.

sarcona, is common in man and animals. A case is shown in a photograph in Fig. 103 from a Barred Plymouth Rock hen.

In the case illustrated in Figs. 103 and 104, numerous round white tumors were observed involving the viscera. The larger tumors measure $38 \times 30 \times 23 \mathrm{~mm}$.; this larger tumor is attached to the side of the right lobe of the liver. The second larger of the tumors indicated by 2 , measure $28 \times 22 \times 15 \mathrm{~mm}$.. and are attached to the anterior and inner part of the right lobe of the liver. There are several, twenty or more, smaller tumors varying in diameters of from 10 to $15 \mathrm{~mm}$. There are still smaller tumors imbedded in 
the liver substance as indicated at 4. These smaller tumors vary from 1 to $4 \mathrm{~mm}$. in diameter. In addition to this tumors were distributed over other organs, there being three involving the pericardium and the base of the heart, and two of the same membrane near the apex. The balance of the organs were apparently in a normal state.

\section{SARCO-CHONDRO-OSTEOMATA}

Another form of compound tumor is a combination of the structures of a sarcoma, a chondroma or cartilage tumor, and an osteoma, or a bony tumor. This has been observed in a Single Comb Rhode Island Red hen. In Fig. 105 will be seen

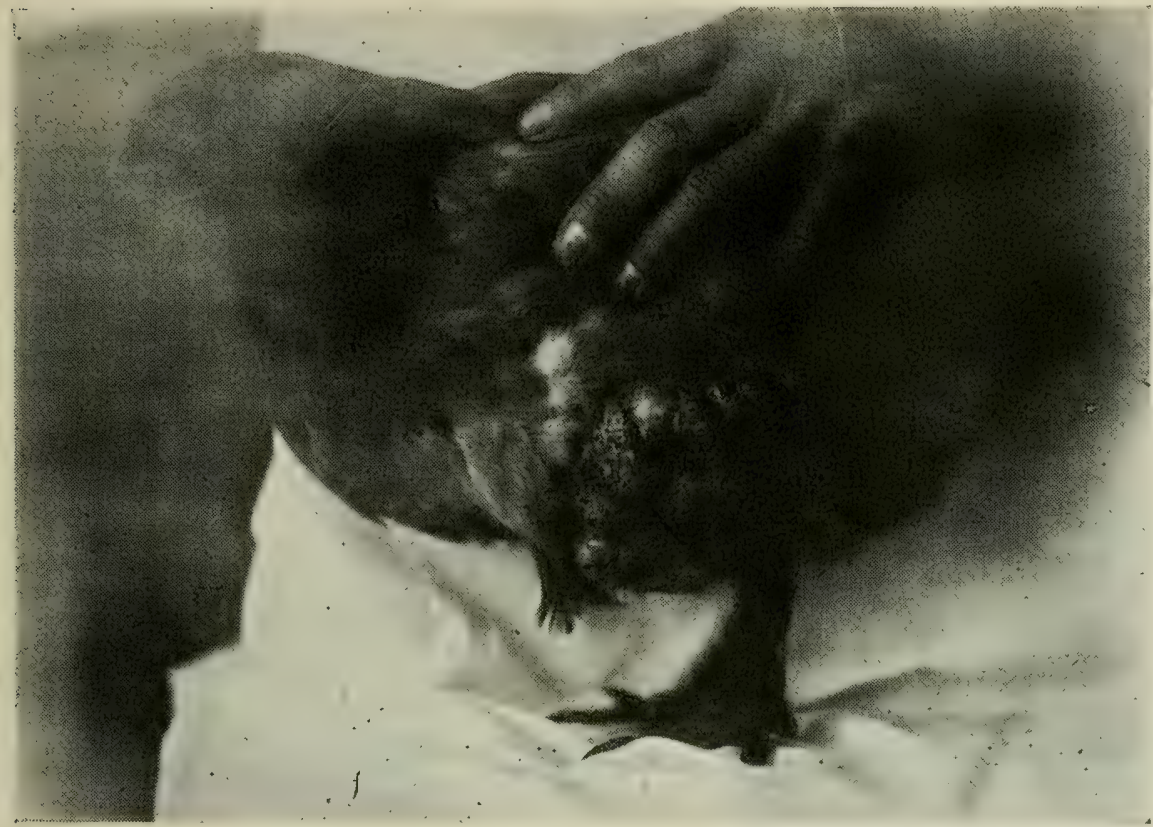

Fig. 105. Sarco-Chondro-Osteomata of the Tibial Region of a Single Comb Rhode Island Red Hen.

a tumor of the thigh and drum-stick region of a hen. This is a sarco-chondro-osteomata. This enlargement had been of gradual occurrence. The bird was thin in flesh. There were also other tumors on the sides of the thoracic walls. The bird seemed to suffer no great inconvenience, having a good appetite, and appearing happily disposed.

At autopsy there was observed an irregular-shaped tumor on the left thoracic wall measuring $2 \times 2 \times 1.5 \mathrm{~cm}$. There is also an irregular-shaped tumor on the right thoracic wall measuring $7 \times 6$ $\mathrm{x} 2 \mathrm{~cm}$. These two tumors were attached to the ribs. A third hard tumor, irregular in shape, was observed on the leg just above the hock. This tumor measured $7 \times 10 \times 11 \mathrm{~cm}$. This tumor had no capsule; on the other hand, presented rather a raw surface. It sprang from the subcutancous connective tissue. In a cross sec- 
tion the tumor showed islands, the center of which contained bone tissue surrounded by a hyaline cartilage the balance of the structure being made up of sarcomatous elements. The sarcomatous elements are of the large spindle-cell variety.

\section{TERATOMA}

A teratoma is a congenital tumor, usually occurring in the sacral region, called coccygeal tumor; the head and neck, midiastinum, or the abdominal cavity. They have also been observed in the kidney, ovary, and testes.

The teratoma is composed of epithelial and connective-tissue types, adult and embryonic; and originates in rudiments de-

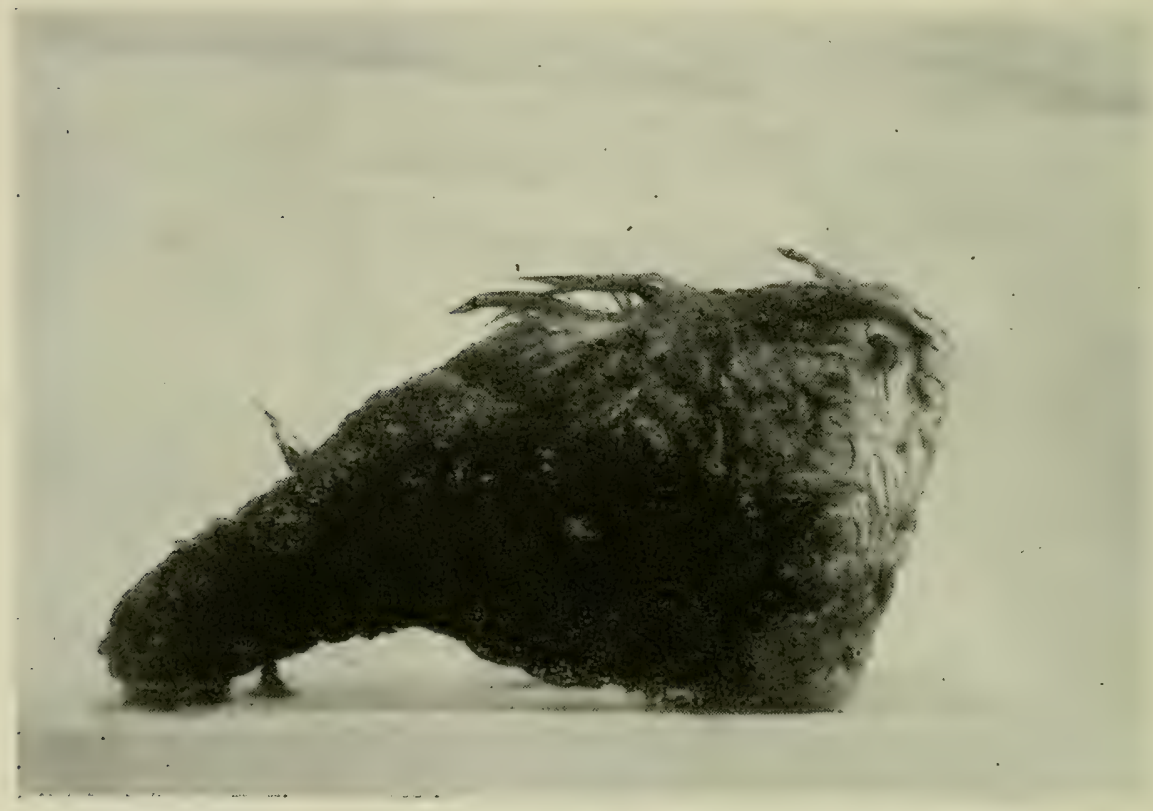

Fig. 106. A Horny Growth From the Thigh of a Fowl.

rived from the three layers of the blastoderm, namely: ectoderm, mesoderm, and entoderm.

Teratomas have been observed in the fowl. Such a tumor was described in Vol. XXIV. Part 2, of the Journal of Comparative Pathology and Therapeutics.

The tumor was observed in the abdominal cavity and involved the mesentery. The left testicle was absent. The growth measured $3 \times 4 \times 6$ inches and was enclosed in a thin, fibrous membrane. The tumor mass consisted of a multitude of cysts ranging up to the size of a pea. Islands of cartilage, cavities lined with stratified epithelium and minute feathers. The conclusion was drawn that this tumor probably originated from the left testicle. 


\section{HORNY GROWTHS ON THE CUTICULAR SURFACE OF FOWLS}

Horny growths upon the skin surface of fowls have been recorded by Gadow and also from this laboratory.

Fig. 106 shows a photograph of a horny growth removed from the outer thigh region of a two-year-old hen. The specimen measured $14 \mathrm{~cm}$. long and $7.5 \mathrm{~cm}$. in diameter at the base. The outer structure is horn-like, resembling the horny structure of the spur or claw. Feathers are noted, here and there, over the surface. The inner core consists of connective tissue and is soft in consistency.

\section{CYSTS}

A cyst is made up of a connective-tissue membrane or supporting wall lined by epithelium or endothelium, and forming a cavity the contents of which may be fluid or semi-fluid, uniform in composition, or made up of a mixture of similar or dissimilar substances.

When the wall of the cyst is passive and influenced by the retained or extravasated contents in a mechanical way only, the cyst is said to be simple or unilocular. When several simple cysts occur together, all arising from the same cause, and identical in structure, they are called multiple cysts. When one cyst springs from the wall of another and thus gives rise to a second or a third cyst, the process resembling proliferation, the resulting cysts are known as proliferus cysts; the cyst walls may be destroyed by pressure, and thereby many of the cyst cavities communicate. When the cysts remain distinct, they are spoken of as multilocular. When the cysts communicate they are spoken of as cavernous. When the lining membrane of the cyst develops papillomatous masses, the process is called papilliferous. A cyst may be a retention cyst. These include all cysts due to occlusion of excretory ducts of glands with accumulation of glandular secretion and exudative fluids in the closed cavity resulting from obstruction of the passage by which, normally, evacuations occur. Such cysts occur in connection with the gall bladder, pancreas, and mucus cysts and in connection with the kidney. Such cysts have a wall composed of condensed and thickened connective tissue, and the lining cells are epithelium. The contents are derived from the functional activity of the gland structure, altered from the absorption of a part of the fluid and the degenerative changes that the cellular elements wall and content may have undergone.

Exudation cysts embrace cysts arising from accumulation in closed cavities not supplied with an excretory duct. Such cysts may be observed in connection with bursae, tendon 
sheaths, and the cyst wall is formed of a thick connectivetissue membrane lined with endothelium.

Extravasation cysts are a variety of cysts formed around distended or ruptured vessels, or in areas of hemorrhage, and may occur in any tissue.

Dermoid cysts are always of congenital origin, and arise as the result of cutaneous inclusion. The wall may contain elements of the skin, the lining cells are epithelial, and the contents feathers and fluid.

Parasitic cysts may be observed as sarcocysts and possibly other animal parasites infest the fowl and may become encysted.

\section{CYSTOMA}

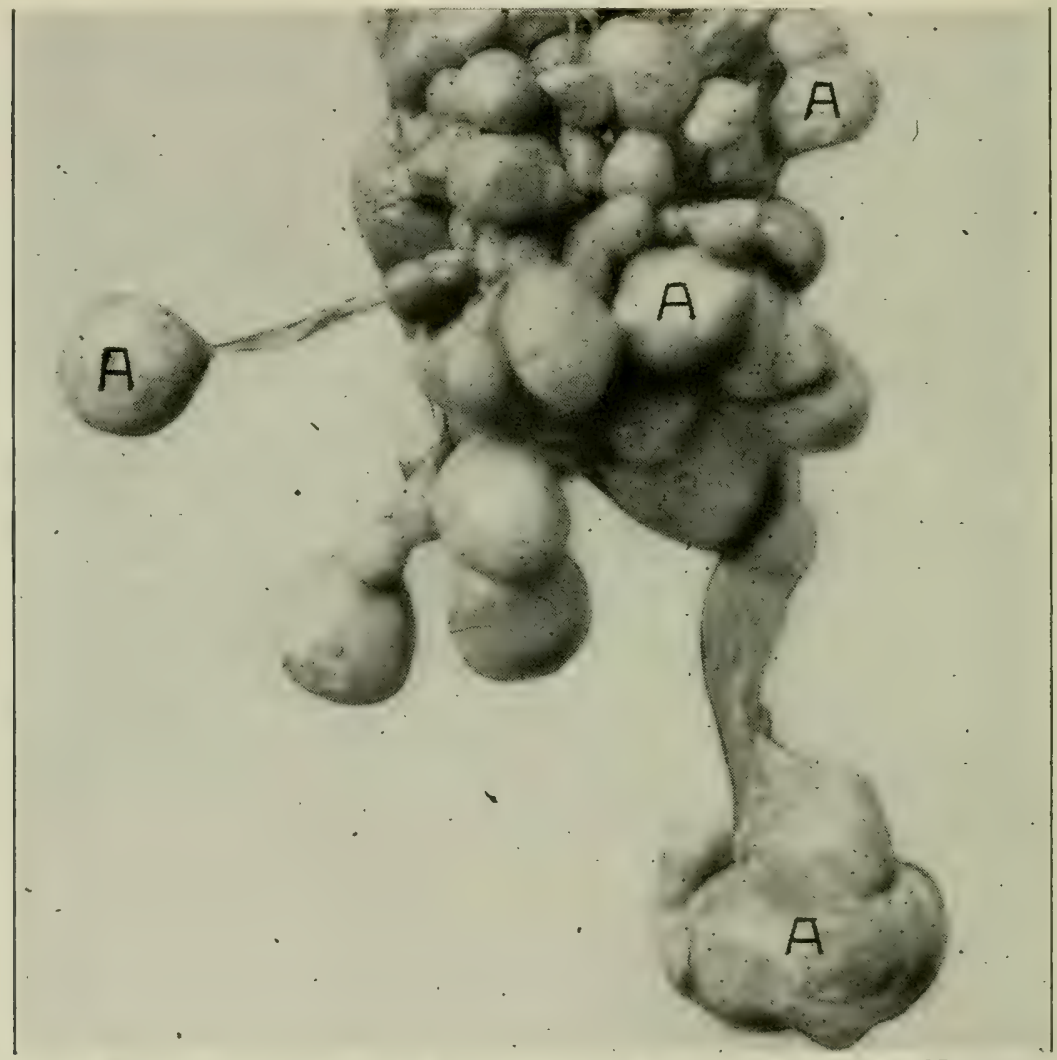

Fig, 107. Multiple Tumors or Cysts of the Ovary of a Hen, Natural Size. a, ova that have undergone degeneration. Note the pedicle-like structure joining to the ovarian mass.

A cystoma is a cyst resembling the above described cysts. The wall is of mesoblastic origin, lined with a flattened layer of connective-tissue cells and endothelium. The contained fluid is usually clear, straw-colored, slightly albuminous, and of low specific gravity; the fluid may be colored, cloudy, and contain flocculi. The contents may be grumous, cheesy or semi-solid. 
The most frequent site of a cystoma is the ovary. Multiple cysts or tumors of the ovary is the most common and consist of yolks, or ova, which were forming, but failed of maturity and to enter the oviduct, disease processes overcoming them. Later these masses become hard and irregular in shape, yellowish in color, consisting of more or less dried or inspissated yolk much of the liquid being absorbed. Ovarian infection with the Bacterium pullorum is a common cause of this condition. Fig. 107 illustrates natural size of one of these cases.

Many times cysts are observed in connection with the ovary that contain a colorless fluid. These cysts vary in size and are attached to the ovarian mass by a pedicle.

\section{CASEOUS ABDOMINAL TUMORS OR CYSTS}

Caseous abdominal tumors or cysts are quite frequently observed. Such cysts may be in connection with the air sacs,

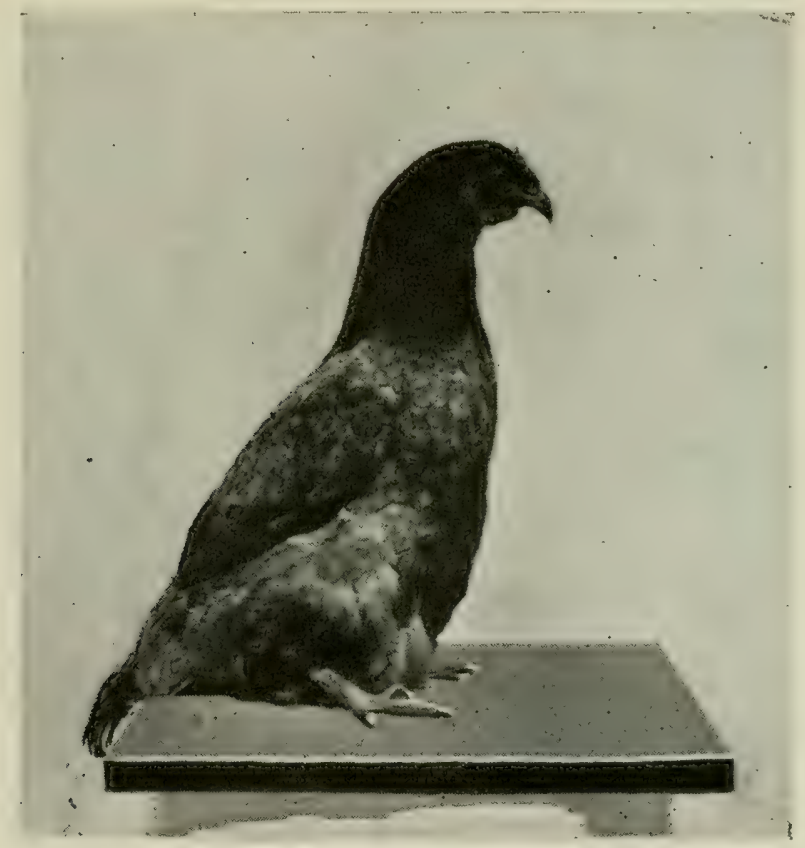

Fig. 108. A S. C. Rhode Island Red Hen With an Abdominal Cyst.

The upright attitude is a diagnostic symptom of an abdominal tumor, ruptured oviduct with eggs in the abdominal cavity, or, of an injured spine.

ovary, oviduct with aborted formative eggs, or in connection with other structures.

Symptoms.-A hen with quite a large abdominal cyst may appear in good health, the appetite and general appearance remaining excellent and only the symptoms of such a condition as to attitude be the only sign of the disease. If the cyst is of 
considerable size, as was the case in the hen photographed and shown in Fig. 108, she will assume an attitude like a penguin and when she walks her tail drags the ground and when standing still she assumes almost an upright position. Upon palpation of the abdomen there will be felt the tumor-like mass, which may be freely movable or stationary, depending on location and structures involved. Such a tumor the size of a hen egg may cause such symptoms.

Treatment.-The bird can be placed under chloroform and the cyst removed by making an incision three or four inches

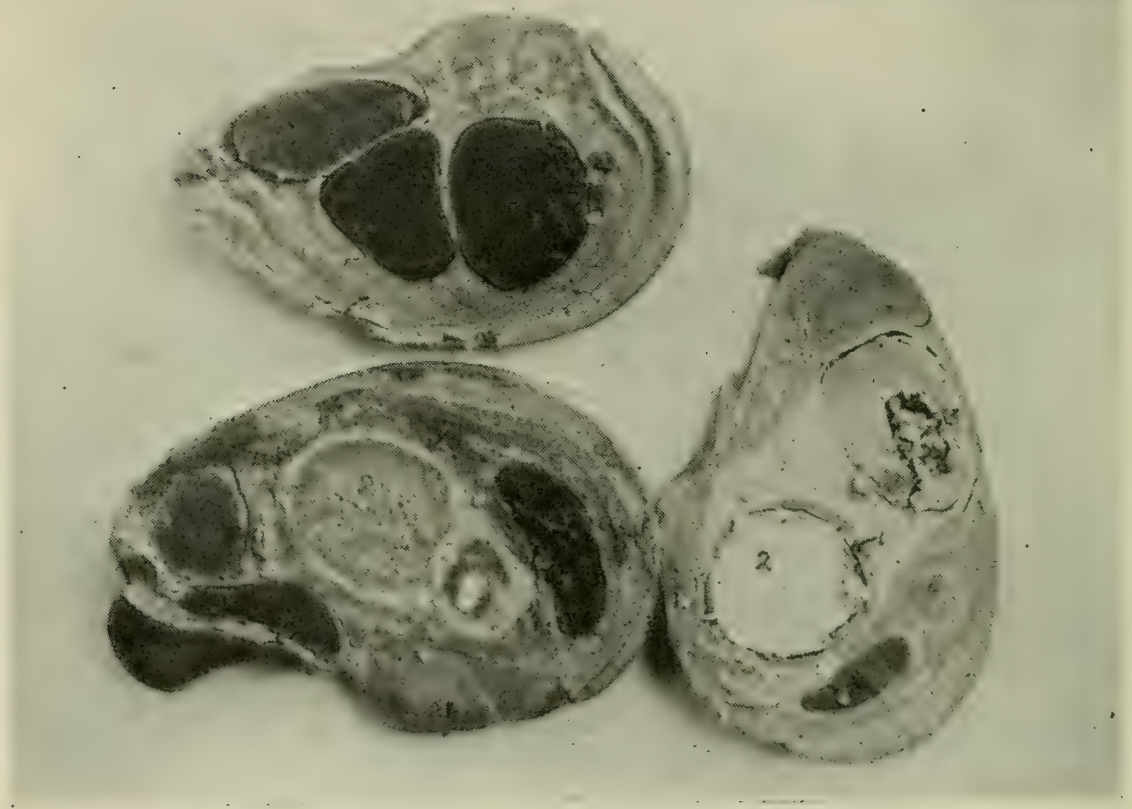

Fig. 109. Photograph Showing Sections Through the Oviduct Mass of Arrested Formative Eggs.

1, shell; 2, yolks; 3, dirty, grayish, putty-like mass.

long, in the abdominal wall. After making the incision through the skin and controlling the hemorrhage, a mass of retroperitoneal fat, three-fifths of an inch may be encountered. Incising this fat causes very little hemorrhage and in fact, some of the fat, lipectomy, is necessary in order to be able to remove the cyst. Usually the cysts are easily dissected out. Many of these cysts contain a granular cheesy material. Birds take chloroform anesthesia very readily and care must be exercised to not give too much. A bird soon comes out of the effects and shows no ill after effects of the anesthesia. After the tumor is removed the wound may be sutured by a continuous suture and the bird placed in a comfortable coop and 
given a light diet for a few days. Birds die readily from shock and some operations are followed in a short space of time with death.

Formative Egg Accumulations Due to Weakness of the Muscular Walls of the Oviduct.-Weakness of the muscles of the walls of the oviduct and a weakness of the walls of the ovarian pocket often result in the accumulation of formative eggs in the oviduct or in the abdominal cavity outside of the oviduct. The attitude of a hen in such a condition is similar to that of cysts in the abdominal cavity, the hen assuming an upright position when at rest. The abdominal wall is usually tense and there may be an accumulation of some fluid in the abdominal cavity. Tumefactions may be felt upon palpating the abdomen. In one such case the mass entirely filled the oviduct, extending from the juncture of the first portion through the second or albumin-secreting portion, and through the third or isthmus and through the fourth or shell gland portion. The tumor mass measured $14.5 \mathrm{~cm}$. in the anterio-posterior diameter, $9.2 \mathrm{~cm}$. in its lateral diameter, and $9.4 \mathrm{~cm}$. in its dorso-ventral diameter. In addition to this large mass there was a small one in the vaginal portion.

Ten eggs, two of them with shells, made up the entire tumor-like mass. Degenerative changes had taken place in some of them, and there was much dirty, grayish, granular material laid down in layers, alternating with layers of yellow and layers of white albuminous material surrounding the yolks.

\section{Polypus}

Polypus is a name given to tumors which are attached by means of a pedicle. They occur in mucous membranes especially, and which have been compared to certain zoophytes. Polypi may form on every mucous membrane, but are most common in the pharynx, oviduct and intestine. A polypus was observed by the writer in the third portion or isthmus of the oviduct of a hen. The hen had laid two eggs the day previous, one in the morning and one at $4 \mathrm{p} . \mathrm{m}$. and at the time of the death of the hen there had been arrested, by the polypoid obstruction three yolks with their albumin surrounding them. The main polypus was $1.5 \mathrm{~cm}$. in diameter, containing a fatty liquid. In addition to the main polypus there were four degenerating polypi. Each of these were $4 \mathrm{~m} . \mathrm{m}$. in diameter. The ovary was still functioning.

Polypi vary in size, number, mode of adhesion, and intricate nature. The polypi may be soft, mucus type, myxomatous, hard fibrous, and dense or compact and whitish in color. 



\section{DISEASES OF THE BRAIN AND NERVES OF THE FOWL LEG WEAKNESS}

This is a condition in which the bird cannot bear its own weight or it has difficulty in doing so. It occurs in young as well as in old birds but the causes as generally observed in the very young bird is entirely different from that of the old bird or those nearing maturity. Knowledge as to leg weakness, so common at times in certain localities, is imperfect. The conditions are being investigated and are gradually being cleared.

Canse.-One cause of leg weakness or lameness in chicks may be food deficiencies. Feed deficient in water soluble B vitamine will not only show slow growth and malnutrition but will also show lameness with possibly complete loss of the use of the limbs or paralysis. The chick may hold its head sidewise, or even curled under its body till the top of the head rests on the floor.

In young chicks some of the causes are believed to be improperly heated brooders, too much bottom heat, damp and badly ventilated houses and keeping chicks constantly on wooden floors. Chicks developing weak legs on board floors recover as soon as they are placed on ground and allowed to run out doors. Leg weakness has also been reported where chicks were on cement floors but it is not believed to be so fruitful a source as board floors. There is little doubt but that overheating and too much under heat are among the common causes. There has not been near so much leg weakness among chicks since the days when top, instead of under, heat was adopted. It appears to be the artificially brooded chicks that develop leg weakness and the disease is supposed to be unknown among the natural brooded chicks, especially where the hen and brood are provided with the combination sitting and brooding coop and the chicks are out doors all day.

Leg weakness may be observed in birds that are heavily fed and grow rapidly and where the bird's weight appears to increase faster than its strength under such conditions the chick may show spells of dizziness, run sidewise, fall over on its side, and, after a few moments get up and stagger off. The chick will now for a while appear better only to suffer another attack and if not changed in its feed and environment may die. In fact one after another of the flock will become affected. 
Overcrowding and close ventilation are no doubt contributing factors.

Leg weakness among baby chicks at times appears suddenly and with a change in environmental conditions it usually disappears just as suddenly. The worst cases die and the milder ones may recover. The condition may affect only one, or at most a few birds. There is unsteadiness in walking, and in badly affected cases the bird sits around till finally the muscular function is entirely arrested and in baby chicks the legs may present a shriveled appearance.

Postmortem Findings.-No definite lesions can be found in the baby chick that has died of leg weakness.

Treatment.-Leg weakness of baby chicks is due either to environmental conditions or to improper feed. Correct any feed error and look to sanitary conditions and get chicks out on clean grassy plot as soon as weather will permit.

Proper ventilation of the quarters is essential, keep the runs clean, change litter once a week and under the hover once a day. If the cause is a lack of lime salts supply lime water or chick size oyster shell. Ground bone meal to baby chicks and place the chicks on the ground, at least, through the warmer part of the day. Even temperature and proper heat must be maintained at all times for the baby chicks. Fifteen minutes chilling may cause congestion of the lungs and kidneys and result fatally.

Remove the affected birds, in case of disease among young chicks, and reduce the amount of fat forming feeds. Give the proper protein ration. A narrow ration is needed, one with a nutritive ratio of about $1: 3$. Feed oatmeal and oats, cracked corn, and whole wheat, plenty of green feed, sour milk, or meat or fish meal, and one teaspoonful fluid nux vomica to each pint of water. In case birds are "fed off their feet" widen the ration and feed more grain and fewer number of feedings. Throw the grain in fine litter so that the chicks will be kept busy much of the time scratching for it.

\section{PARALYSIS OF THE DOMESTIC FOWL}

Paralysis, or partial or complete loss of one or both legs, wings, or similar affection of the head and neck occurs among fowls and a wing paralysis has been described among pigeons.

In these outbreaks the disease may appear sudden and appear each year among the young birds after its first appearance, and in one instance it was reported to have skipped a year and then reappear among the young birds of that premise. It may appear on new ground and in others upon ground that has been used for various periods by poultry, 
varying from one to several years. Ploughing and seeding and even changing these same birds to new quarters does not stop the disease.

In many cases there is a history that new birds have been introduced into the flock. In others such definite information cannot be secured.

Birds, both male and female, from three months of age to two-years-old may be affected. The most number of cases range in chicks from four to six months old.

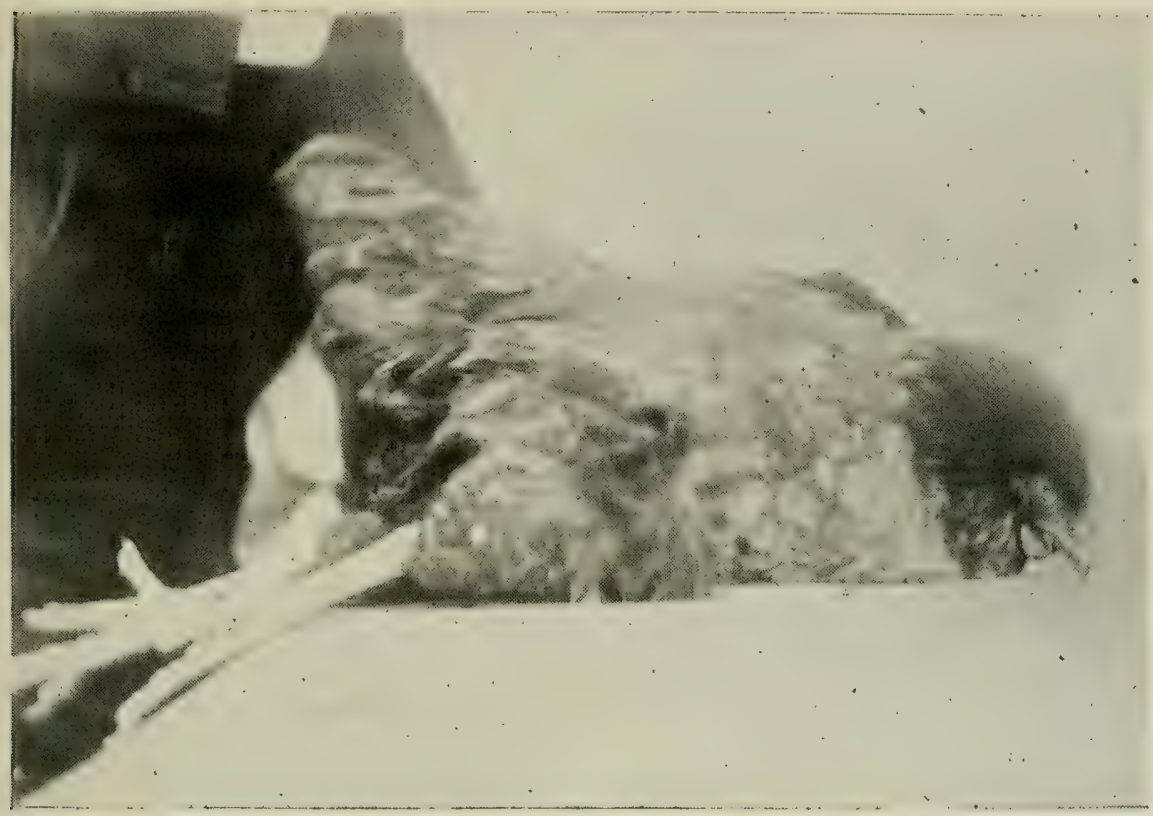

Fig. 110. Photograph of a Single Comb Rhode Island Red Hen With ParalySIS OF Both LEgs.

Note diarrhea and the distressed appearance of the face.

Symptoms.-The disease is always fatal. The symptoms are sudden in their onset. A bird apparently well today and laying may show slight lameness in one leg tomorrow, later both legs become affected, complete loss of the use of both legs, diarrhea, possibly a loss of appetite, though in some cases the appetite remains normal, emaciation and finally death. One or both wings may be the first to be attacked or the disease may first show its appearance in the head, neck, or eyes when blindness occurs. The most common type we have seen is that affecting the legs. The duration of the disease varies. A bird may die in a few days, ten days to two weeks, or it may live for many weeks.

Postmortem Findings.-At autopsy usually the organs appear quite normal, sometimes congestion and a possible cloudy swelling shown by the grayish appearance of the sectioned surface. 
The microscopic diagnosis is one of degeneration and myelitis. The changes present will depend on the stage of the disease and its extent. There may be only degenerating motor ganglionic nerve cells, or in case of lameness in one leg a unilateral myelitis, or, in case of lameness in both legs a transverse myelitis.

A microscopic examination of sections of the various organs and nerve tissues shows the following: The heart musculature is always normal. The kidneys may be normal or there may be different degrees of parenchymatous nephritis, cloudy swelling, active and passive congestion, and cellular invasion. The liver may show no alteration or may show cloudy swelling, congestion and cellular invasion. Changes in the nervous system will be observed in those ganglionic areas where the nerves supplying motor impulses arise. If the legs are affected structural changes will occur in the lumbosacral segment of the spinal cord: If the wings are affected then lesions will be observed in the ganglion of the brachial segment, and if the neck or eyes are affected then in the centers presiding over these parts.

The cellular invasion in some cases is perivascular and in others diffuse. Complete degeneration and solution of areas of the spinal cord may occur-a liquefaction necrosis. The cellular invasion or myelitis may be entirely absent in cases of paralysis. Secondary changes may or may not be present in the parenchymatous organs. In acute, extensive cases, with severe paralysis and diarrhea and a rapid course cloudy swelling may be noted in the liver and kidneys. Both active and passive congestion may be present. Cellular invasion as seen in the spinal cord may be present, in some cases diffused and in others perivascular. Parenchymatous nephritis and hepatitis then may accompany the disease though these changes may be absent or only a slight degree.

Birds from flocks never known to have had the disease before, placed with affected flocks, is followed, by several of the healthy susceptible birds introduced, coming down with the disease. A flock or premise once having paralysis birds upon it will always cause paralysis, even when a new flock of birds are placed upon the ground or in the building. The disease may occur on new ground or on ground where birds have been reared for many years.

Treatment is impossible. A new flock on new land and new buildings is advised.

\section{RHEUMATISM}

Rheumatism may affect birds and especially adult birds. This will cause lameness or loss of function of a wing, and will have a tendency to shift. Birds kept on (lamp) low ground are especially liable to become affected.

Treatment.-Give, to adult birds, 5 grains aspirin three times a day.

\section{PARALYSIS OF THE WINGS OF PIGEONS}

The shoulder and ellow of the wing of carrier pigeons are sometimes affected by arthritis, which has been described as 
infectious. The affected wing droops and the bird is unable to fly.

Predisposing causes are, housing in cold lofts and allowing the birds to roost in drafts. Wet fly may also be mentioned as well as bad sanitation.

The affected birds should be isolated from the balance of the flock.

Abscess formation sometimes occurs, pus being of a caseous consistency. Birds may recover, but if abscesses form they may lose the power of flight.

\section{DIZZINESS-VERTIGO}

Affections of the brain are comparatively common in the fowl. Vertigo has been known where the brain is congested, especially in very fat, plethoric birds. Excessive heat in hot summer weather; absorption of poisonous substances, as toxins, from the intestinal tract; irritation due to intestinal worms; injury to the head, as by a blow, etc., are among the causes of dizziness among birds.

Symptoms.-Vertigo is observed among baby chicks. The birds appear dizzy, finally falling over on their sides helpless and in a few minutes may recover to later suffer another attack and may finally die. The adult bird throws its head upward, backward, and to one side. It may walk sidewise or backward, and have an unsteady walk-staggery. The bird may be drowsy, and even have epileptiform symptoms.

Treatment.-Place the affected bird in cool, well ventilated, comfortable quarters, free from drafts, and for the adult bird give thirty grains epsom salts, dissolved in warm water. Give also two-grain doses of strantium bromide every hour. Thorough purging is one of the first essentials.

In case of limber neck, due to eating rotten meat, and prostration, give one-fifth grain doses of strychnin three times a day.

\section{HEMORRHAGE OF THE BRAIN}

Hemorrhage into the brain substance is called apoplexy. This condition is thought to be common in the domestic fowl but on further investigation it is thought that it is not common. Hemorrhage of the brain may be due to over-straining, as in egg-laying, it very fat birds. Injury to the head, as a blow; and over stimulating feed.

Symptoms. - The hen may be obesrved dead on the nest. The symptoms are of short duration; the attack comes on suddenly, as the hemorrhage soon presses on the brain structures so that the function of that part stops and the animal is seen to stagger, fall, and immediately die. 
Postmortem Findings.-Upon opening the brain cavity and examining the brain, there will be observed hemorrhages, or clots, in the brain substance.

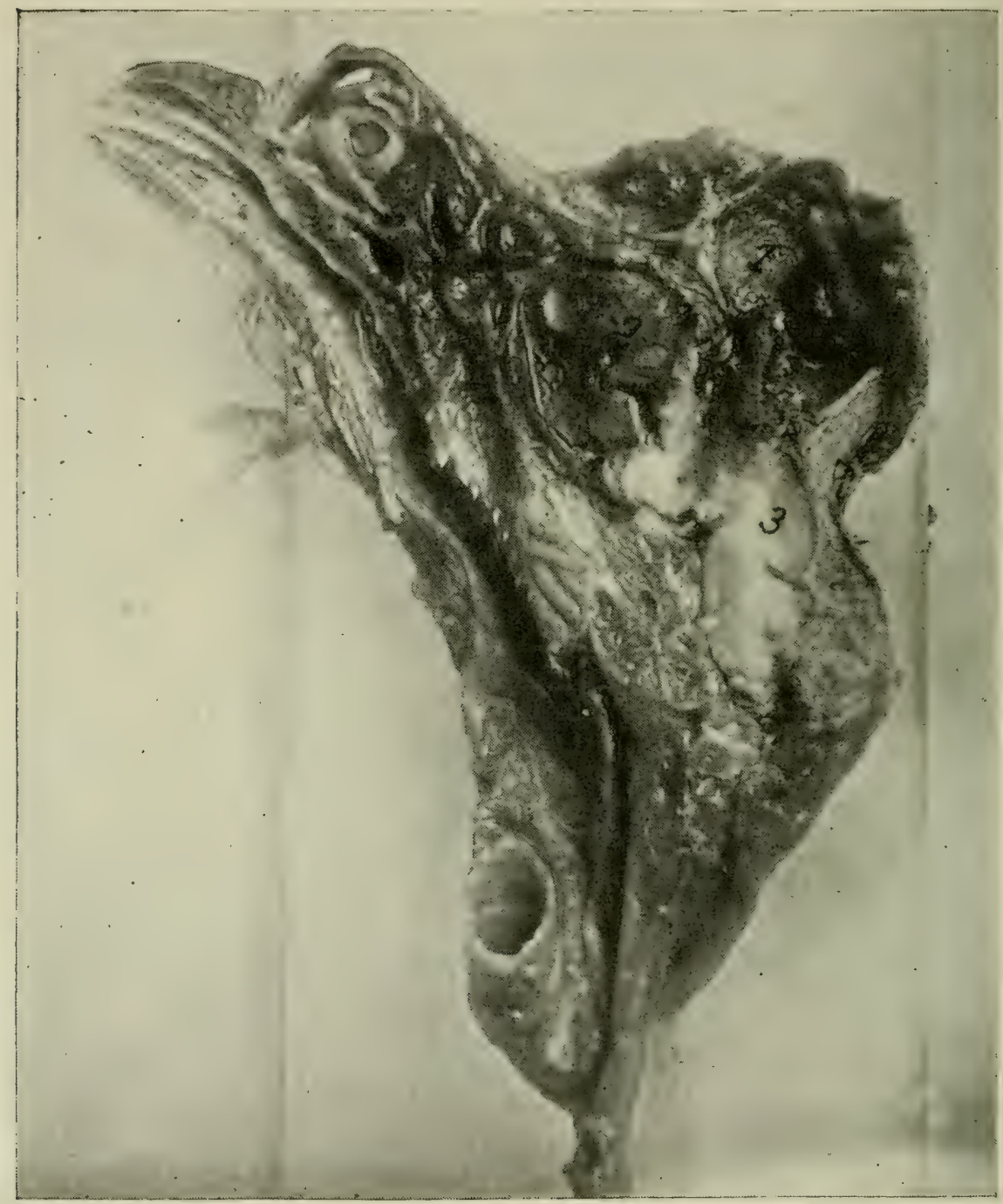

Fig. 111. Photograph of a Longitudinal Section Through the Head of a Mottled Houdan Sirowing Hemorrhage Into the Brain Substance Due to a Blow From a Small Stone Thrown by a Boy.

1, hemorrhage into the brain; 2 , hemorrhage into the eye orbit; 3 , brain tissue in which there is no hemorrhage.

\section{EPILEPSY}

Epilepsy is a disease of the brain and spinal cord, occurring in paroxisms, characterized by the loss of consciousness and convulsive motions of the muscles, with uncertain intervals between them.

In the attack the bird emits sharp sounds, makes flopping 
movements with its wings, falls on its side or back, moves its feet rapidly, rolls its eyeballs, bends its neck round to one side, opens and shuts its beak alternately, and moves the whole body to and fro.

After one or two minutes the spasms cease and the bird gets up, staggers and may at first support itself with its beak and outstretched wings and finally falls because of a fresh attack or it may gradually recover from the first attack without any immediate renewal of the spasms.

Hemiplegia, or loss of motion on one side of the body, sometimes follows as a sequel of this disease.

Epileptiform attacks are sometimes caused by internal parasites.

Treatmont.-The treatment should consist of a physic. During the attack the bird should be cared for to prevent its injuring itself. True epilepsy is incurable and such a bird should not be bred from.

\section{MYELITIS}

Myelitis is an inflammation of the spinal marrow or its membranes. There are indications of a deep seated burning pain. It is accompanied by various nervous and vascular irregularities of function.

Myelitis in the cervical region of the fowl has been observed and is noted to manifest itself by paresis and hyperesthesia of the wing, lateral flexion of the neck during repose so that the beak may be directed backward. During feeding the head may be carried in a normal position.

\section{POLYNEURITIS}

Polyneuritis is a multiple neuritis, and neuritis is an inflammation of a nerve.

Birds fed on a diet wholly of wheat bread develop a polyneuritis similar to polyneuritis gallinarum as produced in fowls with a diet of polished rice. The disease made its appearance in from twenty-one to eighty-two days, with an average of about forty days.

In the large majority of the fowls the first symptoms of polyneuritis is a slight unsteadiness, together with evidence of an involvement of the nerves supplying the exterior muscles of the legs, manifested by a high step and a tendency to bring the foot down with a flop. In many birds there seems to be difficulty in co-ordination early in the disease. In such case the fowl teeters slightly forward as if trying to balance on its toes and walking is with a decided ataxic gait. As the disease progresses in all cases walking becomes more and more 
difficult, until the bird can only squat in the cage, and this condition is soon followed by one of complete paralysis. The disease usually begins with peripheral paralysis and later an involvement of the higher nerve centers. When fowls are fed on white bread without yeast, they come down with polyneuritis sometime sooner than when fed with bread containing yeast. The same holds true of pigeons fed in a similar manner, except that the pigeons were fed on ship biscuits. When fowls are fed on whole wheat bread, they remain perfectly healthy for as long as seventy-five days. It is apparent that whole wheat bread contains some element or elements, lacking in the white bread, which is necessary to maintain the proper bodily metabolism. Fowls force-fed come down fully as soon as those that are allowed to eat at will, indicating that it is not a matter of how much feed is taken, but its quality.

Fowls fed on whole corn remain perfectly well for a period of sixty-three days, but when they are fed on the inside of the corn kernel alone they come down with the disease as when fed on wheat bread.

Tests were run to determine the effects of a starvation diet. Two fowls were started on normal diet, then the amount was gradually cut down, until after about twenty-one days the birds were receiving nothing but water. One fowl went for thirtyeight days without food and the other for fifty-six days. In neither fowl was there a typical picture of paralysis; the symptoms presented appeared to be due to muscular weakness.

Polyneuritis has also been produced by feeding exclusively on one of the following foods: sago, boiled white potato, cream of wheat, corn starch, wheat flour, corn grits, boiled sweet potato, puffed rice, and macaroni.

It is apparent according to tests that pigeons fed on biscuits baked of rye flour, either with or without yeast, do not develop polyneuritis, while pigeons fed on wheat bread do.

The clinical picture of a case of polyneuritis is as follows: During the first ten days the bird eats heartily of the feed and appears at all times active and well, except for more or less diarrhea. On the eleventh day the fowl begins to refuse feed and on the fifteenth day forced feeding must be resorted to. The first symptoms of paralysis is noted on the twentysecond day. On this day the fowl is slightly unsteady and stands teetering forward and backward on its toes; when forced to walk it does so with a high step pushing its feet well forward as if trying to brush something from in front of it. Three days later there will be noted a slight head tremor, and the bird walks with a decided staggery gait, but 
does not fall. The next day both wings droop, and it walks with great difficulty, and every now and then the joint formed by the tibia and the metatarsus seemed to give way and the fowl suddenly assumes a squatting position. It remains in the cage in a squatting position most of the time on the flexed tarsometatarsus, with the extremities also flexed. On the following day the bird cannot stand. The comb is cyanotic, there is convulsive movements, now and then, with retraction of the head. The following day the bird is prostrate. The duration of the disease is six or seven days.

\section{PARALYSIS OF THE AUDITORY NERVE OF THE DOMESTIC FOWL}

\section{ANATOMY OF THE PARTS}

In the fowl the facial, or seventh pair of cranial nerves, and the auditory or eighth pair, are so intimately associated, it appears well to give the origin and distribution of both at the same time.

The facial nerve originates, with the auditory, from the cerebellum. It divides into three parts, the first probably from the complex ganglion with the posterior roots of the auditory. This root belongs to the somatic group of nerves. From this same group originates the auditory which spreads out into the cochlea and takes the impression of sound. This nerve is short and thick, and at the point where it loses its medullary covering on entering the cochlea there is developed a ganglion. This ganglion is similar to the spinal ganglion.

The second part originates by one root which is located medially and ventrially from the deeper ganglion cells. Some of the fibers from this root constitute the vestibular branches and accompany the auditory and supply the anterior part of the ear labyrinth and semicircular canals. The larger part of the fibers of this trunk make up the intermediate part of the facial. The geniculate ganglion is formed at their fusion. The sympathetic sphenopalatine nerve emerges from this ganglion, coming out of the aqueduct of Fallopius.

The third part is called the portio dura and is the main facialis. It is located opposite the auditorius intermedius. Its roots may be traced to the complex ganglion, from which they take a ventral direction.

\section{PARALYSIS OF THE COCHLEAR NERVE}

The paralysis of the cochlear nerve, the true organ of hearing, may be a congenital defect and has been observed in ambliotic animals. It is due to a defective condition of the spinal ganglion with resulting degeneration of the organs of Corti. Paralysis of the nerve may also be caused by inflammatory changes in the internal ear or intracranial disease in the neighborhood of the medulla oblongata. It has been observed as a symptom in coccidiosis and also in fowl plague. 


\section{PARALYSIS OF THE VESTIBULAR NERVE}

This condition is frequently observed in fowls. It is often caused by inflammation of the middle or internal ear. It has been observed in fowl pest. It is also brought about in caries of the petros temporal bone. Concussion of the brain and hemorrhage of the internal ear is also a causative factor. Certain disease conditions of the cerebellum and medulla oblongata may cause an interruption in the conductivity of the vestibular nerve. In pigeons it is observed in contagious meningitis.

The symptoms of bilateral disease of the cochlear nerve are easily recognized owing to the fact that there is complete deaf-

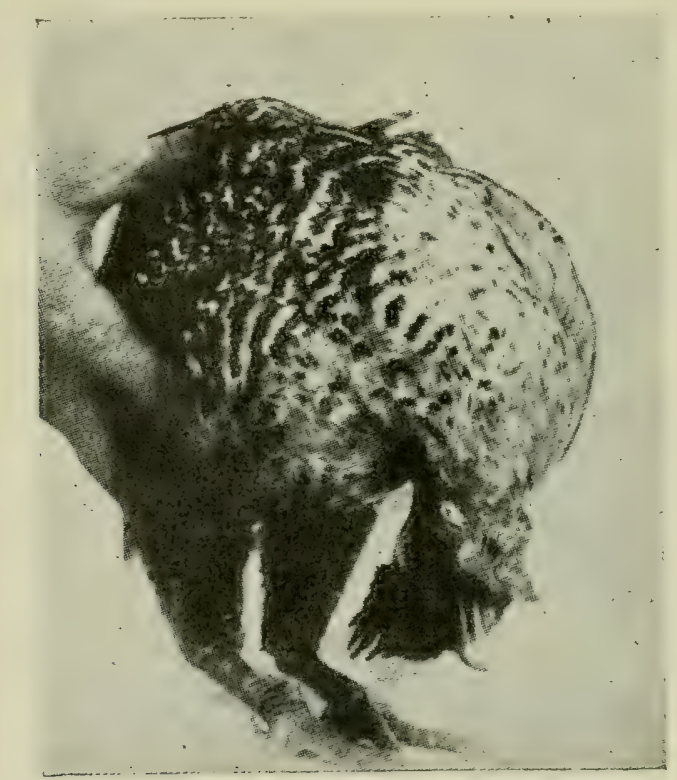

Fig. 112. Paralysis of the Auditory Nerve of a Silver Campine Hen.

ness. If it is unilateral the symptoms may be so meagre that its presence entirely escapes the observation of the owner.

Unilateral paralysis of the vestibular nerve is evidenced in all species by the head being held in an oblique manner. The head may be held in a position under the front part of the body with the lower part of the head turned back toward the sound side. This turning of the head may be slight or $45^{\circ}$ to $75^{\circ}$, but in birds it may be as much as $180^{\circ}$, or even more. If the head be carried under the body the dorsum of the cranium may touch the ground. There is frequently a horizontal rolling of the eyeballs toward the sound side. There may be 
difficulty in taking food, and in birds it is commonly quite impossible. If the disease is bilateral it closely resembles cerebellar ataxia, only the symptoms are limited to the head and neck.

If the paralysis is due to an injury, the disease as a rule is not permanent and the symptoms all disappear in a few days or weeks. If the condition is due to pathological changes in the auditory nerve or nerves the symptoms are persistent, depending on the nature of the primary cause. The bird may, after a while, die.

In traumatic causes the bird needs to be kept in a quiet place free from annoyances, and, if necessary, artificial feeding reserted to. If there has been an injury and hemorrhage takes place in the tissues, surgical interference may be indicated In cases in pigeons due to contagious meningitis an attempt should be made to isolate the diseased bird and carry out thorough disinfection. Calomel should be given to the bird and cold water packs applied to the head.

In Fig. 112 shows a photograph of a Silver Campine hen which was sent to the laboratory for study. The hen was from a farm flock, and was two years old. There was not present among the flock any contagious disease nor was it certain that any accidental or mechanical injury had taken place. The bird holds her head under the body with the top of the head nearly touching the ground. Other such cases have been brought to the attention of the writer. In the case just related there was a thorough examination made of the external auditory canals but results were negative for evidence of violence or parasitism. Eating by the bird was accomplished with difficulty and the bird died after suffering in this manner for a period of sixty days.

In a second case the bird recovered after a period of about five weeks. In this latter case examination of the skull and auditory canals were negative. 



\section{BACTERIA OF THE INTESTINAL TRACT OF THE FOWL}

The bacterial flora of the intestinal tract of fowls has been receiving considerable study during recent years. The alimentary tract of man and animals contains many millions of bacteria, of many varieties. Many of these are constantly present and constitute what is known as the normal intestinal flora. To the newly-born child or animal the intestinal tract is sterile, that is, it contains no germs, but as soon as the young partakes of food and water the intestines are seeded and ever after contain bacteria in large numbers. The same can be said of the chick.

Some of these germs are not harmful, but give off ferments similar to the cells of the accessory glands of digestion; these ferments may aid in the splitting up of foodstuffs and in preparing it for absorption. Ferments of this kind have been called organized ferments, but we have now learned that such ferments do not in any way differ in action from those secreted by the stomach, pancreas, or intestinal glands. It is their ferments, and not the germs themselves, that cause the splitting up of the food nutrients.

Some of the bacteria are at times injurious, and oftentimes pathogenic organisms gain access to the intestinal tract and may produce disease, if the bird is susceptible. There are also, at times, protozoa present, especially those belonging to the coccidia group.

The following organisms have been found in the normal mouth and pharynx of the fow1: Bacillus subtilis, Bacillus coli communis, Bacillus lactis bulgaricus, Bacillus viscosus, Bacillus cloacae, Pnemmococcus, Streptococcus pyogenes, Staphylococcus pyogenes aureus, Micrococcus magmus, Micrococcus tetragenes, Pseudo pyocyaneus, Bacillus prodigiosus.

The following germs have been found as normal inhabitants of the duodenum, or first portion of the intestine, of birds: Bacillus mesentericus, Bacillus subtilis, Bacillus ramosus, Bacillus screus, Bacillus asterosporus, Bacillus fusiformis, Bacillus coli communis, Streptococcus lacticus, Bacillus lactis acrogenes, Bacillus prodigiosus, Sarcina aurantiaca, Sarcina lutea, Sarcina ventriculus, Cladothri.r asteroides, Micrococcus rosettaceus, brown, white and green molds, coral and white 
yeasts. Micrococcus roseus and Clamydothrix fermgenes.

In the third portion of the small intestine, or ileum, may be found green and white molds, Cladothrix astcroides, Bacillus cloacae, Bacillus ramosus, Sarcina lutca, and sarcina aurantiaca, Staphylococcus pyogenes albus, and citreus, Staphy'lococcus cereus albus, Bacillus fluorescens liquefaciens, Micrococcus asterosporus, Streptococcus lacticus, Bacillus lactis aerogenes, Bacillus coli communis, Bacillus prodigiosus, Bacillus mesentericus, Bacillus corcus, Bacillus megatherium, Bacillus fusiformis, Bacillus subtilis.

Practically the same microorganisms are to be found in the caecum. The same may be said of the cloaca, with possibly the addition of the Bacillus aerogenes capsulatus and the staphylococcus pyogenes aureus.

It must be remembered that the intestinal flora is probably not the same for all birds, as different surroundings or environments, different sources of feed, as well as different feed and water, play a part in carrying germs to the intestinal tract. 


\section{CHAPTER XV}

\section{THE EGG \\ COMPOSITION}

An average-sized hen egg weighs about two ounces, of which eleven per cent is shell, thirty-two per cent yolk, and fiftyseven per cent white. The principal chemical constituents of the egg are as follows: Ash (mineral matter) nine per cent; fat (hydrocarbon) nine and three-tenths per cent; proteids (nitrogenous matter) eleven and nine-tenths per cent, and water, sixty-five and five-tenths per cent.

\section{COMPOSITION OF THE EGG SHELL}

Gadow gives the composition of the egg shell as follows:

Per Cent

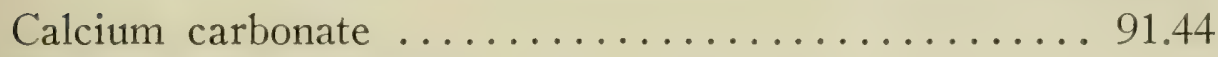

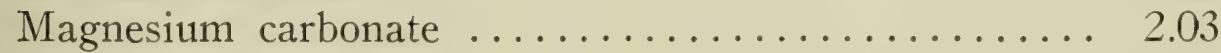

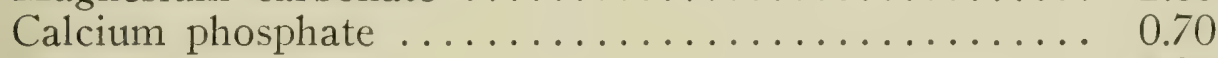

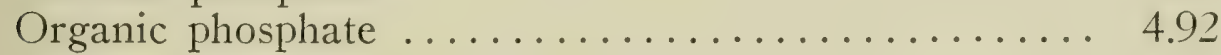

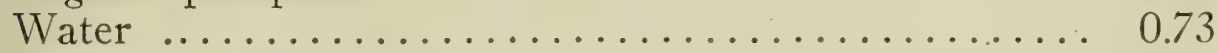

Loss (or traces of other salts) ............ 0.18

Total

100.00

\section{ANIMAL PARASITES IN EGGS}

Worms have been found in eggs. It is probable that intestinal worms as the Ascaris inflexa, and the Heterakis papillosa, and other round worms, normally inhabiting the intestines, may find their way up the egg canal and be incorporated with the egg as it is formed. By referring to Fig. 2, it will be seen that a live worm, possessing power of movement as these worms do, passing into the cloaca, 16 , from the rectum, 15 , can pass up the egg canal, 23, and thus be incorporated in the albumin of the egg, as it is formed around the yolk. These conditions are rare.

\section{BACTERIA OF EGGS}

Several investigators have, of recent years, devoted much time to the investigation of the bacterial flora of eggs. It is needless to say that all understand that the spoiling of eggs is due to the multiplication of bacteria in them, when the egg is brought under the proper temperature. The cold storage of eggs holds them under conditions unfavorable for the rapid growth of these bacteria. When eggs are kept cold the bac- 
teria within them are in a more or less dormant state and hence by reason of this retardation of germ growth the eggs keep longer.

Eggs can be successfully desiccated or dried and such powdered product is on the market. The moisture in it is so reduced that germs do not grow and, like any other dried product, it keeps well. This desiccated products retains the quality of the fresh egg for a long time. One pound represents about three and one-half pounds raw egg or an amount obtained from thirty eggs. The egg contains considerable fat and because of this the dried product gradually undergoes a change at warm temperatures, much as butter does, finally giving off a rancid, fish-like odor.

It is not probable that the yolk or ovum becomes infected while it is being formed in the ovary, unless the ovary, from which it is developed, is diseased. It has been shown that birds that have had white diarrhea while chicks and recovered. grown to maturity, and commenced laying, have diseased ovaries, ovaries which harbor the Bacterium pullorum, the cause, or at least one of the causes, of white diarrhea, and this germ is incorporated within the yolk of the egg. This is an important means of spreading this disease and one before which sanitation is powerless.

Ordinarily the internal organs, as the ovaries, kidneys, spleen, etc., are sterile unless diseased, as just stated. However, Conradi maintains that he has found bacteria in these supposedly sterile organs in seventy-two cases out of one hundred sixty-two.

The germs that have been alluded to under the intestinal flora of chickens can easily find their way into the cloaca and up the oviduct, as illustrated in Fig. 2. The yolk or ovum when fully developed in the ovary is delivered, in a similar manner as in higher animal life, into the first portion of the oviduct or, in animals the fallopian tube, which at its free extremity is rather funnel shaped and is called the ostium infundibulum. This egg canal which can be likened to the uterus of higher animals is about eighteen to twenty inches long and is lined with tubular glands which secrete albumen. and in the posterior portion the shell membranes, then the shell. This material is formed from foods carried by the blood, which is very abundant in these walls. As the egg passes through the last portion of the oviduct, called the uterus, the pigment of shell coloring is put on it. As the egg passes through the cloaca in being passed out or layed, it is exposed to contamination by germs which may be taken up into the oviduct with the male elements or spermatozoa, after copula- 
tion. Bacteria are not so common in non-fertile eggs, a fact that supports this theory.

Many of the organisms found in eggs are non-motile, so that they must find their way up this canal by extension by growth or be carried mechanically. Among the germs that have been found in eggs are: Micrococcus, non-liquifaciens, Staphylococcus pyogenes aurous and albus, Bacillus protidigiosus, Bacillus riolaceus, Bacillus putridis, Bacillus putridis non-liquifaciens, Streptococci, Micrococcus luteus, Micrococcus candicans, Micrococcus flavus tardigradus.

The colon bacillus is ever present in the intestinal tract of fowls and is found on the outer shell, yet contamination of the egg content by it does not occur. This has led some to think that there may be a substance present in the egg canal that is bacteriacidal for this germ and the matter is being further investigated.

Among those germs which find their way through the pores of the egg shell after it is layed in the Bacillus paratyphosis, the cause of paratyphoid in man.

\section{BACTERIA IN FRESH EGGS}

Of 1,894 yolks from fresh eggs that were examined in the month from February to September 7.7 per cent contained germs other than the Bacterium pullorum and over 16 per cent contained the Bacterium pullorum.

A total of three years, including all months, and a total of 3,510 yolks of fresh eggs, 9.5 per cent contained bacteria, not including the Bacterium pullorum. Nearly 20 per cent contained the Bacterium pullorum.

Of 105 tested with the fermentation tube for the Bacillus coli, all were negative.

Of the whites of 582 eggs examined, only 1.2 per cent contained bacteria.

Lange placed eggs, after sterilizing with corrosive sublimate and ether, in bouillon cultures of Bacillus coli, Bacillus typhosis, Bacillus paratyphosis, Bacillus enteritidis and Bacillus botulinus. The Bacillus typhosis required two days to enter the white and three days to penetrate the yolk at $37^{\circ} \mathrm{C}$. The Bacillus coli may reach the white in one and the yolk in five days.

The secretions of the glands of the mucous lining of the oviduct have some bacteriacidal action as well as the egg white itself.

Eggs that have been incubated artificially for three weeks remain relatively free from bacterial decomposition, provided they were fresh and clean when placed in the incubator. 



\section{CHAPTER XVI}

\section{MALFORMATIONS}

Malformations among birds are occasionally observed. A complete discussion of the dozens of various forms of malformations that may be found cannot be given here for the lack of space, but a few facts will be given.

In higher animal life, including man, malformations have been attributed to the following causes:

External mechanical influences, such as falls, blows, or severe shock of any kind, by affecting the general health of the

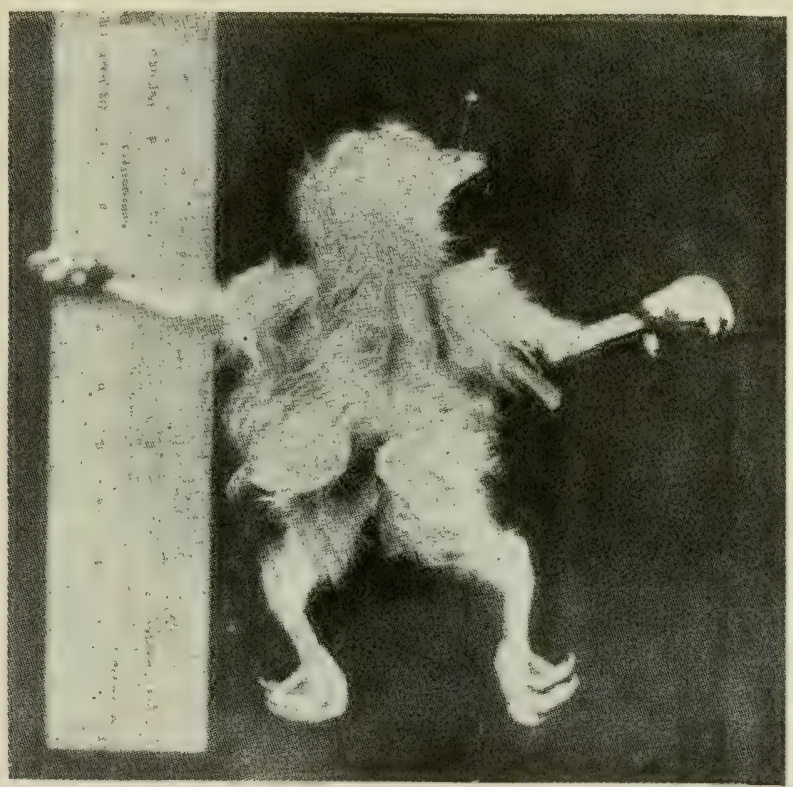

Fig. 113. Dipygus Tetrabrachium or Monster Showing two bodies, four legs, four wings, and one head.

pregnant female, may have power to arrest, retard or otherwise disturb the normal development of the embryo or fetus.

There may be spontaneous amputation, during incubation, by a coil of the umbilical cord finding its way around a part of the fetus or adhesions, causing pressure and amputation, as shown in Fig. 123. The more often eggs are turned each day during incubation up to nine times a day the fewer malformations will occur.

It has been observed that a hen will turn her eggs, on an 
average, nine times a day. In artificial incubation where eggs are turned but once a day there are far more cripples or deformed legs and cross beaks due to adhesions, than where the eggs are turned six or more times a day.

The percentage of malformations in the human family is one to three or four thousand births; in the lower animals and in birds the percentage is much smaller.

During the formation of the fetus an arrest of development of the bud which forms the wing may result in a malformed wing; the same can be said of any other part, as the leg, beak, etc.

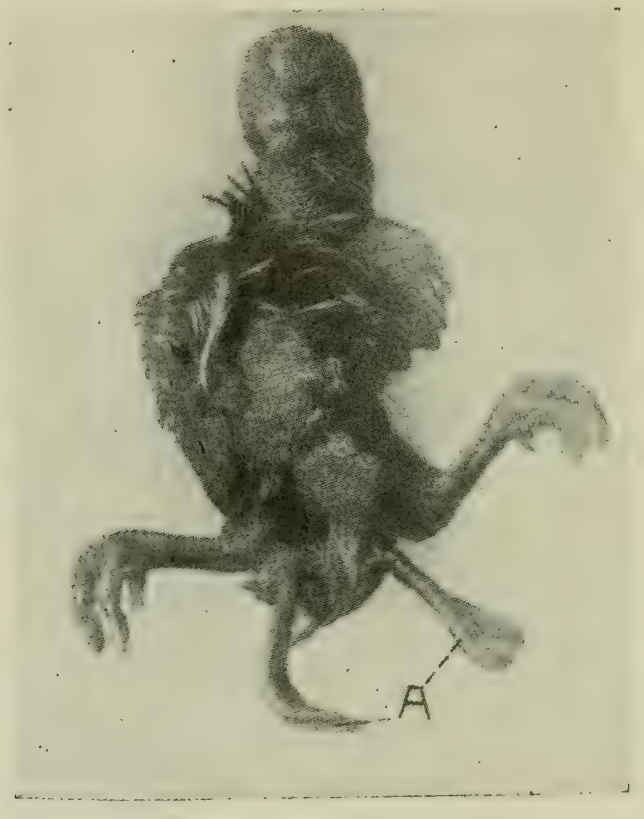

Fig. 114. Polymelus.

2 , the two supernumerary legs.

If the arrangement of the groups of cells during development does not follow the normal type, then malformations, as atresia, imperforation of a natural opening, as an imperforate anus, may result; abnormal position of viscera, a failure of the closure of the abdominal or thoracic plates may take place.

The germ or embryo is first developed as a manifold membraneous expansion, the free margins of which incline towards each other, and eventually meet to form two cavities. A failure to mect results in malformations. Fusion of parts may also take place.

Those malformations in which there are supernumerary parts or duplications of almost an entire body are sometimes called composite or compound malformations and monsters. 
Hermaphroditism is a complete duplication of both male and female genital organs; that is, a single individual possessing both male and fcmale genital organs. Pseudo-hermaphroditism is a condition in which the duplication is only partial. It is desirable that more scientific observations be made along these lines, in birds, and recorded.

In Figure 115 is shown a photograph of the genital organs of a hen. It will be noted that there has developed two ovaries, two oviducts, and that both are functioning. In the embryo chick two

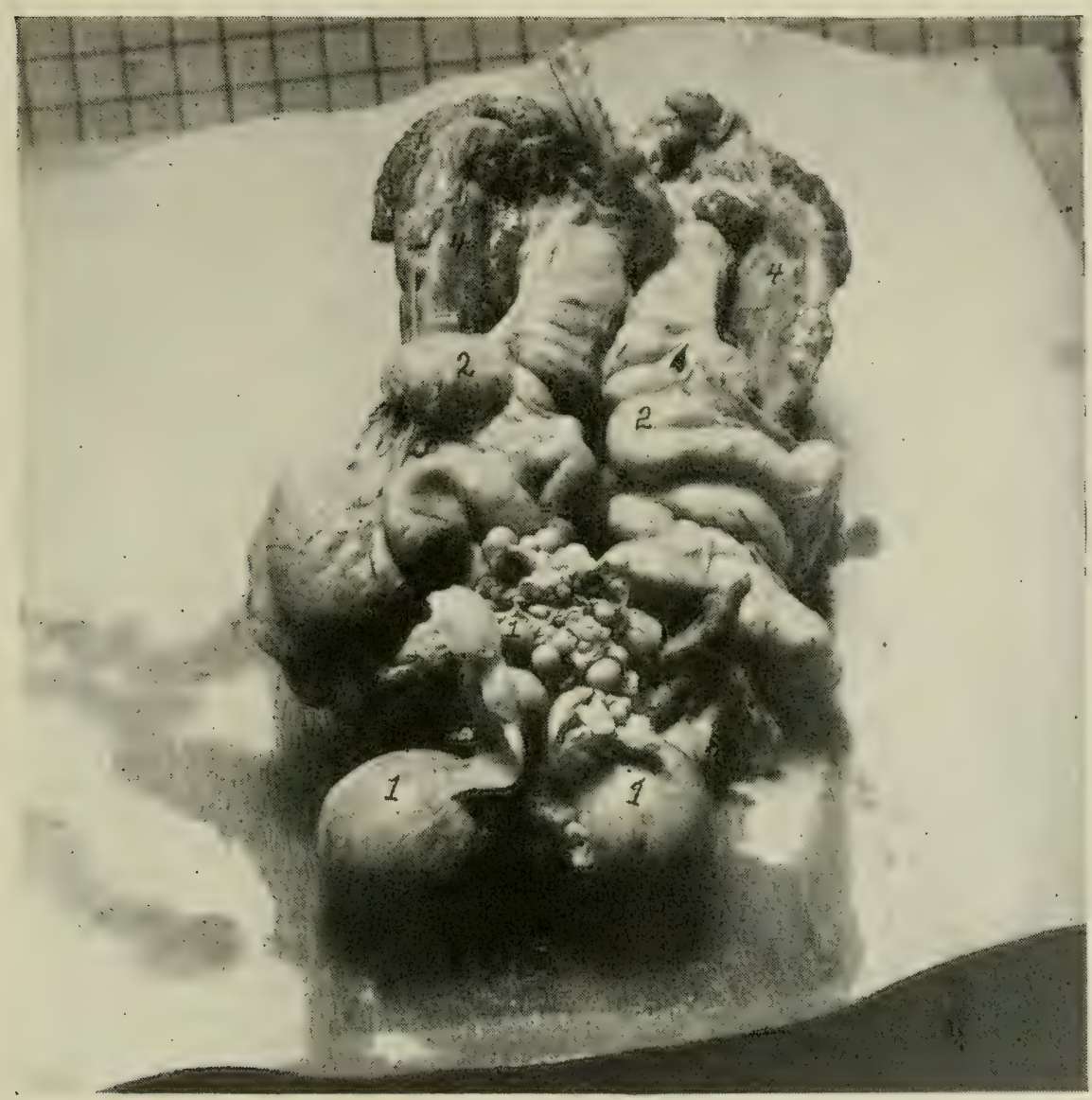

Fig. 115. A Photograiph of the Genital Organs of a Hen Showing Two Complete Ovaries and Oviducts.

Courtesy of Prof. Rice.

ova and oviducts, a right and a left, start to develop but the right ovary and oviduct atrophy and disappear before the chick is hatched, presumably because there is not space for two such organs to function at the same time.

Figure 116 shows a drawing of a case of Holoacardius acephalus in a fowl. A holoacardius acephalus chick is one without a heart or a head. This is one of the extreme forms of acardius acephalus which fall under the head of omphalosites. These latter constitute a group of composite monsters in which the two individual partsan antosite and a parasite-are in vascular communication by way 
of a common placental region, or, in case of non-placental animals, as birds, through the vasculosa of the common yolk sac.

The case being described was hatched from the same egg as a normal duckling. Externally the specimen consisted of very little more than a pair of hind limbs, which in contrast with the re-
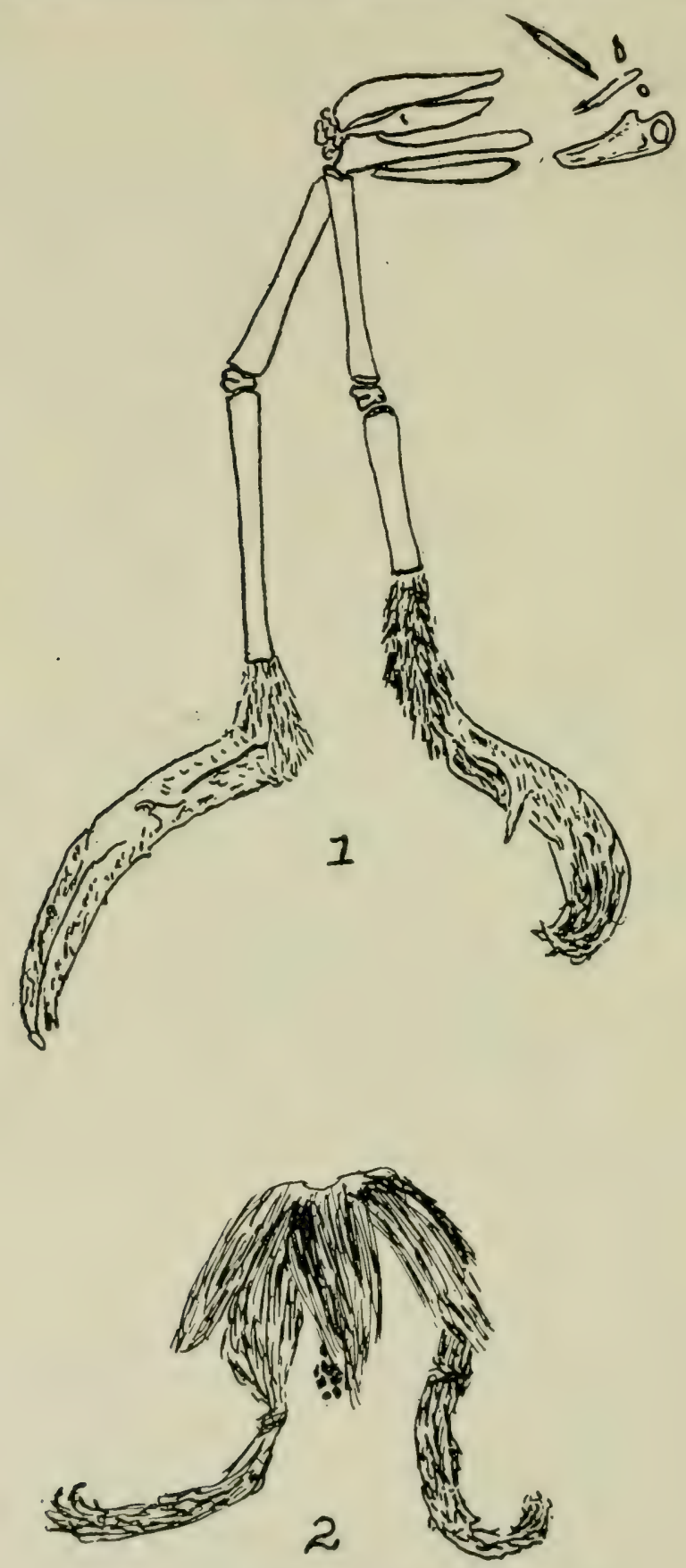

Fig. 116. Hotoacardius Aoephalus.

1. Pelvic rudiments; 2 , fore limb rudiments. 
mainder of the bird-if it is possible to term it such-showed a wonderful degree of development. The feet were scaled, the legs and the rudiment of the body were clad in down. The limbs had suffered considerable rotation which were not easy to analyze, and at one end of the body were shrivelled remains of the yolk sac.

The axial skeleton was entirely absent. The main bones-the limbs excluded-were those of the pelvic girdle. A small lamina of semi-cartilaginous bone represented the sternum, while a few minute rods were apparently ribs. The pelvic rudiments consisted of two pairs of elements. Of these, two bones, presumably morphologically dorsal and the representatives of the ilia, were compressed rods fused into an irregular spong* mass as their acetabular ends, crossing at an acute angle. Below these and parallel to them were a second pair of fairly cylindrical bones distal to the acetabulum were free, and, to judge from the insertion of the omphalic remains between them and the supposed sternum, were directed posteriorally. The acetabula were very imperfectly formed, there being only rough depressions for the femora.

The genesis of acardii is supposed to originate in the following manner: It is supposed that two embryos develop in one ovum, but that one develops more rapidly than the other. Vascular connections are established which in some way encompass the degeneration of the heart of the less advanced embryo, and therefore an only partial nutrition of its body. In the case of birds anastamoses of the vessels of the vasculosa would probably be the cause of the malformation, while in the case of mammals the earlier formed allantois interpolating itself between the second allantois and the chorion would furnish anastamoses with the former.

Others consider that the inequality of the twins to a primary inefficiency of the parasite so incompatible with life that any development is only possible when a secondary connection, respiratory and nutritive, is established between the two unequal twins.

Such monsters as those having a double head, or four legs, etc., come from a single yolk which has two or more distinct germ vesicles. The double development of the anterior end is called an Anadidymus, the doubling of the posterior end is called an Katadidymus, the doubling of both the anterior and posterior ends is called an Anakatadidymus, and the doubling in the middle is called an Mesodidymus or Hemididymus. As hinted before, the cause is due to conditions inherent in the fertilized germ cell. The origin of double and multiple forms in the vertebrates, common in birds, are the appearance of two or more embryonic rudiments on a single blastoderm, or, the presence on the egg of two or more separate blastoderms, germ vesicles, quite often seen in birds; the fusion, or dichotomy on the part of a single embryonic rudiment accounts for certain examples of duplication, such as two heads. The formation of certain axial structures, in two parallel sets on a single embryonic rudiment is apparently not found in birds, or, if found is exceedingly rare. In the formation of certain monsters it is probable that the two female germinal cells of a single yolk are both fertilized by separate spermatozoa and then during the period of ovogenesis fusion takes place, and through disturbance of nutrition one zygote becomes the more fully developed autosite, and the other, the imperfectly developed zygote, becomes the parasite.

In Fig. 113 is shown a chick with two bodies, four wings, and one head, this is a Dipygus tetrabrachium. 
In Fig. 114 is shown a rather common monstrosity, two supernumerary legs have developed just posterior to the normal legs This is a Polymelus.

In Fig. 117 is shown a photograph of a fowl $4 \frac{1}{2}$ months old, in which two supernumerary legs have developed. There is partial

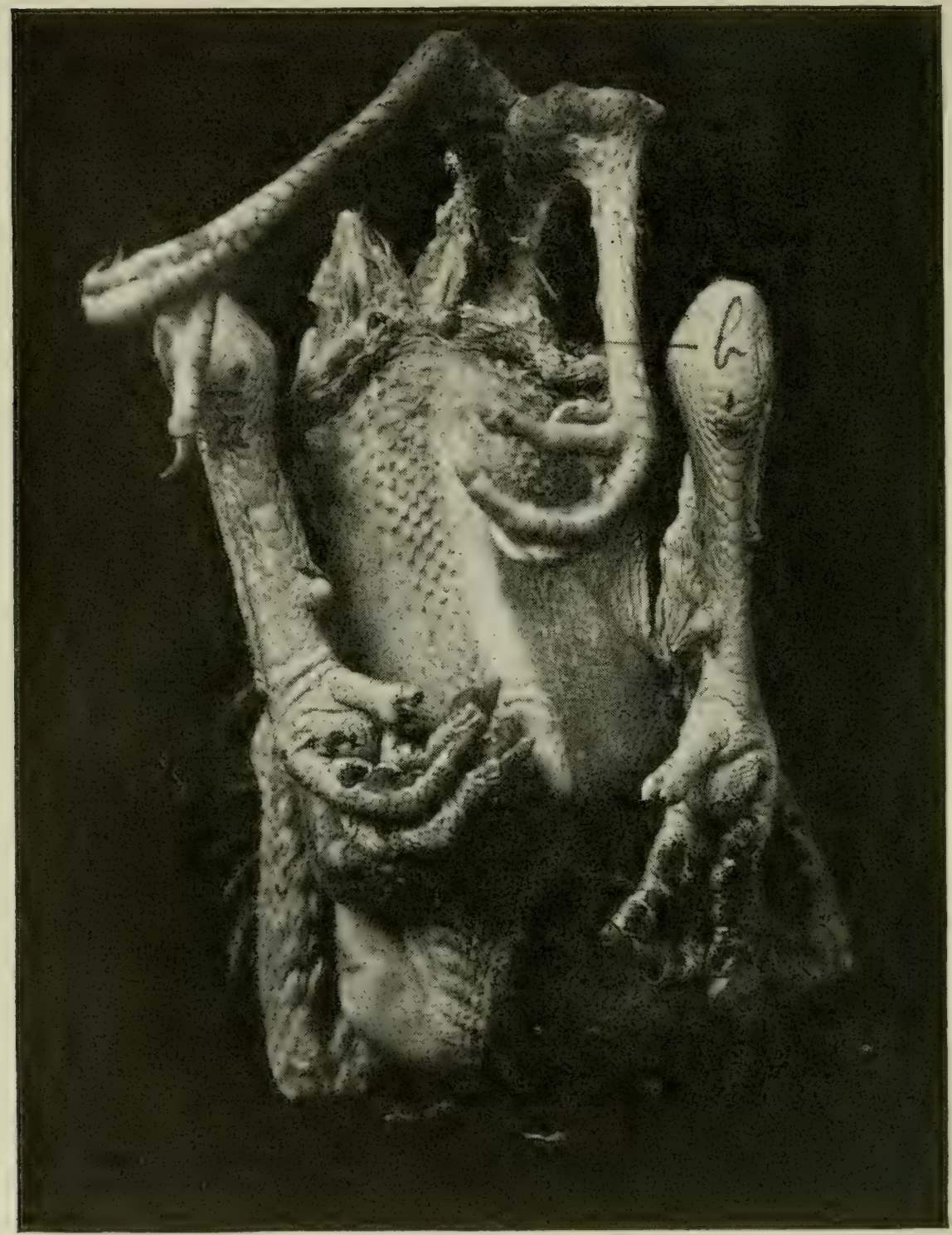

Fig, 117. A Dipygus of A Fowl, 41/2 Months Old.

Supernumerary hind limbs. Posterior dichotomy. Dorsal attachment. Courtesy of Mr. Laurie.

fusion of the parasitic limbs. a, indicates a normal anus and an accessory anus is indicated at $b$.

Figure 118 shows a photograph of a common white chicken in which there were developed two heads and two necks, a dicephalus. This is a photograph of the dorsal view. The two bodies are 


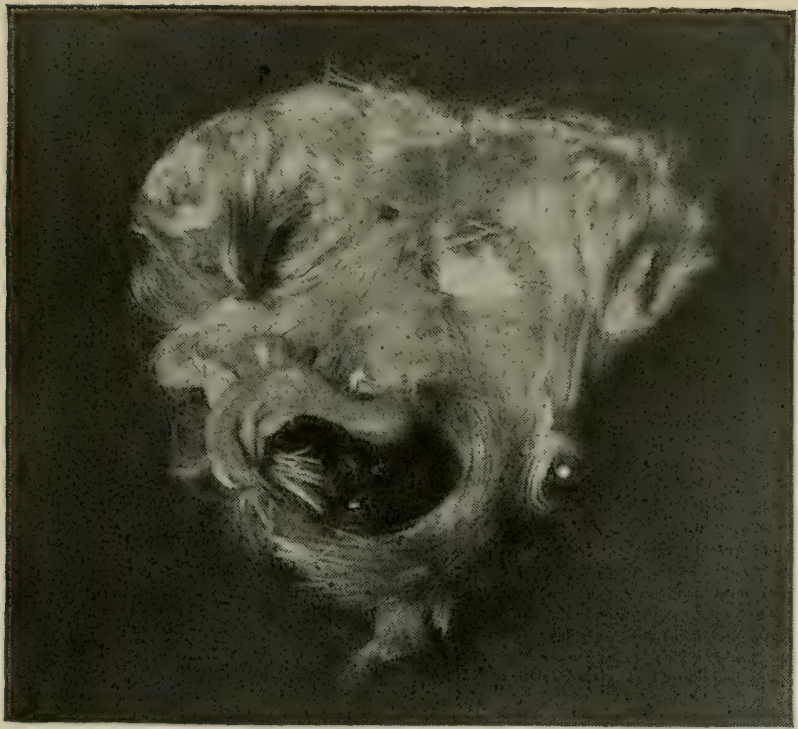

Fig. 118. A Dicephalus, Dorsal View of a Monstrosity Showing Two Necks and Two Heads.

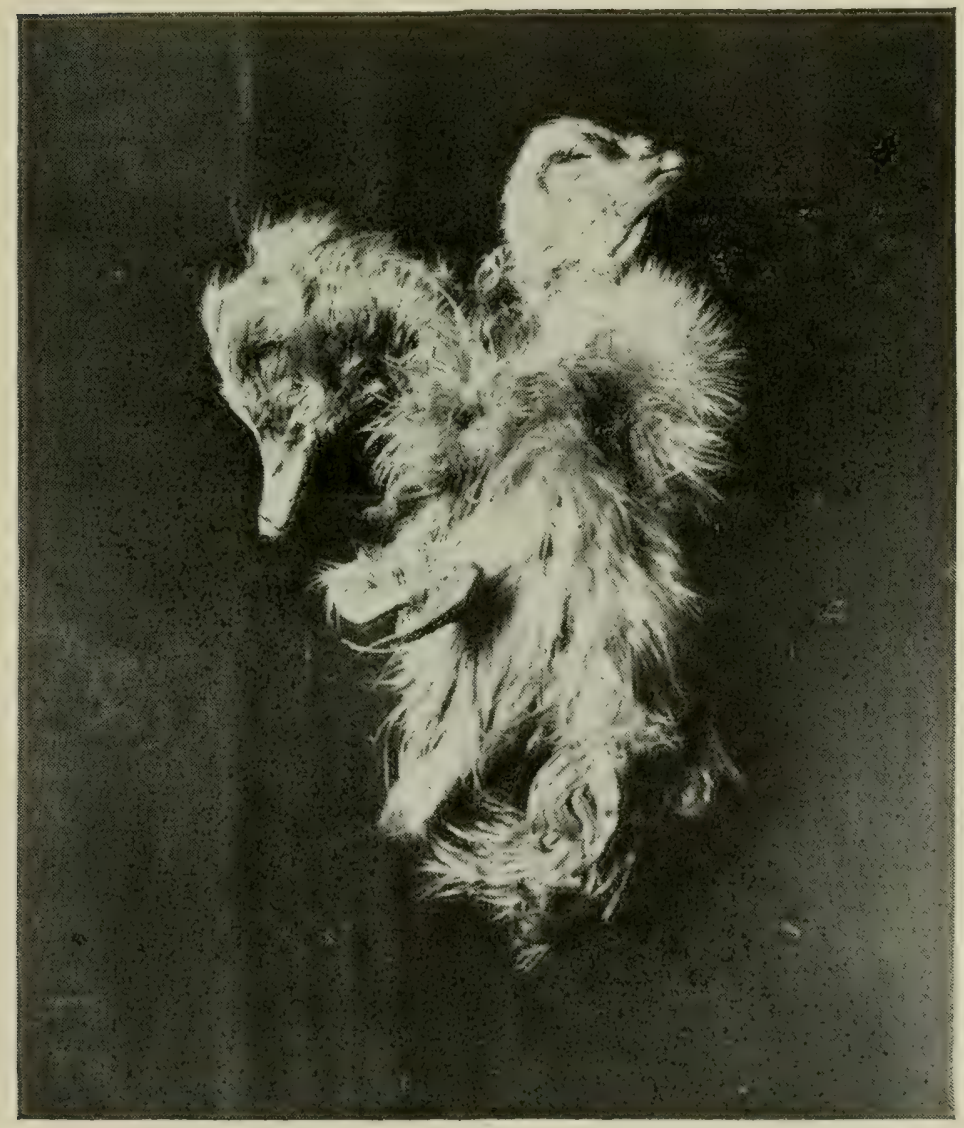

Fig. 119. A Dicephalus of a Muscovy Ducking With Two Heads AND Two Necks. 


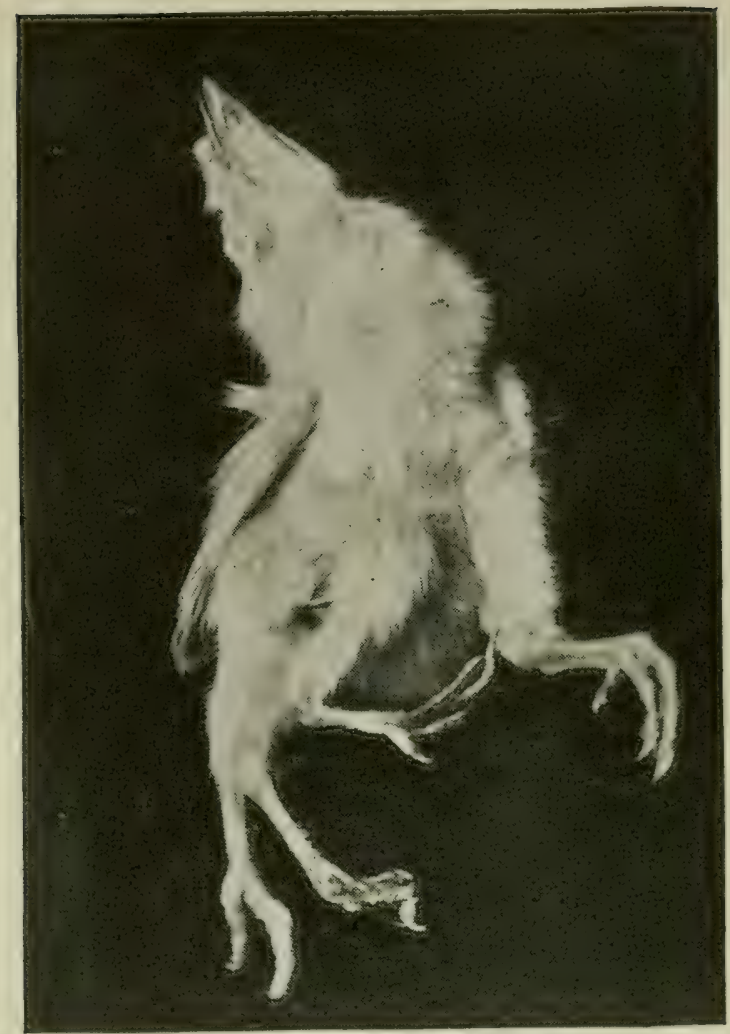

Fig. 120. A Dipygus of a White Leghorn Chick With Supernumerary Hind Limbs.

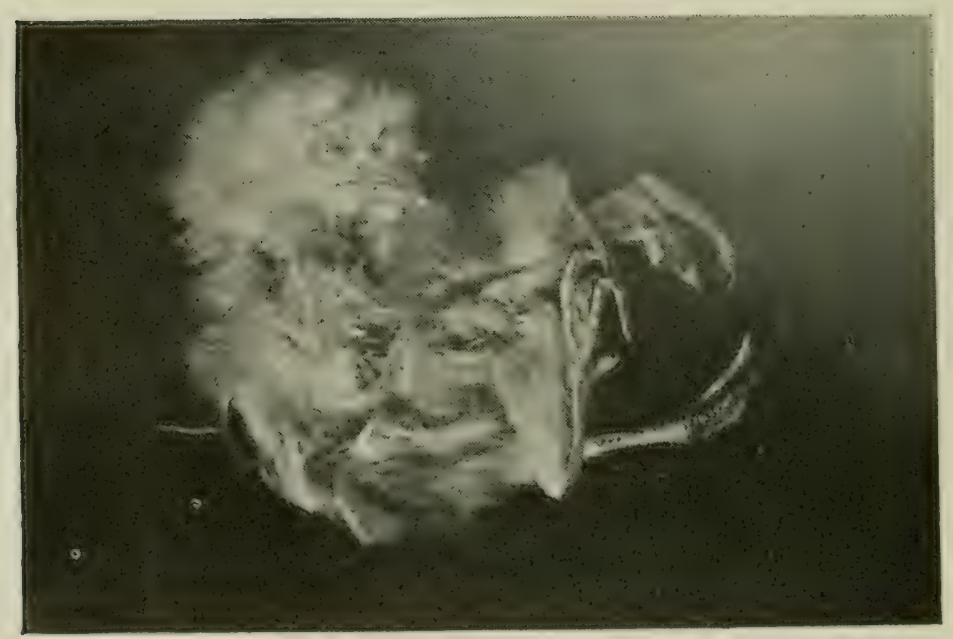

Fig. 121. Dipygus, the Supranumerary Hind Limbs are Ventrally Attached. 
joined ventrally. There was one umbilicus. The legs are normally developed as also is the caudal vertebrae. There are two ani.

In Fig. 119 is shown a photograph of a common white duckling in which there are two heads and two necks. This is a picture of the ventral surface. This is another case of conjoined twins with separate ani and a common umbilicus.

In Fig. 120 is a photograph of a White Leghorn chick in which there are developed two supernumerary hind limbs. This is a photograph of the ventral side. The limbs are ventrally attached. The supernumerary limbs are normally developed.

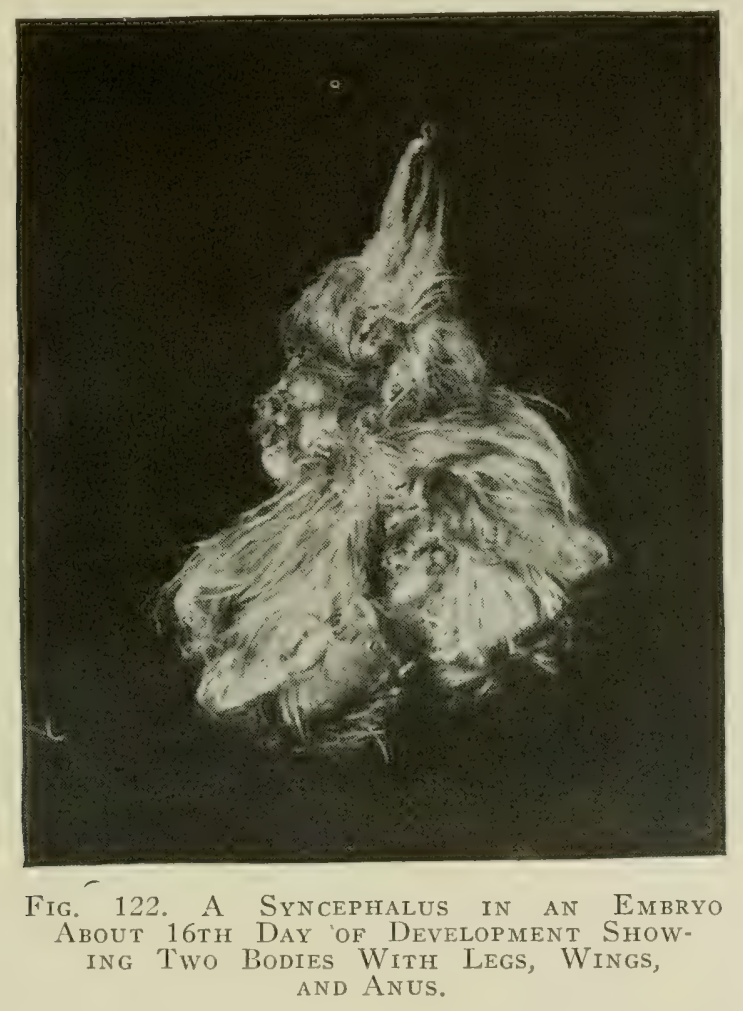

In Fig. 122 there is shown an immature chick embryo about the sixteenth day of development. There are two complete bodies joined near the top of the sternum. In each twin the legs, wings, and anus are normal. The cervical vertebrae are not fused. Both necks are included in an outer skin. The head is malformed. There is one beak and the mandibles are long and abnormal. The orbits have a frontal aspect. When more or less perfect separation of the trunks and fusion of the heads occur the syncephalus results.

The double-yolked eggs, in cases where two ova have been delivered into the oviduct at the same time, and both being surrounded by albumen and finally one membrane and one shell, have been supposed to produce double monsters, but this is not the case as is shown by the records of eighty such eggs which were incubated, all from the domestic fowl, and in each separate twins were produced. In some eggs both chicks were males, and in others both chicks were females, and in still others. one chick of 
each sex. In one case out of eighty one yolk developed a single chick and the other a double monster.

A study was made of a double embryo in the egg of a goose, which had been incubated five days. This study showed a double primitive trace is actually formed on a single blastodermic layer proceeding from a single vitellus and vitelline membrane. This same work has been corroborated by other studies by those so fortunate as to find these monstrosities in early stages of development.

Compound monsters proceed from single germs which have subsequently undergone different degrees of dichotomy. They are governed in their development by certain fixed and invariable laws among which are unity of sex, homologous fusion and bilateral symmetry. In each case there is single sexuality.

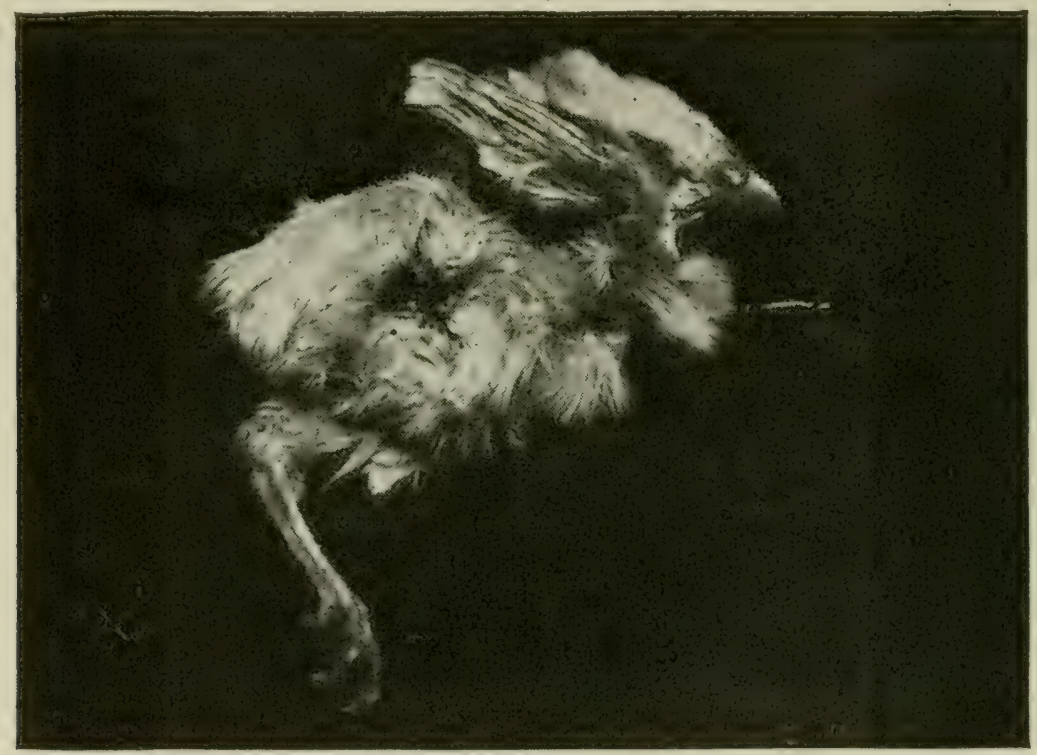

Fig. 123. A Chick With Only the Left Leg, the Other leg Bud Must HAVe ABorted.

The various forms of duplex development are determined by the extent to which the primitive trace is cleft, and also by the limitations of the dichotomy to the cephalic or caudal extremity of the neural axis. Either or both extremities may become bifid alone, as has been shown, and a double head, or still further bifid and the posterior extremities single or the posterior extremity become bifid and the anterior single.

\section{UMBILICAL HERNIA OF THE BABY CHICK}

Near hatching time the abdominal walls or plates come together after the intestines and abdominal yolk sac has receded within its walls. In many cases it will be observed that apparently chicks die, from lack of vigor and in some cases from improper development, while piping out of the shell. If the shell is carefully removed, some of the cases may show a rupture, or, a lack of closing of the abdominal walls with evidence 
of injury in straining while piping out of the shell. At the umbilical entry into the abdominal cavity the part is very delicate and the abdominal wall is very thin, and is not reinforced with elastic tissue, as in quadrupeds, and if the membranes be-

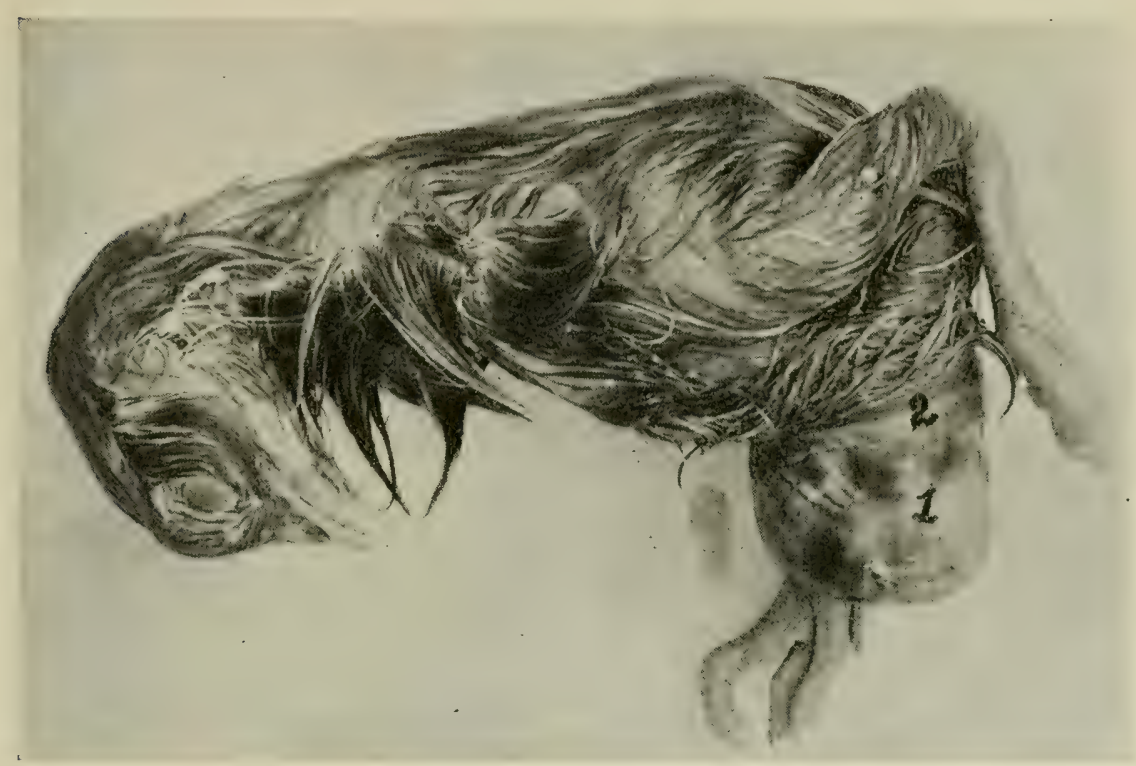

Fig. 124. Umbilical Hernia of a Baby Chick.

come very dry and tough, the straining by the chick in its effort to free itself may prove fatal. Fig. 124 shows a hernia as a result of excessive straining in forcing its way out of the shell. No. 1 is the abdominal yolk sac which has been forced down through the umbilicus, at 2. 



\section{CHAPTER XVII}

\section{FRACTURES-WOUNDS-ANESTHESIA FRACTURES}

Fractures or broken bones among birds in the poultry yards are of rather common occurrence, especially where birds are allowed to run with large animals on the farm or ranch, as is usually the custom, or where they are allowed to run in driveways and public roads.

\section{REPAIR OF THE FRACTURE OF BONES OF THE DOMESTIC FOWL}

This study is divided into three groups as follows: First, the structure and development of the bones of the domestic fowl; second, the kinds of fractures and the reparative processes; third, means of control of the bird and care of the fracture.

\section{THE STRUCTURE OF BONES}

In the gross study of bones we find that in the limbs as the legs and wings they form levers which have to sustain weight of the body and confer the power of locomotion, either during walking, running, or flying. The femur, tibia, and humerus are examples. The shaft of the long bone is narrow and contracted, which affords greater space for the bellies of the muscles. The extremities are generally somewhat expanded for greater convenience of motor connections, for the purpose of articulation and to afford a bony surface for muscular attachments. Some long bones are slightly curved, thus affording greater strength.

Where there is required great strength and at the same time motion it is divided into a number of small bones. As an example we have the patella and the two carpal bones.

Where the principal requirement is that of extensive protection or the need of large bony surface for the attachment of muscles, we find osseous structures expanded into flat bony plates, as is the case in the skull and pelvis.

The respiratory apparatus of the domestic fowl consists of two lungs, which occupy the upper thoracic region, pushing out between the ribs, and is made up of a series of air tubes and bladder like structures extending from them called air sacs. Some of the bronchi or air tubules communicate with air sacs or bladder-like structures located at the anterior thoracic region, others at the diaphragmatic region. Many of the bones of the fowl, as the head, vertebrae, and humerus, contain air cavities. The air sacs send extensions into these cavities.

Bones of fowls nearly always develop from a connective tissue foundation. The inorganic substance of the bone is compressed in or between fibers of the connective-tissue, while cells of the latter are transformed into bone cells. Between fibers are calcified 
bone cells, each of which rests in a cavity of the matrix, called lacuna.

The bone cells have processes that anastamose with the processes of other cells. They lie in special canals known as canaliculi.

The histological structure of the bone of the domestic fowl is similar to that of mammals, with the exception given above, and the reader is referred to any histology for further study and to Anatomy of the Domestic Fowl.

\section{REPARATIVE PROCESSES OF BONE}

A fracture of a bone may be defined as a sudden solution of continuity in a bone. The cause of fractures in a fowl are: First, injury or trauma, receiving a blow as from a stick or

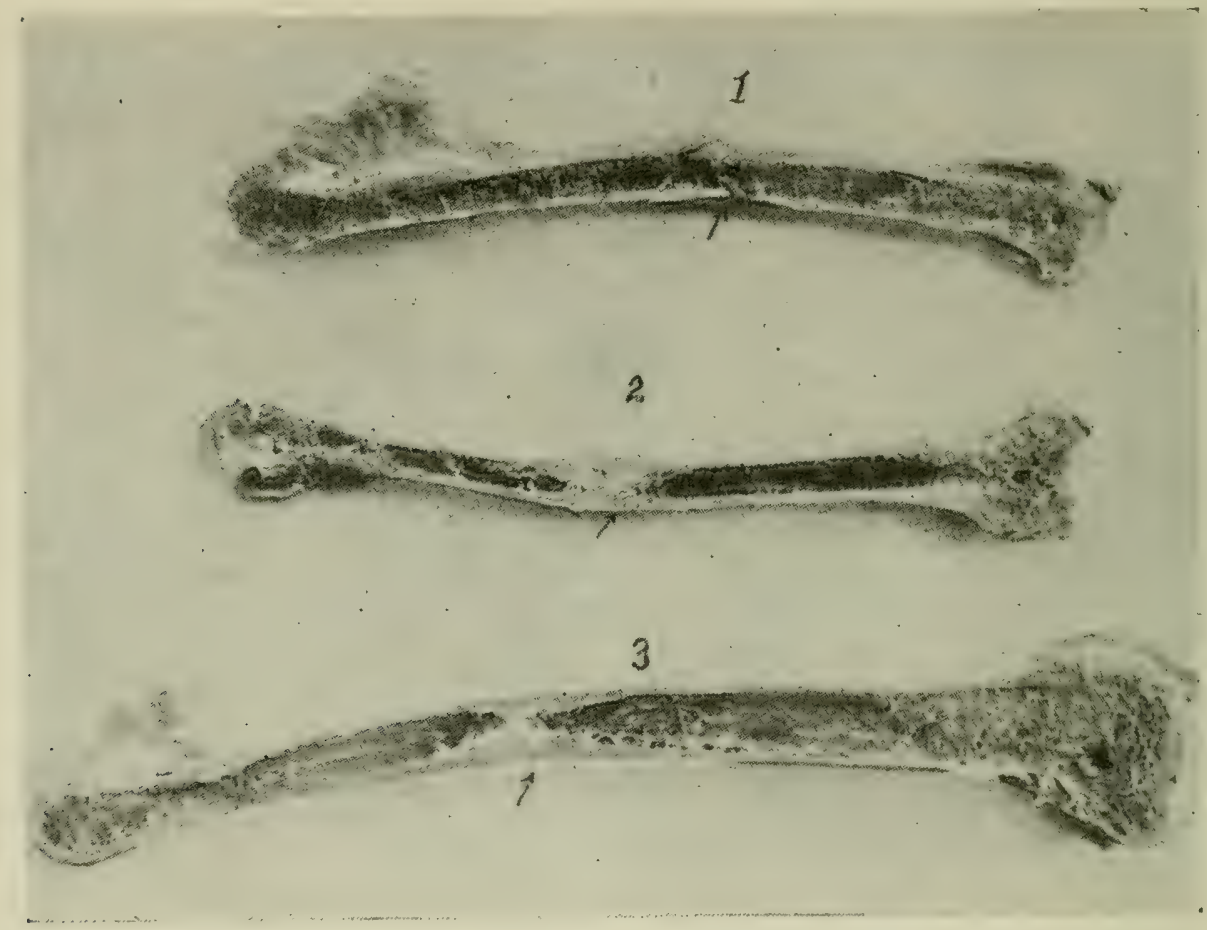

Fig. 125: Section Through Bones of Different Stages of Fractures.

1, five days standing; 2 and 3 , thirteen days standing. Note the provisional plug and the provisional callus.

stone or being stepped upon by a large animal, as a horse or cow, or by the infliction of a gun shot wound; second, muscular action. Bones are most resistant to traction, next to pressure and less risistant to flexion or bending and least of all to torsion. External violence may be direct or indirect. In fracture from direct violence the bone is broken at or near the spot where violence is applied. As a rule the soft structures surrounding the fracture are more or less injured and more serious results may follow than in fractures by indirect vio- 
lence. In this kind of fracture the bone may be comminuted or fissured and perhaps driven into vital parts or organs, as the liver or lungs, if the fracture be near these regions, or into the brain if in the cranial region.

External violence is the most common cause of fracture in the fowl. The most common bones that are fractured are those of the leg and next those of the wing.

Fractures may be classified as follows: First, simple frartures - those breaks in the continuity of the bone where the skin is not broken; second, compound, also called open, or complicated fracture-those where the break is accompanied

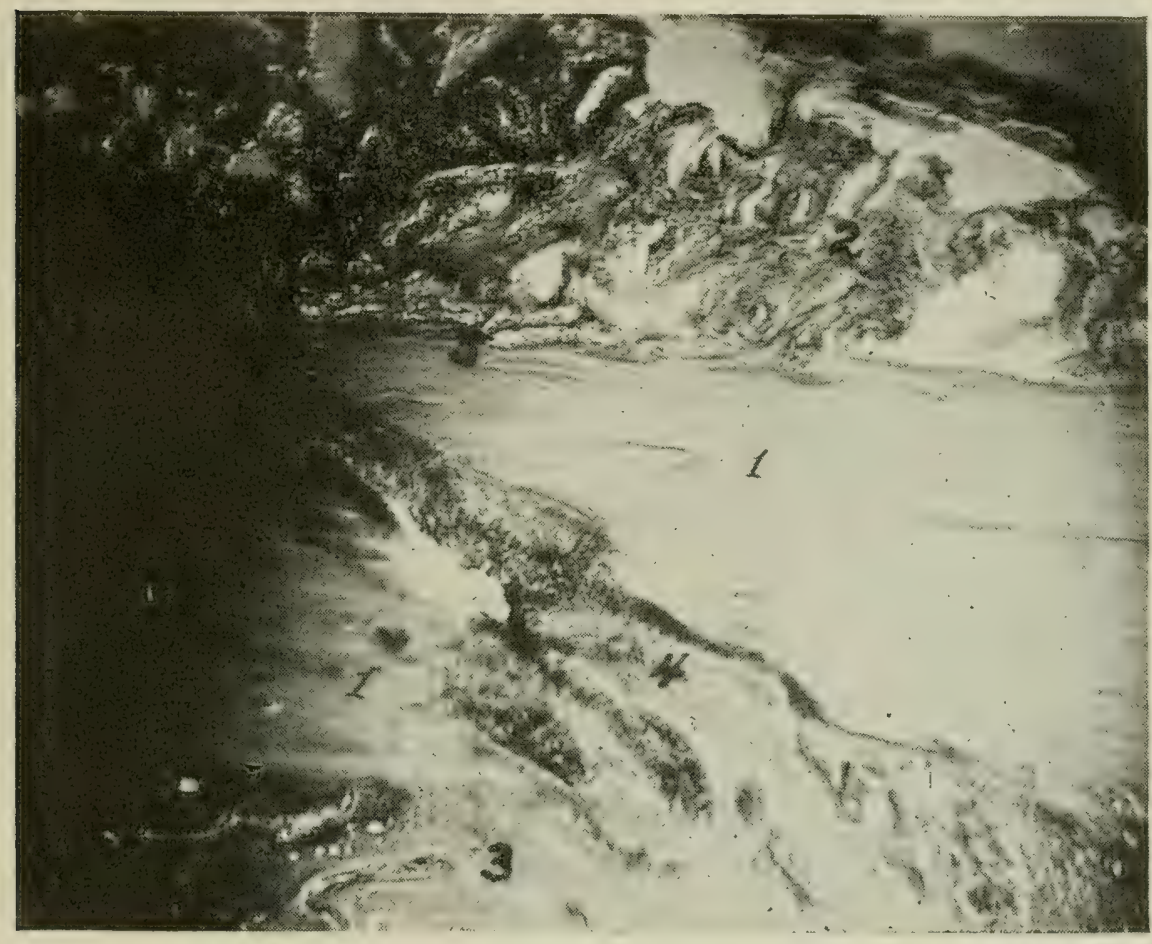

Fig. 126. A Photomicrograph of a Prepared Section of an Oblique Fracture OF THE ULNA OF A FOWL.

1 , the fractured ends which have been placed in perfect apposition in the process of setting; 2, the provisional callus; 3 , the provisional plug; 4 , the intermediary callus; 5 , the periosteum, one of the sources of new bone cells.

by a break through the skin and soft parts extending to the seat of the fracture.

A series of studies was made in this laboratory, of repaired fractures of fowls, of long standing, after which a series was made of the nature and rapidity of repair of fractured bones of the domestic fowl. The birds were chloroformed and the bones fractured and set while the birds were still under the anaesthesia. The metatarsus and ulna were selected. The 
appliance that was used to hold the bones in place was cotton, one inch muslin bandage, wooden splints and glue. At the end

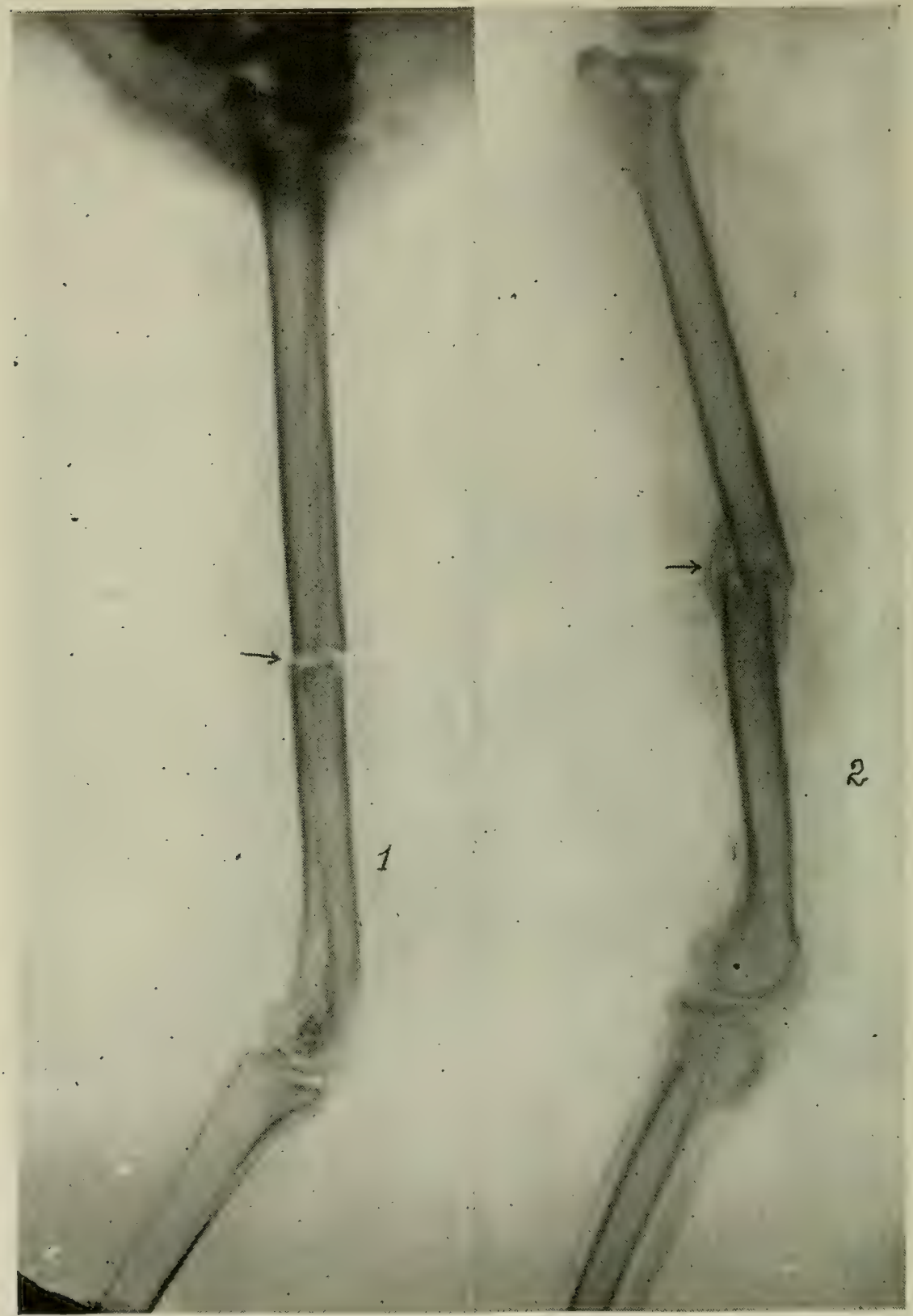

Fig. 127. Roentgengraph of a Bone Just Fractured and Properly Set Indicated by an Arrow at 1, and a Fracture Improperly Set Showing Provisional Callus filling in Around the ENdS of the Bone and Around the Fracture.

of each experiment the bird was chloroformed and the bone removed. After a physical examination, the bone was sec- 
tioned longitudinally, photographed and the lesion of one-half cut out and placed in a ten per cent solution of hydrochloric acid for forty-eight hours for decalcification, and then passed through three changes of absolute alcohol; then alcohol and ether equal parts; then embedded in paraloid and sectioned.

The sections were stained in hematoxylon and eosin and clarified in beechwood creosote, and mounted in Canada balsam for microscopic study.

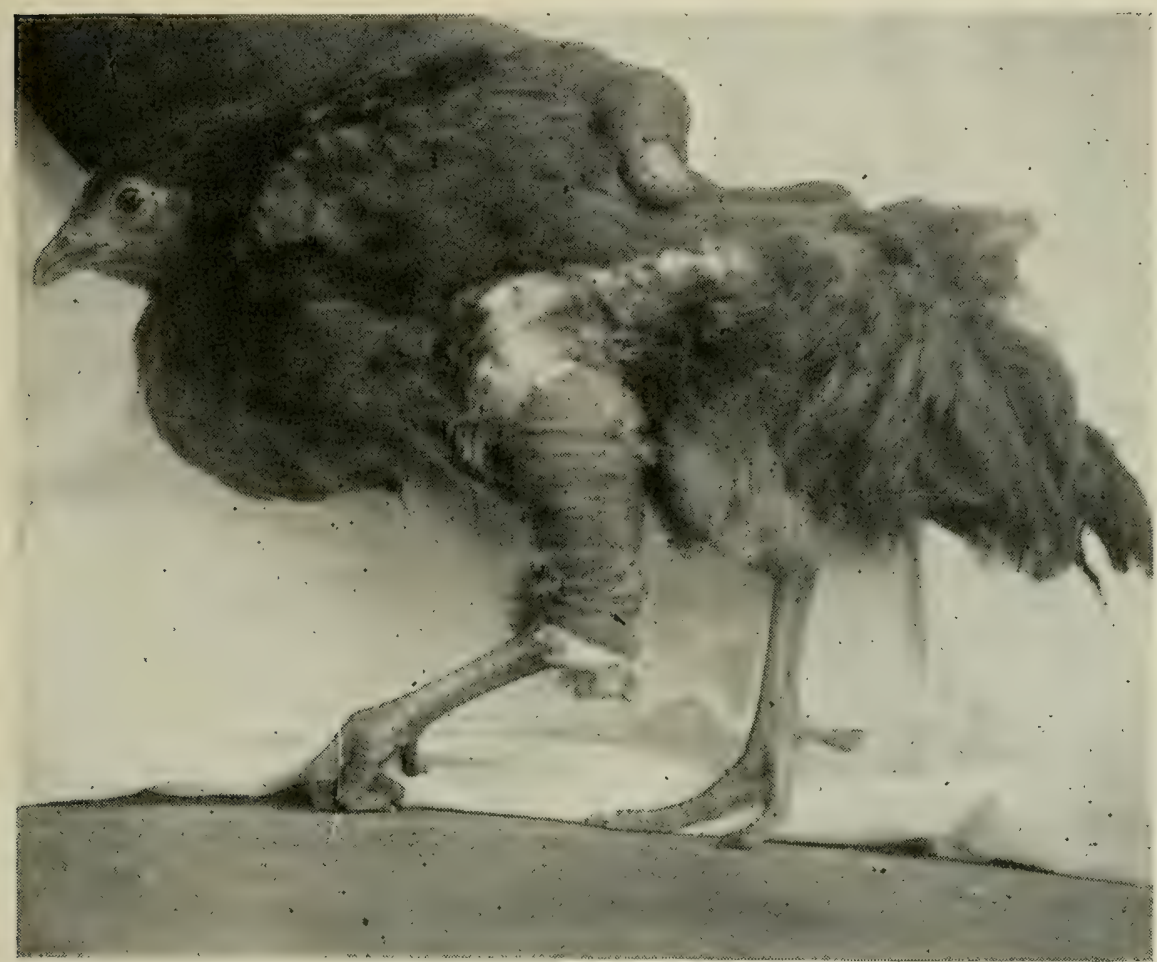

Fig. 128. A Rhode Island Red Pullet With a Broken leg Properly Set Úsing Light Wood Splints, Cotton, One-Inch Muslin

Bandage, AND Glue.

In a study of a fracture of the metatarsus of a Single Comb Rhode Island Red of eight days' standing, the following picture presented itself. The gross specimen showed a mottled reddish white zone in the region of the fracture indicating that immediately following the fracture there was extravasation of blood which had collected around and between the fragments and between the ends of the compact portion of the bone and had also invaded, to a certain extent, the marrow cavity. The fluid at this time did not give evidence of advance organization and was rather jelly-like allowing the fractured ends of the bone to fall apart by slight traction. There was present the initial hyperemia or congestion of repair. This hyperemia was most marked in the periosteum. Leucocytes had invaded this part, às shown by microscopic examination. Proliferative changes had taken place in the connective tissue 
and in fact this was observed in cases of only forty-eight hours standing. The most active cellular multiplication was in connection with the fibrous structure of the periosteum. This forms the germinative or reparative tissue from which arises osteoblasts. The nature of the new formed structure was that of connective tissue and is plainly shown in the photomicrograph. This picture shows this organization into trabecular-like arrangement forming the periosteal callus and the provisional plug. It can be seen that this had been formed and poured out from the periosteum. This field showed many fibroblasts and was packed with osteoclasts and in still other fields of the trabeculae a homogeneous matrix with formative bone cells in their lacunae. The repair was apparently one of intramembraneous bone formation with islands of newly formed bone at the end of the fifth day.

Another study of a fractured metatarsus of thirteen days' standing was in a one-year-old Single Comb White Leghorn hen, that was of low vitality and the reparative processes were more tardy than in a similar section of another case in which the bird was in a good state of health. After the metatarsus was removed it could, with considerable force, be made to spring, which was not the case with the latter specimen. Both birds showed the reparative process far enough advanced to have the cast removed with safety.

From these two studies it is rather indicative that repair in the bone of the domestic fowl is quite rapid and that ten days to two weeks is ample time to allow the bandage or cast to remain on the fractured part.

\section{THE TREATMENT OF FRACTURES AND CARE OF THE FOWL}

In simple or so-called subcutaneous fracture of bones the fragments of the bone should be placed in perfect apposition. The normal shape of the bone should be restored as far as possible. The loose arrangement of the muscles in the fowl makes this an easy task.

The next step is to apply an apparatus holding the parts firmly in place while the reparative processes are being accomplished. In applying the apparatus, the circulation must not be interfered with and the nerves must be safeguarded. After the setting is complete the bird must be provided with a clean coop and a grassy run where other birds cannot interfere. Good food and water should be provided and an occasional examination made to determine if all is well with the bone undergoing repair.

If the fracture is on a feathered part, the feathers that are in the region to be manipulated should be removed. Next apply a thin layer of cotton, carefully holding the fractured parts in the proper position; then apply about three thin, narrow splints of wood of proper length in such a manner that they cannot chafe the leg and do injury to the skin. Next apply a one-inch cotton bandage, at the same time saturating 
it with glue. In a few hours the liquid glue will become hard and the parts will be firmly held in position.

At the end of fourteen days, carefully remove the bandage. Confine the bird for a few days longer and then allow it to run in its accustomed quarters.

As to feed, for the first two or three days after injury give easily digested feed, such as bread soaked in milk or wet mash. Later some grain may be fed, but only two light feeds should be given while the bird is in confinement. Pure, clean water should be kept before the bird at all times.

\section{WOUNDS}

Birds possess a high immunity to pyogenic infection, the germs that ordinarily infect the wounds of animals, and wounds, whether accidental or surgical, unless very serious, heal with great rapidity. The degree of tolerance of infection that the peritoneum of birds possess is probably not equalled by the peritoneum of any other domestic animal. For example, birds seldom die from infection after caponizing. Death when it does occur as a result of this operation is ordinarily due to hemorrhage. Men and animals, except the dog, survive abdominal operations only when done under aseptic conditions.

Wounds should be cleansed with antiseptics as in mammals. Full strength iodin favors healing and is an excellent antiseptic. This is to be applied after the wound has been cleansed with water.

\section{ANESTHESIA AND RESTRAINT OF THE FOWL}

Chloroform may be administered by means of the carton a one-fourth pound can or bottle comes in. A small hole is cut in the bottom of the box to admit air and a small pledget of cotton placed in the box to absorb the chloroform.

The hen is very susceptible to chloroform, quickly going under its influence and quickly coming out again. Care needs to be exercised lest too much be given and the bird die from an overdose. In administrating the chloroform the head of the bird is first thrust into the box. The bird usually struggles very little and is soon completely under its influence. The cover is then removed or held one or more inches from the nostrils as indicated by appearance of the bird. If an overdose be given, open the mouth and resort to artificial respiration as with animals; many are thus revived.

Note is made of the respirations, or breathing, and eye reflexes as in anaesthesia of animals.

After the limbs are entirely relaxed an assistant holds the bird on its back or it may be tied to the operating table. 
A poultry operating table has been designed by the writer. This table consists of a top, see $a$ in Fig. 129. The top is two feet wide and thirty inches long. This table is provided with four-inch cross-pieces as shown in the picture at $c$. These cross-pieces are located about half-way from the top to the

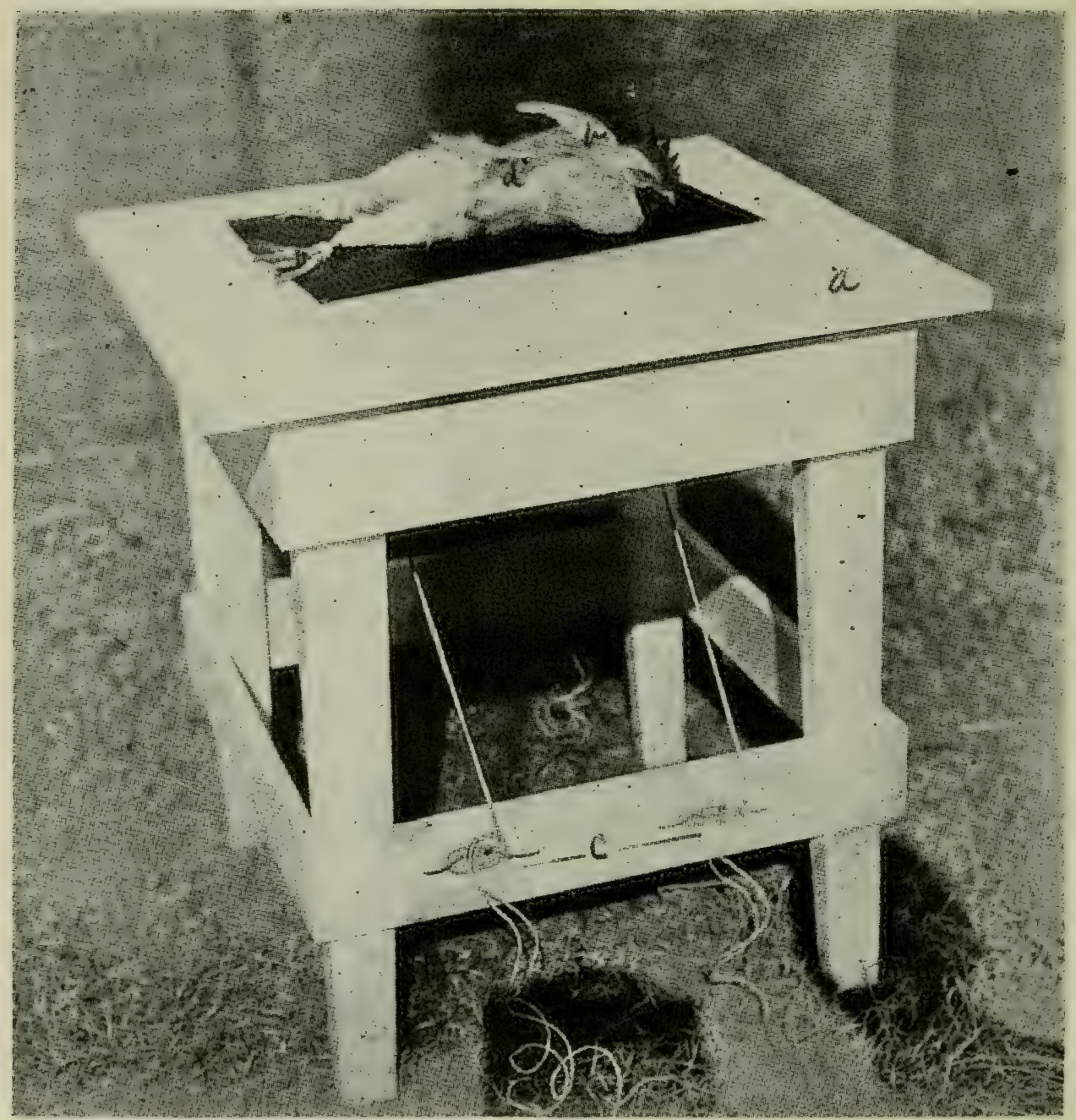

Fig. 129. A Convenient Poultry Operating Table.

ground. These cross-pieces are provided with two awning hooks on either side. Holes are bored through the top at suitable locations. The loop of the string is run through the hole on its respective side and over the legs or base of the wings shown at $h$, in the picture. The legs and wings are drawn down snugly to the top of the operating table and the free portions of the string wrapped around the hook and given a half hitch. 


\section{INJURY TO THE STERNUM OR BREAST BONE}

If chicks are allowed to roost on small limbs of trees or sharp poles before the breastbone becomes sufficiently ossified there is likely to be a dent or curvature of the free margin.

Fig. 130 illustrates this kind of an injury. The dressed carcass from such a bird would bring at least two cents a pound less on the large city markets than if the breast was straight and presented a pleasing appearance.

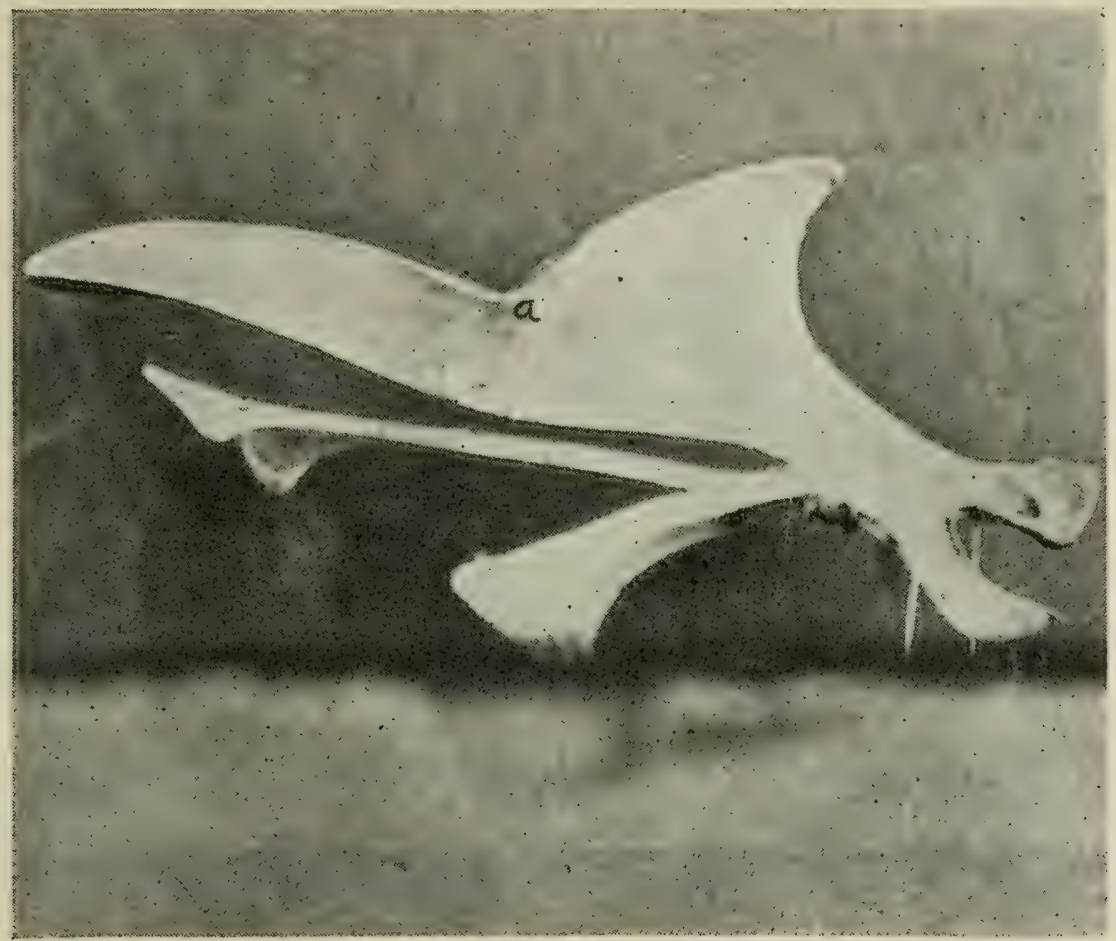

Fig. 130. Injury to a Sternum, a Result of Roosting on Some Sharp Object While QUite IMMATURE.

Other injuries as broken limbs with healing of the injured limb in a crooked position. This makes a deformed part which detracts from the nice appearance of a carcass.

\section{CURVATURE OF THE SPINE}

Deformed chicks may result from insufficient turning of eggs in the incubator during hatching. This deformity is partly due to adhesions and turning six to eight times a day largely prevents this trouble, or, the eggs may lie with small end up not giving the embryo sufficient space. Eggs, during incubation, should lie on their sides. If allowed to stand on end in the last days of incubation there is a possibility of some 
chicks being deformed. The major deformity is of the legs, feet and toes.

Curvature of the spine has been noticed as appearing rather suddenly during their developing stage and its true cause,

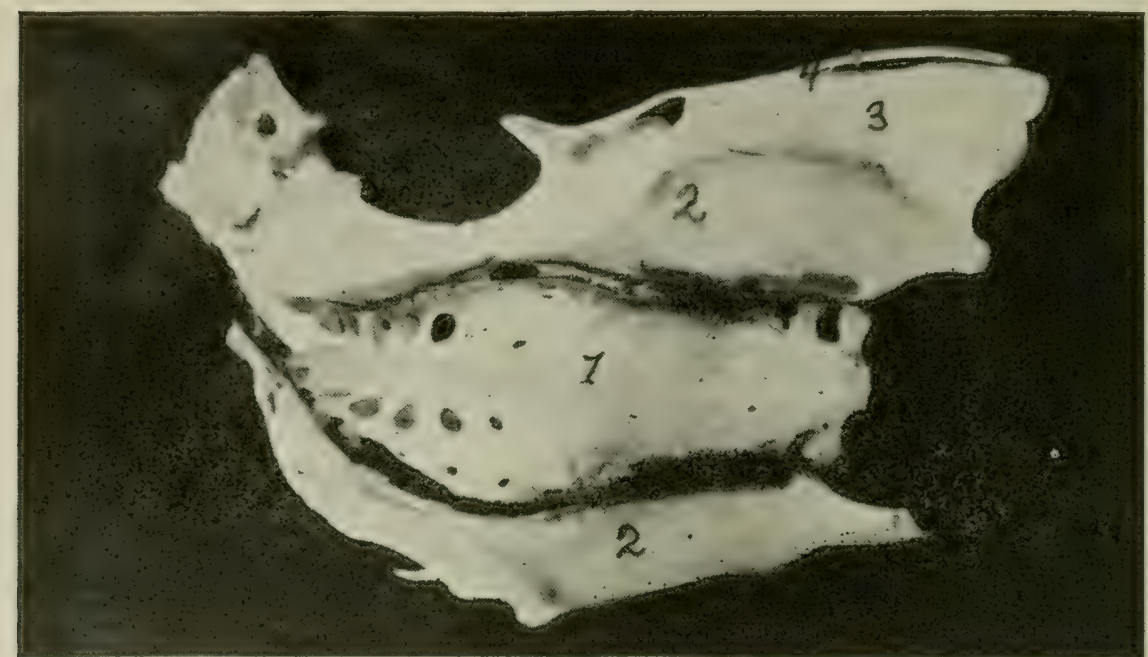

Fig. 131. Curvature of the Spine of a Young Developing Chick.

1, the Vertebra Showing a Decided Curve; 2, the Ilium; 3, the Ischium; 4, the Pubis.

whether due to injury or otherwise, has not been determined.

This curvature is no respecter of breeds or varieties. It attacks the developing bird most at twelve to twenty weeks of age or at a weight of from two to four pounds. 


\section{CHAPTER XVIII}

\section{CASTRATION OF BIRDS OR CAPONIZING}

There are great possibilities in the more extended practice of capon production. The fact that there is a growing demand, making their value as a meat product superior to that of the cock or cockerel, and the fact that they bring about thirty cents a pound while the uncastrated bird brings only about fifteen cents a pound, together with the fact that they become very much larger, makes this phase of poultry production work a very remunerative one.

The male bird after the removal of his reproductive organs loses his masculine appearance, takes on more of a juvenile look, becomes sluggish and gains weight rapidly as a result of his inactivity.

From the loss of that internal secretion manufactured in the testes we note that there is the same difference which is observed in other animals under the same treatment. The development of the gelding as compared to the horse, and of the barrow as composed to the boar, are some concrete examples.

The same improvement in meat is noted in the castrated or caponized bird as in the meat of the steer over that of the bull, or the meat of the barrow over that of the boar, hence capon raising is highly desirable and if properly managed is a profitable undertaking.

A capon of the Plymouth Rock, Wyandotte, or Rhode Island Red breed should weigh fully seven or eight pounds when eight months of age.

Light capons are produced from Plymouth Rocks, Wyandottes and Rhode Island Reds, while the heavy capons are produced from the Brahmas and Cochins.

The cockerel should be caponized when he weighs from one to one and one-half pounds, which will probably be about the eighth week if force fed or the tenth week if not force fed.

If the birds are allowed to become too old before operation testes are too large and the removal of which may prove fatal to the bird. If the birds are hatched from March to May the operation could be performed in the months of June to September and with proper feeding and care these birds should then be ready to market from December to March.

The equipment needed to perform this operation is a table as illustrated in Fig. 129. This table is provided with a means of confining the bird on the table. The instruments needed 
consist of a knife or scalpel with which to make the incision or cut through the abdominal wall, a hook for tearing through the peritonetm and abdominal air sac walls and, at times, through the mesentery and a spreader for holding the wound open while the removal of the testes is accomplished.

The intestines may be pushed to one side by aid of the end of the scalpel.

An improvised table may be made by taking a barrel, using two cords and two half bricks, or weights of sufficient size to hold the bird down. Such an improvised table is shown in Fig. 132.

If the bird or birds are to be operated on in the forenoon, no feed should be given on the previous day. It is also well to withhold water as an abundance of water causes more hemorrhage, owing to the increased amount of liquid in the body tissues. It is rather difficult to accurately and satisfactorily operate when the intestines are full of feed.

The operation is best performed in the bright sunlight unless the operator is provided with a head reflector.

The instruments should be kept in a shallow pan of antiseptic, or zenoleum, or a formaldehyde solution. A small amount of absorbent cotton should also be at hand. After the bird is confined pluck a few feathers from over the field of operation which is between the last two ribs. It is well to place a small piece of ice in the pan of antiseptic and use the ice water in sponging the field of operation. The cold water thus acts as an antiseptic as well as causing a contraction of the blood capillaries of the region and less hemorrhage will result. As the blood of the fowl coagulates in 30 seconds the hemorrhage soon stops.

When ready make the incision through the skin, then pull the skin over so that after the cut through the abdominal wall has been made the skin will close the hole in the abdominal wall. If it is the desire to make the cut through the skin and abdominal wall at one time pull the skin orer, make the cut. and when the skin is released it will cover the hole in the wall of the abdomen.

In making the incision through the skin the bird will struggle very little. The knife or scalpel should be very sharp and the incision made quickly to minimize the pain. The upper point of the incision should be about one-half inch from the center of the backbone or vertebrac. The incision should be about one inch long. As a nerve, artery and a vein pass along the posterior border of each rib, it is necessary not to cut to the anterior border of the last rib.

A second incision is now made in the same wound, this time 
cutting through the abdominal muscles. Care must be exercised not to cut too deep and injure the internal organs. If the peritoneum is not cut through this can be broken by aid of a hook and the spreaders inserted. Now tear through the walls of the air sac and push the intestines to one side and the uppermost testis will be in plain view. The testis will appear bean-shaped, about one-half inch long and yellowish-white in color. It lies close to the body of the vertebrae and large abdominal blood vessels, being attached by connective tissue. If it is the desire to remove both testes through one opening

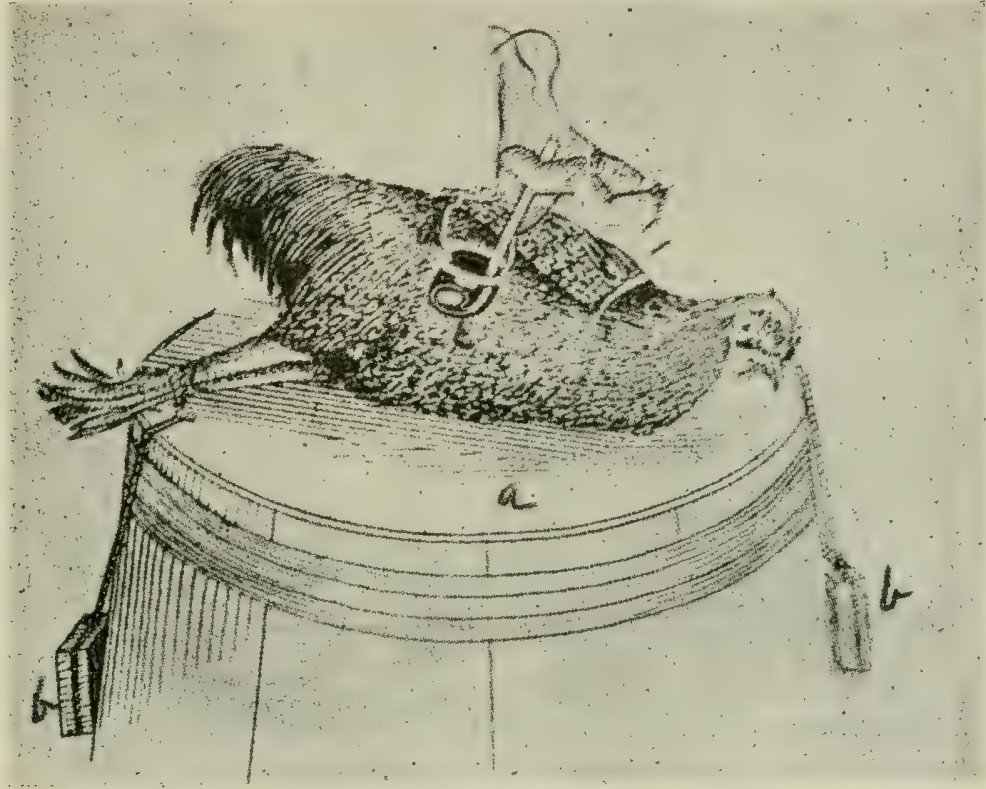

Fig. 132. The Top of a Barrel Can Be Used as an Operating table.

it is necessary to tear through the mesentery or web-like membrane supporting the viscera, care being taken to not make the opening too close to its attachment to the vertebrae or a large vessel may be torn and fatal hemorrhage may take place as a result of rupturing these delicate vessels.

If the opening has been properly made the lower testis will be in plain view. Always remove the lower testis first as, if the upper one be removed first, some hemorrhage may take place that will make it very hard to find the lower one later. For beginners it is better to operate from both sides, each time removing the upper testis. To reach the lower testis tear through the mesentary about an inch from the vertebral attachment so as to avoid hemorrhage. The extractor can be passed through this opening and the lower testis readily reached. 
The testicular tissue is very soft and it is necessary to use great care to remove all of the tissue. If the testis is crushed it will be very difficult to successfully remove all of it so that it is necessary that the operation be clone with skill. If care is not used often the end of the testis will break off and this part remaining will make a slip. This small particle will furnish some internal secretion and the bird can be regarded neither a cockerel or a capon. Slips are undesirable and when older can be recognised by the fact that they are neither the extreme juvenile appearance of a capon or the appearance of a cock. The accident may occur as follows: On account of the testis lying close to the vertebrae in close proximity to the abdominal aorta and other large blood ressels and the capsule of the testis being attached to them, too much traction or improperly applying the tractors may result in rupture of the vessel and fatal hemorrhage occur at once. If the aorta is ruptured there will be heard a hissing sound because the blood from the artery comes out in spurts as the heart forces it into the arteries in jets. Immediately the bird becomes pale in the face and comb and it immediately collapses. In this case cut off the head of the cockerel and the bird can be used as food. If the bird is allowed to struggle after the operation a large vessel already injured by the operation may rupture and fatal hemorrhage result.

After the operation if the incision has been properly made no suture is necessary, but if the opening in the abdominal wall be large it is well to take one or two sutures with clean strile cotton or silk thread. A half curved needle with common white thread will be satisfactory.

After the operation is completed remove the bird as carefully as possible and quietly place it in a clean coop or run bedded down with clean straw. Care must be observed in removing the bird from the table as often when they find their strings of restraint are loosened they struggle and such struggles often result in fatal hemorrhages. Do not allow the birds that have been operated on in coops or inclosures where they can jump upon boxes, perches or fly, as they must be kept down on the floor for a few days.

Supply the birds with clean, fresh water and give them ground feed mixed with milk as soon as they are placed in their runs. They apparently do not suffer any inconvenience from the operation and will eat heartily immediately after.

On the third day examine each bird to make sure there are no "wind puffs" or emphysematous conditions, that is, air worked under the skin from the edge of the wound or incision. In some cases cluring the act of breathing, the cut acting as a 
valve, air is gradually worked in between the skin and the body wall. If "wind puffs" or emphysema is present puncture with a clean, sterile, sharp knife and allow the air to escape. It is best to make an $\mathrm{X}$-shaped incision so that air will not again work in between the skin and body wall. Birds have a great resistance against the common germs of wound infection, as the staphylococci and streptococci and fatalities from this cause are very rare, if at all.
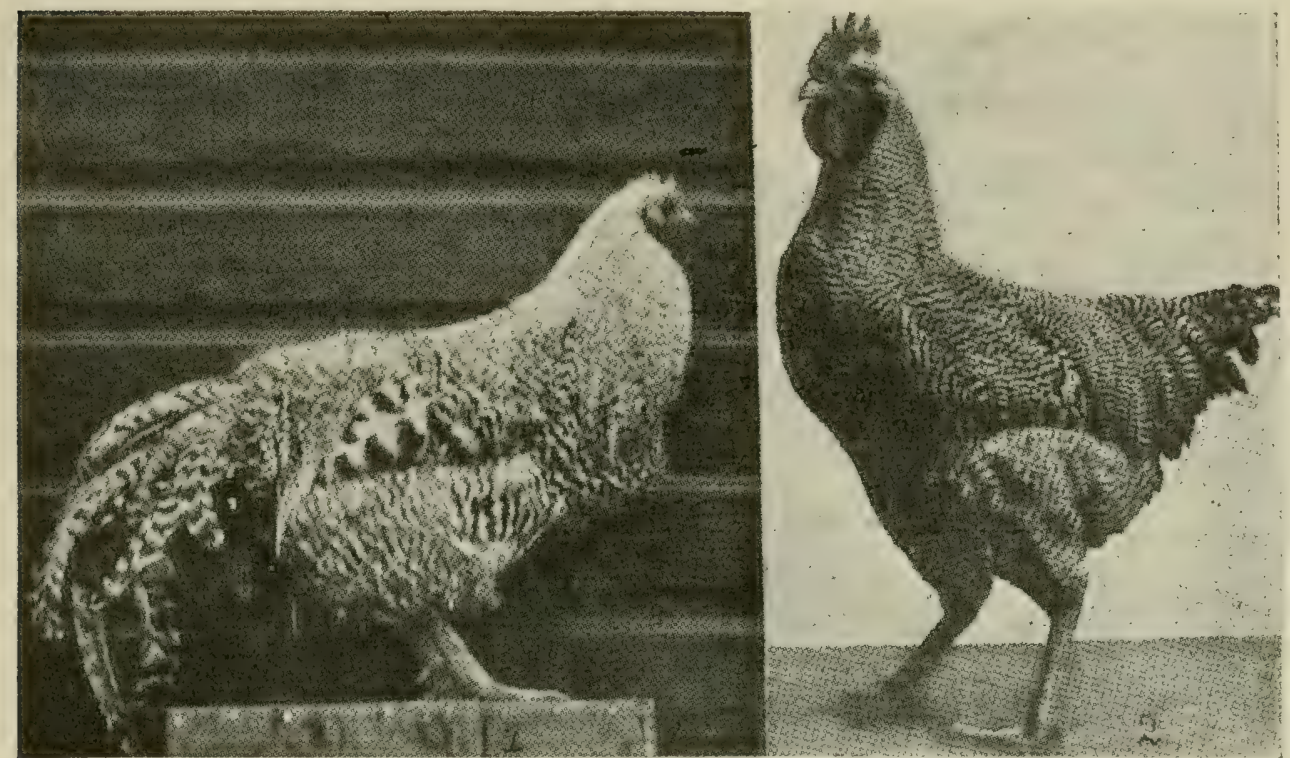

FIG. 133. 1, a capon; 2, a cock.

The wound should be entirely healed in less than three weeks' time.

Fig. 133 shows a Barred Plymouth Rock capon and a Barred Plymouth Rock cock. Notice the difference in the head, plumage and general appearance between the capon and the cock. Capons are usually marketed at about ten months of age. Force feeding is sometimes resorted to when they are marketed in a less time and are called soft roasters.

\section{OVARIECTOMY OF THE PULLET}

An unsexed, or spayed, pullet is called a poulard. Spayed pullets make more rapid growth without the handicap of egg production, at a later stage, and the meat is of improved quality and flavor. The spayed pullet takes on some of the appearance of a cockerel. The poulard, like the capon, becomes an outcast in the poultry yard, being shumned by hens and cocks alike, and is never known to cackle. 
The pullets are usually operated upon at about the same age as in caponizing the cockerel and usually in the late spring or early summer. The pullets are prepared in the same manner as the cockerels for caponizing. The incision is made in a similar manner as in the cockerel and the undeveloped egg cluster or ovary in the pullet is found in a similar location to that of the testes in the cockerel. With a pair of artery forceps grasp the undeveloped oviduct, which will be observed to be about the size of a broom straw, and remove about one inch of this and the ovary. To do this a curved pair of scissors will be required. Care must be taken not to cut or rupture any of the large abdominal blood vessels lying just back of the ovary and against the vertebrae, a similar precaution as in caponizing. The removal of a section of the oviduct and ovary prevents the further development of the egg canal and functionating of the cells of the canal and the formation of eggs. Pullets that have begun to develop eggs cannot be successfully operated upon.

The after treatment is the same as for capons which see. 


\title{
CHAPTER XIX \\ FOODS POISONOUS TO FOWLS
}

\author{
THE ROSE CHAFER
}

\section{(Macrodactylus Subspinosus Fab.)}

In the spring of the year when the grapes are in bloom, large numbers of the common rose-chafers, or rose bugs, often appear to feed upon the blossoms and later attacking the young

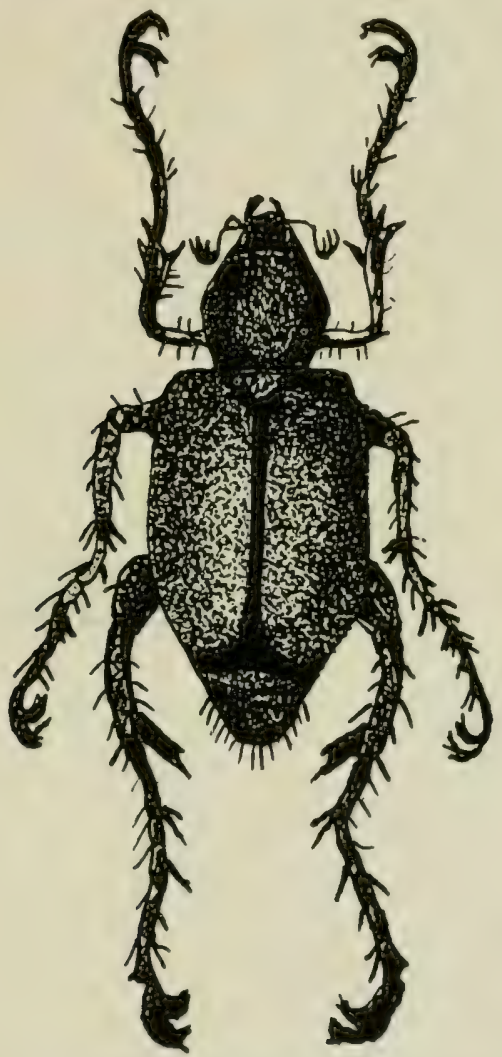

Fig. 134. The Rose Chafer or Macrodactylus SUBSPINOSUS, ENLARGED.

and developing fruit and leaves of the plant. The beetle is about one-third of an inch long, of a light brown color and is covered by numerous lighter hairs. It is provided with long, spiny legs. This bug may also be found on roses, from which its common name is derived. It may also be found on other shrubs and upon apple, plum, cherry and peach trees. When numerous and its preferable food scarce, it may attack different grasses and grains. 
The bug passes through the four stages, namely: ova, larva, pupa and adult.

It has been shown that when large numbers of these bugs are eaten by small chicks death may occur from their poisonous effects in from nine to twenty-four hours. It was found that fifteen to twenty rose chafers were sufficient to kill chicks seven days old in seven days; twenty-five to forty-five proved fatal to chicks twenty-one days old. Chicks over ten weeks old did not die from their effects.

The symptoms usually appear an hour after the bird eats a large quantity of these bugs. The first symptoms noted are those of a dozing attitude, the bird becomes weak, and finally prostrate and is unable to walk. Some may recover from the poison. Occasionally convulsions are noted in the dying chick. The poison appears to be a neuro-toxin. Post mortem does not reveal any lesions.

Prophylactic treatment consists of keeping the young chicks away from parts of the premises that are infested by these bugs.

\section{ARSENICAL POISONING}

Arsenical poisoning may occur from the birds drinking spray mixtures containing paris green or other arsenical compounds, from eating rat poison, etc. Cases have been brought to our attention where birds had been poisoned by eating grasshoppers. The grasshoppers had been given arsenic in bran, and the birds, devouring large numbers of them, became ill, and many birds died.

Sy'mptoms.-Loss of appetite, black comb, dullness, sitting, moping and unsteady gait, increasing weakness and death. Judging from the effects of poisonous doses of arsenic on higher animals, the poisoned birds must have been in considerable pain, but they did not show it; birds do not manifest pain as other animals do.

Autopsy.-The liver was normal, except that it was a trifle dark in color. There were no noticeable changes in the other abdominal organs, except the intestinal tract. Upon opening the intestines there were noted patches of hemorrhage and areas of congestion and inflammation.

Treatment.-This is scarcely worth while. Demulcent drinks, as water, in which slippery elm bark has been soaked, or even milk, are indicated, after a full dose of castor oil.

\section{SALT POISONING}

Chicks do not stand a very high percentage salt. We found in some tests that the poisonous dosage was between one and one-quarter to one and one-half per cent in the feed. Older birds have not been tested for the danger line, but it is 
safe to say that the percentage in mash should not exceed one per cent.

Salt seems to be a common poison, irate neighbors use, in wishing to kill the neighbors' hens which bother them. Many such cases have been sent to laboratories for confirmatory tests.

Poisoning among chickens and turkeys from eating common salt or drinking brine is quite common and the losses from it are large. It may occur from eating salt pork, or fish, or from drinking the brine left from freezing ice cream, and in many other ways. The symptoms and treatment vary but little from arsenical poisoning.

A classical case has often been told in which a newly wed in baking a cake made a mistake and used common table salt instead of sugar. After the cake was baked and the mistake discovered the young housewife concluded to feed it to her nice flock of hens, consisting of twenty-three hens and one cock. All of the birds except the cock died.

It has been determined that twenty-five grains of common table salt per pound live weight is sufficient to produce death in an adult bird.

\section{OTHER MINERAL POISONS}

Saltpeter poisoning, from eating fertilizer; phosphorus poisoning, from eating rat poison; lead and zinc poisoning, from eatting paint, and copper poisoning, from drinking bordeaux mixture, have been described; all are infrequent.

\section{PTOMAIN POISONING}

Limber neck is one of those convenient generic terms which poultrymen sometimes apply to any ailment in which the bird is too sick to hold up its head. It is a very prominent symptom in all forms of ptomaine poisoning. However the term limber neck has been scientifically understood to mean only that condition due to a specific germ the Bacillus botulinus.

Cause.-Ptomaine poisoning may be due to eating any kind of food in which putrefaction has set in, but is usually the result of eating decaying meat or fish.

Because of the more favorable conditions for the rapid putrefaction of meat in very hot weather, ptomaine poisoning occurs chiefly in mid-summer, and on farms where the fowls have an extended range, including patches of high weeds that effectually conceal dead animals, as rats, cats and chickens, from the caretaker, until the loss of a large number of individuals of the flock compels cutting the weeds and a diligent search for the carcass. 
The beginning of ptomaine poisoning in a flock is usually something like this: During very hot weather a bird dies in the tall weeds, it may be from disease or from violence, and in three or four days its carcass is filled with maggots and in an advanced stage of decomposition; it is found by the other birds and devoured, with the consequent death of many of them. some of them dying in out of the way places and remaining undiscovered by the keeper, and in turn poisoning others.

Oftentimes the keeper is responsible for the beginning of the trouble by thoughtlessness, throwing some small animal which he has killed, opossum, weasel or rat, where the fowls find it. If the weather conditions are favorable to rapid decomposition, potmaine poisoning in the flock will result and the "vermin" dead will destroy more birds than ten of its kind would destroy during life.

Eating maggots from manure piles where the organic matter is decomposing is a fruitful cause of death among fowls.

Maggots are usually found in the crops of birds dying from eating putrid flesh, and if the poultryman holds autopsies on the dead birds, he is quite apt to conclucle that the maggots have killed them.

Treatment.-Give a tablespoonful of castor oil and one-fifth grain doses of sulphate of strychnin, the latter every four to six hours.

Experiments have been conducted to determine the exact dosage of strychnin for an average-sized hen. It has been found that the dose should be from one-sixth to one-fifth of a grain, for an average sized hen, three times a day. The writer has given, in solution, one grain on a full crop without ill effect. If the drug be given on an empty crop the absorption is much more rapid and larger doses are required where drugs are given with mash or on full crops.

\section{BOTULISM (Limber Neck)}

Botulism is a nervous disease in which there is prostration due to infection, through the food, of the Bacillus botulinus or germ causing so-called sausage poisoning. The germ lives in decaying animal or vegetable matter.

Outbreaks of botulism have been studied among birds as well as among man and animals. In one case which is related fifty fowls were fed home-canned corn which had caused the death of a woman who had tasted it. In another case between fifty and one hundred fowls became paralyzed and died at the same time that a woman who cared for them had died of bulbar paralysis. Eight fowls, in another case, showed the same symptoms and died after eating home-canned string beans which had caused the death of a woman who had eaten them. And in still another case seven fowls died after eating home-canned apricots which had also caused the death of five people. 
In all cases in human and fowls the symptoms and the course of intoxication were the same as those of botulism and in the histologic examination of one case, after autopsy, showed the rypical thrombosis and hemorrhages which have been shown to be characteristic of the condition. The organism recovered from the fowls were identical in morphological and cultural characteristic to the Bacillus botulinus. The toxin from these cultures produced typical symptoms.

Symptoms.-The symptoms of limber neck or botulism are as follows: The birds become dull, inactive, refuse to eat, remain quiet; their feathers are ruffled, and the bird gradually becomes weaker. This is manifested in the legs, wings and finally in the neck, so that the bird is unable to stand, drops the wings; the muscles of the neck are out of use and the neck is limber, the bird is sitting on its breast with its beak on the floor and finally complete prostration results and the bird lies listless on its side and in a state of coma or unconsciousness dies. Death usually occurs in less than twenty-four hours after taking in the poisonous feed.

\section{CORN COCKLE POISONING}

Chickens eating large quantities of corn cockle, in ground form, incorporated in their feed in the form of a mash, have been poisoned.

The seed contains a poison, sapotoxin, which causes a severe inflammation of the entire digestive tract, including the crop. The bird gradually becomes weak, and as the poisonous effects advance the bird gradually becomes prostrate and dies in a state of unconsciousness or coma.

\section{ERGOT POISONING}

Ergot is a diseased condition of rye and other cereals, in which the grain becomes black, and often spur-shaped and several times its normal size. It is caused by a parasitic fungus the claviceps purpurea.

Should ergot be ground and mixed with feed as described in the case of corn cockle sufficient quantities may be eaten to cause poisonous results.

When sufficient quantities of ergot is taken in with the food an acute poisoning will occur. There may be convulsions, twitching of the muscles, a staggery gait, partial paralysis, intense thirst, and unconsciousness or coma followed by death.

When a less quantity is taken in continuously there occurs a chronic ergot poisoning which is manifested by indigestion, diarrhea or constipation, and gangrene, especially of the dependent parts as the comb and wattles. Ergot is a powerful constrictor of the blood vessel walls and in these parts the 
circulation is gradually cut off and the comb or wattles turn black and falls off, usually a dry gangrene. Death of the bird may follow.

\section{WEEVILY WHEAT}

Weevily wheat, due to the small brownish-black weevil, if abundant in wheat, together with their dust which adheres to the grains, kill baby chicks. It does not noticeably injure older birds.

\section{BLACK LOCUST LEAF POISONING}

Birds become poisoned by eating the leaves of the black locust.

Symptoms.-The affected fowl lies down, apparently paralyzed, eyes half closed, comb quite red, and droppings thin, watery, slimy, and greenish in color, and containing strings of mucus and blood. The breathing is deep and heavy. Locust leaves fed to fowls experimentally cause death in from 12 to 24 hours.

Treatment.-Do not allow fowls to consume the leaves. Give cathartics, as epsom salts, and stimulants, as strychnine. 


\section{POULTRY REMEDIES}

The average temperature of the domestic fowl is $107^{\circ} \mathrm{F}$. The temperature of the fowl is easily disturbed by high or low atmospheric temperature, exercise, worry or fright.

The average number of respirations of fowls are, for the male 20 , and the female 36 . The number of respirations is easily disturbed by worry, fright or high atmospheric temperature.

Blood pressure of the average sized utility cock, taken from the femoral artery, is $135 \mathrm{~m}$. $\mathrm{m}$. The average pulse rate of the average size utility cock is 350 times a minute.

With the development of Veterinary Medicine there has come a knowledge of drugs and their applicability to diseases of the domestic fowl. The writer has devoted much time and thought to rational medication of fowls and has worked out a dosage of about fifty drugs based on the physiological and therapentical application in disease.

The following brief discussion of poultry materia medica and its therapeutic application is thought best in this volume.

In medicating birds it will be well either to give the drug by the mouth in capsules or in tablet form, since in a large number of cases the liquids administered as such by the mouth find their way down into the trachea and bad results follow. Liquids may be mixed with mash or soaked in bread in cases where the fowl has not completely lost its appetite or where it does not object to the taste.

In large flocks it is more practical to give the medicine either in the drinking water or mixed with the mash.

The larynx of the fowl is not provided with an epiglottis and, in struggling birds, stands more or less open.

Birds require a comparatively larger dosage to obtain the full physiological results than do mammals. A large amount of unsatisfactory medication of fowls in the past has come through the lack of rational medication.

\section{POULTRY MATERIA MEDICA}

\section{ALOIN}

Source-A neutral principle obtained from aloes.

Properties-Small acicular crystals, in color yellow to yellowish brown; odorless and bitter taste.

Use-Cathartic. 
Indications-Constipation.

Dose-For adult fowls, 1 to 2 grains.

\section{AMMONIUM CARBONATE (Smelling Salts)}

Source-A mixture of ammonium chlorid or sulphate, and calcium carbonate, is sublimed and resublimed.

Properties-White, hard, translucent, striated masses, having a strong ammoniacal odor and a sharp salty taste.

Action-A heart and respiratory stimulant.

Indications-Acute bronchitis, pneumonia, or in colds.

Dose-For an adult fowl, 20 grains given in a capsule.

\section{ARECA NUT (Betal Nut)}

Source-From the seed which resembles nutmeg in shape and color.

Properties-A brownish powder.

Action-Destroys intestinal parasites.

Indications-Infestation of intestines with worms, and acts as a cathartic.

Dose-For adult fowls, 5 to 10 grains, given in wet mash.

\section{ARGENTI NITRAS FUSUS (Lunar Caustic)}

Source-Melted silver nitrate, 3 parts, and potassium nitrate, 6 parts, cast in suitable moulds.

Properties-White, hard, solid pencils which appear finely granular at a broken end.

Action-A caustic.

Indications-The pencil, lunar caustic, may be used to lighty burn ulcers in the mouth or head as in roup or sorehead.

\section{BARBADOES ALOES}

Source-The dried or inspissated juice of the Aloe vera.

Properties-A hard mass, orange brown and opaque. Odor, saffron-like and a bitter taste.

Action-A cathartic.

Indications-Constipation.

Dose-For adult fowl, 10 to 20 grains.

\section{BELLADONNA FLUID EXTRACT (Deadly Nightshade)}

Source-An extract of the leaves of Atropia belladonna.

Properties-Blackish-brown liquid of characteristic odor.

Preparations-Extract; fluid extract, and tincture.

Action-A stimulant. Lessens mucous secretions. Small doses do not affect respiration but large doses make breathing quicker and deeper.

Indications-Acute inflammation of the air passages as bronchi and lungs.

Dose-To adult hen, five drops, repeated every three hours. May be dropped in the mouth by aid of a medicine dropper.

\section{CARBO LIGNI (Wood Charcoal)}

Source-Soft wood is charred by piling it in a heap, igniting, and covering it with sand and dirt to prevent rapid combustion.

Properties-A black, odorless and tasteless powder or bits, free from gritty matter. 
Action and Indication-It is indicated in indigestion, chronic gastritis, and intestinal catarrh and diarrhea.

How Given-May be kept in compartment of dry mash hopper where the birds have access to it at all times. Use large size pieces for fowls and chick size for chicks.

\section{CASTOR OIL}

Source-A fixed oil expressed from the castor oil bean.

Properties-A pale, yellowish and almost odorless, transparent viscid fluid and possessing an offensive taste.

Use-A cathartic.

Indications-Constipation.

Dose-Use tablespoonful to each four fowls. May be given in wet mash.

\section{CHLOROFORM}

Source-Alcohol and water are heated in a still to $37.7^{\circ} \mathrm{C}$., when chlorinated lime is added and choloform is evolved.

Properties-A heavy, clean, colorless, mobile and diffusable liquid of a characteristic etherial odor, and a burning sweetish taste.

Action-Anaesthetic.

Use-It is used as an anaesthetic in preparing birds for operation. Chloroform and ether are an excellent combination for anaesthetic purposes.

\section{COAL-TAR DISINFECTANT DIPS (Standardized)}

A standardized coal-tar disinfectant dip is a coal-tar distillate. Coal-tar dips may be standardized. In standardizing the dip its germacidal power as compared to carbolic acid is determined and its co-efficient expressed. Thus, for example, a dip with a coefficient 5 would mean that its power to destroy germs is five times as great as carbolic acid.

Source-Coal tar distillation products, the active principles of which are cresols and hydrocarbons.

Properties-They are of rather thick, black tarry liquid which turns the water a milky color, due to the soapy emulsion that results upon the addition of the water.

Use-A destroyer of germs and parasites. A dip of a co-efficiency of 5 , as Zenoleum, is used in 4 per cent solution and at a temperature of 100 to $110^{\circ}$, or near body heat, as a dip for fowls to rid them of lice, or as a dip for other animials except cats to which it is fatal. To make this per centage consider that there are 128 ounces in a gallon and to each gallon there must be added approximately 5 ounces or a large teacup full. It may be used in this same strength in spraying against lice and mites or as a disinfectant to destroy infective germs.

\section{CONVALLARIA FLUID EXTRACT (Lily of the Valley)}

Source-An extract of the lily of the valley plant.

Preparations-Extract, fluid extract, and tincture.

Action and Indication-Similar to digitalis.

Dose-For adult fowl, ten to twenty drops.

\section{CREOLIN}

Source-Obtained from soft coal by dry distillation, its composition is very complex. It is said to contain cresol and higher homologues of phenol. 
Properties-A dark-brown syrupy, alkaline liquid of a tarry taste and odor.

Action-A powerful disinfectant, antiseptic, and parasiticide.

Use-As a spray for poultry houses and equipment use five ounces to the gallon of water.

\section{CREOSOTE}

Source-A mixture of phenols and phenol derivatives, chiefly guaiacol and cresol, obtained during the distillation of wood tar, preferably of that derived from the beech.

Properties-An almost colorless, yellowish or pinkish, highly refractive, oily liquid, having a penetrating, smoky odor, and a burning, caustic taste. It usually becomes darker in tint on exposure to the light.

A similar product can be obtained from the gas plants at a cheaper price for poultry use.

Action-It is a strong preserver of woods as it is a disinfectant and a parasiticide.

Indications-Infestation of the perch poles, dropping boards, nests, and other parts of the building with mites or other harmful parasites as chicken ticks, and fleas.

Use-Use in a pure state. Paint on, with brush, all sides of the perch poles and if necessity requires, the dropping boards, nests, and walls.

\section{DIGITALIS FLUID EXTRACT (Fox Glove)}

Source-From the leaves of digitalis.

Preparations-Extract, fluid extract, and tincture.

Action-It strengthens the heart beat.

Indications-It has a tendency to correct the rapid, weak, irregular pulse.

Dose-For adult hen ten to twenty drops.

\section{ETHER (Sulphuric Ether)}

Source-Obtained by distillation of alcohol with sulphuric acid.

Properties-A transparent, colorless, mobile liquid, having a characteristic odor and a burning, sweetish taste.

Action-To produce anaesthesia.

Use-It is used as an anaesthetic in preparing for operations on birds.

\section{EUCALYPTUS, OIL OF}

Source-A volatile oil distilled from the leaves of the eucalyptus tree.

Properties-A colorless or faintly yellowish liquid. It has a characteristic aromatic odor, and a pungent, spicy and cooling taste.

Action-It is an antiseptic and a disinfectant, being three times as effective as carbolic acid.

Indications-Catarrhal conditions of the nasal mucous membranes, and mucous membranes of the trachea and bronchi.

Use-As a steam inhalation or as a spray or injection in catarrh and roup combined with other drugs, as follows:

Oil of eucalyptus................30 drops

Oil of thyme...................... drops

Menthol ....................10 grains

Oil petrol .................. 2 ounces

Mix and inject into the nasal passage. 


\section{FERROUS SULPHATE, COPPERAS (Green Vitriol)}

Source-Iron wire is dissolved by boiling in dilute sulphuric acid.

Properties-Large, pale, bluish green, monoclinic prisms, without odor and having a salty taste. Changes to a fine powder on being exposed to the air.

Action-Externally it is an astringent and stimulant.

Indications-In a solution of one ounce to one gallon of water for wounds of any kind in the fowl.

\section{FORMALDEHYD (Formic Aldehyd)}

Source-Obtained by partial combustion of wood alcohol, without ignition, by evaporation of the spirit in contrast with a hot, platinized, asbestos plate.

Properties-A pungent gas. Sold in aqueous solution, 40 per cent gas in water. It is volatile.

Uses-A strong disinfectant. Used in about the same dilution as lysol for disinfection purposes.

\section{GENTIAN (Powdered)}

Source-Obtained from the gentian roots.

Properties-A yellowish brown powder possessing a bitter taste.

Action-Improves the appetite and stimulates digestion.

Indications-Indigestion, loss of appetite, and malnutrition.

Dose-Same as for ginger, which see.

\section{GINGER (Powdered, Zingiber)}

Source-From the roots of the Zingiber officinale.

Properties-A yellowish-brown powder with bitter taste.

Action-A bitter tonic.

Indications-Indigestion, loss of appetite, malnutrition.

Use-Combined for a tonic with other drugs, as follows:

Powdered ginger ............. 2 ounces

Powdered gentian .............. 2 ounces

Powdered nux vomica .......... 2 ounces

Mix.

Give one teaspoonful to each twelve fowls. Given twice daily in wet mash.

\section{GLYCERIN}

Source-A liquid obtained by the decomposition of vegetables or animal fats or fixed oils.

Properties-A clear, colorless liquid of a thick, syrupy consistency, oily to the touch, odorless, very sweet and slightly warm to the taste.

Action-Its affinity for water causes it to keep moist the surface to which it is applied.

Indication-Inflammation or injury of the mouth, stomatitis, pip.

\section{HYDROCHLORIC ACID (Muriatic Acid)}

Source-From a distillation of sulphuric acid, sodium chlorid and water. The resulting gas is passed into distilled water.

Properties-A colorless, fuming liquid of pungent odor, and an intensely acid taste. 


\section{HYDROCHLORIC ACID (Dilute)}

Made by taking 100 parts concentrated hydrochloric acid, 219 parts distilled water.

Action-It aids digestion by stimulating the formation of secretin in the stomach and intestines and excites the activity of all the glands giving rise to the secretions concerned with digestive activity.

In concentrated form it is caustic.

Indications-Indigestion.

Dose-One tablespoonful to each gallon of drinking water.

\section{LINSEED OIL}

Source-A fixed oil expressed from linseed or flaxseed.

Properties-A yellowish, oily liquid, peculiar odor and bland taste.

Use-A cathartic.

Indication-Constipation.

Dose-One tablespoonful to each six fowls. May be given in wet mash. Raw oil must be used. Boiled oil is poisonous.

\section{LYSOL}

Source-From that part of tar oil which boils between $190^{\circ}$ and $200^{\circ} \mathrm{C}$., by dissolving in fat and saponifying in alcohol.

Properties-A clear, brown, oily liquid of a feeble creosote-like odor.

Action-Destroys germs and parasites.

Use-One-half to two per cent solution in water to disinfect water or feed containers and to spray houses as a disinfectant.

\section{MAGNESIUM SULPHATE (Epsom Salts)}

Source-It is obtained from native dolomite, a double carbonate of magnesium and calcium.

Properties-Small, colorless, rhombic prisms, without odor, and having a cooling, saline and bitter taste. Slowly becomes a fine powder in dry air.

Action-A hydrogogue cathartic. A feeble diuretic.

Indications-Constipation.

Dose-For adult fowls as a mild laxative, one tablespoonful to each twelve fowls. Best given in solution in water and this mixed with dry mash. One teaspoonful given by the mouth in solution will produce action in about four hours.

\section{MENTHOL (Peppermint Camphor)}

Source-Obtained from the official oil of peppermint.

Properties-Colorless, acicular or prismatic crystals. It possesses a strong, pure odor of peppermint.

Action-Allays irritation.

Indication-Catarrhal condition of the mucous membranes of the head.

Use-Same as the oil of eucalyptus, which see.

\section{MERCURIC CHLORIDE, CORROSIVE (Corrosive Sublimate)}

Source-A heated mixture or mercuric sulphate 20 parts, sodium chlorid 16 parts, magnesium dioxid 1 part. 
Properties-Heavy, colorless, rhombic crystals, odorless, and having an acrid or persistent metallic taste. Permanent in air.

Action-A severe caustic.

Indications-May be used in contagious bowel diseases of fowls used in the drinking water. To each gallon of water add 6 grains mercuric chlorid and 3 grains citric acid. It may be used in a solution of one, to one-thousand, as a disinfectant.

\section{MERCURIC CHLORID, MILD (Calomel)}

Source-Heat mercurous sulphate and sodium chlorid. Calomel sublimes.

Properties-A white, impalpable powder; odorless and tasteless, permanent in air. Insoluble in water.

Action-A cathartic.

Dose-For adult fowl, 3 to 5 grains.

\section{NAPHTHALENE (Naphthalin)}

Source-A hydrocarbon obtained from coal tar by distillation between $356^{\circ} \mathrm{F}$. and $482^{\circ} \mathrm{F}$. The impure naphthalin resulting is treated with sulphuric acid and sodium hydroxid, and is further purified by distillation with steam, and then by a mixture with strong sulphuric acid and finally by distillation.

Properties-Colorless, shining, transparent laminae, having a strong characteristic odor resembling that of coal tar, and a burning aromatic taste. It is slowly volatilized on exposure to air. It will kill lice in three-quarters of an hour.

Action-An excellent destroyer of parasites.

Uses-As a powder in nests to destroy lice. Dissolved in kerosene, in from 5 to 10 per cent, to saturate perches to kill mites. Five per cent in vaseline as an ointment in scaly legs. This ointment gives good results in sore head.

\section{NUX VOMICA, POWDERED}

Source-From the seed of the Nucis vomicae.

Indications-Indigestion, paralysis, loss of appetite.

Action-Powdered nux vomica is a bitter tonic, increasing the appetite, gastric secretion, and motion. A nerve stimulant.

Dose-For adult fowl, ten to twenty grains, repeated three times a day. May be given in capsule or dough ball. As a tonic, onehalf ounce in mash to each 12 hens given three times a day. Twelve, "stroke measure," teaspoonfuls of nux vomica makes one ounce.

\section{PETROL OIL}

A neutral thick oil used as a vehicle in which to mix liquid preparations.

\section{PETROLATUM, VASELINE (Cosmolene)}

Source-A mixture of hydrocarbons, chiefly of the marsh gas series, obtained by distilling off the higher and more volatile portions from petroleum and purifying the residue when it has the desired consistency.

Properties-A colorless or more or less yellowish, oily transparent liquid, without odor or taste; or giving off when heated, a faint odor of petroleum.

Use-As a vehicle for other drugs in the preparation of ointments. 


\section{PHENOL (Carbolic Acid)}

Source-Obtained from crude carbolic acid by agitation with caustic soda, heating to $338^{\circ} \mathrm{F}$. and adding hydrochloric acid; then by agitation with sodium chlorid, digestion with calcium chlorid and distillation at a temperature between $336^{\circ}$ and $374^{\circ} \mathrm{F}$. and finally by crystallization, phenol results.

Properties-Colorless, interlaced or separate, needle-shaped crystals, or a white crystalline mass, sometimes acquiring a reddish tint. It has a characteristic and somewhat aromatic odor and when it is greatly diluted with water has a sweetish taste. It goes into solution with a very small amount of water.

Action-An antiseptic, and in five per cent solutions it is a parasiticide.

Use-As an intestinal antiseptic, use one to two teaspoonfuls to each gallon of water. As a spray for poultry houses, use five ounces to each gallon of water.

\section{PODOPHYLIN (May Apple)}

Source-Obtained from the roots of the may apple.

Properties-A fine non-crystallizable powder. It is yellowish in color and possesses a peculiar, faintly, bitter taste.

Action-A powerful, though slow, acting cathartic. In an experiment on baby-chicks, one-eighth grain doses proved fatal in 42 out of 43 tests.

Indications-Constipation.

Dose-For adult fowl, one-fourth grain.

\section{POTASSIUM DICHROMATE}

Properties-It is a reddish-yellow crystalline potash product.

Use-A pale, straw-colored solution in water is an astringent and is indicated in sore throat or in sore mouth.

\section{POTASSIUM PERMANGANATE}

Source-Caustic potash, chlorate of potassium and black oxid of manganese are fused together.

Properties-Slender, monolithic prisms, of a dark purple color, odorless, and having a taste first of sweet but afterwards disagreeable and astringent. Permanent in dry air.

Action-An intestinal antiseptic.

Indications-In contagious bowel diseases, as fowl cholera. Make the drinking water bright red nearing a purple. About one ounce to each four gallons of water. In ulcers or sores use the pure crystals on the sore.

\section{PYRETHRUM (Persian Insect Powder)}

Source-The powdered flowers of the Pyrethrum roses.

Properties-A coarse, greenish-yellow, pungent powder.

Use-Destroys fleas and lice. It gives off a gas when exposed to the air that is a slow poison. It requires about forty-five minutes to kill lice.

Indications-May be used to dust in hen's nests and on birds for lice. Oniy the pure product will give satisfactory results, and too liberal quantities should not be employed. Some depressing results have been observed when used too freely upon birds. 


\section{QUININE SULPHATE}

Source-Obtained by boiling cinchona bark with hydrochloric acid and adding lime to the filtered decoction.

Properties-White, silky, light, and fine needle-shaped crystals, with bitter taste.

Action-Retards metabolism and thus lessens the production of heat in the tissues. It dilates the vessels of the skin and therefore induces loss of heat.

Indications-Cold, bronchitis, and pneumonia.

Dose-For adult fowl, 2 to 3 grains three times a day.

\section{SANTONIN}

Source-A neutral principle obtained from santonica. Santonica is derived from worm seed.

Properties-A colorless, flattened, prismatic crystal, odorless and almost tasteless.

Action-Destroys intestinal parasites.

Indications-Infestation of intestines with worms.

Dose-For adult fowls, one-fourth grain.

\section{SODIUM CHLORID (Common, or Table Salt)}

Source-Mined in native state or obtained by evaporation of brine, spring, or sea water.

Properties-Colorless, transparent, cubical crystals or a white crystalline powder with salty taste, permanent in dry air.

Action-Essential constituent of the food, since it is necessary to the composition of hydrochloric acid in the gastric juice and of blood plasma, from which it is constantly eliminated in the urine. Herbovorous and grain-eating animals require sodium chlorid in addition to that contained in their food. While the blood is rich in sodium salts, vegetables are particularly rich in potassium salts.

Birds deprived of salt suffer from anemia and general weakness. Birds cannot tolerate large doses of table salt.

Use-The mash should contain from one-half to one pound sodium chloride to each 100 pounds mash, and should be thoroughly mixed.

\section{SODIUM FLUORIDE}

Source-Obtained by the action of hydrofluoric acid upon sodium hydroxid, sodium carbonate, or sodium, with the formation at the same time of water and carbon dioxid, or hydrogen, respectively.

Properties-Sodium fluoride is a white powder consisting of small crystals.

Action-It is destructive to parasite life.

Use-It is used as an insect powder working it into the skin and among the feathers. When put on the heads of young chicks it may cause ill effects upon the chicks. It will kill lice.

\section{SODIUM SULPHATE (Glaubes Salts)}

Source-The neutralized residue left in the manufacture of hydrochloric acid from salt with sodium carbonate.

Properties-Large, colorless, transparent monoclinic prisms or granular crystals, odorless and possessing a bitter, salty taste. Upon exposure to the air it gradually becomes a fine powder and loses its water of crystallization.

Action-A cathartic producing a watery stool. 
Dose-One tablespoonful to each 12 adult fowls. Younger birds in proportion. It is best given dissolved in water and this mixed with mash. It is dangerous to attempt to pour liquids down the throats of fowls, as there is great danger of their passing into the superior larynx and down the trachea into the lungs. By opening the mouth of a bird and looking into the throat one can see the entrance into the air passage as it opens and closes.

\section{STRYCHNIN SULPHATE}

Source-The alkaloid or active principle of the nux vomica button or seed. Strychnin sulphate is formed by the action of sulphuric acid on strychnin.

Properties-Colorless, transparent, octohedral or prismatic white crystalline powder. Odorless and with an intensely bitter taste.

Action-A nerve stimulant.

Indications-Paralysis.

Dose-For adult fowl, one-fifth to one-fourth grain three times a day.

SULPHOCARBOLATES OF CALCIUM, ZINC AND SODIUM

Source-The action of sulphuric acid and carbolic acid on metallic zinc, sodium, or calcium.

Properties-A white, crystalline powder.

Use-In diarrhea, as an intestinal antiseptic.

Dose-Of the mixture of equal parts of sulphocarbolates of calcium, sodium, and zinc, dissolve five grains in a pint of water and use as drink or with which to mix the mash.

\section{SULPHUR SUBLIMATUM (Sulphur)}

Source-Obtained from native sulphur by sublimation.

Properties-A fine, yellow powder, having a slightly characteristic odor and a faintly acid taste.

Action-Dissolved sulphur as in the lime and sulphur dip, in which seven pounds of unslaked lime is dissolved in twenty-one pounds of sulphur, and one-hundred gallons of water, is an excellent destroyer of parasites. In a dry state it has no effect upon mites and perhaps none on lice.

\section{THYME OIL}

Source-A volatile distillate from the Thymus vulgaris.

Properties-A thin liquid of characteristic odor.

Use-In catarrhal conditions. See discussion under oil of cucalyptus.

\section{THYMOL}

Source-A phenol occurring in the volatile oil of thyme.

Properties-Large, colorless, translucent crystals of the hexagonal system.

Action-Destroys intestinal parasites.

Indications-Infestation by round worms.

Dose-For adult fowl, three grains, followed by a physic.

\section{TOBACCO}

Properties-From a golden-yellow to a chestnut-brown, leaf containing an active principal, nicotine. The leaves of tobacco contains from 0.6 per cent nicotine in good grade, to 8.0 per cent nicotine in poor grade tobacco. 
Use-Nicotine is very destructive to parasites, as lice and mites. Tobacco stems and leaves have no effect upon these parasites. Dry leaves and stems placed in nests are useless so far as destroying parasite life is concerned. A decoction made bv boiling tobacco stems or leaves in water is destructive to parasites and contains nicotine in solution. As a spray, nicotine in one-fourth of one per cent solution will kill parasites. One-fourth of one per cent pure nicotine in dusting powder will kill lice. Finely triturated leaves and stems, as in the case of snuff, give off sufficient poisonous gas for lice that it will be destructive used as a dusting powder. Such finely pulverized tobacco should be incorporated in some vehicle as plaster of paris using not more than 10 or 15 per cent of the tobacco.

\section{WORM SEED, OIL OF}

Source-Obtained from santonica.

Properties-A volatile oil, yellowish in color with characteristic odor.

Action-Destroys worms.

Indications-Intestinal worms.

Dose-For adult fowl, one teaspoonful to each twelve birds.

\section{ZINC SULPHATE}

Source-Prepared by dissolving metallic zinc in sulphuric acid.

Properties-Colorless, transparent, rhombic crystals, without odor, and having an astringent, metallic taste. Changes to a fine powder when exposed to the air.

Indications--One per cent solution in distilled water and two or three drops in the eye in case of inflammation or conjunctivitis. 


\section{LIST OF DOSAGE OF DRUGS FOR ADULT FOWLS}

Name of drug.

Quinine sulph. \& bisulph.

Ferrous sulphate.....

Carbo ligni .........

Phenol

Magnes. sulph

Pott. dichromate and permang. ........ 30 grains

Sodium chloride...... 2.5 drams

Thymol

Calcium phos. precip.. 1.25 drams

Potass. chlorate, sulph. and iodid

Sodium bicarb........

Ferrous oxide........

Mercury bichloride....

Potass. tartrate ......

Oil turpentine........

Sulphur

Mustard

Nux vomica.........

Gentian

Ginger

Wormseed oil........

Capsicum

Anise

Quassia

Strychnine sulph

Aloin

Ammonia carb.......

Areca nut ..........

Belladonna F. E.....

Castor oil...........

Convallaria F. E.....

Digitalis F. E........

Calomel

Podophyllin

Santonin

Sulphocarbolate of calc.

Sulphocarbolate of zinc

Sulphocarbolate of sodium
Lethal dose.

Maximum dose.

3 grains

30 grains

10 grains

2 grains

1 dram

15 grains

1 dram

3 grains

0.5 dram

$1 / 20$ grain

20 grains

5 grains

4 grains

1 grain

10 grains

1 dram

5 grains

10 grains

1 ounce

2 drams

4 drams

5 drams

10 minims

5 grains

$1 / 3$ grain

I/8 grain

$1 / 4$ grain

2 grains

20 grains

10 grains

10 minims

1 tablesp'nful

20 minims

20 minims

10 grains

$1 / 2$ grain

$1 / 2$ grain

$1 / 2$ grain

$1 / 2$ grain

$\mathrm{I} / 2$ grain
Minimum dose.

1 grain

10 grains

5 grains

0.5 grain

0.5 dram

2 grains

0.5 dram

0.5 grain

0.25 dram

$1 / 40$ grain

10 grains

1 grain

1/10 grain

5 grains

0.5 dram

2 grains

5 grains

0.1 dram

0.2 dram

0.3 dram

5 minims

1 grain

1 grain

1/16 grain

$1 / 6$ grain

1 grain

10 grains

5 grains

5 minims

1 teasp'nful

10 minims

10 minims

5 grains

$1 / 4$ grain

$1 / 4$ grain

$\mathrm{I} / 4$ grain

$\mathrm{T} / 4$ grain

$1 / 4$ grain 


\section{INDEX}

Page

\section{A}

Abdominal tumors, caseous.......269 Yolk sack ...............6 63

Abnormal eggs ..............226

Abortion, epiornithological ......230

Abscess of the foot............233

Acantha inodora .................103

Acanthocephala .........................

Acarids .................. 87

Achorion schoenleinii .......... 105

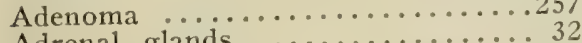

Adrenal glands .............. 32

Adult connective tissue tumors....254

Epithelial tumors ...........252

Air sacs $\ldots \ldots \ldots \ldots \ldots \ldots \ldots . . .61$

Air sacs, disease............ 91

Inflammation $\ldots \ldots \ldots \ldots \ldots, 33,325$

Aloin

Ameba meleagridis ............ 134

Anaesthesia $\ldots . . . \ldots \ldots . . . .309$

Anatomy of fowl............. 17

Visceral $\ldots \ldots \ldots \ldots \ldots \ldots \ldots{ }_{257}^{22}$

Angioma $\ldots \ldots \ldots \ldots \ldots \ldots \ldots \ldots \ldots \ldots 221$

Animal food ............... 56

Antiseptic, intestinal ........66 61

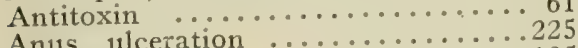

Anus, ulceration ......................

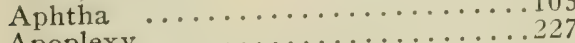

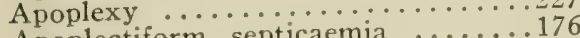

Apoplectiform septicaemia .........320

Arsenical poisoning ................

Argas miniatus ............... 101

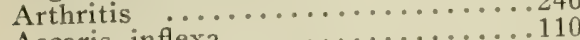

Ascaris inflexa .................155

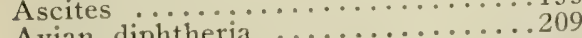

Avian diphtheria $\ldots \ldots \ldots \ldots \ldots \ldots 6,192$

Aspergillosis $\ldots \ldots \ldots \ldots . \ldots \ldots, \ldots \ldots .203$

Asthma $\ldots . . . \ldots \ldots \ldots \ldots . . . . . . .218$

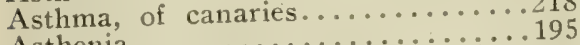

Asthenia $\ldots . . . \ldots \ldots$................. 64

\section{B}

Baby chick, health.......... 62

Bacterial diseases .............165

Bacterium sanguinarium .........169

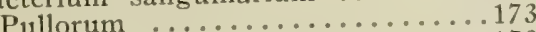

Chick infection ...........173

Adult fowl infection.........175

Bacteria, intestinal ...........287

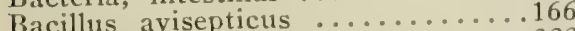

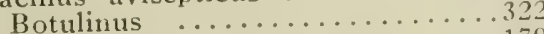

Coscoroba .........................

Tuberculosis ................187

Bacillary white diarrhea.........173

Baldness .........................

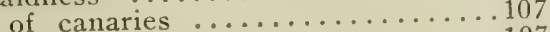

of fowls ....................

Beaks, broken ................246

Bed bug of poultry............... 103
Page

Bee stings ....................104

Bermuda grass ............... 56

Birds, fever ................... 183

Number per acre........... 56

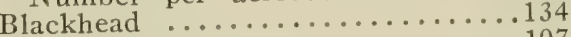

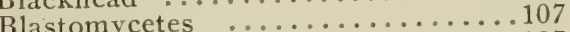

Blastomycosis of goose............107

Blood

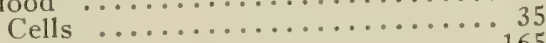

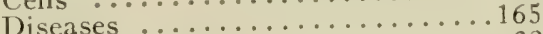

Vascular system ........................

Vessels, rupture $\ldots \ldots \ldots \ldots \ldots \ldots 241$

Body lice ....................

Bones, of skeleton............. 14

Structure ...................303

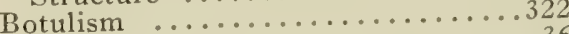

Brain ................... 36

Parts ...................... 36

Brooder disease ................ 192

Broken beaks .............246

Bronchitis $\ldots \ldots \ldots \ldots \ldots \ldots \ldots 20.27$

Bursa of fabricus............ 27

Bursitis

C

Caecal worms ................112

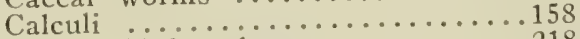

Canary bird asthma............218

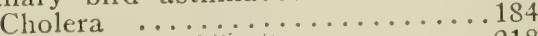

Respiratory diseases ..........218

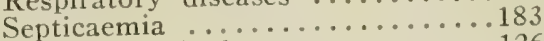

Canary, intestinal worms.........126

Louse

Typhoid $\ldots \ldots \ldots \ldots \ldots \ldots \ldots \ldots \ldots \ldots \ldots \ldots \ldots$ 184

Canker of anus................155

Fowls ..................209, 213

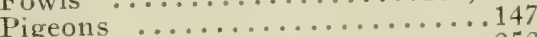

Cancer $\ldots . . . \ldots \ldots \ldots \ldots \ldots . . .252$

Catarrh $\ldots \ldots \ldots \ldots \ldots \ldots \ldots \ldots \ldots \ldots \ldots \ldots \ldots, 202$

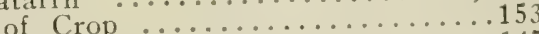

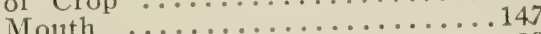

Cavities, nasal .............. 28

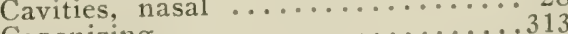

Caponizing $\ldots \ldots \ldots \ldots \ldots \ldots \ldots . .252$

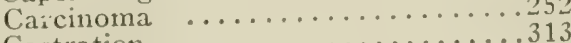

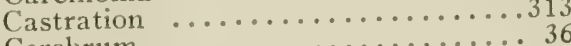

Cerebrum ................. 36

Cerebellum $\ldots \ldots \ldots \ldots \ldots \ldots \ldots . .36$

Chanota infundibuliformis .....1121

Chicken bug ...........................

Cholera $. . \ldots \ldots \ldots \ldots \ldots \ldots . .203$

Pox $\ldots \ldots \ldots \ldots \ldots \ldots \ldots \ldots \ldots \ldots \ldots$

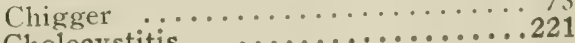

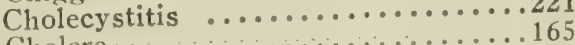

Cholera .............................

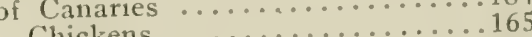

Chickens $\ldots \ldots \ldots \ldots \ldots \ldots \ldots \ldots \ldots$

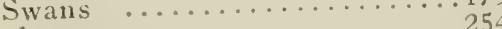

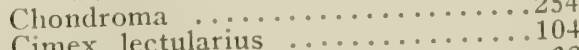

Cimex lectularius ................ 32

Circulatory system ............. 26

Cloaca $\ldots \ldots \ldots \ldots \ldots \ldots \ldots \ldots \ldots \ldots$

Cloaca, eversion ...............155

Cloacitis 
Coccidian white diarrhea.........128

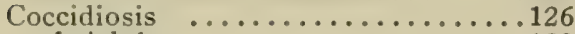

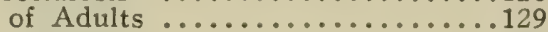

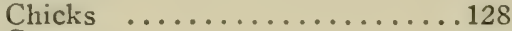

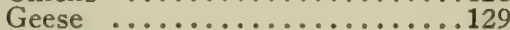

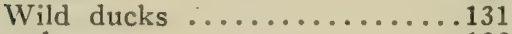

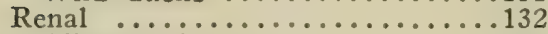

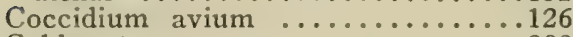

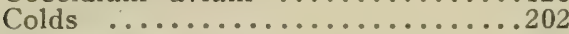

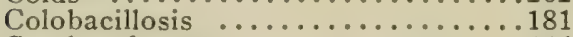

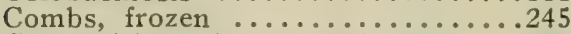

Composition of eggs and shell....28

Compound tumors ............260

Commercial unit ............ 57

Congestion of liver............220

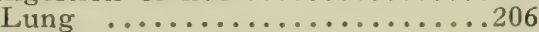

Connective tissue tumors........254

Conjunctivitis ................245

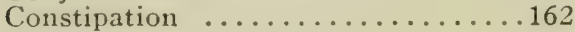

Contagious Bronchitis .........206

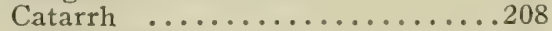

Epitheliomas ................211

Inflammation of air sacs of geese.208

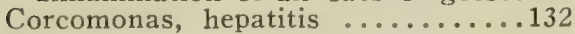

Cord, spinal ............... 36

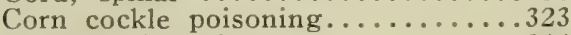

Cornea, ulceration .............244

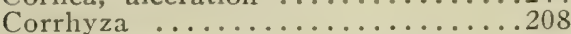

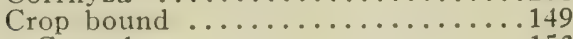

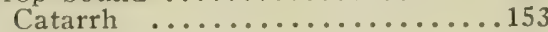

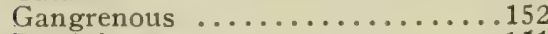

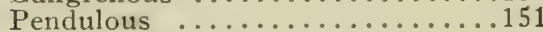

Crossbeak ...................246

Croupous inflammation of pigeons..147

Curvature of spine .....................

Cytodites nudus ................ 91

\section{D}

Davainea echinobothrida ........124

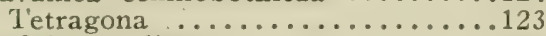

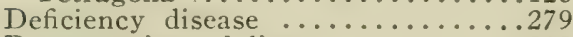

Degeneration of liver...........219

Depluming lice .............. 81 Scabies .................. 87

Dermanyssus Gallinae ........... 93

Dermis ................... 42

Diarrhea $\ldots \ldots \ldots \ldots \ldots \ldots \ldots . . .64,162$

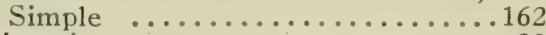

Digestive tract ............. 22

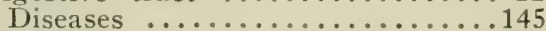

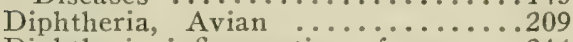

Diphtheric inflammation of eye....244

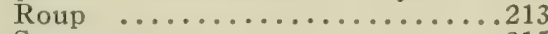

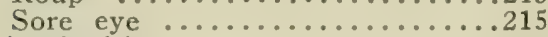

Diphtheritis $\ldots \ldots \ldots \ldots \ldots . \ldots . \ldots 209$

Diseases of liver..............219

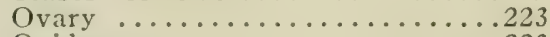

Oviduct $\ldots \ldots \ldots \ldots \ldots \ldots \ldots \ldots . \ldots \ldots 223$

Disinfection ...........59, 67, 70 of Houses ................. 67 Yards ................... 70

Disinfestation ................... 67

Dispharagus laticeps . . . . . . . . 119

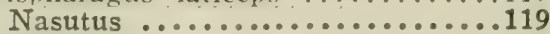

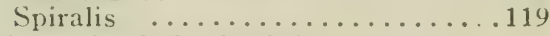

Disposal of dead birds......... 72

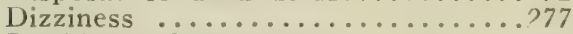

Double yardage system.......... 56

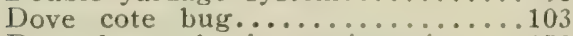

Dove hemorrhagic septicaemia.....179

Drepanidotaenia infundibulus .....121

Drinking vessels ............6 65

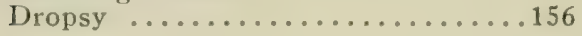

Droopy wings ............126, 128

Duck lice .................. 79

Dusting hens for lice........... 85

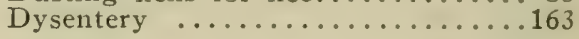

\section{E}

Echinorhynchus polymorphus ....125

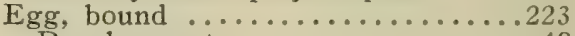

Development ..............48 48

Eggs, abnormal ...............226

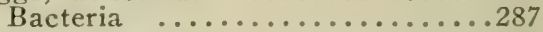

Blood specks ...............228

Composition ................287

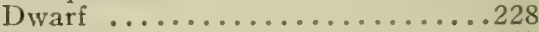

Double ...................227

Multiple shell membranes......227

Parasites ................289

Shell composition ..........289

Soft shells ...............226

Triple yolks ................227

Yolkless ..................226

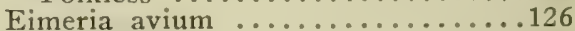

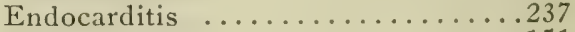

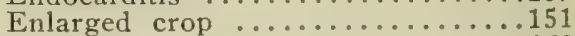

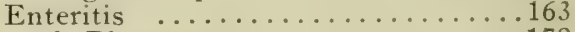

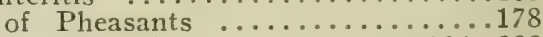

Entero-hepatitis $\ldots \ldots \ldots \ldots \ldots 134,222$

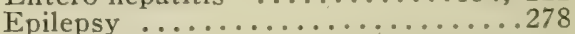

Epiornithological pulmo-pericarditis

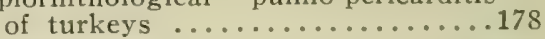

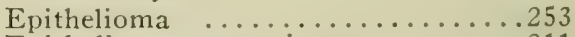

Epithelioma contagiosum ........211

Epithelial tumors .............252

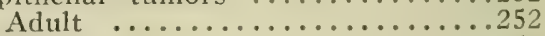

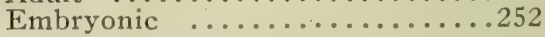

Equipment, poultry house....... 58

Esophagus ................. 24

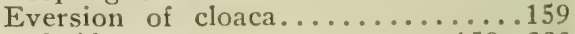
Oviduct ..............159, 223

Exercise essential ............. 57

External parasites .............. 75

Eyes ..................... 39

Diphtheric inflammation ......244

\section{F}

Fabricus, bursae of.......... 27

False tuberculosis ...................... 191

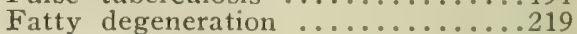

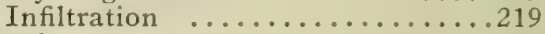

Feathers ...................... 42

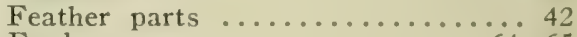

Feed $\ldots \ldots \ldots \ldots \ldots \ldots \ldots 64,65$

Fibroma ..................256

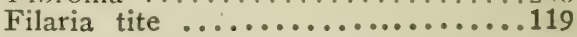

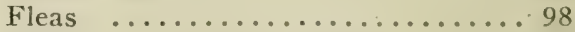

Floor of poultry houses........ 58

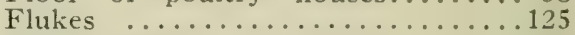

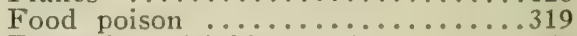

Fountains, drinking .......... 68

Foot, mange ............... 88

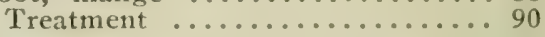

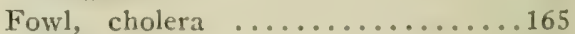

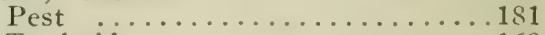

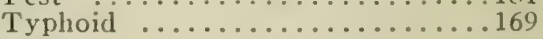

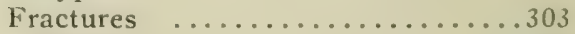

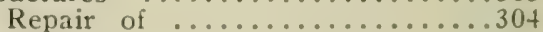

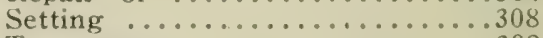

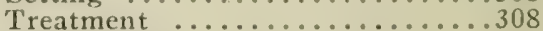

Frozer combs ................245

Fruit trees for shade .......... 57

Fungi ...................... 
G

Page

Gall bladder ................ 28

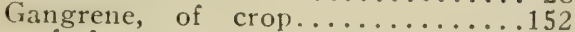

of foot $\ldots \ldots \ldots \ldots \ldots \ldots \ldots \ldots \ldots \ldots 242$

Dry $\ldots \ldots \ldots \ldots \ldots \ldots \ldots \ldots \ldots \ldots \ldots \ldots \ldots, 245,242$

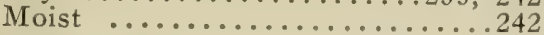

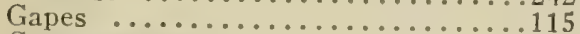

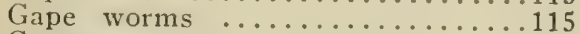

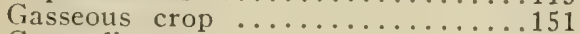

Geese lice .................. 79

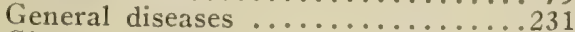

Gizzard ..................25

Inflammation $\ldots \ldots \ldots \ldots \ldots \ldots .157$

Worms ............................

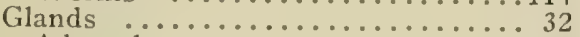

Adrenal ......................... 32

Carotid .................. 32

Thymus .................. 36

Glandular stomach ............ 25

Going light ............................

Goniocotes gigas ........... 77

Goniodes damicornis .........79 79

Dissimilla ............... 78

Stylifer $\ldots \ldots \ldots \ldots \ldots \ldots \ldots \ldots \ldots \ldots \ldots \ldots \ldots 78$

Gout of fowls................ 197

Grass runs $\ldots \ldots \ldots \ldots \ldots \ldots \ldots . \ldots 56$

Grouse disease $\ldots \ldots \ldots \ldots \ldots 18$

\section{$\mathrm{H}$}

Head ................... 17

Lice ..................... 80

Health of chicks ............6 62

Heart ..................... 33

Rupture .............. 241

Hearing, organs .............. 40

Hematoma ...............257

Hemangioma ...............257

Hemorrhage of brain..........277

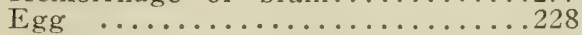
Internal ................241

Hemorrhagic septicaemia of the

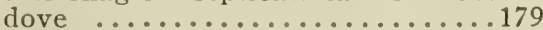

Hemiplegia ...............279

Hermaphrodite ...............119

Hepatitis ................221

Heterakis compressa ...........118

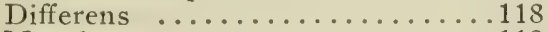

Maculosa ....................118

Papillosa ..................112

Perspicillum ..................110

Honey comb ringworm...........105

Horny growth ..............267

Houses, poultry ............ 57

Hyperplasia ............... 239

Hyperplasia of Intestine .......239

Hypertrophy .................239

Hyphae ....................

I

Immunity $\ldots \ldots \ldots \ldots \ldots \ldots 60,61$

Acquired $\ldots \ldots \ldots \ldots \ldots \ldots, 60,61$

Natural ..............60, 61

Impaction of baby chicks.......151

Canaries ....................151

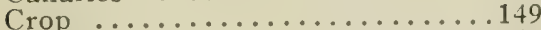

Intestine ................150

Incubation, effects on health of chick ..................6 62

Indigestion $\ldots \ldots \ldots \ldots \ldots \ldots \ldots \ldots \ldots \ldots \ldots \ldots$

Infectious blood diseases.........165 Constitutional diseases .......887

Enteritis of pheasants ........178
Leukemia Page

169

Inferior larynx canaries...........183

Infiltration

Inflration, fatty of liver.......219

Crop

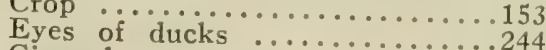

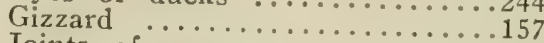

Joints of geese...............

Ducks $\ldots \ldots \ldots \ldots \ldots \ldots \ldots \ldots \ldots 185$

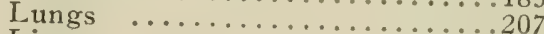

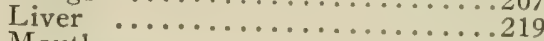

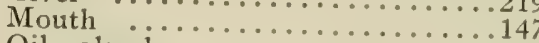

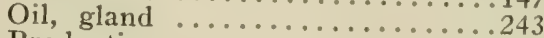

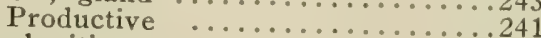

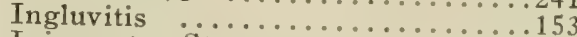

Injury to Sternum.....................

Internal hemorrhage $\ldots \ldots \ldots \ldots \ldots 241$

Internal, acanthocephala ...............

Echinorhynchus ................125

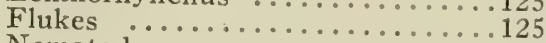

Nematodes .............109, 124

Notocotyle verrucosum ..............

Parasites ....................109

Worms .........109, $110,111,112$

Intestine $\ldots \ldots \ldots \ldots \ldots \ldots \ldots \ldots, 26$

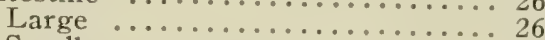

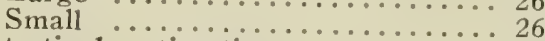

Intestinal antiseptics $\ldots \ldots \ldots \ldots \ldots .66$

Bacteria ....................285

Diseases of canaries..............

Intussusception ................161

Invagination of bowel...............

\section{J}

Jaundice

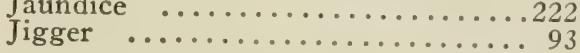

\section{K}

Keratitis .................215

Kidney $. . . \ldots \ldots \ldots \ldots \ldots \ldots . . . .63,54$

Kline's bacillus .................. 170

Disease ..................... 169

L

Large intestines ........... 26

Larynx $\ldots \ldots \ldots \ldots \ldots \ldots \ldots \ldots 29$

Inferior $\ldots \ldots \ldots \ldots \ldots \ldots \ldots \ldots 29$

Superior $\quad . \ldots \ldots \ldots \ldots \ldots \ldots \ldots \ldots \ldots \ldots 29$

Leg weakness ...........................

Leucocytozoon of ducks............133

Leukaemia $\quad . . \ldots \ldots \ldots \ldots \ldots . . .169$

Leukaemia, myeloid .................172

Lice $\ldots \ldots \ldots \ldots \ldots \ldots \ldots 75,76$

Lice, body ....................... 77

Effects on fowls of ............8 83

Examination for ............. 84

How eradicate ............. 84

Multiplication of $\ldots \ldots \ldots \ldots \ldots 82$

of ducks ................ 79

Geese ..................... 79

Head and neck............. 79

Pigeons .................. 79

Turkeys ............... 78

Lime $\ldots \ldots \ldots \ldots \ldots \ldots \ldots \ldots, 71$

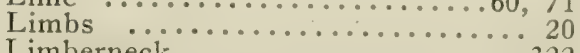

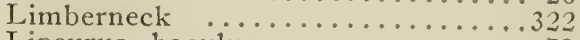

Lipeurus baculus ............... 79

Infuscatus $\ldots \ldots \ldots \ldots \ldots . . .60$ 
Page

Jejunus ............... 79

of canaries .................. 80

Polytrapezius ............. 79

Squalidus ................. 79

Variabilis .................. 81

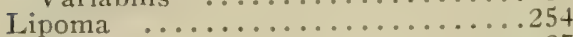

Liver ....................27

Abscess ...............222

Amyloid degeneration .......222

Aspergillosus ............222

Congestion ................220

Cercomoniasis .............222

1)iseases ..................219

Inflammation ............221

Nutmeg ................221

Jaundice .................222

Rupture .................220

Tumors .................222

Location of plant ............56,54

Locust poisoning .....................

Lousiness ................. 75

Lungs ................. 30

Congestion .........................

Inflammation .................207

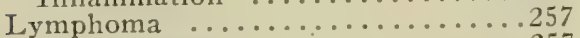

Lymphangioma .............257

Lymph vessels .............. 36

Lympho-sarcoma .............261

\section{M}

Malaria .....................134

Male reproductive organs....... 51

Malformations ..................291

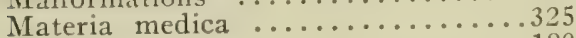

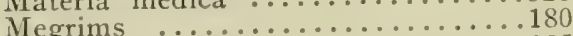

Medicines $\ldots \ldots \ldots \ldots \ldots \ldots \ldots . . . .325$

Menopon biseriatum .......... 77

Obscurum ................. 77

Pallidum $\ldots \ldots \ldots \ldots \ldots \ldots \ldots .6 \%, 93$

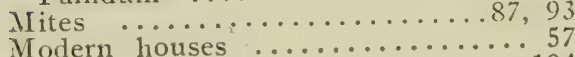

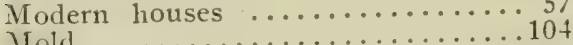

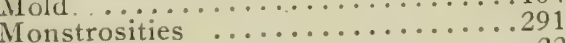

Nouth ..................22

irycelium ...................105

Mycosis of pigeons............... 106

Myeloid leukaemia .............172

IIyelitis ...................279

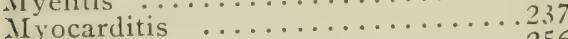

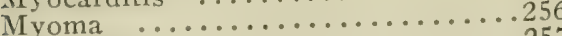

Myxoma ..................257

Myxo-sarcoma $\ldots \ldots \ldots \ldots \ldots \ldots . . .263$

\section{N}

Nasal cavities .............. 28

Natural resistance ............6 60

Neck lice ................. 80

Nephritis .................. 157

Nerve diseases ............. 36

Nervous system ............. 36

Nests $\ldots . \ldots \ldots \ldots \ldots \ldots . . .123$

Nodular taeniasis ................ 123

Notocotyle verrucosum $\ldots \ldots \ldots \ldots .556$

Nutmeg liver .............221

\section{O}

Obstruction of beak.............146

Crop ..................... 149

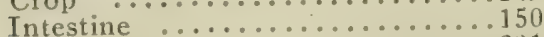

Trachea ..................201
Page

Oidium albicans .............105

Oil glands, inflammation of ......243

Olfactory nerve ............4 41

Open front house............ 57

Joint ..................247

Operating table ..............310

Organs of the fowl................ 15

Hearing ............4 40

Hen ................45 45

Pullet .............44 44

Reproduction .......43, 51

Smell .............. 41

Special sense .......... 39

Taste .............4 42

Touch .............. 42

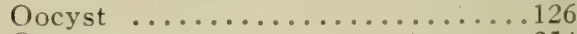

Osteoma ...................254

Osteitis ....................246

Osteo arthritis .................. 185

Osteology ................... 14

Ossification of tendons..........256

Ovary ....................45

Ovariectomy ........................

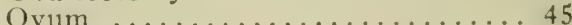

Development ............. 45

Oviduct .................. 47

Broken Eggs in............225

Eversion of ..............159, 223

Function of $\ldots \ldots \ldots \ldots \ldots \ldots+7$

Obstruction of .............223

Prolapse of ................223

Parts of $\ldots . . \ldots \ldots \ldots \ldots \ldots . \ldots 47$

Rupture of ...............225

Ulceration of $\ldots . . . \ldots \ldots \ldots \ldots . . .225$

\section{$\mathrm{P}$}

Pancreas ..................28

Papilloma $\ldots \ldots \ldots \ldots \ldots \ldots \ldots \ldots \ldots \ldots \ldots \ldots .252$

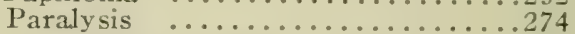

Anterior cocklear nerve.......281

Cochlear nerve .............281

Vestibular nerve $. . . . \ldots \ldots \ldots . . .281$

Wings of pigeons ............276

Parasites ................ 75

External ................. 75

Internal .................... 109

Parasiticides ................... 95

Parrot Septicaemia ............. 180

Parched tongue ........................

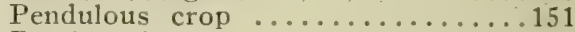

Perch poles ..............59, 60

Proof against mites.........6 60

Pericarditis ...................236

Peritonitis .................156

Pest, fowl ................181

Pharynx .................23

Pharyngitis $\ldots \ldots \ldots \ldots \ldots \ldots \ldots \ldots .203$

Ulcerative $\ldots . \ldots \ldots \ldots \ldots . .203$

Phthiriasis .................... 75

Pica $\ldots \ldots \ldots \ldots \ldots \ldots \ldots \ldots \ldots \ldots \ldots$

Pigeon lice ................79, 80 Paralysis of wings ..........276

Septicaemia ................ 180

Pip ...............................

Plasmodium danielewskyi ........134

Pneumonia ................106, 207

Pneumomycosis ..........106, 192

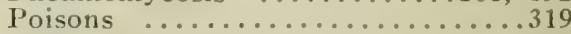

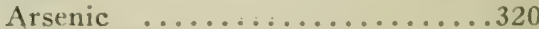

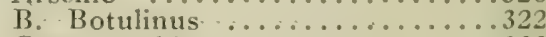

Corn cockle ...............323

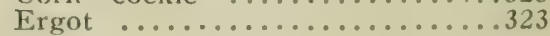


Page

Locust leaves ................324

Ptomain ......................................

Rose chafer ......................

Salt ...............................

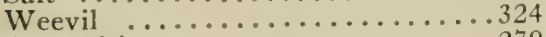

Polyneuritis ...............279

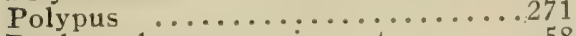

Poultry house equipment....... 58

Poultry plant site............ 55

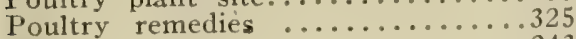

Pox of turkeys................243

Proctitis $\ldots . . . \ldots \ldots \ldots . . . . . .155$

Prolapse of Caecum ............159

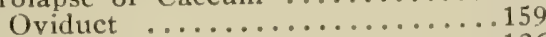

Protozoa ...................126

Ameba meleagridis ............133

Blackhead ............................

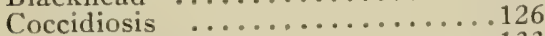

Entero-hepatitis .............133

Liver of pigeon................... 132

Leucocytozoon of ducks........133

Malaria of fowls...............133

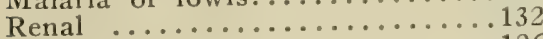

Trichomoniasis ............. 126

Trychosomiasis ................. 133

Pseudo-tuberculosis ..............191

Psittacosis .............................

Ptomain poisoning ............. 321

Pulex avium ................. 99

Purulent inflammation of abdomen..231

Purulent inflammation air sacs...233

$P$ is of fowls $\ldots \ldots \ldots \ldots \ldots \ldots \ldots 157$

\section{Q}

Quail diseases 181

$\mathbf{R}$

Rectum ................ 26

Red mites .................. 93

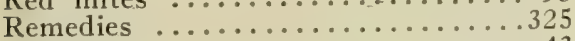

Reproductive organs ..........44 43

Female ................. 43

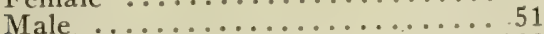

Renal coccidiosis ..................

Resistance, natural .........6 60

Respiratory disease of canaries...218

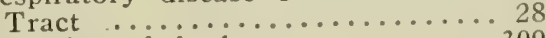

Restraint of fowls ................

Rheumatism ................276

Ringworm ........................... 105

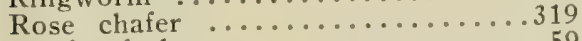

Roof of house.............. 59

Roup ...............209, 215

Chronic carrier.$\ldots \ldots \ldots \ldots \ldots 217$

Diphtheric $\ldots \ldots \ldots \ldots \ldots \ldots \ldots \ldots \ldots$

Runs and buildings........... 56

Rupture, blood vessels..........241

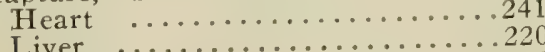

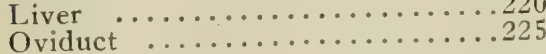

S

Sacs, air ............... 31

Sacs, air $\ldots$...................... 105

Salt poisoning $\ldots \ldots \ldots \ldots \ldots \ldots . . .621$

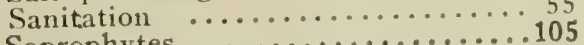

Saprophytes $\ldots \ldots \ldots \ldots \ldots \ldots . . .65$

Sarcoptes mutans ................ 859

Sarcoma ...................255

Sarco-chondro-osteoma ..............................
Sarcopsylla gallinacea ........100 Laevei ...................87

Scabies .................. 87

Depluming $\ldots \ldots \ldots \ldots \ldots \ldots \ldots .87$

Scaly eggs $\ldots \ldots \ldots \ldots \ldots \ldots .68$

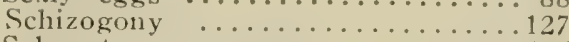

Sclerostoma syngamus .............115

Scratch material ................5 57

Secretion, kidney .............. 53

Urinary $\ldots . \ldots \ldots \ldots \ldots \ldots \ldots . \ldots \ldots{ }_{53}$

Segmented worms .................119

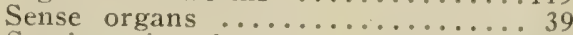

Septicaemia of canary birds. 183, 184 Parrots ................... 180

Pigeons .................... 180

of fowls .......................

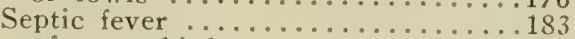
of cage birds................ 184

Shade, kind ................. 55

Sick birds, disposal of ............. 72

Site for poultry plant......... 55

Skeleton .................. 14

Skin ......................... 42

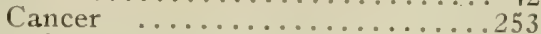

Sleeping disease $\ldots \ldots \ldots \ldots \ldots \ldots . . .177$

Small intestine .................. 26

Smell organs $\ldots \ldots \ldots \ldots \ldots \ldots \ldots+1$

Sod disease (dry gangrene of feet) .235

Soil best suited for fowls........ 56

Sore eye ............................

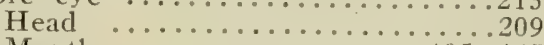

Mouth ..................105, 147

Special Sense ................ 39

Spermatozoa ................. 53

Spinal cord $\ldots \ldots \ldots \ldots \ldots \ldots \ldots \ldots \ldots \ldots \ldots \ldots \ldots$

Spirillum Metchnikovi ...........187

Spirochetosis ...............186

Spiroptera hamulosa .............114

Spirocheta gallinarum ..........186

Spleen ......................28

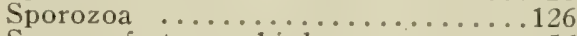

Square feet per bird............. 56

Staphylococcus pyogenes aureus....185

Stick tight flea.................. 100

Stomach ......................... 25

Glandular ................... 25

Muscular $\quad . . \ldots \ldots \ldots \ldots \ldots . . \ldots 25$

Stomatitis ............................

Streptococcus capsulatus .........176 Gallinarum .....................

Sunshine in house................ 59

Swollen eye ...............209

Head $. . . \ldots \ldots \ldots \ldots . . .209$

of Turkeys ............... 243

Joints of geese $\ldots \ldots \ldots \ldots \ldots \ldots \ldots \ldots$

Syngamus bronchialis ........... 115

Trachealis ................... 115

\section{$\mathrm{T}$}

Taenia, description ...........121

Echinobothrida .................

Infundibuliformis $\ldots \ldots \ldots \ldots \ldots \ldots \ldots 121$

Life history of ................ 122

Treatment of $\ldots \ldots \ldots \ldots \ldots \ldots 122$

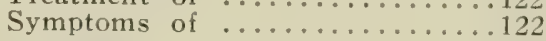

Tapeworms ..................119

Taste organs ................ 42

Testes ..................... 51

Tendon Ossification ...........256

Thornheaded worm ..........124, 125

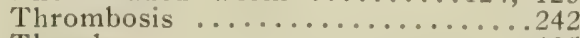

Thrush ....................... 105

Ticks ...................... 
Tinea favosa .................................

T'ouch organs .............. 42

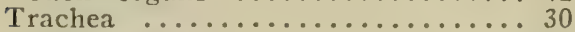

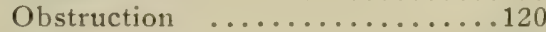

Trees ..................... 56, 57

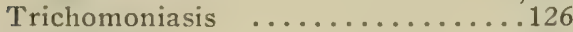

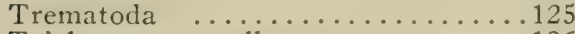

Trichomonas pullorum .........126

Trichosomum contortum ........118

Trinoton continuum ........... 79

Trombidium holosericeum ........ 93

Trunk .................... 19

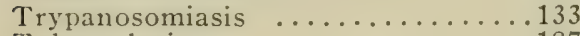

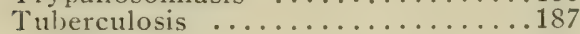

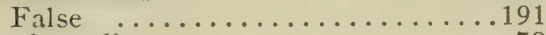

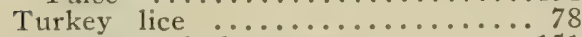

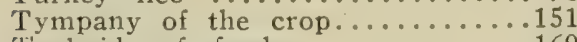

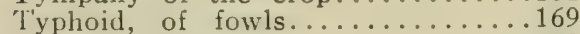

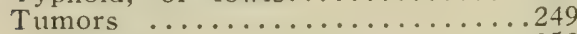

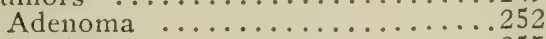

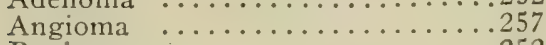

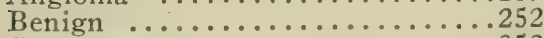

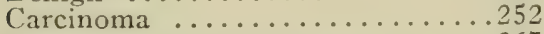

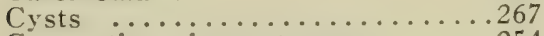

Connective tissue tumors.......254

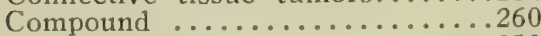

Classification of $\ldots \ldots \ldots \ldots . . .250$

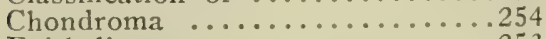

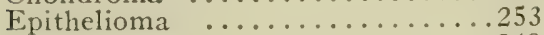

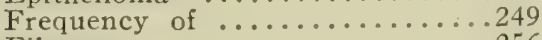

Fibroma ..................256

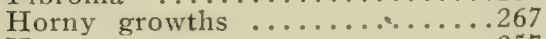

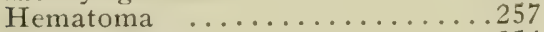

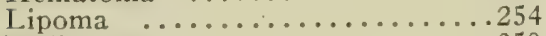

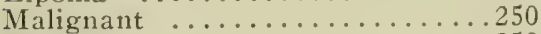

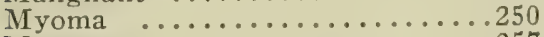

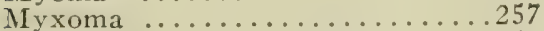

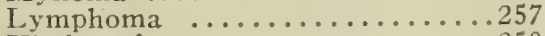

Kinds of $\ldots \ldots \ldots \ldots \ldots \ldots 250$

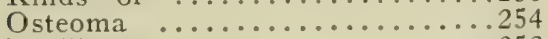

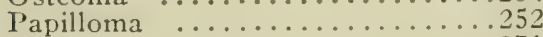

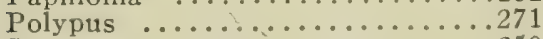

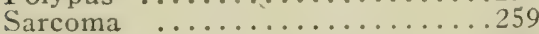

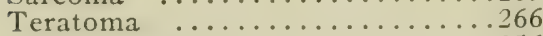

Tendon ossification ............256

\section{U}

Urinary Secretion ........... 53

Urine $\ldots \ldots \ldots \ldots \ldots \ldots \ldots . . . . . . . .54$

U1cerative Page

sore mouth...........147

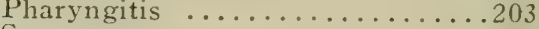

Sore eye ..................211

Ulceration of anus........... $15 \dot{5}, 225$

Cornea .................244

\section{V}

Vaccination ..................6 61

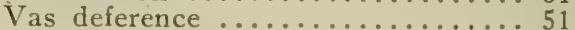

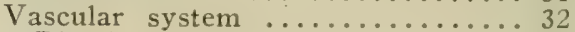

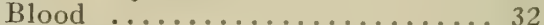

Lymph $\ldots \ldots \ldots \ldots \ldots \ldots \ldots \ldots . . . . . . . .32$

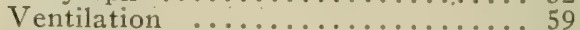

Vertigo .....................277

Vessels, drinking ............ 65

Vibrio metchnikovi ................ 187

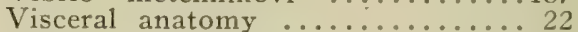

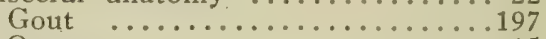

Organs $\ldots \ldots \ldots \ldots \ldots \ldots \ldots . \ldots \ldots 15$

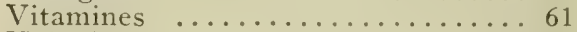

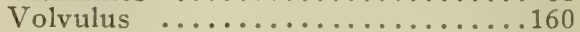

\section{W}

Walls of house.............. 59

Water supply ................ 65

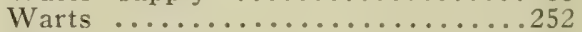

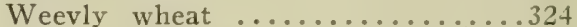

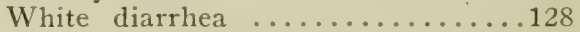

Bacillary ........173

Chicks ...........173

Adults ...........176

Scale Coccidian ..................

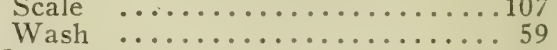

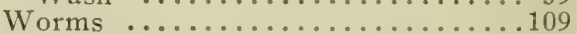

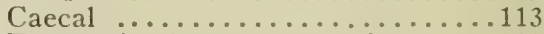

Economic importance of........109

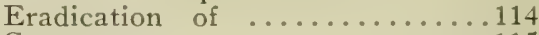

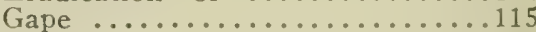

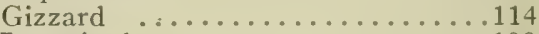

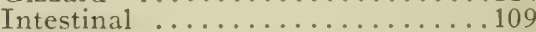

Round ................................ 110

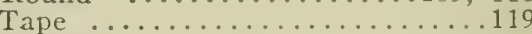

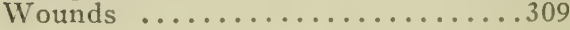

\section{$\mathrm{Y}$}

Yardage system ............ 56 

Printed by CRAFTSMEN PRINTERS

701 South La Salle Street

Chicago 





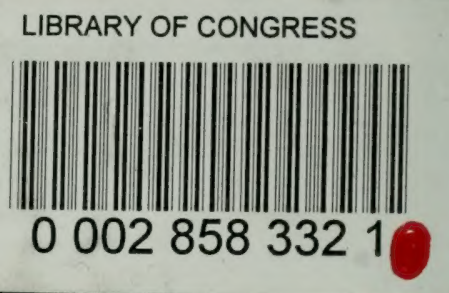

